Walter B. Cannon

\title{
Conférences sur les émotions et l'homéostasie, Paris, 1930
}

Édition, introduction et notes par Mathieu Arminjon

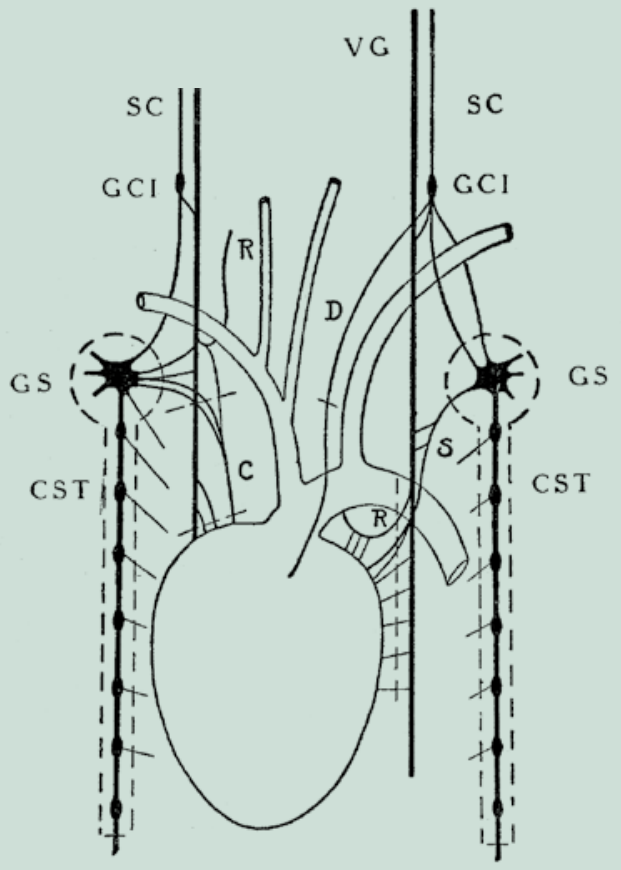


Conférences

sur les émotions

et l'homéostasie,

Paris, I930 
Walter B. Cannon

\section{Conférences sur les émotions et l'homéostasie, Paris, 1930}

L'Institut des humanités en médecine édite BHMS, Bibliothèque d'Histoire de la Médecine et de la Santé.

BHMS comprend trois collections:

- Bibliothèque d'histoire de la médecine et de la santé

- Sources en perspective

- Hors-série

BHMS publie des travaux, essais et documents relatifs à l'histoire de la médecine et des sciences de la vie, de I'Antiquité à l'époque contemporaine. BHMS entend ainsi

encourager un dialogue interdisciplinaire et transversal entre sciences humaines, médecine et sciences du vivant.

Ses publications, expertisées par des spécialistes internationalement reconnu.e.s, s'adressent aussi bien aux chercheuses et chercheurs, universitaires et professionnel-le·s concerné'e·s, qu'à un public élargi, soucieux d'alimenter sa réflexion sur les questions les plus actuelles à partir d'études et de documents historiques.

Publié avec le soutien du Fonds national suisse de la recherche scientifique.

Les recherches d'archives concernant Walter B. Cannon ont été menées à la Francis A. Countway Library of Medicine, Havard University, Cambridge, Massachusetts.
Édition, introduction et notes par Mathieu Arminjon 
Direction Vincent Barras

Responsable éditoriale Alba Brizzi

Relectrices Valérie Bucheli et Isabelle Sbrissa

Graphisme Eva Rittmeyer

Mise en page Claire Goodyear

Coordination fabrication Alain Berset

Diffusion promotion Médecine \& Hygiène / Georg

Crédits images page 379

Figure de couverture Schéma de l'anatomie des nerfs

cardiaques chez le chat. Les traits interrompus indiquent

les parties de la chaîne sympathique thoracique sectionnées

ou excisées afin de mettre en évidence le rôle de la sécrétion

d'adrénaline au cours des états d'excitation émotive.

ISBN 978-2-940527-12-0

ISSN 1662-2421

C 2020 Éditions BHMS

Éditions BHMS

c/o CHUV

Institut des humanités en médecine

Av. de Provence 82

$\mathrm{CH}-1006$ Lausanne

T. +41213147050

bhms@chuv.ch

www.chuv.ch/bhms
Table des matières

Introduction par Mathieu Arminjon

Homéostasie, stress et société :

Walter B. Cannon aux fondements des

déterminants sociaux de la santé

"Le stress »: mythe $e t$ réalité sociale

Walter B. Cannon: de la digestion aux émotions

L'humain hormonal

Un premier séjour en France

38

La parenthèse de la guerre

Physiopathologie en temps de guerre, physiopathologie en temps de paix

Un second séjour en France

Un nouveau concept pour une "nouvelle physiologie»

Des « Sorbonne Lectures » à La Sagesse du corps: genèse d'un livre

La Sagesse du corps: un destin contrarié

Sagesse, crise et égarement 
Sélectionner les plus résistants ou préserver

la vitalité: de l'hygiénisme aux déterminants

sociaux de la santé

Homéostasie et déterminants biopsychosociaux

de la santé

Établissement du texte

\section{Les émotions fortes et leur influence}

\section{sur l'organisme}

Première conférence

Modifications physiologiques accompagnant

les états de grande excitation

Deuxième conférence

Interprétation des réactions émotives en fonction de leur utilité

Troisième conférence

Mécanismes nerveux de l'expression des émotion

Quatrième conférence

Théories de l'origine des émotions

Cinquième conférence

Troubles émotifs des fonctions de l'organisme

\section{Cinq leçons sur l'homéostasie}

Première conférence

- Homéostasie. La stabilité des organismes;

- Définition de l'homéostasie;

- Importance de la constance du milieu intérieur;

- Mécanismes stabilisant la composition du liquide nourricier;

- Mécanismes correctifs après hémorragie.
Deuxième conférence

- Homéostasie par mise en réserve et par mise en liberté

- La soif et la faim considérées comme moyens d'assurer l'approvisionnement;

Inondation et séparation comme types de mise en réserve;

- Exemples de mise en réserve par inondation.

Troisième conférence

- Homéostasie par la mise en réserve et libération consécutive (suite), et par inondation;

- Exemple de séparation avec comme type les hydrates de carbone;

- Problèmes relatifs aux réserves de protéines, graisse et calcium.7

Quatrième conférence

- Le débordement, processus limitatif

dans l'homéostasie des matériaux

- Homéostasie par augmentation ou réduction de la vitesse des processus continus

- Utilité du système sympathico-surrénal dans le contrôle du pH sanguin et de

la constance de la température.

\section{Cinquième conférence}

Revue des conditions homéostatiques et rôle du système sympathico-adrénal dans leur maintien;

- Physiologie des animaux dont le système sympathique a été enlevé;

Division extérofective et intérofective

du système nerveux

Interprétation des fonctions intérofectives

du système autonome.

\section{Bibliographie}


Le monde actuel est dans un état lamentable. Je pense tous

les jours à mes amis en France, en Angleterre, en Finlande, en Suède et en Belgique, dont les travaux scientifiques sont

profondément perturbés par leurs devoirs militaires [...].

Le cortex cérébral semble avoir été développé dans le seul

but d'être dirigé par les aspects les plus sauvages

du diencéphale.

Walter B. Cannon, lettre à Zénon M. Bacd

du 2 janvier 1940, (Cannon, 1940).

\section{Homéostasie, stress}

et société: Walter B. Cannon

aux fondements des déterminants

sociaux de la santé

Mathieu Arminjon 
Ce projet d'édition a été initié lors d'un séjour de recherche à la Mailman School of Public Health de l'Université de Columbia. Nous tenons à exprimer notre reconnaissance aux différentes institutions qui en ont permis la réalisation: la Fondation Agalma à Genève, grâce au soutien de Christian de Saussure, de Pierre Magistretti et de François Ansermet; l'Institut éthique histoire humanités (IEH2) à Genève, où nous avons reçu l'appui de Samia Hurst et d'Andrea Carlino: I'Institut des humanités en médecine (IHM) à Lausanne, où Vincent Barras a apporté son soutien au projet en mettant notamment à notre disposition les ressources et le personnel de la bibliothèque; la Haute École de santé Vaud à Lausanne, ainsi que le Fonds national suisse pour la recherche (FNS) qui a soutenu la publication de cet ouvrage.

Les recherches d'archives concernant Walter B. Cannon ont été menées à Boston à la Francis A. Countway Library of Medicine de Harvard. Nous avons également consulté le fonds Georges Canguilhem au Centre d'archives de philosophie et d'histoire des sciences de l'École normale supérieure (archives Georges Canguilhem) à Paris. Nous tenons particulièrement à remercier Jon Arrizabalaga (Institución Milà i Fontanals, Barcelone) d'avoir attiré notre attention sur les liens complexes que Cannon a entretenus avec l'unitarisme, ainsi que François Ansermet d'avoir mis à notre disposition un des rares exemplaires de La Sagesse du corps.

Nous remercions chaleureusement toutes celles et ceux qui ont contribué aux recherches de documents d'archives et de ressources bibliographiques, en particulier Jessica B. Murphy de la Francis A. Countway Library of Medicine de Harvard à Boston; Muriel Leclerc de la bibliothèque de l'Université de Genève (UniCMU); Maika Casse, Magdalena Czartoryjska Meier, Roxane Fuschetto et Caroline Hofer de la bibliothèque de l'Institut des humanités en médecine à Lausanne; Anne Bréaud et Saskya Cachemaille de la bibliothèque de la Haute École de santé Vaud à Lausanne.

Nous tenons à exprimer toute notre reconnaissance à Alba Brizzi des Éditions BHMS/Georg pour le travail éditorial qu'elle a réalisé. Nous tenons encore à exprimer notre gratitude envers Valérie Bucheli, Jean-Claude Dupont, Pierre-Nicolas Oberhauser, Vincent Barras, Anne-Frédérique Schläpfer et Marie-Louise. Leurs commentaires et leur soutien nous on été précieux.

\section{«Le stress »: mythe et réalité sociale}

Le 25 mai 1972 se tient au Downstate Medical Center de Brooklyn un symposium célébrant le centième anniversaire de la naissance du physiologiste américain Walter Bradford Cannon (I87I-I945). Sur la photo qui immortalise le moment ${ }^{1}$, prise sur le parvis du centre, on reconnaît, au milieu du premier rang, Bradford Cannon (1907-2005), le fils du physiologiste, professeur de chirurgie à l'École de médecine de Harvard. À sa droite se tient Zénon M. Bacq (1903-1983) ${ }^{2}$. Le physiologiste belge a assisté Walter Cannon de 1929 à I 930 et, comme on le verra, s'est vu confier la traduction française de certains travaux de Cannon. On reconnaît aussi d'anciens élèves et collaborateurs. À la gauche de Bradford, Philip Bard ${ }^{3}$, qui a mené avec Walter Cannon des études sur les chats décortiqués et décérébrés. À la gauche de Bard, on reconnaît Hans Selye ${ }^{4}$,

Brooks/Koizumi/Pinkston 1975, 220.

2 Zénon Bacq reçoit le titre de Docteur en médecine de l'Université libre de Bruxelles en I927. De retour de son séjour de recherche à Harvard (I929I930) il enseigne la physiologie animale à l'Université de Liège. Il y est nommé professeur en I944 puis, en I949, doyen de la Faculté de médecine. Ses travaux portent tout particulièrement sur la transmission chimique de l'influx nerveux.

3 Philip Bard (I898-1977) est un physiologiste américain. Il entre à Harvard en I924 où il travaille sous la direction de Cannon. Il est chargé d'identifier les sections du tronc cérébral nécessaires au phénomène de réaction pseudo-effective. Il obtient son doctorat en I927 et est nommé professeur et directeur du département de physiologie de l'Université Johns Hopkins en I933. Il garde des rapports amicaux avec Cannon et devient le I8 8 ème $^{\text {pré- }}$ sident de l'American Physiological Society de I94I à I946.

4 Hans Selye (1907-1982) est un médecin et endocrinologue d'origine autrichienne. Il étudie la médecine à Prague. Diplômé en I929, Selye commence ses recherches sur le stress à l'Institut de pathologie de Prague. Une bourse de recherche lui permet de séjourner aux États-Unis, à l'Université Johns-Hopkins, ainsi qu’à l'Université McGill, au Canada où il obtient, en I934, un poste d'interne de médecine. En I945, il rejoint l'Université de Montréal où il fonde et dirige l'Institut de médecine et de chirurgie 
le physiologiste canadien d'origine hongroise. Dans la publication issue du symposium, ce dernier admet n'avoir jamais étudié ni collaboré avec Walter Cannon. Cela ne l'empêche pas de considérer celui-ci comme «l'un des plus grands physiologistes non seulement de ce siècle, mais de tous les temps ${ }^{5}$. Bien qu'il s'agisse ici d'introduire les textes des deux cycles de conférences que Walter Cannon délivre en I930 à Paris, il nous paraît utile de nous attarder un moment sur le cas de Selye.

Auteur prolifique, Selye est souvent considéré comme un pionnier de l'étude de la physiologie du "stress", sinon le "créateur du concept biologique du stress ${ }^{6}$. Il a en effet décrit comment certaines hormones sécrétées par le système adrénergique circonscrivent les processus inflammatoires lorsqu'un organisme est confronté à un pathogène. Les agents syntoxiques (corticoïdes), d'une part, limitent l'inflammation en favorisant la tolérance de l'organisme au pathogène. Les agents catatoxiques (stéroïdes), de l'autre, détruisent le pathogène. Sur la base de ces travaux liminaires, Selye en est venu à conceptualiser les trois phases du "syndrome général d'adaptation». Toute confrontation à un pathogène, nous dit Selye, impose un stress à l'organisme hôte. La réaction d'alarme est suivie d'une phase d'adaptation. Si l'exposition est chronique, ou si un dérangement hormonal se produit, l'organisme peut entrer dans une phase d'épuisement (exbaustion). Cette réaction non spécifique - c'est-à-dire commune à l'ensemble des situations susceptibles d'induire un stress - rend l'organisme vulnérable à un vaste ensemble de maladies s'étendant des troubles émotionnels aux maladies cardio-vasculaires.

Selye reconnaît qu’à l'instar de nombreuses autres innovations conceptuelles, son "syndrome général d'adaptation » a mis quelque temps à s'imposer dans la communauté médicale. Pour ce polyglotte qui, selon les sources, parlait couramment

expérimentales. En 1979 il crée l'Institut international du stress. Il est notamment l'auteur de livres sur le stress destinés au grand public et traduits en plusieurs langues dont The Stress of Life (Selye 1956) ou encore Stress Without Distress (Selye 1974).

Selye 1975,89 , notre traduction.

Beer $1977, \mathrm{I} 35$ entre huit et dix langues, un problème d'ordre linguistique se serait posé. À McGill, université montréalaise principalement anglophone, l'emploi de la notion de stress, qui désigne originellement la contrainte physique qu'une force exerce sur un matériau, n'a selon lui pas posé de problème. À l'Université de Montréal, une institution francophone, on se serait accommodé du terme en l'absence de solution alternative. Il en est allé tout autrement en France. Selye rapporte que, durant la première conférence qu'il donne au Collège de France en 1946, il perçoit une certaine agitation au sein de l'auditoire. L'impression est confirmée au terme de sa communication, lorsque la discussion porte principalement sur l'anglicisme retenu. Des alternatives sont évoquées en vain - «agression", "stimulation», «irritation »- mais Selye reste inflexible, persuadé qu'aucune ne rend compte du phénomène. C'est l'usage qui tranchera: Selye note que la «bible de la terminologie française », le Larousse, lui a finalement donné raison en incluant une nouvelle entrée - «le stress » - à son dictionnaire médical. Selye se vante alors d'avoir ajouté un mot à la langue française, une victoire qui aura selon lui très largement contribué à généraliser l'emploi du terme dans bien d'autres pays.

Si l'on en croit cette anecdote, Selye semble avoir intuitivement intégré ce que Canguilhem a analysé dans son étude sur le réflexe: la formation d'un concept scientifique nécessite de forger un terme sous lequel est subsumé un ensemble d'observations. L'acte de dénomination joint aux observations confère aussitôt une unité phénoméno-technique aux observations ${ }^{7}$. Mais pour donner vie à cette existence phénoméno-technique, encore faut-il que le concept se vulgarise. Il doit non seulement devenir un "outil» d'expertise (les troubles associés aux réflexes, tout comme le stress, existent puisque le médecin les traite), mais il doit encore infuser la culture populaire: $«$ tu as de bons réflexes » ou "tu stresses ${ }^{8}$. Le phénomène de laboratoire doit parvenir à dénommer, aussi, une expérience usuelle. Dans le cas de Selye, la langue française semble garantir la traductibilité

\footnotetext{
Canguilhem 1977, I6I.

Canguilhem 1977, I63.
} 
du concept de stress et, avec elle, légitimer l'universalité de l'état somatique et psychologique dénoté.

Mais les remarques que Selye rapporte peuvent surprendre le lecteur contemporain, tout comme elles auraient pu surprendre l'assemblée réunie pour le centenaire de la naissance de Cannon. Si Selye est considéré comme celui à qui l'on doit la "découverte» du stress ${ }^{9}$, il n'a pas fallu attendre les années 1950, ni même la fin des années I930, pour voir apparaître la notion ${ }^{10}$. Il n'a pas fallu non plus attendre que des physiologistes et médecins lui donnent le statut de concept scientifique pour que la notion trouve une place dans le langage courant. Un bref retour sur l'historiographie du stress s'impose ici.

Au tournant des années I980, la notion de stress s'est largement répandue dans la culture. Certains n'ont d'ailleurs pas manqué d'appréhender le concept comme un "mythe social». La thèse selon laquelle le "stress", a fortiori le stress «social", induirait des maladies ne repose, selon Kristian Pollock par exemple, sur aucune base scientifique solide. Quand les études ne sont pas contradictoires, estime-t-il, elles reposent au mieux sur de "vagues associations ${ }^{11}$. "Fait social» plutôt que physiologique, le stress serait avant tout un mode de subjectivation visant «à favoriser une anxiété généralisée et à saper la confiance que les gens ont dans leur santé et dans leur capacité à faire face aux problèmes ordinaires de la vie ${ }^{12}$. Allan Young ${ }^{13}$ soutient de son côté que les recherches portant sur le rôle du stress dans l'étiologie des maladies sont aveugles au caractère historiquement situé (occidental et capitaliste) des catégories sociales qu'elles mobilisent (statuts socio-économique, marital, ethnique, etc.). Ce naturalisme naif, qui présuppose que les individus "expérimentent» le stress comme la véritable cause de leurs maux, contribuerait, là encore, à reproduire et à justifier l'ordre social lui-même.

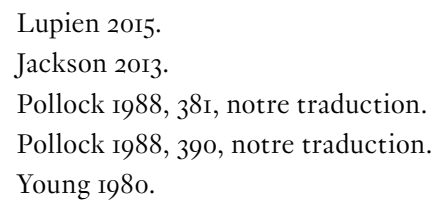

Se dégageant de postures critiques confinant au réductionnisme social, Mark Jackson note, dans The Age of Stress, que les analyses passées ont minimisé la dimension hybride du stress - comme coproduction sociale et biologique -, voire ont cantonné la notion au monde occidental de l'après-guerre, en particulier anglo-saxon. Jackson nous invite au contraire à nous dégager de l'apparente actualité du concept de stress :

L'anxiété face aux pics épidémiques de stress et de tension parmi les populations modernes, l'appréhension de la prolifération de la fatigue et de l'épuisement chez les travailleurs et les soldats, et la crainte que les niveaux croissants de démence et d'instabilité nerveuse ne soient alimentés par des modes de vie sous haute pression, ne se limitent pas [...] au monde occidental, comme le supposaient de nombreux observateurs contemporains. Des études historiques récentes ont révélé que le sentiment de crise ou d'anxiété morbide provoqué par l'àge des machines et exacerbé par la Grande Guerre et l'effondrement subséquent du marché boursier américain a eu des répercussions dans le monde entier: les patients et leurs médecins, ainsi que les employeurs et l'État, étaient également préoccupés par la prolifération de l'épuisement nerveux, la folie et le choc des obus, ainsi que par une augmentation associée des angines de poitrine et autres maladies du cœur, en Grande-Bretagne, en Suède, en Chine, au Japon, aux Pays-Bas, en France, en Allemagne, en Argentine et en Russie. Le stress et la tension mentale et physique provoqués par le choc perçu de la modernité, ou par ce que Wells appelait le «sentiment d'un choc à venir", étaient globaux ${ }^{14}$.

La figure de l'humain stressé ne renvoie donc pas uniquement à la construction d'un individu hypermoderne porté à la fatigue d'être soi. Si la science est une solution aux problèmes politiques, l'ordre du savoir et l'ordre politique doivent alors se penser dans un rapport de coproduction ${ }^{15}$. Dans ce cadre, le stress désigne bien un état physiologique objectivable. Mais cette objectivation même témoigne de l'émergence d'un souci social et intellectuel, du besoin de penser un ordre social alternatif permettant de limiter les effets pathogènes des modes de vie modernes.

À l'évidence, Selye s'attribue la paternité d'une notion de stress, dont on peut pourtant recenser tout au long du XIX ${ }^{e}$

14 Jackson 2013, 24, notre traduction.

15 Jasanoff 2004, 3I 
siècle des occurrences et qui recouvrent déjà, peu ou prou, l'idée d'un inconfort psychologique et somatique occasionné par un ensemble de facteurs: l'insécurité économique, la fatigue au travail, la perte de repères induite par le progrès technique, la peur de la guerre, si ce n'est la terreur du soldat sur le front. Au tournant du $\mathrm{XX}^{\mathrm{e}}$ siècle, la notion de stress était donc bien ancrée dans le lexique populaire anglo-saxon. Et si, au-delà du monde anglo-saxon, on ne disposait pas encore d'un terme unique, on ne s'inquiétait pas moins de voir se généraliser des états similaires que l'on dénommait de manière différente mais connexe: «trouble émotionnel ", " névrose de guerre », « anxiété », etc.

Est-ce à dire que Selye a simplement fourni les bases biologiques et médicales qui manquaient à un concept populaire? Non plus. Et c'est ce qui nous ramène à Walter Cannon. Selye rapporte avoir utilisé le terme pour la première fois en 1935 , dans un article sur les effets d'un "stress sévère " sur l'activité ovarienne de rats ${ }^{16}$. L'article a été soumis pour publication le I3 mai 1935 et publié le 2 décembre de la même année. Walter Cannon a quant à lui usé du terme dans une publication intitulée "Stresses and Strains of Homeostasis ${ }^{17}$, parue dans le Journal of the Medical Sciences quelques mois auparavant, en janvier 1935. Ce n'était d'ailleurs pas la première fois. On le retrouve sous sa plume dans «The Mechanism of Emotional Disturbance of Bodily Functions", un article publié six ans plus tôt, en 1928 . Cannon y parle déjà d'emotional stress ${ }^{18}$, de stress of excitement ou encore de stresses of the war ${ }^{19}$. En d'autres termes, Cannon, comme bien d'autres physiologistes et médecins de la même génération, a déjà eu recours au concept de stress, non plus seulement pour désigner un sentiment psychologique, voire une impression somatique, mais en le reliant à un ensemble de processus hormonaux. Il n'a donc pas fallu attendre Selye pour qu'une toute jeune science biomédicale - l'endocrinologie - transforme une notion vague en un concept scientifique à part entière.

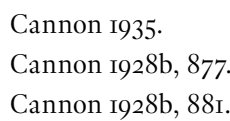

Il ne s'agit pas ici de minimiser le rôle que Selye a pu jouer dans la formation, la stabilisation et la popularisation du concept. Il serait tout aussi absurde de lui dénier la paternité du terme «stress » que de l'attribuer, sans autre forme de procès, à Cannon. Mais il importe de préciser le rôle de Cannon dans la formation du concept et de se demander pourquoi ce n'est pas son nom que l'on associe aujourd'hui au concept de stress, en particulier dans le monde francophone où ses travaux sont peu étudiés. Or ce sont justement des éléments de réponse à ces questions que nous fournissent les deux conférences que nous présentons ici. Elles nous éclairent sur le fait que la contribution de Selye à la formation du concept de stress commence là où celle de Cannon s'arrête et qu'elle n'a pu se déployer que parce que Cannon a posé, avant lui, les bases d'un cadre expérimental et théorique.

Le lecteur pourra s'étonner d'une telle affirmation dès lors que l'on ne relève aucune occurrence du mot "stress " dans les deux séries de conférences. Mais cette absence n'enlève rien au fait que ces conférences représentent une étape dans les cheminements intellectuels, mais aussi culturels et sociaux, qui vont mener inéluctablement au concept. Le lecteur qui parcourt ces conférences a rapidement l'intuition que le stress en est bien l'objet et qu'il se cache à l'intersection des deux thématiques connexes, mais encore indépendantes, qu'elles traitent: les émotions d'une part, l'homéostasie de l'autre. Et si on peut l'affirmer avec certitude, c'est que Cannon aurait pu revendiquer, lui aussi, l'introduction du concept de stress en France, à l'occasion de ses conférences, s'il n'avait pas tenu, à la différence de Selye, à adapter son propos à son public du moment. Comme nous le verrons plus bas, c'est en effet à des contingences de traduction - de l'anglais au français - que l'on doit la disparition du mot stress de ces conférences. Or ces contingences confortent deux des principales propositions évoquées jusqu'ici : celle de Jackson, d'une part, selon qui le concept de stress doit être replacé dans une histoire globale, et celle de Selye, d'autre part, selon qui la France a constitué une étape majeure dans le processus de globalisation du concept. Car c'est bien à Paris que nous ramènent encore ces deux séries de conférences. Nous ne sommes cependant plus avec Selye, au 
Collège de France, en I946. Nous sommes cette fois au premier semestre 1930, à l'Université de Paris: à la Faculté de médecine, où Cannon délivre cinq conférences sur les émotions ainsi qu'à la Faculté des sciences, où il donne cinq conférences sur l'homéostasie.

Dans la première série de conférences, Cannon synthétise les résultats de ses recherches sur les émotions déjà consignés dans un ouvrage, Bodily Changes in Pain, Hunger, Fear and Rage, publié en $1915^{20}$ et profondément remanié en $1922^{21}$. Les cinq conférences de Cannon reprennent l'essentiel des données et analyses de la seconde édition de son ouvrage. Bien que largement commenté par la communauté scientifique française, l'ouvrage ne fut, comme on le verra, jamais traduit en français. Rédigées en français, par Cannon lui-même, ces conférences inédites constituent la seule traduction permettant de s'immerger dans le parcours expérimental et intellectuel de Cannon. On peut y suivre le cheminement par lequel il a été amené à établir une physiologie des émotions, tout en réfléchissant aux implications médicales et sociales de sa conception de la vie affective.

La deuxième série de cinq conférences nous plonge cette fois dans les recherches récentes de Cannon. Si celui-ci n'a pas introduit la notion de stress en France, son séjour parisien l'amène bien, en revanche, à ajouter un mot à la langue française. Dans cette deuxième série de conférences, Cannon progresse dans l'élaboration de sa théorie de la régulation physiologique et introduit le terme d'homéostasie. Or lorsqu'il élabore ses conférences, à l'automne I929, il sait déjà que celles-ci serviront à la composition de ce qui deviendra son ouvrage phare, paru en 1932 sous le titre The Wisdom of the Bod ${ }^{22}$. Comme nous aurons l'occasion de le voir plus en détail, ce sera le seul ouvrage qui bénéficiera, non sans mal, d'une traduction française.

Si les conférences que Cannon délivre à Paris constituent un moment clef dans son itinéraire intellectuel, c'est qu'elles attestent que le physiologiste opère un glissement de la

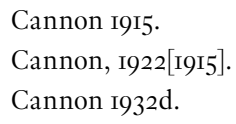

UNIVERSITÉ DE PARIS -- FACULTÉ DE MÉdECINE

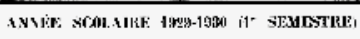

M. WMLTER BRADFORI CAXVoX, prolessenr de Physiologie at PUniversité Ilarvard, lera, à Ptumphilheailre Vulpian, à 16 heures, cinc conférences e'n framais sur'

\section{Les Émotions fortes et leur influence sur l'organisme}

Jeuli, a fetries 1930 - MODIFICATIONS PHYSIOLOGIQUES ACCOMPA GNANT LES ETATS DE GRANIE ÉMOTION (Avec projectionsi.

SuUn EN FONGTION DE LEUR UTILITE.

Harit, $1 /$ fitrie 1130. - MEGANISME NERVEUX DE L'EXPRESSION DES MOTIONS (Avec projections).

Jezti, to ferrier ty30. - THÉORIE DE L'ORIGINE DES EMOTIONS Avec projectionst-

Sinmerti. 15 firrier $19 \% 0$ - TROUBLES EMOTIFS DES FONGTIONS DE

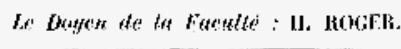

physiologie des émotions à la conceptualisation de l'homéostasie. Il ne s'agit pas d'abandonner une thématique au profit d'une autre. De nombreux éléments issus de ses travaux sur les émotions sont repris dans les conférences sur l'homéostasie. Cannon ne change pas d'objet, mais il élargit sa focale. Car, si le concept d'homéostasie est nouveau, il n'en est pas moins le produit d'un mouvement initié au tournant du $\mathrm{XX}^{\mathrm{e}}$ siècle, par une génération de physiologistes, dont l'ambition est de fonder ce que John Scott Haldane (I860-1936) ${ }^{23}$ nomme une

23 John Scott Haldane est un médecin, physiologiste et philosophe britannique, diplômé de médecine à l'Université d'Édimbourg en I884. En I887, il est engagé comme démonstrateur de physiologie à l'Université d'Oxford. Il y entame des recherches sur les gaz libérés dans les mines durant les coups de grisou. Il met au jour les effets physiologiques du monoxyde de carbone et met au point un hémoglobinomètre permettant de mesurer le taux d'hématies et la quantité d'hémoglobine dans le sang. Ses recherches sur les déficiences en oxygène, menées durant son expédition à Pike's Peak, dans le Colorado, en IgII, l'amènent à étudier l'acclimatation aux hautes altitudes. Durant la Grande Guerre, il étudie les effets des gaz utilisés sur le front par l'armée allemande. Il établit encore des tables de décompression permettant d'éliminer, durant leur remontée, l'azote que les plongeurs emmagasinent à la descente. Auteur d'un important traité sur la respiration (Haldane I922), il consacre ses derniers 
«nouvelle physiologie ${ }^{24}$. Comme ce dernier, ces chercheurs sont convaincus de poursuivre l'œuvre fondatrice de Claude Bernard (I8I3-I878) ${ }^{25}$ et de prendre part au deuxième âge d'or de la physiologie moderne qui durera une quarantaine d'années. Cette nouvelle physiologie, dont Cannon est peut-être le dernier représentant, sera supplantée et invisibilisée, au sortir de la Seconde Guerre mondiale, par l'essor de la génétique et de la biologie moléculaire ${ }^{26}$.

Sous cet angle, les deux séries de conférences sont d'autant plus intelligibles qu'on les replace dans leur contexte d'énonciation. Il est fort possible, et c'est l'hypothèse que nous tenterons de conforter dans les pages qui suivent, que la notion de stress n'ait pu naître que dans cette courte fenêtre temporelle, à la croisée de deux exigences : l'ambition de certains

travaux à la philosophie de la biologie (Haldane 193i). Haldane et Cannon se connaissent bien,tous deux ayant travaillé durant la Grande Guerre pour le Gas Defense Service.

24 Haldane 1929

25 Claude Bernard est un médecin et physiologiste français. Il entame des études de médecine en I834, en tant que préparateur de François Magendie (1782-I855), qui occupe alors la chaire de physiologie expérimentale du Collège de France. Il passe successivement les concours de l'externat et de l'internat en I836 et en I839. Il obtient le titre de Docteur en médecine en I843. Nommé Chevalier de la Légion d'Honneur en I849, il se voit décerner en 1853 un doctorat en sciences naturelles pour sa thèse portant sur la fonction glycogénique du foie. En I854, on crée pour lui à la Sorbonne la chaire de physiologie générale. À la mort de Magendie, en I855, Bernard est nommé professeur de médecine expérimentale au Collège de France, place qu'il cumule avec son enseignement à la Sorbonne. Bernard est élu membre de l'Académie de médecine en I86I. En I868, il est élu à l'Académie française, puis, en I879, Napoléon III le nomme sénateur. Cannon a tout à fait conscience, a fortiori lorsqu'il s'exprime à la Sorbonne, que ses vues sur l'homéostasie s'inscrivent dans la voie ouverte par le père de la physiologie expérimentale. C'est ce qu'il exprime tout particulièrement en I939, dans la préface qu'il rédige spécialement pour la version française de La Sagesse du corps: « C'est pour moi une grande satisfaction de voir mon livre traduit en français, car sa composition est mêlée à des souvenirs intimes et agréables de la France. L'idée centrale de ce livre, "la stabilité du milieu interne de l'organisme chez les vertébrés supérieurs", est directement inspirée des vues précises et de la compréhension profonde de l'éminent physiologiste français Claude Bernard. Ce livre peut même être considéré comme un hommage à sa mémoire » (Cannon 1946[1939], 5). physiologistes, engagés dans le contexte social troublé du début du $\mathrm{XX}^{\mathrm{e}}$ siècle, de placer la physiologie au centre de la médecine moderne et, par conséquent, de repenser le rôle social et politique des physiologistes. Or cette hypothèse n'est pas sans lien avec la question que nous avons soulevée plus haut, relative au fait que c'est Selye, et non Cannon, que l'on associe habituellement à la conceptualisation du stress. Il nous semble important de considérer les raisons pour lesquelles ces conférences représentent une étape dans une série de rendez-vous manqués entre Cannon et la France. Des problèmes relatifs à l'édition des traductions françaises de ses livres, pourtant dérivés de ses conférences, les critiques à l'encontre des extrapolations sociales et politiques de sa théorie de l'homéostasie, et surtout des différences culturelles et sociales entre la France et les États-Unis de l'entre-deux-guerres, ont sans doute porté préjudice à la réception des travaux de ce physiologiste francophile qui, pourtant, n'a jamais cessé d'inscrire ses travaux dans la tradition physiologique française. Il ne fait pour nous aucun doute que les problématiques qui agitent les sciences biomédicales contemporaines ainsi que le champ de la santé publique nous invitent à redécouvrir l'œuvre et la postérité de Cannon. À ce titre, les deux séries de conférences que nous présentons ici constituent une voie d'entrée privilégiée dans la trajectoire intellectuelle de celui que Selye considérait comme l'un des plus grands physiologistes de tous les temps. Mais elles éclairent aussi certains aspects du tournant social que les sciences biomédicales contemporaines sont en train d'opérer. 
Walter B. Cannon:

\section{de la digestion aux émotions}

Walter Bradford Cannon ${ }^{27}$ naît le I9 octobre I87i à la Prairie du Chien, une petite ville du Wisconsin, située à quatre heures de route de Chicago. La ville tient son nom des explorateurs français qui y débarquèrent en $\mathrm{I} 673$, à la recherche d'une route permettant de rejoindre le Canada français et le Mississippi ${ }^{28}$ Son père, Colbert Cannon, travaille pour les chemins de fer. Après avoir été blessé par un cheval, il devient fermier, avant de s'établir à St. Paul, dans l'État du Minnesota, à quelques kilomètres de Minneapolis. Il y est à nouveau employé par la compagnie de chemin de fer locale. Sa mère, Wilma Cannon, décède d'une pneumonie en I88I, quelques semaines après avoir donné naissance à son troisième enfant. Colbert Cannon se remarie avec une amie d'enfance qui assure l'éducation de Walter et de ses trois sœurs, Bernice May, Ida Maude et Jane Laura.

Walter est âgé de quatorze ans lorsque son père le retire de l'école, estimant qu'il est plus utile de le former à un travail. Après deux années passées à la Compagnie des chemins de fer, Walter convainc son père de le laisser retrouver les bancs de l'école. Il rejoint la St. Paul High School en I888. Il s'y passionne pour le darwinisme et pour le débat opposant Thomas Huxley et William Wilberforce. Au grand désarroi de son père, fervent calviniste, il se retire de toute activité religieuse. Il se lie alors d'amitié avec Samuel McChord Crothers (1857-1927), un jeune pasteur diplômé de Harvard. Ancien presbytérien, Crothers se convertit en I 882 à l'unitarisme. Cannon va rester proche de Crothers puisque ce dernier, qui officie à St. Paul de I886 à I894, va prendre les rênes de la First Parish de Cambridge. Sise au I446 de l'avenue du Massachusetts, à Cambridge, l'église unitarienne fait face à l'Université de Harvard et n'est qu'à quelques mètres du domicile que Cannon occupera

27 Pour les éléments biographiques, nous suivons principalement Benison/ Barger/Wolfe I987; Wolfe/Barger/Benison 2000.

28 Le chef du groupe qui occupait les lieux se prénommait Alim, ce qui signifie en amérindien «chien». lorsqu'il sera nommé professeur à l'École de médecine de cette même université.

Il n'est pas inutile de s'attarder sur l'unitarisme. Ce mouvement naît au XVI ${ }^{\mathrm{e}}$ siècle dans l'Europe de l'Ouest, avant de s'implanter en Angleterre à la fin du XVIII ${ }^{\mathrm{e}}$ siècle, puis aux États-Unis, en particulier dans la Nouvelle-Angleterre. Branche libérale et universaliste du christianisme non trinitaire, cette Église est composée de croyants, mais aussi d'agnostiques, voire d'athées. Les actions des adeptes s'ordonnent autour de sept principes, reconnaissant notamment la dignité de toutes les personnes et visant à établir la justice, la paix et la liberté dans le monde. Le quatrième principe, qui revendique «la liberté et la responsabilité de chacun dans la recherche de la vérité et du sens ", est certainement à l'origine de la popularité de cette Église auprès de nombreux savants et intellectuels de la côte Est. L'Église unitarienne voue un culte tout particulier à la recherche de la vérité, qu'elle n'oppose aucunement, bien au contraire, à l'activité scientifique. Walter Cannon ne se réclame pas explicitement de l'unitarisme. Mais on notera que les unitariens refusent généralement toute forme de prosélytisme ${ }^{29}$. Comme on le verra, les écrits et les actions politiques de Cannon, tout au long de sa carrière, reflètent cet éthos humaniste libéral, qui entend construire le bien commun sur les avancées de la science.

En I892, Cannon achève sa scolarité et envisage de poursuivre ses études à l'Université du Minnesota. Son père n'a pas les moyens de lui payer des études ailleurs que dans une université d'État, mais ses professeurs l'encouragent à rejoindre une grande université. Moyennant quelques lettres de recommandation vantant l'excellence de ses résultats, Cannon se voit octroyer une bourse qui lui permet de s'inscrire à l'Université de Harvard. Elle couvre 250 des 372 dollars de budget annuel nécessaires à la vie sur le campus de Harvard. En I892, il réussit les examens complémentaires en trigonométrie, grec, allemand et français, qui lui ouvrent les portes de la prestigieuse

29

On note que la Harvard Square Library - bibliothèque numérique dédiée
aux archives de l'universalisme unitarien - consacre bien une notice bio-
graphique à Walter Cannon (Lescouflair 20I2). Au sujet de Cannon et de l'unitarisme, voir Martínez-Vidal/Arrizabalaga (à paraître). 
institution. Durant les premières années, Cannon y suit divers cours et s'intéresse tout particulièrement à la médecine, à la philosophie et à la psychologie. Il suit assidûment les cours de William James (I842-I9I0) ${ }^{30}$. En I896, Cannon est diplômé summa cum laude de l'Université de Harvard. Entre-temps son choix s'est arrêté: il poursuivra des études de médecine.

En I896, Cannon entre à l'École de médecine de Harvard, située dans le centre de Boston, à l'angle de Boylston et d'Exeter street. Dans son autobiographie, il rapporte que les quatre années consacrées à l'étude de la médecine se sont avérées particulièrement pénibles. Il décrit de longs et ennuyeux aprèsmidi passés à étudier passivement la physiologie, alors que son colocataire, étudiant en droit, participait à de stimulantes séances d'étude de $\operatorname{cas}^{31}$. Son insatisfaction aura deux conséquences. Comme en témoignent ses premières publications, Cannon va militer pour que l'École de médecine réorganise son enseignement afin de mieux articuler savoir théorique et savoir clinique. Il propose que soient fournies :

aux étudiants des données imprimées issues de cas réels. Ces données doivent ensuite être étudiées et analysées par les étudiants, qui devront examiner en détail le diagnostic différentiel, les principes du pronostic et le traitement rationnel approprié au cas. Après avoir étudié le cas, les étudiants participeront à une conférence durant laquelle des problèmes particuliers seront discutés avec l'instructeur. Telles sont les grandes lignes de ce plan d'étude ${ }^{32}$.

L'École de médecine adoptera effectivement ce plan d'étude. De 1924 à 1935, un proche de Cannon, Richard Clarke Cabot

William James (I842-I9I0), philosophe et psychologue américain, a étudié et enseigné la physiologie à l'École de médecine de Harvard, avant de devenir en I876, professeur de psychologie puis, en I88I, de philosophie. Rattaché au département d'anatomie, James a notamment étudié les effets de la stimulation électrique sur le cortex. Dans son autobiographie, Cannon rapporte que James a contribué à son orientation. Le philosophe, à qui il aurait exposé son envie de s'orienter vers l'étude de la philosophie, lui aurait répondu de ne pas le faire au risque de n'avoir que le "vent d'est pour se remplir le ventre» (Cannon I945, I9, notre traduction).

32 Cannon 1900,33 , notre traduction.
(I868-I939) ${ }^{33}$, parviendra à faire publier certains des cas « clinicopathologiques »étudiés durant ces conférences dans le Boston Medical and Surgical Journal. Dans ses conférences sur les émotions, Cannon se réfère à certains d'entre eux afin d'illustrer son propos (Conférence I/5).

L'intérêt de Cannon pour la clinique se double d'un intérêt pour la recherche fondamentale. Dès i 896 , Cannon propose ses services à Henry Pickering Bowditch (I840-I9II). Ce professeur de physiologie à Harvard, ancien élève de Claude Bernard, cofondateur et premier président de l'American Journal of Physiology, lui propose de mener des recherches sur les mécanismes de déglutition, au moyen d'un tube à rayon $\mathrm{X}$ tout juste acquis par l'école. Jusqu'ici, les recherches les plus récentes, comme celles de Samuel James Meltzer (I85I-I920) ${ }^{34}$, avaient conclu que les liquides et les

33 Richard Clarke Cabot (I868-I939) est un médecin, cardiologue et éthicien américain. Cabot travaille pour le Massachusetts General Hospital de I 898 à 192I. En 1908, il est nommé professeur de médecine clinique à l'Université de Harvard, puis professeur d'éthique sociale en I920. Cabot fréquentait aussi l'église First Parish de Brookline, à quelques encablures de l'Université de Harvard. Il embrassait les principes de l'unitarisme.

34 Samuel James Meltzer est un médecin et physiologiste d'origine russe, spécialiste des mécanismes de déglutition. Il étudie la médecine à l'Université de Berlin. Après l'obtention de son diplôme de médecine en I882, il s'installe aux États-Unis et pratique la médecine privée à New York. Il mène en parallèle des recherches au laboratoire W. H. Welch's du Bellevue Hospital Medical College de New York City, ainsi qu'au laboratoire J. G. Curtis du College of Physicians and Surgeons. En I904, il prend la direction du département de physiologie et de pharmacologie de l'Institut Rockefeller pour la recherche médicale. De I9II à I9I3, il est le cinquième président de l'American Physiological Society. C'est notamment à Meltzer que l'on doit d'avoir montré que l'iris se dilate sous l'effet de l'adrénaline (Meltzer I904). C'est aussi l'hypothèse de Meltzer, selon laquelle les muscles de la bouche suffisent à la déglutition, sans que les muscles péristaltiques n'interviennent, que Cannon teste au moyen des rayons $\mathrm{X}$ lors de ses premières recherches. Cannon a l'occasion de faire une démonstration prouvant le rôle des mouvements péristaltiques en présence de Meltzer, lors de la réunion annuelle de l'American Physiological Association qui se tient à Boston le 28 décembre i896. Meltzer et Cannon vont trouver d'autres occasions de s'opposer, comme sur le rôle hypotenseur ou hypertenseur de l'adrénaline ou sur la cause physiologique des chocs. Pour autant, Meltzer, convaincu de l'apport des recherches de laboratoire vis-à-vis de la clinique, fondateur de la Society for Experimental Biology and Medicine en I903 et de l'American Society for Clinical Investigation en I908, a l'occasion de défendre 
solides se déversent dans l'estomac non pas grâce aux mouvements péristaltiques, mais par la contraction rapide des muscles de la bouche. Cannon et Albert Moser (1870-1903) ${ }^{35}$, un étudiant de seconde année, vont montrer le contraire. Ils vont réaliser la première observation des mouvements péristaltiques de l'estomac d'un chat vivant, à qui ils ont fait avaler divers aliments rendus opaques aux rayons par l'adjonction de nitrate de bismuth. C'est la première fois que le mécanisme de déglutition est observé en "condition normale", c'est-à-dire sans que des dispositifs soient introduits dans le corps des animaux d'expérience, ni que le réflexe de déglutition soit artificiellement induit par stimulation électrique du nerf laryngé supérieur. Les résultats sont publiés dans l'American Journal of Physiolog $y^{36}$.

Bien que l'étude des mécanismes de déglutition ne l'intéresse pas vraiment, ces premières recherches vont grandement orienter la carrière de Cannon. Ce n'est pas pour rien que Cannon, dans son autobiographie, insiste pour remettre au goût du jour le concept de " sérendipité ${ }^{37}$, forgé originellement en I754 par l'homme politique et écrivain anglais Horace Walpole (I7I7-I797) afin de désigner la capacité active du chercheur à faire des découvertes accidentelles. Comme il le rappelle dans la première série de conférences, c'est

les travaux de Cannon. Invité à discuter la conférence que Cannon délivre le 4 mai Igı́ à l'Académie de médecine de New York, portant sur les maladies somatiques ayant pour origine des émotions (Cannon igi6), Meltzer note que «le laboratoire du docteur Cannon possède une influence humanisante [bumanizing] et est animé par un esprit clinique pas moins que ne l'est la personne du docteur Cannon elle-même. Il a été très instructif et agréable d'entendre des faits aussi précis que ceux contenus dans l'allocution, tous étant les résultats de ses propres observations. Il a présenté des faits, et lorsqu'il a présenté des théories, elles concordaient étroitement avec ses faits 》 (Meltzer I9I6, 873, notre traduction).

35 Albert Moser entre à l'École de médecine en I895. Diplômé en I899, il pratique la médecine à Lima, sa ville natale de l'État de l'Ohio. La même année, il s'engage dans l'armée et est envoyé en Chine durant la Révolte des Boxers. Souffrant de tuberculose pulmonaire, il est rapatrié en Igor. Il meurt au Saranac Lake Sanitarium de New York en 1903.
Cannon/Moser I898.

Cannon I945, 68 . bien la bonne fortune qui a amené Cannon à s'intéresser aux émotions:

J'ai commencé à m'intéresser aux effets des états d'excitation émotive sur le fonctionnement de l'organisme en mille huit cent quatrevingt-dix-sept (I897). Je faisais alors ma première année de médecine et j'utilisais la découverte (alors récente) des rayons $\mathrm{X}$ pour l'étude des mouvements de l'estomac et de l'intestin. À mon grand étonnement, le moindre signe de malaise, d'inconfort ou de colère chez les chats que j'observais était aussitôt accompagné d'une abolition totale des mouvements du tractus digestif. On observe les mêmes phénomènes chez le chien et le lapin. Cette inhibition ne s'observe pas exclusivement chez les animaux inférieurs. La même ressemblance qui existe entre les manifestations superficielles des émotions chez le chien et chez l'homme se retrouve pour leurs manifestations internes. (Conférence I/I)

Cannon constate qu'il ne peut étudier le phénomène de la déglutition chez les divers animaux d'expérience qu'à la condition que ceux-ci bénéficient d'un calme absolu. Sans cela, le mécanisme étudié cesse. Or Cannon va inverser son rapport à ses objets d'étude: l'analyse des processus émotionnels, ceux-là mêmes qui font disparaitre son objet de recherche initial, va devenir l'objet principal de ses recherches futures. De processus parasites interférant avec les fonctions biologiques normales qu'il étudie, les états d'excitation émotionnelle vont se changer en phénomènes positifs. Comme on le verra, ce n'est que près d'une vingtaine d'années plus tard qu'il reviendra, lors d'un second renversement, sur cette normalité qu'il reconceptualisera dans les termes d'une fonction physiologique homéostatique. À ce stade, il importe de voir dans quelle mesure le premier renversement ne va pouvoir être opérationnalisée qu'à la faveur d'un contexte scientifique précis, celui du développement d'une science nouvelle: l'endocrinologie. Cette dernière va contribuer à transformer les intérêts de Cannon, autant que les recherches de Cannon vont contribuer à définir les contours de cette nouvelle discipline. 


\section{L'humain hormonal}

En juin I905, Ernest Henry Starling (I866-I927) ${ }^{38}$ est invité à délivrer les Croonian Lectures, devant la prestigieuse assemblée du Royal College of Physicians of London ${ }^{39}$. Le 22 juin, Starling décrit les résultats de ses études sur la digestion, menées avec William Bayliss (I860-I924) ${ }^{40}$. Ivan Pavlov (I849-I936) ${ }^{41}$, rap-

38 Ernest Henry Starling est un physiologiste britannique, élu à la Royal Society en I899. Il débute des études au Guy's Hospital de Londres en I882 puis y devient démonstrateur en physiologie en I889. En I899, il est nommé professeur de physiologie à l'University College de Londres. Sterling et Bayliss découvrent la sécrétine, une hormone qui régule, via le pancréas, le taux d'acidité gastrique. Starling est aussi connu pour ses travaux sur la fonction régulatrice du cœur, qui adapte son débit en fonction des besoins (repos, exercice, émotions, etc.), afin de garantir l'apport en oxygène des tissus et d'éliminer les déchets. Sa préparation « cœur-poumon » lui permet de formuler la loi dite de Starling, selon laquelle plus le volume diastolique d'un cœur est grand, plus la force de contraction qui en résulte est grande.

39 Starling I905.

40 William Maddock Bayliss (I860-I924) est un physiologiste britannique, diplômé du Wadham College à Oxford. Bayliss est nommé professeur de physiologie générale à l'University College de Londres en IوI2. Il découvre avec Starling la secrétine et son rôle dans les mouvements péristaltiques de l'intestin. Il met au jour l'effet dit de Bayliss, soit la réponse des cellules musculaires lisses artérielles permettant de réguler le débit sanguin par vasoconstriction des artères.

41 Ivan Petrovich Pavlov est un médecin russe. Il est nommé à la chaire de pharmacologie de l'Académie de chirurgie et de médecine de SaintPétersbourg en I89o. En I895, il est nommé professeur et directeur de l'Institut de médecine expérimentale de Saint-Pétersbourg. Ses travaux sur la fonction gastrique du chien le mènent à mettre au jour les mécanismes de réflexes conditionnels, pour lesquels il reçoit le Prix Nobel de physiologie en I904. Cannon s'est intéressé aux travaux de Pavlov dès le début de sa carrière, lorsqu'il étudie les processus digestifs au moyen des rayons X. En octobre I920, la communauté scientifique américaine apprend que Pavlov, comme beaucoup de scientifiques russes, vit dans une grande pauvreté et souffre de la famine qui sévit en URSS. Cannon participe à des collectes de fonds destinées au physiologiste russe, ainsi qu'à l'organisation de la première visite de Pavlov aux États-Unis. Cannon rapporte qu'à leur arrivée en 1923, Pavlov et son fils, avec qui il voyage, se font voler tout leur argent dans le train reliant New York à Boston. Un don de 800 dollars du Rockefeller Institute permet à Pavlov de poursuivre son voyage. Pavlov revient aux États-Unis en 1929, à l'occasion de l'International Physiological Congress tenu à Boston. Pavlov et Cannon se rencontrent une dernière fois en I935, durant l'International Physiological Congress organisé à Leningrad et à Moscou. pelle-t-il, a réussi, grâce à un dispositif chirurgical complexe, à étudier le processus digestif. Il a notamment pu montrer que le canal digestif est doté de glandes qui s'activent en présence de nourriture, via le système nerveux. Le passage d'un aliment de l'estomac au duodénum, par exemple, active, via le nerf vague et les nerfs splanchniques, un réflexe de sécrétion du suc pancréatique. Il a par ailleurs montré que l'activation de l'un de ces nerfs est invariablement suivie d'une sécrétion de suc gastrique ou pancréatique. Des études ultérieures, menées en France par le physiologiste Émile Wertheimer (I852-I924) et en Russie par Léon Popielski (I866-I920), vont cependant montrer que le mécanisme réflexe se déclenche aussi bien après section du vague ou des splanchniques. Ces travaux laissent penser que la sécrétion de suc gastrique est assurée par des nerfs courant tout au long des parois de l'intestin ou du pancréas. Si Bayliss et Starling vont confirmer que les mouvements péristaltiques de l'intestin sont bien activés par les nerfs des parois du canal alimentaire, il en va tout autrement du réflexe de sécrétion du suc gastrique qui, lui, n'est pas activé par un influx nerveux, mais par voie chimique. L'entrée d'un aliment dans l'intestin induit la sécrétion d'une substance chimique, la sécrétine. Produite par la muqueuse duodénale, celle-ci induit les sécrétions de suc par le pancréas. Dans la conférence qu'il a délivrée deux jours auparavant devant le Royal College of Physicians, le 20 juin, Starling a donné un nom à ces substances qui, comme la sécrétine, ont une fonction similaire aux influx nerveux:

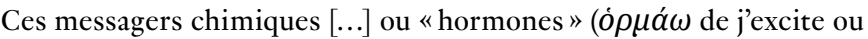
je stimule), comme on peut les appeler, doivent être transportés de l'organe où ils sont produits à celui qu'ils affectent, par le moyen du

Pavlov, qui préside le congrès, et Cannon y délivrent les deux conférences inaugurales. Dans son autobiographie, Cannon (1945, I84) consacre quelques pages à son amitié avec Pavlov, soulignant la proximité de leurs travaux; tous deux s'attachent à explorer les mécanismes par lesquels des processus psychiques influent sur la physiologie. Cette amitié, ainsi que son excellente connaissance de la physiologie russe, conduiront Cannon à accepter, en I943, la proposition émanant de Henry Sigerist (I89I-1957) de présider l'American-Soviet Medical Society. La société se donne comme principale mission de diffuser les travaux de chercheurs soviétiques via sa revue l'American Review of Soriet Medicine. 
flux sanguin, et les besoins physiologiques continuellement renouvelés de l'organisme doivent déterminer leur production réitérée et leur circulation dans le corps ${ }^{42}$

Pour Starling, la mise au jour de ces «messagers chimiques» et leur conceptualisation sous le terme d'« hormone » n'est pas une simple découverte s'ajoutant au savoir physiologique acquis jusque-là. La physiologie hormonale est supposée renouveler non seulement la physiologie, mais aussi, plus fondamentalement, la médecine. Pour Starling, le discours médical s'est toujours appuyé sur «l'hypothèse tacite que les fonctions de l'organisme, qu'il s'agisse de la croissance ou de l'activité des organes, peuvent être contrôlées par des moyens chimiques ${ }^{43}$, et c'est ce présupposé qui a pu justifier l'idée même d'une pharmacopée susceptible d'influencer la restauration de la normalité des fonctions physiologiques au moyen de substances chimiques.

Si la mise au jour de messagers chimiques est perçue comme une étape cruciale dans le développement de la médecine moderne, elle est aussi censée sortir la physiologie d'une impasse. Le XIX ${ }^{e}$ siècle a été marqué par la figure de Claude Bernard ${ }^{44}$ qui annonçait les promesses d'une scientifisation de la médecine passant par l'abandon de l'observation au profit de l'expérimentation physiologique. Or, quelque vingt années après la mort de Bernard, les promesses médicales de cette physiopathologie n'ont pas encore été tenues. Si c'est bien du laboratoire que sont issues certaines des avancées médicales les plus spectaculaires, celles-ci ne proviennent pas des laboratoires

42 Starling I905, 6 , notre traduction.

43 Starling 1905,3 , notre traduction.

44 Dans ses Croonian Lectures, Starling n'identifie pas les grandes figures qui composent son panthéon de la physiologie. Il se montre plus précis à ce sujet lorsqu'il délivre la conférence intitulée "The Wisdom of the Body" dans le cadre de la Harveian Oration: «Et c'est pourquoi aujourd'hui, dans nos esprits et nos souvenirs, nous comptons parmi nos saints non seulement l'illustre lignée des membres du Collège, mais aussi des hommes comme Boyle, Mayow, Hales, Cavendish, Lavoisier, Bernard, Pasteur, et une foule d'autres, qui étaient seulement en esprit nos collègues et dont la plupart n'étaient même pas des étudiants en médecine " (Starling 1923, 685, notre traduction). de physiologie, mais de ceux d'infectiologie. L'avènement de la figure de l'humain hormonal, complétant celle de l'humain nerveux, constitue un enjeu central: Starling entend convaincre l'illustre assemblée qu'un nouvel âge d'or de la physiologie s'annonce, qui réalisera, enfin, le programme de Bernard:

J'espère pouvoir vous faire valoir l'hypothèse qui est à la base de la pratique médicale et montrer que les activités de la grande majorité des organes du corps sont, dans tous les cas, coordonnées entre elles par la production et la circulation de substances chimiques. Les résultats des recherches physiologiques effectuées jusqu'à présent nous confortent dans la conviction que, dans un délai raisonnable, nous serons en possession de substances chimiques qui sont des produits physiologiques normaux et grâce auxquelles nous serons en mesure de contrôler non seulement les activités mais aussi la croissance d'un grand nombre des organes du corps ${ }^{45}$.

Cette physiologie hormonale renouvelle la représentation du corps. La théorie nerveuse rendait compte d'une distribution de l'influx nerveux en divers points du corps, mais pas dans l'ensemble du corps. L'approche hormonale va plus loin en donnant corps à la notion bernardienne de "sécrétion interne ". Ces substances chimiques, sécrétées par les glandes, se déversent dans le "milieu intérieur ", soit dans l'ensemble du corps, via les liquides comme le sang et la lymphe. Ce faisant, elles activent, à distance, un ensemble de processus physiologiques.

Sur quelles bases Starling peut-il fonder, en 1905 , les promesses médicales de cette physiologie hormonale? La découverte de l'insuline dans le courant de l'année I92I, qui vaudra à Frederick Banting (I89I-I94I) et à John Macleod (I876-I935) ${ }^{46}$

45 Starling 1905,3 , notre traduction.

46 John James Richard Macleod est un médecin et physiologiste d'origine écossaise. Diplômé de médecine en I898, il est nommé professeur de physiologie à la Western Reserve University de Cleveland en I903. En 1916, il est nommé à l'Université McGill à Montréal. En I9I8, il est nommé à l'Université de Toronto, où il dirige le laboratoire de physiologie et codirige la Faculté de médecine. Frederick Grant Banting est un médecin canadien diplômé en 1916. Durant la Grande Guerre, il s'engage comme médecin. Il est blessé durant la bataille de Cambrai. En 1922, il est nommé démonstrateur de médecine à l'Université de Toronto. Il s'engage durant la Seconde Guerre mondiale, mais l'avion supposé l'amener au Royaume-Uni 
le Prix Nobel de 1923, représente sans doute la meilleure illustration des débouchés cliniques de la physiologie hormonale. Mais en 1905, nous dit Starling, on ne connaît la nature et les effets exacts que d'un seul de ces messagers chimiques ${ }^{47}$.

L'adrénaline, l'hormone produite par les glandes surrénales, est originellement découverte par George Oliver ( $184 \mathrm{I}^{-}$ I9I5) et Edward Schäfer (I850-I935) de l'University College de Londres ${ }^{48}$. Mais c'est le biochimiste et pharmacologue américain John Abel (1857-1938) qui réalise, en I897, une première extraction grossière de l'hormone qu'il nomme epinephrin ${ }^{49}$. En I90I, le chimiste japonais Jokichi Takamine (I854-I922) réalise une nouvelle extraction ${ }^{50}$. Elle sera brevetée par l'entreprise pharmaceutique américaine Parke, Davis \& Co. sous la marque Adrenalin. C'est pour éviter l'utilisation d'une marque déposée que les milieux médicaux et pharmaceutiques étatsuniens prendront l'habitude d'utiliser le terme epinephrin, bien que la substance extraite par Abel n'ait jamais eu les propriétés de l'adrénaline. Conscient que cette epinepbrin ne désigne pas la substance active, Schäfer propose de substituer le terme adrenin à celui d'Adrenalin ${ }^{51}$. Au Royaume-Uni, Henry Dale (I875-1968) - futur corécipiendaire du Prix Nobel de médecine obtenu en I936 pour des travaux sur l'acétylcholine -, employé par les Wellcome Physiological Research Laboratories, y ajoute un «e » en I906, afin de se conformer aux usages des chimistes. Bien que l'Adrenalin ne soit pas vendue en Europe, le "a» minuscule et l'ajout d'un «e» doivent surtout permettre aux laboratoires d'éviter tout problème relatif à l'utilisation d'une marque déposée ${ }^{52}$. On notera que sur le tapuscrit de ses conférences, Cannon biffe systématiquement le terme "adrenine» au profit du terme "adrénaline», afin de s'adapter aux usages

s'écrase en I94I. En I923, Macleod et Frederick Banting obtiennent le Prix Nobel de physiologie et de médecine pour leur découverte de l'insuline.

Starling I905, 9 .

Oliver/Schäfer I894.

Abel I898.

Takamine I9oI.

Schäfer 1908 .

Voir à ce sujet Tansey 1995; Aronson 2000. européens. Or c'est très précisément cette substance extraite des surrénales qui va permettre à Cannon d'opérer un déplacement de l'étude des mécanismes physiologiques normaux à celle des facteurs émotionnels susceptibles de perturber le cours normal des fonctions biologiques.

Dans le cadre du développement du département de physiologie de l'École de médecine de Harvard, Bowditch et William Porter (1862-1949) ${ }^{53}$ demandent au président de Harvard, Charles Eliot (I834-1926) ${ }^{54}$, que Cannon soit nommé professeur assistant. Il était jusqu'alors engagé en tant qu'instructeur en physiologie à l'École de médecine de Harvard. Leur souhait sera exaucé en 1902. En 1906, l'École de médecine se trouve dans une situation délicate. Bowditch souffre de la maladie de Parkinson et envisage de démissionner durant l'été. Porter espère lui succéder à la tête du département de physiologie, mais ses relations avec ses collègues et avec la direction sont de plus en plus

William Townsend Porter est un médecin et physiologiste américain. Il obtient son diplôme de médecine à l'Université de médecine de St. Louis en I885. Après des séjours de recherche dans les universités de Berlin, Kiel et Breslau, il exerce la médecine au St. Louis City Hospital et est nommé professeur de physiologie à l'Université de médecine de St. Louis en I888. En I893, Bowditch l'engage à l'École de médecine de Harvard. Porter est chargé de réformer l'enseignement de physiologie, notamment en développant la pratique de l'expérimentation. Parallèlement, il reçoit l'aide d'Eliot afin de mettre sur pied la Harvard Apparatus Company. Cette entreprise à but non lucratif a pour mission d'importer, de développer et d'améliorer du matériel de laboratoire destiné à l'Université de Harvard, ainsi qu’à d'autres universités du pays.

54 Charles William Eliot (I834-I926) est diplômé de Harvard. En I858, il est nommé professeur adjoint de mathématiques et de chimie. Son contrat n'étant pas renouvelé, Eliot hésite à poursuivre une carrière dans l'industrie. Après un séjour en Europe, il devient président de l'Université de Harvard en I869, poste qu'il occupe jusqu'en I909. Eliot lance un vaste programme de réforme de l'université dont les grandes lignes sont énoncées dans un manifeste, «New Education », publié dans l'Atlantic Monthly (Eliot 1869). En accord avec ses convictions unitariennes, Eliot entend former des étudiants capables de surmonter les défis des mutations sociales qui touchent les États-Unis. Un concours d'entrée est créé afin de favoriser la diversité des étudiants. Il est ouvert aux minorités, en particulier afro-américaines. L'enseignement se veut pratique et tourné vers les besoins de l'industrialisation du pays. Les salaires des professeurs sont revus à la hausse afin de garantir un haut niveau de formation. 
difficiles. Une pétition exprimant le mécontentement des étudiants contre les réformes de l'enseignement qu'il a engagées a par ailleurs circulé au sein de l'école. Parallèlement, Cannon se voit offrir la possibilité d'être nommé professeur de physiologie à l'École de médecine de Cornell. Cannon s'empresse de faire part à Bowditch et Eliot qu'il acceptera l'offre si rien n'est fait pour résoudre les problèmes liés au caractère de Porter. De son côté, Porter menace de donner sa démission si la direction du département de physiologie ne lui revient pas. À l'automne 1906, il est décidé que Cannon succédera à Bowditch au poste de professeur de physiologie et à la tête du département, tandis qu'un nouveau poste de professeur de physiologie comparée sera créé pour Porter. Cette décision impactera durablement les relations entre les deux hommes, jusqu'au départ à la retraite de Porter en 1928. Mais, dès 1906, Cannon, qui s'était jusque-là presque exclusivement consacré à réformer l'enseignement de l'École de médecine ainsi qu'à la rédaction d'un manuel de physiologie ${ }^{55}$, va pouvoir reprendre ses recherches.

L'année I9II atteste de ce tournant. Une première publication fait état de ce que Cannon considère comme une avancée significative. S'il était en effet admis qu'un état émotionnel induit un ensemble de réactions typiques d'une activation du sympathique - dilatation des pupilles, inhibition de l'estomac et des intestins, accélération du rythme cardiaque, hérissement des poils - l'implication de l'adrénaline dans l'activation des réactions somatiques en situation de choc n'avait pas encore été mise au jour expérimentalement. Dans une première étude ${ }^{56}$, Cannon montre qu'en réinjectant dans la circulation sanguine d'un chat calme du sang prélevé juste après qu'il a été exposé à des chiens, on réactive son état d'excitation. Fort d'avoir expérimentalement démontré que la sécrétion des surrénales augmente durant la peur, Cannon publie la même année les résultats d'autres études ${ }^{57}$ confortant la thèse selon laquelle

Cannon i9ıo.

Cannon/de la Paz rgir. Daniel de La Paz (?-?) est un chercheur d'origine philippine qui mène ses recherches post-doctorales dans le laboratoire de Cannon. Cannon/Hoskins I9II. Roy Graham Hoskins (I880-I964) est un médecin endocrinologue américain. Diplômé de l'Université du Kansas en des états induisant des réactions de défense - asphyxie, hyperpnée ou stimulation électrique du nerf sciatique - augmentent le taux d'adrénaline dans le sang. Une autre étude atteste du souci constant de Cannon de relier ses travaux expérimentaux à la clinique. Effectivement, dans un troisième article ${ }^{58}$, Cannon et ses collaborateurs rappellent que de nombreux médecins ont pu observer l'apparition de diabète ou de glycosurie faisant suite à de fortes émotions. Les cas mobilisés ne sont pas sans rappeler ceux que Cannon rapporte dans ses conférences sur les émotions: un patient découvrant les infidélités de son épouse, des patients ayant subi des bombardements ou qui ont été marqués par le suicide d'un proche, etc. Cannon démontre le bien-fondé de ces observations cliniques, souvent contestées, en produisant une "glycosurie émotionnelle» expérimentale chez des chats effrayés, état qui disparaît aussitôt après ablation chirurgicale des capsules surrénales. Ce faisant, Cannon s'inscrit de plainpied dans cette révolution hormonale que Starling annonce en 1905. C'est l'ensemble des méthodes de mesure et des procédés chirurgicaux élaborés dans son laboratoire afin de consolider, contre ses détracteurs, la thèse d'une sécrétion d'adrénaline dans les états émotionnels que Cannon expose dans ses deux conférences, en particulier dans celle portant sur la physiologie des émotions. Mais cette médecine hormonale va prendre une dimension toute particulière lorsque Cannon va être amené, comme nombre de ses collègues, à poursuivre ses recherches sur les champs de bataille de la Grande Guerre.

I905, il obtient un Ph. D. de l'Université de Harvard en IgIo. En I920, il est nommé directeur du Département de physiologie de l'Université de l'Ohio. En I927 il retourne à Harvard où il est nommé directeur de la Memorial Foundation for Neuro-Endocrine Research.

58 Cannon/Shohl/Wright I9II. Alfred Theodore Shohl(I889-I946) est un médecin et physiologiste américain diplômé de l'Ecole de médecine de Harvard en I9Io. Spécialiste de la nutrition et du métabolisme, il est nommé professeur associé au Département de pédiatrie de Harvard en I934. Nous n'avons trouvé aucune information concernant W. S. Wright. 


\section{Un premier séjour en France}

Dans sa première conférence sur l'homéostasie, Cannon se réfère aux expériences qu'il a menées à Béthune et à Strasbourg. En effet, lorsque la guerre éclate en Europe, à l'été I9I4, les ÉtatsUnis décident de ne pas prendre part au conflit ${ }^{59}$. Ils conserveront cette neutralité jusqu'en avril I9I7. Mais, dès novembre I9I4, le gouvernement autorise que du personnel médical volontaire provenant d'écoles de médecine américaines soit envoyé en France afin de soutenir les forces alliées. Les équipes de «l'ambulance américaine », dirigées par George Washington Crile (I864-1943) ${ }^{60}$ et Harvey Cushing (I869-1939) ${ }^{61}$, sont basées en France, à Neuilly-sur-Seine, mais elles développent aussi, proches des lignes de front, des Casualty Clearing Stations. Ces centres médicaux militaires sont situés en retrait des lignes de front, hors de portée de l'artillerie ennemie. Les blessés y sont stabilisés avant d'être évacués vers les hôpitaux de campagne.

Cannon, qui s'est joint aux intellectuels opposés à la guerre, décline l'invitation de Cushing à rejoindre l'Europe en I9I5. En I9I6, il rejette à nouveau la proposition du Rockefeller Institute de rejoindre le laboratoire du chirurgien français Alexis Carrel (1873-1944). Installé à Compiègne, Carrel met au point des techniques de prévention des infections chez les soldats ayant

59 Nous suivons ici la chronologie de l'engagement de Cannon durant la Grande Guerre telle que l'a établie Katy Ryan (2018).

60 George Washington Crile est un chirurgien américain diplômé du Wooster Medical College en I887. Il rejoint le St. Alexis Hospital de Cleveland où il réussit la première transfusion sanguine en I9o6. C'est à la demande de Crile que l'Université de Harvard crée les équipes de médecins destinées à rejoindre «l'ambulance américaine ». Il s'engage dans l'armée durant la Grande Guerre où il étudie, en France, les chocs et les empoisonnements au gaz. À son retour aux États-Unis, il crée et préside la clinique de Cleveland.

Harvey Williams Cushing est un neurochirurgien américain, diplômé de l'École de médecine de Harvard en I895. En I902, il est nommé professeur au Johns Hopkins Hospital à Baltimore. En I9I2, il est nommé professeur de chirurgie à l'Université de Harvard et chirurgien en chef au Peter Bent Brigham Hospital à Boston. En I933, Cushing quitte Harvard pour enseigner la neurologie à Yale. Cushing fait partie de la première équipe de médecins de Harvard à rejoindre «l'ambulance américaine». été blessés par des éclats d'obus ${ }^{62}$. Quelques jours après l'entrée en guerre des États-Unis, Cannon fait part à Cushing de son désir de s'engager, mais en tant que chercheur. Le 2I avril I9I7, Cannon est incorporé en tant que premier lieutenant. Après une courte préparation militaire, il embarque en avril sur le S.S. Saxonia. Il débarque en Angleterre d'où il rejoint le British General Hospital $\mathrm{n}_{5}^{\circ}$ à Camier, dans le Pas-de-Calais, à une centaine de kilomètres du front. Du 3I mai au 23 juin I9I7, il effectue quelques allers et retours vers Londres, où il assiste aux réunions de la Royal Society et du Medical Research Committee (MRC). Le centre de recherches sur la tuberculose s'est recentré sur les pathologies de guerre liées aux éclats d'obus ainsi qu'aux gaz de combat. Ne pouvant mener des recherches dans de bonnes conditions, Cannon demande à être réaffecté dans un lieu plus proche du front. Du 23 juin au 23 octobre 19I7, il est affecté à la Casualty Clearing Station $\mathrm{n}^{\circ} 33$, celle de Béthune, située à dix kilomètres du front. Cannon dispose alors des conditions lui permettant de mener des recherches sur les chocs traumatiques des soldats blessés au front. Au même moment, Bayliss lui propose de rejoindre Starling, Dale et Charles Sherrington (I857-I952) ${ }^{63}$ au sein du Special Committee on Shock and Allied Conditions. Le comité se rencontre pour la première fois à Londres le I7 août 19I7. Cannon demande à nouveau une réaffectation afin de tester certaines hypothèses sur des animaux de laboratoire. Il séjourne à Londres d'octobre à février $19 \mathrm{I} 8$, dans le laboratoire de physiologie de Bayliss, à l'University College. Après un séjour

62 Durant la Grande Guerre, l'utilisation massive d'obus pose de réels problèmes aux médecins et chirurgiens. Les blessures par balle sont nettes et circonscrites, contrairement à celles occasionnées par les éclats d'obus. Celles-ci favorisent la gangrène, ainsi que des états de choc (wound shocks) dont les causes sont, au début du conflit, encore incertaines.

63 Charles Scott Sherrington est un médecin et physiologiste britannique. Il participe aux recherches de Friedrich Goltz de I 884 à I 885 , consistant à étudier les effets de l'hémisphérectomie pratiquée sur des chiens. Sherrington est nommé professeur de physiologie à l'Université de Liverpool en I895, avant d'occuper la chaire de physiologie à l'Université d'Oxford. Sherrington et Edgar Adrian (I889-I923) se voient décerner le Prix Nobel de physiologie et de médecine en 1932, pour leurs travaux sur la fonction des neurones. En I9o6, il introduit le terme «synapse» afin de désigner la zone de contact entre deux neurones ou entre un neurone et une cellule (Sherrington I906). 
à Paris fin février I9I8, où il assiste à la Interallied Conference on Gas Warfare, Cannon se voit proposer la direction du Central Medical Department Laboratory basé à Dijon. Mis en place par l'American Expeditionary Force (AEF), le centre est destiné à étudier les chocs, ainsi qu'à enseigner au personnel médical les modalités de prise en charge des soldats blessés. Cette dernière affectation dure du $\mathrm{I}^{\mathrm{er}}$ avril au 25 décembre $19 \mathrm{I} 8$. L'armistice étant signé le II novembre I9I8, Cannon retourne aux ÉtatsUnis le is janvier 1919. Entre-temps, il participe à deux conférences : la British Expeditionary Force (BEF) Conferences on Treatment of Shock, tenue à Boulogne le I5 novembre I9I8, l'Interallied Surgical Conference at the Red Cross Research Society, tenue à Paris les 22 et 23 novembre 1918 .

Par ailleurs, une lettre datée du 2I mai I9I8 nous apprend que Cannon a pris contact avec Charles Richet (I850-I935) ${ }^{64}$, qu'il n'avait pas réussi à rencontrer lors de son premier séjour à Paris en février $1918^{65}$. Richet, alors président de la Société de biologie, l'invite à Paris. Lors de la séance du I9 octobre I9ı6, Cannon délivre à la Société de biologie une conférence intitulée Rapport destiné à servir de base pour une discussion sur le choc traumatique ${ }^{66}$. Il y définit le choc traumatique comme « un état somatique général qui s'observe après les blessures et qui est caractérisé par une pression artérielle basse persistante, un

Charles Richet est un physiologiste français. Élève de Bernard, il est agrégé de physiologie à la Faculté de médecine de Paris en I878 et nommé professeur de physiologie en I887. Nommé à l'Académie de médecine en I 898 , puis à l'Académie des sciences en I9I4, Richet obtient le Prix Nobel de physiologie pour sa découverte de l'anaphylaxie en I9ı2, à partir de laquelle il développe les premières sérothérapies. Ses travaux sur l'anaphylaxie s'inscrivent dans une approche globale des fonctions physiologiques de défense du corps, en particulier des réflexes médullaires et bulbaires qui permettent à l'organisme de résister aux agressions environnementales (température parasites, poisons, etc.). Si Richet est un savant incontournable au début du $\mathrm{XX}^{\mathrm{e}}$ siècle, ses activités dépassent le champ de la physiologie. Il introduit notamment le terme de "métapsychique » afin de désigner l'étude scienti fique des phénomènes paranormaux, tels que la télépathie ou la clairvoyance. Auteur de nombreux ouvrages sur le sujet, dont un Traité de métapsychique (Richet 1922), il préside l'Institut métapsychique international de I930 à I935. Auteur prolifique, il est aussi connu pour ses écrits politiques.

66 Cannon igr8c pouls accéléré, de la pâleur, des sueurs, enfin par une respiration superficielle rapide ${ }^{67}$. Le wound schock, déjà décrit lors de conflits plus anciens, constitue une énigme médicale. Il annonce la mort des soldats ayant subi une hémorragie importante, aussi bien que de certains autres dont les blessures ne semblent pourtant pas fatales à première vue. Il arrive pourtant que ces derniers développent les mêmes symptômes et meurent à leur tour, parfois plusieurs heures après avoir été blessés. Les chocs traumatiques, seconde source de mortalité chez les blessés, après les infections, sont jugés suffisamment préoccupants pour que la Royal Army Medical Corps crée le London Schock Committee, placé sous la présidence de Starling ${ }^{68}$.

Plusieurs théories ${ }^{69}$ ont été avancées afin d'expliquer l'hypotension caractéristique des chocs traumatiques dont, entre autres, celle de Crile, qui évoque un épuisement des centres vasomoteurs, ou de Dale, qui soutient que l'hypotension est l'effet de l'action vasodilatatrice d'une sécrétion interne. L'hypothèse d'une hypersécrétion d'adrénaline induisant un épuisement des surrénales, et donc une sous-stimulation du système cardiovasculaire, est aussi envisagée, tout comme celle d'une libération de substances toxiques par les tissus ou les os des blessés. Quoi qu'il en soit des causes, on imagine aussi des traitements possibles. Bayliss tente l'injection de gomme d'acacia visant à restaurer le volume du sang.

Curieusement, Cannon n'évoque pas encore l'hypothèse d'un choc émotionnel impliquant le système sympathique. Mais, durant leurs expérimentations communes menées à Londres, Cannon et Bayliss remarquent que les chocs expérimentaux entraînent une acidose du sang. Ils ont tôt fait d'y voir la cause de l'hypotension, thèse qui sera infirmée par le constat que l'injection d'acide ne diminue aucunement la pression artérielle. Mais les expériences que Cannon mène durant son séjour en France, relatées dans ses conférences sur l'homéostasie, le conduisent progressivement à établir que l'acidose, soit la baisse

67 Cannon I9I8c, 854 .

68 Voir à ce sujet Geroulanos/Meyers 2018.

69 Pour une vue d'ensemble des théories, voir Fraser 1920. 
du pH du sang, n'est sans doute pas la cause du choc traumatique. Elle est plutôt l'effet d'une réaction en chaîne: à l'abaissement de la pression sanguine succède l'exhémie, soit la stagnation du sang dans les capillaires et non pas dans les veines abdominales, comme on a pu le croire auparavant. Cannon observe par ailleurs que cette stagnation du sang entraîne une hyperglobulie, soit une concentration des globules rouges, qui s'avère, chez les animaux expérimentaux, accélérée par le froid. Cannon établit qu'en deçà d'une pression systolique de 80 millimètres de mercure «la pression du sang passe par un point critique ${ }^{70}$. L'acidose n'étant plus régulée en raison de l'abaissement progressif des réserves alcalines et l'apport d'oxygène n'étant plus assuré en raison de l'abaissement de la tension et de la concentration des globules rouges dans les capillaires, les cellules nerveuses supposées activer le tonus vasoconstricteur sont irrémédiablement lésées. En d'autres termes, ni la blessure, ni à proprement parler l'hémorragie ne sont la cause des décès suivant le traumatisme. Le traumatisme externe induit, au contraire, un déséquilibre au sein des mécanismes compensateurs qui assurent, d'ordinaire, le maintien des paramètres vitaux. Ce n'est donc pas tant le traumatisme externe que l'effondrement progressif des mécanismes de compensation qui cause la mort. Que la cause externe soit seulement l'occasion d'un tel effondrement est attesté par l'imprédictibilité des risques. Certains blessés graves ne développent pas les symptômes du choc traumatique, tandis que d'autres, bien que légèrement blessés, succombent. C'est que l'état de l'organisme - la qualité de son équilibre homéostatique pourrait-on dire - au moment du traumatisme et durant les quelques heures qui le suivent joue un rôle central.

Les études de Cannon et des autres membres du Schock Committee vont permettre d'établir des recommandations. Outre les mesures plus proprement médicales, comme l'édiction des bonnes pratiques du garrot ou le développement de techniques de collecte et de conservation du sang permettant la transfusion sanguine, le comité va surtout insister sur la

70 Cannon I9I8c, 856 prévention de l'hypothermie, qui hâte l'hyperglobulie des capillaires. Le dispositif est simple, mais il va faire drastiquement baisser le taux de mortalité: le soldat blessé doit être immobilisé, si possible au chaud, sur quatre couches de couvertures et recouvert de deux autres couvertures. Un réchaud peut être ajouté sous son brancard. Le blessé doit encore être hydraté, si possible, de boissons chaudes. Il faut pour ainsi dire que l'environnement externe reproduise artificiellement cette "serre chaude ${ }^{71}$ qui, selon Bernard, garantit l'indépendance du milieu intérieur vis-à-vis des vicissitudes cosmiques.

\section{La parenthèse de la guerre}

Dans son autobiographie, Cannon écrit: «Je suis retourné, en I9I9, aux recherches que j’avais abandonnées. L'expérience vécue au cours de l'intervalle de deux ans a été isolée, elle a constitué une sorte de parenthèse dans l'exercice courant de mes responsabilités universitaires. $»^{72}$ À la lecture des conférences, nous pouvons sans doute concéder que la guerre a manifestement mis entre parenthèses la vie académique de Cannon. Mais nous sommes en droit de douter qu'elle ait véritablement interrompu le cours de ses recherches. Mais pour rétablir cette continuité, il importe de se pencher à nouveau sur les travaux de Cannon portant sur les émotions, ainsi que sur leurs implications cliniques. À cet égard, une remarque de Young peut s'avérer particulièrement éclairante:

il pourrait sembler que Cannon a dépathologisé la peur, en transportant ses associations traumatiques dans le domaine de la biologie évolutionnaire et en redéfinissant sa neurophysiologie comme un état de mobilisation [arousal] adaptative. Mais il suffit de suivre Cannon quelques étapes plus loin pour voir que le contraire est vrai ${ }^{73}$.

Revenons en effet quelques années auparavant. Juste avant I9I5, date de l'entrée en guerre des États-Unis, Cannon a fait

71 Bernard $1879,6$.

72 Cannon 1945 , I30, notre traduction.

73 Young $1995,22$. 
paraître un livre, Bodily Changes in Pain, Hunger, Fear and Rage ${ }^{74}$, dans lequel il compile, dans un style semi-grand public, les résultats des recherches menées depuis ses premiers travaux sur la digestion. Nous nous contenterons ici de rappeler les grandes lignes de la théorie des émotions que Cannon y expose, dès lors que les conférences sur les émotions en présentent une synthèse d'autant plus précieuse que l'ouvrage n'a jamais bénéficié, pour des raisons que nous expliciterons plus loin, d'une traduction française.

Cannon insiste avant tout sur la division entre les systèmes nerveux crânial, sympathique et sacral, auxquels il attribue les fonctions respectives de stockage de l'énergie, de mise en place de réactions de défense et, enfin, de gestion des fonctions de préservation de l'espèce. C'est la fonction sympathico-surrénalienne qui y occupe une place centrale, dès lors que ses recherches ont principalement contribué à démontrer que les états d'excitation émotionnelle primitifs - la douleur, la faim, la peur, la rage - s'accompagnent tous d'une sécrétion d'adrénaline par les surrénales. L'hormone a pour effet d'induire, bien plus profondément et durablement que le système nerveux central, un ensemble de réactions physiologiques. L'adrénaline favorise la libération de sucre dans le sang, réduit la fatigue musculaire, contribue à faire affluer le sang de l'abdomen vers les poumons, le cœur et les muscles. Mieux encore, elle favorise la coagulation du sang. L'ensemble de ces effets physiologiques accompagnant les émotions semble converger vers ce que Cannon, suivant une interprétation darwinienne, nomme la réponse "combat ou fuite ${ }^{75}$ (fighting or flight). Darwin (I809I882) s'était bien attaché à mobiliser sa théorie de la sélection naturelle afin d'expliquer l'origine des expressions des émotions comme l'érection des poils, la dilatation des pupilles, la sécrétion de la sueur, l'accélération de la respiration et du pouls et certaines attitudes corporelles. Mais, dans son livre comme dans ses conférences sur les émotions, Cannon n'hésite pas à préciser qu'il n'entend pas rendre compte, comme Darwin, de l'origine

74 Cannon 1915.

75 Cannon I9I5, 2II, notre traduction. des expressions émotionnelles superficielles. Il estime pouvoir expliquer que les états de peur et de rage sont des états physiologiques ayant une fonction adaptative beaucoup plus vitale, celle de préparer le corps à l'action.

Les conférences sur les émotions présentent l'avantage d'intégrer les nouvelles données et expériences que Cannon a ajoutées en 1922, à l'occasion de la seconde édition de Bodily Changes in Pain, Hunger, Fear and Rage ${ }^{76}$. Cette refonte substantielle est importante, car elle vient combler les lacunes de la première édition. Comme l'attestent les références mobilisées dans ses conférences sur les émotions, Cannon n'est de loin pas le seul, dans le courant des années I9I0, à tenter d'élucider l'origine, la nature et les fonctions de la vie émotionnelle. Pourtant, dans la première édition de l'ouvrage, Cannon ne fait que très rarement référence aux théories alternatives. Il ne se donne même pas la peine de confronter sa théorie à celle de son ancien professeur et mentor, James ${ }^{77}$, que nombre de ses contemporains, comme Ralph Barton Perry (I876-1957) ${ }^{78}$, défendent encore. Cette lacune est comblée dans la seconde édition. Nous pouvons nous contenter de résumer les arguments que Cannon y

76 Cannon I922[1915].

77 James est notamment connu pour sa théorie des émotions, qu'il expose pour la première fois en I884. Il écrit: « Si nous nous représentons une forte émotion, et qu'ensuite nous tentons d'abstraire de la conscience que nous en avons toutes les sensations de ses symptômes corporels, nous trouvons qu'il ne reste plus rien. Nulle "étoffe mentale" pour constituer l'émotion; tout ce qui persiste, c'est un état froid et neutre de perception intellectuelle.» (James I9I7[1884], 63) Le nom de James est généralement associé à celui de Carl Lange.

78 Ralph Barton Perry est un philosophe américain. Il étudie la philosophie à Harvard et y devient professeur en I9I3. Figure de proue du néoréalisme, il est l'auteur d'une biographie de William James (Perry 1935), dont il a été l'élève, pour laquelle il obtient le Prix Pulitzer en I936. Perry est l'auteur d'une General Theory of Value dont la troisième partie du chapitre X est consacrée à l'analyse critique des différentes théories relatives aux émotions. Perry est un voisin et ami proche de Cannon. Il lui dédie son livre et Cannon lui dédiera, à son tour, The Wisdom of the Body (Cannon 1939[I932]). Cela n'empêchera pas Perry de discuter la portée des critiques que Cannon adresse à la théorie de James, notamment le fait que les changements viscéraux ne seraient pas assez subtils pour déterminer des émotions spécifiques. Pour Perry, James est le premier à soutenir que les émotions ont toutes 
développe contre la théorie dite "James-Lange » 79 et qu'il synthétise ici dans la quatrième conférence sur les émotions:

On peut résumer comme il suit la théorie de James, à peu près dans les termes employés par son auteur: un objet extérieur excite un organe des sens ou plusieurs; des influx afférents sont envoyés à l'écorce cérébrale et la perception de l'objet en résulte; aussitôt, les influx descendent aux muscles et aux viscères et les modifient de façon complexe; des influx afférents venant de ces organes troublés reviennent au cortex et, quand ils sont perçus, réalisent la transformation de l'objet « simplement perçu » en objet « émotivement senti ». (Conférence I/4)

En d'autres termes, pour James, l'émotion n'est rien d'autre que la perception des changements physiologiques (des muscles et de la peau, aussi bien que des viscères), associée à une représentation. Pour Carl Georg Lange (I834-I900) ${ }^{80}$, l'émo-

des sensations organiques communes et que la rage et la peur peuvent se différencier selon que les changements organiques touchent la propriocep tion ou l'intéroception. En définitive, Perry estime que Cannon contribue à affiner plutôt qu’à saper la théorie de James (Perry 1950[I926], 296).

79 La théorie James-Lange désigne l'approche «périphéraliste » des émotions, pour laquelle ce ne sont pas les émotions qui induisent un ensemble de réactions somatiques, mais les états somatiques qui donnent une coloration affective aux représentations intellectuelles.

80 Carl Georg Lange (I834-I900) est un neurologue et psychiatre danois. En I885, il devient professeur d'anatomie pathologique à l'Université de Copenhague. En I885, il expose dans son livre Om Sindsbevagelser, et Psyko Fysiologisk Studie (Lange 1885) une théorie physiologique des émotions similaire à celle que James avait exposée, un an auparavant, dans la revue Mind (James I884). Lange et James partagent le même présupposé de base: «Supprimez dans la peur les symptômes physiques, rendez le calme au pouls agité, au regard sa fermeté, au teint sa couleur normale, aux mouvements leur rapidité et leur sûreté, à la langue son activité, à la pensée sa clarté, que restera-t-il de la peur? » (Lange I895[I885], I02) Bien que l'on parle de la «théorie James-Lange », il subsiste entre les deux auteurs quelques diffé rences. Là où James assignait à l'ensemble des parties du corps le pouvoir de donner une coloration émotionnelle aux représentations, Lange limite son analyse aux muscles de la vie de relation, aux viscères, ainsi qu'au système vasomoteur. Il estime, par exemple, que les émotions gaies sont induites par des mécanismes de vasodilatation, tandis que les vasoconstrictions causeraient les émotions tristes. Si Cannon se réfère encore positivement à James dans son principal ouvrage sur les émotions (Cannon 1915), il s'en démarque nettement dès 1927, dans un article (Cannon 1927) reprenant l'ensemble des données expérimentales qui semblent, selon lui, infirmer la théorie James-Lange. La présente conférence reprend et approfondit les tion est encore plus étroitement associée aux modifications du système circulatoire.

Selon Cannon, cette théorie de l'origine périphérique de l'émotion n'a pas de fondement positif. Au mieux, elle repose sur des fondements neuro-anatomiques dépassés, infirmés par de nombreuses observations tant expérimentales que cliniques. Premièrement, la séparation chirurgicale des viscères du système nerveux central, notamment chez les chats sympathectomisés, n'abolit aucunement les manifestations habituelles associées aux états émotionnels alors que, selon James ou Lange, ils devraient avoir disparu. Deuxièmement, les modifications viscérales comme l'augmentation de la fréquence du cœur, la contraction des artérioles, la dilatation des bronchioles, l'accroissement du sucre sanguin, l'inhibition de l'activité des glandes digestives ou du péristaltisme gastro-intestinal, la sudation, la décharge d'adrénaline, la dilatation des pupilles et le hérissement des poils sont des manifestations communes à des états aussi distincts que la peur ou la rage. Aussi, conclut Cannon, «les réponses dans les viscères semblent trop uniformes pour offrir un moyen satisfaisant de distinguer entre des émotions si différentes au point de vue subjectif». (Conférence I/4) Troisièmement, le fait que les viscères soient des organes relativement insensibles semble condamner la possibilité d'en faire l'origine des riches sensations émotives. Quatrièmement, les changements viscéraux sont trop lents pour qu'on y voie la source unique des sentiments émotionnels. Cinquièmement, la modification expérimentale de l'état des viscères - par injection d'adrénaline, par exemple - n'induit pas, chez les sujets expérimentaux, d'états émotionnels spécifiques ${ }^{81}$.

arguments exposés dans l'article de 1927. La théorie de "Cannon-Bard», désignée ainsi en référence à l'élève et collaborateur de Cannon, est qualifiée de théorie "centraliste", en opposition à la théorie périphéraliste de James et de Lange.

81 Grand défenseur de la théorie James-Lange, le neurologue Antonio Damasio réévalué chacune des critiques énoncées par Cannon (Damasio 2004). Les ouvrages de Damasio n'ont pas vocation à offrir une vision historique et épistémologique cohérente des travaux des figures historiques qu'ils mobilisent. On ne peut cependant s'empêcher de noter l'ambivalence qu'ils entretiennent vis-à-vis de Cannon, a fortiori dans le présent ouvrage qui 
La conclusion de Cannon laisse entrevoir que sa théorie des émotions est indissociable de sa conception homéostatique $\mathrm{du}$ fonctionnement neurophysiologique - que l'ensemble des transformations viscérales qui accompagnent les états émotionnels n'ont donc pas pour fonction « d'ajouter de la richesse et de la saveur à l'expérience, mais plutôt d'adapter l'économie interne de telle manière que, malgré des changements de circonstances extérieures, le déroulement uniforme de la vie intérieure ne soit pas profondément troublé». (Conférence I/4) Mais elle atteste encore d'une évolution centrale dans l'histoire de la neurologie.

La théorie des émotions de James est fondée sur le modèle cortico-centrique ${ }^{82}$ développé par le neuropsychiatre viennois Theodor Meynert (I833-1892). Sur cette base, James soutient l'hypothèse qu'il n'y a aucune raison de postuler l'existence de centres cérébraux spécifiquement dévolus à la vie affective. Il suffit que l'organisme dispose de voies nerveuses afférentes et efférentes reliant les organes de perception aux cellules corticales pour qu'une émotion soit associée à la perception d'un objet ${ }^{83}$. Avant les recherches du neuroanatomiste améri-

entend insister sur l'étroite interdépendance des travaux de Cannon sur les émotions, d'une part, et sur l'homéostasie, de l'autre. D'un côté, Damasio apporte une contre-critique détaillée des arguments que Cannon soulève contre la théorie jamesienne des émotions. De l'autre, il place le concept d'homéostasie aux fondements de sa théorie neurophysiologique des émotions, de la conscience, voire de la société, puisqu'il n'hésite pas à soutenir, dans des extrapolations proches de celles de Cannon, qu'étudier des institutions politiques et économiques revient à étudier les développements de l'« homéostasie socio-culturelle» (sociocultural homeostasis) (Damasio 2010 43). La lecture de Damasio nourrit l'impression que les travaux de Cannon sur les émotions, d'une part, et sur l'homéostasie, de l'autre, sont dissociables. Une lecture plus intégrative aurait pourtant permis à Damasio de donner, via le concept de stress, une vision moins idyllique de cette homéostasie socio-culturelle. À l'exception de quelques "ratés », c'est à elle que l'on doit, selon Damasio, les plus belles réalisations de la civilisation, comme «les systèmes juridiques, économiques et les organisations politiques, les arts, la médecine et la technologie » (Damasio 2010, 43, notre traduction). C'est justement cette pensée que l'on attribue souvent a Cannon et que nous déconstruirons plus loin, celle de concevoir l'homéostasie, aussi bien biologique que sociale, comme une stabilité harmonieuse. Meynert I888[1884].

83 James I89I, 72 cain James Papez (1883-1958), qui feront du système limbique le «substrat" anatomique des émotions ${ }^{84}$, et bien avant que le cortex insulaire et ses interconnexions étroites avec le thalamus et l'amygdale ne soient identifiés comme jouant également un rôle central dans la vie affective et les régulations homéostatiques, Cannon va défendre l'hypothèse selon laquelle le thalamus représente la base anatomique de la vie émotionnelle. Il peut étayer ses hypothèses sur les travaux de son collaborateur, Bard, qui a développé des méthodes d'ablation de quantités variables de la substance cérébrale. Les deux hommes ont pu observer les comportements des animaux décortiqués (suite à une ablation du cortex) ou décérébrés (ablation du tronc cérébral). Confirmant les thèses jacksoniennes de la hiérarchisation des fonctions cérébrales et les nombreuses observations de dérèglements émotionnels chez les patients atteints de lésions sous-corticales, Bard a pu montrer que la décortication supprime l'inhibition des réactions affectives. Les animaux décortiqués entrent dans des états de "fausse rage " (sham rage) accompagnés de décharges d'influx sympathique. Or les chercheurs notent que ces réactions cessent dès que la moitié postérieure du diencéphale est à son tour retirée. La persistance de ces états, après enlèvement d'une grande partie de la portion dorsale du tronc cérébral, conduit Cannon à avancer que le centre principal de la vie émotionnelle est situé dans l'hypothalamus.

Si l'on s'en tient aux thèses principales exposées dans ses conférences sur les émotions, en particulier celles reprises des deux éditions de Bodily Changes in Pain, Hunger, Fear and Rage, Cannon semble bien avoir contribué, comme l'indique Young, à dépathologiser la peur et, avec elle, les émotions. Comme ses illustres prédécesseurs - Herbert Spencer (I820-1903), Charles Darwin (I809-I882), James - et comme nombre de ses contemporains - Crile, McDougall (I87I-I938) ${ }^{85}$, Georges Dumas (I866-

84 Papez ${ }_{1995}[\mathrm{I} 937]$. Cannon est le premier auteur cité dans un article dans lequel Papez étend, plus qu'il ne les remet en cause, les théories de celui-ci. Pour Papez, les émotions relèvent d'interconnexions entre l'hypothalamus, les noyaux thalamiques antérieurs, le girus cinguli et l'hippocampe.

85 William McDougall est un psychologue d'origine britannique. Il est chargé de cours à l'Université d'Oxford en I904. Il défend l'apport de la physiologie 
1946) ${ }^{86}$, etc. -, Cannon s'inscrit dans une liste déjà longue de penseurs et de physiologistes qui ont œuvré à faire de la vie émotionnelle cette composante essentielle et normale de la vie humaine que la sélection naturelle a donnée en partage à l'ensemble du règne animal. En ce sens, Cannon a bien contribué à dépathologiser les émotions. Ce n'est pas pour autant, comme le note Young, que Cannon rompt avec une tradition de pensée qui, de Platon aux philosophes rationalistes du XVII ${ }^{e}$ siècle, assimile les émotions à des passions de l'âme susceptibles de détourner de la saine raison. Comme en témoignent ses conférences sur les émotions, Cannon se distingue des positions de physiologistes qui, à la manière d'Angelo Mosso (I846-I9I0) ${ }^{87}$, estimaient à peine trente ans plus tôt que la sélection de la peur, au travers de l'évolution, ne peut " excuser une imperfection si grave de l'organisme ${ }^{88}$. La normalité de la vie affective n'en est pas moins étroitement associée, chez Cannon, aux pathologies.

vis-à-vis des sciences psychologiques. Trouvant peu d'écho dans le milieu académique anglais, il quitte Oxford en I920 pour l'Université de Harvard où il reprend la chaire de psychologie anciennement occupée par William James. En I927, il rejoint enfin l'Université de Duke, à Durham, où il développe des travaux sur la parapsychologie. Évolutionniste atypique, qui tente de concilier le darwinisme avec la théorie de l'hérédité des caractères acquis de Lamarck, McDougall écrit une influente introduction à la psychologie sociale (McDougall I908). Il y soutient que l'ensemble des comportements humains trouve sa source dans des instincts innés. Si McDougall adopte les grandes lignes de la théorie des émotions développée par William James, il s'y oppose sur certains points. Selon McDougall, «l'émotion est la part affective d'un processus instinctif » (McDougall I908, VIII, notre traduction). Georges-Alphonse Dumas est un médecin et psychologue français. Élève de Théodule Ribot (I839-I9I6), Dumas devient en I9I2 professeur de psychologie à la Sorbonne, où il succède à Pierre Janet.

87 Angelo Mosso est un médecin et physiologiste italien. Avant d'occuper la chaire de physiologie de l'Université de Turin, il étudie auprès de Carl Ludwig en Allemagne, puis, à Paris, auprès de Jean-Martin Charcot. Il s'in téresse principalement aux rapports entre physiologie et psychologie. Son livre La paura, publié en I884, est traduit en français (Mosso I886[I884]), puis en anglais (Mosso I896[I884]). Bien qu'il y rende un vibrant hommage à Darwin, Mosso ne peut se résoudre à voir dans la peur autre chose qu'une imperfection de l'organisme. Seule une éducation fondée sur la physiologie et visant à forger chez l'enfant une âme robuste et courageuse saurait selon lui contrecarrer les défauts de la nature.

88 Mosso I886[I884], 99
Si ce point n'apparaît pas forcément dans Bodily Changes in Pain, Hunger, Fear and Rage, il ressort clairement des conférences sur les émotions que Cannon délivre à Paris. Pour s'en convaincre, il suffit de suivre les nombreuses vignettes cliniques qu'il expose. Dans celles-ci, les émotions ne sont certes pas considérées comme étant des pathologies en soi, mais comme des sources potentielles de pathologies somatiques et mentales. Ce sont précisément ces pans des travaux de Cannon, particulièrement mis en avant dans ses conférences sur les émotions, qui nous mènent à nous demander sous quelles conditions Cannon participe bien d'une pathologisation des émotions et, par conséquent, ce qui le distingue de ses prédécesseurs. À cet égard, c'est certainement en s'intéressant au contexte dans lequel Cannon construit ses théories que l'on comprend que, plutôt que de pathologiser les émotions en tant que telles, il élabore un modèle physiologique permettant de penser une étiologie émotionnelle. Ceci nous amène à nouveau à douter que la guerre ait constitué une réelle parenthèse dans les recherches de Cannon. Au contraire, Cannon a contribué à populariser l'idée selon laquelle la pathophysiologie traumatique observée en temps de guerre n'est, en définitive, pas fondamentalement différente de celle observée en temps de paix.

\section{Physiopathologie en temps de guerre, physiopathologie en temps de paix}

Les vignettes cliniques que Cannon mobilise dans ses conférences sur les émotions ne sont pas toutes issues des conférences «clinicopathologiques" qu'il a contribué à mettre en place à l'École de médecine de Harvard. L'une d'elles provient d'un article publié par Rollin Woodyatt $(\mathrm{I} 878-\mathrm{I} 953)^{89}$, intitulé « Psychic and Emotional Factors in General

89 Rollin Turner Woodyatt (I878-I953) est un médecin américain. Il étudie la médecine au Rush Medical College, puis travaille au Presbyterian Hospital de Chicago. Il est nommé président de l'Association of American Physicians en 1936. À la suite de la découverte de l'insuline en I92I, il est l'un des premiers médecins américains à préparer ses propres synthèses de l'hormone afin de l'injecter à ses patients diabétiques. 
Diagnosis and Treatment » et paru en I927 dans le Journal of The American Medical Association ${ }^{90}$. Woodyatt y rapporte le cas d'un homme d'affaires de soixante-cinq ans, diabétique, hospitalisé, dont la diète est contrôlée et qui, moyennant des injections d'insuline, présente une urine normale. Un jour, ses analyses d'urine révèlent une augmentation soudaine du taux de sucre. Ni la diète, ni les injections d'insuline n'ayant été modifiées, aucune cause biomédicale ne semble pouvoir expliquer la glycosurie. Pour Woodyatt, l'explication ne fait aucun doute: « Nous avons constaté que le patient avait reçu des nouvelles, ce qui l'avait amené à craindre (à juste titre) que la société dans laquelle il avait été dirigeant pendant une vingtaine d'années avait pris des mesures pour le mettre à la retraite, et c'est sa réaction à cette situation qui a fait baisser sa tolérance. " ${ }^{91}$ Woodyatt vient à nouveau conforter, sur le plan clinique, les données de Cannon sur la glycosurie émotionnelle expérimentalement induite ${ }^{92}$. Woodyatt peut alors conclure que «le pouvoir des émotions de produire des altérations physiques du corps ne semble pas irréel dans ces conditions $"{ }^{93}$. Mais il semble surtout vouloir convaincre son lecteur qu'une telle étiologie émotionnelle ne peut plus être ignorée aujourd'hui. Si les médecins d'avant-guerre avaient de bonnes raisons d'en douter, précise-t-il, cela ne lui semble plus possible en 1927. Non seulement les observations cliniques et expérimentales se sont accumulées, mais encore les médecins ne peuvent ignorer qu'un peu plus d'une dizaine d'année s auparavant, durant la guerre, les hôpitaux ont vu défiler tous ces soldats souffrant de « choc de bombardement " (shell shock) ou « obusite».

Au début de la guerre, les chocs des bombardements (shell shock) sont considérés comme distincts des chocs traumatiques (wound shock) que Cannon a étudiés durant son premier séjour européen. Certains font l'hypothèse que les symptômes hystériques ou psychasthéniques que les soldats développent

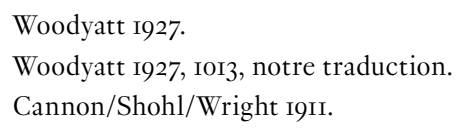

pourraient être dus à une atteinte du système nerveux induite par le souffle des bombes. On note par ailleurs que les soldats atteints ne présentent pas pour autant de blessure précise ${ }^{94}$. Ces chocs viennent s'inscrire dans la catégorie très large des névroses de guerre, recouvrant un ensemble de symptômes comme la neurasthénie, les maux de tête, une extrême fatigabilité, une capacité de concentration limitée ainsi qu'une hypersensibilité aux bruits soudains activant des réflexes de défense exagérés. Mais, comme dans le cas de wound shock, les soldats souffrant d'obusite présentent aussi des symptômes somatiques, notamment une pression artérielle très basse. Dans sa cinquième conférence sur les émotions, Cannon mentionne cette caractéristique sous l'expression «cœur de soldat» (soldier's heart).

Woodyatt n'hésite pas, en 1927, à évacuer une possible origine somatique et à affirmer que la guerre a finalement été l'occasion de confirmer que les névrosés de guerre, même ceux dont les symptômes sont principalement somatiques, souffrent principalement de troubles émotionnels. Or, s'interroge-t-il, comment se fait-il que, malgré l'expérience de la guerre, la médecine persiste, au sortir du conflit, à n'appréhender les maladies que sous un angle somatique, sans considération des facteurs psychiques. Si toutes les maladies ne peuvent être exclusivement attribuées à des facteurs émotionnels, il n'en reste pas moins que certaines le peuvent et que, quelle que soit leur étiologie, tous les malades ont une vie affective susceptible d'interagir avec leur maladie. En définitive, Woodyatt tente de rallier le corps médical à une position encore loin d'être dominante; il n'y a selon lui pas de raison que la médecine en temps de paix soit différente de celle que l'on pratiquait en temps de guerre. Or, si nous suivons la trajectoire intellectuelle et expérimentale de Cannon, depuis son retour de la guerre jusqu'à ses derniers travaux, en passant bien évidemment par ses conférences parisiennes, nous avons toutes les raisons de penser que la guerre n'était définitivement pas une parenthèse pour lui. Au contraire Cannon a été le principal promoteur d'une continuité entre la

94 Hurst, I9I7. 
pathophysiologie en temps de guerre et la pathophysiologie en temps de paix.

C'est en effet ce dont témoignent les conférences sur les émotions. La «glycosurie émotionnelle» chez le patient qui découvre les infidélités de son épouse ou chez la femme qui aperçoit son mari au bras d'une inconnue n'est pas différente de celle des soldats revenant du front. Tous ces cas illustrent une sensibilisation du "contrôle sympathique du cœur». D'autres pathologies permettent d'établir une continuité entre médecine en temps de guerre et médecine en temps de paix. Cannon cite notamment Gregorio Marañon (I887-I960) ${ }^{95}$, qui a rapporté plusieurs cas d'hyperthyroïdisme chez les soldats revenus du front. Ces derniers ne sont pas sans rappeler celui de cette femme qui développe un goitre ainsi qu'un syndrome métabolique après avoir assisté au suicide de son mari. On notera que c'est dans les passages des conférences sur les émotions où Cannon égraine les cas de pathologies induites par des états émotionnels, tous rapportés par ses confrères ou tirés de la littérature populaire, qu'émerge une question toute spéciale: se pourrait-il qu'une vive excitation émotionnelle suffise, via une surexcitation du système sympathico-surrénal,

Gregorio Marañon est un endocrinologue et écrivain espagnol. Il étudie la médecine à la Faculté de l'Hôpital San Carlos à Madrid, où il est l'élève de Santiago Ramón y Cajal. Sa rencontre avec Freud en igıo l'oriente vers l'étude de la sexualité (Martín-Moruño, 20I3). Il est nommé professeur d'endocrinologie à l'Université Complutense de Madrid en 193I. Marañon rap porte avoir injecté de l'adrénaline à de nombreux sujets humains. Ils se sont sentis « comme s'ils» étaient effrayés ou émus, mais faisaient une claire distinction entre les états somatiques périphériques, induits par l'adrénaline, et l'état psychologique perçu. L'état somatique n'a induit des sentiments de chagrin que chez les sujets émotifs, les patients hyperthyroïdiens, ou lorsque l'adrénaline était injectée juste après que les sujets avaient évoqué des souvenirs tristes relatifs à leurs enfants malades ou à leurs parents décédés. On notera encore que Marañon était un fervent républicain. Ses critiques contre la dictature de Miguel Primo de Rivera (I870 - I930) le conduisent à faire un mois de prison. Il soutient la Seconde République espagnole, avant de se distancier des communistes. De I936 à I942, il fuit la dictature de Franco et s'installe en Argentine, puis à Paris. Il publie alors des «biographies biologiques» visant à décrire le comportement de personnalités historiques en fonction de leur profil endocrinien. Cannon et Marañon se connaissent bien. à induire la mort ? ${ }^{96}$ Dans ses conférences sur les émotions, Cannon répond par la négative: «Il est douteux, écrit-il, que la mort n'ait jamais été la conséquence d'une grande excitation émotive, sauf en présence d'une faiblesse organique du cœur ou des vaisseaux sanguins. » (Conférence I/5) Or la question n'a jamais cessé de préoccuper Cannon. Il y a une bonne raison à cela.

La possibilité que de vives émotions puissent causer des maladies, a fortiori la mort, entre en contradiction avec les présupposés darwinistes de Cannon. C'est précisément le paradoxe que relève Piéron (I88I-I964) ${ }^{97}$, lors du symposium international qui se tient au Wittenberg College de Springfield du

96 On ne peut s'empêcher ici de relever quelques similarités entre le questionnement de Cannon et celui que Freud développe dans Au-delà du principe de plaisir. Freud y pointe du doigt un paradoxe: le principe de plaisir - tendance de l'appareil psychique à maintenir aussi bas que possible sa quantité d'excitation - semble contredit par l'existence des «névroses de guerre», qui, comme les «névroses traumatiques du temps de paix (Freud 198I[1923], 49), attestent que le psychisme peut entretenir, par la répétition, la réminiscence de souvenirs déplaisants. S'interrogeant sur le sens d'une telle fonction psychique qui irait à rebours de l'instinct de conservation, Freud mobilise des travaux de biologistes afin de déterminer dans quelle mesure il est légitime d'attribuer au vivant un «instinct de mort». C'est au cours de ce raisonnement que Freud en vient à exposer une conception du vivant qui n'est pas sans rappeler la théorie homéostatique de Cannon: «Une pulsion serait une poussée inhérente à l'organisme vivant vers le rétablissement d'un état antérieur que cet être vivant a dû abandonner sous l'influence perturbatrice de forces extérieures; elle serait une sorte d'élasticité organique.» (Freud I98I[I923], 80) C'est en poussant le raisonnement jusqu'au bout que Freud en vient à extrapoler que si le vivant tend à restaurer un état d'excitation minimum, alors le retour à l'état inanimé est le but de la vie. Il conclut alors que le vivant contient en lui-même la nécessité interne de la mort. Il est tout à fait frappant qu'une réflexion sur l'angoisse, que Freud définit dans $A u$-delà du principe de plaisir comme «un état caractérisé par l'attente du danger et la préparation à celui-ci» (Freud I98I[1923], 50), alimentée par les apports de la physiologie et des connaissances acquises sur les névroses de guerre, mène les deux auteurs à remettre en cause la thèse selon laquelle la mort survient en raison de causes exogènes (des lésions causées par un événement externe), mais pourrait être due à des causes endogènes.

97 Piéron 1928. Henri Piéron est un psychologue français. En I9I2, il prend la succession de Binet à la direction du laboratoire de psychologie physiologique de la Sorbonne. Il occupe, dès I930, la chaire de physiologie des sensations au Collège de France. 
I9 au 23 octobre 1927 et auquel participe Cannon ${ }^{98}$. Piéron s'y montre particulièrement sceptique vis-à-vis des explications darwinistes relatives à l'utilité supposée des expressions émotionnelles faciales : «Lorsqu'on lit Darwin, on sourit de la puérilité de son explication détaillée et utilitariste de l'expression des émotions. ${ }^{99} \mathrm{Il}$ ajoute :

Les biais finalistes ont conduit à une interprétation de ces réactions sécrétoires, circulatoires et viscérales comme étant des éléments utiles dans l'activité de défense. C'est le cas de la conception de Cannon, dont Watson a tiré l'idée de l'émotion définie comme «instinct viscéral et glandulaire». Mais, quand on voit que ces réactions végétatives sont parfois mortelles, qu'elles paralysent généralement les mécanismes de défense et qu'elles conduisent à des états pathologiques souvent graves, on devient sceptique quant à la valeur des interprétations finalistes ${ }^{100}$.

Effectivement, si les émotions sont issues de la sélection naturelle, elles devraient favoriser la survie des organismes. Pourquoi l'évolution aurait-elle alors sélectionné des fonctions physiologiques susceptibles d'induire des maladies, voire la mort des organismes qui les possèdent?

Cannon relaye ce paradoxe dans ses conférences sur les émotions. Et c'est finalement sa théorie du stress, formée à l'interface de ses travaux sur les émotions, d'une part, et de ceux sur l'homéostasie ${ }^{101}$, de l'autre, qui lui permettra de dépasser cette contradiction. Ce sont bien les mécanismes homéostatiques qui, bien que sélectionnés, peuvent, dans des conditions de stress, causer des maladies, voire la mort de l'organisme. Mais c'est du côté de l'anthropologie que Cannon va trouver les moyens de reconsidérer définitivement l'hypothèse d'une cause sium de nombreuses figures internationales dont certaines sont citées par Cannon dans ses conférences (Bekhterev, Janet, McDougall, Stratton, Woodworth). Cannon participe lui aussi au symposium, avec une interven tion intitulée «Neural Organization for Emotional Expression» (Cannon I928a).

99 Piéron, 1928, 290, notre traduction.

100 Piéron, I928, 290, notre traduction.

101 Cannon, 1935 émotionnelle de la mort, à laquelle il donne encore une réponse négative dans ses conférences parisiennes.

Trois ans avant sa disparition, Cannon publie dans la revue American Antbropologist un article intitulé "Voodoo Death» 102 (La Mort vaudoue). Cannon revient sur les nombreux témoignages relatifs à des cas de morts suspectes, que des anthropologues ${ }^{103}$ auraient principalement observés dans des tribus aborigènes du Brésil, de l'Afrique, de la Nouvelle-Zélande et de l'Australie. Il note que, dans l'ensemble des cas rapportés, les victimes ont été préalablement mises à l'index par les membres de leur groupe. Ces parias, rapporte Cannon, se retrouvant « totalement privés de la confiance et du soutien social de la tribu ${ }^{104}$, cessent alors de s'alimenter et meurent en proie à de grandes terreurs, persuadés d'être poursuivis par des esprits malins.

Cannon ajoute que les recherches sur les chocs et les fonctions des systèmes sympathique et sympathico-surrénalien peuvent fournir des explications scientifiques à ces phénomènes. Les animaux décortiqués, en état de fausse fureur durable, montrent les mêmes symptômes que les soldats souffrant de choc (wound schock). Dans les deux cas, l'accélération du rythme cardiaque, l'augmentation de la pression artérielle et la glycosurie laissent progressivement place à une hypotension artérielle due à une réduction du volume du sang circulant. Létat de «stress émotionnel », induit par une « terreur évidente

102 Cannon, I942.

103 Il correspond notamment avec Lévy-Bruhl (I857-I939), qui lui confirme dans sa lettre du 26 octobre I924 (Lévy-Bruhl I934) avoir connaissance de cas de morts vaudoues: «Les ethnologues se fondant sur un grand nombre de témoignages parfaitement indépendants les uns des autres, et recueillis dans des tribus de toutes les parties du monde, admettent que dans certains cas bien nets, un indigène qui se croit ensorcelé et par conséquent condamné (doomed) sans remède, meurt effectivement au bout de quelque temps. Au contraire, les physiologistes et les médecins - qui n'ont aucune expérience dans les faits ethnologiques - tendent à considérer la chose comme impossible et mettent en doute les témoignages les plus formels.» Cependant, Lévy-Bruhl note que le fait que les indigènes se croyant ensorcelés cessent de s'alimenter et de s'hydrater doit en partie expliquer leur décès.

104 Cannon 1942 , I75, notre traduction. 
ou refoulée [repressed] ${ }^{105}$ entraîne un cercle vicieux. La sécrétion de diverses hormones, dont l'adrénaline, qui doit contribuer à maintenir le débit sanguin dans les organes comme le cœur et le cerveau, induit une constriction des capillaires, qui favorise à son tour une perte de plasma sanguin dans l'espace périvasculaire. Le volume du sang circulant se réduisant, les organes vitaux souffrent d'un défaut d'oxygénation qui, à son tour, provoque une sécrétion d'adrénaline. Ce sont finalement les mécanismes de rétroaction, censés pourtant protéger l'organisme, qui entraînent la mort. Cannon remarque enfin que, durant la guerre, des cas de mort ont été rapportés, « dans lesquels ni le traumatisme ni aucun des facteurs de choc connus ne pouvaient expliquer la situation désastreuse ${ }^{106}$. En d'autres termes, Cannon semble revenir sur l'étiologie des chocs traumatiques qu'il avait lui-même contribué à élaborer durant la Grande Guerre, alors même que l'idée d'une cause émotionnelle, médiée par le système sympathico-surrénal, n'avait pas été envisagée à l'époque. La distinction entre les deux types de chocs traumatiques - wound schock et shell schock - s'évanouit, et avec elle l'opposition entre une médecine en temps de guerre et une médecine en temps de paix.

C'est ce revirement que Cannon est en train d'opérer durant les conférences parisiennes. Il atteste non seulement que l'expérience de la guerre n'a aucunement été une parenthèse dans le parcours intellectuel de Cannon, mais encore que, comme l'a bien noté Young, en inscrivant la vie émotionnelle dans la nature physiologique commune aux humains et aux animaux, Cannon n'a pas dépathologisé les émotions. Mais doit-on pour autant en déduire, à la manière de Young, que Cannon s'est finalement contenté de poursuivre la pathologisation des émotions en «biologisant» les névroses traumatiques?

Il ne fait aucun doute que les cas que Cannon rapporte l'inscrivent dans un style de pensée typique de la première moitié du XX $\mathrm{X}^{\mathrm{e}}$ siècle qui, à la manière de Sigmund Freud (I8561939), n'a pas attendu l'apparition des névroses de guerre pour

105 Cannon I942, I80, notre traduction, nous soulignons.

106 Cannon 1942 , I79, notre traduction. thématiser la possibilité que des patients souffrent de souvenirs traumatiques. Les dernières lignes de la cinquième conférence sur les émotions sont éloquentes. Cannon y exprime le danger que représente la recherche systématique de causes anatomopathologiques en réponse aux plaintes des patients, a fortiori lorsque celles-ci s'étayent sur des symptômes somatiques. Pour des raisons de prudence, précise-t-il, «il ne faut recourir aux agents émotifs pour l'explication des troubles de l'organisme qu'en dernier ressort, et seulement lorsqu'on n'a pas pu trouver, malgré tous ses efforts, une autre explication ». (Conférence $\mathrm{I} / 5$ ) Mais la recherche d'une telle "altération structurale " du système nerveux ne doit pas prendre le dessus sur la recherche d'une "altération fonctionnelle i imprimée sur le système nerveux par de fortes réactions émotionnelles. Dans ces derniers cas, "quand la cause des perturbations n'apparaît pas, on peut parfois la trouver par une enquête soignée ou par l'analyse». (Conférence I/5) Et Cannon de préciser enfin «qu'une explication complète de la façon dans laquelle le trouble a été causé suffit souvent à supprimer ce trouble rapidement et complètement ". (Conférence I/5) Il ne fait aucun doute que Cannon se réfère ici à la psychanalyse, en voie de popularisation aux États-Unis ${ }^{107}$.

Mais cette référence ne doit pas laisser penser que Cannon n'appréhende les troubles émotionnels que sous l'angle de parcours de vie idiosyncratiques, susceptibles d'expliquer, à la manière de la clinique psychanalytique, l'étiologie symbolico-émotionnelle de certaines pathologies. Les soldats traumatisés au front, l'homme de soixante-cinq ans poussé à la retraite, ou plus évidemment encore les victimes de mort vaudoue, mises à l'index et privées de «soutien social ", laissent transparaître une réflexion étiologique plus large. L'origine du traumatisme ne s'épuise ni dans le souvenir, ni dans la neurophysiologie du système adrénergique, ni à proprement parler dans l'événement spécifique (bombardement, accident, scène traumatisante). C'est le contexte social qui, en soi, devient la cause du

En 1909, Granville Stanley Hall (I846-1924) invite Freud à délivrer des conférences sur la psychanalyse à la Clark University. Dans la foulée, ces conférences, connues aujourd'hui sous le titre "Cinq leccons de psychanalyse" (Freud I923), sont publiées dans l'American Journal of Psychology (Freud I9Io). 
traumatisme. Pour Cannon, la mort vaudoue n'est pas qu'une curiosité ethnologique. Elle représente la forme épurée de l'étiologie sociale des maladies, qu'il a œuvré à appréhender et à expliquer. La vie émotionnelle peut d'autant plus provoquer la mort dans ces cultures dites primitives où «l'environnement social, en tant que soutien moral, est probablement beaucoup plus important et impressionnant [...] en raison de leur profonde ignorance et de l'insécurité qu'inspire un monde peuplé de fantômes ${ }^{108}$. Mais l'expérience est transposable: tout «milieu extérieur» n'est pas propice au bon développement du «milieu intérieur». Aussi l'idée d'une normalité physiologique, dont les réactions émotionnelles, héritées de la sélection naturelle, s'avèrent naturellement « adaptatives », ne conduit-elle pas à proprement parler à pathologiser les émotions, mais à pathologiser certains environnements sociaux. Les pathologies émotionnelles ne relèvent pas tant de faiblesses individuelles que de conditions sociales anormales. Mais surtout, les conditions sociales en temps de paix ne sont pas moins pathogènes que celles qui prévalent en temps de guerre.

Cannon et Woodyatt ne reconfigurent pas à eux seuls la clinique et la physiologie. Ils s'inscrivent dans un contexte plus général, un réseau d'acteurs décidés à faire de la médecine une science sociale. On a vu qu'une grande partie des vignettes cliniques mobilisées par Cannon sont tirées des conférences « clinico-pathologiques" dont il a eu l'idée et que son ami Cabot a mises en place dans le cadre des réformes des plans d'étude de l'École de médecine de Harvard. C'est ce même Cabot qui, dans son projet de développement d'une médecine dévolue aux populations les plus démunies, au Massachusetts General Hospital, va engager des assistants sociaux [social workers] qu'il payera de sa poche pendant plusieurs années, le temps de convaincre la direction de l'hôpital de pérenniser un « service social ». Créé en 1905 , le service est le premier du genre aux États-Unis. Dès I907, il comptera parmi ses social workers Ida Maud Cannon (I877I960), la sœur cadette de Walter Cannon, qui en prendra la tête en I908. D'autres vignettes cliniques citées par Cannon sont tirées d'articles de Charles Phillips Emerson (I872-I938) ${ }^{109}$, à qui l'on doit aussi, dès I90I, l'ouverture d'un centre social à l'École de médecine de Johns Hopkins. Pour Emerson, il s'agissait de développer, à côté des services de chimie clinique ou de psychologie clinique déjà existants, un service de medical sociology. Par «sociologie médicale», précise Emerson, «nous voulons dire l'application des outils de la sociologie dans la résolution des problèmes rencontrés au chevet du malade ${ }^{110}$. Anticipant sur ce que Robert Straus ${ }^{111}$ appellera une "sociologie dans la médecine ", distincte d'une sociologie de la médecine, les sociologues engagés par Emerson ont pour tâche de se documenter sur la vie des patients, afin de replacer leurs problèmes médicaux dans leurs contextes environnementaux et sociaux. Emerson met ses équipes en quête des événements, dans le milieu familial ou professionnel, qui coïncident avec l'apparition de troubles somatiques et, selon lui, les expliquent ${ }^{112}$.

En définitive, la fusion des wound schock et des shell schock en une seule et même catégorie clinique, comme le développement parallèle d'une pathophysiologie émotionnelle et d'une prise en compte progressive des facteurs sociaux dans l'étiologie des maladies, doivent attirer notre attention sur le contexte dans lequel s'est développée la médecine dans les États-Unis de l'après-guerre. Car, s'ils n'ont été qu'indirectement impliqués dans les deux conflits majeurs qui se sont principalement déroulés en Europe, les États-Unis n'en ont pas moins été touchés par une crise d'importance, qui a eu pour effet de révéler

Charles Phillips Emerson (I872-1938) est un médecin américain. Diplômé de médecine de l'Université Johns Hopkins en I899, il devient en IgıI le second doyen de l'École de médecine de l'Université d'Indiana à Indianapolis.

110 Emerson 1927,348 , notre traduction.

111 Straus 1957.

112 Il s'agit notamment d'être attentif à l'apparition d'un «malajustement»: soit un changement d'attitude dans le milieu familial ou professionnel, coïncidant avec l'apparition de troubles somatiques chez le patient. L'endocrinologie joue un rôle théorique fondamental dans cette sociologie médicale, puisque les glandes endocrines sont considérées comme constituant « les organes de liaison qui fonctionnent dans la zone entre les organes de notre vie émotionnelle et le corps extracérébrospinal » (Emerson 1927, 350 , notre traduction) 
les problèmes sociaux structurels propres au contexte étatsunien. La crise économique de 1929 est la toile de fond du séjour parisien de Cannon, et elle a aussi largement contribué à façonner ses engagements politico-scientifiques.

\section{Un second séjour en France}

Un soir de l'année I928, Cannon annonce à sa famille avoir décliné une offre d'échange interuniversitaire à Paris ${ }^{113}$. Face aux reproches unanimes des siens, il s'empresse d'informer David Linn Edsall (I869-I945), professeur de médecine au Massachusetts General Hospital et doyen de l'École de médecine de Harvard, qu'il est prêt à reconsidérer l'offre. Ne souhaitant pas s'éloigner trop longtemps de son laboratoire, Cannon décide de ne séjourner que six mois en Europe plutôt qu'une année complète. Son épouse, Cornelia James Cannon ${ }^{114}$ (I876-1969), entend en profiter pour visiter l'Europe, en particulier l'Italie. Écrivaine, elle planifie la rédaction d'un récit de voyage centré sur l'art ${ }^{115}$. Initialement prévue en novembre 1928 la tenue du Congrès international de physiologie à Boston, à l'été I929, impose de repousser le voyage au tout début de l'année 1930.

113 Nous suivons ici les grandes lignes du voyage familial de la famille Cannon à Paris et en Europe, telles qu'elles sont rapportées dans le chapitre XI, «Exchange Professorship in France», de la biographie inédite de Cannon rédigée par sa femme Cornelia. Le manuscrit, intitulé $A$ Servant of Science, Walter Bradford Cannon (James-Cannon tapuscrit inédit) ne sera jamais publié et n'est pas daté.

114 Cornelia James Cannon est originaire de St. Paul. En septembre 1895, elle est admise au Radcliffe College à Cambridge, considéré comme l'annexe de Harvard réservée aux femmes. Elle y suit notamment les cours de William James. Elle connaît Walter Cannon depuis les années passées à St. Paul. Ils se marient le 25 juin rgor. À l'occasion de leur voyage de lune de miel dans le Montana, Walter et Cornelia sont les premiers à gravir le Goat Mountain. Le sommet de 2657 mètres est renommé le Mont Cannon. Cornelia et Walter Cannon ont cinq enfants: Bradford, Wilma, Linda, Marian et Helen. Cornelia poursuit une carrière de romancière. De ses huit romans publiés, Red Rust (James-Cannon, I928) est celui qui rencontre le plus grand succès.

115 Cornelia Cannon écrira bien un manuscrit intitulé Art Awbeel in Italy qu'elle ne parviendra pas à publier, comme en témoignent les échanges de Cannon avec Norton, auprès de qui il prend conseil pour le compte de sa femme (Cannon I93IC).

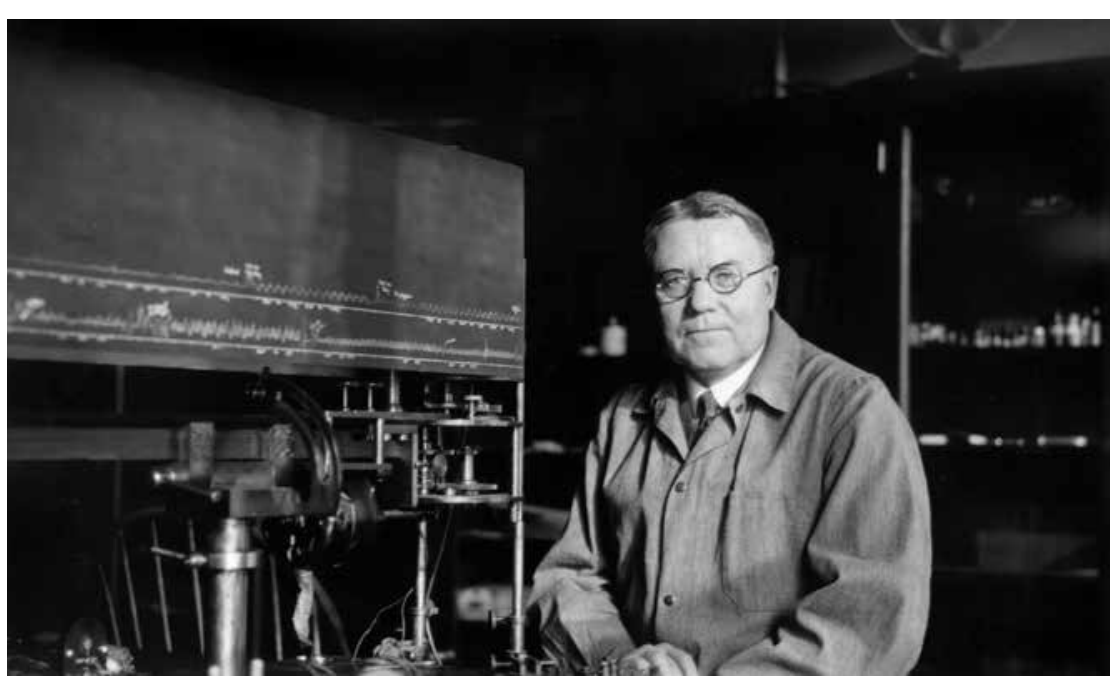

Cannon dispose ainsi de plus de temps pour préparer les deux séries de conférences qu'il prévoit de donner à Paris. En séjour de recherche à Harvard, le biochimiste belge Marcel Florkin (1900-1978), futur doyen de la Faculté de médecine de Liège (I948-1949), l'aide à améliorer sa prononciation du français.

Cannon est accueilli à la Sorbonne par Louis Lapicque (I866-I952) ${ }^{116}$, qu'il a déjà rencontré à l'Université de Harvard en I929, où il avait été invité à délivrer les Dunbam Lectures

116 Louis Lapicque est nommé maître de conférences à la Faculté des sciences de Paris en I899, puis professeur titulaire de la chaire de physiologie générale. Il est membre de l'Académie de médecine et de l'Académie des sciences. Il joue un rôle central dans le développement de la neurophysiologie expérimentale française. Avec Marcelle de Heredia, avec qui il se marie, Lapicque entreprend des recherches sur l'excitabilité nerveuse. En I909, il crée le terme de «chronaxie» afin de désigner le temps nécessaire à une cellule nerveuse ou musculaire pour réagir à l'application d'un courant électrique (Lapicque I942). Selon les Lapicque, les excitabilités (chronaxies) entre un nerf moteur et son muscle, ou entre deux neurones connectés par des synapses, dépendent de leur isocbronisme (voir à ce sujet Barbara 2005). En I923, Cannon est nommé à la tête du comité destiné à organiser les «Dunham Lectureship for the Promotion of the Medical Sciences». Des chercheurs sont invités à Boston dans le but de délivrer une série de conférences, pour lesquelles ils touchent 2500 dollars d'honoraires. Pour l'année I928-I929, il est prévu de recevoir Otto Warburg (I883-1970), professeur de physiologie et de médecine nobélisé en I93I pour ses travaux sur la 


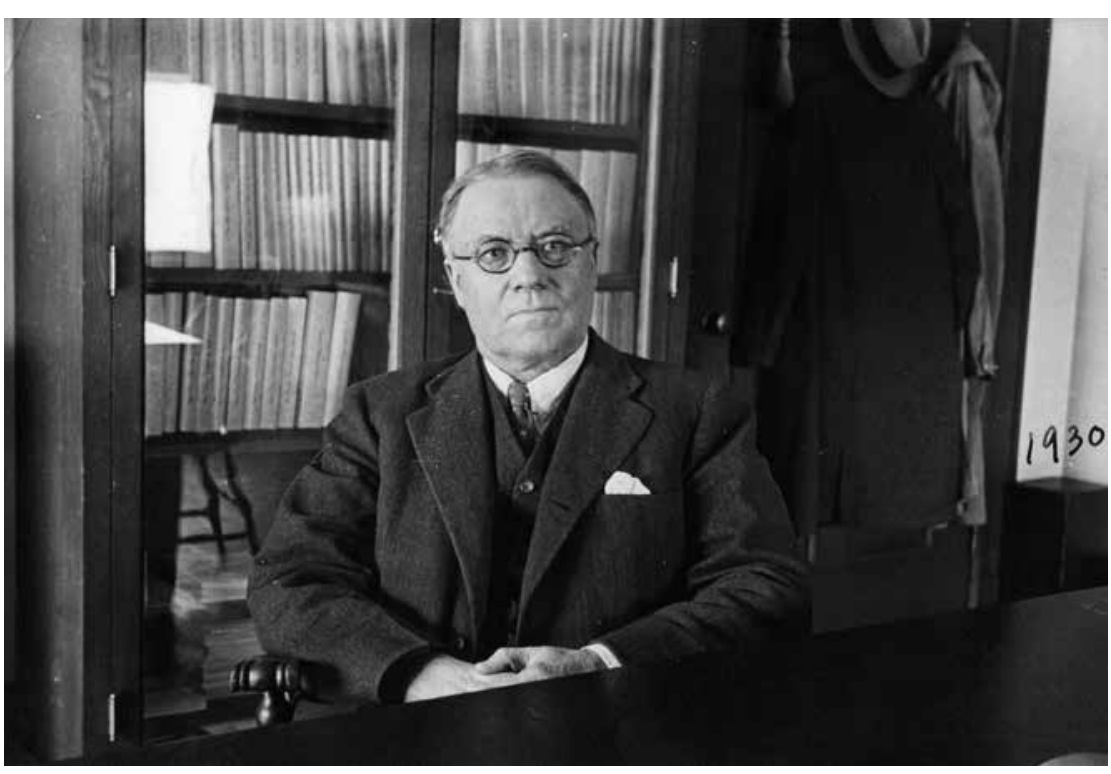

juste avant de participer au congrès international de physiologie. Cannon se voit attribuer un bureau et Lapicque lui met à disposition deux étudiants avec qui il répète les conférences. La première série de cinq conférences, intitulée "Les émotions fortes et leur influence sur l'organisme", est délivrée à $\mathrm{I} 6 \mathrm{~h}$, les 6, 8, II, I3 et I5 février 1930, dans l'amphithéâtre Vulpian de la Faculté de médecine de Paris. Les " 5 leçons sur l'homéostasie " sont délivrées à I 7 h les 2I, 26, 28 février et les 5 et 7 mars I930, dans l'amphithéâtre de physiologie de la Faculté des sciences. Les conférences sont un succès. Si nous ne sommes pas parvenus à identifier précisément les personnalités présentes lors des conférences - nous n'avons de certitude qu'au sujet de Lapicque et de Dumas, alors titulaire de la chaire de psychologie expérimentale à la Sorbonne -, il est en revanche possible d'évoquer quelques-unes de celles que Cannon rencontre durant son séjour parisien.

respiration cellulaire. Suite à son désistement, Louis Lapicque est invité à le remplacer. Le choix de Lapicque est d'autant plus judicieux qu'il est prévu qu'il participe aussi à l'International Congress of Physiology dont l'édition de I929 a lieu à Boston.
Une soirée est organisée en l'honneur du couple Cannon chez André Mayer (I875-1956) ${ }^{117}$, à laquelle assistent Lapicque et Lévy-Bruhl ${ }^{118}$. Dans sa lettre datée du io janvier $1930^{119}$, Charles Richet invite le couple Cannon à dîner le 22 janvier, en présence de quelques collègues. Le couple dîne encore chez les Lapicque, en présence d'Eugène Gley (1857-1930) ${ }^{120}$. Le 22 février 1930, Cannon participe à une séance de la Société française de psychologie à la Sorbonne, dans l'amphithéâtre Guizot. Avant de délivrer une conférence intitulée «Quelques observations sur le comportement maternel d'animaux privés du système sympathique ${ }^{121}$, suivie d'une projection cinéma-

117 André Mayer est un biologiste français. Il obtient un doctorat de médecine en I90o. Durant la Grande Guerre, il dirige le laboratoire de physiologie des Services chimiques de guerre. Il est nommé professeur de physiologie à la Faculté de médecine de Strasbourg, avant de rejoindre le Collège de France en 1922. Ses travaux portent principalement sur le maintien de l'équilibre physico-chimique cellulaire et sur la nutrition. Mayer est un ami de Cannon. Les deux hommes se rencontrent pour la première fois lors du premier séjour de Cannon à Paris, en I9I8. À son arrivée, Cannon, qui doit poursuivre ses travaux sur les chocs, ne dispose d'aucun laboratoire. Mayer l'accueille dans son laboratoire du Collège de France, situé à quelques mètres de celui où Claude Bernard menait ses recherches. En I94I, Mayer et sa famille, de confession juive, fuient la France. Cannon s'occupe de lui trouver, via l'entremise de Henderson, un poste d'enseignement à l'Université de Cambridge. C'est aussi sur la recommandation de Mayer que Cannon accepte que Bacq passe l'année I929-I930 dans son laboratoire.

118 Lucien Lévy-Bruhl (I857-I939) est un philosophe et anthropologue français. Il étudie la philosophie à l'École normale supérieure, où il obtient l'agrégation de philosophie en I879. En I888, il est nommé à la Sorbonne où il reprend la chaire d'histoire de la philosophie moderne. André Maver n'est autre que le neveu de Lévy-Bruhl. La lettre que Lévy-Bruhl adresse à Cannon le 26 octobre I934 (Lévy-Bruhl I934), au sujet de la «mort vaudoue", atteste que la réception en l'honneur du couple Cannon a bien eu lieu au début de l'année r930. On y apprend aussi que Lévy-Bruhl avait déjà eu l'occasion d'entendre Cannon lors d'un séjour à Harvard en I919-I920.

119 (Richet, 1930).

120 Émile Marcel Eugène Gley est un physiologiste et endocrinologue français, nommé professeur au Collège de France en I908. Les travaux de Gley et Quinquaud semblent indiquer que la sécrétion d'adrénaline par les surrénales n'a pas d'effet sur le système vasomoteur ni sur le cœur. Les observations de Cannon sur le cœur dénervé vont clore la controverse. Il semblerait que Gley ait aussi assisté aux conférences parisiennes de Cannon sur les émotions.

121 Cannon I930c. 
tographique illustrant les comportements des animaux sympathectomisés, Cannon est élu membre associé étranger de la société. Lapicque et Gley sont présents, ainsi que le psychologue Ignace Meyerson (I888-I983) qui, en sa qualité de secrétaire général de la société, rédige le compte rendu de la séance (Meyerson, 1930). Une lettre datée du 27 février nous apprend que, à l'initiative d'Henri Laugier (I988-I973), médecin et futur secrétaire général adjoint de l'ONU, Édouard Toulouse (I8651947) ${ }^{122}$ invite Cannon à délivrer une conférence à l'Institut de psychiatrie et de prophylaxie mentale qu'il dirige ${ }^{123}$. Nous ne disposons pas de plus d'informations sur cette conférence, prévue le lundi 3 mars I930 à Iohž, dans l'amphithéâtre Magnan de l'Hôpital psychiatrique Henri Rousselle. Les deux hommes n'ont vraisemblablement pas entretenu de correspondance. Mais on peut supposer que c'est à cette occasion que Cannon a eu vent des travaux de Toulouse sur la «biocratie», qui, comme on le verra, vont influencer ses réflexions sur l'homéostasie sociale.

Lors de son séjour parisien, Cannon a aussi l'occasion de rencontrer des compatriotes impliqués dans des réseaux internationaux. Alan Gregg (I890-I957) est l'une des premières personnes qu'il rencontre à Paris. Ce médecin, diplômé de Harvard, ancien étudiant de Cannon, a lui aussi servi en France de I9I7 à I9I9. Convaincu que la prévention et la santé publique sont l'avenir de la médecine, Gregg rejoint ensuite l'International Health Board mis sur pied par la Fondation Rockefeller. De I924 jusqu'à I930, il en dirige le bureau parisien, à partir duquel il suit l'évolution des différents projets financés par la fondation et coordonne des programmes européens d'éducation médicale.

122 Édouard Toulouse est un médecin et psychiatre français diplômé de l'Université de Marseille. Il est nommé interne des asiles de la Seine en I89o, médecin adjoint de l'asile de Saint-Yon en I892 et chef de clinique à l'asile Sainte-Anne à Paris en I894. En I927, il fonde l'Institut de psychiatrie et de prophylaxie mentale de l'École des hautes études. Il y développe des instruments de mesure psychophysiologiques visant notamment à définir les aptitudes professionnelles. Il est l'auteur de nombreux travaux scientifiques, ainsi que d'essais dans lesquels il expose ses théories eugénistes et hygiénistes et son projet politique «biocratique».

123 Toulouse rig30.
Cannon entre aussi en contact avec James Hazen Hyde (I876-1959), un riche héritier américain. Cet ancien étudiant de Harvard, francophile, a quitté les États-Unis suite à un scandale financier. Il s'établit en France de 1905 à 1940 et il crée et dirige, de 1906 à 1953, la Fédération des Alliances françaises, dont la mission consiste à promouvoir la langue française dans le monde. L'organisation finance notamment les échanges interuniversitaires de professeurs. L'entremise de Hyde va être déterminante, puisqu'elle va permettre à Cannon d'étendre son réseau, à Paris comme en province. Dans une lettre datée du i6 janvier $1930^{124}$, nous apprenons que Hyde organise une rencontre avec le philosophe Henri Bergson, le 22 janvier à I6h30. Cannon est prié de retrouver Hyde chez lui, au i8 de la rue Adolphe Yvon, dans le $\mathrm{XVI}^{\mathrm{e}}$ arrondissement. Les deux hommes se rendent ensemble au domicile de Bergson, situé à quelques pas, au 47 boulevard de Beauséjour. Dans sa lettre du 24 janvier, Bergson ${ }^{125}$ rapporte avoir trouvé la discussion «intéressante et instructive ». Il précise par ailleurs qu'il est en train de lire une sélection de travaux que Cannon lui a fait parvenir.

Hyde et Cannon sont entrés en contact plusieurs mois auparavant. Une lettre remontant au 28 décembre $1928^{126}$ nous apprend que le premier a proposé au second de profiter de son séjour parisien pour délivrer ses conférences en province. Ce serait, selon Hyde, l'occasion de séjourner quelques jours dans chacune des villes et d'y visiter leurs écoles de médecine. La Fédération des Alliances françaises verserait à Cannon 2500 francs par conférence, ce qui devrait, au dire de Hyde, largement couvrir les dépenses. Avec l'accord de Cannon, Hyde a mobilisé ses contacts. Dans une lettre datée du I5 mars I930, Charles Petit-Dutaillis (I968-I947) ${ }^{127}$, directeur de l'Office national des universités et écoles françaises, indique à Cannon la liste des recteurs des universités où il donnera des conférences. La liste, annotée par Cannon, permet de retracer son agenda.

\footnotetext{
124 Hyde 1930

125 Bergson 1930.

126 Hyde I928.

127 Petit-Dutaillis I930.
} 
Le mois de mars est particulièrement chargé. Le I5 octobre 1929, Perrin Comstock Galpin (I889-I973), secrétaire de l'Educational Foundation de la Commission for Relief in Belgium ${ }^{128}$, avait aussi proposé à Cannon de prendre en charge une tournée en Belgique rétribuée ioo dollars par conférence. Cannon commence à Louvain, le II mars, où il donne deux conférences. Il rejoint ensuite l'Université libre de Bruxelles, où il délivre trois conférences, les I7, I9 et 2I mars. Le I8 mars, il se rend à l'Université de Liège pour une dernière conférence ${ }^{129}$. Une fois sa tournée belge terminée, la famille Cannon embarque dans la Cadillac pour Lyon, où Cannon donne deux conférences les 27 et 29 mars. La famille rejoint Montpellier le $\mathrm{I}^{\text {er }}$ avril, puis séjourne à l'Université Aix-Marseille les 4 et 5 avril. La tournée provinciale est une première fois interrompue. La famille Cannon traverse les Pyrénées et atteint Barcelone, puis Madrid où Cannon rencontre Marañon et Juan Negrin (I892-1956). Negrin, ancien étudiant de Cannon, deviendra en 1937 le président du gouvernement de la Seconde République d'Espagne jusqu'en I939 où, contraint à l'exil, il s'établit à Paris ${ }^{130}$.

Cannon reprend sa tournée française à Toulouse le 29 avril, puis à Bordeaux le 2 mai. Le tour des universités de province est une nouvelle fois interrompu. De Bordeaux, la famille Cannon rejoint le Royaume-Uni. Cannon a en effet été invité à intervenir,

128 La Commission for Relief in Belgium est créée en IgI4 afin d'acheminer, via l'ambassade des États-Unis, une aide alimentaire à la Belgique qui, envahie par l'Allemagne, n'est plus en mesure de subvenir aux besoins de sa population. Sous la responsabilité de Herbert Hoover, la commission est dotée d'un budget annuel de I2 millions de dollars, composé essentiellement de dons et de subventions. À la fin de la guerre, les 30 millions de dollars sont alloués à l'Educational Foundation qui les redistribue aux universités et écoles supérieures afin de promouvoir l'enseignement en Belgique.

129 Cannon a vraisemblablement donné une conférence à Gand également.

130 C'est lors de ce voyage que Cannon se lie plus profondément d'amitié avec Negrin. Ils resteront en contact notamment lorsque Cannon rejoindra le Spanish Medical Bureau. Cette branche de l'American Friends of Spanish Democracy, que Cannon préside de 1937 à 1939, apportera une aide médicale au camp républicain. Les réseaux internationaux de l'église unitarienne permettront, par l'entremise de Cannon, de contourner l'embargo décrété en I937 par les États-Unis qui, dans le contexte international tendu et avec la montée du nazisme, préfèrent rester neutres. le 6 mai, dans le cadre des Linacre Lectures de Cambridge. La conférence intitulée «The Autonomic Nervous System: an Interpretation » est publiée dans la foulée dans la revue The Lancet $^{131}$. Le I6 mai, Cannon est de retour en France. Il délivre une nouvelle conférence à Lille. S'ensuivent deux conférences à Strasbourg les I9 et 2I mai, puis les deux dernières à Nancy, les 24 et 25 mai.

La tournée provinciale terminée, la famille Cannon dispose de temps libre. Elle remonte vers Reims puis Verdun, afin de visiter les champs de bataille de la Grande Guerre. Puis la Cadillac prend la direction de la Suisse. Un document non daté remontant probablement à 1929 ou au début de l'année 1930, dans lequel Cannon liste ses contacts dans les universités européennes, laisse penser que Cannon a un temps envisagé d'étendre à la Suisse sa tournée de conférences. Cannon y indique deux personnalités: Maurice Arthus (I862-I945) ${ }^{132}$ à Lausanne et Charles Dhéré ${ }^{133}$ à Fribourg. Le projet a vraisemblablement été abandonné. En Suisse, Cannon se contente finalement de quelques visites de courtoisie. La famille s'arrête tout d'abord à Berne, où Cannon retrouve son ami Leon Asher (I865-I943) ${ }^{134}$ qui lui fait visiter son laboratoire. Ils rejoignent ensuite Zurich où ils prennent le thé avec Walter Rudolf Hess $(\mathrm{I} 88 \mathrm{I}-\mathrm{I} 973)^{135}$. Les vacances en Suisse vont prendre une tournure

131 Cannon I930e.

132 Arthus est un physiologiste d'origine française. Il étudie la médecine à la Sorbonne, où il est nommé maître de conférences en I8go. Il est nommé professeur de physiologie à l'Université de Fribourg en I896. Après quelques années à l'Institut Pasteur de Lille puis à l'École de médecine de Marseille, il est nommé en I907 professeur de physiologie et directeur du laboratoire de physiologie à Lausanne.

133 Charles Dhéré (I876-I955) est un physiologiste d'origine française. Il obtient un doctorat de médecine à la Sorbonne en I 898 . Il succède à Maurice Arthus à la chaire de physiologie de Fribourg en Igoo et devient doyen de la Faculté des sciences naturelles de Fribourg en I933-I934.

134 Asher est un physiologiste d'origine allemande. Il étudie la médecine à l'Université de Leipzig de I885 à I890. En I894, il devient assistant à l'Institut de physiologie de Berne. Il y est nommé professeur de physiologie en 1895, puis professeur de médecine en 1925 .

135 Ophtalmologue de formation, Hess devient assistant à l'Institut de physiologie de Zurich en I9I2, puis professeur et directeur de l'institut en I9I7. 
inattendue. Alors qu'ils rejoignent Ragaz, la seconde semaine de juin, Cannon présente des symptômes d'hématurie. Un médecin de Ragaz diagnostique une néphrite hémorragique et lui recommande de prendre du repos. Fin juin, alors que la famille remonte le Rhin vers l'Allemagne, puis la Hollande où leur fils, Bradford, doit les rejoindre, Cannon fait une nouvelle crise. Le médecin qu'il consulte à Bruxelles lui diagnostique un papillome vésical. La famille Cannon se rend alors à Londres où le diagnostic est confirmé. Walter et Cornelia Cannon décident alors de prendre le premier bateau pour Boston où Cannon est opéré le 3I juillet. De petites tumeurs malignes ont été repérées et retirées, mais l'opération est un succès. Par précaution, Cannon devra toutefois être examiné tous les deux mois.

Si Cannon en a terminé avec sa tournée européenne, il n'en a pas pour autant fini avec ce que son éditeur nomme encore en I93 I ses « Sorbonne Lectures» ${ }^{136}$.

\section{Un nouveau concept}

pour une "nouvelle physiologie»

Bien que le terme "homéostasie" n'apparaisse jamais dans les conférences sur les émotions, la conception holiste du fonctionnement physiologique à laquelle il renvoie n'en est pas moins suggérée. Cannon y achève sa réflexion sur les altérations structurales et fonctionnelles par ces mots: «Il est certain que c'est seulement lorsque toutes deux sont considérées comme des perturbations d'un tout unique, l'organisme, qu'on peut en avoir une conception adéquate et qu'on peut les traiter d'une manière effective. » (Conférence I/5) Pour Cannon, la conception "adéquate" des modifications structurelles et fonctionnelles s'avère dépendante d'une compréhension tout aussi adéquate du fonctionnement de l'organisme. À cet égard, introduire la notion d'homéostasie nécessite que l'on

En 1949, il reçoit le Prix Nobel pour ses travaux sur le rôle du diencéphale, en particulier de l'hypothalamus, dans la régulation des fonctions végétatives.

136 Norton I93IC. s'attarde sur ce que serait une représentation «inadéquate » du fonctionnement physiologique.

Dans ses conférences sur l'homéostasie, Cannon se réfère à la polémique opposant Haldane à Lawrence Joseph Henderson (I878-1942) au sujet du livre Blood que ce dernier publie en I92 $8^{137}$. La controverse révèle l'ambition de Cannon et de ses collègues de défendre, dans la lignée des réflexions amorcées

(Henderson 1928). Lawrence Joseph Henderson est un biologiste, philosophe et sociologue américain. En I898, il entre à l'École de médecine et il en est diplômé en I902. Il débute ses recherches sur les propriétés de l'hémoglobine durant un séjour de deux ans dans le laboratoire de Franz Hofmeister (I850-I922), à Strasbourg. Il est nommé professeur de chimie biologique de I9I9 à I934, puis professeur de chimie en I934. Durant les années I9IO, Henderson publie deux ouvrages philosophiques qui témoignent de son intérêt pour les questions relatives à l'ordre du monde et à l'adaptation à l'environnement. Mais c'est dans Blood. A Study in General Physiology, publié en I928, que ces thématiques sont plus précisément abordées. Il s'y inscrit dans la droite ligne de Claude Bernard, à qui l'on doit d'avoir délimité les contours disciplinaires de la physiologie, et du physicien et chimiste Willard Gibbs (I839-I903), dont la publication de "On the Equilibrium of Heterogeneous Substances» (Gibbs 1878) fait de lui le père de la physiologie américaine. Bien que tous deux aient fondé la physiologie comme l'étude des processus physico-chimiques qui se trouvent "harmonieusement organisés et intégrés " (Henderson I928, 4 , notre traduction) dans la matière vivante, Henderson estime que la physiologie est une science qui se trouve «toujours dans son enfance» (Henderson I928, 4, notre traduction). Les mécanismes de régulation automatique permettant au sang de maintenir ses propriétés stables, comme son alcalinité, via les mécanismes d'oxygénation, constituent pour Henderson un objet d'étude de choix pour le physiologiste. Par ailleurs, la lecture des travaux du sociologue et économiste Wilfredo Pareto, auxquels il consacre un livre (Henderson 1935), en particulier ses théories de l'équilibre, vont l'amener à s'intéresser à la sociologie. Cet intérêt, commun à Cannon, va être catalysé par les intentions de la Harvard School of Business de former un comité de recherche spécialisé dans l'étude de la production industrielle. Henderson est chargé du Fatigue Laboratory. Dans ce cadre, il participe aux expériences menées de 1928 à I 932 à l'usine «Hawthorne» de la Western Electric, qui marquent les débuts de la sociologie industrielle. Dès les années I930, il enseigne à la Harvard School of Business une sociologie qu'il veut concrète, basée sur les faits et tournée vers l'action (voir Desmarez 1986). Son enseignement vise avant tout à former des experts en interactions humaines, soit une forme d'élite scientifique proche de l'idéal technocratique imaginé par Cannon. Comme pour ce dernier, il s'agit de constituer des experts capables de s'émanciper des idéologies politiques ainsi que des intérêts privés, afin de mener le pays vers l'équilibre social. 
par Starling, une physiologie holiste. Pour Haldane, le problème tient davantage à l'entreprise même de Henderson, qui tend à expliquer les mécanismes de régulation permettant de conserver la stabilité des propriétés du sang sous une perspective physico-chimique, qu'au fait de se réclamer de la physiologie de Bernard. Appréhender le sang comme un système physico-chimique contrevient, selon Haldane, à l'esprit des théories de Bernard, pour lesquelles la stabilité du milieu intérieur est maintenue non pas seulement par des mécanismes impliquant les molécules composant le sang ou celles des tissus vasculaires, mais, plus généralement, par la coordination de l'ensemble des organes. La stabilité du sang ne relève pas des quelques mécanismes permettant de compenser des variations physico-chimiques locales, mais dépend plus largement de l'action des reins et du système respiratoire, dont les actions régulatrices dépendent elles-mêmes de l'ensemble des facteurs physiologiques assurant la stabilité de l'organisme.

La critique est l'occasion d'identifier l'objet authentique de la physiologie, autant que d'en définir les contours disciplinaires. À cet égard, Haldane écrit : « Si, suivant L. J. Henderson, nous négligeons la coordination organique active, nous faisons, il me semble, un pas en arrière. ${ }^{138}$ En d'autres termes, l'approche d'Henderson régresse vers une conception mécaniste et pré-bernardienne de la physiologie. Or la physiologie n'est pas un mécanisme, soit une science des parties de l'organisme (cellule, organe, etc.), mais une science de la coordination des parties. La perspective de Haldane n'est pas nouvelle. Il ne fait ici que reprendre les arguments qu'il a déjà eu l'occasion d'exposer en I9I6, dans un article défendant les fondements épistémologiques de ce qu'il nomme la "nouvelle physiologie»:

Je ne connais rien de plus désespéré que les tentatives d'explication des caractéristiques remarquables des sécrétions d'urine sur les bases de la physique et de la chimie ordinaires. Dans l'état actuel de nos connaissances, nous pouvons toutefois avoir une compréhension pratique des activités rénales, si nous attaquons le sujet du point de vue du maintien actif de la composition sanguine normale ${ }^{139}$.
En d'autres termes, une analyse des mécanismes physiques et chimiques ne peut rien apprendre en physiologie. Ce n'est qu'une fois la fonction d'un organe déterminée, eu égard à l'ensemble de l'organisme, que l'on peut attribuer un sens aux mécanismes physiques et chimiques en question. À ce titre, Haldane va même jusqu'à soutenir que la physiologie pré-bernardienne n'est tout simplement pas une physiologie, puisque le mécanisme n'a selon lui jamais été une étape dans le développement de la physiologie, mais «l'échec le plus colossal de toute l'histoire de la science moderne $"{ }^{140}$. La "nouvelle physiologie " doit donc être vue comme un retour, succédant à une période d'égarement, à la seule physiologie possible: "une physiologie biologique", qui n'est ni une biophysique ni une biochimie ${ }^{141}$. C'est sur cette nouvelle physiologie que Haldane entend aussi définir l'objet réel de la médecine:

Quel est l'objet pratique de la médecine? Il s'agit de promouvoir le maintien et d'aider au rétablissement de la santé. Mais qu'est-ce que la santé? C'est très certainement ce qui est normal pour un organisme. Par «normal", on entend non pas ce qui relève d'une moyenne, mais ce qui est normal au sens biologique, c'est-à-dire les conditions dans lesquelles l'organisme maintient l'intégrité de toutes les normalités interconnectées qui [...] se manifestent aussi bien dans les structures du corps que dans les activités corporelles ${ }^{142}$.

Il faut retenir cette définition de la normalité pour comprendre ce que Cannon a lui-même en tête lorsqu'il utilise le terme «normal». Il se réfère à une normalité plastique, dynamique et active. Mais surtout, pour cette nouvelle physiologie, profondément holiste, il s'agit moins de penser la normalité, que des "normalités interconnectées».

Bien que Cannon et Henderson aient été très proches ${ }^{143}$, le premier ne manque pas de prendre parti pour

140 Haldane I9I6, 630, notre traduction

141 Haldane I9I6, 630, notre traduction.

142 Haldane iqi6, 630, notre traduction.

143 En I923, Cannon rapporte que Henderson fait partie des collègues du département de physiologie qui ont pris l'habitude de se réunir pour prendre le thé tous les mardis à I6h3o dans la bibliothèque. Cannon qualifie ces 
Haldane. Les lignes de conclusion de ses conférences sur l'homéostasie ne font que reprendre les arguments de Haldane quant à l'objet de la physiologie et à ses rapports à la biophysique et à la biochimie: «Les analyses ultérieures de processus biologiques exprimées en termes physiques et chimiques doivent attendre une compréhension complète des voies que suivent les processus homéostatiques pour remplir leurs fonctions. » (Conférence II/5) Cannon affirme encore plus clairement que Haldane l'antériorité épistémique de la physiologie biologique sur toute autre approche réductionniste des fonctions biologiques normales, puisque c'est la première qui informe la seconde. Aussi, lorsque Cannon forme le concept d'homéostasie, n'apporte-t-il à proprement parler rien de nouveau, si ce n'est qu'il range, sous un terme unique, l'holisme mis au jour par la physiologie hormonale de Starling et la hiérarchisation disciplinaire inversée de Haldane, selon laquelle, en biologie, l'avancée des sciences ne procède pas, comme dans les autres champs des sciences naturelles, par réduction des sciences spéciales à des niveaux explicatifs supposés plus fondamentaux. En formant le terme d'«homéostasie », Cannon évite par ailleurs les incohérences historico-épistémologiques qu'évoque le terme de "nouvelle physiologie ». Vis-à-vis de quoi serait-elle nouvelle? Vis-à-vis du "nouveau mécanisme » que défendrait Henderson, mais qui reconduit par ailleurs le mécanisme pré-bernardien? Mais est-elle si nouvelle, dès lors qu'elle se réclame de la physiologie d'un physiologiste, Claude Bernard, disparu près d'un demi-siècle plus tôt? La physiologie doit être biologique, certes, mais Cannon entend identifier, plus précisément, ce qui caractérise les phénomènes biologiques, du plus bas niveau d'explication (le sang par exemple), au niveau le plus global. L'homéostasie est à cet égard le métaconcept qui dénote ce que tous les phénomènes physiologiques ont en commun et auquel tous les niveaux explicatifs ont affaire: l'autorégulation. Sans cette dernière, le biologique n'est tout simplement plus du biologique. À cet égard, il conclut dans

réunions de particulièrement stimulantes. En I943, c'est Cannon, membre depuis I9I4, qui rédige et prononce la notice biographique en mémoire de Henderson, durant l'assemblée d'automne de l'Académie (Cannon 1943). ses conférences que «la régulation de l'organisme est le problème central de la physiologie ». (Conférence II/5)

Mais revenons à cette définition de la physiologie comme une science de l'homéostasie. Celle-ci nous ramène encore à la France. Cannon introduit pour la toute première fois le concept en I926, dans un court article en anglais, paru à l'occasion du jubilé du physiologiste français Charles Richet. L'article s'ouvre sur deux références, citant d'abord Richet qui écrivait :

L'être vivant est stable; et il faut qu'il le soit pour n'être pas détruit, dissous, désagrégé par les forces colossales, souvent adverses, qui l'entourent. Mais, par une sorte de contradiction qui n'est qu'apparente, il ne maintient sa stabilité que s'il est excitable, capable de se modifier suivant les irritations du dehors et de conformer sa réponse à l'irritation; de sorte qu'il n'est stable que parce qu'il est modifiable. La défense n'est compatible qu'avec une certaine instabilité. Celle-ci doit s'exercer sans cesse, mais dans d'étroites limites; et cette modérée instabilité est la condition nécessaire de la véritable stabilité de l'être ${ }^{144}$

Puis l'article convoque Claude Bernard, pour qui « tous les mécanismes vitaux, quelque variés qu'ils soient, n'ont toujours qu'un but, celui de maintenir l'unité des conditions de la vie dans le milieu intérieur ${ }^{145}$.

Dans ce bref article, Cannon entreprend de lister ce qu'il nomme les postulats de l'homéostasie. Mais c'est surtout à partir de l'été 1929, alors que son séjour parisien s'annonce, que Cannon entre plus en détail. En juillet, il publie dans le Physiological Review «Organization for Physiological Homeostasis" puis, en février, dans les Archives of Neurology छ Psychiatry "The Sympathetic Division of the Autonomic System in Relation to Homeostasis ${ }^{146}$. Ses conférences parisiennes sur l'homéostasie sont en grande partie extrapolées de ces deux articles.

Il est frappant de constater que ces deux publications, en particulier la seconde, témoignent d'un nouveau déplacement

144 Richet I899, 72I.

145 Bernard I878, I2I.

146 Cannon ig29e. 
dans la carrière de Cannon. Comme on l'a vu, il a tout d'abord constaté que les émotions vives interfèrent avec, voire abolissent, le fonctionnement normal de la digestion. Il a dès lors œuvré à mettre au jour les mécanismes, en particulier le rôle du système sympathique-surrénal, susceptibles d'expliquer ces interférences. En définitive, c'est une fois déplacé son intérêt du fonctionnement normal vers ce qui l'abolit que Cannon en est revenu au fonctionnement physiologique normal - mais pas pour faire de la normalité cette sorte de stabilité immuable à laquelle les critiques du concept l'associent trop promptement comme nous aurons l'occasion de le voir. La normalité ne se définit pas comme un état physiologique dans lequel le système sympathique-surrénal serait inactif. Au contraire, celui-ci ne cesse jamais d'assurer les « ajustements mineurs de l'organisme requis afin de répondre à l'environnement $"{ }^{147}$. La normalité à laquelle revient Cannon n'est donc aucunement un état de stabilité parfaite, mais une modalité des réactions d'adaptation - l'une des normalités possibles, dirait Haldane -, dont les états de grande excitation émotionnelle ne sont que des cas extrêmes.

C'est ce nouveau tournant que Cannon est en train d'opérer durant la fin de l'année I929, alors qu'il s'apprête à rejoindre la France. À cet égard, son voyage n'est pas l'occasion d'aller exposer au public français et européen une théorie achevée. L'imperméabilité des deux cycles de conférences indique qu'il faudra encore quelques années à Cannon pour que la notion de stress vienne définitivement achever l'articulation entre sa théorie des émotions et sa théorie de l'homéostasie. À la fin de l'année 1929, Cannon voit plutôt dans son séjour parisien l'occasion d'avancer dans l'élaboration d'une théorie générale de l'homéostasie, voire de profiter du temps passé loin de son laboratoire et de ses obligations académiques pour écrire un nouveau livre.
Des « Sorbonne Lectures"

à La Sagesse du corps: genèse d'un livre

Le 24 mai I929, Cannon reçoit une lettre de William Warder Norton (I89I-1945), directeur de publication et fondateur, en I923, de la W. W. Norton \& Company ${ }^{148}$. La maison d'édition s'est spécialisée dans la publication d'anthologies, mais aussi d'essais universitaires. On apprend dans cette lettre que les deux hommes se sont récemment rencontrés à Boston. Norton confirme l'entente verbale: Cannon écrira un livre sur les «facteurs de stabilisation" (factors of stabilization). Les termes du contrat, que Norton joint à sa lettre, sont déjà précis : Cannon touchera Iooo dollars la première année de vente du livre et ce, même si le livre ne se vend qu'à une centaine d'exemplaires. Norton ne doute cependant pas que le livre, écrit dans un style «semi-populaire» [semi-popular], se vendra. Il ne doute pas non plus que Cannon remettra le manuscrit autour du $\mathrm{I}^{\text {er }}$ septembre I930, dès lors que Cannon «a pris des dispositions précises pour donner les conférences et envisage de les rédiger sous forme de livre ${ }^{149}$. Quatre jours plus tard, Cannon répond qu'il ne signera pas le contrat: non seulement le livre n'est pas encore écrit, mais il doute que ses conférences puissent faire l'objet d'une publication grand public ${ }^{150}$.

Norton revient à la charge le 24 novembre 1930. Il propose de rencontrer Cannon à Boston. Cannon est en effet rentré de son séjour parisien et s'est remis de son opération ${ }^{151}$. Malgré l'insistance de Norton, ce n'est qu'au début du mois de mai I93 I que les deux hommes parviennent à se rencontrer ${ }^{152}$. Dans sa lettre du II mai I93I, Norton ${ }^{153}$ confirme que, à l'issue de la rencontre du 9 mai 193i, Cannon s'est définitivement engagé à lui remettre un manuscrit. Un contrat amendé lui est envoyé, repoussant la date de remise du manuscrit au 3I décembre I93I.

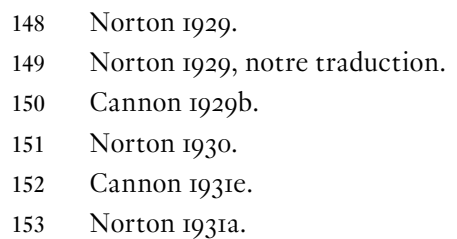


Les conditions de publication résolues, c'est surtout la question du titre qui préoccupe Cannon. Dans sa lettre du i mai, Norton propose à Cannon de reconsidérer le titre qu'il lui a proposé, "Organization for Stabilization in Human Beings". Il juge la conjonction des termes organization et stabilization trop longue et euphonique. Il propose d'opter pour stability. Dans sa lettre du I2 mai I93I, Cannon ${ }^{154}$ propose à Norton deux nouveaux titres: "How We Stay Normal», proche du titre d'une conférence donnée le I9 mars I930 à Bruxelles, ou "Our Arrangement for Stability ».

Dans sa lettre du 7 août, Cannon ${ }^{155}$ annonce à Norton qu'il a terminé la première version des seize premiers chapitres de son livre, qui devrait en compter dix-neuf. Il en profite pour exposer de nouvelles idées de titres: "Stabilitas", "Homeostasis», "Our Internal Balance», «The Self-Regulating Stability of Man", "Automatic Human Stabilization", "Organized Stabilization in man". Dans les trois derniers cas, Cannon précise qu'il serait envisageable de remplacer man par Human Being ou Human Economy. Le I2 août, Norton exprime ses doutes concernant ces titres qu'il juge trop techniques. Aussi, à moins qu'une idée brillante n'émerge, préférerait-il en rester au titre «How We Stay Normal ${ }^{156}$. Il faut attendre la lettre que Cannon adresse à Norton le 3 décembre $193{ }^{157}$ pour apprendre que Cannon a envoyé le manuscrit final à Norton, à l'exception du dernier chapitre, et, surtout, que l'ouvrage sera intitulé The Wisdom of the Body. Le titre est tout simplement emprunté à la conférence que Starling a délivrée au Royal College of Physicians de Londres, le I8 octobre I923, et publiée dans le British Medical Journal ${ }^{158}$. Or la reprise de ce titre et les raisons pour lesquelles Cannon diffère la livraison du dernier chapitre de l'ouvrage à son éditeur sont liées.

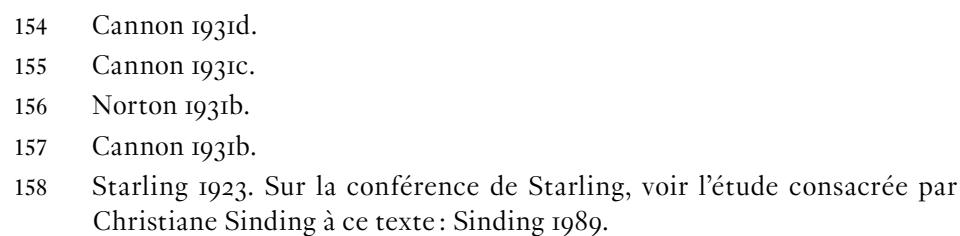

Dans sa conférence au Royal College of Physicians de Londres, Starling ne se contente pas de confirmer l'apport de la physiologie hormonale, comme il le faisait quelques décennies plus tôt, en 1905 , lorsqu'il délivrait ses Croonian Lectures. L'émergence d'une physiologie hormonale est perçue comme participant à une reconfiguration de la médecine, ainsi que du rôle social du médecin. C'est en effet en ces termes qu'il conclut sa conférence:

En tant que médecins, nous nous tenons du côté opposé au bien et au mal, nous savons que le péché pour lequel l'homme paie continuellement la pénalité n'est pas nécessairement le non-respect de l'un ou de l'autre des brutaux ajustements tribaux à l'environnement que nous appelons la moralité, mais que c'est toujours et dans tous les cas l'ignorance ou le mépris du travail immuable des forces de la Nature qui nous est continuellement révélé par la recherche scientifique. Malgré l'augmentation remarquable des connaissances, sur laquelle j'attire votre attention, la souffrance est encore très répandue parmi nous. Seulement, en suivant les injonctions de notre grand prédécesseur [Harvey] - rechercher et étudier les secrets de la nature au moyen de l'expérimentation -, nous pouvons espérer parvenir à une compréhension de « la sagesse du corps et du cœur » et, partant, à la maîtrise de la maladie et de la douleur, qui nous permettra de soulager le fardeau de l'humanité ${ }^{159}$.

De son côté, si Cannon tarde à rendre à Norton son dernier chapitre, c'est qu'il a décidé, contre l'avis de son éditeur, de ne pas en faire le dix-neuvième chapitre de l'ouvrage, mais un épilogue, "afin de le distinguer des parties biologiques principales du livre " ${ }^{160}$. Dans cet "épilogue», intitulé «Relations of Biological and Social Homeostasis ", Cannon s'interroge quant à l'utilité de se référer aux principes de la stabilité homéostatique du corps physiologique pour penser la stabilité du corps social. Cannon avance que les cellules de l'organisme, comme les membres de la société, possèdent des vies propres bien que liées aux autres par des liens de dépendance mutuelle. Même les cellules œuvrant, par division du travail, à maintenir la fluid matrix dépendent elles-mêmes de l'organisation totale. Pour

159 Starling 1923,690 , notre traduction.

160 Cannon 1932, notre traduction. 
les mêmes raisons, nous dit-il, le bien-être des individus qui composent une communauté dépend de l'homéostasie sociale. Il faut entendre par là que, dans les larges communautés où la division du travail a atteint un point de spécialisation extrême, seul l'État peut produire la stabilité du corps social garantissant à tous un égal accès aux ressources primaires, aux soins médicaux ou au travail, et peut éviter, par un système de redistribution, la captation des richesses par quelques individus. Bref, Cannon estime que, en s'inspirant des mécanismes de régulation biologique, les dirigeants pourraient mettre en place les mécanismes de contrôle et de planification permettant de réguler la vie sociale et économique.

Indépendamment des positionnements politiques respectifs de Starling et de Cannon - ce dernier prône une forme de technocratie socialiste contrastant fortement avec la séquence de politique économique libérale qui caractérise les États-Unis jusqu'à la crise de $1929-$, les deux hommes partagent l'idée que la physiologie, la médecine et plus généralement les sciences permettent de fonder des principes moraux et des directions politiques échappant aux idéologies " tribales ». Cet imaginaire sociotechnique - nourri, dans le cas de Cannon, par ses convictions unitariennes - prône l'avènement de sociétés technocratiques. On verra que Cannon aura d'autres occasions de défendre cette utopie «biocratique» dans ses textes politiques.

Ainsi, il semble que plus Cannon s'est convaincu que son ouvrage devait s'achever sur un chapitre portant sur la pertinence du concept d'homéostasie dans le champ politique, moins les titres imaginés ont été centrés sur la physiologie. Pour autant, l'écriture de cet épilogue n'a pas été une formalité. Cannon a même estimé préférable de faire relire les pages à certains de ses collègues de Harvard, le professeur de sociologie Pitirim Sorokin (I889-1968), les économistes Frank William Taussig (1859-1940) et Edwin Francis Gay (I867-I946), ainsi qu'à un ami de Norton, Thomas Nixon Carver (I865-196I). Dans sa lettre du 9 janvier $1932^{161}$, Cannon informe Norton qu'il est tout juste en train de modifier le texte en fonction des commentaires de ces

161 Cannon $\mathrm{I932b}$ derniers. Si Cannon ne les détaille pas, il précise, presque amusé, que Carver a finalement été le plus « indulgent » de tous. Il faut dire que les sympathies que Cannon exprime dans son épilogue pour le socialisme, voire le communisme, ont pu paraître déconcertantes pour Sorokin, par exemple, expulsé d'URSS quelques années plus tôt, en I923, pour dissidence.

Quoi qu'il en soit, les épreuves du futur livre sont envoyées à Cannon à la mi-janvier et renvoyées à Norton dans la foulée. Début février, les deux hommes s'attellent à établir un plan de communication en vue de la sortie officielle du livre. Dans sa lettre du io février I932, Cannon se dit «agréablement choqué » ${ }^{162}$ [agreeably shocked] de savoir que le livre est sur le point de paraître et il confirme en avoir bien reçu six exemplaires. Comme prévu, le livre paraît le i8 février. Pour autant, Cannon n'en a toujours pas fini avec ses «Sorbonne Lectures». Début novembre, Norton informe Cannon qu'une maison d'édition française a manifesté son désir d'acquérir les droits pour l'édition française de The Wisdom of the Body.

\section{La Sagesse du corps: \\ un destin contrarié}

À ce stade, nous sommes en mesure de donner quelques éléments de réponse aux questions soulevées en introduction: pourquoi Cannon est-il un auteur méconnu du public français et pourquoi son rôle dans l'histoire du stress n'est-il pas aussi reconnu que celui de Selye? Nous avons mentionné que Cannon, dans ses conférences comme dans la traduction française de son livre, a éliminé le terme de "stress». Comme nous l'indiquons en note, la cinquième conférence reprend des parties d'un ancien article ${ }^{163}$. La comparaison des deux textes révèle que les termes emotional stress sont traduits par «trouble émotif " et stressfull event, par "circonstances pénibles». L'absence du terme «stress » a donc très certainement contribué à invisibiliser le rôle de Cannon dans l'introduction du concept biologique

162 Cannon $1932 \mathrm{c}$

163 Cannon I928b. 
de stress dans les milieux francophones. Pour autant, on doutera que cette absence explique pourquoi le phénomène physiologique que le concept de stress dénote n'a pas non plus marqué les esprits. Fallait-il en effet connaître le terme de stress pour comprendre ce à quoi il renvoie, soit à l'hypothèse selon laquelle des conditions environnementales, en particulier sociales, pourraient induire de vives émotions, et que ces dernières pourraient, à leur tour, provoquer des dérégulations physiologiques susceptibles d'induire des pathologies somatiques? Il nous semble que des contingences contextuelles, tant intellectuelles que sociales, expliquent plus profondément la difficile réception des travaux de Cannon en France. Parmi celles-ci, nous pouvons d'abord considérer les obstacles que Cannon a rencontrés dans la publication des versions françaises de ses ouvrages phares.

Durant son séjour parisien, Cannon est entré en contact avec les Éditions Masson. C'est ce dont atteste la lettre que lui adressent les éditeurs le i6 juin I930. Nous y apprenons qu'ils sont intéressés à publier à leurs frais la traduction française de son livre sur les émotions, Bodily Changes in Pain, Hunger, Fear and Rage. Il est déjà prévu que Cannon touchera des honoraires forfaitaires à hauteur de 5000 francs, somme qui devrait couvrir ses droits d'auteur ainsi que les frais de traduction ${ }^{164}$. Cette dernière devrait par ailleurs être confiée à Zenon Bacq. La lettre précise enfin qu'il ne manque plus qu'une réponse d'Appleton, l'éditeur américain, qui n'a pas encore communiqué le montant de cession des droits de traduction. En l'absence de nouvelles, les éditeurs français supposent qu'il n'en demandera pas. Dans leur lettre du I8 octobre $1930^{165}$, les Éditions Masson informent Cannon qu'Appleton s'est enfin manifesté. Il réclame cependant I05 dollars pour céder le matériel d'illustration, ce qui semble justifié, mais il ajoute encore I50 dollars de droits de traduction. La conclusion des éditeurs français est sans appel: «Par la période de crise universelle que nous traversons actuellement, il nous serait impossible de prendre cette charge nouvelle à notre compte. » À l'occasion d'un échange entre Cannon

164 Éditions Masson I930a.

165 Éditions Masson I93ob. et Dumas ${ }^{166}$, nous apprenons que le projet de publication est définitivement abandonné. Les deux maisons d'édition, américaine et française, ne sont pas parvenues à s'entendre, et ce malgré la proposition de Cannon de renoncer à ses droits d'auteur. Il ne paraitra donc pas de version française de Bodily Changes in Pain, Hunger, Fear and Rage. Or, il s'en est fallu de peu que la publication de la traduction française de The Wisdom of the Body achoppe elle aussi.

Le 4 novembre $1932^{167}$, Cannon reçoit une lettre des Éditions Payot qui souhaitent publier la version française du livre. Quelques jours plus tard, il contacte René Gayet ${ }^{168}$ pour le prévenir qu'il a recommandé son nom auprès des éditeurs. Gayet accepte par retour de lettre de réaliser la traduction de l'ouvrage, mais précise qu'il ne pourra s'y mettre qu'une fois passé le concours de l'agrégation prévu en juin $1933^{169}$. Le 8 mai I933, Norton transmet à Cannon la copie d'une lettre envoyée par les Éditions Payot ${ }^{170}$. Après examen de l'ouvrage, les éditeurs renoncent finalement à la publication, sous le prétexte que « les circonstances économiques deviennent de plus en plus défavorables ici en France». Norton tente de négocier en personne avec les éditeurs lors d'un séjour en Europe ${ }^{171}$. S'il refuse que Cannon paye de sa poche la première édition, comme c'est l'usage en France, il propose cependant de baisser les droits d'auteur sur les mille premières copies. La proposition est finalement refusée.

Ce n'est que le 24 mai I 938 que Norton annonce à Cannon qu'une nouvelle maison d'édition manifeste son intérêt pour la publication de la version française du livre ${ }^{172}$. Il s'agit cette fois

166 Cannon i93ra.

167 Payot 1932

168 René Gayet (1892-1939) est le gendre du médecin et neurologue français Louis Hallion (I862-I940). Ce physiologiste, professeur de physiologie à la Faculté de médecine et successeur de Hallion à l'École des Hautes Études est un spécialiste de la fonction endocrine et exocrine du pancréas. Cannon l'invite en I932-I933 à l'École médecine de Harvard.

169 Gayet 1932.

$170 \quad$ Payot 1933

171 Norton I933a.

172 Norton $1938 \mathrm{~b}$. 
des Éditions Albatross \& Tauchnitz. La lettre fait mention de deux demandes: le livre ayant déjà six ans, Cannon se voit proposer d'y apporter des additions et corrections, d'une part, et, de l'autre, une préface spécifique à l'édition française. Cannon accepte volontiers les deux demandes. Mais, pour des raisons que les archives ne permettent pas d'identifier, les échanges entre Cannon, Norton et Albatross \& Tauchnitz cessent soudainement et un nouvel acteur entre en jeu à peine quelques jours plus tard. Le 15 juin 1938 , en effet, Norton fait suivre à Cannon une réponse à une lettre datée du 2 juin, signée de Fernand Lelle ${ }^{173}$. Ce dernier, président des Éditions de la Nouvelle Revue critique, sises au 12 rue Chanoinesse dans le $\mathrm{IV}^{\mathrm{e}}$ arrondissement de Paris, fait part à Norton de son enthousiasme à publier la traduction.

Il faut s'intéresser à Albatross \& Tauchnitz pour comprendre ce revirement apparent et trouver quelques rares informations concernant les Éditions de la Nouvelle Revue critique ${ }^{174}$. Tauchnitz est une ancienne maison d'édition allemande, spécialisée dans la publication de livres en anglais, d'auteurs anglo-américains, réservés au marché européen, hors RoyaumeUni. La maison d'édition Albatross a été quant à elle fondée en 1932 par John Holroyd-Reece (1897-1969), Max Christian Wegner (I893-1965) et Kurt Enoch (I895-1982), avec des capitaux anglais. Albatross rachète Tauchnitz en 1934, afin d'intégrer son principal concurrent. Contre toute attente, l'arrivée au pouvoir d'Adolf Hitler (I889-I945) ne signe pas la cessation des activités d'Albatross \& Tauchnitz. La maison d'édition, qui vend dans toute l'Europe, permet au régime nazi de faire entrer des devises étrangères qui alimentent le vaste plan de militarisation de l'Allemagne. Moyennant la censure de certains passages, les livres en langue anglaise, principalement lus par l'élite allemande, ne sont pas perçus par les autorités comme

173 Lelle I938b. Nous n'avons trouvé aucune information concernant Fernand Lelle, dont la signature tapuscrite est clairement identifiable. Il n'y a donc pas de confusion possible entre Fernand Lelle et Fernand Keller qui, en 1938, est le secrétaire général des Éditions de la Nouvelle Revue critique.

174 Nous suivons ici l'étude détaillée sur les Éditions Albatross durant le Troisième Reich de Michele K. Troy (2017). susceptibles d'interférer avec l'endoctrinement du peuple. La montée du nazisme contraint deux des fondateurs - Enoch et Holroyd-Reece, qui sont d'ascendance juive - à installer, dès 1936 , le bureau éditorial à Paris. Le siège financier restant à Hambourg, le régime laisse l'entreprise poursuivre ses activités tant que les devises étrangères entrent en Allemagne. C'est pour éviter cette dépendance envers le marché allemand que la maison d'édition se diversifie en I937. Holroyd-Reece achète des parts importantes d'autres maisons d'édition, dont les Éditions de la Nouvelle Revue critique, fondées en I923 à Paris par Alfred et Fernand Keller, qui tiennent la librairie de L'Arc-en-ciel. Spécialisée dans la publication de roman, la maison d'édition avait commencé en 1932 à éditer quelques auteurs anglo-américains. En d'autres termes, les lettres que Cannon et Norton reçoivent du I2 rue Chanoinesse sont en fait envoyées du bureau parisien d'Albatross \& Tauchnitz ${ }^{175}$.

Quoi qu'il en soit, la lettre du 2 juin 1938 atteste que l'accord financier est déjà fixé. Cannon ne déboursera pas un sou pour la première édition et il touchera des droits d'auteur à hauteur de cinq pour cent, puis dix pour cent, lorsque les cinq mille premières copies auront été écoulées. Conformément aux demandes révélées lors des échanges avec les Éditions Albatross \& Tauchnitz, Cannon va apporter des additions et corrections à l'ouvrage et préparer une préface spécifique à l'édition française. Le 2 juin, Norton propose à Cannon de profiter de la parution de la traduction française pour établir une nouvelle édition

175 Si l'on suit Troy (2017), les services secrets français ne parviendront jamais à déterminer si, depuis son bureau parisien, Holroyd-Reece servait la propagande nazie et/ou renseignait, en qualité d'agent double, le RoyaumeUni. Ses arrangements auront quoi qu'il en soit permis d'assurer la pérennité de la maison d'édition jusqu'à ce que la France soit envahie. En I939, Enoch fuit la France pour les états-Unis et John Holroyd-Reece rejoint le Royaume-Uni, où il continue à diriger, péniblement, les activités d'Albatross. Les éditions Tauchnitz sont quant à elles reprises par les autorités allemandes et réorientées vers la promotion de la culture aryenne. Au sortir de la guerre, les éditions Albatross parviennent difficilement à reprendre leurs activités. Malgré la publication de livres dont les droits avaient été acquis avant le conflit, la société ne parvient pas à échapper à la faillite à la fin de l'année 1950. Le destin des Éditions de la Nouvelle Revue critique n'est pas clair. Elles cessent aussi leurs activités en I95I. 
américaine de The Wisdom of the Body ${ }^{176}$. Dès juillet, Cannon envoie à Norton la liste des modifications qu'il entend réaliser. Quelques additions doivent être apportées au chapitre XVI. Un nouveau chapitre sera ajouté, intitulé «The Aging of Homeostatic Mechanism » (Le vieillissement des mécanismes homéostatiques). Enfin, Cannon montre un certain engouement à l'idée d'écrire la préface à l'édition française:

J'aurai beaucoup de plaisir à la préparation d'une préface pour l'édition française, car j’ai largement construit sur les fondements posés par le physiologiste français Claude Bernard. Je pense que, si les lecteurs français sont satisfaits par ce que j'ai écrit concernant l'homéostasie, ils seront ravis de constater que mes idées sont le prolongement de celles du grand physiologiste français ${ }^{177}$.

La lettre du I 8 juillet 1938 que Lelle adresse à Norton ${ }^{178}$ confirme la réception du contrat d'édition signé et que les Éditions de la Nouvelle Revue critique, qui pensaient faire appel aux services du Docteur Albert Brousseau ${ }^{179}$ (I888-I955) pour la traduction, acceptent que Cannon choisisse le traducteur. Nous ne sommes pas parvenus à déterminer si Cannon a recontacté Gayet, qui avait pourtant accepté de traduire l'ouvrage pendant l'été 1933, avant que le projet de publication des Éditions Payot n'achoppe ${ }^{180}$.

Dans sa lettre datée du 25 novembre 1938 , Bacq écrit : "L'affaire est réglée. Je vais traduire votre dernier livre pour la Nouvelle Revue Critique à Paris. ${ }^{181}$ Bacq, qui devait traduire la version française de Bodily Changes in Pain, Hunger, Fear and Rage avant que le projet ne tombe lui aussi à l'eau, estime pouvoir achever la traduction pour la fin mars. Dans sa lettre

176 Norton I938a.

177 Cannon 1938 , notre traduction.

178 Lelle, 1938a.

179 Il s'agit vraisemblablement d'Albert Brousseau, interne aux Asiles de la Seine. Il obtient un doctorat en médecine en 1920. Sa thèse est intitulée Essai sur la peur aux armées, I9I4-I9I8 (Brousseau I920).

180 Le destin tragique de Gayet n'explique donc pas le fait que la traduction ne lui soit pas confiée. Il meurt un soir de décembre I939 dans un accident d'automobile impliquant son véhicule ainsi qu'un camion de l'armée allemande.

181 Bacq 1938 , notre traduction. du 23 janvier $1939^{182}$, il informe Cannon qu'il a traduit les trois quarts de l'ouvrage. Le 2 février 1939, Bacq confirme avoir bien reçu la préface et que la traduction devrait être achevée début mars, ce qui lui laissera le temps de «l'oublier quelques semaines avant de la relire non pas comme une traduction mais comme un manuscrit en français ${ }^{183}$. C'est avec un peu d'avance sur son planning que Bacq annonce à Cannon, dans sa lettre du 23 mars $1939^{184}$, qu'il a pu apporter les dernières corrections au manuscrit et qu'il entend l'envoyer l'après-midi même aux éditeurs parisiens. Les circonstances semblent enfin favorables à la publication de cette traduction française, que Cannon annonce dans la préface destinée à la seconde édition américaine de The Wisdom of the Body, rédigée au même moment :

La perspective d'une édition française de "The Wisdom of the Body", qui devrait inclure les derniers résultats des études sur la régulation de la stabilité dans la fluid matrix, a conduit à une discussion avec M. Norton concernant la possibilité d'une traduction française simultanée et d'une deuxième édition en anglais ${ }^{185}$.

Mais cet optimisme contraste avec le pessimisme dont Bacq fait preuve à un tout autre sujet. Le contexte géopolitique s'invite dans cette lettre du 23 mars I939, dans laquelle Bacq annonce vouloir remettre le manuscrit l'après-midi même. En référence à la dernière lettre de Cannon, il poursuit :

L'optimisme relatif de votre autre lettre, quant à une possible pression économique sur les États totalitaires, est probablement dépassé compte tenu du comportement récent des nazis en Tchéquie-Slovaquie. Vous vous rendrez compte à quel point nous ne nous sentons pas en sécurité à Liège, avec ces hommes juste à notre frontière. Je n'ai jamais été pessimiste, mais je suis profondément convaincu que cela ne durera pas longtemps avant que les scientifiques de ce pays (moi y compris) soient obligés d'appeler l'Amérique à l'aide ${ }^{186}$.

182 Bacq 1939b.

183 Bacq I939a, notre traduction.

184 Bacq I939c.

185 Cannon 1939[1932], XVII, notre traduction.

186 Bacq I939, notre traduction. 
Le I5 mars, l'armée allemande a envahi la Tchécoslovaquie. La France et le Royaume-Uni entrent en guerre le $\mathrm{I}^{\mathrm{er}}$ septembre 1939. La Belgique est envahie le io mai I940.

La guerre rend la correspondance entre Cannon et Bacq plus difficile. Dans sa lettre datée du 2 janvier 1940, Cannon retourne un courrier des Éditions Nouvelle Revue critique que Bacq lui a fait parvenir quelques jours plus tôt. On peut en deviner le contenu aux commentaires de Cannon: «J'espère qu'une fois la guerre terminée, la France ne sera pas épuisée au point que le livre ne puisse plus paraître. ${ }^{187} \mathrm{Il}$ faut attendre une année pour que les échanges portant sur la traduction française du livre reprennent. Le 28 janvier I94I, Bacq informe Cannon qu'il a pu discuter de la traduction avec l'éditeur le I2 décembre I940, à Paris, et que ce dernier «n'a pas l'intention de la publier bientôt ${ }^{188}$. Dans la France occupée depuis le 22 juin 1940 , le monde de l'édition doit s'astreindre aux mesures de censure de l'administration allemande. Otto Abetz (1903-1958), ambassadeur du Reich à Paris, fait paraitre le 28 septembre 1940 une liste des cent quarante-trois livres politiques interdits de publication. Les éditeurs signent dans la foulée une convention de censure qui les engage à respecter la « liste Otto ». Environ quatre-vingts librairies et maisons d'édition se voient interdire toute activité, dont les Éditions de la Nouvelle Revue critique ${ }^{189}$. La liste Otto ne va pas cesser de s'allonger, incluant les livres d'auteurs juifs, mais aussi, dès juillet I94I, toute édition ou réédition de livres d'auteurs anglo-américains postérieurs à I87I.

Toujours dans sa lettre du 28 janvier I94I, Bacq ajoute cependant qu'« un éditeur belge très sérieux désire reprendre les droits à NRC. ${ }^{190}$. Comme en témoigne la correspondance entre Cannon et Norton, les 28 et 29 mars ${ }^{191}$, la piste de l'éditeur belge va bien être étudiée. Celle-ci n'aboutira cependant pas, puisque La Sagesse $d u$ corps paraîtra finalement aux

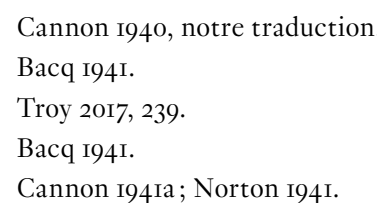

Éditions de la Nouvelle Revue critique, à l'automne 1946, soit deux ans après la Libération. Il s'agit bien de la traduction de Bacq. La préface à l'édition française, datée de I939, y est présente. Cannon y rappelle que le livre est extrapolé des conférences délivrées en I930, lors d'un échange à l'Université de Paris. Il y rend aussi hommage à Bernard: "L'idée centrale de ce livre, écrit-il, "la stabilité du milieu interne de l'organisme chez les vertébrés supérieurs", est directement inspirée des vues précises et de la compréhension profonde de l'éminent physiologiste français Claude Bernard. ${ }^{192}$

La quatrième de couverture indique quant à elle que le livre expose une «vision étonnamment claire et actuelle de ce que devrait être l'organisation sociale si l'on prenait exemple sur les dispositifs de sécurité dont l'évolution a progressivement enrichi l'organisme animal », enfin, qu'il s'agit là de «l'un des derniers ouvrages du vénéré maître physiologiste de Harvard, décédé en $194{ }^{» 193}$. Cannon n'aura en effet jamais pu se féliciter de la parution du livre, annoncée dès I939 dans la seconde édition de la version américaine. Il meurt le I ${ }^{\text {er }}$ octobre I945, à l'âge de soixante-quatorze ans, des suites d'un mycosis fongoïde associé à une leucémie lymphoïde. La maladie, diagnostiquée dès I932, a très certainement été induite par l'exposition prolongée et non protégée de Cannon aux rayons $\mathrm{X}$, lorsqu'il réalisait ses premières expériences sur les mouvements péristaltiques ${ }^{194}$. Par un hasard de l'histoire, La Sagesse du corps paraît en 1946, l'année même où Selye délivre au Collège de France ses conférences sur le syndrome général d'adaptation et sur le stress.

\section{Sagesse, crise et égarement}

Il ne fait aucun doute que l'échec de la traduction du livre Bodily Changes in Pain, Hunger, Fear and Rage, conjugué à la parution

192 Cannon 1946[1939], 5.

193 Cannon I946[1939], quatrième de couverture.

194 La maladie a commencé à impacter la vie de Cannon dès la fin des années I930. Il développe notamment des crises de paralysie faciale, des démangeaisons cutanées sévères, une surdité bilatérale, des lésions ulcérées et un cancer de la peau (Diedrich 20II, 227). 
tardive de La Sagesse du corps, quinze ans après sa première parution américaine et seize ans après le séjour parisien de l'auteur, n'a pas facilité la diffusion des travaux de Cannon en France, ainsi que dans le monde francophone. Mais la réception de La Sagesse du corps contribue aussi à expliquer le faible impact des travaux de Cannon. La réception française de La Sagesse $d u$ corps ne peut se limiter à Georges Canguilhem (1904-1995). Mais elle nous semble particulièrement instructive tant la figure de Canguilhem a dominé les champs de l'histoire et de la philosophie dans la France de l'après-guerre. Ses critiques récurrentes de l'homéostasie, en particulier de l'homéostasie sociale, assorties d'une valorisation des travaux de Selye, éclairent sous un autre angle le rendez-vous manqué entre Cannon et le public français.

On ne compte pas moins de quatre articles dans lesquels Canguilhem se montre particulièrement critique vis-à-vis de Cannon: « Le problème des régulations dans l'organisme et dans la société »195, "Sur les normes organiques chez l'homme» 196 l'article qu'il publie dans l'Encyclopeedia Universalis sous l'entrée «RÉGULATION, épistémologie » ${ }^{197}$, puis « La régulation comme réalité et comme fiction ", tiré de la série de conférences inédites que Canguilhem délivre à l'Université de Louvain en I973, sous le titre général de «Fin des normes ou crise des régulations ${ }^{198}$. En d'autres termes, Canguilhem n'a eu de cesse, tout au long de sa carrière, de mettre en cause les thèses exposées dans La Sagesse $d u$ corps. Or ces critiques sont d'autant plus surprenantes que Canguilhem reste par ailleurs un ardent défenseur de l'holisme, allant jusqu'à affirmer, dans un style proche de celui de Cannon, que «la régulation, c'est le fait biologique par excellence. ${ }^{199}$

À vrai dire, ce n'est pas tant la physiologie holiste de Cannon qui l'interpelle que les liens que Cannon tente d'établir entre homéostasies biologique et sociale:

\footnotetext{
Canguilhem 2002[1955].

Canguilhem I966[1943].

Canguilhem I974[I968].

Canguilhem 1973 .

Canguilhem 1974[I968], 402
}

L'analyse de ces rapports est un tissu de lieux communs de sociologie libérale et de politique parlementaire concernant l'alternance - dans laquelle Cannon voit l'effet d'un dispositif de compensation - entre conservatisme et réformisme. Comme si cette alternance, loin d'être l'effet d'un dispositif inhérent, même à l'état rudimentaire, à toute structure sociale, n'était pas en fait l'expression de l'efficacité relative d'un régime inventé pour canaliser et amortir les antagonismes sociaux, d'une machine politique acquise par les sociétés modernes pour différer, sans pouvoir l'empêcher à la fin, la transformation de leurs incohérences en crises. À observer les sociétés de l'âge industriel on peut se demander si leur état de fait permanent ne serait pas la crise, et si ce ne serait pas là un symptôme franc de l'absence en elles d'un pouvoir d'autorégulation. ${ }^{200}$

Pour comprendre ce passage, il peut s'avérer utile de distinguer les perspectives sociologiques relevant des «théories du conflit», d'une part, de celles relevant des "théories de l'ordre " 201 , de l'autre. Canguilhem s'inscrit clairement dans la première catégorie, tandis qu'il rejette la pensée politique de Cannon dans la seconde ${ }^{202}$

Pour Canguilhem, il y a bien une continuité entre le biologique et le social. Lorganisme vivant est normatif, au sens où son rapport à l'environnement institue des valeurs. Il existe des conditions propices à la vie, que les choix sociaux et politiques sont supposés organiser et garantir. À cet égard, la politique relève pour Canguilhem d'une « biologie de la technique ». Il faut entendre par là que, selon lui, l'organisme humain se dote des institutions politiques qui, comme tout autre outil, sont construites afin de favoriser la vie. Or cette solution de continuité est remise en cause par le passage d'une normativité individuelle à une normativité collective. En société, les valeurs collectives n'expriment pas les valeurs de tous, mais celles d'un groupe social restreint qui fait prévaloir ses propres valeurs sur celles des autres. Pour Canguilhem, la vie sociale est marquée du sceau du dissensus. Aussi, malgré la continuité entre le biologique et le social, l'absence même de consensus politique

200 Canguilhem 1966[1943], 250.

201 Horton 1966.

202 Arminjon 2020 
«naturel » entre les humains rend-elle impossible l'identification du biologique au social. Dans le corps biologique, les normes de régulation sont immanentes. En l'absence de loi immanente au corps politique, la norme devient un problème. C'est justement le rôle de la politique que de produire les instruments permettant de réguler l'ordre social, là où le corps possède spontanément ses propres règles de fonctionnement.

Sur cette base, Canguilhem estime que le fait d'assimiler le corps politique à un corps biologique conduit les théoriciens de l'ordre à se méprendre sur la notion de crise sociale. Pour ces derniers, les crises sont des déviations pathologiques accidentelles de l'ordre social naturel, qui sont automatiquement régulées par des mesures compensatrices. Si Canguilhem vise particulièrement les théories politiques de Thomas Malthus (I766-I834), la critique peut tout aussi bien s'étendre aux philosophies libérales, pour lesquelles les volontés individuelles et égoïstes tendent, comme portées par une main invisible, à établir un équilibre naturel qui s'apparente au bien commun. S'inscrivant dans les théories sociales du conflit - celles de Karl Marx (I8I8-I883), voire d'Auguste Comte (I798-I857) ${ }^{203}-$, Canguilhem soutient que les crises ne sont aucunement des déviations minimes vis-à-vis d'un ordre social naturel, mais l'expression du dissensus propre à toute communauté humaine. Les crises ne sont donc pas assimilées à ses petites déviations devant être compensées afin que la société recouvre son intégrité, mais au moteur des mutations sociales qui font l'histoire des sociétés, sans que cette histoire ne puisse d'ailleurs être assimilée à un progrès. Une fois exposée la position de Canguilhem, on peut se demander dans quelle mesure celui-ci a raison d'associer les thèses politiques que Cannon développe dans le dernier chapitre de La Sagesse du corps aux théories de l'ordre.

Cannon a bien estimé déceler dans certains processus sociaux «des traces de dispositifs grossiers de stabilisation" sociale ${ }^{204}$. La lecture de Canguilhem ne semble pas pour autant résister à l'analyse. Le fait même que Cannon propose de

203 Canguilhem $1974[$ [1968], 403.

204 Cannon I946[1939], 248 s'inspirer de la physiologie afin de trouver les «outils» permettant, potentiellement, d'atteindre une homéostasie sociale indique, justement, qu'il ne souscrit aucunement aux principes de l'organicisme classique, pour lequel corps biologique et corps social entretiennent une stricte relation d'identité. Sous cet angle, la critique de Canguilhem se révèle particulièrement paradoxale. Mais, plus que les biais d'interprétation de Canguilhem à l'endroit de La Sagesse du corps, il nous semble opportun d'analyser la manière dont sa lecture met en exergue certains aspects de la physiologie de Cannon et, surtout, comment ces aspects constituent une réponse scientifique aux problèmes posés par un contexte historique spécifique.

La lecture canguilhémienne nous laisse en effet le sentiment que, pour Cannon, le corps biologique est, comme le corps politique, un organisme dont la stabilité, supposément fixe et harmonieuse, n'est qu'accidentellement soumise aux crises. Par exemple, Canguilhem, en I968 puis en 1973, s'étonne que certains jeunes contestataires, constatant l'échec de l'autorégulation supposée des sociétés libérales, nourrissent l'espoir de refonder des modes de vie archaïques normés par le mythe. Or, écrit-il, «le mythe, c'est l'instrument d'boméostasie sociale de la société dite primitive, il est la représentation de l'ordre social sans lacune et sans résidu, ou, si l'on veut, d'un ordre social trop peu riche en rôles pour donner naissance à plusieurs idéologies ${ }^{205}$. Est-ce à dire que, pour Cannon, les corps homéostatiques, qu'ils soient biologiques ou sociaux, jouissent d'un ordre "sans lacune ni résidu»? Qu'ils sont incapables de sécréter les causes internes de leurs propres crises? Bref, qu'ils n'ont pas d'histoire? ${ }^{206}$

Si Canguilhem en vient là, c'est que la question de l'historicité du vivant est au cœur de sa critique de la physiologie. On connaît les grandes lignes de son analyse des concepts de normal et de pathologique : la maladie est, écrit-il, « une capacité

205 Canguilhem $\mathrm{I973}, 8$, nous soulignons.

206 Cette représentation fixiste et anhistorique de l'homéostasie, a fortiori sociale, est notamment reconduite dans la critique de l'homéostasie sociale à laquelle procède Gilbert Simondon (Simondon I989[1958], I5I) dont la thèse a été dirigée par Canguilhem (voir à ce sujet Le Roux 2007). 
transitive ${ }^{207}$. Il faut entendre par là qu'il n'y a pas pathologie en soi, mais toujours pathologie pour un organisme donné, en rapport avec un milieu. C'est ce qui l'amène à évoquer dans Le Normal et le Pathologique le cas d'une bonne d'enfants ${ }^{208}$ qui découvre son hypotension et les troubles neuro-végétatifs qui l'accompagnent lors d'un séjour en montagne. Cet état pathologique n'est pas absence de norme, mais expression d'une norme alternative, une forme de normativité répulsive qu'il faut opposer à la normativité propulsive ${ }^{209}$ de la grande santé, celle de l'alpiniste par exemple, capable de pousser son organisme au-delà de ses limites. Curieusement, c'est lorsqu'il rapporte ce cas de la bonne d'enfants que l'on trouve chez Canguilhem sa seule référence positive aux travaux de Cannon. Il s'agit en fait de sources de seconde main, empruntées au neurologue Kurt Goldstein (1878-1965). Les dimensions normatives, adaptatives et transitives de l'état pathologique sont exemplifiées selon lui par les chats sympathectomisés de Cannon. L'absence de thermorégulation, tout comme la perte de leur instinct de survie, sont normales tant que l'animal évolue dans un environnement domestique, soit dans les limites protectrices du laboratoire. Elles ne sont anormales que dans la vie non domestiquée, où « s'imposent les exigences soudaines de l'adaptation au milieu $»^{210}$.

Cette référence est d'autant plus surprenante que c'est à Selye et non à Cannon que Canguilhem attribue le mérite d'avoir théorisé le stress et les pathologies de l'adaptation. Dans la préface de la seconde édition de Le Normal et le Pathologique, Canguilhem écrit en effet:

Je devrais, si j'écrivais aujourd'hui cet essai, faire une grande place aux travaux de Selye et à sa théorie de l'état d'alarme organique [...]. Selye a établi que des ratés ou des dérèglements du comportement, comme les émotions et la fatigue qu'elles engendrent, produisent, par leur réitération fréquente, une modification structurale du cortex surrénalien analogue à celle que détermine l'introduction dans le milieu intérieur de substances hormonales, soit impures soit pures mais à hautes doses ou bien de substances toxiques. Tout état organique de tension désordonnée, tout comportement d'alarme et de détresse (stress) provoque la réaction surrénalienne. Cette réaction est «normale», eu égard à l'action et aux effets de la corticostérone dans l'organisme. [...] Mais ces réactions normales (c'est-à-dire biologiquement favorables) finissent par user l'organisme dans le cas de répétitions anormales (c'est-à-dire statistiquement fréquentes) des situations génératrices de la réaction d'alarme. [...] C'est ainsi qu'on a observé, dans la population des villes anglaises soumises aux raids aériens de la dernière guerre, une multiplication notable des cas d'ulcère gastrique ${ }^{211}$.

Or, pour Canguilhem, il ne fait aucun doute que l'activation chronique des mécanismes normaux d'alarme, puisqu'elle est susceptible d'induire des pathologies, nous amène « assez loin de la sagesse du corps $»^{212}$.

Pourtant, si les deux séries de conférences présentent bien un mérite, c'est celui d'infirmer la thèse selon laquelle l'homéostasie renverrait à un idéal de stabilité physiologique fixe et parfaitement harmonieuse. Les vignettes cliniques mobilisées par Cannon, son approche des chocs, en temps de guerre comme en temps de paix, tout comme l'hypothèse d'une mort endosomatique, qui préfigure ses recherches sur la mort vaudoue, témoignent du contraire. Il ne peut y avoir de stabilité organique parfaite, dès lors que l'organisme en vient, dans certaines circonstances, à sécréter les causes de ses propres pathologies, voire de sa propre mort. On ne saurait assimiler l'homéostasie à une fixité organique, dès lors que les relations familiales, les conditions économiques ou la guerre produisent, via le système sympathico-surrénalien, des régimes de fonctionnement physiologique différenciés. En d'autres termes, Canguilhem aurait pu trouver dans les cas cliniques présentés par Cannon, non seulement de quoi reconsidérer sa compréhension des thèses de celui-ci, mais encore, et surtout, de quoi étayer ses conclusions sur l'historicité biosociale de la normalité. Comme le note en effet Canguilhem, «la physiologie humaine est [...] toujours plus ou moins physiologie appliquée, physiologie du travail, du sport, du loisir, de la vie en altitude, etc., c'est-à-dire étude

211 Canguilhem I966[1943], 4.

212 Canguilhem I966[I943], 264. 
biologique de l'homme dans des situations culturelles génératrices d'agressions variées ${ }^{213}$. Or les vignettes cliniques mobilisées par Cannon nous démontrent que sa physiologie est bien une physiologie de l'adaptation et une pathophysiologie des troubles de la fonction d'adaptation, en particulier aux situations sociales, et, par conséquent, que le fonctionnement physiologique est toujours historiquement situé.

Canguilhem aurait sans doute eu une vision différente des travaux de Cannon, s'il avait disposé d'une synthèse permettant de relier ses recherches sur les émotions d'une part, et sur l'homéostasie, de l'autre. Les présentes conférences comblent partiellement ce vide, que l'article « Stress and Strain in Homeostasis » comblera définitivement ${ }^{214}$, mais qui, parce qu'il a été publié en anglais, n'aura certainement pas atteint le public français. Or c'est peut-être à Jean Starobinski qu'il revient d'avoir reconnu le rôle de Cannon dans la mise en équation des concepts d'homéostasie, d'émotions et de stress, et d'en avoir saisi les implications sociales.

En 1952, Jean Starobinski publie dans la revue Critique une recension du livre The Physiology and Pathology of Exposure to Stress que Selye publie en $1950^{215}$. Dans cette recension, intitulée "La "Sagesse du corps" et la maladie comme égarement ${ }^{216}$, il rappelle que Selye se réclame explicitement des thèses de Cannon. Selye parle de "réactions d'alarme" en lieu et place des "réactions de défense " de Cannon et, bien que chacun mobilise des circuits neurologiques et des hormones distincts, il n'en reste pas moins que le modèle de Selye complète plutôt qu'il ne supplante celui de Cannon. Starobinski évoque aussi les limites de la théorie du stress. En effet, le syndrome d'adaptation général est une réponse stéréotypée au stress, mais il n'explique ni les différences interindividuelles de vulnérabilité au stress, ni les raisons pour lesquelles le stress induit divers types de pathologie. En définitive, invoquer le stress, nous dit Starobinski, risque de devenir la «solution de paresse » 217 dans toute recherche des causes des maladies. Mais, pour ce qui nous intéresse, Starobinski a bien perçu que les maladies $\mathrm{du}$ «stress» nous placent dans un contexte historique, voire une pathocénose bien précise:

Selye évoque à plusieurs reprises les maladies que l'on groupe volontiers sous le nom générique de «maladies de civilisation": thyréotoxicose, ulcère gastro-duodénal, hypertension dite essentielle. La fréquence de ces maladies augmente en effet de façon impressionnante dans les centres urbains et dans les conditions psychologiques de la vie civilisée [...]. Mais on aura peine à se tenir entièrement satisfait par cette simple explication. Si on la considérait comme valable, on admettrait implicitement que la civilisation a multiplié les agressions auxquelles les individus doivent répondre en s'adaptant. Est-ce bien vrai? La civilisation, d'abord, n'apporte-t-elle pas une protection perfectionnée contre les agressions du milieu extérieur? ${ }^{218}$

La diffusion limitée des traductions françaises des livres de Cannon a sans doute restreint la circulation de ses idées, par ailleurs réduites, durant l'après-guerre, à la critique de ses extrapolations politiques. Mais, suivant Starobinski, on peut se demander si dans le contexte social et politique français du début des années 1930, le public n'était tout bonnement pas disposé, intellectuellement et culturellement, à évaluer la pertinence potentielle des théories de Cannon. Principalement élaborées dans le but de contribuer à la compréhension et à la prise en charge des maladies chroniques, celles-ci répondaient davantage aux préoccupations des institutions de santé états-uniennes que françaises.

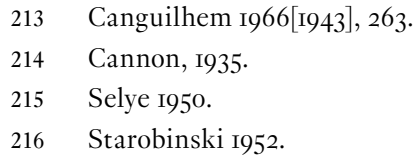

217 Starobinski 1952, 358.

218 Starobinski 1952, 359 
Sélectionner les plus résistants ou préserver la vitalité: de l'hygiénisme aux déterminants sociaux de la santé

George Weisz a montré qu'il faut attendre les années 1960 pour que les institutions de santé publique françaises commencent à s'intéresser aux maladies chroniques et transforment, très progressivement, les anciens sanatoriums et hospices en hôpitaux dévolus aux malades chroniques ${ }^{219}$. Aux États-Unis, en revanche, la préoccupation pour les maladies chroniques remonte au moins à I908, lorsque le président Theodore Roosevelt ( 1858 I919) confie à Irving Fisher (I867-I957), économiste de l'Université de Yale, la préparation d'un rapport sur l'état de santé de la population américaine. Dans son Report on National Vitality, Fisher ${ }^{220}$ évalue à un milliard de dollars les pertes économiques occasionnées par les maladies chroniques. Ces maladies étant considérées comme dues aux modes de vie, Fisher estime qu'elles pourraient être évitées moyennant l'application de mesures médicales connues, tel le suivi de l'état de santé des citoyens, la mise en place de mesures eugéniques et, surtout, le développement de l'hygiène. La parution de The Challenge of Chronic Diseases ${ }^{221}$, l'année même où Cannon prépare son séjour parisien, témoigne du fait que les maladies chroniques sont devenues un enjeu central des instances de santé publique états-uniennes. Le mouvement est amplifié par le développement de l'industrie assurantielle et par la réorganisation des institutions hospitalières. Or nous avons de bonnes raisons de penser que ce décalage culturel entre les États-Unis et la France des années 1930 a pu jouer un rôle dans la réception des conférences de Cannon. Ce hiatus ressort tout particulièrement de l'analyse comparative des préoccupations intellectuelles de Cannon et de celles de ses collègues français contemporains. Nous limiterons notre analyse à ceux avec qui Cannon entretenait des rapports plus ou moins directs et qui, pour certains, ont assisté à ses conférences.

219 Weisz 20I4, 2II.

220 Fisher 1909

221 Boas/Michelsohn I929.
Georges Dumas, avec qui Cannon entretient une correspondance régulière et qui est présent durant ses conférences parisiennes, représente un interlocuteur privilégié. Ses nombreux articles sur les émotions, synthétisés dans La vie affective. Physiologie - psychologie - socialisation ${ }^{222}$, ont contribué à populariser les théories de Cannon dans le monde francophone. C'est aussi à lui que l'on doit les traductions françaises des textes de James touchant à la question des émotions, ainsi que la traduction française du livre de Lange ${ }^{223}$. Dumas s'oppose tout particulièrement aux thèses finalistes de Darwin, de Wundt et de Spencer, pour qui les expressions des émotions (la toux dans l'embarras, le hérissement des poils qui impressionne l'ennemi, etc.) auraient été d'abord volontaires, puis associées, par répétition, aux impressions sensorielles qui les ont originellement causées. On note que, pour Dumas, « les théories de l'expression que Darwin, Spencer et Wundt ont formulées, et qui sont restées si longtemps célèbres, avaient, en dépit de la part de vérité qu'elles contiennent, le grave défaut de n'être ni assez physiologiques ni assez sociales ${ }^{224}$. L'antifinalisme de Dumas vise surtout à conforter sa propre théorie de l'origine sociale de l'expression émotionnelle. Il estime qu'il est impossible de séparer l'expression sociale de l'expression physiologique de l'émotion, tant la collectivité impose des schémas à la physiologie de l'émotion. En d'autres termes, les différentes expressions émotives ne relèvent pas de réflexes innés, mais sont acquises au travers des usages sociaux, comme le sont les «signes » d'un langage.

Même si Dumas concède que Cannon a contribué à reléguer la théorie James-Lange dans «l'histoire de la psychologie » ${ }^{225}$, il reproche tout autant à sa théorie des émotions d'être empreinte de finalisme et de manquer de fondements scientifiques. Dumas n'hésite pas à convoquer les divers travaux semblant indiquer ${ }^{226}$ que l'adrénaline ne se diffuse pas dans le sang

222 Dumas 1948

223 Lange I895[1885].

224 Dumas 1922, 55

225 Dumas I948, I73.

226 Gley/Quinquaud I9I8; Stewart/Rogoff I9I6. 
mais qu'elle s'y dégrade rapidement, ou que les états de fureur apparaissent indépendamment de la sécrétion d'adrénaline ${ }^{227}$. La thèse de Cannon selon laquelle les manifestations somatiques des émotions résulteraient d'une innervation sympathique d'origine nerveuse, augmentée chimiquement par la circulation d'adrénaline, serait donc contestable. Dans ses conférences sur les émotions, Cannon conteste très précisément les données mobilisées par Dumas. Mais ce qui nous importe ici, c'est que l'intérêt de Dumas pour les mécanismes permettant à la collectivité d'imposer "des schémas » à la physiologie de l'émotion ${ }^{228}$ n'est abordé que dans le cadre d'une critique du finalisme et de l'origine des expressions faciales. De même, si Dumas établit des liens entre des pathologies et la vie émotionnelle, ce n'est que lorsqu'il se réfère à des pathologies cérébrales susceptibles de mettre au jour, dans une perspective anatomopathologique, les bases cérébrales de la vie émotionnelle. Dans tous les cas, la possibilité que des situations sociales induisent, via la physiologie des émotions, des pathologies mentales ou somatiques, n'est jamais évoquée par Dumas. Pourtant la thèse qu'il défend, celle d'une sociabilisation de la vie émotionnelle, pourrait parfaitement mener à concevoir des troubles émotionnels d'origine sociale. Est-ce à dire que les physiologistes français de l'entre-deux-guerres ne se souciaient pas, comme c'était le cas aux États-Unis, de ce qui était susceptible de réduire la «vitalité nationale" ? Bien sûr que non. Mais les savants préoccupés par la baisse de vitalité des Français ne vont, pas plus que Dumas, évoquer l'hypothèse que la physiologie des émotions puisse constituer une piste de réflexion intéressante. Et pour cause puisque, dans la France de l'entre-deux-guerres, ce n'est pas le mode de vie propre aux sociétés en voie d'industrialisation que l'on tient responsable de la dégradation de la vitalité du peuple français.

Il n'y a qu'à s'intéresser aux écrits politiques de Richet pour s'en convaincre. Si Cannon admire le physiologiste, qu'il rencontre à plusieurs reprises, leurs orientations sociales et politiques s'avèrent radicalement opposées. Dans La Sélection bumaine, Richet défend un anarchisme libéral ${ }^{229}$ radical, visant à réduire toute intervention de l'État au minimum, si ce n'est pour encadrer les «lois naturelles» du darwinisme social qui permettront aux «meilleurs ", la race blanche, de survivre et de se reproduire ${ }^{230}$. Nul besoin ici de détailler les thèses racistes que Richet soutient dans L'Homme stupide ${ }^{231}$. Il voit un danger de dégénérescence tout particulier dans la reproduction des Blancs avec ce qu'il nomme les «Noirs", les «Jaunes» et les "Rouges ". A contrario, on ne trouve pas chez Cannon de thèses racistes ni eugénistes et, bien évidemment, pas de promotion d'un anarchisme libéral, bien au contraire ${ }^{232}$. Mais il importe

229 Pour Richet, «l'anarchie, c'est une société extrêmement libérale, beaucoup plus libérale qu'aucune de nos sociétés actuelles. C'est un État dans lequel l'individu ne sera pas écrasé par la collectivité. Tout homme pourra s'y mouvoir librement, à ses risques et périls, devenir riche ou pauvre, selon ses talents ou son travail. L'État ne lui commandera rien; mais l'État ne lui donnera rien. Il sera responsable de ses actes. Tant pis pour lui s'il a été paresseux ou imprévoyant. » (Richet I919a, 99) Pour Richet, l'anarchie est un mode de gouvernement qui, se rapprochant de la sélection naturelle, doit permettre d'améliorer la race. Le rôle de l'État serait limité au minimum, si ce n'est pour accélérer la sélection des plus aptes, en empêchant la reproduction des Blancs avec des races qu'il considère inférieures (les « Noirs", les «Jaunes» et les «Rouges") et en contribuant à l'élimination des individus malades, infirmes ou débiles. Richet aura l'occasion de défendre ses positions en prenant la présidence de la Société française d'eugénisme de I920 à 1926.

230 Richet I9I9a, 99.

231 Richet I9I9b.

232 Notre analyse des positions de Cannon contraste avec celle de Maria Dietrich, à qui l'on doit une biographie de Cornelia Cannon (Diedrich 20II). L'eugénisme, visant notamment à préserver la vitalité de la « race blanche», trouve de nombreux défenseurs dans l'élite états-unienne des années I930, en particulier dans l'entourage de Cannon. Son épouse, Cornelia Cannon, assiste à la First National Birth Control Conference, qui se tient du II au I3 décembre I92I au Plaza Hotel de Boston. À une époque où il est encore interdit de parler de méthodes de contraception dans bien des États du pays, Cornelia est durablement marquée par la conférence de Margaret Sanger (I879-1966), fondatrice de l'American Birth Control League, qui donnera naissance au planning familial. Cornelia Cannon embrasse cette cause féministe et pro-eugéniste, un engagement qu'elle mène notamment dans ses romans. Certes, dans la première partie du XX siècle, l'information sur la sexualité et sur les méthodes de contraception encore limitées 
surtout de s'intéresser aux raisons qui justifient, toujours selon Richet, que l'État, dont il entend pourtant limiter l'intervention, se voit cependant chargé d'améliorer la race. Pour Richet, c'est la Grande Guerre qui a dévitalisé la France, en opérant une sélection darwinienne «à rebours » et, ce faisant, en éliminant « les braves, les jeunes, les forts, les vigoureux, les beaux " pour ne laisser survivre, « pour perpétuer l'espèce, que le rebut humain ${ }^{233}$.

Ce puissant sentiment de dévitalisation de la nation n'est pas l'apanage d'une élite intellectuelle qui, comme Richet, incarne les excès d'une idéologie libérale et eugéniste. On le retrouve chez Toulouse, dont la sensibilité politique est pourtant radicalement opposée à celle de Richet. En I932, Toulouse rejoint notamment le cabinet de Justin Godart (I87I-I956), ministre de la santé publique sous le gouvernement du Front Populaire ${ }^{234}$. Le cas de Toulouse offre un éclairage particulièrement intéressant sur le contraste entre les solutions scientifiques que Cannon entend donner aux problèmes sociaux de son époque et celles qu'imaginent ses collègues français. Il le permet d'autant mieux que Cannon a rencontré Toulouse lors

représentait le seul moyen à disposition des femmes pour maîtriser des grossesses perçues comme une charge, voire un danger de propagation des maladies. Le féminisme de Sanger, auquel adhère Cornelia Cannon, n'en reste pas moins controversé. Les mesures radicales comme la stérilisation des malades ou des "dégénérés ", sont assorties de thèses racialistes. Maria Dietrich laisse cependant penser que Walter Cannon partageait les convic tions de sa femme (Diedrich 20II, 72). Une exploration thématique des archives s'imposerait. On ne trouve cependant aucune référence à l'eugénisme ni aux minorités raciales dans les textes politiques de Cannon, tout au contraire. Par exemple, une lettre datée du 4 juin I909, adressée à William James, atteste que Cannon attribue une origine sociale et non biologique à l'infériorité des minorités ethno-raciales. Reprochant à James ses préjugés contre les défenseurs de la vivisection, Cannon écrit: «Ils appartiennent à cette classe de préjugés déraisonnables portant sur la race et la condition sociale, selon lesquels les hommes sont regroupés, classés et labellisés empreints [les préjugés] de notions doctrinales sur ce qu'ils doivent être et les vies qu'ils doivent mener. Nous plaçons inexorablement le nègre [negro] dans une position inférieure, puis nous nous écrions: "Voyez, il est incapable de s'élever".» (Cannon I909, notre traduction)

233 Richet I9I9b, 44

234 Pour Toulouse, voir Huteau 2002. de son séjour en France et lui emprunte le terme de «biocratie ${ }^{235}$ dans une conférence donnée au MIT en I933 et publiée dans la foulée (Cannon, I933a) ${ }^{236}$.

Pour Toulouse aussi, pour qui tout phénomène social trouve sa cause dans la physiologie, la guerre a bien diminué la capacité de travail des Français. Selon lui, ce n'est pas tant un effet de sélection à rebours qui l'explique qu'« un phénomène normal de réaction physiologique » : « À toute excitation, ajoute$\mathrm{t}$-il, - et la guerre força les nerfs à se tendre au maximum - succède une période de dépression compensatrice. $\gg{ }^{237}$ Plus que Richet, Toulouse accorde un rôle important aux causes sociales. Les mesures qui permettraient selon lui aux individus de se reproduire en toute responsabilité - qu'il nomme prophylaxie ou hygiène mentale - n'en sont pas moins eugéniques :

L'eugénique répond évidemment au premier desideratum de la prophylaxie mentale qui veut que l'on s'oppose à la procréation d'êtres dégénérés et par là voués aux psychopathies. Mais la prédisposition héréditaire ou congénitale n'est pas le seul facteur des troubles mentaux.

235 Le concept de «biocratie» apparait chez Auguste Comte dans le $\mathrm{I}^{\text {er }}$ tome du Système de politique positive (Comte 1967 [1851]) où il est intimement lié à celui de «sociocratie »: «Le développement normal de l'unité biocratique ne pourra être pleinement systématisé que quand l'unité sociocratique sera devenue irrévocable. (Comte 1967 [1851], 630) Pour Comte, le terme désigne ainsi une forme de vie sociale fondée sur l'égoïsme naturel sur laquelle la sociocratie pourra dans un second temps s'appuyer afin de constituer un système politique juste et équitable. Il est peu probable que Cannon ait trouvé son inspiration chez Comte puisqu'il utilise le terme au sens de Toulouse, soit comme un type de technocratie fondée sur les connaissances de la biologie.

236 Ayant eu vent de la conférence du 5 février 1933 au MIT, Norton écrit à Cannon le 7 février: «Je serais très heureux de vous envoyer instantanément un contrat pour un livre de vous sur la Biocratie.» (Norton I933b) Dans sa réponse du 8 février, Cannon exprime son étonnement quant au fait que la conférence ait eu un tel écho et ses craintes que le Tecbnological Revierw veuille le publier. Il ne laisse en conclusion aucun espoir à Norton quant à l'écriture d'un livre sur le sujet: «J'ai dit à la plupart des personnes qui m'ont consulté qu'elles trouveraient l'histoire complète dans l'Épilogue de "La Sagesse du Corps". Vous pouvez être certain que je ne suis pas prêt de signer un contrat pour un livre sur la "biocratie". (Cannon I933, notre traduction) Dépité, Norton lui répond le I5 février qu'il espère que « la biocratie n'est pas aussi morte que la technocratie» (Norton I933, notre traduction).

237 Toulouse I92I, I4I. 
Les causes occasionnelles méritent d'être retenues plus qu'on ne l'a fait jusqu'ici, surtout au sortir d'une période particulièrement difficile, où les organismes physiques et mentaux ont été ébranlés par les fatigues, les privations et les émotions de la guerre. La mise en œuvre des lois eugéniques appartient au domaine de l'hygiène générale; la lutte contre les causes occasionnelles demande au contraire une culture psychiatrique très spéciale ${ }^{238}$.

Il est bien question que la France, détruite par la guerre, « soit restaurée dans son génie propre et agrandie dans sa force vive ${ }^{239}$. Or, pour revitaliser ce peuple dégénéré, il s’agit de doubler la sélection biologique d'une sélection sociale.

Par ailleurs, la revitalisation du peuple ne peut pas se faire dans un système politique démocratique - qui confierait le pouvoir à des incompétents -, ni dans un système aristocratique - qui ne peut être mû que par des intérêts égoïstes. C'est donc au biologiste que Toulouse accorde la prérogative de diriger le peuple: «Le point de vue biologique, écrit-il, doit être à la base de toute organisation de la société, et c'est pourquoi j’ai appelé Biocratie ce système d'organisation rationnelle de toute nation moderne. ${ }^{240}$ Technocratie totale, la biocratie confie aux sciences de la vie l'autorité sur la vie des administrés : choix des aliments, des vêtements, du nombre d'enfants, du type d'éducation, etc. ${ }^{241}$

On peut dès lors mettre au jour ce qui différencie les visées politiques, pour ne pas dire biopolitiques ${ }^{242}$, de Cannon d'une

238 Toulouse/Genil-Perrin I920, 5 II.

239 Toulouse I92I, 284

240 Toulouse I92I, 305

241 Toulouse I92I, 285

242 Pour Michel Foucault, la biopolitique est un type de gestion politique du vivant qui s'exerce sur l'«homme-espèce», et non sur l'individu comme dans la société disciplinaire. On note que la pensée homéostatique est, selon lui, indissociable de l'émergence de la biopolitique: «Il s'agit surtout d'établir des mécanismes régulateurs qui, dans cette population globale avec son champ aléatoire, vont pouvoir fixer un équilibre, maintenir une moyenne, établir une sorte d'homéostasie, assurer des compensations; bref d'installer des mécanismes de sécurité autour de cet aléatoire qui est inhé rent à une population d'êtres vivants, d'optimaliser, si vous voulez, un état de vie.» (Foucault 1997[1976], 219) L'existence de biopolitiques alternatives (celles d'un Cannon, qui contraste radicalement d'avec celles d'un Richet part, et de Richet et Toulouse de l'autre. Pour ces deux derniers, la sélection naturelle et/ou sociale constitue une force permettant au biologiste de modeler une population capable de résister, physiologiquement et intellectuellement, aux défis de la modernité ${ }^{243}$. Dans le cas de Cannon, la biocratie ne consiste aucunement à utiliser le savoir biologique afin de construire de toutes pièces l'individu moderne idéal. La politique doit fournir les outils sociaux permettant de protéger les ressources vitales des travailleurs, dont les limites, mises à l'épreuve par le développement de l'industrialisation dans le contexte particulièrement libéral des États-Unis des années 1920, sont révélées par les soignants et les physiologistes. On ne s'étonne donc pas que Cannon défende des mesures visant à préserver le travailleur de la fatigue et de l'épuisement au travail tandis que Richet, comme Toulouse, voient les revendications sociales en faveur d'une diminution du temps de travail comme les symptômes mêmes d'une dégénérescence. Comme Jackson l'a noté, le concept de stress n'est pas chez Cannon qu'une construction sociale qui viserait à légitimer le mode de production et de consommation des sociétés modernes. Il est aussi la marque d'une prise de conscience sociale de "l'impact de l'évolution des modes de vie et du travail sur la santé » ${ }^{244}$. À cet égard, les biocraties de Cannon et de Toulouse sont bien différentes. Si les deux chercheurs s'accordent sur la nécessité de fonder un nouvel ordre social technocratique, l'état biocratique de Toulouse entend réguler l'ensemble des activités humaines, alors que pour Cannon il n'intervient qu'à un niveau structurel:

Les conséquences néfastes de l'instabilité de la nation apportent en dernier ressort la misère aux ultimes unités sociales [...]. Mais la

ou d'un Toulouse) amène à se demander si certaines politiques de la vie ne peuvent pas être perçues comme des résistances biopolitiques (Renault 2008), visant moins à normaliser qu'à objectiver des inégalités sociales, a fortiori celles de santé (Fassin 2009).

243 Dans un article sur la « Méthode du profil psychologique », visant la « reconnaissance et la mesure de la vigueur intellectuelle», V. Kovarsky attribue très clairement ces vues à Toulouse: " "Tout le progrès ne peut se réaliser que par des cerveaux capables de résister au travail intensif lié à la vie moderne", dit M. Toulouse.» (Kovarsky 1930, 805, nous soulignons).

244 Jackson $2013,16$. 
stabilité de la société dans son ensemble et la stabilité de ses membres sont aussi intimement liées. Dans la mesure où la stabilité sociale favorise la sécurité, tant physique que mentale, des membres de l'organisation sociale, elle favorise également leur plus grande liberté, en leur assurant l'opportunité de subvenir à leurs besoins, en remplaçant la peur par la confiance et en leur assurant une santé et une sécurité raisonnables ${ }^{245}$

La biocratie de Cannon se limite à réduire l'insécurité dans trois principaux secteurs : l'économie, le travail et la santé. Cet environnement social doit pouvoir favoriser la stabilité de l'environnement intérieur, sans que des conduites précises soient, comme pour Toulouse, édictées. On peut se demander dans quelle mesure la position de Cannon n'ouvre pas ici une voie alternative aux idéologies eugénistes et hygiénistes héritées du XIX ${ }^{e}$ siècle. Il ne s'agit plus de fonder, sur la biomédecine, les mesures politiques et sanitaires destinées à sélectionner ou à encourager les comportements individuels s'accordant aux besoins socioéconomiques du moment. Il s'agit d'imaginer, à partir du savoir biomédical, les refontes institutionnelles qui garantiront la mise en place d'un environnement social capable de neutraliser les déterminants sociaux susceptibles d'affecter l'intégrité physiologique et sociale de tous.

Il est dès lors très probable que les thématiques abordées par Cannon, révélant sa solution personnelle aux problèmes de santé publique tels qu'ils se posaient dans le contexte étatsunien, ne pouvaient trouver aucun écho dans la culture de santé publique de la France de l'entre-deux-guerres, occupée à reconstruire le pays et à construire, littéralement, ses forces vives. Il ne s'agit pas de négliger la prégnance des idéologies eugénistes et hygiénistes aux États-Unis, bien au contraire. Cependant, l'évolution du contexte sociopolitique et sanitaire américain va $\mathrm{y}$ favoriser plus rapidement qu'en France la diffusion des thèses de Cannon.

\section{Homéostasie et déterminants biopsychosociaux de la santé}

Si cette série de rendez-vous manqués entre Cannon et la France contribue à expliquer la faible diffusion et réception de ses travaux, il n'est pas impossible que les mutations sociopolitiques qui touchent actuellement le monde et la France nous amènent cette fois à redécouvrir le rôle que Cannon a joué dans l'histoire de la santé publique.

Revenons en 1930, lorsque le couple Cannon rentre de son séjour parisien. Cela fait près d'une année que l'économie américaine a été ébranlée par le krach boursier de 1929. D'innombrables banques ont fait faillite, entrainant dans leur chute de nombreuses entreprises. On estime qu'en I933, quinze millions de travailleurs ont perdu leur emploi. On ne compte plus les paysans expropriés et les locataires expulsés, qui viennent remplir les boovervilles, du nom que l'on donne aux bidonvilles formés sous la présidence d'Herbert Hoover (I874-I964). Franklin Delano Roosevelt est élu président en 1932. Il lance successivement les deux Nerw Deals, en I933 puis en I935, afin de sortir les États-Unis de la Grande Dépression. Le beau-fils de Cannon, Arthur Meier Schlesinger Jr (I9I7-2007), historien de Harvard et conseiller de Roosevelt, aura tenté de convaincre Cannon que les mesures mises en place dans le cadre du Nerw Deal ('Emergency Banking Act, l'Agricultural Adjustment Act, le National Labor Relations Act et le Social Security Act) s'apparentent à une mise en place des protections sociales que Cannon invoquait dans son épilogue à La Sagesse du $\operatorname{corps}^{246}$. En républicain convaincu, Cannon ne votera jamais pour Roosevelt ${ }^{247}$.

246 Valenstein 2005, 220.

247 On notera par ailleurs que c'est le Parti républicain, fondé par Abraham Lincoln, qui ratifia le $\mathrm{I}^{\mathrm{e}}$ amendement de la Constitution, garantissant les droits civiques de tous, sans considération de race. Le parti républicain s'est inscrit dans une lignée progressiste remportant le vote des plus déshérités (principalement la communauté afro-américaine). Ce n'est qu'à partir de l'élection de Roosevelt, en 1932, et du lancement du New Deal, que le Parti démocrate est parvenu à capter les votes progressistes, comme c'est encore le cas encore aujourd'hui. Cannon est très certainement resté attaché aux valeurs sociales que défendait 
Pourtant, les travailleurs américains n'auront jamais bénéficié d'autant de protection sociale que dans cette courte période de l'histoire américaine. Le 25 mai 1972, lorsque se tient à Brooklyn le symposium célébrant le centième anniversaire de la naissance de Cannon (I87I-I945), le monde a quelque peu changé. La crise est loin et la Seconde Guerre mondiale a très largement contribué à redresser l'économie américaine. Les mouvements de 1968, conjugués au retour des gouvernances libérales qui succèdent à la présidence de Lyndon B. Johnson (1908-1973), attisent cependant une autre crise, celle suscitée par les inégalités, touchant en particulier les minorités raciales.

On insiste souvent sur le rôle que Cannon a joué dans le développement de la cybernétique ${ }^{248}$. Mais c'est dans le champ de la santé publique que les travaux de Cannon ont joué, et jouent toujours, un rôle central. C'est en particulier dans celui de l'épidémiologie sociale, cette branche de l'épidémiologie qui étudie «la distribution sociale et les déterminants sociaux de la santé ${ }^{249}$, que Cannon reste une référence incontournable. Comme le rappelle Nancy Krieger, "au milieu des années I950, les notions d'"homéostasie", de "stress" et de "déséquilibre", en lien avec ce que l'on nommait explicitement la privation matérielle et psychologique, sont devenues des concepts

le Parti républicain dans la première partie du $\mathrm{XX}^{\mathrm{e}}$ siècle (voir à ce sujet Becker 2016, 9).

248 Le physiologiste mexicain Arturo Rosenblueth (I900-I970) travaille auprès de Cannon de I930 à I944. Cannon aurait souhaité qu'il reprenne son poste. Il retourne finalement au Mexique où il est nommé chef du laboratoire de physiologie de l'Institut national de cardiologie de Mexico. Rosenblueth est considéré comme l'un des fondateurs de la cybernétique, tout comme le mathématicien Norbert Wiener (I894-1964), qui est lui aussi un proche de la famille Cannon. Wiener, se référant à La Sagesse du corps, écrit: « Tout manuel complet sur la cybernétique devrait contenir une analyse détaillée et approfondie des processus homéostatiques, dont de nombreux cas individuels ont fait l'objet de discussions assez détaillées dans la littérature." (Wiener 196r[I948], II5) Dans ses travaux sur l'histoire du concept de régulation, Canguilhem est le premier à limiter la postérité de la notion d'homéostasie au seul champ de la cybernétique et à omettre de signaler le rôle central qu'elle va jouer dans le champ de la physiologie, notamment celle du stress.

249 Berkman/Kawachi 2000, 6, notre traduction. fondamentaux de la théorie épidémiologique psychosociale ${ }^{250}$. C'est en se référant au modèle homéostatique que différents acteurs clefs dans le développement de la santé publique étatsunienne, comme Iago Galdston (I895-I989) ${ }^{251}$, Harry Kruse (1900-I977) ${ }^{252}$ ou René Dubos (I90I-I982), s'accordent à «envisager la possibilité que des facteurs sociaux puissent accroître le risque de maladie en augmentant la susceptibilité générale à la maladie ${ }^{253}$. Dubos s'intéresse principalement aux effets du stress sur le développement des maladies infectieuses. Mais il ne faut pas attendre longtemps pour qu'une figure centrale de l'épidémiologie sociale moderne, John Cassel (I92I-I976), mobilise à son tour le modèle homéostatique, afin de fournir un cadre théorique général aux données épidémiologiques et expérimentales révélant le lien causal entre le stress psychosocial et l'apparition de maladies de civilisation comme l'hypertension ${ }^{254}$. La question des causes sociales de l'hypertension artérielle dans la population en général et, en particulier, dans la population afro-américaine va, dans le contexte des mouvements sociaux des années I970, assurer à nouveau la pérennité du concept d'homéostasie et ses mutations ${ }^{255}$. Peter Sterling (1940-) et Joseph Eyer (194420I7), reprochant à l'homéostasie de conceptualiser une «stabilité au travers de la constance ", vont forger le concept d'allostasie afin d'insister sur la capacité de l'organisme à maintenir une «stabilité au travers du changement » ${ }^{256}$. Bruce McEwen (1938-) tente quant à lui de forger une théorie générale susceptible d'intégrer plusieurs niveaux de régulation (homéostasie, allostasie, charge allostatique, surcharge allostatique) et leurs pathologies respectives ${ }^{257}$. C'est dans ce contexte que Richard Wilkinson (1943-) et Michael Marmot (I945-), qui prend la présidence de la Commission pour les déterminants sociaux de l'OMS de 2005

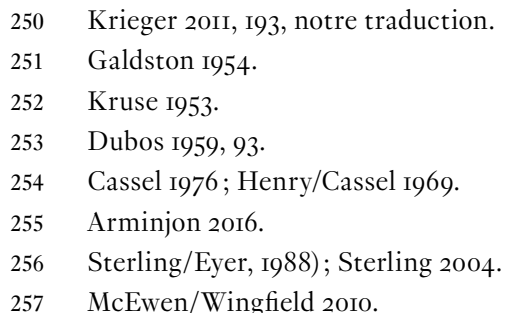


à 2008, donnent comme mission à l'épidémiologie sociale de déterminer les "différences individuelles et sociales qui, en réponse à un même stimulus, peuvent menacer ou non l'homéostasie " ${ }^{258}$. Dans tous ces cas, les auteurs restent fidèles à l'esprit de Cannon, qui veut que l'homéostasie désigne moins un mécanisme de régulation interne qu'un modèle physiologique intégratif, visant à expliquer les conditions de stabilité du milieu intérieur et les pathologies que l'organisme est susceptible de développer en réponse aux pressions du milieu extérieur en général et de l'environnement social, en particulier.

Dans la France de l'après-guerre, on l'a vu, Cannon n'a pas bénéficié de la même postérité. Il faut dire que les mesures sociales qu'il pensait tirer de la physiologie, appelant l'État à préserver les citoyens de l'anxiété ou du stress, ont finalement été elles aussi réalisées, mais dans un contexte historique bien différent de celui du New Deal. L'effondrement du régime nazi a entraîné avec lui la chute des idéologies eugénistes telles que celles d'un Richet ou d'un Toulouse ${ }^{259}$. Mais surtout, le 15 mars 1944, le Conseil national de la Résistance est parvenu à s'entendre sur un "plan d'action immédiate » visant à fixer les grandes lignes des réformes politiques devant être réalisées à la Libération. Outre la reconnaissance d'un gouvernement provisoire, créé par le Comité français de Libération nationale et par son dirigeant, Charles de Gaulle, le programme d'action entend mettre en place un ensemble de mesures politiques telles que le rétablissement de la démocratie et du suffrage universel, la nationalisation des moyens de production dans des secteurs économiques clefs (banques, énergie, assurances), le réajustement des salaires, le rétablissement du syndicalisme, ainsi que la mise en place d'une sécurité sociale visant à garantir à l'ensemble des citoyens leurs moyens d'existence. Dans la foulée, l'assurance maladie est mutualisée. Il n'est pas impossible que l'on trouve ici aussi une des raisons de la faible postérité de Cannon en France. C'est du moins l'hypothèse à laquelle nous amène l'histoire du développement tardif de l'épidémiologie sociale en France.

258 Marmot/Wilkinson 2005, 24, notre traduction.

259 Ohayon 200I
Contrairement au reste du monde, en particulier les États-Unis et le Royaume Uni, il a effectivement fallu attendre le tournant des années 2000 pour qu'un groupe de travail du Haut Conseil de la santé publique s'étonne que la France n'ait pas fait "partie des pays qui ont signé et participé aux travaux de la Commission sur les déterminants sociaux de la santé " de l'OMS ${ }^{260}$. Cette prise de conscience suivait un revirement amorcé dès 1995 , avec la commande d'un rapport sur les inégalités sociales en France. Dans ce rapport, les auteurs s'autorisent quelques hypothèses susceptibles d'expliquer le retard français en la matière:

La demande sociale et politique ne s'est guère manifestée sur ces questions au cours du dernier demi-siècle, probablement en grande partie parce que la création de la Sécurité sociale après la Seconde Guerre mondiale a laissé penser, dans un contexte de croissance économique où la pauvreté était considérée comme "résiduelle», que l'intégration de la population dans un système universel de protection sociale résoudrait le problème des inégalités de santé, là encore appréhendées essentiellement en termes d'accès aux soins ${ }^{261}$.

Doit-on s'étonner que, dans ce même rapport, la figure de Cannon resurgisse dès que les contributeurs évoquent les mécanismes biologiques susceptibles d'expliquer l'étiologie sociale des maladies?

En réponse aux pressions de l'environnement qu'il perçoit et interprète comme des menaces pour son intégrité, l'organisme procède à un ensemble d'ajustements psychologiques, comportementaux et neuro-endocriniens, auquel fait référence le concept de stress. La notion de stress social désigne, dans ce cadre, les ajustements liés à la présence, ou à l'action particulière des congénères. Les ajustements neuro-endocriniens permettent le maintien de l'homéostasie et participent à l'expression des ajustements comportementaux et psychologiques ${ }^{262}$

La prise de conscience que ni la sécurité sociale ni l'égal accès au soin ne suffisent à compenser l'impact des conditions sociales

260 HCSP 2009, 5

261 Fassin/Grandjean/Kaminski/Lang/Leclerc 2000, I8.

262 Berton/Mormède 2000, 378. 
sur la santé aura certainement été alimentée par le contexte de crises sociales auxquelles les pays européens, dont la France, doivent faire face au tournant du XXI ${ }^{\mathrm{e}}$ siècle : la montée du chômage et de la précarité, voire les attaques répétées contre un système de protection sociale jugé trop coûteux.

La France, comme d'autres pays occidentaux, entame une réflexion sur les déterminants sociaux de la santé et des maladies dans une période de crise. Bien qu'historiquement éloignée, cette crise n'en partage pas moins, en ce qui touche la santé des travailleurs, quelques ressemblances avec celle qu'ont pu traverser les États-Unis de l'entre-deux-guerres. À la faveur de ce contexte, il n'est pas impossible que les thèses que Cannon exposait dans ses conférences parisiennes trouvent enfin l'écho qu'elles n'ont pas trouvé en I930. Aussi le lecteur contemporain trouvera-t-il dans ces conférences une photographie des sciences biologiques de l'époque; mais il découvrira également que la France a en quelque sorte joué un rôle, quoique indirect, dans l'élaboration de certains des concepts clefs que mobilisent aujourd'hui les institutions de santé nationales et internationales.

\section{Établissement du texte}

Les tapuscrits originaux des deux cycles de conférences présentés ici sont conservés à la Countway Library of Medicine de l'Université de Harvard, dans le fonds Walter Bradford Cannon Papers. Les deux séries de conférences, sur les émotions et sur l'homéostasie, sont respectivement composées de cent soixante-quatre et de cent cinquante et un feuillets. Chacune des conférences compte environ une trentaine de feuillets. Comme l'usage d'un stylo et d'un crayon à papier en témoigne, Cannon a, à deux reprises au moins, apporté des modifications et des ajouts. Comme indiqué en notes, l'une de ces relectures a principalement consisté à marquer les liaisons entre les mots. Ces annotations témoignent du soin que Cannon a apporté à améliorer sa prononciation du français. Au fil des pages, Cannon cesse progressivement de recourir à ces aides phonétiques. Son français a sans doute gagné en assurance au cours des cinq semaines qu'ont duré les deux premiers cycles des conférences parisiennes.

Lors de la retranscription, nous nous sommes attaché à nous rapprocher au plus près du texte que Cannon a vraisemblablement lu devant le public parisien. Nous avons éliminé les parties raturées, en reportant les passages supprimés dans des notes de bas de page, lorsque ceux-ci nous paraissaient pertinents ou qu'ils nous ont semblé favoriser la compréhension du texte. Afin de favoriser la lecture, nous nous sommes contenté de corriger les coquilles ou fautes de grammaire et de syntaxe les plus manifestes, et, à quelques occasions, les erreurs de ponctuation.

On notera encore que trois feuillets de la première conférence sur l'homéostasie sont manquants. Nous avons pu reconstituer le texte, en empruntant les passages tirés du texte publié dans le premier fascicule des Cours de la Facultédes sciences de Paris $^{263}$, intitulé «L'Homéostasie». Le raccord parfait entre les pages manquantes laisse penser que cette reconstruction du texte s'avère proche du tapuscrit original.

Cannon a ajouté à la main, dans les marges gauches et droites, de nombreuses annotations. Nous les rapportons en notes de bas de page lorsqu'elles nous paraissent pertinentes. Certaines indiquent le temps qu'il lui reste, d'autres qu'une diapositive doit être projetée. Les diapositives ne sont pas référencées dans le tapuscrit, pas plus que ne le sont les nombreux articles et ouvrages que Cannon mobilise tout au long de ses conférences. Nous nous sommes efforcé d'identifier chacune des diapositives et références, en nous appuyant sur les articles et les livres qui ont servi à l'élaboration des conférences ou en ont été dérivés. Malgré le soin apporté à la reproduction de ces images, il n'a pas été toujours possible d'en obtenir une lisibilité parfaite. Sauf mention contraire, toutes les traductions d'extraits d'auteurs anglophones sont de notre fait. Pour chacune des références citées, nous avons tâché de donner quelques éléments biographiques (tirés des dictionnaires biographiques, nécrologies parues dans les revues scientifiques, encyclopédies

263 Cannon i930a. 
et monographies usuelles), afin de renseigner le lecteur sur les auteurs cités, leurs liens éventuels avec Cannon, ainsi que le contexte intellectuel et social dans lequel les auteurs menaient leurs recherches. Lorsque nous l'avons jugé nécessaire, nous avons aussi apporté en notes quelques définitions et explications de termes appartenant au jargon biomédical, qu'ils soient toujours usités de nos jours ou non. Par ailleurs, lorsque, dans les notes, nous nous référons au livre The Wisdom of the Body, nous renvoyons, sauf précision, à la seconde édition parue en 1939 . Nous nous référons à La Sagesse $d u$ corps lorsqu'il s'agit de référencer les sources des figures légendées en français qui en sont issues ou de préciser le texte des conférences. La numérotation des figures de la traduction française est identique à celle de la seconde édition de The Wisdom of the Body.

Enfin, la publication de ces deux cycles de conférences nous paraît d'autant plus importante que les textes en français de Cannon sont devenus difficiles d'accès. Si les conférences sur les émotions sont inédites, les diverses publications dont ont fait l'objet les conférences sur l'homéostasie sont devenues rares. Les deux fascicules des Cours professés à la Faculté des sciences de Paris reproduisent les cinq conférences sur l'homéostasie, mais présentent un texte déjà modifié. Une recherche bibliographique menée sur WorldCat ${ }^{264}$ ne révèle d'ailleurs l'existence que d'un seul exemplaire des fascicules consultable publiquement, celui-là même qui est conservé à Harvard, dans le fonds Cannon.

Deux documents dérivés du séjour parisien de Cannon nous sont donc accessibles. Les conférences qu'il a données à Nancy les 24 et 25 mai 1930 ont fait l'objet d'une publication dans la Rerue médicale de l'est ${ }^{265}$. Celle-ci ne constitue cependant qu'une synthèse d'une quinzaine de pages des conférences sur l'homéostasie. La version française de l'ouvrage dérivé de ces conférences, La Sagesse du corps, constitue donc l'accès le plus aisé aux travaux qui occupaient Cannon en 1930. Cette accessibilité est toute relative, dès lors que quinze exemp laires de La Sagesse du corps sont aujourd'hui répertoriés dans les bibliothèques du monde. La faillite de la maison d'édition Albatross \& Tauchnitz en I950, entraînant avec elle celle des Éditions de la Nouvelle Critique à la fin de l’année 195I, explique très certainement la diffusion limitée de l'ouvrage. collections de plus de 7o'ooo bibliothèques dans le monde.

Cannon I93od. 
Les émotions

fortes et leur influence

sur l'organisme 


\section{Modifications physiologiques accompagnant} les états de grande excitation

Je voudrais exprimer en français ma reconnaissance la plus profonde pour votre accueil si cordial, si amical, si généreux; mais je ne pourrais pas le faire même en anglais! Je ne peux que dire, simplement, «je vous remercie de tout mon cœur ${ }^{1}$.

L'étude des émotions constitue un sujet singulièrement approprié pour un conférencier qui apparaît pour la première fois devant un auditoire aussi distingué, dans une institution médicale aussi ancienne. L'orateur lui-même devient alors une illustration de son sujet. Il est profondément impressionné et un peu exalté, peut-être, de l'honneur qu'il a de se trouver où beaucoup de grands hommes se sont trouvés avant lui, et à cela se mêle une certaine appréhension ${ }^{2}$ et la crainte de ne pas atteindre au degré d'excellence de ces prédécesseurs.

Permettez-moi de dire d'abord, pourtant, que je n'ai aucune intention d'étudier des états aussi compliqués et d'une nature aussi subtile que l'orgueil et l'appréhension. Je me propose plutôt d'examiner d'abord des états affectifs plus primitifs, la peur et la colère, en insistant naturellement sur leur côté physiologique.

Ces émotions présentent quelque chose d'assez mystérieux. Subjectivement, elles semblent prendre naissance dans un domaine distinct du domaine conscient et faire irruption dans la conscience indépendamment de notre volonté. C'est là, évidemment, le fondement des expressions courantes: "être saisi par la peur", "être possédé par la colère», ou "avoir un sentiment de terreur qui s'élève en nous ». Une impulsion étrange et puissante à l'action naît et, parfois, prend complètement possession de l'organisme avec une telle force et un tel

Ce paragraphe est écrit à la main, sur une feuille volante.

2 Dans l'ensemble du passage, Cannon a biffé le mot «anxiété » en faveur de celui d'« appréhension". 
pouvoir qu'il en résulte un comportement tout à fait imprévu. Dans les états d'excitation émotive ${ }^{3}$ on se comporte d'une manière dont on peut, dans la suite, être fier parfois sans grande raison, ou dont on peut être mécontent d'une façon aussi injustifiée.

Depuis des siècles, ceux qui étudient la nature humaine se sont passionnés à l'étude des émotions. Des moralistes, des philosophes, des psychologues ont discuté de l'origine des émotions, de leur rôle dans notre conduite, et des différents modes de contrôle auxquels elles sont soumises. Des naturalistes ont fait remarquer la ressemblance qu'il y a entre l'expression des émotions chez l'homme et chez les animaux ${ }^{4}$. Mais il n'est pas nécessaire de recourir aux descriptions données par les savants en ces matières. Nous pouvons discuter des grandes émotions et arriver à nous comprendre parfaitement à ce sujet parce que nous connaissons tous quelque chose en ce domaine. Tous, nous avons été en colère ou effrayés, ou nous avons vu des gens en état de colère ou de peur, ou nous avons pu observer des animaux domestiques présentant des signes de semblables émotions. Il n'est besoin de personne pour nous faire remarquer les ressemblances entre l'homme en colère, les dents serrées, les sourcils froncés, les poings serrés ou crispés sur une arme, marmottant, et le chien en colère avec les babines retroussées découvrant les dents, le poil hérissé, et grognant de façon menaçante. Ces modes d'expression sont tellement connus qu'ils constituent un langage commun à l'homme et au chien. L'un sait ce que l'autre veut dire. L'ouvrage classique de Darwin, The Expression of Emotion in Man and Animal ${ }^{5}$, traite des nombreuses ressemblances entre les réactions émotives des hommes et des animaux.

Dans l'ensemble du texte, Cannon remplace «réaction émotionnelle» par "réaction émotive».

Cannon supprime ici «animaux moins élevés».

5 Darwin I890 [1872]. Dans The Expression of the Emotions in Man and Animals, Charles Darwin entend expliquer, au moyen de la théorie de l'évolution, l'origine fonctionnelle des expressions émotionnelles communes aux animaux et aux humains. Son approche naturaliste tranche avec celle de Charles Bell (I774-I842) ou encore celle de Guillaume-Benjamin Duchenne de Boulogne (I806-I875), pour qui les expressions émotionnelles, principalement celles de la face, auraient été arbitrairement fixées par Dieu.
Les réactions dont parle Darwin sont pour la plupart superficielles; par exemple, l'érection des poils, la dilatation des pupilles, la sécrétion de la sueur, l'accélération de la respiration et du pouls, et des attitudes corporelles caractéristiques. À côté de ces changements, cependant, il y a d'autres changements internes très importants qui accompagnent toute forte émotion et qui ont été mis en lumière par des recherches relativement récentes. J'ai l'intention de vous décrire ces changements plus profonds et moins visibles des fonctions physiologiques. À la façon dont je vous les présenterai, ces manifestations pourront vous sembler chaotiques, désordonnées, et sans signification. Cependant, ce sera là le fondement de la discussion et de l'interprétation que je vous présenterai dans les leçons suivantes.

J'espère mettre en évidence que, normalement, ces diverses altérations fonctionnelles concourent à former un complexe de modifications physiologiques essentiel pour la compréhension aussi bien des fonctions émotives que des désordres émotifs, et nous permettant d'apprendre quelque chose sur la nature du phénomène "émotion". Au cours de mon exposé, je parlerai souvent des animaux inférieurs ${ }^{6}$, mais l'emploi de ceux-ci pour l'étude des principales émotions, celles que William James ${ }^{7}$ appelait les plus grossières, est justifié par la similarité d'expression de ces états chez l'homme et les animaux. Cette ressemblance est l'un des arguments en faveur de l'existence d'un passé lointain commun. L'importance de ce point apparaîtra petit à petit, je l'espère, au cours des prochaines leçons.

J'ai commencé à m'intéresser aux effets des états d'excitation émotive sur le fonctionnement de l'organisme en mille huit cent quatre-vingt-dix-sept (I897). Je faisais alors ma première année de médecine et j'utilisais la découverte (alors récente) des rayons X pour l'étude des mouvements de l'estomac et de l'intestin $^{8}$. À mon grand étonnement, le moindre signe de malaise,

Cannon ajoute en marge «le chien et le chat par exemple».

Pour William James, voir l'introduction.

Dans son tapuscrit, Cannon opte pour une double notation des nombres et des dates (en toutes lettres et en chiffres arabes), sans doute pour s'assurer de leur bonne prononciation en français. 


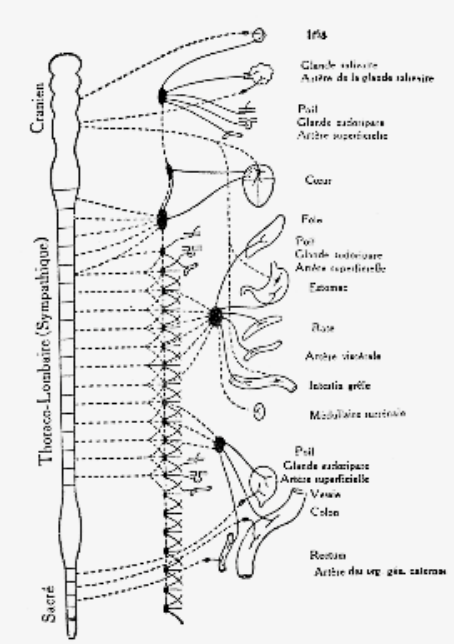

Figure 34. - Diagramme de l'anatomie générale du système hés à gauche. Les nerfs du système somatique ne figurent pas sur ce schéma. Les fibres préganglionnaires sont en traits interrompus, les fibres postganglionnaires en traits pleins. Pour détails, voir texte. (Bard, d'après Cannon, « Foundations of experimental Psychology », avec l'autorisation de la « Clark University
Press ».)

d'inconfort, ou de colère chez les chats que j'observais était aussitôt accompagné d'une abolition totale des mouvements du tractus digestif. On observe les mêmes phénomènes chez le chien et le lapin. Cette inhibition ne s'observe pas exclusivement chez les animaux inférieurs. La même ressemblance qui existe entre les manifestations superficielles des émotions chez le chien et chez l'homme se retrouve pour leurs manifestations internes. On connaît des exemples chez l'homme, de l'arrêt rapide de la motilité gastrique et intestinale dans la peur et la colère. Cette inhibition s'étend également aux sécrétions des glandes digestives.

Fig. $1^{9} \quad$ De nombreuses observations, faites par des chercheurs différents sur l'homme et les animaux, montrent que la fureur et la peur sont ordinairement accompagnées d'une suppression de la sécrétion salivaire, de la sécrétion gastrique, et de la sécrétion des sucs pancréatique et intestinal. Un caractère intéressant et important de ce phénomène est sa persistance pendant un temps considérable après la disparition de l'émotion.

Comment expliquer cette persistance? Cette question a été l'origine d'une série de recherches. La portion du système nerveux qui entre en action dans les états de grande excitation est la partie sympathique du système nerveux autonome. Comme le montre le schéma actuellement à l'écran ${ }^{9}$, l'un des caractères importants du système nerveux sympathique est d'être constitué de ganglions disposés en série aux deux côtés

$9 \quad$ Cannon projette la figure reproduite dans La Sagesse du corps (Cannon I946[1939], 203). La figure est aussi reproduite dans Bodily Changes in Pain, Hunger, Fear and Rage (Cannon 1915, 454). de la ligne médiane. Ces ganglions sont reliés entre eux et à la moelle épinière par ce qu'on appelle les «fibres préganglionnaires » qui sont les axones de cellules nerveuses situées dans la substance grise entre le renflement brachial et sacré ${ }^{10}$. Dans les ganglions eux-mêmes on trouve les corps cellulaires d'autres neurones dont émanent les fibres "postganglionnaires", indiquées sur le schéma par des lignes continues, et qui fournissent aux muscles et aux glandes des viscères, à l'estomac et aux intestins ${ }^{11}$, au cœur et aux vaisseaux sanguins, au foie, aux muscles érecteurs des poils, aux glandes sudoripares, aux bronchioles, et à la portion médullaire des capsules ${ }^{12}$ surrénales. Les influx conduits par les nerfs sympathiques exercent sur chacun de ces différents organes un effet particulier. Dans certains cas l'effet est inhibiteur, par exemple, sur les fonctions du tractus digestif. Dans d'autres cas il est excitant, comme au niveau du coeur, des vaisseaux, des poils, du foie et d'autres organes. D'autre part, fait extrêmement intéressant, si on excite le sympathique, on détermine aussitôt la production par la portion médullaire des surrénales, d'une décharge de son produit de sécrétion, l'adrénaline ${ }^{13}$, dans le courant sanguin. Il existe une relation remarquable entre cette substance et les organes innervés par le sympathique. Elle est capable de produire au niveau de ces organes le même effet que l'excitation des nerfs sympathiques. C'est ainsi que l'adrénaline, injectée dans le courant sanguin, peut accélérer le cour, contracter les vaisseaux, hérisser les poils, produire la libération de sucre par le foie - en un mot, imiter, pratiquement, sous tous ses aspects,

10 La moelle épinière compte deux renflements ou élargissements. Les nerfs qui innervent les membres supérieurs émergent du renflement cervical ou brachial, situé au niveau des cervicales. Les nerfs qui innervent les membres inférieurs émergent quant à eux du renflement sacré, situé au niveau des lombaires.

11 Tout au long du tapuscrit, Cannon a ajouté à la main des aides phonétiques. Ici, il a par exemple barré le «c» d'« estomac» ou encore matérialisé la liaison entre « aux» et «intestins ».

12 Dans l'ensemble du texte, Cannon biffe «glande» en faveur de « capsule».

13 Sur l'ensemble des conférences, Cannon biffe systématiquement le terme d'«adrénine " au profit d'«adrénaline ", pour désigner le produit de sécrétion des surrénales (sur ce point, voir l'introduction). 
l'influence des nerfs sympathiques. De plus, cette substance est extraordinairement active. Par exemple, diluée dans du sang à un pour un milliard quatre cents millions, elle a le pouvoir d'accélérer le cœur isolé du chat ${ }^{14}$. La question se pose naturellement de savoir si dans les états de grande excitation il se produit dans le courant sanguin une sécrétion d'adrénaline. Ce sujet a donné lieu à de nombreuses années de discussion et de controverse ${ }^{15}$.

Stewart et Rogoff ${ }^{16}$, de Cleveland, ont été les principaux adversaires de la doctrine de la production d'une quantité supplémentaire d'adrénaline au cours des états d'excitation émotive, (ou dans la douleur, ou dans l'asphyxie, qui sont également des phénomènes accompagnés de production d'influx sympathiques). De plus le professeur Gley et le docteur Quinquaud ${ }^{17}$ ont soutenu que l'adrénaline, bien que sécrétée de façon plus marquée

14 La méthode d'isolement du cœur est décrite plus bas.

15 Cannon revient longuement sur ce point, dans un chapitre de son autobiographie consacré aux bienfaits ainsi qu'aux effets pervers des polémiques sur l'avancement de la science (Cannon 1945, I00).

16 Stewart/Rogoff igi6. Julius Moses Rogoff (I884-I966) est médecin et endocrinologue. Il a enseigné la médecine expérimentale à la Western Reserve University School of Medicine, à Cleveland, de I9I5 à 1939, puis l'endocrinologie à l'Université de Pittsburgh de 1939 à 1950. D'origine anglaise, George Neil Stewart (I860-I930) enseigne la physiologie et l'histologie à Harvard, de I893 à I894, puis, de I894 à I930, à la Western Reserve University School of Medicine à Cleveland. Son Manual of Physiology, with Practical Exercises (Stewart I895) influence plusieurs générations de physiologistes. Dans la septième édition de son manuel, parue en I9I4, Stewart mentionne la théorie de Cannon relative à l'implication du système adrénergique dans les réactions adaptatives de peur, de rage et de douleur. Cependant, dès I9I6, Stewart et son assistant, Rogoff, contestent les résultats de Cannon (Cannon/Hoskins I9II). Stewart et Rogoff ont utilisé une méthode permettant de collecter le sang issu de la veine cave inférieure de chiens asphyxiés ou dont les nerfs splanchniques sont activés au moyen d'électrodes. Mais, contrairement aux thèses de Cannon, le sang collecté ne présentait pas une concentration d'adrénaline supérieure à celui des animaux contrôles respirant normalement. Ils en concluent: «Cannon et Hoskins ont affirmé que la stimulation des nerfs sensoriels et l'asphyxie produisent une si grande libération d'épinéphrine dans le sang que le sang prélevé dans la [veine]

Gley/Quinquaud I9I8. Alfred Quinquaud (I884-I96I) est médecin et physiologiste. Les travaux de Gley et Quinquaud semblent indiquer que la sécrétion d'adrénaline par les surrénales n'a pas d'effet sur le système vasomoteur ni sur le cœur. Les observations de Cannon sur le cœur dénervé vont dans les conditions qui provoquent une excitation du sympathique, n'atteint pas alors dans le sang à une concentration suffisante pour exercer une action sur les viscères. Je n'ai pas l'intention de faire actuellement un exposé critique de cette longue controverse. Peut-être suffira-t-il de noter que notre thèse de l'existence d'une décharge d'adrénaline dans la douleur et l'asphyxie a trouvé dans la suite l'appui des expériences de divers chercheurs qui ont travaillé avec des techniques différentes en Angleterre et en Russie, en Algérie, en Argentine, aux ÉtatsUnis et au Japon. Personne encore n'a confirmé les vues de Stewart et Rogoff selon lesquelles la sécrétion d'adrénaline est fixe et invariable. En fait, des chercheurs japonais ${ }^{18}$ qui ont employé les méthodes de Stewart et Rogoff ont confirmé nos propres résultats. J'exposerai dans la suite les arguments qui parlent en faveur de l'existence d'une adrénalinémie vraie - c'est-à-dire, d'une décharge physiologique d'adrénaline dans le sang, en quantité suffisante pour agir sur le fonctionnement des viscères.

Pour mettre en évidence a sécrétion d'adrénaline au cours des états d'excitation émotive, nous avons dans les derniers temps - quand je dis «nous", il s'agit aussi bien de mes collaborateurs que de moi-même - nous avons pris comme test le cœur

clore la controverse. Gley assiste aux conférences parisiennes de Cannon sur les émotions.

18 Satake/Watanabé/Sugawara I927. L'étude est menée par les équipes de Yasaturo Satake, du laboratoire de physiologie de l'Université du Tohoku à Sendai. Elle montre notamment que l'utilisation d'anesthésiques diminue l'activité des surrénales. Yasaturo Sataké (I884-1959) est un médecin et physiologiste japonais. Il est nommé professeur à l'École de médecine de Kyoto en IوIo puis, en I9I5, à l'Université du Tohoku à Sendai, où il devient le directeur du département de médecine en I928. Il prend la présidence de l'Université du Tohoku en I946. Tadashi Sugawara et Masanosuke Watanabe sont des physiologistes diplômés de l'Université du Tohoku, respectivement en I9I6 et en I9I9. Ils sont tous deux rattachés au laboratoire de physiologie de Sataké. Cannon a conservé des contacts avec Sataké, dont les travaux plaidaient en faveur de sa thèse, contre ceux de Stewart et Rogoff pour qui les fortes émotions n'entraînent pas de libération d'adrénaline. En avril I935, Cannon prend le bateau de San Francisco à Kobe, au Japon. Il repart aussitôt pour rejoindre la Chine, où un séjour de recherche au Peking Union Medical College l'attend. En juin I935, il retourne au Japon, où Sataké lui a organisé un cycle de conférences à Sendai, Tokyo et Kyoto. 


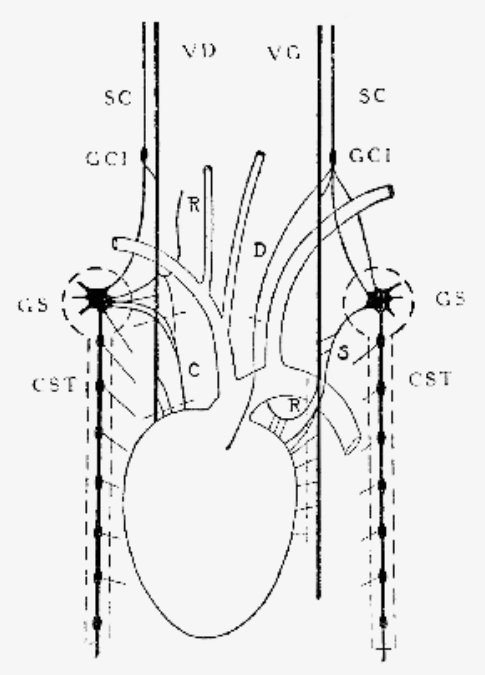

Figure 17. - Schéma de l'anatomie habituelle des nerfs cardiaques chez le chat. V D, vague droit; $\mathrm{V} \mathrm{G}$, vague gauche; $\mathrm{SC}$, récurrent laryngé; D, nerf dépresseur; G S, ganglion stellaire; C, nerf commun cardiaque; $;$, fibre sympathique; $\mathrm{CST}$, chaîne symraits interrompus indiquent les partie sectionnées ou excisées. énervé ${ }^{19}$. Pour séparer le cœur du système nerveux central nous utilisons un procédé chirurgical: nous coupons le nerf vague droit en dessous du rameau récurrent laryngé, comme le montre le schéma; nous coupons tous les rameaux cardiaques du vague gauche et nous enlevons les chaînes sympathiques thoraciques des deux côtés depuis le ganglion stellaire jusqu'à la huitième côte environ. Le larynx reste ainsi innervé d'un côté, le vague gauche continue à exercer son action sur les organes de la digestion, et le cœur n'étant plus sous le contrôle du système nerveux, continue à pomper le sang dans la circulation, mais n'est Fig. $2^{19}$ plus soumis qu'à l'action des modifications du sang lui-même. Les animaux ainsi opérés se rétablissent vite de l'opération et continuent à vivre normalement dans le laboratoire. On peut les employer beaucoup de fois sans qu'il soit besoin de les anesthésier. On place un tambour sur la paroi thoracique au niveau du cœur. Et on les relie à un tambour enregistreur. On peut ainsi facilement enregistrer les mouvements du cœur ${ }^{20}$.

Après avoir pris de ceux-ci un tracé analogue à celui que montre cette photographie d'un de nos tracés originaux, on approche du chat servant à l'expérience un chien agressif. On observe alors chez le chat une dilatation des pupilles, une érection des poils de la queue et du dos, une rétraction des oreilles, des grincements de dents. Le chat siffle, grogne, crache, sort ses griffes et cherche à en frapper le chien. En

19 Cannon projette la figure reproduite dans La Sagesse du corps (Cannon 1946[1939], 87), initialement parue dans Cannon et al., I926: 331.

20 Cannon projette très vraisemblablement la figure reproduite dans La Sagesse $d u$ corps (Cannon I946[1939], I22), initialement parue dans Cannon/Britton I927.

fait le chat reste tranquille sur un coussin. Les mouvements mettant en jeu les muscles du squelette, cependant, sont peu importants. Les mouvements viscéraux sont plus étendus, comme le montre l'érection des poils sur toute la longueur du corps. L'accélération de fréquence du cœur énervé était dans le cas présent, de quarante (40) battements par minute. Habituellement l'accélération varie entre quinze (15) et trente (30) mouvements par minute avec une moyenne de vingt-deux (22) dans quarante-cinq (45) expériences. Aux variations d'accroissement cardiaque correspondent des varia-

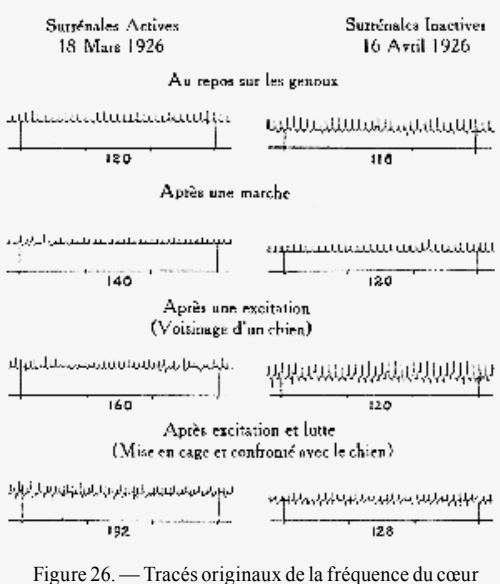

Figure 26. - Tracés originaux de la fréquence du cœur
(n) tivation des surrénales ( 6 avril). Temps en intervalles de 5 secondes. La fréquence normale de l'animal au repos sur les genoux est comparée à la fréquence après une marche, apres une excitation determinee par la présence d'un chien aboiements du chien. tions dans les autres signes de la réaction émotive. Après enlèvement d'une des capsules surrénales et énervation de l'autre capsule, la reproduction de la même expérience avec le même animal a produit, comme vous le voyez, une accélération légère seulement - dans ce cas, deux (2) battements par minute. En fait, au cours de trente-neuf (39) expériences de cette espèce la moyenne a été une augmentation de fréquence de seulement deux (2) battements par minute.

Quand l'état de grande excitation est accompagné d'une lutte vigoureuse, on obtient un effet maximum; le cœur énervé peut alors augmenter sa fréquence de plus de cent (I00) battements par minute. Dans le cas présent, il y avait un accroissement de soixante-douze (72) battements. Vous pouvez voir qu'après l'inactivation des capsules surrénales l'accroissement de fréquence a été de dix (Io) battements seulement, ce qui est probablement dû à l'augmentation de température résultant de l'activité musculaire.

\section{Fig. $3^{20}$}




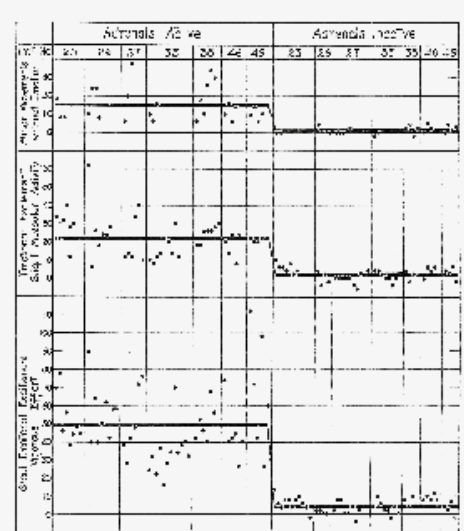

Fig. 2. Graphic record of the results presented in tables 1 , 2 and 3 . The dots representincreases of rate of the denervated heart above the basallevel that followed a, minor movements
without notable emotion; $b$, emotional excitement withslight muscular activity, and c, great emotional excitement with average of the changes of heart vigorous effort, before and after inactivation of the adre-

Le cliché ${ }^{21}$ suivant vous montre les résultats de nombreuses expériences faites sur les mêmes animaux avant et après suppression de la sécrétion surrénale. Vous noterez que les accroissements moyens de fréquence du cœur énervé quand il $\mathrm{y}$ avait en même temps excitation et lutte étaient de quarante-neuf (49) mouvements par minute, et seulement cinq (5) mouvements par minute après suppression de la sécrétion de l'adrénaline.

Le cliché suivant ${ }^{22}$ se rapporte à la persistance de l'excitation après la disparition de la cause de cette excitation. Sous ce rapport, ce cliché est intéressant. Le chat rendu inquiet Fig. $4^{21}$ par les aboiements d'un chien pendant une minute a montré un accroissement de plus de soixante-dix (70) mouvements cardiaques par minute; et même après vingt (20) minutes il n'était pas revenu à son rythme cardiaque normal, bien que pendant ce temps l'animal fût resté couché sur un coussin. Deux (2) minutes d'excitation dans les mêmes circonstances après inactivation des surrénales ont augmenté la fréquence cardiaque de huit (8) battements seulement et après cinq (5) minutes environ l'état primitif était rétabli.

Je tiens à insister sur le fait que le cœur de ces animaux se comportait comme un instrument introduit dans le corps de l'animal et perfusé par son sang. Le seul fait d'enlever aux capsules surrénales la possibilité de secréter a produit cette différence si marquée entre les résultats de l'excitation émotive; d'une part, cette augmentation dramatique de fréquence cardiaque et d'autre part, cette absence d'augmentation. Ce

Cannon projette vraisemblablement la figure parue dans Cannon/Britton 1927. Il ajoute à la main, dans la marge gauche: «Les lignes épaisses représentent les moyennes.»

22 Cannon projette vraisemblablement la figure parue dans Cannon/Britton 1927. fait, rapproché d'autres observations dont je vous parlerai dans la suite, peut être, à mon avis, interprété comme montrant qu'une forte émotion telle que la peur ou la colère est accompagnée d'une production d'adrénaline dans le courant sanguin à une concentration suffisante pour exercer une action sur les viscères.

Comme je l'ai dit déjà, l'adrénaline est capable de produire au niveau des viscères les mêmes effets que l'excitation des nerfs sympathiques. La décharge d'adrénaline dans le sang au moment où les nerfs

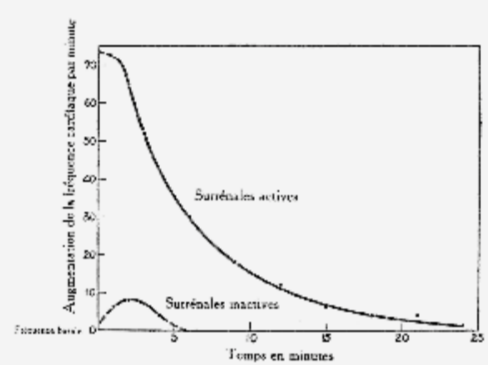

Figure 35. - Longue persistance de l'accélération cardiaque quand les surrénales sont actives et que l'animal (chat) a été excité pendant une minute par les aboieque le chat ait été excité pendant un temps deux fois plu de la cage après l'excitation et mis pendant la durée du retour à la normale. ments d'un chien. Légère accélération du cour et retour que le chat ait été excité pendant un temps deux fois plev
long. Dans les deux cas, l'animal fut rapidement enleve

sympathiques envoient des influx aux viscères coopère avec les mécanismes nerveux. Le mécanisme nerveux et le mécanisme humoral coopèrent à étendre leurs effets dans l'organisme. Nous pouvons parler d'un système sympathico-surrénal.

Quand le système sympathico-surrénal est excité, il y a, comme nous l'avons vu, une suppression des fonctions des organes digestifs; mais il peut y avoir d'autres effets très marqués dans l'organisme, comme le montre le résultat d'une injection d'adrénaline dans le courant sanguin. Mais c'est là un procédé artificiel. Nous devons, au moyen d'autres expériences et d'autres observations, savoir si les conditions naturelles qui excitent le système sympathico-surrénal sont accompagnées d'une influence sur les viscères.

On sait depuis de nombreuses années que l'injection d'adrénaline produit une augmentation du taux du sucre sanguin. Elle agit ainsi en libérant du sucre mis en réserve dans le foie. Y a-t-il un accroissement du sucre sanguin au cours d'excitations émotives?

Nous avons entrepris l'étude de cette question en mille neuf cent onze ${ }^{23}$ (I9I I), et nous avons vu que les chats, excités par la mise en cage et par la présence d'un chien qui aboie, ont un

23 Cannon/Shohl/Wright I9II. 


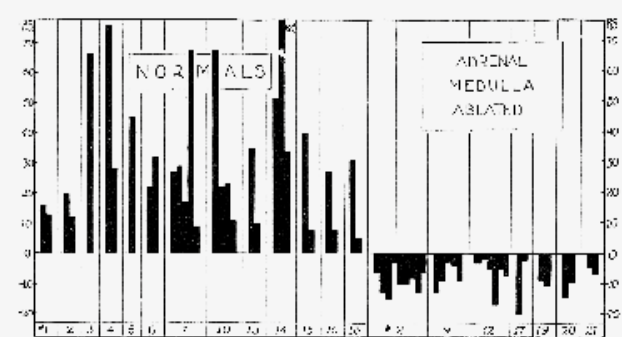

Fig. 2. Chart indicating hyperglycemic reactions in normal animals and decrements in blood sugar in animals without adrenal medulla, following emotional excitement. Ordinates, changes in blood sugar
from fasting (0) level, abscissae, animal numbers. All cases are listed. At a glance the striking difference in reaction may be appreciated. accroissement du sucre sanguin si marqué que le sucre apparaît dans l'urine: il y a glycosurie. Ce résultat a été confirmé au moyen d'autres observations sur le sucre sanguin lui-même. Nos expériences sur ce point ont montré un accroissement de trente-quatre (34) pour cent en moyenne après trois $(3)$ à dix (I0) minutes d'excitation.

Fig. ${ }^{25}$ Dans les expériences d'un de mes anciens élèves, Britton ${ }^{24}$, l'accroissement du sucre sanguin était de vingt-six (26) pour cent en moyenne au cours des trois (3) à six (6) minutes, après seulement deux (2) minutes d'excitation. Comme le montre le cliché $^{25}$ que vous voyez sur l'écran, l'accroissement de sucre sanguin observé par Britton ne s'est pas produit après l'inactivation des surrénales, bien que les nerfs du foie soient restés intacts et bien que les animaux aient été soumis à la même excitation que les autres : en d'autres termes, l'adrénaline circulante non seulement agit sur le foie, mais elle agit sur lui d'une façon plus marquée que les influx nerveux.

Ces résultats obtenus sur des chats ont été confirmé dans des expériences sur des lapins, des chiens et des hommes. Une large proportion des étudiants se présentant à un examen difficile ont de la glycosurie, ils étaient étudiants en médecine ${ }^{26}$

24 Britton I925. Sidney William Britton (I892-I977) est un physiologiste américain. Il rejoint le laboratoire de Cannon de I924 à I926. Dès 1928 , il prend la tête du département de physiologie de l'Université de Virginie. 25 Cannon projette vraisemblablement la figure parue dans Britton I928.

26 Folin/Denis/Smillies I9I4. Otto Folin (I867-I934) est un chimiste d'origine suédoise. Il travaille pendant deux ans dans l'industrie, avant de rejoindre en Igoo le McLean Hospital, premier établissement hospitalier américain disposant d'un laboratoire de chimie. Il y mène des recherches sur les bases biochimiques des maladies mentales. Cannon, particulièrement favorable au développement d'un département de biochimie à Harvard, intervient en faveur de Folin auprès d'Eliot, président de l'Université de Harvard depuis I869 (voir Benison/Barger/Wolfe 1987, 126). En 1906, Folin devient le et nous avons trouvé également une glycosurie chez environ quarante-cinq (45) pour cent des membres d'une équipe de football de l'Université Harvard après le match le plus passionnant de la saison - celui entre les Universités Harvard et Yale ${ }^{27}$. Observez ici encore qu'il s'agit d'un phénomène commun aux hommes et aux animaux inférieurs.

L'injection d'adrénaline produit des effets marqués sur le système circulatoire. Si on ne la donne pas à dose trop élevée, elle produit une augmentation de fréquence cardiaque, elle contracte les artérioles, particulièrement dans l'aire splanchnique, et par ce fait même elle augmente les résistances périphériques et elle peut produire une dilatation des vaisseaux des muscles squelettiques. Les conséquences d'une augmentation de fréquence cardiaque et d'une constriction de l'aire splanchnique non compensée par une dilatation à un autre endroit, sont une augmentation de la pression sanguine et une accélération de la vitesse d'écoulement du sang dans les vaisseaux qui ne sont pas contractés.

premier professeur de biochimie de Harvard. Il y développe des méthodes de chimie clinique basées sur l'analyse du sang et de l'urine des patients. Willey Glover Denis (I879-1929) est une biochimiste et physiologiste américaine. L'Université de Chicago lui décerne un doctorat de chimie organique en I905. En I909, elle rejoint le laboratoire de Folin, avec qui elle collabore jusqu'en I920, date à laquelle elle est nommée professeure assistante au département de physiologie de la Tulane Medical School. Elle est la première femme à obtenir un tel poste dans une institution états-unienne d’importance. Wilson George Smillie (I886-I97I) est un épidémiologue américain, diplômé de l'École de médecine de Harvard en IgI2. Il travaille deux ans au Peeter Brent Brigham Hospital de Boston puis, en I9I4, comme instructeur au département de médecine préventive de Harvard. En I9I7, il rejoint l'International Health Division de la Rockefeller Foundation. En I9I9, il prend la direction de l'Institut d'hygiène de Sao Paulo. En I927, il est nommé professeur de santé publique à l'École médicale de Harvard puis, en I937, professeur de santé publique à la Cornell University.

27 Cette étude ne semble pas avoir fait l'objet d'une publication. Elle est aussi relatée dans la première édition de Bodily Changes in Pain, Hunger, Fear and Rage: «O. H. Fiske et moi avons examinél'urine de vingt-cinq membres de l'équipe de football de l'Université de Harvard, immédiatement après le dernier et le plus excitant combat de la saison I9I3, et avons trouvé du sucre dans douze cas. Cinq de ces cas positifs faisaient partie des remplaçants non appelés à entrer en jeu. Le seul spectateur excité ayant assisté à la victoire de Harvard et dont l'urine a été examinée avait également une glycosurie marquée, qui avait disparu le lendemain.»(Cannon 1915, 75, notre traduction). 


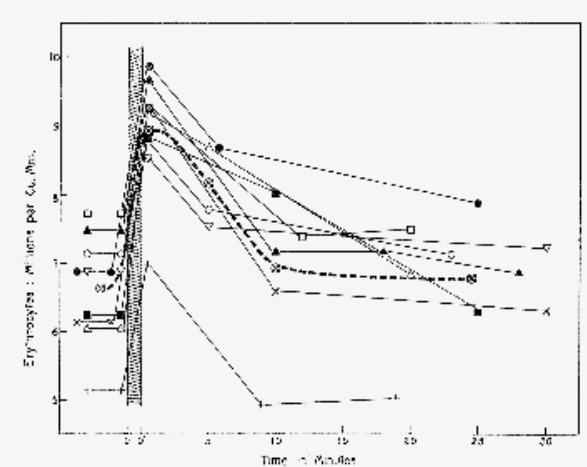

Fig. 1 The course of emotional polycythemia,- a graphic record the one-minute period of emotional excitement; the thick dash-line, the average of the 9 cases.
Déjà en mille huit cent quatre-vingt-dix-sept (I897) Binet et Vaschide ${ }^{28}$ ont noté qu'une émotion soudaine s'accompagne d'une brusque élévation de pression sanguine chez l'homme. Gallavardin et Haour ${ }^{29}$ rapportent que la pression sanguine des malades est, lors du premier examen clinique, de vingt-cinq (25) à trente-cinq (35) millimètres de mercure plus élevé que dans la suite lorsqu'ils sont habitués à cet examen. La

Fig. $7^{30} \quad$ crainte d'entendre poser un diagnostic sévère peut se traduire par une pression sanguine de trente-trois (33) millimètres de mercure plus élevée que dans la suite, lorsque le malade est rassuré. Dans l'extrême colère ou dans la terreur, il peut se produire une élévation de quatre-vingt-dix (90) millimètres. En réalité, le conseil qu'on donne aux personnes âgées et ayant des vaisseaux fragiles et durcis d'éviter les états d'excitation, repose sur la connaissance du danger de rupture des vaisseaux comme conséquence de l'élévation de pression qui accompagne les états d'excitation.

Bien que la plupart des arguments que nous avons cités soient de nature clinique, nous pouvons admettre qu'ils démontrent une vérité générale; c'est-à-dire que l'excitation du système sympathico-surrénal au cours des émotions a les effets des autres exemples d'excitation de ce système - un accroissement de la

Binet/Vaschide I897. Alfred Binet (I957-I9II) est un psychologue et pédagogue français, connu pour ses travaux de psychométrie. Il prend la direc tion du laboratoire de psychologie physiologique de la Sorbonne en I892. Il contribue à introduire en France la théorie des émotions de James (Binet 1902). Nicolas Vaschide (I874-I907) est un psychologue d'origine roumaine dont le livre posthume (Vaschide 19I8) a contribué à introduire la théorie freudienne en France.

29 Gallavardin/Haour, I9I2. Louis Bénédict Gallavardin (I875-1957) est un cardiologue français, médecin des Hôpitaux de Lyon. En 1946, il succède à Alexis Carrel à l'Académie des sciences. Nous ne sommes pas parvenus à identifier Jean Haour. pression sanguine et une augmentation de la vitesse d'écoulement du sang dans les vaisseaux qui ne sont pas contractés.

En connexion avec cet écoulement plus rapide du sang il y a dans les émotions un accroissement du nombre des globules rouges dans le sang et, comme nous le verrons, il y a une relation entre ces deux effets. Chez le chat nous avons observé qu'en moyenne le nombre de globules rouges

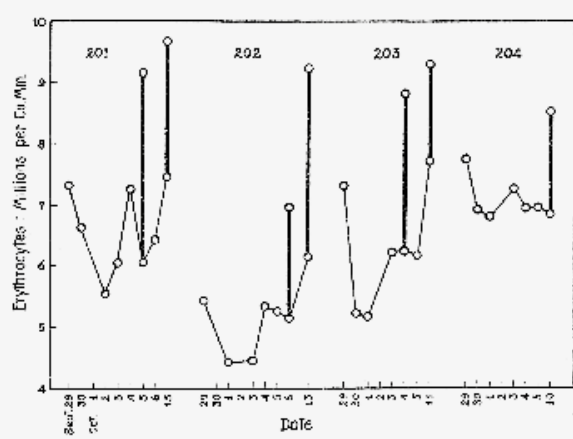

Fig. 2. Graphic record of the daily variations of the erythrocyte count of the first five cats of table 1 , and the rises due to one minute of
emotional excitement (thick vertical lines). See footnote, table 1 , for explanation of the high basal count of 203 on October 11 : it i s noteworthy that the first counts as a rule were high est d'un peu plus de six millions et demi par millimètre cube. Il s'agit du sang pris au moyen d'une toute petite coupure dans l'oreille de l'animal en état de tranquillité. Ensuite, on met l'animal pendant une minute dans une cage étroite et on approche un chien aboyant violemment. Aussitôt que possible après cette expérience on prélève de nouveau du sang à l'oreille et on compte les globules. Comme le montre le diagramme actuellement projeté ${ }^{30}$, il y a alors accroissement marqué du nombre des érythrocytes ${ }^{31}$ circulants; la moyenne de cet accroissement a été dans quinze (15) expériences de vingt (20) pour cent. Remarquez qu'après cinq (5) minutes ce nombre a commencé à diminuer et que dans l'espace d'une demi-heure de calme et de tranquillité, l'état primitif a été rétabli.

Le cliché suivant ${ }^{32}$ montre que ces variations dépassent de beaucoup celles qui se produisent chez l'animal en état de

Cannon projette vraisemblablement la figure parue dans Izquierdo/Cannon I928. José Joaquín Izquierdo (I893-1974) est un médecin, physiologiste et historien mexicain, diplômé de médecine de l'Université de Puebla en I9I7. En I927, une bourse de la Fondation Rockefeller lui permet de poursuivre ses recherches à l'École de médecine de Harvard, sous la direction de Cannon. De retour au Mexique en I930, il œeuvre au développement de la physiologie expérimentale.

31 «Érythrocyte» est le nom savant pour globule rouge.

32 Cannon projette vraisemblablement la figure parue dans Izquierdo/ Cannon I9282. 
tranquillité. Sur ce diagramme les lignes épaisses désignent les effets de l'excitation. Ces changements remarquables de concentration du sang ne se produisent pas après enlèvement de la rate, la reproduction du même essai n'a pas montré d'action sur le nombre de globules du sang. Sans aucun doute la polyglobulie ${ }^{33}$ était due à une décharge de sang concentré de la rate dans la circulation à la suite de l'action du système sympathico-surrénal.

Voilà de nouveau reproduit chez l'homme un phénomène observé chez les animaux. Il y a déjà beaucoup d'années que Ferrari $^{34}$ a vu qu'après un examen les étudiants avaient en moyenne, par millimètre cube de sang, quatre cent soixantequinze (475) mille globules rouges de plus qu'avant l'examen. Et l'année dernière Benhamou, Marchioni et Nonchi ${ }^{35}$ ont rapporté à une réunion de la Société de Biologie de Paris que la peur cause la production d'un accroissement d'environ un million cinq cent mille globules chez un malade ayant sa rate, et n'a pratiquement pas d'effet sur un autre malade dépourvu de rate.

Un autre effet de l'excitation sur le sang est un accroissement relatif du nombre de cellules mononucléaires. Au laboratoire de physiologie de Harvard, Menkin ${ }^{36}$ a montré qu'il y a un

33 Le terme désigne une production anormale de globules rouges.

34 Ferrari I897. Giulio Cesare Ferrari (I867-1932) est un psychiatre et psychologue italien. Après des études de médecine, il séjourne à Paris et travaille dans le laboratoire de Binet à la Sorbonne. Il fonde en I905 la Rivista di Psicologia Applicata alla Pedagogia ed alla Psicopatologia. Dès 1922, il dirige l'Hôpital psychiatrique de Bologne. On lui doit la traduction italienne des Principes de psychologie de William James (I90I [I890]).

35 Benhamou/Marchioni/Nouchy I929. Edmond Benhamou (I882-I973) est un médecin français, président de la Société française de la transfusion sanguine dès 1962 et membre de l'Académie de médecine. Nous n'avons pas trouvé d'informations relatives à Raoul Marchioni (I892-?), qui obtient un doctorat de médecine à la Faculté de médecine d'Alger en I920, ni surNouchy, dont Cannon écorche d'ailleurs le nom. Les auteurs de l'article rapportent l'observation par radiographie des effets de la peur sur la rate. Ils se demandent si les contractions de la rate, ou splénocontraction, ne valident pas les thèses de Cannon sur l'hyperadrénalinémie accompagnant les émotions.

36 Menkin 1928. D'origine russe, Valy Menkin (I90I-1960) mène ses recherches à l'Université de Harvard, depuis la fin des années I920 jusqu'à I946. Il est l'auteur d'un ouvrage sur les mécanismes de la réaction inflammatoire (Menkin 1940). accroissement de treize (I3) pour cent en moyenne après une courte période d'excitation émotive et que la proportion normale est rétablie après environ une demi-heure, comme celle des globules rouges. Si la rate est enlevée ou énervée le phénomène ne se produit pas.

Une autre action remarquable de l'adrénaline est la dilatation des bronchioles. Vous savez qu'une préparation commerciale d'adrénaline est employée couramment dans le traitement de l'asthme et souvent produit une amélioration soudaine et un soulagement étonnant chez le malade en détresse respiratoire. Il semble que dans l'excitation émotive le système sympathico-surrénal produise le même effet. Un de mes amis de Londres, le docteur Hurst ${ }^{37}$, qui souffre parfois de grave accès d'asthme m'a dit qu'il avait le souvenir dans son expérience personnelle de cas de disparition brusque et miraculeuse de sa détresse respiratoire à la suite de la production d'un état de frayeur. Et un pharmacologiste américain m'a raconté un cas dans lequel un accès d'asthme avait été brusquement interrompu par un accès de colère.

D'anciennes recherches sur les glandes surrénales ont montré que l'enlèvement de ces organes a pour conséquence un affaiblissement de la puissance musculaire et que l'injection de l'extrait de la glande exerce un effet fortifiant. Ces recherches anciennes, cependant, n'ont pas pris en considération la structure complexe de la capsule surrénale. On peut y distinguer deux parties - le cortex et la moelle. Quand on a reconnu l'influence du cortex dans le maintien de la puissance musculaire,

37

Cannon fait très certainement référence à sir Arthur Frederick Hurst (I8791944). Ce médecin britannique a travaillé au Guy's Hospital puis au département de neurologie du Netley Hospital, où il s'occupe du traitement des combattants de la Grande Guerre. Hurst s'appuie sur les travaux de Cannon dans sa clinique des «névrosés de guerre». Les soldats montrent des symptômes de neurasthénie accompagnés de maux de tête et de fatigue physique et mentale, ainsi qu'un ensemble de manifestations somatiques, dont, principalement, les symptômes du « cœur de soldat» (soldier's heart). Le cœur de soldat se manifeste par une pression artérielle anormalement haute et une hyperthyroïdie due à un hyperadrénalisme (Hurst I9I7). À la fin de la guerre, Hurst s'installe à Windsor, où il pratique la médecine en cabinet privé 


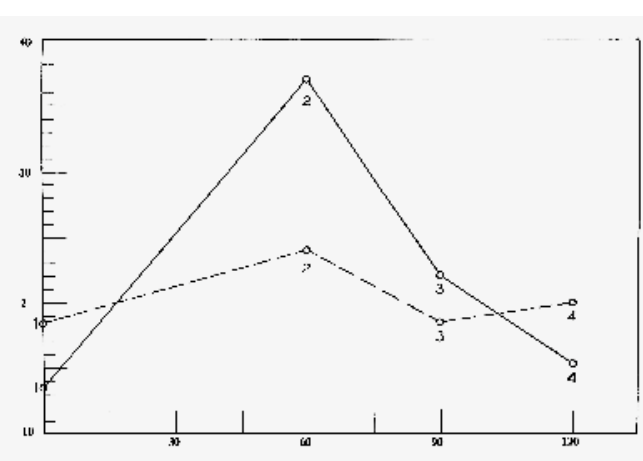

Figure 1. A curve plotted from the data of one experiment. The time interval in minutes is represented on the abscissa; the value of the threshold in $\beta$ units is represented on the ordinate. The continuous lin $\beta$ of the curve-
of the muscle, the broken line that of the nerve-muscle. The $\beta$ of nerve muscle is magnified ten times, that of the muscle is normal.

(1) Normal threshold stimulus.

ue $120 \mathrm{gm} .240$ times per minute.

(3 and 4) The thresholds after rest.

on s'est demandé si la substance médullaire ${ }^{38}$ exerçait une action.

Panella ${ }^{39}$ a montré que l'adrénaline peut exercer une action favorable sur des muscles fatigués de poïkilothermes ${ }^{40}$, et aussi sur des muscles d'homéothermes quand on les amène à l'état poïkilotherme. La question a été reprise par un de mes élèves, Gruber ${ }^{41}$. Quand on excite rapidement et pendant longtemps un muscle d'un chat - par son nerf, le muscle étant en place et dans ses conditions normales de circulation sanguine - le muscle perd graduellement son pouvoir de répondre au stimulus, ce pouvoir étant mesuré par les variations de seuil.

Le graphique ${ }^{42}$ suivant, que vous voyez sur l'écran, montre cet effet. Vous remarquez que le seuil s'est élevé aussi bien quand le muscle était excité directement que lorsqu'il était excité par l'intermédiaire de son nerf. Si on permet au muscle fatigué de se reposer il récupère graduellement son excitabilité primitive,

38 Cannon remplace «moelle» par «substance médullaire».

39 Panella 1907. Nous n'avons trouvé aucune information relative au physiologiste italien A. Panella.

40 Les animaux poïkilothermes sont des animaux dont la température corporelle varie avec celle du milieu, contrairement aux animaux homéothermes, dont la température reste stable. Les hétérothermes, les animaux hibernants par exemple, sont des homéothermes que l'on peut amener à l'état poïkilotherme.

41 Charles Michael Gruber (I887-I974) est un médecin et pharmacologue américain. Diplômé de l'Université de Harvard, il assiste Cannon dans ses recherches durant l'année I9I2. En I953, il est nommé professeur et chef du département de pharmacologie et de thérapeutique expérimentale à l'Université de Loma Linda, en Californie.

42 Cannon projette très probablement la figure parue dans Gruber igiz. mais cela peut demander un temps assez long, pouvant aller jusqu'à deux heures. La situation est toute différente si on fait entrer en jeu l'adrénaline.

Comme le montre le tracé suivant ${ }^{43}$, si on fait une injection intraveineuse d'une très petite quantité d'adrénaline, cette petite quantité est capable de rétablir immédiatement dans le muscle fatigué le pouvoir de répondre à l'excitation. Quand un muscle d'un animal à sang chaud a été enlevé du corps pendant un temps prolongé et est

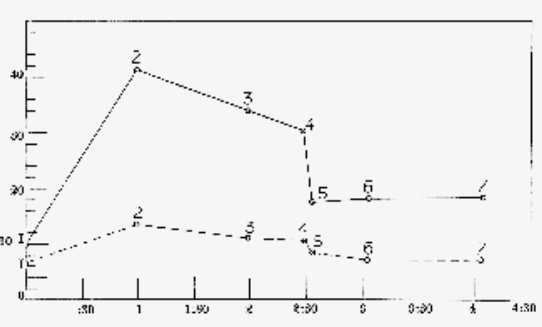
Figure 2. - A curve plotted from the data of one experiment the value of the threshold in $\beta$ units is represented on the ordinate. The continuous line is the curve of the muscle, the broken line that of the nerve-muscle. The curve of the nerve-muscle is magnified en times; that of the muscle is normal.

(1) Normal threshold stimulus. (2) The threshold after one hour's fatigue. The muscle contracted 120 times per minute against spring having an initial tension of $120 \mathrm{gm}$. (3 and 4) Threshols five minutes after an injection of adrenalin (0.1 (cc. of a 1:100,000 solution). (6 and 7) Thresholds after rest; after 60 minutes (6), and after 90 minutes (7) devenu insensible au choc électrique auquel on le soumet, l'addition d'une très petite quantité d'adrénaline à la solution saline au moyen de laquelle on perfuse les vaisseaux du muscle produit immédiatement un changement qui se traduit par une réponse après chaque stimulus.

Récemment, Redfield et Hunt ${ }^{44}$, dans le laboratoire de Harvard, ont remarqué qu'un muscle cardiaque qui ne se contracte plus après un séjour dans une atmosphère d'azote, continue à battre si on lui fournit de l'adrénaline. De plus, nous avons publié l'année dernière des expériences qui montrent

43 Cannon projette très probablement la figure parue dans Gruber igi4.

44 Bien que Redfield et Hunt aient tous deux travaillé sur l'adrénaline, nous n'avons pas trouvé de trace d'une publication commune aux deux auteurs. Alfred Clarence Redfield (I890-1983) est nommé professeur de physiologie à la Faculté de Harvard, en I92I, et directeur du laboratoire de biologie en I934. Il est un proche ami de Cannon. Il a soutenu un doctorat en I9I7, portant sur le rôle de l'adrénaline dans le blanchiment de la peau de crapauds à cornes à des fins de camouflage. Incidemment, les recherches postdoctorales de Redfield ont contribué à mettre au jour les effets radioactifs des radiations ionisantes sur les organismes vivants exposés aux rayons X (Redfield/Bright, I9I9). Reid Hunt (I870-I948) est un médecin et pharmacologue américain. Il devient professeur de pharmacologie à Harvard en I9I3. Il publie de nombreux travaux sur les effets hypertenseurs de l'adrénaline et les effets hypotenseurs de l'acétylcholine. 
que lorsqu'un chien a été soumis à une fatigue très prononcée, il est tellement exténué qu'il semble incapable de courir ${ }^{45}$. $\mathrm{Si}$ à un tel chien on donne une injection sous-cutanée d'une petite dose d'adrénaline diluée on augmente d'une façon étonnante sa capacité de travailler encore. Il recouvre à un degré étonnant sa capacité de courir. Dans nos expériences ces chiens ont produit un supplément de dix-sept (I7) à quarante-quatre (44) pour cent de ce qu'ils avaient déjà produit en énergie.

Ces observations posent une question intéressante en ce qui concerne la relation des fibres sympathiques avec les muscles du squelette. L'observation d'Orbeli ${ }^{46}$ d'après laquelle l'excitation des fibres sympathiques augmente l'amplitude de contraction d'un muscle fatigué, est en rapport avec l'action similaire de l'adrénaline. Ce serait là (de nouveau) une action sympathico-surrénale. De quelle manière l'adrénaline est-elle capable de faciliter la contraction, nous n'en savons rien encore. Gruber $^{47}$ a noté que l'adrénaline raccourcit de façon marquée la longueur de la période latente et de la période de contraction et produit des contractions plus élevées, effets qui furent considérés comme étant vraisemblablement dus à un accroissement d'irritabilité, ou à la libération d'une plus grande quantité d'énergie utilisable, ou à l'accélération du métabolisme du muscle.

Dans nos expériences avec les chiens travaillant sur un tapis roulant ${ }^{48}$, l'adrénaline n'avait d'effet que si l'animal était fatigué. Elle n'améliorait pas la capacité de travailler de l'animal

Campos/Cannon/Lundin/Walker, I929. Nous n'avons trouvé aucune information concernant H. Lundin, T. T. Walker et A. De Campos, rattachés au laboratoire de physiologie de l'École de médecine de Harvard au moment de la publication.

46 Orbeli 1923. Leon Abgarovich Orbeli (I882-I952) est un médecin d'origine arménienne, qui rejoint le laboratoire d'Ivan Petrovich Pavlov (I8491936) à Saint-Pétersbourg, en I899. Cannon décrit ici l'« effet d'Orbeli» soit le fait que la fatigue d'un muscle soit réduite par la stimulation des fibres sympathiques. Les travaux d'Orbeli ont contribué à conforter la thèse d'un système de stimulation humorale, empruntant notamment la voie sanguine et impliquant une substance spécifique, l'adrénaline. Gruber 1922.

48 Campos/Cannon/Lundin/Walker I929. si elle était injectée avant qu'il commençât à courir sur le tapis roulant. Le professeur Lapicque ${ }^{49}$ a trouvé que l'adrénaline pourrait favoriser le rétablissement de la fonction du muscle fatigué en rétablissant l'isochronisme du muscle et du nerf troublé par l'accroissement de la chronaxie du muscle. Ce point intéressant devrait être examiné de plus près dans des conditions normales d'effort prolongé. Le fait que l'adrénaline exerce un effet favorable sur un muscle fatigué, dont les nerfs ont été coupés et ont dégénéré, montre que l'hétérochronisme entre le nerf et le muscle probablement ne peut pas expliquer complètement le phénomène.

Il reste encore beaucoup à faire en ce qui concerne l'étude de l'influence de l'excitation émotive sur le travail musculaire. On peut trouver dans la littérature beaucoup d'exemples de grande puissance ou d'endurance prolongée, ou de suppression soudaine de la fatigue sous l'influence d'une intense excitation. Nous ne savons pas à quel degré nous pouvons avoir confiance en ces descriptions, et même s'il était prouvé qu'elles soient complètement exactes, l'importance relative de l'innervation directe des muscles intéressés, d'une part, et, d'autre part, d'une influence humorale, est problématique. Les procédés expérimentaux qu'il faut mettre en jeu pour obtenir des résultats définis sont difficiles, mais ils ne sont pas irréalisables, et il faut espérer qu'avant peu nous aurons en cette matière des résultats précis.

Quand on injecte de l'adrénaline dans le courant sanguin, la coagulation du sang se fait plus rapidement qu'avant injection. Cette observation a été faite incidemment par Elliott ${ }^{50}$,

49 Lapicque, I9II, 4.

50 Thomas Renton Elliott (I877-I96I) est un médecin et physiologiste britannique. Il devient professeur de médecine à l'University College Hospital en I9IO. De I90 à I906, il mène des recherches au département de physiologie de l'Université de Cambridge, sous la direction de John Newport Langley (I852-I925). On doit à ce dernier plusieurs découvertes. Il remarque premièrement que l'adrénaline induit des effets similaires à ceux d'une stimulation électrique des nerfs sympathiques, soit une augmentation de la pression artérielle par contrition des artères, une accélération du rythme cardiaque, une dilatation des bronchioles, etc. Il introduit la distinction entre les systèmes sympathique et parasympathique. Il décrit aussi leur structure, 


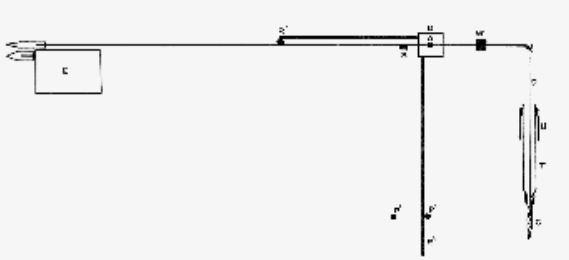

Figure 1. Diagram of the graphic coagulometer. The cannula at the right rested in a water bath not shown in this diagram. For further description see text.

moment de la coagulation, et pour cela nous avons employé un instrument qui enregistre graphiquement le fait que le sang est coagulé. Le cliché ${ }^{52}$ que vous voyez sur l'écran vous montre ce

notamment leur composition en deux types de nerfs, pré- et postganglionnaires. Cette dernière découverte l'amène à s'interroger sur le type de mécanisme assurant la transmission entre les synapses du ganglion et celles situées à la jonction des organes et des fibres postganglionnaires. Enfin Langley remarque que l'injection d'adrénaline, chez des animaux dont le système sympathique a été retiré, induit une contraction des muscles. Or, s'il était connu que les surrénales produisent de l'adrénaline, Langley estime que la vitesse de diffusion de l'adrénaline est trop lente pour agir sur les muscles dans des conditions normales. Il en vient à faire l'hypothèse que l'adrénaline n'agit ni sur les nerfs ni sur les muscles, mais sur les terminaisons nerveuses, sur lesquelles elle agit comme un transmetteur. Langley et Elliott sont ainsi les premiers à formuler l'hypothèse moderne d'une neurotransmission chimique. Elliot montre que la stimulation des nerfs splanchniques cause une augmentation de l'adrénaline, sans que le contenu des glandes médullaires ne diminue. Les terminaisons nerveuses du sys tème sympathique produisent donc bien l'adrénaline assurant la transmission entre le nerf et le muscle effecteur. Ce point va jouer un rôle important dans la controverse opposant Cannon à Stewart et Rogoff. Ces derniers vont se baser sur les résultats d'Elliott, afin de réfuter la thèse de Cannon selon laquelle le système adrénergique libérerait, durant de fortes émotions, de l'adrénaline, stimulant à son tour l'ensemble des fonctions corporelles afin de favoriser les réactions motrices.

51 Vosburgh/Richards I903. Alfred Newton Richards (I876-I966) est physiologiste et pharmacologue. Il se voit décerner un doctorat en chimie physiologique de l'Université de Columbia en I9or. Il devient professeur au département de pharmacologie de l'Université de Pennsylvanie en IgIo et joue un rôle central dans le développement de la pharmacologie expérimentale. Nous n'avons trouvé aucune information au sujet de Charles H. Vosburgh.

52 Cannon projette la figure parue dans Cannon/Mendenhall igr4a. Walter Leslie Mendenhall (I88I-I953) est pharmacologue, il assiste Cannon au département de physiologie de Harvard. De I92I à I946 il occupe le premier

coagulomètre. Le sang est recueilli dans le courant sanguin au moyen d'une petite canule; l'extrémité de la canule est immédiatement fermée au moyen de mastic. On l'attache alors au support et le tout est suspendu à un statif, la canule étant immergée dans un bain d'eau à vingt-cinq (25) degrés. Au-dessus du tube se trouve un levier inscripteur en rapport avec un cylindre

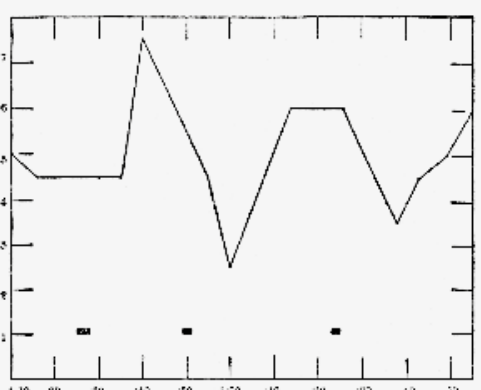

igure 4. - Résultats de la stimulation des nerfs splanchnique gauches $(4$ h. 25 à 4 h. 28$)$ après enlèvement de la capsule sur(4h. 49 i 4 h. 51 et 5 h. 23 i 5 h. 25). La caplanchich enfumé. Le bras du levier qui inscrit est légèrement plus lourd que l'autre bras avant qu'on suspende sur ce dernier un fil de cuivre portant un petit bouton à son extrémité inférieure. Ce bras est alors seulement légèrement plus lourd que l'autre. Le bouton se trouve au voisinage du niveau supérieur du sang dans la canule. Si on libère le bras inscripteur, le bouton s'enfonce dans le sang et inscrit par ce fait même l'absence de coagulation du sang. Quand la coagulation se produit, le bouton est encastré dans le caillot, le fil est alors supporté et le bras inscripteur étant plus lourd que l'autre ne s'élève plus. Par ce fait même, la présence du caillot est enregistrée. Évidemment, on prend la précaution de maintenir semblables autant que possible les conditions de plusieurs tests successifs.

La valeur de la méthode est démontrée par le cliché ${ }^{53}$ suivant qui montre le graphique d'une expérience dans laquelle le temps de coagulation du sang a été déterminé à plusieurs reprises. Si on injecte de l'adrénaline par voie intraveineuse ou si on excite un nerf afférent de manière à provoquer une décharge d'adrénaline, le sang coagule rapidement.

poste de professeur et de directeur du département de pharmacologie et de thérapie expérimentale de l'École de médecine de Boston.

53 Cannon projette très vraisemblablement la figure reproduite dans La Sagesse du corps (Cannon 1946[1939], 34), initialement parue dans Cannon/ Mendenhall igrua. 


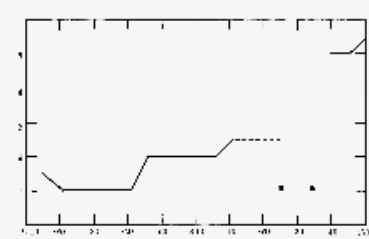

Figure 4. Rapid clotting after emotioal excitement, with slowing of the proin thorax (the left at 3.26 , the right a 3.35 .

L'accélération ne se produit pas si les surrénales sont inactivées et nous avons observé également que l'injection d'adrénaline n'accélère pas la coagulation si les intestins et le foie sont enlevés.

Le cliché ${ }^{54}$ suivant montre l'influence de l'excitation émotive sur la coagulation du sang. Dans cette expérience, avant de prélever le sang, le chat a été fixé à l'appareil de contention et l'artère fémorale découverte sous anesthésie locale. Pendant cette période l'animal présentait les signes d'une vive excitation. Le premier échantillon de sang prélevé a coagulé en une demi-minute. L'animal a été alors rapidement éthérisé, procédé toujours accompagné d'une grande agitation. Le graphique montre que le sang coagule en moins d'une demi-minute après le prélèvement. Aussitôt après la section des nerfs des surrénales le temps de coagulation s'est allongé jusqu'à trois minutes et demie. D'autres résultats analogues nous ont permis de tirer la conclusion que l'excitation du système sympathico-surrénale produite par une forte émotion est capable de produire une accélération marquée du processus de coagulation.

L'adrénaline en très petite dose est capable d'augmenter le métabolisme. Boothby ${ }^{55}$ a calculé qu'un milligramme d'adréna-

Cannon projette très probablement la figure parue dans Cannon/ Mendenhall, r9I4b.

55 Boothby/Sandiford I923. Walter M. Boothby (I880-I953) est un chirurgien et anesthésiste américain. Il poursuit ses études à l'Université de Harvard. Il devient en I9I3 anesthésiste et directeur d'un laboratoire spécialisé dans l'étude de la respiration au Peter Bent Brigham Hospital de Boston. Il est surtout connu pour avoir développé, avec Frederic Jay Cotton (I869-I938), un appareil d'anesthésie permettant de réguler en continu un mélange d'oxyde d'azote, d'oxygène et d'éther. En I9I6, il se voit offrir l'opportunité de développer un laboratoire dédié à l'étude du métabolisme, à la Mayo University de Rochester. Outre les travaux qu'il mène sur le rôle de l'adrénaline dans le maintien du métabolisme de base, il contribue au développement du «Boothby-Lovelace-Bulbulian mask», qui sera utilisé comme masque à oxygène médical ainsi que dans l'aviation militaire. Boothby est un ancien étudiant de Cannon. C'est lui qui propose à Cannon de diriger le laboratoire du Gaz Service mis en place à Paris, position qu'il occupe dès line donné en injection sous-cutanée libère chez un homme cinquante (50) calories supplémentaires. Aub et ses collaborateurs ${ }^{56}$, et McIver et Bright ${ }^{57}$ ont montré, dans le laboratoire de Harvard, que l'enlèvement des capsules surrénales est accompagné d'un abaissement du métabolisme et que l'injection d'adrénaline le rétablit à son niveau primitif. D’autre part l'adrénaline sécrétée par les surrénales à la suite de l'excitation des nerfs splanchniques accélère les processus de combustion de l'organisme tout comme l'injection d'adrénaline. Malheureusement, il y a très peu d'observations au sujet de l'influence de perturbations émotives - la peur par exemple - sur le métabolisme. Selon Landis ${ }^{58}$

février 1918. Irene Sandiford (1889-1978) est une médecin et biochimiste américaine, spécialisée dans le métabolisme et l'endocrinologie. Elle est nommée directrice assistante du laboratoire de métabolisme de la clinique Mayo et de la Fondation Mayo, de rgı6 à 193o. Elle se voit décerner un doctorat de chimie par l'Université du Minnesota en I9I9. En I930, elle est nommée professeure de médecine à l'Université de Chicago.

56 Aub/Forman/Bright I922. Joseph Charles Aub (I890-I973) est un endocrinologue américain. Il est nommé professeur à l'École de médecine de Harvard en I924, ainsi que directeur du laboratoire médical du Massachusetts General Hospital en 1928. Il est un des plus anciens étudiants de Cannon. En I9I7 et I9I8, il séjourne en France et étudie avec Cannon les chocs traumatiques que subissent les soldats. Jonathan Forman (I887-1974) est un médecin américain. Il passe l’année Igrg au Harvard Medical College, où il travaille sous la direction de Cannon. De I920 à I934, il pratique la médecine privée, avant d'être nommé professeur d'histoire de la médecine à l'Ohio State University de Columbus. Elizabeth Bright (I893-1975) est une physiologiste de l'Université de Harvard. Elle travaille auprès de Cannon, puis avec Alfred C. Redfield.

57 McIver/Bright 1924. Monroe Anderson McIver (I890-?) est un chirurgien américain. Il rejoint le laboratoire de Cannon durant les années I92I-I923.

58 Landis 1925. Carney Landis (1897-1962) est un psychologue américain. Dès 1930, il est nommé chercheur associé au New York State Psychiatric Institute and Hospital et professeur au département de psychologie de l'Université de Columbia, à New York. Landis remarque que l'anticipation d'un choc électrique augmente le métabolisme de ses sujets et que les variations du métabolisme en lien avec des émotions sont surtout d'ordre musculaire et vasculaire. Il observe encore qu'une émotion comme la colère peut aussi bien induire une augmentation qu'une diminution du métabolisme. Selon lui, il n'y a donc pas de lien causal nécessaire entre un stimulus émotionnel et une modification métabolique, pas plus qu'une variation du métabolisme basal ne peut être le marqueur d'une émotion spécifique. On doit à Landis toute une série de travaux qui infirment la thèse James-Lange, 
l'attente d'une excitation électrique douloureuse a augmenté le métabolisme de trois sujets de six (6), dix-sept (I7), et trentesept (37) pour cent. Augar ${ }^{59}$ a donné des résultats inconstants. Segal et ses collaborateurs ${ }^{60}$ a vu que le métabolisme des malades présentant un goitre exophtalmique est augmenté de vingt (20) à trente-cinq (35) pour cent quand on leur annonce qu'ils vont être opérés. Ici, de nouveau, il faut évidemment encore du travail et des faits nouveaux avant que nous puissions comprendre les causes internes qui produisent cette accélération du métabolisme dans les états d'excitation.

Si nous jetons un coup d'œil d'ensemble sur ces changements profonds et délicats que produisent les émotions fortes, nous voyons que tous - inhibition des mécanismes digestifs, sécrétion d'adrénaline, augmentation de fréquence du cœur, contraction des vaisseaux sanguins, dilatation des bronchioles, élévation du taux de sucre sanguin, élévation du nombre de globules rouges et blancs, aussi bien que le rétablissement des muscles fatigués, l'accélération de la coagulation du sang, et peut-être aussi l'accélération des processus d'oxydation dans l'organisme - nous voyons que tous ces phénomènes remarquables accompagnant l'état de grande frayeur et de colère sont

notamment celle portant plus spécifiquement sur l'expression faciale des émotions (Landis 1924). Ces travaux montrent notamment que, si les individus utilisent préférentiellement certains groupes de muscles faciaux, des différences interindividuelles marquées interdisent d'associer une expression faciale typique à une émotion particulière. Landis en déduit qu'il n'y a pas de correspondance entre l'émotion en tant que mot auquel est socialement attribué un sens et le patron d'expressions faciales qui l'accompagne. Contre Darwin, il estime que l'étude des émotions devrait donc plutôt s'apparenter à une "philologie des réactions expressives" (Landis I924, 484, notre traduction) plutôt qu'à une description «naturaliste» des comportements émotionnels.

59 Nous ne sommes pas parvenu à identifier la référence, ni l'auteur.

60 Segal/Binswanger/Strouse 1928. Harry Louis Segal (I900-I990) est un médecin et gastroentérologue américain. Il est nommé médecin en chef du Genesee Hospital à Rochester en 1955. Herbert F. Binswanger (I899I973) est un médecin et physiologiste américain. Dès I930, il enseigne à l'École médicale de la Northwester University, où il est nommé professeur en 1946. Solomon Strouse (I882-I966) est un médecin américain. Il enseigne à l'Ecole médicale de la Northwester University jusqu'en I937, puis à l'École de médecine de l'University of Southern California. plus ou moins directement les conséquences de l'action du système sympathique, ou, si nous considérons la médullaire surrénale comme une partie de ce système, les conséquences de l'excitation du système sympathico-surrénal. Ce système n'est pas sous le contrôle de la volonté, c'est-à-dire, qu'il n'est pas sous le contrôle du cortex cérébral.

Quelle est maintenant la signification de ces changements profonds dans l'organisme? C'est la question que nous examinerons dans la prochaine conférence. 
Deuxième conférence

\section{Interprétation des réactions émotives en fonction de leur utilité}

Au cours de notre dernière réunion, je vous ai décrit un groupe remarquable de changements physiologiques accompagnant les états de grande excitation, et j'ai montré que ces changements sont la conséquence de l'excitation du système sympathico-surrénal. Vous vous souvenez que parmi ces changements on trouve une mobilisation de sucre dans le sang; une redistribution du sang dans l'organisme à la suite de laquelle le sang est enlevé à l'aire splanchnique pour être plus abondamment fourni au cœur, au poumon, au système nerveux central et aux muscles des membres ; une abolition rapide des effets de la fatigue musculaire, et une coagulation plus rapide du sang.

Nous avons associé ces effets remarquables aux émotions et aux instincts les plus élémentaires : l'émotion de la peur et l'instinct de fuir ou d'échapper au danger, et l'émotion de la fureur ou de la colère et l'instinct d'attaquer. On peut considérer ces émotions et ces instincts comme élémentaires parce qu'ils conditionnent la lutte pour l'existence. Dans l'état de vie primitive, échapper au danger et attaquer dans le but de trouver de la nourriture ou d'assurer sa sécurité, sont des conditions essentielles pour la persistance de la vie.

De plus, ces émotions et ces instincts sont étroitement liés entre eux. Comme William James le disait il y a de nombreuses années, " nous craignons et en même temps nous désirons tuer ce qui voudrait nous tuer ${ }^{1}$. Et en dernière analyse, ces émotions et ces instincts sont en relation avec de nombreux autres instincts. Chaque fois qu'on porte atteinte à un sentiment puissant, ou à un comportement profondément enraciné, on peut être certain de voir se développer des tendances agressives ou de la colère. Si on approche d'un chien très occupé à ronger un

1 «We both fear, and wish to kill, anything that may kill us (James I89I, 4I5). os, ou si on dérange une mère occupée à témoigner sa tendresse maternelle, et si on fait quoi que ce soit qui puisse empêcher la satisfaction de ces tendances primitives, la faim et l'amour maternel, on provoque aussitôt la colère et en même temps la tendance à combattre.

Comme les exemples choisis le montrent, ces phénomènes élémentaires sont communs à l'homme et à l'animal. Quelle est leur signification?

Le caractère dominant de ces réactions aux causes qui provoquent les émotions, est, me semble-t-il, qu'elles appartiennent à la catégorie des réflexes : ce ne sont pas des mouvements volontaires. En fait elles causent souvent de la détresse en échappant au contrôle de la volonté. Nous n'apprenons pas à montrer de la peur ou de la colère. Dans ces émotions, comme dans d'autres, le schéma de la réaction est tracé dans les mécanismes du système nerveux central et quand survient une cause inadéquate, la réponse organique s'accomplit par la voie d'un automatisme inné.

On sait depuis longtemps qu'un des traits les plus typiques des réflexes est leur caractère adapté à un but ${ }^{2}$, leur caractère d'utilité, soit dans la préservation du bien-être de l'organisme, soit dans sa protection contre les atteintes extérieures. Il suffit de mentionner les réflexes de succion, de déglutition, de vomissement et de toux, par exemple, pour montrer par quelles voies variées les réflexes peuvent concourir à assurer le bien-être de l'organisme. Lorsque nous étudions les significations des réactions automatiques qui accompagnent la peur et la colère, il est donc raisonnable de nous demander d'abord quelle est leur utilité.

Les biologistes et les philosophes ont suggéré de nombreuses explications ingénieuses rendant compte des manifestations les plus apparentes des états d'émotion. Comme l'a fait remarquer le professeur Dumas ${ }^{3}$, les auteurs de ces tentatives ont souvent abusé de leur imagination pour trouver des interprétations compliquées de l'utilité du froncement des sourcils, de la dilatation des narines, et d'autres expressions faciales sans

Cannon remplace «intentionnel » par «adapté à un but ».

Dumas 1922. 
importance qu'on trouve dans les états de grande excitation. Nous pouvons admettre que certains muscles de l'expression sont intéressés dans la réaction sans utilité apparente et cela sans doute à la suite d'irradiation d'influx nerveux. D'autre part, comme j'espère pouvoir vous le montrer, l'explication qui s'applique de la façon la plus générale aux réactions viscérales profondes qui présentent le caractère des réflexes, est qu'elles se sont développées pour pouvoir être mises en jeu rapidement dans la lutte pour l'existence.

À ce propos, il est intéressant de noter que les manifestations viscérales accompagnant la peur et la colère, sont, dans les grandes lignes, les mêmes que celles résultant d'un travail musculaire important. Quand nous faisons des exercices violents, la fréquence du cœur augmente, la pression sanguine s'élève, il y a une libération du sucre du foie et un accroissement du nombre de globules rouges, la respiration est plus fréquente, la transpiration apparaît et il peut y avoir des perturbations des mécanismes digestifs. Le fait que ces manifestations apparaissent également dans la peur et dans la colère vient à l'appui de la manière de voir des anciens auteurs qui, traitant de l'évolution organique, ont noté le caractère anticipatoire des réactions émotives. Il est également en concordance avec l'idée émise par $\mathrm{McDougall}^{4} \mathrm{et}$ dont j'ai déjà fait mention, selon laquelle il y a une relation entre l'émotion de la peur et l'instinct de fuir et l'émotion de la colère et l'instinct d'attaquer. Selon cette manière de voir, les émotions préparent l'organisme à la lutte, car aussi bien pour celui qui attaque que pour celui qui est attaqué, aussi bien pour celui qui poursuit que pour celui qui est poursuivi, la lutte peut être décisive : son dénouement peut être la vie ou la mort.

Il semble alors que nous pourrions réunir les modifications physiologiques dont nous avons parlé dans la dernière leçon et qui nous ont paru assez dépourvues de relations en un groupe parfaitement ordonné, systématisé, et utile de modifications physiologiques, si nous pouvions avoir accès à leur signification.

4 Cannon s'appuie sur les travaux de McDougall, afin de montrer que James insiste beaucoup trop sur la dimension sensitive de l'émotion et ne prète pas ou peu d'intérêt à la part impulsive et instinctive de l'expérience affective (Cannon 1927, I23).
Je vous propose de les considérer sous l'angle des rapports de ces émotions avec les instincts correspondants et avec la lutte qui peut être la conséquence de ces instincts. Quelle est alors la valeur que peuvent avoir ces manifestations des émotions au cours d'un grand effort physique?

Tout d'abord, on sait maintenant très bien que le travail musculaire s'accomplit aux dépens de matériaux carbonés. Cela est démontré par l'accroissement au cours d'un travail musculaire important, de l'anhydride carbonique éliminé qui peut atteindre jusqu'à vingt (20) fois la quantité éliminée au repos, tandis que l'élimination azotée n'est pour ainsi dire pas augmentée. De plus, les travaux récents ont démontré l'importance des hydrates de carbone, et surtout du glycogène, comme source d'énergie. Le glycogène est mis en réserve dans les muscles. Il disparaît des muscles qu'on excite. Il est diminué par un travail prolongé et les muscles isolés ont une moins grande capacité de travail si leur réserve de glycogène est préalablement réduite. De plus, le sucre est apporté aux muscles par le sang et si cet apport descend en dessous d'un certain niveau, la capacité de travail est réduite parallèlement.

De plus, Chauveau et Kaufmann ${ }^{5}$, Quinquaud ${ }^{6}$, Morat et Dufourt ${ }^{7}$ ont montré qu'à l'état d'activité le muscle enlève au sang plus de sucre qu'à l'état de repos. Si on fait passer une série de fois du sang ou du liquide de Ringer ${ }^{8}$ à travers un muscle cardiaque qui se contracte, on voit que le sucre circulant disparait de façon plus ou moins marquée. Et en outre, de nombreuses

5 Chauveau/Kaufmann I886. Jean-Baptiste Auguste Chauveau (I827-I9I7) est un vétérinaire, médecin et physiologiste français. Il est nommé à la chaire de médecine expérimentale et comparée de la Faculté de médecine de Lyon en I877. Il montre notamment que les muscles consomment du glucose. Maurice Kaufmann (I856-I924) devient professeur à l'École vétérinaire d'Alfort en I888.

6 Quinquaud I886

7 Morat/Dufourt I892. Jean-Pierre Morat (I846-I920) est un médecin et physiologiste français. Il devient professeur à la Faculté de médecine de Lyon en I882. Nous ne sommes pas parvenus à identifier E. Dufourt, qui soutient une thèse de médecine à Lyon en 1892 .

$8 \quad$ La composition du liquide de Ringer, mise au point par le physiologiste britannique Sidney Ringer (I835-I9I0), s'approche de celle du milieu intérieur. Il permet de conserver provisoirement un organe en dehors du corps. 
preuves montrent qu'on peut augmenter directement la capacité de travail musculaire en augmentant la teneur du sang en sucre.

Si on perfuse un cœur isolé de lapin, dont les battements commencent à s'affaiblir avec du liquide de perfusion additionné de dextrose, on voit aussitôt les battements devenir plus forts et le cour peut continuer à battre pendant un temps allant jusqu'à sept heures.

Et enfin, comme l'ont montré Mann et Magath ${ }^{9}$, l'extirpation du foie a pour résultat, trois à huit heures après l'opération, une réduction marquée du sucre sanguin, accompagnée d'une grande faiblesse. Les muscles de l'animal peuvent devenir tout à fait mous et il semble incapable de les contracter, à l'exception du diaphragme. Si on lui fait une injection de glucose, il se remet sur ses pattes en moins d'une minute, marche, remue la queue, boit de l'eau, et se comporte tout à fait normalement. L'ensemble de ces faits viennent à l'appui de la thèse de la possibilité d'une utilisation rapide du sucre circulant par les muscles. On peut donc admettre que l'accroissement du sucre sanguin circulant que produit une forte excitation émotive peut être un grand appoint pour l'organisme dans les efforts violents qu'entraînent la fuite, le combat, ou la fuite pour se libérer.

Rappelons que dans notre dernière réunion nous avons vu que l'adrénaline elle-même, quand elle est déchargée dans le courant sanguin, peut avoir un effet favorable au cours de contractions musculaires marquées, en rétablissant, dans les muscles fatigués, l'excitabilité diminuée ou perdue. Nous avons vu aussi que, tandis qu'il faut de quinze minutes à deux heures de repos, selon la longueur de la période d'excitation, pour rétablir l'excitabilité normale, le même effet peut être produit par l'adrénaline en trois à cinq minutes.

9 Mann/Magath I922. Frank Charles Mann (I887-I962) est un chirurgien amé ricain. Il est nommé professeur de chirurgie expérimentale à la Mayo Clinic à Rochester, en I92I. De I935 à 1937, il préside l'American Physiology Society, que Cannon a présidée de rgr4 à ror6. On lui doit de nombreux travaux sur le rôle du foie dans la production d'urée, ainsi que sur la glycogénèse. Thomas Byrd Magath (I895-198I) est un médecin et physiologiste américain. Il mène l'ensemble de sa carrière au département de pathologie clinique de la Mayo Clinic.
De tout ce qui précède nous sommes autorisés à conclure que l'adrénaline déchargée dans le sang, non seulement aide à faire appel à du sucre aux dépens de la réserve glycogénique du foie, mais encore exerce une remarquable influence en rétablissant dans les muscles fatigués qui ont perdu leur excitabilité primitive, la faculté de répondre qu'ils avaient à l'état frais. À la suite de la libération d'adrénaline dans la peur et dans la fureur, les muscles fatigués sont donc remis complètement à la disposition du système nerveux. La difficulté que les influx nerveux pourraient avoir à mettre les muscles en activité complète est donc pratiquement abolie et ce fait, en combinaison avec l'apport de nouvelles sources d'énergie dans les quantités supplémentaires de sucre appelées dans le courant sanguin, concourt à mettre l'animal chez qui ces mécanismes fonctionnent de la façon la plus parfaite, dans les conditions les meilleures pour la production de grands efforts musculaires.

Des expériences faites récemment dans le laboratoire de Harvard ${ }^{10}$ montrent que l'injection continue de petites doses d'adrénaline - un millième de milligramme par kilo et par minute - réduit considérablement les processus d'oxydation dans l'organisme. Cela pourrait indiquer une diminution de la capacité d'utiliser le sucre sanguin en la présence d'adrénaline dans le sang. Il faut cependant noter que dans ces expériences, l'action déprimante de l'adrénaline s'observait

10 Colwell/Bright 1930. Arthur Ralph Colwell Sr. (I897-I978) est un médecin et physiologiste américain. Il obtient un doctorat de médecine du Medical College de Rush, dans l'Illinois. Il fait son internat au Presbyterian Hospital de Chicago, auprès de Woodyatt. Après un séjour de recherche de deux ans auprès de Cannon, au laboratoire de physiologie de l'École de médecine de Harvard, il retourne au Presbyterian Hospital de Chicago. En 1933, il est nommé à l'École de médecine de l'Université de Northwestern, à Chicago. L'étude montre que l'oxydation du glucose est abolie par l'administration prolongée d'adrénaline en intraveineuse. Les auteurs en concluent que ces données «suggèrent que le diabète mellitus ordinaire est le résultat d'un trouble fonctionnel du pancréas qui est dépendant d'une maladie du système nerveux sympathique, et qu'une sécrétion continue et excessive d'épinéphrine peut constituer un facteur intermédiaire important dans ce mécanisme » (Colwell/Bright 1930, 566, notre traduction). Les auteurs ne vont pas jusqu'à formuler l'hypothèse selon laquelle une émotion prolongée pourrait, via une sécrétion excessive d'adrénaline, favoriser le développement d'un diabète de type 2. 
dans le cas d'injection continue. Une injection unique d'une petite quantité d'adrénaline libère immédiatement du sucre dans le sang. L'action de l'adrénaline comme le met en évidence l'action sur le cour énervé, passe rapidement. L'effet sur le sucre sanguin, cependant, se prolonge longuement au-delà de cette brève période. L'adrénaline libérée au cours de l'excitation n'empêchera donc pas l'utilisation postérieure du sucre également libéré.

Des observations sur le métabolisme d'êtres humains après l'injection d'adrénaline ont montré que la présence temporaire de cette substance peut avoir non pas une action déprimante, mais une action excitante. Des soldats à qui on a injecté de l'adrénaline ont montré non seulement un métabolisme augmenté, mais encore augmenté d'une façon qui révélait une consommation plus marquée de glucose. En fait, l'adrénaline cause régulièrement une augmentation du métabolisme, ce qui ne se produirait pas si elle avait une action déprimante. Nous pouvons donc conclure que lorsque, par suite d'un état d'excitation il y a eu, dans le sang, libération d'adrénaline et augmentation du sucre, le pourcentage de sucre n'est pas plus grand à la suite de l'arrêt ou de la diminution de l'utilisation du sucre par les tissus; et une décharge temporaire d'adrénaline n'a pas d'effet nuisible sur l'utilisation postérieure du sucre comme source d'énergie.

Il y a d'autres faits en concordance avec la thèse d'après laquelle le sucre et l'adrénaline introduits dans le sang au cours de l'excitation émotive concourent à donner à l'animal plus de puissance dans la lutte corporelle : ce sont les changements vasculaires que produit le système sympathico-surrénal. Comme nous l'avons vu, la mise en action de ce système provoque la soustraction de sang des viscères au bénéfice des organes auxquels il est fait appel dans le combat: le système nerveux central, les poumons, le cœur et les muscles du squelette. L'absence de vasoconstriction effective dans le cerveau et les poumons quand la pression générale est augmentée, et la poussée plus grande de pression qui chasse le sang à travers ces organes et aussi à travers le cœur et les muscles squelettiques pendant les périodes d'activité augmentée, font dépendre l'apport de sang dans ces régions de l'accroissement de la pression sanguine générale. Dans les états de grande excitation, la pression est très élevée et par conséquent le courant de sang à travers les vaisseaux non contractés ou dilatés est particulièrement abondant.

L'importance de l'accélération du cœur, avec en même temps une augmentation de l'apport sanguin au cœur au cours d'un exercice musculaire a été démontrée par une expérience sur un chien au cœur complètement énervé. Alors qu'il avait encore son innervation cardiaque normale, ce chien a été habitué à pouvoir fournir, en deux heures trois quarts, vingt mille kilogrammètres de travail avant de montrer des signes d'épuisement. Sa fréquence cardiaque s'augmentait de quatre-vingtseize à cent cinquante-six battements par minute. Ensuite, sous l'influence de l'adrénaline la fréquence cardiaque est montée de quatre-vingt-quatre à cent cinquante-six par minute, l'accroissement étant donc de soixante-douze battements par minute, et l'animal s'est montré capable de courir pendant trois heures trois quarts et de fournir trente-trois mille kilogrammètres avant d'être épuisé. On a ensuite énervé le cœur. À la suite de cette opération l'accroissement maximum qu'on ait pu observer en faisant courir le chien, a été de douze battements par minute. Bien qu'on l'ait de nouveau habitué à travailler, le chien n'a pas été ensuite capable de courir assez longtemps pour qu'apparaissent des signes de fatigue. Cependant, si on lui donnait de l'adrénaline et que par ce fait même on accélérait considérablement le rythme cardiaque, l'animal devenait capable de courir pendant une période de temps considérable, une heure et demie, et de fournir un travail de douze mille kilogrammètres. Comme ces expériences le montrent clairement, l'adaptation que le cœur subit est de première importance pour l'effort musculaire, et l'accélération du pouls peut être considérée comme une préparation à un travail vigoureux.

Dans le combat et dans la fuite, il y a un besoin urgent d'un apport considérable d'oxygène pour brûler l'acide lactique qui résulte de la contraction musculaire et il est nécessaire que l'anhydride carbonique soit rapidement éliminé. L'oxygène et l'anhydride carbonique sont tous deux transportés par les globules rouges du sang. Quand la pression sanguine est élevée à la 
suite de la constriction des vaisseaux splanchniques et de l'augmentation de fréquence du rythme cardiaque, le sang, comme nous l'avons vu, coule plus rapidement à travers les vaisseaux non contractés du cerveau et de la moelle, et à travers les vaisseaux actuellement dilatés et des muscles en activité. Un plus grand parti est donc tiré des globules rouges utilisables à la suite de l'augmentation du nombre de leurs voyages des poumons aux organes en activité.

Outre ce mécanisme pour le transport d'un plus grand volume des gaz respiratoires, il y a libération par la rate d'une partie des globules qu'elle tient emmagasinés. Comme nous l'avons vu, une période courte d'excitation peut augmenter de vingt pour cent ou plus le pourcentage de ces globules dans la circulation. Ces transporteurs supplémentaires d'oxygène et d'anhydride carbonique ne sont pas seulement ajoutés à la circulation: ils y apparaissent alors que le cœur bat rapidement et que la poussée de pression artérielle est élevée. Les services qu'ils rendent à l'organisme sont multipliés par le fait de leur mobilité augmentée. On sait que la fatigue est largement dépendante de la quantité d'acide lactique présente dans les muscles qui travaillent. L'accroissement du nombre de globules rouges, produit pas l'état d'excitation, sera donc favorable à un exercice musculaire prolongé, parce que leur présence se traduit par l'apport de plus d'oxygène pour brûler l'acide lactique non volatil en anhydride carbonique volatil. Par ce fait même aussi l'anhydride carbonique est plus complètement enlevé aux muscles et rejeté par les poumons.

Lors du début d'un exercice vigoureux, la respiration se modifie de façon à provoquer une ventilation pulmonaire plus complète. Une respiration profonde et rapide est l'un des caractères les plus marquants d'une intense excitation émotive. Ici encore la réponse naturelle est celle qui rend le plus de services à l'organisme si la peur ou la colère sont accompagnées ou suivies par les efforts importants d'une fuite ou d'un combat. On sait qu'une respiration augmentée, comme celle dont il est question ici, peut réduire la teneur en sang en anhydride carbonique à un tel point que le besoin de toute respiration peut être supprimé pendant une minute et même plus.
De plus, une respiration augmentée modérément pendant plusieurs minutes avant un travail musculaire marqué, réduit considérablement la détresse respiratoire qui accompagne l'exercice subséquent. La quantité d'anhydride carbonique rejeté et la quantité d'air inspiré sont réduites; le cœur bat moins rapidement après l'exercice et revient plus rapidement à la normale. Bref, le renforcement anticipé de la respiration a eu une influence favorable prononcée sur le travail musculaire subséquent. On peut donc interpréter de façon semblable l'augmentation de profondeur de la respiration qui se produit au cours d'une émotion profonde. Elle produit une diminution préparatoire de l'anhydride carbonique dans le sang et prépare ainsi le terrain pour l'augmentation de production d'anhydride carbonique dans le sang dès que commence un exercice vigoureux.

À chaque respiration, l'air, pour entrer dans les poumons et pour en sortir doit traverser les fines ramifications des canaux aérifères ou bronchioles. Les bronchioles sont pourvues de muscles lisses qui, comme les muscles lisses des vaisseaux, par exemple, sont probablement maintenus dans un état permanent de contraction tonique. Quand cette contraction tonique est fortement augmentée, comme dans l'asthme par exemple, la respiration devient difficile. Dans ces conditions, même quand l'organisme est au repos, la ventilation pulmonaire minimale exige un effort anormal. Pendant un exercice fatigant, l'air doit pénétrer dans les bronchioles sous un volume plus grand et avec une vitesse plus grande à chaque respiration. Donc chez une personne époumonée par une course, les bronchioles doivent devenir relativement trop étroites pour le courant d'air qui les traverse, exactement comme elles sont trop étroites chez une personne souffrant d'asthme. Dans ces conditions une quantité supplémentaire d'énergie doit être dépensée pour faire mouvoir l'air dans les poumons et hors des poumons avec assez de vitesse pour satisfaire aux besoins de l'organisme.

Il est probable que, même dans les conditions les plus favorables, le travail de la respiration dans l'exercice marqué comporte, à un certain degré, le travail d'accélération de la circulation de l'air courant. Ce travail supplémentaire est évidemment réduit lorsque la contraction tonique des bronchioles est 
réduite et lorsque les conduits peuvent se dilater. De nombreux expérimentateurs ont montré au moyen de méthodes diverses que l'une des actions certaines de l'adrénaline injectée dans le courant sanguin est de dilater les bronchioles. Ladrénaline déchargée au cours de l'excitation émotive est, aussitôt après avoir quitté le cour, transportée aux artères des bronchioles et délivrée aussitôt à leur territoire. Le premier effet peut être le relâchement des muscles lisses des poumons. Ceci serait un autre moyen direct de mettre l'organisme dans une situation plus avantageuse lorsque les besoins d'une lutte soutenue réclament une ample fourniture d'oxygène et une élimination rapide de $\mathrm{CO}_{2}$.

Lorsqu'une forte émotion est suivie d'un exercice vigoureux et que les mécanismes circulatoires et respiratoires ne fournissent pas les quantités voulues d'oxygène, l'asphyxie survient. Nous avons fait des expériences ${ }^{11}$ qui montrent que l'asphyxie est capable de provoquer à peu près toutes les modifications provoquées par l'émotion elle-même. L'asphyxie peut donc agir de concert avec l'excitation émotive, ou en prolongeant l'effet de celle-ci, pour provoquer une sécrétion d'adrénaline, enlever du sucre au foie, et pour venir en aide aux muscles en action, comme nous l'avons vu. Le phénomène bien connu du second wind, qui consiste dans le rafraîchissement et le retour miraculeux de la vigueur chez un individu qui a continué un exercice violent bien qu'il fût hors d'haleine, peut être dû à la mise en jeu des mécanismes adjuvants qui semblent jouer un rôle si important en augmentant la vigueur de l'organisme.

Les modifications physiologiques que nous avons jusqu'à présent interprétées seraient favorables aux nécessités organiques de la fuite et du combat, dans la vie à l'état sauvage. L'augmentation de la vitesse de coagulation du sang dans les mêmes circonstances peut être également interprétée comme un processus d'adaptation favorable à l'organisme. Il n'est pas nécessaire d'insister sur l'importance de la conservation du sang, particulièrement au cours des efforts d'un combat acharné. L'effet du traumatisme local, favorisant la formation d'un caillot

11 Cannon rqI4a; Cannon/Hoskins igiı. obturant les vaisseaux ouverts, est évidemment un mécanisme d'adaptation pour la protection de l'organisme contre les hémorragies. La blessure qui provoque l'ouverture des vaisseaux sanguins est rendue moins dangereuse quant à l'éventualité d'une hémorragie sérieuse, par deux effets produits par la blessure dans l'organisme: l'effet local sur la coagulation dans la région de la blessure, et l'effet général sur la rapidité du processus de coagulation, amené par la sécrétion réflexe d'adrénaline.

Les effets inhibiteurs de l'excitation émotive sur les processus de la digestion ne sont pas favorables à l'action musculaire. Comment alors les interpréter? Il est possible et raisonnable, me semble-t-il, de considérer l'arrêt de la digestion par les émotions fortes comme l'accompagnement naturel de la constriction de l'aire vasculaire splanchnique. Le processus de digestion ne peut pas se dérouler de façon effective en l'absence d'une irrigation sanguine importante. Dans l'état de grande excitation, le sang est enlevé au tractus digestif. L'activité des organes digestifs est abolie quand les conditions ne sont plus favorables à la persistance de cette activité.

$\mathrm{Si}$ vous me permettez de tirer une conclusion de la discussion que je vous ai exposée, je dirai que les émotions fortes, comme la peur ou la colère, sont accompagnées de modifications physiologiques qui peuvent être raisonnablement interprétées comme rendant les plus grands services au cours de l'action violente qui peut faire suite à la naissance de ces émotions. Si on prend en considération l'association de l'effort avec l'excitation émotive, les modifications prennent aussitôt un sens, sinon on ne peut y voir que des perturbations nuisibles qui interviennent sérieusement dans les processus favorables à l'organisme. Ces modifications physiologiques accompagnant l'accès émotif sont fortement analogues à celles qui se produisent dans la douleur et dans l'effort. À tel point que, ainsi que je l'ai fait remarquer déjà, les anciens auteurs qui traitaient de l'évolution ont suggéré que les émotions pouvaient être considérées comme un tableau anticipé des souffrances et de l'intensité de la lutte. Parmi les organismes, donc, ceux qui possèdent au plus haut degré ces préparatifs physiques au combat et aux blessures possibles posséderont aussi les chances les plus grandes dans les 
combats acharnés dont peut dépendre la persistance de la vie de l'individu.

Une critique a été présentée à la conclusion que nous venons d'énoncer: c'est que la peur, par exemple, n'est pas toujours suivie de la fuite, mais que parfois l'animal se cache ou reste paralysé. Le professeur Dumas ${ }^{12}$, et avant lui le physiologiste Mosso ${ }^{13}$, ont insisté sur ce point. Sans doute, la frayeur peut être paralysante. Il me semble cependant que, dans le long processus de l'évolution, l'effet paralysant de la peur ne peut pas avoir été général. Il aurait infailliblement eu pour résultat la disparition rapide des organismes présentant la frayeur. De plus, il est probable que l'effet paralysant se prolonge seulement aussi longtemps qu'il y a indécision ou impossibilité d'évasion. Comme l'a montré Darwin, "un homme ou un animal poussé par la terreur au désespoir est pourvu d'une force étonnante et il est bien connu qu'il est extrêmement dangereux ${ }^{14}$. Ici encore, même si dans certains cas une grande frayeur peut se traduire par l'indécision et l'inaction, ce n'est pas là un argument contre les services rendus par les préparatifs à l'effort

12 Dumas I922, 39. Reprenant la terminologie de Wilhelm Wundt (I832-I920), Dumas distingue les émotions sthéniques des émotions asthéniques. Pour Dumas, l'expression émotionnelle procède d'une irradiation générale de l'excitation émotionnelle. Or ce type d'explication rend difficilement compte du fait qu'une excitation émotionnelle peut, à un certain moment, favoriser l'activité motrice et, à un autre, induire une paralysie motrice.

13 Mosso I886[I884]. Par exemple: «Je citerai, à mon tour, un autre phénomène qui semble en désaccord avec quelques hypothèses soutenues par Spencer et Darwin. S'il est vrai que, dans la lutte pour la vie, les animaux ont toujours perfectionné les attitudes propres à leur défense et abandonné progressivement, dans les générations qui succombaient, les dispositions de l'organisme nuisibles à la conservation de l'espèce, pourquoi ne sont-ils pas parvenus à se défaire du tremblement? Pourquoi, en présence d'un danger, lorsque l'existence est menacée, au moment le plus grave, quand rien ne serait plus nécessaire que la fuite, l'attaque ou la défense, nous voyons invariablement les animaux paralysés par le tremblement, incapables de lutter et succombant sans pouvoir faire usage de leur force? 》 (Mosso I886[I884], 99).

14 La citation originale est la suivante: «A man or animal driven through terror to desperation, is endowed with wonderful strength, and is notoriously dangerous in the highest degree. (Darwin 1872,86 ) Cannon ne suit pas la traduction française disponible à l'époque: «L'homme ou l'animal poussé au désespoir par la terreur acquiert une force prodigieuse, et devient dangereux au plus haut degré» (Darwin I890[I872], 86). quand ceux-ci sont mis en action, lorsque la frayeur est suivie d'une activité puissante et prolongée des muscles de l'organisme. Il est intéressant à ce sujet que les recherches psychologiques récentes de Stratton ${ }^{15}$ l'ont amené à conclure que « l'excitation et l'émotion ne sont pas généralement des causes d'incapacité, ou de réaction provoquant l'incapacité. Elles sont plutôt d'habitude des réactions qui augmentent notre capacité : elles complètent nos modes habituels de réponse, insuffisants en la circonstance. L'émotion elle-même, dans sa phase sthénique du moins, aide à nous mettre dans les conditions que réclament les circonstances. Elle nous aide à surmonter le danger en rendant mieux utilisables et plus parfaitement organisés les éléments de succès, dans tout ce qui est à notre disposition, que ce soit naturel ou acquis ou que ce soit sensoriel, moteur, intellectuel ou impulsif, que ce soit instinctif ou d'habitude. Derrière et à l'appui d'un but vivifié par l'émotion, une série de pouvoirs de caractère varié sont soudainement prêts à être utilisés. ${ }^{16}$

15 George Malcolm Stratton (I865-1957) est un philosophe et psychologue américain. Il est nommé professeur de psychologie à l'Université de Californie en 1908. Élève de Wundt, il développe à l'Université de Berkeley l'un des premiers laboratoires de psychologie expérimentale des États-Unis. On lui doit de nombreuses études sur la vision binoculaire, ainsi que sur les émotions et les maladies associées aux émotions. Si Stratton reconnaît la nécessité d'étudier la part physiologique de la vie affective, il ne se résout pas à réduire les émotions aux instincts, à la manière de McDougall, ni à un ensemble de sensations somatiques, à la manière de William James et de Carl Lange. Comme Cannon le soutiendra sur la base de données expérimentales, l'instinct et les déterminants somatiques constituent un ensemble de facteurs affectifs trop indéterminés pour rendre justice à la complexité des émotions.

16 On trouve le passage original dans Stratton $\left(1928,3_{3} 63\right)$ : «Excitement and emotion generally are not-primarily and usually causes of inadequacy, or reactions which reduce one's adequacy. They rather usually are reactions which increase our adequacy; they supplement our routine modes of response which at the moment appear inadequate. They may arise when calm action seems inadequate, indeed when it actually is inadequate. But the emotion itself normally, at least in its sthenic phase, helps us on toward a condition in which we are more nearly equal to the occasion. It helps us to meet an emergency by making more easily available, and by more perfectly organizing, whatever is of promise for success, of promise in our entire equipment, native and acquired, and whether it be sensory, motor, intellectual, hedonic, or of impulse, whether instinctive or of habit. Behind and in 
En donnant aux modifications physiologiques qui accompagnent l'excitation émotive une explication utilitariste, je désire insister sur le point qu'une semblable explication ne doit nullement être regardée comme définitive et finale ${ }^{17}$. Considérons la situation simplement. Nous avons été mis en présence d'un groupe de faits et nous avons essayé de les comprendre. Les faits prennent aussitôt une signification si nous les considérons à la lumière du long combat livré par les organismes pour la continuité de l'existence. Dès un passé éloigné, cette lutte a discipliné et sélectionné les plus aptes. Comme je l'ai dit, si la peur avait toujours pour conséquence la paralysie, son seul résultat serait de mettre l'organisme en danger de destruction. Les arguments que j'ai passés en revue me paraissent indiquer que la peur et les tendances agressives, en tant que réponses anticipées à des situations critiques, et en tant que préparation à l'action, peuvent avoir eu une grande influence dans la survivance des organismes au cours des âges.

Ainsi, me semble-t-il, les modifications accompagnant un état de grande excitation peuvent être interprétées raisonnablement, mais si on offre une explication plus raisonnable, sans doute serions-nous tous très heureux de l'accepter.

$\mathrm{Si}$ nous admettons que les modifications physiologiques accompagnant les états de grande excitation sont des préparatifs à une production soutenue et prolongée d'effort musculaires, nous trouvons une explication des nombreux exemples connus de force et d'endurance remarquables accompagnant l'excitation émotive. L'un des plus intéressants parmi ces exemples est récemment venu à ma connaissance. Il s'agit d'un pionnier américain, nommé Colter ${ }^{18}$, qui était en mille huit cent huit

support of a purpose enlivened by emotion, there is suddenly made ready for use an enlarged array of powers of most varied character.»

17 Cannon remplace «finaliste» par «définitive et finale».

18 John Colter (I770 ou I775-I8I2 ou I8I3) est un explorateur américain, membre de l'expédition Lewis and Clark (I804-I806). Il est le premier à explorer la région de l'actuel parc national de Yellowstone. Le récit de ses aventures, notamment sa course visant à échapper aux Indiens «Pieds-Noirs" (Blackfeets Indians), a fait l'objet de plusieurs narrations. Cannon cite ici la version de Thomas James. Ce dernier situe l'événement en I807 et non en I808: «Colter, naked and defenseless as he stood before him and made a en exploration dans l'ouest du Montana, avec un compagnon. Tous deux furent capturés par une bande d'Indiens sauvages. Colter fut mis nu. Son compagnon, qui résistait, fut tué et mis en pièces sous ses yeux. Le chef indien fit alors par signes à Colter l'ordre de s'en aller à travers la prairie. Quand il eut parcouru une courte distance, il vit les jeunes Indiens se débarrasser de ce qu'ils portaient, sauf de leurs armes et s'apprêter à la poursuite. Il se rendit alors compte de ce qui se passait: il s'agissait d'une course dont le prix devait être sa propre vie et son scalp. Il s'enfuit avec la vitesse du vent. Le cri de guerre des Indiens s'éleva aussitôt. Regardant derrière lui, Colter vit un groupe nombreux de jeunes guerriers le poursuivant rapidement, un javelot à la main. Il courut alors avec toute la vitesse que la nature, excitée au dernier degré, peut fournir. Comme le dit le récit: «La peur et l'espoir prêtèrent à ses jambes une vigueur surnaturelle, et la rapidité de sa fuite l'étonnait lui-même. ${ }^{19}$ Après trois milles environ ses forces commencèrent à faiblir. Il s'arrêta et regarda derrière lui. Un seul de ses poursuivants était proche. L'Indien se précipita vers lui, tenta de lancer son javelot, mais tomba de tout son long. Colter saisit le javelot, tua son ennemi et se remit à fuir «avec une vigueur renouvelée», comme il le dit à l'auteur du récit, "se sentant comme s'il n'avait pas fait

desperate lunge to transfix him. Colter seized the spear, near the head, with his right hand, and exerting his whole strength, aided by the weight of the falling Indian, who had lost his balance in the fury of the onset, he broke off the iron head or blade which remained in his hand, while the savage fell to the ground and lay prostrate and disarmed before him. Now was his turn to beg for his life, which he did in the Crow language, and held up his hands imploringly, but Colter was not in a mood to remember the golden rule, and pinned his adversary through the body to the earth by one stab with the spear head. He quickly drew the weapon from the body of the now dying Indian, and seizing his blanket as lawful spoil, he again set out with renewed strength, feeling, he said to me, as if he had not run a mile. A shout and yell arose from the pursuing army in his rear as from a legion of devils, and he saw the prairie behind him covered with Indians in full and rapid chase. Before him, if anywhere, was life and safety; behind him certain death; and running as never man before sped the foot, except, perhaps, at the Olympic Games, he reached his goal, the Madison river and the end of his five mile heat» (Thomas Igr6[I846], 6I).

19 «Fear and hope lent a supernatural vigor to his limbs and the rapidity of his flight astonished himself» (Thomas I9I6[I846], 60). 
un mille $»^{20}$, voilà donc un témoignage direct de la puissance physique extraordinaire que l'homme peut déployer en cas de détresse. William James, dans un de ses derniers essais ${ }^{21}$, a suggéré que chaque personne a des "réservoirs de puissance " auxquels il n'est pas fait appel en temps ordinaire, et qui cependant sont prêts à entrer en jeu quand l'occasion se présente. Cette expression figurée du psychologue peut recevoir une illustration définie et concrète dans les modifications physiologiques hautement utiles que nous avons examinées.

En rapport avec l'idée que les émotions fortes ont une valeur dynamogénique ${ }^{22}$, il est intéressant de noter que lorsqu'il est fait appel au système neuro-musculaire pour l'accomplissement d'un effort inhabituel, une excitation émotive est souvent présente. Dans les jeux que réclame la mise en action continuelle de la valeur physique, comme par

20 «He quickly drew the weapon from the body of the now dying Indian, and seizing his blanket as lawful spoil, he again set out with renewed strength, feeling, he said to me, as if he had not run a mile» (Thomas I9I6[I846], 6I). second souffle [second wind], en essayant de trouver une théorie physiologique. Il est évident que notre organisme dispose de réserves d'énergie accumulées qui ne sont généralement pas mobilisées, mais qui peuvent être utilisées: des couches de plus en plus profondes de matière combustible ou explosive, disposées de façon discontinue, mais prêtes à être utilisées par quiconque puise si profondément, et qui se réparent au repos » (James I9II, 230).

22 Le concept de dynamogénie a été formé par le physiologiste franco-britannique Charles-Édouard Brown-Séquard (I8I7-I894), qui succède en I878 à Claude Bernard à la chaire de physiologie expérimentale du Collège de France. Le concept est vraisemblablement introduit lors des trois communications que Brown-Séquard délivre à la Société de biologie le 8 janvier I88I, résumée dans le deuxième numéro de la Gazette hebdomadaire de médecine et de chirurgie, paru en janvier i88I. Pour Brown-Séquard, le terme «dynamogénie» est l'antonyme d'«inhibition »: «Les influences exercées sur le diaphragme et les nerfs phréniques par le chloroforme versé dans une narine ont été très souvent tout à fait semblables à celles qu'exerce ce liquide appliqué sur la peau d'un côté du thorax et sur une épaule. Il y a eu rupture d'équilibre entre les deux moitiés de l'appareil respiratoire diaphragmatique. En effet, le nerf phrénique et la moitié du diaphragme du côté correspondant à celui de la partie irritée par le chloroforme gagnent en énergie et en durée d'action ou d'excitabilité, après l'ouverture du thorax, tandis que le nerf phrénique et la moitié du diaphragme du côté opposé à celui de l'irritation ont une diminution notable de force et de durée d'excitabilité exemple le jeu de football, les conditions de la préparation du jeu et du jeu lui-même sont hautement favorables à la production d'un profond sentiment émotif. Le combat se passe, aux États-Unis, en présence d'un nombre immense de spectateurs, hommes et femmes, qui encouragent continuellement les joueurs, crient et chantent des chants entraînants. Chaque avantage remporté par un camp est le signal d'applaudissements de la part de ses partisans. En un mot, tout est mis en ouvre pour faire naître chez les joueurs cette excitation émotive qui est, comme nous l'avons vu, si favorable à la mise en jeu des forces de réserve pour un combat acharné. Ce que nous avons vu dans la dernière conférence montre que ces conditions sont effectives. Nous avons vu que la glycosurie existe chez presque cinquante pour cent des joueurs, ce qui signifie que le sucre sanguin est à peu près doublé par rapport à la normale. Comme le système sympathique fonctionne, dans la grande excitation, comme un tout, nous pouvons prendre l'accroissement du sucre sanguin comme le témoignage de l'action du système sympathique tout entier. Ce qui est vrai de l'excitation accompagnant les grandes compétitions sportives est également applicable à la frénésie prolongée

Il y a donc alors dynamogénie du côté irrité et inhibition du côté opposé.» (Brown-Séquard I88I, 28) Avec le temps, Brown-Séquard ne va plus restreindre le concept aux phénomènes d'inhibition et de dynamogénie induits dans une partie de l'organisme à la suite de l'administration d'une substance active ou d'une lésion du système nerveux. Il désigne plus généralement un état général de l'organisme. Brown-Séquard est aussi connu pour avoir commercialisé la « Séquardine », soit des extraits d'hormones testiculaires provenant de chiens, dont les injections sont supposées induire un certain tonus, en particulier sexuel: «Malgré l'insuffisance de défauts dans toutes les observations publiées jusqu'ici, malgré nombre de critiques que l'on a incontestablement le droit d'adresser à la plupart d'entre elles, il en ressort néanmoins, et d'une manière évidente, que le suc testiculaire employé a produit sur les centres nerveux tous les effets dynamogéniques que j'ai observés sur moi-même » (Brown-Séquard I889, 746), à savoir, entre autres, une baisse de la fatigue, une augmentation de la souplesse (Brown-Séquard I889, 743), une amélioration de l'humeur, une augmentation de la force et des capacités érectiles (Brown-Séquard I889, 744), etc. Bien que BrownSéquard ait été professeur de physiologie et de pathologie à Harvard de I864 à I869, Cannon semble plutôt se référer à Charles Richet (I850-I935) lorsqu'il utilise le terme. 
des danses cérémoniales primitives et dans les exemples d'endurance prolongée qu'on peut trouver dans les annales de la manie religieuse ${ }^{23}$. Dans de semblables exhibitions, il y a une excitation intense chez ceux qui y participent et qui peuvent prolonger continuer leurs efforts pendant des périodes étonnamment longues.

Il semble hautement probable que, tout comme chez les joueurs de football, des modifications physiologiques favorables à l'exercice neuro-musculaire soient développées par le fanatisme et que les performances anormales accomplies dans ces circonstances proviennent d'emprunts faits à ces réservoirs de puissance dont nous avons parlé, par l'intermédiaire de l'excitation émotive. J'ai seulement pu vous mentionner un petit nombre d'exemples de combinaisons d'excitation émotive extrême avec une vigueur musculaire étonnante. Dans de nombreuses autres conditions, ces deux groupes de phénomènes se montrent simultanément - dans la vie des pompiers et des policiers, dans les évasions de prisonniers, dans les récits de marches et de retraites forcées - et sans aucun doute beaucoup d'autres exemples pourraient être trouvés où l'émotion peut être regardée, comme nous l'avons fait, comme préparatoire à un effort musculaire effectif et à des déploiements anormaux de puissance et d'endurance.

Sous ce rapport, il est hautement significatif que, dans les moments de forte excitation, les témoignages montrent qu'il y a souvent l'apparition de la conscience d'une puissance

23 Cannon développe plus longuement ce point dans Bodily Changes in Pain Hunger, Fear and Rage. Parmi les exemples cités, on trouve celui des «manies dansantes » ou «chorémanies ": «Dans l'histoire des manies religieuses, il $\mathrm{y}$ a beaucoup de cas où un grand nombre de personnes deviennent fréné tiques et font preuve d'une endurance extraordinaire en dansant. En I374 une folie s'est répandue en Allemagne, aux Pays-Bas et en France, où les victimes prétendaient danser en l'honneur de saint Jean. Des hommes et des femmes dansaient main dans la main, par deux ou en cercle, dans la rue, dans les églises, chez eux, ou n'importe où, heure après heure, sans repos. Alors qu'ils dansaient, ils chantaient, poussaient des cris et avaient des visions. Des groupes entiers de ces fanatiques sont allés dansant le long des routes publiques et dans les villes.» (Cannon 1915, 222) Cannon décrit la manie telle qu'elle est présentée dans The Schaff-Herzog Encyclopedia of Religious Knowledge (Herzog/Schaff/Hauck 1909, 346). écrasante qui apparaît comme une marée soudaine et met l'individu à un niveau élevé de puissance.

Un de mes amis, qui est naturellement assez colérique, m'a dit que lorsqu'il est saisi par la colère, il a en même temps une intense conviction qu'il pourrait écraser et détruire entièrement l'objet de sa fureur. J'ai entendu dire par un joueur de football qu'avant le match final, un tel accès de puissance semble survenir en lui, qu'il se sent capable, à un signal donné, de se baisser et de passer d'un saut à travers une porte de résistance ordinaire. Il existe une satisfaction intense dans ces moments d'exaltation, lorsque le corps est au summum de sa puissance. Et, somme toute, il est possible que les dangers de l'aventure aient de l'attrait parce que la peur est excitante et que l'appel des ressources physiques pour surmonter les difficultés produit beaucoup de joie de la conquête. Pour cette raison, des hommes vigoureux s'en vont à la recherche du danger et s'exposent à des blessures dangereuses. «Le danger nous rend plus vivants. Nous aimons tellement à combattre que nous en arrivons à aimer la peur qui nous donne de la force pour le combat. La peur n'est pas seulement une chose dont il faut se débarrasser par la fuite, mais elle doit être accueillie comme un arsenal de nouvelles forces. ${ }^{24}$

Et c'est ainsi que dans les sports dangereux, dans les ascensions de montagnes et dans la chasse au gros gibier, les risques, l'excitation et le sentiment de puissance s'unissent pour libérer des énergies nouvelles et pour apporter à la conscience la révélation fraîche et vivante de nos possibilités d'accomplissement.

«Danger makes us more alive. We so love to strive that we come to love the fear that gives us strength for conflict. Fear is not only something to be escaped from to a place or state of safety but welcomed as an arsenal of augmented strength. » (Hall I9I4, I54) On doit cette citation à Granville Stanley Hall (I844-I924), philosophe et psychologue américain. Élève de Wundt et de James, il est principalement connu pour ses travaux sur la psychologie de l'adolescence. Il devient le premier président de l'American Psychological Association en I892, et le premier président de la Clark University en I889. Il est notamment à l'initiative du seul séjour de Freud aux États-Unis, en I909, durant lequel celui-ci délivre à la Clark University une série de cinq conférences originalement publiées dans l'American Journal of Psychology (Freud 1923). 
À la conférence prochaine je discuterai l'organisation nerveuse de l'expression des émotions et je montrerai des clichés des animaux complètement dépourvus du système sympathique ${ }^{25}$.
Troisième conférence

\section{Mécanismes nerveux}

de l'expression des émotions

J'ai montré dans ma première leçon qu'au cours d'une grande excitation émotive il se produit non seulement des phénomènes typiques d'innervation de certains des muscles squelettiques mais aussi une décharge d'influx dans les muscles lisses et dans les glandes des viscères par la voie de la portion sympathique du système nerveux autonome. D'autre part, nous n'avons de contrôle que sur nos muscles squelettiques; nous ne pouvons pas d'habitude exercer de contrôle volontaire sur nos viscères. Nous pouvons contracter ou relâcher nos biceps quand nous le voulons, mais nous ne pouvons pas directement produire d'accélération ou de ralentissement de notre cœur ou produire à volonté des contractions ou des relâchements de notre estomac. Il y a là une dissociation curieuse : d'une part, un contrôle rapide et effectif des systèmes squelettique et viscéral lorsqu'une émotion forte prend naissance, et d'autre part, un contrôle limité au système squelettique quand il s'agit d'un ordre froidement considéré ou d'un ordre intellectuel. En faisant mention de cette distinction, j'ai employé la terminologie des psychologues; en fait, j'ai employé les mots du langage courant. Ces mots suggèrent qu'il peut y avoir un double contrôle ou peut-être un contrôle partant de deux niveaux différents du système nerveux, c'està-dire d'un niveau inférieur ou les actions d'ordre émotif non volontaire peuvent prendre leur origine, et d'un niveau supérieur - l'écorce cérébrale - dont partiraient les actions volontaires. Un argument intéressant en faveur de cette manière de voir se trouve dans des expériences sur des animaux et dans des observations sur l'homme placé par la maladie dans des conditions analogues aux conditions expérimentales.

En partant du point de vue physiologique, une réaction émotive est un modèle typique de réaction. Ses traits sont les signes habituels que nous employons pour juger si le sujet est 
triste ou heureux, fâché ou effrayé. En considérant aujourd'hui l'organisation des émotions, je me propose de limiter d'abord la discussion aux réactions typiques de la colère ${ }^{1}$.

Le complexe des modifications physiologiques qui apparaissent dans les émotions fortes, dans la colère, par exemple, a beaucoup de traits communs avec les réflexes simples comme l'éternuement, la toux, et la succion. D'abord, le fait qu'ils apparaissent au cours des premiers mois de la vie d'un organisme aussi développé que le petit enfant indique que les voies nerveuses, comme celles des réflexes que nous venons de mentionner, sont tracées congénitalement dans les mécanismes du système nerveux central. Deuxièmement, comme nous le voyons chez les réflexes, il y a une réponse rapide au stimulus adéquat. De plus, il s'agit d'une réponse constante et uniforme, à tel point qu'on ne se trompe pas sur sa nature, que cette réponse soit montrée par les diverses races d'hommes ou par les animaux. Ce complexe est également analogue aux réflexes dans le fait qu'il constitue un mode de réaction permanente; pendant toute la durée de la vie, les signes caractéristiques de la colère peuvent être soudainement provoqués dans toute leur complexité. De plus, il s'agit là d'une réponse à un stimulus bien défini: c'est un stimulus interne qui naît chaque fois qu'il y a un empêchement à un mouvement ou une opposition à une impulsion de nature primitive. Enfin, comme les réflexes simples, la réaction de colère est utile.

Dans les deux dernières leçons j’ai appelé votre attention sur le grand nombre de mécanismes d'adaptation physiologique qui se produisent chez l'animal en fureur - l'accélération du rythme cardiaque, la redistribution du sang, l'accroissement de globules rouges dans la circulation, l'augmentation de la profondeur de la ventilation pulmonaire, la dilatation des bronchioles, la libération de sucre par le foie, la sécrétion d'adrénaline avec son action favorable sur les muscles fatigués, autant de réactions qui peuvent être considérées comme rendant l'organisme plus

1 Cannon biffe une phrase: «Plus tard nous discuterons les enseignements que les faits se rapportant à ce système de réactions peuvent nous donner à propos d'autres systèmes.» puissant dans la lutte, lutte qui peut être nécessaire pour surmonter une opposition extérieure et pour permettre la prédominance des impulsions naturelles. Il semble donc clair que les caractères principaux des réflexes simples, - leur nature innée, leur rapidité de production, leur constance, leur uniformité, leur permanence, et la nature utile de leur réponse à un type défini de stimulus - tous ces caractères des réflexes simples sont reproduits dans ceux d'un accès de colère.

Il y a de nombreuses raisons de penser que tandis qu'une réponse retardée - incertaine, temporaire et parfaitement modifiable - met en jeu le cortex cérébral, les réactions rapides, uniformes, et stéréotypées s'accomplissent à des niveaux moins élevés du cerveau et de la moelle. Il devient intéressant de nous demander où est le siège des mécanismes nerveux mis en jeu dans le complexe de la colère. Ce mécanisme est-il localisé dans les neurones corticaux ou dans les portions les plus primitives du névraxe ? ${ }^{2}$

Il y a dans le tronc cérébral un groupe de centres qui, chez les vertébrés inférieurs sans cortex, accomplissent des fonctions élémentaires pour le maintien de l'existence. Dans les formes plus élevées, ces centres, bien que normalement sous le contrôle de l'écorce, sont capables de fournir une réponse énergique quand les conditions réclament une action immédiate. Quand on supprime le contrôle cortical, ces mécanismes secondaires deviennent prédominants. Les chiens de Goltz ${ }^{3}$ et Rothmann ${ }^{4}$

2 Le névraxe désigne l'ensemble du système nerveux central, soit l'encéphale et la moelle épinière.

3 Goltz I892. Friedrich Goltz (I834-I902) est un physiologiste allemand. Il est nommé professeur à l'Université de Strasbourg en I872. Il développe une technique d'hémisphérectomie consistant à injecter de l'eau sous pression dans la boîte crânienne de chiens. Il montre notamment que les chiens hémisphérectomisés continuent à se mouvoir presque normalement. Ces faits valident selon lui la conception holiste du système nerveux, qu'il défend contre David Ferrier (I843-I928), pour qui il existe des zones cérébrales spécialisées, notamment motrices.

4 Rothmann 1923. Hans Rothmann (I899-I970) est un médecin et physiologiste d'origine allemande, beau-frère du célèbre neuropsychiatre Kurt Goldstein (I878-1965). Il mène des recherches au Dutch Brain Research Centre à Amsterdam, avant de pratiquer la médecine à Halle. Il fuit l'Allemagne nazie et s'installe à San Francisco. 
et les chats de Dusser de Barenne ${ }^{5}$, dépourvus d'hémisphères cérébraux mettent ce point en évidence. Dans des conditions variées ces animaux ont réagi habituellement avec des manifestations de colère. Le chien de Goltz montrait un accès typique de fureur quand on le retirait de sa cage pour le nourrir. Il aboyait violemment, cherchant à mordre et se débattant vigoureusement. Des stimulus légers comme celui de tirer ou d'appuyer sur la peau donnait naissance à des réactions émotives exagérées du même ordre. Le chien de Rothmann se comportait d'une façon analogue. Dès qu'on égratignait légèrement son dos, il se mettait à grogner, et la présence d'une mouche sur son museau le faisait entrer dans un accès de fureur. Chez le chat de Dusser de Barenne le pincement des pattes ou de la peau produisait de violents mouvements de défense et des réactions analogues à celle du chat en colère - des crachements, des grognements et le hérissement des poils de la queue et du dos. Ici, de nouveau, de très légères excitations se montraient capables de provoquer la même réponse; elle se produisait même si on soulevait simplement le chat du plancher.

Ces différents exemples de réactions de colère typique paraissant chez des animaux dépourvus de cortex cérébral peuvent être interprétés comme des exemples de «phénomène de libération » pour employer l'expression introduite par le grand neurologiste anglais, Hughlings Jackson ${ }^{6}$ - c'est-à-dire,

$5 \quad$ Dusser de Barenne I9I9. Johannes Gregorus Dusser de Barenne (I885-I940) est un physiologiste d'origine hollandaise, spécialiste de la physiologie du cervelet et des hémisphères cérébraux. Il fait carrière aux États-Unis, à la Yale University de New Haven.

6 Hughlings Jackson I888. John Hughlings Jackson (I835-I9II) est un neurologue anglais. Cannon fait ici référence à sa théorie de l'évolution et de la dissolution. Pour Jackson, le système nerveux est composé de trois strates successivement formées au cours de l'évolution. Le premier niveau, comprenant le bulbe rachidien et la moelle épinière, contiendrait les centres des mouvements simples. Le niveau intermédiaire contiendrait les aires motrices du cortex. Les aires préfrontales constitueraient le niveau supérieur. Pour Hughlings Jackson, les centres supérieurs inhibent ou contrôlent les niveaux inférieurs. Sur cette base, il appréhende les maladies psychiatriques comme procédant d'une dissolution des fonctions, terme qu'il emprunte au philosophe et sociologue anglais Spencer. Les symptômes négatifs des maladies mentales seraient des phénomènes résultants de la mise en activité de centres inférieurs du système nerveux et produits par des stimulus légers quand la prédominance des centres supérieurs est supprimée. Il a semblé raisonnable d'espérer que ces centres inférieurs feraient montre de leurs activités typiques après l'enlèvement de l'écorce cérébrale au cours d'une expérience. De plus, l'enlèvement du cortex supprime une condition essentielle pour la sensation et par conséquent nous dispense d'employer une anesthésie qui peut avoir une action troublante ou modificatrice.

Conformément à cette idée, nous avons entrepris (sur des chats) l'étude de quelques effets immédiats de la suppression de l'écorce cérébrale ${ }^{7}$. Un stylet comprimé à travers le quadrant supérieur et interne de l'orbite gauche et poussé en suite au côté opposé au crâne est promené vers le bas et sur les côtés et ramené le long du plancher de la tente osseuse. Une opération analogue est faite à travers l'orbite droite. Nous avons employé une opération très rapide par laquelle (l'opération ne durant que deux ou trois minutes) le cerveau est pratiquement décortiqué et la plus grande partie des ganglions de la base reste intacte. Aussitôt après suppression de l'anesthésie, un remarquable ensemble de phénomènes se produisent, analogues à ceux que l'on observe chez l'animal en fureur - une sorte de fausse colère.

Voici la liste complète des effets pseudo-émotifs que nous avons observés: la queue s'agite violemment, le tronc se courbe, il y a des soubresauts des membres dans les liens qui maintiennent l'animal sur l'appareil de contention, les griffes se découvrent dans les pattes antérieures et l'animal tente de griffer pendant un temps prolongé; le chat miaule, agite la tête d'un côté et de l'autre en essayant de mordre et sa respiration est extrêmement rapide et haletante. Ces manifestations se produisent sans que l'on n'exerce aucune autre excitation que le traumatisme opératoire et le maintien de l'animal sur son support, par accès ou par périodes, durant de quelques secondes à plusieurs minutes. Pendant les périodes de repos intermédiaires

dus aux dysfonctions induites par les lésions cérébrales. Les symptômes positifs résulteraient quant à eux de l'absence de contrôle des fonctions inférieures par les fonctions supérieures devenues dysfonctionnelles.

7 Cannon/Britton I925. 


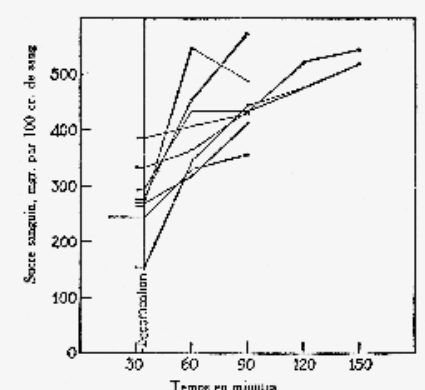

Figure 22. - Sucre sanguin chez des aninaux décortiques présentant des symptômes pseudo-affectifs. on peut produire un accès en manipulant légèrement l'animal, en touchant les pattes ou en remuant la table.

Outre ces manifestations qui mettent en jeu les muscles squelettiques, il y a des effets plus typiques et d'un caractère plus permanent produits par des influx sympathiques : l'érection des poils de la queue qui se rétablit de façon rapide et persistante après qu'on lisse les poils, hérissement des moustaches, transpiration des plantes des pattes, dilatation de

a pupille jusqu'à trois fois ses dimensions des périodes de repos, miction, élévation de la pression sanguine et production d'adrénaline. Ce dernier effet est montré par l'action du cœur énervé. La fréquence cardiaque, normalement de cent quarante (I40) battements par minute chez un chat tranquille, est fortement augmentée dans la fausse colère. Nous avons pu dans ces conditions enregistrer une fréquence de plus de trois cents (300) battements par minute. Également, il y a une tout à fait extraordinaire accélération de la rapidité de la respiration, qui peut être de plus de deux cents (200) mouvements par minute; c'est évidemment une respiration haletante. De plus, comme le montre le cliché suivant ${ }^{8}$, nous avons observé un accroissement notable du sucre sanguin jusqu'à cinq (5) fois la concentration normale.

En excitant des nerfs sensibles, Woodworth et Sherrington ${ }^{9}$ ont pu produire chez des chats décérébrés quelques-uns de ces phénomènes et ils ont appelé les manifestations ainsi obtenues «réflexes pseudo-affectifs». À cause de la ressemblance de ces réactions (apparemment spontanées) de nos animaux avec les

8 Cannon projette la figure reproduite dans La Sagesse du corps (Cannon I946[I939], 93); la figure est initialement parue dans Bulatao/Cannon 1925 b.

9 Woodworth/Sherrington I904. Robert Sessions Woodworth (I869-I962) est un psychologue américain. Il entre à Harvard en I895 et se destine à une carrière de philosophie et de psychologie. Il y côtoie William James, le psychologue Edward Lee Thorndike (I874-I939) ainsi que Cannon, encore étudiant, avec qui il se lie d'amitié. Après avoir obtenu un doctorat en psychologie de l'Université de Columbia en I899, Woodworth activités reflexes décrites par Woodworth et Sherrington, nous avons employé le terme « pseudo-affectif» pour désigner la préparation. Les animaux présentent cet état pseudo-affectif ou de fausse colère (à des intervalles rapprochés), pendant deux ou trois heures avant que la pression artérielle tombe assez bas pour supprimer toute activité.

Les phénomènes réflexes pseudo-affectifs observés par Woodworth et Sherrington, et également par Bazett et Penfield ${ }^{10}$, sur des préparations décérébrées chroniques, se présentaient comme des perturbations d'un état de rigidité par ailleurs parfaitement connu. Dans la décérébration la section passe par

est nommé démonstrateur de physiologie à l'Université de Liverpool, où il mène ses recherches sous la direction de Charles Sherrington. Dès I903, il fait carrière à l'Université de Columbia, où il abandonne la physiologie au profit de la psychologie expérimentale. Pour Sherrington, voir l'introduction. Dans la lignée des travaux de Friedrich Goltz, Woodworth et Sherrington mettent au point des techniques de décortication. Ils montrent que les animaux décérébrés manifestent toujours des réactions "pseudo-effectives » (sham rage) plus ou moins coordonnées, mais qui ne s'apparentent plus à de véritables réactions d'attaque ou de fuite.

10 Bazett/Penfield 1922. Henry Cuthbert Bazett (I885-1950) est un médecin et physiologiste britannique. Chargé de cours de physiologie clinique à l'Université d'Oxford en I9I9, il se voit offrir, en I92I, un poste de professeur de physiologie à l'Université de Pennsylvanie à Philadelphie. Il préside l'American Physiological Society de I885 à 1950. Outre de nombreux travaux sur la régulation de la température, la circulation et le volume du sang, il est surtout connu pour avoir développé une méthode de calcul de l'intervalle Q-T, soit le temps entre la dépolarisation du myocarde ventriculaire et la fin de la repolarisation. Wilder Graves Penfield (I89I-1976) est un neurochirurgien américano-canadien. Il étudie dans plusieurs villes d'Europe, puis à New York, avant de s'installer à Montréal en 1928. En 1932, il devient le directeur du Montreal Neurological Institute and Hospital de l'Université McGill. Penfield est surtout connu pour ses techniques opératoires visant à détruire les foyers épileptogènes de patients souffrant d'épilepsies récalcitrantes. Les opérations sont menées sur des patients sous anesthésie locale, qui peuvent consciemment rapporter les sensations induites par des stimulations électriques, à même leur cortex, au moyen d'électrodes. La méthode, qui permet initialement de localiser les foyers épileptogènes, permet de cartographier le cortex somatosensoriel. Penfield fait réaliser plusieurs "homoncules", dit de Penfield, visant à illustrer comment le cerveau «représente» le corps sensorimoteur (Penfield/Boldrey 1937). La collaboration de Bazett et de Penfield date des années I9I9-I920, lors du deuxième séjour que Penfield passe à Oxford et durant lequel il travaille, sous la direction de Sherrington, sur les animaux décérébrés. 

H. C. - hémisphères cérébraux $; \mathrm{D}=$ diencéphale (indiqués par des points) $; \mathrm{M}=$ mésencéphale; $\mathrm{Ma}=$ moelle allongée;

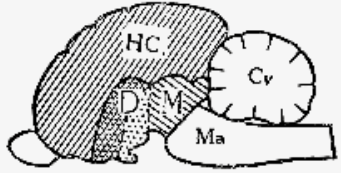

igure 33.-Section médiane d'uncerveau demammifêre.

le cerveau intermédiaire. Elle est représentée par la lettre $M$ dans la projection suivante ${ }^{11}$. Le diencéphale et le cerveau antérieur tout entier sont donc mis hors d'action. Évidemment la portion du système nerveux qui produit directement la réaction de colère est située postérieurement à la section intéressant restante du cerveau intermédiaire ou dans la moelle. Chez ces animaux décérébrés, cependant, par opposition aux animaux décortiqués, la production spontanée de la fausse colère est rare; il faut un stimulus relativement fort pour l'évoquer et il est possible qu'on observe alors certains caractères isolés de la réaction totale et elles peuvent être associées parfois à des éléments de nature différente, comme cela se voit dans l'association de violentes tentatives de griffer avec des ronronnements.

C'est là le point qui s'oppose à la manifestation violente et complète de la fureur que peuvent montrer les animaux décortiqués. La différence de comportement doit être attribuée à une portion du tronc cérébral qui fait défaut chez l'animal décérébré mais qui est présente chez l'animal décortiqué. En d'autres termes, bien que diverses circonstances de la réaction de colère puissent être produites par des influx afférents chez l'animal bulbospinal, l'intégration de ses réponses en un accès de colère énergique et aux manifestations typiques parait être sous le contrôle d'un centre supérieur. Où est situé (parmi ces ganglions basaux) le centre principal? Cette question a été étudiée par un de mes élèves, Bard ${ }^{12}$.

La méthode employée par Bard a été celle de l'ablation de quantités variables de la substance du tronc cérébral après

11 Cannon projette la figure reproduite dans La Sagesse du corps (Cannon I946[I939], I99), initialement parue dans la seconde édition de Bodily Changes in Pain, Hunger, Fear and Rage (Cannon I922[I9I5], 247).

12 Bard 1928. Bard a développé une technique consistant à retirer la voûte du crâne. À partir de là, il supprime le cortex, puis des sections du tronc cérébral, jusqu'à ce que les animaux ne soient plus sensibles aux réactions pseudo-affectives. Pour Bard, voir l'introduction. décortication; il étudia ensuite le comportement de la préparation. Bard a montré qu'une fausse rage typique accompagnée de violentes décharges d'influx sympathique se produit sous la forme d'accès spontanés après enlèvement complet des deux
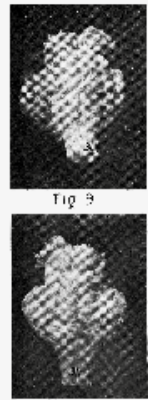

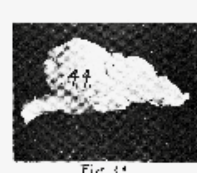

Fiģ 1:

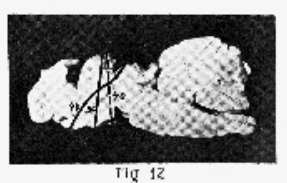

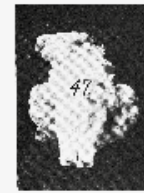

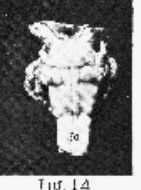

hémisphères cérébraux, des corps striés, et de la moitié antérieure du diencéphale. Sur le diagramme que vous voyez sur l'écran ${ }^{13}$ la ligne de section est représentée par une ligne interrompue. Si en outre on enlève la moitié postérieure du diencéphale l'activité spontanée est rapidement abolie, mais comme elle persiste après enlèvement d'une grande partie de la portion dorsale de la région, nous pouvons conclure que le centre principal est situé dans le voisinage de la base, et probablement dans l'hypothalamus. Sa dimension chez le chat est de moins d'un quart de centimètre cube.

Fulton et Ingraham ${ }^{14}$ ont apporté récemment une confirmation intéressante des résultats de Bard. Par des procédés chirurgicaux ils ont coupé les cordons cortico-thalamiques et par conséquent ont libéré les centres thalamiques du contrôle de l'écorce. Les chats rétablis de cette opération n'étaient plus familiers et ne jouaient plus, mais présentaient un véritable

13 Cannon projette vraisemblablement la figure I2 parue dans Bard I928.

14 Fulton/Ingraham, 1929. John Farquhar Fulton (I899-I960) est un médecin et physiologiste américain. Après avoir achevé sa formation médicale à l'Université de Harvard en I9I7, il obtient un doctorat de l'Université d'Oxford en I92I. En 1929, il est nommé professeur de physiologie à l'Université de Yale. Il contribuera à la création du Yale Aero-Medical Research, visant notamment à étudier l'adaptation physiologique aux vols en haute altitude. En I95I, il renonce à son poste et devient professeur d'histoire de la médecine. Franc Douglas Ingraham (I898-1965) est un neurochirurgien américain. L'article cité par Cannon est le fruit des recherches qu'Ingraham et Fulton ont menées au cours de l'année i928, sous la direction de Sherrington, dans le laboratoire de physiologie de l'Université d'Oxford. 


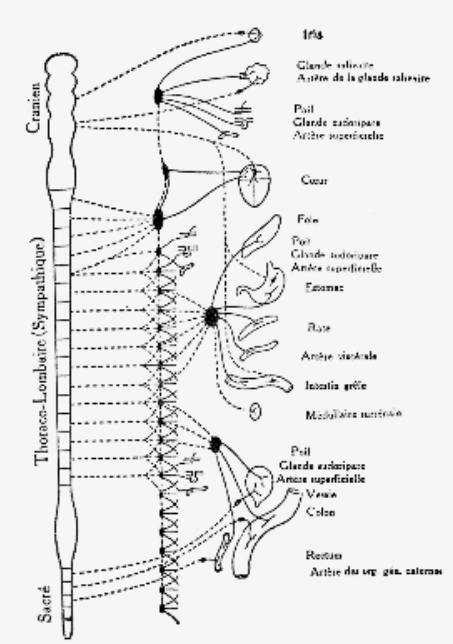

Figure 34. - Diagramme de l'anatomie générale du système nérveux autonome. Le cerveauet la moelle épinière sont représence schéma. Les fibres préganglionnaires sont en traits interrompus, les fibres postganglionnaires en traits pleins. Pour détails, voir texte. (Bard, d'après Cannon, « Foundations of experimental Psychology ", avec l'autorisation de la « Clark University Press $\gg$

état de colère chronique. Si on les caressait doucement sur le dos, par exemple, on déterminait au lieu de manifestations de reconnaissance des crachements et des tentatives de griffer et de mordre. Il y avait en outre des signes d'une décharge diffuse d'influx sympathique - dilatation des pupilles, sudation et hérissement des poils.

Dans les dernières années beaucoup d'arguments ont été apportés en faveur du choix de la région thalamique comme localisation centrale du système sympathique. Isenschmid ${ }^{15}$ et ses collaborateurs ont localisé à cet endroit le mécanisme de la régulation thermique - mécanisme contrôlant, par des

Fig. $17^{18}$ voies sympathiques, les mécanismes de la production et de la perte de chaleur. Karplus et Kreidl ${ }^{16}$ ont observé que l'excitation locale de la même région chez les animaux anesthésiés produit une décharge sympathique. Les observations de Bard sont fortement en faveur de la conclusion qu'il existe dans l'hypothalamus

15 Isenschmid 1926. Georg Robert Isenschmid (I882-I964) est un médecin et physiologiste suisse. Il est nommé professeur à la Faculté de médecine de l’Université de Berne en IgIg. Ses recherches portent essentiellement sur la régulation de la chaleur et la glande thyroïde. Premier chercheur suisse équipé d'un électroencéphalographe, il se spécialise dans l'étude des fonctions cérébrales.

16 Karplus/Kreidl ı9ı. Johann Paul Karplus (I866-I936) étudie la médecine à Vienne et devient assistant du psychiatre Richard von Krafft-Ebing (I840I902). Il dirige la polyclinique générale de Vienne de I9I7 jusqu’à sa mort. Alois Kreidl (I864-I928) étudie lui aussi la médecine à Vienne et travaille sous la direction du physiologiste et clinicien Joseph Breuer (I842-I925), avant de prendre la tête de l'Institut de physiologie comparative en I9I8. Les deux chercheurs mettent au jour l'existence d'un centre de régulation des fonc tions végétatives, en observant qu'une stimulation électrique de l'hypothalamus active les organes via le système sympathique (voir Wang 1965). un centre d'intégration pour les activités sympathiques. Il semble qu'il y a donc dans le diencéphale, c'est-à-dire dans une partie du cerveau ancien (commun à tous les membres de la série des vertébrés depuis les poissons jusqu'aux mammifères), l'appareil nerveux central pour l'intégration du système complexe de réaction de la colère et non seulement de ses manifestations externes mais aussi de la mise en jeu interne des forces de l'organisme pour les efforts physiques violents dans lesquels peut se terminer la colère.

Outre l'organisation nerveuse centrale pour le schéma de réaction de la colère, il y a les organes périphériques par les voies desquelles se réalise la réaction. Comme nous l'avons noté il y a deux catégories parmi ces organes - les muscles squelettiques et les viscères. L'action sur les muscles squelettiques est telle qu'un tableau caractéristique est réalisé. Je viens de décrire l'aspect du chat en fureur ; la description donnée par Darwin ${ }^{17}$ de la colère chez les jeunes enfants n'est pas très différente. Au cours d'un violent accès de colère ils hurlent, ils donnent des coups de pied, égratignent et mordent. Chez l'homme adulte le tableau ne se présente pas d'habitude selon ce type puéril, mais comme je l'ai établi dans la première leçon on peut trouver dans ce tableau le corps replié sur lui-même, les sourcils froncés, les lèvres serrées, les grincements des dents, les grognements, les marmottages et les poings serrés ou tenant une arme pour l'attaque.

J'ai également décrit dans la première conférence les modifications viscérales importantes qui accompagnent la fureur. Comme nous l'avons vu, elles sont profondes et étendues, aussi étendues que la distribution des influx nerveux sympathiques qui modifient les viscères. Les fibres préganglionnaires du sympathique sont en connexion avec de nombreux neurones dans les ganglions. Une seule fibre peut passer à travers différents ganglions et donner des branches aux corps cellulaires de chaque ganglion.

17 Cannon fait certainement référence au chapitre VI de The Expression of the Emotions in Man and Animals, que Darwin consacre à l'expression des émotions chez le jeune enfant (Darwin I890[I872], I54-185). 
Voyez le cliché ${ }^{18}$. La relation des fibres préganglionnaires avec les neurones sympathiques périphériques est donc telle qu'elle donne lieu à une décharge diffuse d'influx nerveux, non pas à une décharge limitée à une région donnée, mais à une décharge dispersée dans tous les organismes innervés. Parmi ces organes se trouvent les glandes surrénales et en même temps qu'une émission diffuse d'influx sympathique, il y a libération d'adrénaline dans le sang.

L'adrénaline qui est libérée a une répartition générale et par le fait même une action très diffuse. Partout où elle agit dans l'organisme, comme vous les savez, elle a le même effet que les influx nerveux; c'est-à-dire les agents humoraux et nerveux coopèrent. Nous pouvons parler alors d'un système sympathico-surrénal. En réalité cette coopération de la distribution généralisée d'adrénaline et de la distribution généralisée des influx nerveux confirme cette conception que les neurones sympathiques sont disposés pour des effets diffus et qu'à l'action localisée de l'agent nerveux se superpose l'action généralisée de l'agent humoral.

Bien que la mise en action du système sympathico-surrénal soit un caractère dominant d'un accès de colère, elle n'en est pas un caractère essentiel. Nous sommes parvenus récemment à enlever complètement les deux troncs sympathiques depuis le ganglion cervical supérieur jusqu'aux ganglions pelviens chez des chats, en gardant les animaux en pleine santé et en pleine vigueur dans le laboratoire pendant de nombreux mois.

Le cliché suivant est la photographie d'un animal neuf (9) mois après l'enlèvement de son système sympathique ${ }^{19}$. Vous voyez le système sympathique propre à l'animal sur le carton placé au-dessus de lui. Dans les circonstances appropriées ces animaux présentent à peu près tous les signes superficiels de

18 Cannon projette la figure reproduite dans La Sagesse du corps (Cannon I946[1939], 203); cette figure est aussi reproduite dans Bodily Changes in Pain Hunger, Fear and Rage (Cannon 1915, 454).

19 Cannon projette la photographie parue dans Cannon/Newton/Bright/ Menkin/Moore I929; cette photographie n'est pas reproduite dans La Sagesse du corps.

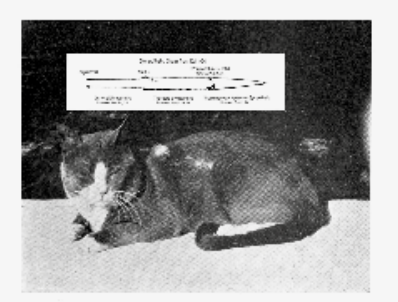

Fig. 5. Photograph of cat 104 taken February 12, 1927, nine monthsafterremovalof thesemilunar ganglia and the last portion of the sympathetic chains. The
parts removed were mounted on the card above the cat.

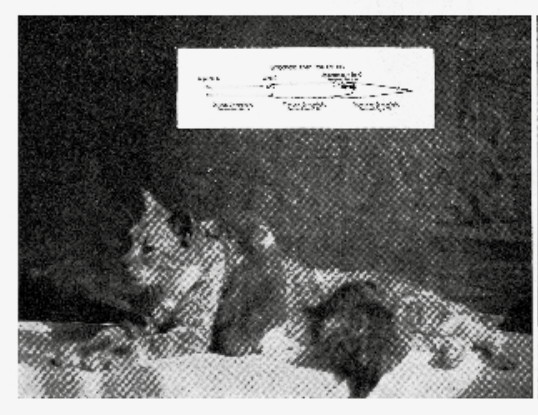

s

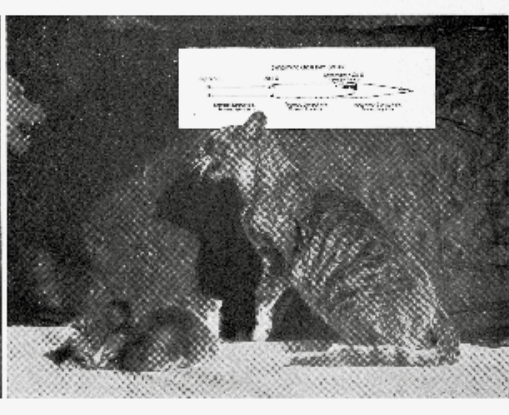

I3
Fig. 3. Photographs of cat 106; her sympathetic ganglionated strands - cervical, thoracic and abdominal (including the semilunar ganglia) - mounted on the card above; and also her kittens born after she was completely sympathectomized.

la colère. Vous voyez dans le cliché suivant une photographie ${ }^{20}$ d'un de ces animaux avec de nouveau son système sympathique placé sur un carton au-dessus de lui. L'animal avait donné naissance à deux chatons et les nourrissait. Quand on approchait de la mère un chien aboyant elle sautait sur ses pattes, montrait les dents, rabattait les oreilles, relevait une de ses pattes antérieures pour frapper, grognait et sifflait. Cependant, les poils ne se hérissaient sur aucune partie du corps ${ }^{21}$. Et comme

20 Cannon projette la photographie 3.A, parue dans Cannon/Newton/Bright/ Menkin/Moore 1929, 9I bis; cette photographie est reproduite dans La Sagesse du corps (Cannon I946[I939], 2I8).

21 Cannon projette la photographie 3.B, parue dans Cannon/Newton/Bright/ Menkin/Moore 1929, 9I bis; cette photographie n'est pas reproduite dans La Sagesse du corps.
Fig. $18^{19}$ Fig. $19 \mathrm{~A}^{20}$ et $19 \mathrm{~B}^{21}$ 
TABLE 2 Results of tests of blood sugar in normal cats (100, 111, and 113$)$ and in sympathectomized cats (104 and 107$)$ be tement and struggle
(For 104,

le montre le cliché suivant, une lutte vigoureuse avec grande excitation ne s'est pas traduite par un accroissement du sucre sanguin $^{22}$. Chez les animaux qui ont les surrénales, le foie et le cœur complètement séparés du système nerveux central, la réaction de colère se pro duit tout à fait comme chez les animaux normaux avec même l'apparition du hérissement de la queue mais en l'absence de toute décharge d'adrénaline ou de notable excitation du cour.
Premièrement, je voudrais insister sur un fait très important: les dispositifs neuro-musculaires et neuro-viscéraux pour les manifestations de la colère ont leur contrôle central organisé de façon congénitale dans ou au voisinage d'une partie du cerveau phylogénétiquement ancien - les couches optiques ou thalamus. Une partie que nous avons en commun avec les animaux inférieurs et qui explique alors pourquoi les expressions des émotions par l'homme et par l'animal sont semblables. Le thalamus n'est pas comme l'écorce cérébrale une région où de nouvelles relations avec le monde extérieur sont enregistrées et d'anciennes relations modifiées; il ressemble plutôt à la moelle en ce qu'il est une région dominée par les centres supérieurs, une région où les influx afférents sont reçus, regroupés, et redistribués, soit à des niveaux plus hauts, soit au voisinage de neurones moteurs qui envoient rapidement des influx aux organes périphériques selon des voies de réaction stéréotypée.

Les postures typiques et les attitudes qui résultent de l'action du thalamus sont plus compliquées que celles produites par les réflexes spinaux, mais ne sont pas essentiellement différentes. J'ai exposé en détail l'organisation physiologique qui établit le tableau réflexe de la colère parce qu'elle peut servir de prototype pour d'autres réponses émotives analogues. Les expressions de la peur, de la joie, et du mécontentement sont analogues. On n'apprend pas leurs caractères essentiels et elles se manifestent si tôt chez l'enfant humain qu'on peut les classer avec la colère comme étant congénitalement imprimées dans le cerveau. Il y a d'excellents arguments en faveur de la localisation du contrôle central de l'expression de ces émotions dans la région sous-corticale, comme pour la colère, et en l'espèce, dans la région thalamique.

Bekhterev ${ }^{23}$ a montré que tandis qu'une excitation douloureuse provoque des cris chez un animal récemment

23 Bekhterev i887. L'ensemble du paragraphe a été biffé par Cannon. Vladimir Bekhterev (I857-I927) est un physiologiste, neurologue et psychiatre russe.

En I885, il est nommé à la chaire de psychiatrie à la clinique psychiatrique
Moore 1929. Dans la marge de gauche, il rajoute à la main: «Normalement l'accroissement était de $34 \%$ et après enlèvement du sympathique presque aucun changement. 


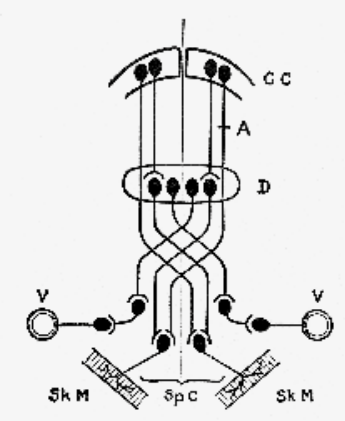

Fig. 40. - Diagram of possible relations of some neurones of the cerebral cortex $(C C)$ and of the thalamic portion muscles $(S k M)$. $S p C=$ spinal cord. The cortico-thalamic tract is regarded as inhibitory. Sensory fibers are not represented. Damage to the cortico-spinal tract at $A$ interrupts cortical control of certain skeletal muscles on one side, but it does not prevent control of these muscles on both sides by the centers in the diencephalon. Unilateral injury of center in the diencephalon may leave bilateral control
from the cortex.

privé de ses hémisphères cérébraux, une excitation légère, une caresse par exemple, peut provoquer des signes de plaisir - par exemple, des ronronnements chez le chat et l'agitation de la queue chez le chien. Chez ces chats ces réponses disparaissent après enlèvement des couches optiques $^{24}$.

Les arguments sont de pharmacologie et de pathologie. Chez l'être humain on peut trouver des indications de l'existence de mécanismes sous-corticaux pour l'expression des émotions dans ce qui se passe au cours de l'anesthésie. Pendant la deuxième phase, celle

Fig. $21^{25}$ d'excitation, dans l'anesthésie à l'éther, il peut y avoir des sanglots comme dans la peine, ou des rires comme dans la joie, ou des réactions agressives vives et énergiques, comme dans la colère - tout cela sans aucune adaptation aux circonstances. Le chirurgien peut ouvrir la poitrine et accomplir des opérations d'une égale gravité pendant que le patient pousse, tire, crie, marmotte, et, cependant, ces événements ne laissent dans l'écorce cérébrale aucune trace capable d'en évoquer le souvenir dans la suite. Un effet particulier de l'anesthésie à l'oxyde nitreux lui a fait donner son nom vulgaire de "gaz hilarant». Pourtant, un anesthésiste expérimenté m'a dit que sous l'influence de ce gaz il y a autant de malades qui pleurent que de malades qui rient; et dans les deux cas lorsque les fonctions de l'écorce ont été si profondément abolies par le gaz que le malade n'éprouve aucune sensation de douleur, il y a une libération

de Kazan. En I908, il est nommé à l'académie médicale militaire de SaintPétersbourg en tant que professeur et directeur de la clinique psychiatrique. La disparition de ce proche de Pavlov reste inexpliquée. Elle survient quelques jours après qu'il a examiné Joseph Staline (I878-I953), chez qui il a diagnostiqué une psychose paranoïaque.

24 Cannon semble avoir biffé l'ensemble du paragraphe. d'expressions typiques de joie et de tristesse.

D'accord avec les arguments expérimentaux et pharmacologiques on trouve également des arguments dans des cas pathologiques. Dans certains cas d'hémiplégie les malades peuvent être incapables de remuer le visage du côté paralysé. Mais si les malades sont soumis

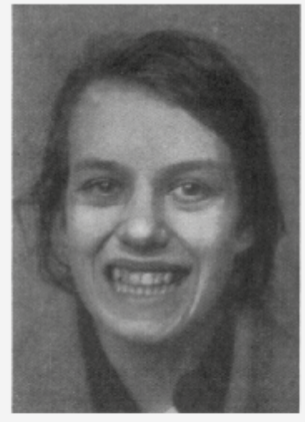

Fig. 5. - Case 9. Normal volitional facia

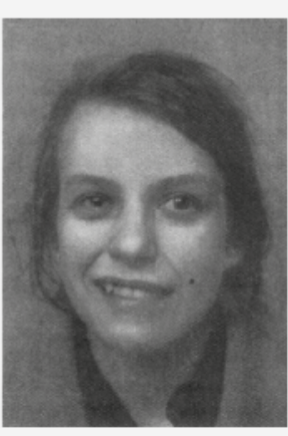

Fig. 6. - Case 9. Emotional or 'mimic' palsy of left side of face in laughing. brusquement à une émotion triste ou joyeuse, les muscles qui n'obéissent pas au contrôle volontaire se mettent en action et donnent aux deux côtés du visage une expression de tristesse ou de gaieté. Le diagramme que montre le cliché ${ }^{25}$ peut aider à rendre ces relations plus claires. Remarquez le double contrôle des muscles squelettiques - celui de l'écorce et celui du thalamus. Des cas semblables à celui que je viens de rapporter se produisent quand les cordons moteurs sont interrompus sous-corticalement et quand le thalamus est laissé intact. Le contraire se voit dans les atteintes unilatérales du thalamus. Un malade de Kirilzev ${ }^{26}$ remuait symétriquement les deux côtés de la figure quand il le voulait mais quand il riait de gaieté ou faisait une grimace de douleur, le côté droit restait immobile. L'autopsie a révélé une tumeur au centre de la couche optique gauche.

Cannon projette la figure parue dans la seconde édition de Bodily Changes in Pain, Hunger, Fear and Rage (Cannon 1922[1915], 249).

26 Cannon renvoie à Kirilzev I89i. Ce neurologue russe a décrit le cas d'un patient qui pouvait mouvoir volontairement et symétriquement les deux côtés de sa face, mais dont la seule partie gauche du visage exprimait le rire ou les grimaces de manière spontanée. Lautopsie révéla une tumeur dans le centre du thalamus optique gauche. 
Ceci est illustré par le cliché suivant d'après Wilson ${ }^{27}$; remarquez le contrôle cortical bilatéral et le contrôle thalamique unilatéral. Cette localisation du système nerveux central pour l'expression du plaisir et de la douleur a de s relations intéressantes avec les phénomènes émotifs qu'on observe communément dans la paralysie faciale bilatérale avec un côté plus entrepris que l'autre. L'acte volontaire (c'est-à-dire, d'origine corticale) d'arrondir les lèvres comme pour siffler ou de plisser le front ou de faire une grimace est impossible. Les muscles faciaux, cependant, fonctionnent normalement dans le rire et dans les pleurs, ou dans le froncement de sourcils. Ces expressions, qui s'accomplissent parfaitement, se produisent en accès incontrôlables et prolongés.

On trouve la description d'un malade qui a commencé à rire à dix heures du matin et a continué presque sans arrêt jusqu'à deux heures de l'après-midi. Tilney et Morrison ${ }^{28}$ qui

27 Cannon projette très probablement la figure parue dans Wilson 1924. Wilson y rapporte le cas d'une femme de dix-sept ans souffrant d'une tumeur intracrânienne. Ses mouvements volitionnels de la face ne sont pas affectés. A contrario, ses expressions émotionnelles sont peu marquées, surtout du côté droit de la face. L'autopsie va révéler une tumeur impactant principalement les côtés gauches du tegmentum et du pont.Samuel Alexander Kinnier Wilson (I878-1937) est un médecin et neurologue d'origine américaine. En I905, il est nommé professeur de pathologie au National Hospital de Londres, puis professeur de neurologie au King's College Hospital. Il est principale ment connu pour avoir étudié plusieurs cas de dégénération des noyaux len ticulaires associés à une cirrhose du foie. Wilson attribue la maladie à une toxine produite par le foie. La maladie de Wilson est liée à une dysfonction du métabolisme du cuivre d'origine génétique. Il introduit la notion de syn drome extrapyramidal, afin de désigner la rigidité ou les mouvements musculaires anormaux et involontaires qui accompagnent la maladie de Wilson.

Tilney/Morrison I9I2. Frederick Tilney (I876-I938) est un médecin et neurologue américain. En I9I5, il est nommé professeur de neurologie au College of Physicians and Surgeons de l'Université de Columbia. Francis Morrison (? - ?) est un médecin et neurologue, chef de la clinique épileptique du College Hospital de Long Island. Tilney et Morrison rapportent des cas de paralysie pseudo-bulbaire. Les symptômes pseudo-bulbaires sont semblables à ceux des atteintes du bulbe rachidien, mais les paralysies musculaires touchent plus spécifiquement les muscles de la face. Ils proviennent d'interruptions partielles ou totales des nerfs reliant le bulbe rachidien aux hémisphères cérébraux. Quelques mois après la paralysie, les patients montrent une transformation de leur état émotionnel, suivie de l'apparition de rires spasmodiques. ont rapporté cent soixante-treize (I73) observations de cette maladie ont trouvé de semblables accès de pleurs et de rire dans dix-sept (I7) pour cent des cas; les pleurs seuls dans seize (I6) pour cent et le rire seul dans quinze (I5) pour cent. Les accès apparaissent généralement sans aucune des causes habituelles et sont, la plupart du temps, intempestifs. Le malade peut paraître convulsé de rire et peut cependant n'éprouver aucun des sentiments justifiant les manifestations de sa figure ou de son corps. Les cas de cette espèce ont été attribués par Brissaud ${ }^{29}$ à des lésions d'une partie déterminée de voies cortico-thalamiques, libérant une partie du thalamus du contrôle cortical. Dans de tels cas il parait probable et d'autres arguments viendront supporter cette manière de voir que les voies thalamo-corticales afférentes sont également déficientes.

Un second point sur lequel je voudrais insister est l'intensité de la réaction de colère qu'on observe chez les animaux pseudo-affectifs. Il y a plusieurs années Hughlings Jackson a suggéré, et cette vue a été récemment appuyée par Head ${ }^{30}$, que

29 Brissaud I895. Édouard Brissaud (1852-1909) est un médecin et neurologue français. Élève de Charcot, il devient médecin des hôpitaux en I884. Le texte cité par Cannon est tiré des leçons dispensées à la Salpêtrière durant les années i893 et i894. Brissaud assure alors l'intérim de la chaire laissée vacante suite au décès de Charcot. La vingt et unième leçon porte «Sur le rire et le pleurer spasmodique» (Brissaud I895, 446-468).

30 Head I92I. Henry Head (I86I-I940) est un neurologue britannique, à qui l'on doit de nombreux travaux sur la neurophysiologie de l'intégration somatosensorielle et de la proprioception. En I898, il est nommé médecin assistant au London Hospital. Pour Cannon, les travaux de Head jouent un double rôle, puisqu'ils justifient à la fois l'existence de zones cérébrales spécifiquement dédiées aux émotions (le thalamus) - ce que contestait James - et la thèse du contrôle du cortex sur les parties sous-corticales. En effet, Head et son assistant Gordon Morgan Holmes (I876-1965) remarquent que les lésions dans la région du thalamus induisent un déficit sensitif dans l'hémicorps controlatéral à la lésion (Head/Holmes I9I2). Pourtant, cette insensibilité s'accompagne d'une hyperpathie: une stimulation d'ordinaire indolore ou superficielle induit une douleur intense et continue. En revanche, une lésion dans les aires sensorimotrices corticales n'a aucun effet sur l'intensité affective du stimulus. Suivant la théorie jacksonienne de la dissolution des fonctions, on peut en déduire que l'absence de contrôle cortical explique la puissance des réactions pseudo-affectives chez les animaux décortiqués. Cannon exprime ailleurs son ralliement à la conclusion de Head, selon laquelle le centre thalamique 
le système nerveux est organisé en une hiérarchie telle que les réactions primitives, qui autrement pourraient troubler les réponses plus délicates qui apparaissent à des niveaux plus élevés, sont réprimées par celles-ci. Quand le contrôle cortical est supprimé, les activités subordonnées, libérées de cette inhibition, deviennent prédominantes. Il suffit alors d'une excitation légère pour produire des résultats exagérés. C'est ainsi qu'on peut expliquer les manifestations violentes et persistantes de la fausse colère chez nos chats décortiqués lorsqu'ils étaient maintenus à un support, les tendances à mordre, les grognements et la résistance des chiens sans hémisphères cérébraux quand on les retire de leur cage, ou les réponses excessives à des excitations affectives légères chez des êtres humains dont le thalamus est libéré de l'influence corticale. L'intensité extraordinaire de ces manifestations simples indique que l'appareil nerveux pour l'expression des émotions est prêt pour une démarche énergique et que si le contrôle supérieur est seulement affaibli ou inhibé, des excitations appropriées provoquent une réponse intense et énergique. Si les conditions extérieures sont telles qu'elles peuvent provoquer une réponse émotive, une innervation définie des organes exécuteurs par l'écorce peut donc ne pas être nécessaire; la suppression de la prédominance corticale peut être la condition principale d'une action rapide et vigoureuse. Mais le contrôle cortical peut ne pas relâcher les neurones excités dans le thalamus. Il y a alors un conflit entre les contrôles supérieurs et inférieurs des activités physiques; il y a des tendances opposées accompagnées d'une certaine confusion. Les neurones corticaux, cependant, peuvent contrôler seulement certaines des activités de l'organisme - celles qui sont normalement sous le contrôle volontaire. Ils ne peuvent pas contrôler les influx impérieux du thalamus qui

gèrerait principalement la dimension émotionnelle des sensations, tout en doutant que l'on puisse attribuer au thalamus l'origine d'une forme de préconscience (awareness) sensorielle (Cannon 1927, II8). On rappellera que, selon Head, le thalamus représente un premier niveau d'intégration des fibres nerveuses, avant que celles-ci n'aillent se projeter dans le cortex pariétal. Cette intégration participerait à la formation d'un «schéma corporel» pré-inconscient, intégrant les données proprioceptives et posturales, avant de donner naissance à une «image du corps» consciente. provoquent le tremblement ou l'évacuation de la vessie ou du rectum. Dans les états de conflit ces phénomènes deviennent prédominants. Nous reviendrons dans une prochaine conférence sur les côtés intéressants de ce contrôle différencié des fonctions physiologiques troublées.

Il y a un troisième point intéressant : comme la conscience est associée au fonctionnement de neurones corticaux, il nous reste comme corollaire des faits rapportés auparavant, que les mécanismes nerveux des émotions primitives s'accomplissent dans une région située en dehors de la connaissance. Cette considération associée avec celle de la tendance de ces mécanismes à provoquer une réponse immédiate à une excitation légère lorsqu'ils sont relâchés de leurs inhibitions, explique à mon avis une des caractéristiques la plus essentielle: la source mystérieuse des émotions. Nous sommes saisis par les émotions, nous rions et nous pleurons et nous nous mettons en colère involontairement; nous nous sentons comme possédés; ce que nous faisons au milieu de l'excitation est surprenant, choquant - quelque chose s'élève en nous et nos actions ne semblent plus être les nôtres. Ces expressions qui sont devenues proverbiales peuvent-elles être expliquées en termes d'une soudaine et puissante prise de possession des forces de l'organisme par les neurones sous-corticaux? Dans les circonstances favorables, dès qu'il $\mathrm{y} a$ a, pendant un instant seulement, une suppression de l'inhibition normale, ces neurones prennent possession des mécanismes d'accomplissement et les entraînent violemment à l'une ou à l'autre de leurs actions variées.

Finalement, il me paraît que les faits que je viens de vous présenter laissent entrevoir une nouvelle source possible pour les sentiments particuliers que nous éprouvons quand nous sommes émus. Vous êtes au courant de l'idée de James, que la perception des changements physiologiques qui apparaissent spontanément dans une situation excitante constitue l'émotion; et vous connaissez également l'idée analogue de Lange que la connaissance des troubles cardio-vasculaires est l'élément essentiel de l'émotion. Comme j'essaierai de le montrer dans la prochaine conférence, cette théorie de l'origine périphérique de l'émotion a peu ou n'a pas de fondement positif. 
De toute façon, pour produire un effet dans le cortex, il n'est pas nécessaire que des influx afférents naissent à la périphérie; ils peuvent être provoqués partout dans les voies afférentes. N'est-ce pas là ce qui peut se produire dans l'excitation émotive? Les neurones des centres sous-corticaux agissent en combinaisons différentes dans les différentes expressions des émotions comme les schémas de réactions typiques des différents états affectifs. Le sentiment ne peut-il pas être dû à des influx venant non des organes périphériques, mais des neurones inférieurs dans la combinaison qui fixe à ce moment le faciès particulier et l'attitude posturale qui caractérisent le système de réaction? Ces neurones, comme nous l'avons vu, sont dans la substance grise basale, dans le cerveau primitif. Ils n'exigent pas une innervation supérieure pour être mis en action. Une condition primordiale pour leur mise en action dans l'organisme est qu'ils soient libérés pour l'action; ils produisent alors des influx précipités et intenses. Nous savons qu'une activité intense dans une partie s'étend aux autres parties par irradiation. Le phénomène se produit dans la substance grise du cortex cérébral; il peut se produire également dans la substance grise des ganglions basaux du tronc cérébral primitif. Ici, dans ou au voisinage du thalamus, les neurones intéressés dans l'expression des émotions se trouvent près du relais, sur la voie sensitive qui va de la périphérie à l'écorce. Nous pouvons admettre que lorsque ces neurones se déchargent en combinaisons variées, ils n'envoient pas seulement des influx nerveux aux muscles et aux viscères, mais influencent également les voies afférentes vers le cortex par irradiation ou par connexions directes. Donc comme accompagnement de toute expression émotive il peut naitre du cerveau primitif vers le cortex cérébral des influx caractéristiques du schéma nerveux alors prédominant. La qualité particulière de l'émotion peut parvenir de (I) la source mystérieuse des influx perturbateurs, (2) du sens du contrôle extérieur des forces de l'organisme, et (3) de différentes combinaisons des neurones afférents excités - chaque combinaison étant caractéristique d'une émotion spéciale. La possibilité d'utiliser cette idée comme base d'une explication de l'expérience émotive sera considérée dans notre prochaine conférence.
Quatrième conférence

\section{Théories de l'origine des émotions}

Dans une conférence antérieure, j’ai mentionné le côté mystérieux de l'expérience émotive, le halo étrange et particulier qui s'ajoute à la sensation simple quand le stimulus approprié se produit. Ce caractère surajouté et inexpliqué, qui a son origine non dans la cause de l'excitation mais dans l'organisme lui-même, a donné naissance à beaucoup de théories relatives à sa nature et à ses sources. Parmi ces théories, la plus remarquable est certainement celle proposée indépendamment par James et Lange. Aux États-Unis tout au moins, elle a été fortement soutenue par les psychologues pour l'interprétation des états affectifs. Elle était fondée sur la physiologie comme on la comprenait il y a plus de quarante (40) ans. De nouveaux faits physiologiques sont, cependant, maintenant à notre disposition et il faudrait les confronter avec la théorie et également avec d'autres théories possibles, avant de reconnaître la valeur absolue et exclusive de la théorie de James-Lange dans ce domaine de la psychologie.

On peut résumer comme il suit la théorie de James, à peu près dans les termes employés par son auteur: un objet extérieur excite un organe des sens ou plusieurs; des influx afférents sont envoyés à l'écorce cérébrale et la perception de l'objet en résulte; aussitôt, les influx descendent aux muscles et aux viscères et les modifient de façon complexe; des influx afférents venant de ces organes troublés reviennent au cortex et quand ils sont perçus, réalisent la transformation de l'objet « simplement perçu » en objet «émotivement senti ${ }^{1}$. En d'autres termes, la

1 «Un objet tombe sur un organe sensoriel, affecte une partie corticale, et il est perçu; ou bien cette dernière partie, excitée intérieurement, donne naissance à l'idée de cet objet. Prompts comme l'éclair, les courants réflexes descendent à travers leurs voies, préétablies, modifiant l'état des muscles, de la peau et des viscères; et ces modifications perçues, comme l'objet original, en autant de parties du cortex, se combinent avec lui en un état de 
sensation des modifications physiologiques, telles qu'elles se produisent dans l'émotion, est entièrement expliquée par les éléments sensitifs et associatifs habituels. Nous sentons les tensions, les palpitations, le fait de rougir, les saisissements, les suffocations - nous les sentons au moment où ils apparaissent et si nous enlevions du tableau d'une émotion ces manifestations physiques, rien ne resterait.

D'après Lange, l'excitation des centres vasomoteurs est la racine de la cause des états affectifs, quelle que soit par ailleurs leur constitution. Il écrit: "Nous devons tout le côté émotif de notre vie mentale - nos joies et nos chagrins, nos heures heureuses et malheureuses - à notre système vasomoteur. Si les impressions qui touchent nos organes des sens n'avaient pas le pouvoir de stimuler ce système, nous traverserions la vie sans sympathie et sans passion; toutes les impressions du monde extérieur enrichiraient notre expérience seulement, accroîtraient notre connaissance, mais ne produiraient ni joie, ni colère, et ne nous donneraient aucun souci ni aucune crainte. $»^{2}$ Étant donné que nous sommes incapables de voir subjectivement la différence entre les sensations d'origine centrale et d'origine périphériques, nous ne pouvons nous fier à des preuves d'ordre subjectif. Mais comme le vin, certains champignons, le haschich, l'opium, une douche froide, et d'autres causes, provoquent des résultats physiologiques qui sont accompagnés de modifications de nos sensations et que, si on supprime les manifestations physiques que présente un individu effrayé, rien ne reste de sa frayeur, l'émotion est seulement une perception de ce changement physiologique. Il est clair que Lange avait la même conception que James, mais l'édifiait

conscience et le transforment, d'un objet simplement représenté, en un objet senti émotionnellement» (James I9I7[I884], I06).

2 Cannon traduit ici un passage tiré de la traduction américaine du livre de Lange : « We owe all the emotional side of our mental life, our joys and sor rows, our happy and unhappy hours, to our vasomotor system. If the impressions which fall upon our senses did not possess the power of stimulating it, we would wander through life unsympathetic and passionless, all impressions of the outer world would only enrich our experience, increase our knowledge, but would arouse neither joy nor anger, would give us neither care nor fear» (Lange I922[I885], 80). sur une base beaucoup plus étroite - sur les modifications du système circulatoire seulement.

Le retour des excitations périphériques auquel James attribue la richesse et la variété des émotions, il le faisait partir de tous les endroits de l'organisme, des muscles et de la peau, aussi bien que des viscères. C'est pourtant à ces derniers qu'il tend à attribuer le rôle principal. Il a écrit: «Il est probable que la plus grande partie de la sensation l'expérience de l'émotion dépende de la partie viscérale et organique de son expression. ${ }^{3}$ Nous pouvons donc distinguer ces deux sources du flux afférent. Considérons d'abord la source viscérale et faisons-en la critique. Par la même occasion, nous examinerons l'idée de Lange sur l'importance des centres vasomoteurs pour l'explication de l'expérience émotive.

Voyons d'abord ce qui se produit lorsqu'on isole les viscères du système nerveux central. Sherrington a coupé la moelle et les nerfs vagues chez des chiens, de manière à détruire toute connexion entre le cerveau d'une part et d'autre part, le cour, les poumons, l'estomac et les intestins, la rate, le foie et les autres organes abdominaux; c'est-à-dire, qu'il a séparé tous les organes dans lesquels les sensations que nous étudions étaient supposées prendre naissance. Comme je l'ai dit dans la dernière conférence, nous sommes récemment parvenus à garder des chats en bonne santé pendant de nombreux mois après enlèvement complet de la division sympathique du système nerveux autonome - celle qui est active dans les états de grande excitation. Toutes les réactions vasculaires sous la dépendance du centre vasomoteur étaient donc abolies; la sécrétion de la moelle des surrénales ne pouvait plus être produite; le fonctionnement de l'estomac et des intestins ne pouvait plus être inhibé, ni les poils hérissés, et il n'y avait plus

3 Cannon cite librement: «The visceral and organic part of the expression can be suppressed in some men, but not in others, and on this it is probable that the chief part of the felt emotion depends. » (James I89I, 465) Il semblerait que Cannon (1927) discute des thèses de James et de Lange, à partir des extraits et traductions parus dans Lange/James 1922. Le livre est édité par Knight Dunlap (I875-1949), professeur de psychologie à la Johns Hopkins University, qui préside en 1922 l'American Psychological Association. 
de possibilité de faire libérer par le foie du sucre dans le courant sanguin. Ces opérations profondément perturbatrices avaient très peu ou pas d'effet sur les manifestations émotives chez ces animaux. Chez un des chiens de Sherrington, un chien qui avait un tempérament très émotif, la réduction par voie chirurgicale du champ sensoriel n'a pas causé de changement apparent dans sa manière d'être au point de vue émotif; sa colère, sa joie, son dégoût, et en tout cas de provocation, sa crainte, restaient aussi visibles, aussi évidentes qu'autrefois. Et chez les chats sympathectomisés, comme nous l'avons vu, tous les signes superficiels de la colère se sont manifestés en présence d'un chien qui aboie; on a vu apparaitre les sifflements, les grognements, le rabattement des oreilles, le fait de découvrir les dents, d'élever la patte pour frapper, - tous exceptée l'érection des poils. Les deux séries d'animaux se sont comportées en manifestant complètement l'expression de l'émotion dans tous les organes encore en rapport avec le cerveau; seules ont disparu les manifestations qui apparaissent dans les organes séparés du cerveau. L'absence de réverbération par les viscères n'a pas modifié le tableau de l'expression. C'est chirurgicalement seulement qu'elle est modifiée.

Comme l'a remarqué Sherrington, en parlant de son chien «tête et épaule ${ }^{4}$, il est difficile de penser que la perception qui donne naissance à l'expression de colère pourrait produire comme conséquence une manière de se comporter correspondant à la colère et avoir été pourtant incapable de produire un sentiment de colère.

Sur ce point, les interprétations diffèrent. Angell ${ }^{5}$ a prétendu que les expériences de Sherrington n'apportent aucune

$4 \quad$ Cannon désigne par là les chiens de laboratoire n'ayant plus de sensibilité au-delà des épaules, suite à une section de la moelle épinière dans la région cervicale inférieure. Celle-ci vise à supprimer toute sortie ou entrée dans le système sympathique. La seule sensibilité restante provient des nerfs situés entre le crâne et les épaules. Voir notamment (Sherrington I899, 400, fig. 2)

5 Angell I9I6. James Rowland Angell (I869-I949) est un psychologue américain. Il poursuit ses études à la University of Michigan. En I89I, il obtient un master sous la direction du philosophe et psychologue américain John Dewey (I859-1952). Il rejoint l'Université de Chicago en I895 puis prend, en I92I, la présidence de l'Université de Yale. Angell défend la théorie de preuve quant à la thèse que les sensations viscérales ne jouent aucun rôle dans la psychose émotive et de plus qu'elles ne prouvent pas que l'état psychique de l'émotion précède son expression. Et Perry ${ }^{6}$ a déclaré que le point de savoir si, en l'absence de sensation partant des organes isolés chirurgicalement, l'émotion est ressentie, reste tout à fait incertain.

Nous devons évidemment admettre qu'il n'existe aucune preuve pour affirmer ou pour nier la présence d'une émotion sentie chez ces animaux mutilés. Nous pouvons, cependant, confronter leur comportement avec la théorie de James-Lange. James a attribué la plus grande partie de l'expression de l'émotion aux sensations parties des viscères, tandis que Lange l'attribue entièrement aux sensations ayant leur origine dans le système circulatoire. Tous deux affirment que, si on enlève par l'imagination ces sensations organiques, il ne reste rien de l'expérience émotive. Sherrington ${ }^{7}$ et nous-mêmes, dans le laboratoire de Harvard, nous avons modifié ce procédé en enlevant les sensations, non par l'imagination, mais par des moyens chirurgicaux. Chez de tels animaux tous les troubles viscéraux produits par la voie du sympathique - c'est-à-dire, par la voie de la décharge nerveuse au cours de la grande excitation - étaient abolis. La possibilité de retour d'influx par ces voies était également supprimée, et chez les animaux de Sherrington le retour

l'émotion de James. Il estime que le fait que les animaux de Sherrington semblent éprouver des états émotionnels, bien que leurs voies de communication provenant des viscères aient été sectionnées, ne signifie aucunement qu'en temps normal les transformations viscérales jouent bien un rôle dans la production de l'état émotionnel. Angell discute aussi l'argument de Cannon adressé à la théorie de James, selon lequel les modifications viscérales, communes à des émotions aussi opposées que la peur ou la rage, ne sont pas assez spécifiques pour jouer un rôle fondamental dans l'émotion. Selon Angell, le fait que les modifications somatiques ne soient pas assez spécifiques ne remet aucunement en cause la thèse générale de James, selon laquelle les transformations viscérales précèdent et causent l'état émotionnel ressenti.

6 Pour Perry, voir l'introduction. Comme Angell, Perry estime que les expériences de Sherrington ne remettent pas en cause la théorie de James. Il conclut "qu'en l'absence de toute expérience sensorielle de ces changements, l'émotion ressentie, reste plus que jamais dans l'obscurité» (Perry 1950[1926], 299).

7 Sherrington 1899 
d'influx par les vagues était également hors de cause. D'après l'exposé que donne James de la théorie, l'émotion sentie devrait avoir largement disparu, et d'après Lange elle devrait avoir complètement disparu. Il déclare en effet, vous vous en souvenez, que sans excitation du système vasomoteur, les impressions produites par le monde extérieur n'éveilleraient ni joie ni colère, et ne nous donneraient ni souci ni crainte. Les animaux se sont pourtant comportés, autant que le permettaient les connexions nerveuses, sans aucune diminution de l'intensité de leurs manifestations émotives. En d'autres termes, des opérations qui, d'après la théorie, détruisent largement ou complètement les sentiments émotifs n'empêchent pas, cependant, les animaux de manifester le même comportement, ou la même joie, ou la même crainte qu'auparavant.

Une autre difficulté pour admettre l'origine périphérique des sentiments émotifs, est que les mêmes modifications viscérales se produisent dans des états émotifs très différents de la même façon que dans les états non émotifs. Comme je l'ai montré dans la dernière conférence, les fibres préganglionnaires de la division sympathique du système autonome sont reliées aux neurones périphériques de telle façon que l'innervation qui en résulte dans les muscles lisses et dans les glandes du corps tout entier, n'est pas spécifique mais diffuse. La distribution diffuse de l'adrénaline renforce les influx sympathiques distribués de façon diffuse. Par suite de cette disposition, le système sympathique fonctionne comme un tout. Il peut y avoir de petites variations, comme, par exemple, la présence ou l'absence de sudation, mais dans l'ensemble l'intégration est caractéristique.

Les changements viscéraux produits par l'excitation du sympathique sont les suivants: accroissement de la fréquence du cour, contraction des artérioles, dilatation des bronchioles, accroissement du sucre sanguin, inhibition de l'activité des glandes digestives, inhibition du péristaltisme gastro-intestinal, sudation, décharge d'adrénaline, dilatation des pupilles, et hérissement des poils. On trouve ces manifestations dans tout état de grande excitation. Elles se produisent dans des états aussi parfaitement distincts que la peur et la colère. Nous avons observé également que la fièvre ${ }^{8}$ et l'exposition au froid ${ }^{9}$ produisent la plupart de ces manifestations - en tout cas, l'accélération de la fréquence cardiaque, la vasoconstriction, l'accroissement du sucre sanguin, la décharge d'adrénaline et le hérissement des poils. Nous avons aussi observé que l'asphyxie ${ }^{10}$, dans la phase d'excitation, provoque les modifications que nous avons énumérées avec l'exception possible de la sudation. Une réduction trop forte du sucre sanguin par l'insuline provoque la réaction hypoglycémique caractérisée par de la pâleur, un cœur accéléré, des pupilles dilatées, une décharge d'adrénaline, un accroissement sanguin, et une transpiration profuse.

Dans ce groupe de conditions qui produisent dans les viscères des modifications caractéristiques de la décharge sympathique, se trouvent des émotions aussi intenses et aussi caractérisées que la peur et la colère, des états affectifs aussi relativement légers que ceux qui accompagnent le refroidissement, l'hypoglycémie, la respiration difficile, et un état aussi différent que celui d'un accès de fièvre. Les réponses dans les viscères semblent trop uniformes pour offrir un moyen satisfaisant de distinguer entre des émotions si différentes au point de vue subjectif. De plus, si les émotions étaient dues à des influx

8 Cannon/Pereira ig24. Nous n'avons trouvé aucune information concernant J. R. Pereira. Il s'agit vraisemblablement d'un chercheur qui a séjourné à la fin des années I920 dans le laboratoire de Cannon, au bénéfice d'une bourse de la Fondation Rockefeller.

9 Cannon/Querido/Britton/Bright 1927. Arie Querido (I90I-I983) est un médecin et homme politique néerlandais. Il débute des études de médecine en I9I8, à l'Université d'Amsterdam, qu'il achève en I926. Il travaille sous la direction de Cannon, lors d'un séjour de recherche financé par la Fondation Rockefeller et la Netherlands-Americans Foundation. En I93I, il rejoint la division des maladies nerveuses et mentales de l'Hôpital d'Amsterdam. En I949, il est nommé directeur de la santé publique de la ville d'Amsterdam, poste qu'il cumule, en I95I, avec la chaire de médecine sociale de l'Université d'Amsterdam. Membre actif du parti socialiste, il joue un rôle central dans la réorganisation des soins psychiatriques et de la médecine sociale. Le concept d'homéostasie (sociale) joue un rôle important dans sa conception des facteurs psychosociologiques à l'œuvre dans le développement des maladies et dans la prise en charge des patients.

10 Cannon/McIver/Bliss I924. Nous n'avons pas trouvé d'informations concernant Sidney W. Bliss, vraisemblablement un candidat au doctorat de physiologie qui assiste Cannon au début des années ig20. 
afférents venant des viscères, nous devrions nous attendre à ressentir de la même façon non seulement la peur et la colère, mais le refroidissement, l'hypoglycémie, l'asphyxie, et la fièvre. Il n'en est pas ainsi. Angell ${ }^{11}$, en commentant cet exposé critique de la théorie de James-Lange, admet qu'il peut y avoir un fondement d'excitation viscérale identique pour certaines émotions, mais attribue les caractères différentiels aux perturbations extra-viscérales, particulièrement aux différences de tonus des muscles squelettiques. Perry ${ }^{12}$ fait aussi appel à la conformation des schèmes proprioceptifs, au mécanisme moteur de l'expression, pour expliquer les éléments distinctifs des différents états affectifs. Nous examinerons dans la suite la part que jouent les muscles squelettiques dans la genèse de la sensation de l'émotion. Pour le moment, nous pouvons insister sur le fait que Lange n'a attribué à cette source aucune partie de l'état affectif et que James lui a attribué un rôle secondaire. Pour lui, la plus grande partie de la sensation de l'émotion dépendait de la partie viscérale et organique de l'expression.

Il y a encore une autre difficulté qui nous empêche d'admettre l'origine périphérique des sensations émotives : c'est que les viscères sont des organes relativement insensibles. On croit habituellement que plus on pénètre profondément dans le corps, plus on le trouve sensible. Tel n'est pas le cas. Tandis que dans les nerfs spinaux les fibres sensorielles sont probablement toujours plus nombreuses que les fibres motrices, dans les nerfs distribués aux viscères les fibres afférentes peuvent être dix fois moins nombreuses que les efférentes. Nous sommes tout à fait inconscients des contractions et des relâchements de notre estomac et de nos intestins au cours de la digestion; du frottement de l'estomac contre le diaphragme, des mouvements de contraction de la rate, des processus qui se passent dans le foie, et il nous a fallu de longues recherches pour savoir ce qui se passe dans ces organes. D'après les chirurgiens, le tractus digestif peut être coupé, déchiré, écrasé, ou brûlé chez l'homme non anesthésié sans produire aucun sentiment

11 Angell 1916, 260.

12 Perry 1950[1926], 300. d'inconfort. Nous pouvons sentir les palpitations cardiaques parce que le cour comprime la paroi thoracique et nous pouvons percevoir des pulsations des vaisseaux sanguins parce qu'ils traversent des tissus largement pourvus en nerfs sensibles; et nous pouvons avoir des douleurs abdominales, mais c'est apparemment à la suite de tiraillements du péritoine pariétal. Normalement les processus viscéraux sont parfaitement dépourvus de manifestations, et même lorsqu'on produit en eux des grands changements comme c'est le cas lors de l'action de l'adrénaline, les résultats sont, comme nous le verrons, des sensations attribuables pour la plupart à des actions au niveau du système cardiovasculaire.

De plus il y a des preuves que les changements viscéraux sont trop lents pour être la source de sentiments émotifs. Les portions actives des viscères sont composées de muscles lisses et de glandes à l'exception du cœur qui est un muscle strié modifié. Les mouvements du cœur avec lesquels nous sommes familiers résultent de l'action rapide d'un muscle strié ayant une période latente de moins d'un millième de seconde. La notion que nous avons de la rapidité des processus physiologiques à la suite de l'observation en action des muscles squelettiques ne peut pas être appliquée aux autres organes. Le muscle lisse et les glandes répondent avec une lenteur relative. Au lieu d'entrer en action en l'espace de quelques millièmes de seconde, ces organes ont une période latente d'environ une seconde, de plusieurs secondes, ou même de plusieurs minutes.

Par opposition à cette longue période de temps qui précède la mise en action des structures viscérales, citons lesobservations de Wells ${ }^{13}$ sur l'apparition des réponses

13 Wells I925. Frederic Lyman Wells (I884-I964) est un psychologue expérimentaliste et clinicien américain. Il devient le chef du laboratoire de psychologie du Psychopathic Hospital de Boston en I92I, puis rejoint en 1938 le département d'hygiène de l'Université de Harvard. Il préside l'American Psychopathological Association, de 1942 à 1944 et de 1946 à 1947. Germanophone, Wells a contribué à introduire la théorie freudienne aux États-Unis. Les expériences qu'il mène sur les temps de latence suivant une stimulation émotionnelle témoignent d'une volonté d'opérationnaliser les modèles et les concepts de la psychologie dynamique. Selon Wells, le temps de latence mesuré permet d'affirmer qu'au-delà d'une seconde, les 
émotives ${ }^{14}$. Il a observé que la période latente des réactions affectives d'hommes et de femmes à l'exhibition d'images, était fréquemment complétée en huit dixièmes de seconde. Selon la théorie de James-Lange, cependant, ces réactions étaient consécutives à des réverbérations à partir des viscères. Mais comment cela peut-il être possible? On doit ajouter à la longue période latente des muscles et des glandes dont nous avons parlé déjà, le temps exigé par l'influx nerveux pour aller du cerveau à la périphérie et revenir de là au cerveau. Il est évident que les modifications organiques ne peuvent pas se produire assez tôt pour être la cause de l'apparition des états affectifs, en tout cas, pas de ceux étudiés par Wells.

Une dernière difficulté d'une haute signification pour l'admission de la théorie de l'origine périphérique des émotions, est que la production artificielle des changements viscéraux caractéristiques des émotions fortes ne produit pas celles-ci. Comme vous le savez, l'adrénaline agit dans l'organisme en imitant les effets des influx sympathiques. Quand on l'injecte directement dans le courant sanguin ou sous la peau, elle produit la dilatation des bronchioles, la constriction des vaisseaux sanguins, la libération de sucre par le foie, l'arrêt des fonctions gastro-intestinales, et d'autres changements caractéristiques des émotions intenses. Si les émotions sont la conséquence des changements viscéraux, nous devrions vraisemblablement nous attendre, en accord avec les postulats de la théorie James-Lange, à voir ces émotions accompagner ces changements viscéraux à chaque fois qu'ils sont produits. Des observations fortuitement faites sur des étudiants qui ont reçu des injections d'adrénaline assez grandes pour provoquer des effets physiologiquement généraux, ont montré qu'ils ne percevaient aucune émotion spécifique. Quelques-uns qui avaient pris part à des joutes athlétiques ont déclaré se sentir nerveux et sur les dents, comme avant une course ${ }^{15}$. Au cours d'une étude

réactions observées en réponse à une stimulation chargée émotionnellement ne relèvent plus de la vie affective.

14 Il s'agit ici des réponses des sujets à qui l'on demande de rapporter verbalement si le stimulus perçu est plaisant ou déplaisant.

15 Peabody/Sturgis/Tompkins/Wearn I92I. Francis Weld Peabody (I88I-I927) très soignée sur les effets de l'adrénaline sur un grand nombre de personnes normales et anormales, Marañon ${ }^{16}$ a observé que parmi les sensations subjectives de ces personnes on trouve des palpitations cardiaques ou épigastriques, des battements artériels diffus, de l'oppression respiratoire, de la constriction de la gorge, des tremblements, des frissonnements, de la sécheresse du gosier, de la nervosité, et des sensations de malaise et de faiblesse. En même temps que ces sensations, il y avait en certains cas un état affectif indéfini froidement perçu et sans émotion réelle. Les sujets disaient: "Je me sens comme effrayé", "Je me sens comme si je m'attendais à une grande joie", "comme si j'étais remué", "comme si j’allais pleurer sans savoir pourquoi", "comme si j'avais une grande frayeur alors que je suis pourtant calme», "comme si on était sur le point de me faire quelque chose ». En d'autres termes, comme le remarque Marañon, il y a une distinction nette entre la perception des phénomènes périphériques de l'émotion végétative, c'est-à-dire les changements physiologiques et l'émotion elle-même, qui n'existe pas et qui permet au sujet de faire mention du syndrome végétatif avec sérénité et sans émotion réelle. Autrement dit, l'adrénaline produit chez l'être humain des modifications physiologiques typiques qu'il traduit comme des sensations et qui, dans certains cas, le font se souvenir d'expériences émotives antérieures, mais ne renouvellent pas ou ne font pas revenir ces expériences.

Les arguments que nous avons rapportés jusqu'à présent montrent que les modifications qui se produisent dans les

est un physiologiste américain, diplômé en Igo7 de l'École de médecine de Harvard, où il est nommé professeur de médecine en I92I. Cyrus Cressey Sturgis (I89I-I966) est un médecin hématologiste, diplômé de l'Université de Washington et de l'École de médecine de l'Université Johns Hopkins. En I925, il est nommé professeur assistant à l'École de médecine de Harvard. En I928, il prend la direction du département de médecine interne de l'École de médecine de l'Université du Michigan. Joseph Treloar Wearn (I893-I984) est un médecin diplômé de l'Université de Harvard en I9I7. Il rejoint en I929 l'École de médecine de la Western Reserve University, dont il devient le doyen en 1945 . Nous ne sommes pas parvenus à identifier Edna M. Tompkins.

16 Marañon I924. 
viscères n'apportent pas de confirmation objective à la théorie de James-Lange. Comme les processus viscéraux ne sont pas, malheureusement, je peux dire, la source de sensation marquée, et comme des perturbations même marquées de leur fonctionnement ne se traduisent pas en émotions appréciables, nous pouvons maintenant comprendre pourquoi ces perturbations ne peuvent suffire comme moyen de distinction entre des émotions aussi marquées que la peur et la colère. Nous comprenons aussi pourquoi la sensation de froid, l'asphyxie, l'hyperglycémie, et la fièvre, bien qu'accompagnées de perturbations profondes, ne sont pas accompagnées d'émotion. Et nous comprenons aussi pourquoi la suppression totale des facteurs viscéraux des expressions des émotions ne produit pas de changement de comportement émotif. C'est parce que les effets renvoyés par les « résonateurs " thoraciques et abdominaux, comme les appelle James, sont très peu marqués, qu'ils jouent un rôle si minime dans le complexe affectif. Les processus qui s'accomplissent dans les organes thoraciques et abdominaux sont bien remarquables et variés; mais leur importance pour l'organisme, cependant, n'est pas d'ajouter de la richesse et de la saveur à l'expérience, mais plutôt d'adapter l'économie interne de telle manière que, malgré des changements de circonstances extérieures, le déroulement uniforme de la vie intérieure ne soit pas profondément troublé.

Dans sa discussion des processus cérébraux accompagnant l'émotion, James a discuté le point de savoir s'il y a pour eux des centres spéciaux, ou s'ils se produisent dans les centres moteurs et sensoriels ordinaires de l'écorce ${ }^{17}$. Et s'ils se produisent, comme il l'admet, dans les centres ordinaires, les processus devraient ressembler à ceux qui accompagnent de façon ordinaire la sensation. Cela seulement et une représentation complète de chaque partie de l'organisme dans le cortex, serait nécessaire pour donner un schéma capable de représenter le modus operandi des émotions. Objet, organe des sens, excitation corticale, perception, réflexes vers les muscles, la peau, les viscères, perturbation dans ceux-ci, excitation corticale consécutive à ces perturbations, perception de celles-ci s'ajoutant aux

I7 Voir le chapitre «No special brain-centres for emotion» (James I89I, 472). perceptions primitives - tels sont les différents états dont l'ensemble constitue l'objet émotivement ${ }^{18}$ senti. Ainsi dit James. Nous ne devons pas nécessairement, cependant, accepter l'alternative déterminée suivante: ou bien processus corticaux et ou bien centres spéciaux. Il peut y avoir des processus corticaux et des centres spéciaux.

Dans la dernière conférence j'ai exposé en détail les preuves expérimentales, pharmacologiques et cliniques qui appuient la conclusion de l'existence de schémas nerveux de manifestations pour les différentes émotions. Nous pouvons à la lumière de ces faits modifier le chapitre de James intitulé « Pas de centres spéciaux pour l'émotion ${ }^{19}$. Il est trop simple de considérer à un bout de la voie nerveuse le cortex comme une surface réflexe, et les organes périphériques à l'autre bout comme source d'influx réfléchis. Entre le cortex et la périphérie se trouve le diencéphale, organe d'intégration sur le plan émotif, station de réception et de décharge, qui après une excitation convenable est capable de provoquer les attitudes stéréotypées de la face et du corps typiques des différents états affectifs. De plus, toutes les voies afférentes conduisant au cortex ont des relais dans le diencéphale. Ceci est un fait de la plus grande importance pour l'explication de la nature des émotions, car il y a des arguments pour penser que les processus thalamiques sont une source de l'expérience affective.

Les relais des voies sensitives dans le thalamus et les preuves que les troubles de cette région sont la cause de sensations profondément affectives sont tout ce dont nous avons besoin pour comprendre son rapport avec la nature des émotions. Head a rapporté de nombreux cas de lésions unilatérales

18 Cannon remplace "émotionnellement" par «émotivement».

19 James souscrit au modèle cortico-centrique de Meynert, selon lequel l'ensemble des sensations corporelles se projette sur la surface du cortex. Pour James, le modèle suffit à donner une explication des émotions, sans qu'il soit nécessaire de postuler l'existence d'une zone cérébrale dédiée: «Supposons que le cortex contienne des parties aptes à être excitées par les modifications de chaque organe sensoriel spécial, de chaque partie de la peau, de chaque muscle, de chaque articulation et de chaque viscère et qu'il ne contienne rien d'autre, il nous reste quand même un schéma capable de représenter le processus émotionnel» (James I9I7[I884], I05). 
dans la région thalamique dans lesquels il y avait une tendance marquée à des réactions excessives aux stimulus affectifs. Des piqûres d'épingle, des compressions douloureuses, une chaleur ou un froid excessifs, produisent plus de manifestations de détresse du côté malade que du côté normal du corps. Les stimulus agréables sont également perçus de façon exquise du côté malade. La chaleur peut provoquer un plaisir intense accompagné de signes de joie au visage et d'exclamation de délices. Des stimulus affectifs, comme le fait de jouir de la musique ou de chanter des hymnes, peuvent provoquer un tel accroissement de la sensation émotive du côté malade qu'ils peuvent être intolérables. Le côté malade est influencé par les états affectifs conscients de la même manière que par les stimulus qui viennent des récepteurs superficiels. Cette influence exagérée des stimulus affectifs, qu'ils viennent d'en haut ou d'en bas, a été attribuée par Head à la libération du thalamus de l'inhibition que l'écorce normalement exerce sur lui. Elle n'est pas d'après lui un effet d'irritation, car elle persiste pendant de longues périodes de temps bien après que les troubles dus au traumatisme ont disparu. Et comme ces états affectifs sont augmentés quand le thalamus est libéré du contrôle cortical, la conclusion de Head est que les centres thalamiques sont impliqués de façon prédominante au côté affectif de la sensation.

Nous sommes maintenant en état d'examiner la thèse d'après laquelle les positions et les degrés de tension des muscles squelettiques sont la base de la différenciation des émotions. Rappelons que bien que James n'ait pas accordé une importance très grande à cet élément dans sa théorie, ses disciples ${ }^{20} \mathrm{y}$ ont attaché une importance plus grande, particulièrement depuis qu'il a paru inexact de faire appel à l'élément viscéral. Les maladies thalamiques nous offrent un moyen de nous rendre compte de la part jouée par les muscles squelettiques, car le tonus affectif d'une sensation est une activité thalamique et le fait qu'une sensation n'est pas accompagnée de tonus affectif, montre que les influx qui sont la condition de cette production ne mettent pas en jeu le thalamus.
Head $^{21}$ a observé que ces malades présentaient des différences marquées dans le tonus affectif de différentes sensations. Un diapason peut ne pas avoir d'effet, tandis qu'une musique patriotique peut être ressentie intensément du côté malade. Tous les stimulus thermiques font appel à la fois au cortex et au thalamus. Les stimulus tactiles quelconques agissent de façon similaire. D'un autre côté, les sensations qui sont à la base de l'appréciation de l'attitude, manquent entièrement de tonus affectif. Ces influx afférents, venant des muscles et des articulations, que James et ses partisans ont pris comme origine de la partie extra-viscérale de l'expérience de l'émotion, sont précisément ceux qui manquent des qualités voulues pour servir à ce but. La qualité particulière des émotions ne doit pas être cherchée dans des répercussions partant des viscères, ni comme venant des muscles innervés.

La discussion qui précède a établi le fait que les dispositifs nerveux pour l'expression des émotions, sont situés dans les centres sous-corticaux et que ces centres sont, à tout moment, prêts à une décharge instantanée et vigoureuse lorsqu'ils sont libérés de l'emprise corticale et sont stimulés de façon convenable. De plus, il apparaît clairement que lorsque ces centres sont libérés, les processus qui naissent en eux deviennent une source de vives expériences affectives ${ }^{22}$. Il est particulièrement heureux pour nous que cette expérience n'existe que dans les cas d'hémiplégie, car on peut ainsi observer chez le même individu l'influence du même stimulus affectif dans les conditions normales et quand on lui a donné libre cours.

Les observations précédentes suggèrent l'adoption de la conception suivante de l'organisation nerveuse qui précède les émotions. Un phénomène extérieur excite les organes récepteurs ; une excitation en résulte qui détermine le transport d'influx vers l'écorce. L'arrivée de ces flux dans l'écorce est associée

21 Head I920, 620. Head rapporte le cas de Mme A. R., 52 ans, qu'il suit dès 1902. Exemple de syndrome thalamique, les états émotionnels de la patiente se manifestent sur la moitié du corps, par des mouvements involontaires accrus et des sensations désagréables.

22 Dans la marge gauche, Cannon indique à la main qu'il a atteint les « $3 / 4 » d e$ son exposé. 
à des processus conditionnés, qui déterminent la direction de la réponse. Soit parce que la réponse est déterminée selon un certain mode qui fait que les neurones corticaux excitent les processus thalamiques, ou soit parce que dans le cours de leur marche centripète les influx, venant des organes récepteurs, excitent les processus thalamiques, ceux-ci sont éveillés et prêts à la décharge. Les types de réactions caractéristiques des différents états affectifs montrent que les neurones thalamiques agissent selon une combinaison spéciale dans une expression émotive déterminée. Ces neurones n'ont pas besoin d'une innervation venant d'un niveau plus élevé pour être mis en action. La condition primordiale pour qu'ils puissent être mis au service de l'organisme est qu'ils soient libérés pour l'action. Ils se déchargent alors instantanément et intensément. Dans ou au voisinage du thalamus, les neurones intéressés dans l'expression de l'émotion sont situés au voisinage du relais de la voie sensitive qui va de la périphérie au cortex. Nous pouvons admettre que lorsque ces neurones se déchargent selon un complexe déterminé, ils n'innervent pas seulement les muscles et les viscères, mais ils excitent aussi par connexion directe et par irradiation les voies afférentes qui viennent au cortex. La théorie qui se présente naturellement à l'esprit est que le caractère particulier de l'émotion est ajouté à la sensation simple quand les processus thalamiques sont éveillés.

La théorie que nous venons de suggérer semble s'appliquer à tous les faits connus. Nous pouvons énumérer brièvement les services qu'elle peut rendre dans l'interprétation de ces faits.

Quand se produit la décharge thalamique, les modifications physiologiques se produisent à peu près au même moment que le fait de ressentir l'émotion. Cette simultanéité des perturbations dans les muscles et dans les viscères avec des frémissements, de l'excitation, ou des dépressions, devait naturellement conduire à l'erreur, car si on ne tient pas compte du rôle du thalamus, la conclusion serait évidemment que la qualité particulière de l'émotion provient des modifications périphériques. C'est en effet là le point vital de la théorie de James-Lange. Les arguments que j'ai présentés montrent, cependant, que le raisonnement est mal fondé. Contrairement à l'opinion de James, les sensations des modifications périphériques sont pâles, décolorées, et dépourvues de chaleur émotive, tandis que les troubles thalamiques ajoutent à des états de simple connaissance, dans les conditions normales, l'éclat et la couleur. La théorie que nous proposons explique comment James et Lange pouvaient raisonnablement faire la suggestion qu'ils ont proposée. Le manque de raison fondamentale pour admettre leurs suggestions nous oblige à chercher ailleurs l'origine des émotions. Nous la trouverons dans le fait que les processus thalamiques peuvent ajouter à la sensation une aura de sentiment.

Un des arguments les plus puissants pour la théorie de James-Lange est la confirmation que le fait de prendre une attitude aide à faire naître les sentiments que cette attitude exprime. Restez assis pendant tout un jour dans une attitude maussade, soupirant et répondant à tout d'une voix lugubre, et votre mélancolie persistera. Au contraire, déplissez votre front, faites vos yeux plus brillants, contractez plutôt le côté dorsal que le côté ventral du tronc, parlez à voix haute, prodiguez des compliments et votre cœur doit être vraiment frigide si vous ne vous dégelez pas rapidement ${ }^{23}$. Ainsi a écrit James. Des gens qui ont mis ce conseil à l'épreuve ont proclamé son efficacité et ont été par là même convaincus de la vérité de l'opinion qui veut que leur humeur ait été une conséquence des attitudes qu'ils ont prises. Tout le monde ne reconnait pas, cependant, que le fait de mimer l'apparence d'une émotion produit l'émotion elle-même. James a suggéré qu'il faut trouver l'explication de ces divergences dans des variations de mises en jeu des viscères au cours de l'expression artificielle. Comme nous l'avons vu, cependant, les modifications physiologiques ${ }^{24}$ apportent un support bien faible à la théorie. Ici, de nouveau, les processus qui ont leur siège dans le thalamus offrent une explication simple et raisonnable. Comme l'ont montré les cas rapportés par Head, les émotions qui ont leur origine dans des souvenirs et dans l'imagination affectent

23 «Smooth the brow, brighten the eye, contract the dorsal rather than the ventral aspect of the frame, and speak in a major key, pass the genial compliment, and your heart must be frigid indeed if it do not gradually thaw! » (James I89I, 463).

24 Cannon biffe «viscérales » en faveur de «physiologiques». 
de façon plus intense la moitié du thalamus qui a été libérée du contrôle central de la moitié normale. Ceci montre que les processus corticaux peuvent donner naissance aux processus thalamiques et par conséquent éveiller une réflexion affective à partir de cette portion du cerveau. Et, en outre, on prend une attitude émotive typique, l'inhibition corticale des neurones thalamiques, en rapport avec cette attitude, est abolie et ils sont complètement relâchés. Dans de telles circonstances l'émotion mimée deviendrait une réalité. D'autre part, une mimique purement intellectuelle de l'expression émotive sans que le thalamus soit intéressé, serait aussi froide et aussi dépourvue d'affectivité que l'ont déclaré certains acteurs. Que l'émotion se produise ou non, la théorie thalamique de l'origine du sentiment donne une explication plus satisfaisante des effets de l'imitation des attitudes que ne l'a fait la théorie de Jame-Lange.

Les cas de libération unilatérale du thalamus du contrôle cortical avec du même côté une augmentation du tonus émotif, présentent un obstacle insurmontable à la théorie de JamesLange. Ni les viscères thoraciques, ni les viscères abdominaux ne peuvent fonctionner dans une de leurs moitiés - le centre vasomoteur est une unité et les malades ne présentent certainement pas de rires ou de pleurs localisés au côté gauche ou au côté droit. Les influx réfléchis à partir des organes périphériques doivent donc être égaux des deux côtés. Pour l'explication de la disposition non symétrique du sentiment, nous devons recourir à la région qui est fonctionnellement non symétrique - c'est-àdire, le thalamus. C'est là que dans la théorie que je vous propose, est localisée la source des émotions.

Une autre difficulté sérieuse pour la théorie de JamesLange est le fait que l'émotion s'accroît en intensité alors que nous en réprimons l'expression. En fait, il y a des psychologues qui prétendent que l'état d'émotion dure seulement aussi longtemps qu'il existe un conflit intérieur entre l'impulsion à l'acte et le contrôle hésitant ou prudent exercé sur cette impulsion. Aussi longtemps que ce contrôle persiste, cependant, les modifications organiques qui sont la source supposée du sentiment, sont supprimées. Comment alors l'émotion peut-elle être ressentie? On peut trouver dans l'argumentation de James deux réponses à cette question. D'abord il nie l'objection. D'autre part, il admet qu'une émotion refoulée peut opérer de façon fâcheuse. Mais ces commentaires indécis ne modifient pas le témoignage courant d'après lequel une frayeur intense, par exemple, peut être ressentie accompagnée de détresse avant que ne se produise aucun acte extérieur, et d'après lequel, à peine le comportement que réclament les circonstances a-t-il commencé à se manifester, que le tumulte intérieur commence à s'apaiser et que les forces de l'organisme sont dirigées vigoureusement et effectivement pour l'accomplissement d'un résultat utile. La difficulté que rencontre la théorie de James-Lange pour expliquer cette situation est évidente. S'il y a un double contrôle, cependant, le conflit intérieur avec son côté émotif net et dans la suite la disparition partielle du sentiment sont parfaitement explicables. Les processus dont le plan est tracé dans le thalamus sont inhérents à l'organisation nerveuse. Ils sont semblables aux réflexes en ce qu'ils sont capables de prendre immédiatement contrôle des réponses motrices, et quand ils le font, c'est avec un grand pouvoir. Ils peuvent être contrôlés, cependant, par les activités du cortex cérébral, activités conditionnées par toutes espèces d'impressions antérieures. Le cortex peut aussi exercer un contrôle sur tous les organes périphériques, sauf sur les viscères. Les processus inhibés dans le thalamus ne peuvent pas mettre l'organisme en action sauf en ses parties qui ne sont pas sous le contrôle volontaire, mais le tumulte qui se produit là peut donner naissance à des émotions à la manière habituelle et même avec une violence augmentée, par suite de l'inhibition. Quand le contrôle cortical est relâché, le conflit est soudainement résolu. Les deux contrôles qui étaient en opposition travaillent maintenant en coopération. Les neurones thalamiques, aussi longtemps qu'ils continuent à fonctionner énergiquement, fournissent à l'émotion ses conditions de persistance, comme James le prétend, pendant la durée des manifestations. La théorie thalamique ne nous épargne pas seulement les difficultés que rencontre la théorie de James-Lange, mais encore nous donne une explication satisfaisante de l'intensité du sentiment au cours de la période d'inaction. 
Il y a un autre point sur lequel on peut insister en ce qui concerne le contrôle double de la réponse. McDougall ${ }^{25}$ s'est opposé à la théorie de James-Lange parce qu'elle se rapporte seulement au côté sensoriel de l'émotion. Elle ne prête peu ou pas d'attention à l'aspect impulsif toujours présent et souvent prédominant d'une expression émotive. La localisation du plan de réaction pour l'expression émotive dans le thalamus - une région qui agit directement, comme la moelle, et par simple automatisme, sauf si elle est contrôlée - ne procure pas seulement une explication pour le côté sensitif, pour l'émotion sentie, mais aussi pour le côté impulsif, pour la tendance des neurones thalamiques à la décharge. Les influx puissants qui naissent dans une région du cerveau qui n'est pas associée à la connaissance et qui par conséquent éveillent d'une manière obscure et isolée les sentiments puissants de l'excitation émotive, expliquent le sens d'être possédé, saisi, contrôlé par une force extérieure et d'être amené à agir sans peser les conséquences de cet acte.

Finalement, la thèse selon laquelle les processus thalamiques ajoutent à la sensation le tonus affectif offre une explication satisfaisante d'une difficulté que rencontre la théorie de James-Lange dans l'explication des émotions délicates. James devait admettre des répercussions indéfinies et hypothétiques pour expliquer les sentiments doux de plaisir et de satisfaction. Si un tube à réaction chauffé est capable de provoquer un sentiment de délice du côté malade dans un cas de lésion thalamique, il est clair que presque tout objet ou toute satisfaction qui peut faire naitre un processus thalamique, peut ajouter une qualité affective à la sensation. Et précisément comme un stimulus peut devenir la condition de certaines réponses motrices ou glandulaires, de même un stimulus peut devenir conditionné pour les schémas d'action de neurones dans le thalamus. Quand ce stimulus se reproduit, l'émotion se reproduit parce que ce schéma est actif. Nous avons là un moyen de nous expliquer comment s'élaborent la richesse et la variété de notre vie émotive.

\section{Cinquième conférence}

\section{Troubles émotifs}

\section{des fonctions de l'organisme}

Dans plusieurs contributions à la théorie des émotions nous avons interprété, en fonction de leur utilité, les faits que nous avions observés dans nos recherches à propos des émotions puissantes de la peur et de la colère. Certains biologistes et psychologues ont été plus impressionnés par les perturbations des fonctions physiologiques que produisent ces émotions que par leur côté utile. Le professeur Piéron ${ }^{1}$, par exemple, a décrit l'émotion comme associée à une décharge affective d'énergie nerveuse anormalement intense. Bien qu'admettant qu'une partie de l'énergie se dépense en réactions utiles adaptées, procurant une force augmentée et une plus grande rapidité de course en cas de besoin, il déclare qu'une partie plus ou moins considérable de l'énergie est dépensée en contorsion inutile de la face et même déversée dans les organes végétatifs où, ajoute-t-il, des réactions de caractère différent se produisent suivant que le système excitateur ou inhibiteur est mis en jeu.

J'ai insisté sur le caractère utile de ces modifications des organes végétatifs dans une discussion antérieure ${ }^{2}$, mais le professeur Piéron cite des cas de maladies et même de mort dans lesquels celles-ci ont été précédées de grandes détresses émotives à laquelle on a toute raison d'attribuer le dénouement. Il a exprimé l'opinion qu'il y a dans les changements physiologiques qui accompagnent les états affectifs marqués une tendance à aller vers le pathologique jusqu'à la limite. Il est donc sceptique quant à la valeur des interprétations utilitaires, qu'il appelle «finalistes ». De même, un physiologiste

Piéron 1928.

2 Nous ne sommes pas certains de cerner ce que Cannon entend ici par « discussion ", s'il se réfère à des publications ou aux différents passages des conférences antérieures où il défend l'utilité des réactions émotionnelles comme préparation physiologique à l'action. 
italien, Mosso ${ }^{3}$, dans son livre sur «La peur » a donné une description vivante des perturbations désorganisantes qui accompagnent un accès de cette émotion - le trouble, le fait de rester muet, le tremblement, le fait de vider la vessie et le rectum; et aussi les réactions sans utilité comme la chair de poule, le hérissement des poils, et la peur ou le rougissement; et finalement les exemples de maladies ou de mort qui ont leur origine dans une frayeur intense.

En examinant ces caractères de la frayeur, puis-je vous rappeler d'abord que certains d'entre eux peuvent être expliqués comme conséquences de la tendance du système sympathique à fonctionner comme un tout. Le hérissement des poils et la constriction des vaisseaux périphériques peuvent n'être d'aucune utilité dans la peur, mais le système sympathique est également mis en action par le froid et dans ces circonstances ces réactions sont utiles à l'organisme. Le tremblement et le frisson, qui sont communément associés à l'innervation sympathique, sont alors utiles en provoquant une plus grande production de chaleur. Le caractère d'inappropriation apparente de certains traits du complexe d'ensemble du fonctionnement sympathico-surrénal est rendu compréhensible par la tendance de ce système à réagir comme un tout, particulièrement quand il est fortement excité. Et en estimant la valeur des résultats nous devons prendre en considération quelles sont les conditions variées qui peuvent provoquer la mise en fonction totale du système.

De plus, il est douteux que la mort n'ait jamais été la conséquence d'une grande excitation émotive, sauf en présence d'une faiblesse organique du cœur ou des vaisseaux sanguins ${ }^{4}$. On connaît la célèbre et tragique remarque de Hunter: "Ma vie est dans les mains de tout coquin à qui il peut venir à l'idée de m'ennuyer ou de me taquiner. ${ }^{5}$ Il souffrait d'angine de poi-

3 Mosso I886[I884]. Voir en particulier le chapitre XII consacré à la frayeur et à la terreur, ainsi que le chapitre XIII consacré aux maladies produites par la peur.

4 Pour les interrogations de Cannon quant à la possibilité qu'une forte émotion entraîne la mort, voir l'introduction.

5 Ces mots sont attribués à John Hunter (I729-I793), médecin britannique trine et possédait malheureusement aussi un tempérament passionné; et en effet, au cours d'une discussion publique, ayant été contredit, il s'emporta violemment, s'évanouit, et mourut en quelques instants. L'élévation de pression sanguine qui accompagne, comme on le sait, un accès de profonde émotion, peut être trop forte pour des vaisseaux fragiles qui peuvent se rompre et amener rapidement la mort. Dans ces conditions l'émotion peut seulement être considérée comme la cause accidentelle de la mort.

Finalement, je veux insister de nouveau sur ce point, que je n'ai aucune espèce d'idée fixe quant au caractère définitif de l'explication utilitaire de la peur et de la colère que je vous ai présentée. Je suis toujours prêt à accepter une autre explication si elle est plus raisonnable. À ce sujet, il peut être à propos de rappeler que jusqu'à présent nous nous sommes seulement occupés des fonctions dans l'excitation émotive; c'est-à-dire, de sa physiologie et non de sa pathologie. Il y a des systèmes hautement utiles qui, étant employés mal à propos ou de façon malheureuse, peuvent être modifiés de telle façon dans les services qu'ils rendent à l'organisme, qu'ils peuvent être dans ces circonstances nuisibles. Il peut s'établir des cercles vicieux de circonstances qui peuvent amener une aggravation des conditions et même la mort. Les reins, par exemple, débarrassent l'organisme des excreta non volatiles. Ils peuvent ne plus fonctionner normalement et alors l'accumulation de déchets dans le sang porte atteinte à l'organisme, les reins y compris. Mais l'apparence de fonctionnements pathologiques ne change rien au fait que ce système fonctionne d'habitude normalement. Il serait absurde de nier le fonctionnement normal d'un organe parce que cet organe peut dans certaines conditions fonctionner anormalement. Quand se présentent des fonctionnements pathologiques, le problème qui se pose est d'essayer de comprendre dans quelles circonstances la transformation se produit. C'est pourquoi, en étudiant les modifications qui caractérisent

considéré comme l'un des pionniers de la chirurgie vasculaire. Il meurt au St. George's Hospital à Londres le I4 octobre I793, lors d'un débat animé (Norton/Barie/Bollinger/Chang/Lowry/Mulvihill/Pass/Thompson 2009). 
les émotions fortes, nous pouvons admettre le caractère habituel d'utilité de ces modifications (comme préparation à l'action), et nous pouvons admettre aussi que ces modifications peuvent devenir persistantes au point d'être une menace et non plus un avantage; et ce contraste rend très intéressante la recherche de la nature de la transformation qui s'est produite.

Avant d'essayer de comprendre la pathologie de l'excitation émotive, nous allons d'abord rappeler brièvement quelques points établis dans la troisième conférence. Nous avons vu que du point de vue physiologique une émotion est typiquement une réaction s'accomplissant selon un modèle déterminé. En étudiant la colère, par exemple, nous avons vu qu'elle est une réponse innée, rapide, constante, uniforme, permanente, et dans l'ensemble utile à une espèce déterminée d'excitation. Nous avons noté aussi que ces caractères sont ceux des réflexes simples comme l'éternuement ou la toux. La différence n'est pas dans la qualité mais dans la complexité.

Il est bien connu que l'homme est supérieur aux animaux moins évolués surtout à cause du développement, important et complexe, de ses hémisphères cérébraux. L'anatomie comparée montre que ces structures ont été superposées à un tronc cérébral qui diffère relativement peu chez les vertébrés supérieurs. Les réactions qui mettent en jeu le cortex cérébral peuvent laisser un temps prolongé, peut-être même des années, entre le stimulus et la réponse; que ces réactions peuvent être complexes au point d'être d'un caractère tout à fait imprévu; qu'elles peuvent disparaître complètement ou être fortement modifiées par d'autres stimulus ${ }^{6}$. Par contre, les réactions qui mettent en jeu les niveaux moins élevés du cerveau et de la moelle sont des réponses rapides à l'excitation, sont simples au point d'être d'un caractère uniforme et ne sont pas facilement modifiables. C'est par suite des différences de leurs hémisphères cérébraux que les individus présentent des différences marquées. L'être humain normal qui a une organisation hautement développée de neurones dans ses hémisphères, se

6 Cannon indique à la main, dans la marge gauche, qu'il a atteint le «1/4»de sa présentation. comporte d'une manière infiniment plus compliquée que l'idiot dont l'organisation des neurones est déficiente.

Dans les vertébrés inférieurs chez lesquels il n'existe aucun organe comparable aux hémisphères cérébraux du mammifère, les organisations nerveuses dans le tronc cérébral sont employées aux fonctions de saisir la proie et d'échapper aux ennemis. Ce sont là des activités qui, chez l'homme, sont associées avec le combat ou avec la fuite loin du danger, et qui sont accompagnées par les émotions de la colère ou de la peur. Chez les formes élevées, les centres, bien que normalement tenus sous le contrôle prédominant du cortex, sont capable de produire des réponses énergiques quand les conditions réclament une action immédiate et indispensable.

Dans la troisième conférence j’ai exposé les arguments en faveur de la localisation des mécanismes nerveux pour la manifestation de la colère (aussi bien en ce qui concerne l'attitude corporelle que les changements viscéraux), dans une petite masse cérébrale située dans la portion ventrale de couches optiques ${ }^{7}$. Cette région, contrairement à l'écorce où s'accomplissent constamment des adaptations nouvelles aux conditions extérieures, est un organe analogue à la moelle où les mécanismes simples des mouvements ordonnés restent et où l'excitation évoque des réponses fixes et régulières. Les attitudes typiques et les modifications viscérales qui résultent de l'action du thalamus sont plus compliquées que le réflexe rotulien ou que d'autres réflexes spinaux, mais ne sont pas essentiellement différentes. Rappelons que la région thalamique n'est pas seulement le siège des schémas de réaction pour la manifestation des diverses émotions. Les arguments que nous avons donnés dans la dernière conférence appuient fortement l'idée qu'elle est également le point de départ des sentiments particuliers qui ajoutent de l'animation

7 Cannon projette la figure reproduite dans La Sagesse du corps (Cannon I946[1939], I99); elle a paru initialement dans la seconde édition de Bodily Changes in Pain, Hunger, Fear and Rage (Cannon I922[1915], 247). 
et de la couleur à des sensations par ailleurs ternes.

Nous avons maintenant passé en revue les arguments qui montrent que les neurones de la région thalamique se déchargent vers le bas, dans la direction des muscles et des viscères, pour produire les changements physiologiques caractéristiques de l'excitation émotive, et qu'ils se déchargent vers le haut, dans la direction du cortex, pour ajouter aux sensations simples, de la richesse et de la chaleur. Je voudrais maintenant insister sur deux autres points.

Le premier a trait aux relaPigrom of possible relations of neurones of the cerebral cortex $(C C)$ and of the thalamic portion of the diencephalon $(D)$ to the viscera $(V)$ and
skeletal muscles $(S k M) . S p C=$ spinal cord The cortico-thalamic tract is regarded as inhibitory. Sensory fiber are not represented. Damage to the cortico-spinal tract at $A$ interrupts cortical control of certain skeletal muscles on one side, but it does not prevent control of these muscles on both sides by the centers in the diencephaleve bilateral control from the cortex.

Fig. $24^{8}$ tions de contrôle cortical et de contrôle thalamique des processus physiologiques. Il est clair que les muscles squelettiques sont contrôlés à deux niveaux - le cortical et le thalamique. Le cliché ${ }^{8}$ que vous voyez actuellement, illustre ce fait. Nous pouvons donc rire spontanément par suite de la présence d'une situation comique - c'est le rire thalamique - ou nous pouvons rire par suite d'une décision volontaire - c'est le rire cortical. Il est également certain que les viscères, d'autre part, sont sous le contrôle thalamique seulement. Nous ne pouvons pas par un acte volontaire accroître notre sucre sanguin, accélérer notre cœur, ou arrêter notre digestion. Quand il existe un contrôle double, les neurones corticaux sont certainement dominants dans les conditions normales et peuvent ne pas relâcher les neurones du thalamus en état d'excitation, bien que, cependant, nous puissions parfois pleurer ou rire malgré nous. Il y a donc un conflit entre les contrôles supérieur et inférieur du fonctionnement de l'organisme; il y a des influences antagonistes avec en même temps une certaine confusion.

8 Cannon projette la figure parue dans la seconde édition de Bodily Changes in Pain, Hunger, Fear and Rage (Cannon I922[I915], 249).
L'écorce cérébrale, cependant, peut seulement contrôler les fonctions qui sont normalement sous le contrôle volontaire. Je voudrais insister particulièrement sur ce point. De même qu'il ne peut pas les provoquer, le cortex ne peut pas empêcher ces processus impérieux qui prennent naissance dans le thalamus et qui augmentent le sucre sanguin, accélèrent le cœur, arrêtent la digestion, ou provoquent les autres troubles caractéristiques d'une grande excitation. Quand une émotion est réprimée, elle est donc réprimée dans ses manifestations extérieures seulement. Il y a certainement des arguments qui montrent que lorsque les manifestations extérieures sont au maximum, le tumulte intérieur est également au maximum; et il est probable que le contrôle cortical, exercé sur les manifestations extérieures de l'excitation, réduit aussi les troubles internes. Pourtant, dans un conflit entre le contrôle cortical et les activités des centres thalamiques, les manifestations internes incontrôlables peuvent être intenses.

Le second point sur lequel je voudrais insister se rapporte aux preuves que les états conscients sont seulement en rapport avec les neurones corticaux. Nous ne sommes certainement pas au courant des réflexes nombreux et compliqués qui déterminent l'attitude du corps, ou les dimensions de la pupille, par exemple, bien que ces réflexes soient réglés par le tronc cérébral. Il s'ensuit que les mécanismes nerveux des émotions primitives, qui ont leur siège dans les ganglions basaux, ne sont probablement pas directement associés avec la connaissance. Comme nous l'avons vu, cette considération explique l'un des traits les plus caractéristiques de l'expérience émotive; c'est-à-dire, son caractère mystérieux et non personnel. À certains moments, il se produit une prise de possession soudaine et puissante des forces de l'organisme, par les neurones sous-corticaux; c'est-àdire, par des neurones dont l'activité n'est pas immédiatement accompagnée par des états conscients. Dans les circonstances favorables, dès qu'il y a une suppression, même momentanée, du contrôle inhibiteur normal, ces neurones inférieurs prennent possession des mécanismes qui causent l'action et les poussent violemment dans l'un ou l'autre de leurs divers schémas de réaction. 
J'ai maintenant rappelé à votre attention les arguments en faveur de la thèse d'après laquelle la région thalamique libérée du contrôle cortical est capable d'avoir une activité indépendante, de caractère stéréotypé; que lorsqu'elle entre en fonction, elle produit des schémas de réaction typiques des diverses émotions fortes au point de vue de l'attitude, de l'expression, et des changements viscéraux; et que l'activité du thalamus provoque les sentiments d'excitation ou de dépression que nous éprouvons au cours du trouble émotif. Comment maintenant rattacher ces considérations à ce qui se passe en pratique? Comment les processus qui s'accomplissent dans les profondeurs de la portion ancienne du cerveau affectent-ils le fonctionnement de l'organisme? Pour montrer comment ce qui se passe dans le thalamus peut déranger profondément les mécanismes délicats de l'organisme normal, je vais vous citer quelques exemples. Je suis certain qu'ils ne paraitront pas inhabituels ou improbables à beaucoup d'entre vous.

Examinons d'abord les fonctions digestives. J'ai commencé à m'intéresser à l'effet des émotions sur l'organisme en observant l'abolition du péristaltisme gastrique au cours de l'excitation. Il y a beaucoup d'exemples d'arrêt total, non seulement de l'action mécanique du tractus digestif, mais aussi du travail des glandes digestives à la suite d'accès émotifs. Des tracas peuvent empêcher la digestion d'un repas pendant toute une nuit et le faire rester dans l'estomac pendant cette période. La sécrétion de salive, de suc gastrique, et de suc pancréatique, peut être arrêtée par la peur. Le processus de la digestion tout entière, qui est sujet au contrôle du système sympathique, peut être profondément troublé par l'anxiété et la détresse, qui sont les aspects les moins marqués de la peur. Des cliniciens compétents estiment qu'un tiers des malades souffrant de troubles des fonctions digestives doivent ces troubles à un manque d'équilibre émotif. Alvarez ${ }^{9}$ cite un cas de vomissement persistant qui a com-

9 Alvarez 1929. Walter Clement Alvarez (I884-I978) est un médecin gastroentérologue américain d'origine espagnole. En I9I6, il est nommé professeur à l'Université de Californie. En 1926, il rejoint la Mayo Clinic à Rochester. Il s'appuie notamment sur les travaux de Cannon pour expliquer les effets des émotions sur le fonctionnement digestif et sur les maladies digestives mencé alors qu'un percepteur d'impôts menaçait le malade de poursuites judiciaires s'il ne payait pas immédiatement ce qu'il devait. Aussitôt qu'Alvarez se fut rendu chez le percepteur par mesure thérapeutique et eut arrangé l'affaire, le trouble a cessé.

Les processus naturels qui se déroulent dans le tractus digestif sont fondamentaux pour toutes les autres fonctions de l'organisme. Toute perturbation du péristaltisme normal et de la sécrétion des sucs digestifs peut avoir des effets nuisibles étendus dans l'organisme. Cabot ${ }^{10}$ a rapporté un cas de fracture de jambe qui ne se consolidait pas. Des recherches ont montré que le malade était extrêmement soucieux de la situation de sa famille pendant son absence à l'hôpital. L'anxiété avait pour résultat une absence du désir de nourriture - une absence de contraction de faim dans l'estomac. De là provenait une nutrition insuffisante qui, à son tour, provoquait le manque d'accomplissement du processus de réunion des fragments osseux. Dès qu'il eut la certitude que sa famille était heureuse et qu'on prenait soin d'elle, l'état du malade s'est rapidement modifié. Ses tracas ont cessé, il a commencé à manger de bon appétit, sa nutrition s'est améliorée, et sa jambe a commencé à guérir ${ }^{11}$.

Le système cardio-vasculaire, comme le système digestif, est sous l'influence des nerfs sympathiques, mais au lieu d'être inhibé, il est excité par eux. L'excitation qui arrête la digestion gastrique fait battre le cœur plus rapidement et élève la pression sanguine en contractant les vaisseaux. Pendant la guerre sont apparus des cas assez nombreux de fonctionnement du cœur, ou

d'origine psychique (suractivité ou inhibition de l'activité intestinale, péristaltique, salivaire, pancréatique, gastrique, etc.). Le cas clinique rapporté par Cannon est le suivant: « Le fait que les flux dans le tube digestif peuvent être inversés par les soucis a été bien montré par une jeune femme névrosée qui a reçu un jour une lettre menaçante du percepteur. Cela l'effrayait tellement qu'au lieu d'aller voir quel était le problème, elle s'est mise au lit et a vomi jour et nuit pendant une semaine. Elle s'est arrêtée seulement quand, après m'être enquis de ce qui pouvait la contrarier, je suis allé au bureau des douanes et ai apaisé l'oncle Samuel avec 3,85 dollars; ce qui montre d'ailleurs que tous les médicaments ne sortent pas des fioles » (Alvarez I929, I234, notre traduction).

10 Cabot 1925. Pour Cabot, voir l'introduction.

11 Cannon indique à la main dans la marge gauche qu'il atteint la «1/2» de son exposé. 
comme on le disait parfois, de « cour de soldat» ${ }^{12}$. La moindre excitation ou la moindre perturbation faisait monter le pouls jusqu'à cent trente (I30) ou cent cinquante (I50) battements par minute. L'état général physique et nerveux des victimes de cette maladie, leur visage anxieux, leurs yeux troublés, les rides au voisinage de la bouche, leurs tremblements, faisait raisonnablement admettre que les épreuves de la guerre leur étaient devenues insupportables et avaient produit une telle sensibilisation du contrôle sympathique du cœur que des excitations, même légères, provoquaient des effets exagérés. Le mécanisme par lequel l'émotion peut produire une sensibilisation semblable est illustré par le cas suivant.

Une femme ne souffrant d'aucune espèce de trouble cardiaque, aperçoit dans la rue son mari, se promenant bras dessus, bras dessous avec une femme inconnue, et se comportant de manière à faire naître chez sa femme la jalousie et le soupçon. Profondément agitée par l'incident, la femme rentre chez elle et reste là plusieurs jours. Elle commence alors à craindre de sortir et de rencontrer son mari avec sa rivale. Après plusieurs jours de cette situation misérable, elle se décide à sortir sur les conseils d'une amie et probablement dans un état de terreur extrême. Mais elle ne va pas loin et court de nouveau à sa maison. Là, elle s'aperçoit que son cœur bat tellement fort qu'elle éprouve de l'oppression dans la poitrine et une sensation d'étouffement. De nouvelles tentatives de sortir produisent les mêmes symptômes alarmants. Elle commence alors à se rendre compte qu'elle pourrait mourir dans la rue si elle sortait. Il n'y avait chez elle aucune espèce de maladie du cour et pourtant l'effort léger qu'elle faisait en sortant de sa maison la mettait dans un état de détresse aiguë.

L'influence de l'excitation sur la pression artérielle peut également être notée. La pression est le résultat de la force de l'écoulement du sang dans les artères et de la résistance que celles-ci opposent à l'écoulement. Les influx sympathiques, en accélérant le rythme cardiaque et en contractant les artérioles, élèvent la pression en modifiant positivement les deux

12 Sur ce point, voir l'introduction. facteurs. J'ai mentionné dans une conférence antérieure le fait que des malades légèrement excités, ont une pression artérielle de vingt-cinq (25) à trente-cinq (35) millimètres plus élevés que leur pression artérielle lorsqu'ils sont calmes, et que dans des cas extrêmes de plaisir, de colère, ou de frayeur on peut observer une modification de quatre-vingt-dix (90) millimètres de mercure. Il n'est pas étonnant que des malades souffrant d'hypertension et d'involution sénile du système circulatoire, puissent souffrir de choc et même mourir dans des circonstances qui provoquent de l'excitation.

Un autre effet des influx sympathiques est l'augmentation du sucre sanguin. J'ai mentionné déjà que les troubles émotifs pouvaient produire de l'hyperglycémie chez l'homme au cours de compétitions sportives ou d'examens importants. Le même phénomène a été observé chez des diabétiques sans doute parce que chez eux on surveille de plus près l'excrétion de sucre. Woodyatt cite le cas suivant, parmi de nombreux autres de même nature, dans sa pratique.

Un homme de soixante-cinq $\left(6_{5}\right)$ ans, diabétique, recevait à l'hôpital une diète quantitativement réglée et contenant une dose quotidienne d'insuline. Son urine était sans sucre. Soudain, un jour, sans aucun changement de régime, il se mit à éliminer quarante-trois (43) grammes de sucre, et un jour il en élimina soixante-seize grammes, et présenta un cas d'acidose légère. La glycosurie ne pouvait donc pas provenir de l'absorption de la nourriture supplémentaire. Un contrôle soigné de toutes les circonstances et de toutes les analyses n'a révélé aucune erreur de technique, et l'examen du malade ne révéla aucune maladie intercurrente. On trouva, cependant, que le malade avait reçu des nouvelles qui le portaient à craindre que la société, dans laquelle il avait été employé pendant plus de vingt (20) ans, puisse le congédier. C'était là la cause déterminante des troubles du métabolisme sucré ${ }^{13}$.

Comme Woodyatt ${ }^{14}$ le remarque, il est intéressant de pou-

13 Woodyatt 1927, I0I3. L'article fait état de plusieurs cas de patients soumis à un «stress émotionnel» (emotional stress).

14 Cannon se réfère à ce passage: «Il est intéressant de pouvoir mesurer le pouvoir de l'émotion en termes aussi tangibles que des grammes [onces] de sucre? 
voir mesurer le pouvoir d'une émotion en termes aussi positifs que des grammes de sucre. Dans ces conditions le pouvoir qu'ont les émotions de produire des altérations physiques de l'organisme ne semble pas fantastique.

Il existe des faits montrant qu'un trouble émotif violent peut produire des effets profonds sur l'organisme par l'intermédiaire d'une action sur la glande thyroïde. Marañon ${ }^{15}$ a réuni de nombreux cas d'hyperthyroïdisme provoqués pendant la Grande Guerre par des expériences pénibles ${ }^{16}$. Emerson ${ }^{17}$ a rapporté récemment quelques exemples frappant d'hyperthyrö̈disme conséquent à des événements profondément émouvants dans la vie des malades.

L'une de ces malades était une femme mariée qui avait eu deux enfants illégitimes et dont le mari s'est suicidé devant elle pour lui reprocher sa manière de vivre. Aussitôt elle s'écroula sur

Dans ces conditions, le pouvoir des émotions à produire des altérations phy siques du corps ne semble pas irréel» (Woodyatt 1927, I0I3, notre traduction).

Marañon I92I. Après avoir rappelé que les cas de maladie de Basedow (maladie thyroïdienne se manifestant généralement par une accélération du métabolisme basal, une hypertension, une hyperthyroïdie et l'apparition d'un goitre exophtalmique) augmentent en temps de guerre, Marañon rap porte quarante-huit cas dans lesquels la vie affective des malades a joué un rôle déclencheur. La clinique, associée aux recherches, en particulier celles de Cannon, attestent selon lui d'une « relation harmonique entre la pathogénie émotionnelle du mal de Basedow et la théorie thyréogène » (Marañon I92I, 90). On notera que Marañon compte parmi les facteurs émotionnels principaux «la vie sexuelle qui, presque toujours, est liée aux épisodes hyperthyroïdiens» et les "contrariétés économiques et professionnelles, etc.» (Marañon I92I, 92).

16 Cannon tire ce passage de son article «The Mechanism of Emotional Disturbance of Bodily Functions »: "Marañon has collected an extensive serie of cases of hyperthyroidism brought on by stressful experiences during the Great War.» (Cannon I928b, 882) On note que le terme stressfull est traduit par «pénible».

17 Emerson 1927. L'article cité par Cannon fait surtout référence à des cas d'hyperthyroïdie pour lesquels des facteurs émotionnels semblent avoir joué un rôle déclencheur. Pour autant, Emerson estime que des facteurs émotionnels rentrent aussi dans l'étiologie de l'hypertension, du diabète et de la polyarthropathie, non pas en tant que cause unique, mais comme facteurs favorisant la «susceptibilité » des patients aux maladies (Emerson I927, 349). L'intérêt d'Emerson pour la biographie de ses patients s'inscrit dans une démarche clinique plus large (à ce sujet, voir l'introduction). le plancher et se mit à hurler jusqu’à être complètement épuisée. Tout à coup, elle sentit son gosier se contracter et sentit qu'elle avalait difficilement. La glande thyroïde se mit à grossir et six semaines après l'incident elle avait un métabolisme soixantecinq (65) pour cent plus élevé que la normale. Dans la suite, des troubles prolongés se présentèrent en même temps qu'une augmentation du sucre sanguin et de la pression artérielle.

Voici un autre cas: un homme de vingt (20) ans s'était querellé avec sa fiancée. Elle, simulant une tentative de suicide, avait avalé devant lui des pilules et était tombée en criant. L'homme s'en alla aussitôt. Pendant une semaine il présenta un gonflement du cou et de la nervosité. Quand il se présenta à l'hôpital quatre mois après, il avait perdu du poids et présentait un goitre volumineux sur lequel on pouvait sentir un tbrill ${ }^{18}$ net, et son métabolisme basal était de vingt-quatre (24) pour cent plus élevé que la normale.

Voici encore un autre cas: une femme mariée avait vu son mari tuer ses deux frères. Le mari lui fit de vifs reproches parce qu'elle ne parut pas au procès pour prendre sa défense. Une semaine après le procès un goitre apparut, et en sept jours devint volumineux. Quelques mois après, quand elle se présenta à l'hôpital, son goitre était énorme, présentait des pulsations visibles, et un thrill palpable, et il provoquait une sensation oppressante de suffocation. Il y avait une exophtalmie prononcée, du tremblement et de l'inquiétude marquée. L'augmentation de métabolisme basal variait de quarante (40) à cent dix-sept (II7) pour cent.

On peut mentionner d'autres influences émotives sur les fonctions de l'organisme, telles que des désordres de la menstruation et de la sécrétion de lait. Nous avons donné assez d'exemples, cependant, pour montrer qu'il y a des effets produits dans les organes innervés par le sympathique - c'est-à-dire, les glandes à sécrétion interne et les glandes à sécrétion externe et les parties contenant des muscles lisses - qui sont tout aussi réels que ceux produits quand on contracte les biceps pour

I8 Le thrill est la sensation de frémissement perçue à la palpation du goitre, due à l'augmentation de la circulation sanguine dans la glande thyroïde. 
soulever un poids. Il y a cependant une différence remarquable dans le niveau de contrôle nerveux de ces deux effets. Alors que le biceps est habituellement sous la dépendance du cortex, les viscères dépendent du diencéphale. Alors que le biceps est soumis à un contrôle volontaire, les viscères ne sont pas sous ce contrôle, mais sont favorablement ou défavorablement influencés par les processus associés aux sentiments et aux émotions. Bien que le centre nerveux pour l'expression des émotions soit sous-cortical - c'est-à-dire, à un niveau bas dans le tronc cérébral - les processus corticaux sont, cependant, intéressés dans la réaction totale à une situation qui provoque des sentiments prononcés. Nous pouvons être effrayés par un ours réel, mais pas par un ours empaillé. La distinction entre les deux est faite par le cortex. Comment pouvons-nous interpréter en termes physiologiques cette relation entre le cortex et le thalamus?

J'ai montré auparavant qu'une réaction émotive présente plusieurs des caractères d'une réponse réflexe. Pour provoquer un réflexe il faut appliquer un stimulus approprié; un corps étranger dans le larynx produit la toux; la nourriture placée dans la bouche provoque un écoulement de salive. De même, pour les expressions émotives. Watson ${ }^{19}$ a étudié des enfants nouveau-nés et il a vu que dès le début les bruits violents et aussi le fait, pour l'enfant, de ne plus se sentir soutenus, sont les stimulus naturels qui provoquent la réaction de peur. La limitation ou l'empêchement de la liberté des mouvements, est, dès le début, le stimulus naturel pour la colère ${ }^{20}$.

19 Watson I924[I925], I2I. John Broadus Watson (I878-I958) est un psychologue américain. Il étudie la psychologie à l'Université de Chicago, où il obtient un doctorat en 1903. Dès 1908, il enseigne la psychologie à la Johns Hopkins University. En I913, il publie un manifeste dans lequel il appelle la psychologie à devenir une science purement objective et expérimentale. Fortement inspirée des travaux de Pavlov, la psychologie comportementale ou behavioriste que Watson contribue à fonder rejette toute forme d'in trospection et entend aligner la psychologie sur les méthodes des sciences naturelles. Dans l'ouvrage cité par Cannon, Watson critique la psychologie de James, en particulier sa théorie des émotions. Il lui reproche notamment son approche théorique, trop peu fondée sur des données empiriques. À cet égard, il cite abondamment les travaux de Cannon et de ses équipes.

20 Cannon indique à la main dans la marge gauche qu'il a atteint les «3/4» de son exposé.
Des agents autres que les stimulus naturels, peuvent, pourtant, produire l'action d'un réflexe s'ils sont étroitement associés aux stimulus naturels. C'est ainsi, comme vous le savez, que si une lumière rouge est montrée éclairée à différentes reprises en même temps qu'un aliment est placé dans la bouche, la lumière rouge seule deviendra aussi efficace que l'aliment dans la production d'un écoulement salivaire. Le stimulus indifférent, en l'espèce la lumière rouge, est alors appelé l'excitation conditionnée, et la sécrétion salivaire réflexe est dans ces circonstances un réflexe conditionné. Toutes espèces d'agents extérieurs d'habitude indifférents - non seulement une lumière, mais un bruit, une forme, un contact, une odeur, en un mot, tout ce qui peut influencer un organe des sens - peuvent devenir un excitant efficace par suite d'associations étroites dans le temps avec l'excitant affectif normal. De cette manière les objets et les phénomènes du monde qui nous entoure prennent constamment une signification différente au point de vue de nos réactions. Tous les processus de conditionnement s'accomplissent dans l'écorce cérébrale. Ces faits, étudiés en détail et d'une façon très instructive par le physiologiste russe, Pavlov, donnent une possibilité d'explication du comportement émotif.

Nos réflexes émotifs, comme le réflexe salivaire, deviennent compliqués par le conditionnement d'excitants indifférents. Lorsqu'on montre un rat blanc à un petit enfant ${ }^{21}$, l'enfant cherche à le saisir et à jouer avec lui. L'enfant n'a pas peur. On présente alors le rat de façon répétée, mais en même temps on produit chaque fois un bruit violent en frappant une barre

21 Cannon renvoie ici à Watson I924[I925], I26). Il s'agit de l'expérience dite du «petit Albert», du nom de l'enfant chez qui Watson a induit un réflexe conditionné de peur des rats. L'expérience et les résultats sont initialement présentés dans Watson/Rayner 1920. Cannon reprend ici le raisonnement de Watson, qui s'oppose à la thèse jamesienne selon laquelle les émotions ne seraient issues que de réflexes héréditaires. Cette thèse est selon lui contredite par la diversité culturelle des objets susceptibles de produire des émotions. Le cas du petit Albert est justement censé démontrer que des réactions émotionnelles peuvent être acquises. On notera que, loin des oppositions contemporaines, Cannon n'a pas de mal à convoquer le comportementalisme et, comme on le verra plus bas, la psychanalyse, afin de rendre compte des pathologies émotionnelles et de leur traitement potentiel. 
d'acier. Le rat devient, donc, un excitant conditionné pour la réaction de frayeur produite par le bruit, et dans la suite, quand on lui montre le rat, l'enfant pleure et se détourne. Il a, maintenant, peur du rat, non point parce que c'est un rat, mais parce qu'il est devenu le signal et le symbole d'une chose à craindre, - le bruit violent. C'est d'une façon analogue que les circonstances indifférentes qui accompagnent une perturbation émotive deviennent des excitants conditionnés ou des signaux de renouvellement de cette perturbation. La femme qui avait vu son mari s'occuper d'une femme étrangère dans la rue, avait eu une émotion intense qui se révéla, non pas en voyant le mari infidèle et sa complice, mais simplement en allant dans la rue. Ainsi à la suite d'associations étendues, les réponses émotives deviennent soumises à des excitants conditionnés de plus en plus compliqués, et il en résulte la grande complexité du comportement affectif.

Bref, les troubles persistants de l'organisme, au cours de fortes réactions émotives, peuvent être interprétés comme étant dus à la persistance des excitants qui provoquent les réactions. Ils peuvent persister parce qu'ils ne sont pas naturellement éliminés par l'accomplissement des impulsions émotives, ou parce que l'accomplissement de ces impulsions est rendu impossible par les circonstances - la mort par exemple -, et dans ces cas, le retour de l'excitant original sous l'action du souvenir, avec des caractères émotifs comme la terreur ou le remords, garde les réactions vivantes; ou ils peuvent persister parce qu'ils deviennent associés avec un objet commun qui, rencontré de façon répétée, est un excitant conditionné répété.

Dans la discussion précédente, j’ai avec intention insisté sur le mécanisme physiologique des troubles émotifs, et cela pour deux raisons: d'abord, je désirais vous montrer que ces troubles remarquables peuvent être décrits en fonction de processus nerveux et aussi je désirais vous persuader que ces phénomènes intéressants ne devraient pas être écartés comme étant des phénomènes mystiques se présentant dans le domaine de la psyché, mais devraient plutôt être considérés comme des mouvements, des inhibitions, ou des troubles de l'organisme, qui appartiennent au domaine des médecins et des biologistes, qui reconnaissent la base physique de notre comportement.

Bien que les médecins aient des occasions fréquentes d'observer des exemples de troubles émotifs dus aux émotions, ils ont une tendance à diminuer cette influence, à nier que ce soit l'affaire du médecin dans les services qu'il doit à son malade de s'occuper de ces troubles simples. Que le malade aille au pasteur et au prêtre pour trouver du réconfort et des consolations, et pour la résolution de ses anxiétés profondes ${ }^{22}$. Le refus, trop fréquent chez le médecin, de considérer sérieusement l'importance de l'élément émotif dans la maladie, me semble dû peut-être à l'influence de deux manières de voir, et à deux disciplines diamétralement opposées. D'une part, il y a l'influence profonde de l'anatomie pathologique. Celle-ci est parvenue à démontrer sous le microscope d'une façon tellement triomphante et tellement générale les altérations structurales qui accompagnent les altérations fonctionnelles, que tout état qui n'a pas une anatomie pathologique définie apparaît comme irréel ou insignifiant. Les peurs, les tracas, et les états de colère et de ressentiment ne laissent pas de traces définies dans le cerveau. En quoi alors regardent-ils le médecin? D'autre part, ces sentiments mystérieux et dominateurs qui s'élèvent en nous à partir de sources inconnues ne sont-ils pas de simples perturbations de la psyché et, dans ce cas encore, en quoi regardentils le médecin? Si les médecins manifestent cette indifférence, cependant, est-il surprenant que les gens souffrant de troubles émotifs ${ }^{23}$ se détournent d'eux et aillent chercher chez les guérisseurs, chez les adeptes de la «Science Chrétienne» (Christian

22 Bien qu'elle soit bancale, nous reproduisons la phrase telle qu'elle figure dans le tapuscrit.

23 Ce passage est repris d'un article publié en I928, intitulé «The Mechanism of Emotional Disturbance of Bodily Functions ». On note que Cannon traduit les termes emotional stresses figurant dans l'article, par ceux de «troubles émotifs »: «Fears, worries and states of rage and resentment leave no clear traces in the brain. What, then, have we physicians to do with them? On the other hand, these mysterious and dominant feelings which surge up within us from unknown sources - are they not pure perturbations of the "psyche". If we show this indifference, however, is it surprising that men and women, beset by emotional stresses, turn from us and go for help to faith healers, to Christian Scientists and to others who recognize the reality of these disturbing states?» (Cannon I928b, 877). 
Science) $)^{24}$ et d'autres qui reconnaissent la réalité de ces états? On trouve, j'en suis convaincu, un moyen d'échapper aux exigences des pathologistes en ce qui concerne les preuves morphologiques évidentes de la maladie, et aussi au mysticisme et au vague des guérisseurs par une compréhension des processus physiologiques qui accompagnent les émotions profondes. En tant que physiologiste, je peux raisonnablement considérer ce qui se passe dans les voies nerveuses du cerveau comme n'étant pas associé à des changements structuraux démontrables. Des conséquences très marquées et désastreuses peuvent être le résultat de réactions habituelles, qui peuvent être considérées comme ne différant pas qualitativement de notre comportement habituel ${ }^{25}$.

La Science chrétienne (Christian Science) est un mouvement religieux protestant non trinitaire, fondé par Mary Baker Eddy (I82I-I9IO) à Boston en I879. De santé fragile, Mary Baker Eddy s'intéresse de près à l'homéopathie ainsi qu'au mesmérisme. Mais c'est dans la Bible et la prière qu'elle finit par trouver le principe de toute guérison. Les principes de la Science chrétienne sont exposés dans l'ouvrage fondateur qu'elle édite en 1875 , Science and Health (Baker Eddy, I875). L'auteure y expose un système métaphysique selon lequel Dieu est tout et la douleur et la maladie ne sont que des idées erronées qui disparaissent dans la prière et la compréhension des Écritures. Le livre, vendu à plus de neuf millions d'exemplaires dans le monde, figure parmi la liste des ouvrages écrits par «des femmes dont les paroles ont changé le monde », établie en I992 par la National Women's Book Association aux États-Unis. Le mouvement compte des milliers de fidèles au tournant du siècle. En I930, le public parisien connaissait très cer tainement le mouvement de la Science chrétienne. En I897, une centaine d'exemplaires de l'ouvrage de Mary Baker Eddy avait en effet été distribué à des personnalités du milieu académique, notamment à la Sorbonne, et du monde culturel. À l'occasion de l'Exposition internationale du livre, tenue à Paris en I907, le livre vaudra à son auteure de se faire nommer «Officier d'Académie » par le gouvernement français, représenté par Aristide Briand (The New York Times, 1907). La revue du mouvement, The Herald of Christian Science (Le Héraut de la Science chrétienne), sera distribuée en France dès I903, et la traduction du livre de Mary Baker Eddy, Science et santé avec la clef des Écritures (Baker Eddy I9I7[I875]), paraitt en I9I7. On note que le mouvement de la Science chrétienne est parfois apparenté au mouve ment unitarien, en raison de visions progressistes communes (Mellone I927). Cependant, la Science chrétienne rejette la médecine moderne sous toutes ses formes. Ceci explique le désarroi de Cannon, qui constate qu'en délaissant les troubles émotionnels, la médecine laisse indirectement prospérer les guérisseurs.

25 Cannon ajoute cette phrase à la main dans le corps du texte.
En tant que physiologiste également, j’ai le droit de considérer les altérations qui se produisent soudainement dans les fonctions des organes innervés par le système nerveux central comme se produisant à la suite de décharges d'influx par ce système. Partant du point de vue physiologique, j'ai donc considéré les émotions en termes d'influx nerveux, tout comme je pourrais considérer les influx nerveux venant de l'aire motrice de l'écorce comme gouvernant les mouvements des muscles squelettiques.

Dans ce que nous avons examiné ensemble en partant du point de vue physiologique, il y a des points qui ont une importance pratique dans les cas de gens qui sont ou ont été profondément troublés en éprouvant une émotion.

D'abord, il y a l'importance d'un traitement prompt. Nous connaissons tous la facilité avec laquelle s'établissent des habitudes dans le système nerveux par la répétition fréquente d'une action. Chaque fois que les influx nerveux traversent une voie déterminée, ils y rendent le passage plus facile pour les influx subséquents. Donc, l'expression habituelle des émotions, aussi bien dans les viscères que dans le visage, peut se fixer et s'imprimer profondément dans l'organisation nerveuse tout comme les mécanismes compliqués de la nage, du patinage, ou de la bicyclette, deviennent imprimés en nous à la suite d'exercices répétés. Il est certain que les réactions émotives habituelles devraient être, autant que possible, prévenues par un traitement rapide.

Comme nous l'avons vu, le cortex n'a pas de contrôle direct sur les fonctions des viscères. Il est donc inutile d'essayer de ralentir un cœur accéléré, ou d'abaisser une expression sanguine élevée ou de rétablir le fonctionnement d'un tube digestif inhibé, simplement en faisant froidement appel par la raison à une manière d'être différente. L'homme dont la jambe ne se réparait pas, parce qu'il avait des craintes au sujet de sa famille, ne pouvait pas être guéri de sa crainte par le raisonnement, mais sa crainte disparut quand il apprit que sa famille était dans une situation convenable. Le cortex, dont l'activité est en rapport avec l'analyse du monde extérieur, ne devrait pas être le seul à qui faire appel quand on tente un traitement. Les occasions de tracas, des anxiétés, des conflits, des haines, 
des ressentiments, et d'autres formes de peur ou de colère qui affectent les centres thalamiques, doivent être supprimés. Bref, les facteurs qui, dans l'ensemble de la situation, sont les sources du sentiment, doivent être découverts et supprimés par le fait de leur explication ou élimination.

Bien que le cortex n'ait pas de contrôle direct sur les viscères, il a un contrôle indirect. Nous pouvons nous mettre dans une situation dangereuse, et en avoir une émotion bien que nous ne puissions pas avoir une émotion simplement parce que nous avons décidé de l'avoir. De la même façon, nous pouvons souvent éviter les circonstances qui éveillent la peur ou la colère ou le dégoût et leurs symptômes viscéraux concomitants? Il suffit de ne pas nous trouver là où se trouvent des causes déterminantes de ces émotions.

Quand la cause des perturbations n'apparaît pas, on peut parfois la trouver par une enquête soignée ou par l'analyse ${ }^{26}$. Il est intéressant de constater qu'une explication complète de la façon dans laquelle le trouble a été causé suffit souvent à supprimer ce trouble rapidement et complètement.

Enfin, un mot d'avertissement peut ne pas sembler ici déplacé. Si on ne trouve pas de raison objective aux plaintes du malade, rien n'est plus facile que de tout attribuer aux facteurs nerveux. Quand quelqu'un insiste sur l'importance des facteurs nerveux comme perturbateurs du calme dans l'organisme, il y a un danger à ce qu'on ne les considère comme écartant la nécessité de rechercher les causes anatomo-pathologiques. Rien n'est plus loin de mes intentions. Je pense plutôt, pour des raisons de prudence, qu'il ne faut recourir aux agents émotifs pour l'explication des troubles de l'organisme, qu'en dernier ressort, et seulement lorsqu'on n'a pas pu trouver, malgré tous ses efforts, une autre explication. Et même quand la cause est trouvée, dans la peur, dans la colère ou dans un autre sentiment profond, on doit chercher la preuve de la validité de cette conclusion très soigneusement, non seulement dans la cause du trouble, mais encore, dans le résultat d'une thérapeutique appropriée. On ne doit non plus perdre de vue qu'il est très

26 Sur ce point, voir l'introduction. possible de trouver une lésion anatomique en même temps que des troubles émotifs profonds. Les deux conditions, l'altération structurale d'un organe et l'altération fonctionnelle du système nerveux, peuvent être causalement reliées l'une avec l'autre, et pourraient être traitées comme un trouble unique. Il est certain que c'est seulement lorsque toutes deux sont considérées comme des perturbations d'un tout unique, l'organisme, qu'on peut en avoir une conception adéquate et qu'on peut les traiter d'une manière effective.

Je voudrais exprimer ma reconnaissance pour la patience dont vous avez fait preuve en m'écoutant, et aussi pour l'attention que vous avez apportée pendant l'audition de ces conférences ${ }^{27}$.

Cette dernière phrase est ajoutée à la main. Cannon a envisagé une autre formule de remerciements: «A la fin de ces conférences je voudrais vous remercier de votre attention et spécialement de votre patience en m'écoutant.» 
Cinq leçons sur

l'homéostasie 
La stabilité des organismes; Définition de l'homéostasie ; Importance de la constance du milieu intérieur; Mécanismes stabilisant la composition du liquide nourricier ;

Mécanismes correctifs après hémorragie.

C'est difficile d'exprimer dans une langue étrangère les sentiments éprouvés à une occasion comme celle-ci. Puis-je dire cependant combien je me sens chez moi d'être encore dans un laboratoire de physiologie, et spécialement dans le laboratoire de mon cher ami, le professeur Lapicque. Et aussi puis-je dire que je regarde comme un témoignage particulier d'amitié et de confiance l'inclusion de mes confrères dans le cours régulier de la Sorbonne. À mes condisciples, je voudrais dire que s'ils trouvent difficile de comprendre mes mots ou s'ils trouvent mes idées obscures, j'espère qu'ils viendront après chaque conférence pour me demander des explications ${ }^{1}$.

Quand l'honneur me fut offert de venir en France comme professeur d'échange, je ressentis profondément la tâche que cet honneur comportait. Je ne pouvais apporter dans ce centre d'étude que peu de choses qui soient neuves ou qui puissent inspirer de nouvelles idées. Cependant, un groupe d'idées qui m'ont intéressé dernièrement se sont trouvées en rapport étroit avec les concepts que le grand physiologiste français, Claude Bernard, fut le premier à émettre il y a quelque soixante-dix ans et qu'il approfondit dans ses écrits ultérieurs. Je parle du maintien des conditions constantes à l'intérieur des organismes. Il m'a semblé qu'un examen des mécanismes grâce auxquels ces conditions sont stabilisées pourrait être intéressant en égard aux idées de Bernard et aussi à cause de l'importance intrinsèque du sujet.

L'aptitude que possèdent les organismes hautement évolués, de se maintenir stables et constants en face de conditions 
qui, à première vue, paraissent devoir amener des troubles profonds, est un fait impressionnant. L'homme peut être exposé à une chaleur sèche de cent quinze à cent vingt-six (I26) degrés centigrades. D'autre part, les mammifères des régions polaires exposés à des froids qui peuvent atteindre trente-cinq (35) degrés au-dessous de zéro, ne montrent aucune chute notable de température corporelle. Dans les régions désertiques ou l'air est extrêmement sec, les habitants ont peu de difficultés à retenir leurs liquides organiques. Et les êtres humains qui, à l'heure actuelle, s'aventurent à de grandes hauteurs, soit dans les montagnes, soit en avion, supportent des tensions d'oxygène fortement réduites sans montrer de troubles sérieux dus au manque d'oxygène. Il n'y a pas seulement résistance aux modifications que pourraient provoquer les circonstances extérieures; il y a aussi résistance aux troubles provenant du fonctionnement de l'organisme lui-même. Par exemple, la chaleur produite dans l'effort musculaire maximum, poursuivi pendant vingt minutes, serait si grande qu'elle occasionnerait la coagulation de quelques-unes des protéines du corps si elle n'était pas rapidement dissipée. Vous me pardonnerez, j'en suis sûr, l'incongruité qu'implique l'hypothèse d'une activité qui continuerait à un tel degré.

De plus, un effort musculaire continu et violent est accompagné d'une telle production d'acide lactique qu'en peu de temps il neutraliserait tous les alcalins du sang si d'autres mécanismes n'entraient en jeu pour prévenir ce désastre. Bref, les organismes bien armés, par exemple, les mammifères, affrontent parfois dans le milieu ambiant des dangers extrêmes, et aussi des dangers également grands qui peuvent résulter de leur activité propre, et malgré cela ils continuent à vivre et à fonctionner sans perturbation considérable.

Des faits tels que ceux que je viens de mentionner pour illustrer la stabilité des organismes ont soulevé de nombreuses questions tendant à faire connaître les moyens ${ }^{2}$ par

2 Trois feuillets des conférences originales sont manquants. Comme indiqué en introduction, nous avons remplacé les passages manquants par ceux tirés du texte publié en I930, dans le cadre de la collection des « Cours des facultés» (Cannon I930a). lesquels cette stabilité est assurée. Comme je l'ai déjà dit, Claude Bernard fut le premier à suggérer un trait hautement important dans ce contrôle de la stabilité. Dans ses Conférences sur la pathologie expérimentale ${ }^{3}$, données en mille huit cent cinquante-neuf et soixante ${ }^{4}$, il montra qu'il y a deux milieux pour les corps animés, un milieu général qu'ils partagent avec les objets inanimés et qui environne l'organisme considéré comme un tout; et un milieu intérieur dans lequel les éléments vivants du corps trouvent leur habitat optimum.

Tout d'abord, il considère seulement le plasma sanguin comme représentant le milieu intérieur. Plus tard, il parla du plasma sanguin et de la lymphe comme constituant le milieu interne ${ }^{5}$, et finalement dans son Traité sur les Phénomènes de la Vie, il classa sous ce nom la «totalité des liquides circulants de l'organisme ${ }^{6}$.

3 Bernard I872. Le titre exact est en fait: Lecons de pathologie expérimentale.

4 Dans la leçon de janvier I865, intitulée «Leçon sur le milieu intérieur comme champ d'action de la médecine expérimentale», Bernard écrit : «Le milieu intérieur n'est autre chose que celui dans lequel vivent les parties élémentaires de l'organisme; les influences du milieu cosmique doivent passer par le milieu intérieur pour atteindre ces parties élémentaires » (Bernard I872, 439).

5 À notre connaissance, Bernard utilise la notion de «milieu intérieur» et n'utilise jamais celle de «milieu interne». Dans The Wisdom of the Body, Cannon utilise indistinctement les notions de «milieu intérieur», «milieu interne» ou, en anglais, de internal environnement. Cannon ne semble pas distinguer l'intérieur de l'interne, ce que Bernard fait lorsqu'il décrit, par exemple, un appareil de laboratoire: «La cloche intérieure est recouverte à sa face interne d'une feuille d'étain, constituant l'armature positive du condensateur » (Bernard I878, 393, nous soulignons).

6 Cannon cite vraisemblablement le passage suivant: «Lorsque le milieu intérieur, c'est-à-dire l'ensemble des liquides circulants se refroidit, chaque élément en contact avec le sang s'engourdit pour son propre compte, révélant ainsi son autonomie et les conditions de son activité propre. " (Bernard I878, I06) Par ailleurs, le titre exact du livre est Leçons sur les phénomènes de la vie, communs aux animaux et aux végétaux, et non Traité sur les phénomènes de la vie, communs aux animaux et aux vééétaux. La référence au livre de Bernard, dans The Wisdom of the Body, apparait en version abrégée: Les phénomènes de la vie (Cannon I939[I932], 40). Il est donc fort probable que, dans la référence abrégée en tête, Cannon ait par erreur utilisé le mot «traité » en lieu et place de celui de «leçon». 
Ce fut une remarquable contribution que Bernard apporta à la physiologie quand il reconnut que le plasma sanguin et les liquides interstitiels ou intra-organiques fournissaient aux éléments vivants de l'organisme un milieu approprié et favorable. Il montra que ce mécanisme permettait la nutrition des cellules cachées dans la profondeur des tissus, loin des surfaces qui séparent la structure vivante du monde extérieur inanimé, et qu'ils fournissaient un véhicule pour le transport des déchets, de ces cellules aux endroits d'excrétion. Il fit remarquer de bonne heure que ce fluide nourricier, cette matrice liquide - fluid matrix ${ }^{7}$ comme nous pouvons l'appeler, est un produit de l'organisme lui-même et qu'il est sous le contrôle de mécanismes qui le maintiennent constant. Il comprit clairement que pour autant que cette stabilité est conservée, l'organisme est libéré des vicissitudes extérieures. "C'est la fixité du milieu intérieur qui est la condition d'une vie libre et indépendante ${ }^{8}$, dit-il. Cette fixité suppose dans l'organisme des mécanismes si parfaitement ajustés que toute tendance à la variation est rapidement compensée.

Ces vues fréquemment défendues par Bernard dans les diverses séries de conférences qu'il fit dans les vingt dernières années de sa vie, trouvèrent bon accueil dans l'esprit d'autres physiologistes. En mille huit cent quatre-vingt-cinq, Frédéricq ${ }^{9}$

7 Cannon introduit la notion de fluid matrix en même temps que celle d'homéostasie (Cannon 1926). Elle est pour lui synonyme de milieu intérieur: «Il [Claude Bernard] a souligné que, chez les animaux à l'organisation complexe, les parties vivantes existent dans les fluides qui les baignent, c'est-àdire dans le sang et la lymphe, qui constituent le "milieu interne" ou "inté rieur", ce que l'on pourrait appeler la matrice fluide du corps » (Cannon I929, 399 , notre traduction).

$8 \quad$ Cannon cite librement le passage suivant: «La fixité du milieu intérieur est la condition de la vie libre, indépendante: le mécanisme qui la permet est celui qui assure dans le milieu intérieur le maintien de toutes les conditions nécessaires à la vie des éléments » (Bernard I878, II3).

9 Léon Frédéricq (I85-1935) est un physiologiste belge dont les travaux portent principalement sur la respiration, la coagulation, la pression et la circulation sanguine. Il obtient un doctorat de sciences naturelles en I87I. En I879, il succède à Théodore Schwann (I8I0-I882) à la chaire de physiologie de l'Université de Liège, où il fonde en I887 l'Institut de physiologie. Zénon Bacq (1903-I983), à qui Cannon confie la traduction de The Wisdom of the Body, lui succède à la chaire de physiologie. Frédéricq et Cannon se sont rencontrés à plusieurs reprises, notamment lors du treizième congrès déclarait: «L'être vivant est agencé de telle manière que chaque influence perturbatrice provoque d'elle-même la mise en activité de l'appareil compensateur qui doit neutraliser et réparer le dommage. À mesure qu'on s'élève dans l'échelle des êtres, ces appareils régulateurs deviennent plus nombreux, plus parfaits, plus compliqués; ils tendent à affranchir complètement l'organisme des influences nuisibles et des changements survenus dans le milieu extérieur. ${ }^{10}$

Plus tard, en dix-neuf cents, Richet attira l'attention sur ce fait général. "L'être vivant est stable", écrit-il, «et il faut qu'il le soit pour n'être pas détruit, dissous, désagrégé, par les forces colossales, souvent adverses, qui l'entourent. Mais par une sorte de contradiction qui n'est qu'apparente, il maintient sa stabilité que s'il est excitable et capable de se modifier suivant les irritations du dehors, et de conformer sa réponse à l'irritation; de sorte qu'il n'est stable que parce qu'il est modifiable, cette stabilité modérée est la condition de la véritable stabilité de l'être. ${ }^{11,12}$

Le pouvoir extraordinaire que possèdent les vertébrés supérieurs, de maintenir leur organisation constante a été le résultat d'une évolution prolongée. Un simple coup d'œil sur les vertèbres inférieures nous le montre. L'amphibien n'est libre ni dans l'eau ni dans l'air. S'il reste dans l'eau il s'asphyxie; s'il reste dans l'air, il se dessèche. Bien plus, il ne peut contrôler sa température et par suite, devient inactif pendant l'hiver. L'activité des espèces immédiatement supérieures aux amphibiens, les

international de physiologie que Cannon organise à l'École de médecine de Harvard en 1929.

10 Frédéricq $1885,35$.

11 Richet I899, 72I. Cannon tronque la citation originale: «L'être vivant est stable; et il faut qu'il le soit pour n'être pas détruit, dissous, désagrégé, par les forces colossales, souvent adverses, qui l'entourent. Mais, par une sorte de contradiction qui n'est qu'apparente, il ne maintient sa stabilité que s'il est excitable, capable de se modifier suivant les irritations du dehors et de conformer sa réponse à l'irritation; de sorte qu'il n'est stable que parce qu'il est modifiable. La défense n'est compatible qu'avec une certaine instabilité. Celle-ci doit s'exercer sans cesse, mais dans d'étroites limites; et cette modérée instabilité est la condition nécessaire de la véritable stabilité de l'être.»

12 Nous reprenons à partir d'ici le fil du tapuscrit original. 
reptiles, est également sous la dépendance de la chaleur extérieure. Quand l'hiver approche ils se refroidissent et s'engourdissent. Cependant, ils ont acquis le pouvoir de retenir leurs liquides organiques et peuvent, par conséquent, s'éloigner des sources sans se dessécher rapidement. Chez les formes encore plus évoluées, les oiseaux et les mammifères, non seulement les liquides de l'organisme sont facilement retenus, mais la température est maintenue très constante, à un niveau optimum. Il est évident que, au cours de l'évolution, le milieu interne s'est trouvé de plus en plus sous le contrôle de mécanismes qui le préservent et le maintiennent stable, et qu'en même temps les organismes se sont libérés de plus en plus des variations considérables du monde extérieur.

Bernard et d'autres ont tout particulièrement attiré l'attention sur cette indépendance vis-à-vis des influences extérieures, indépendance qui est assurée par la constance du liquide nourricier. Il faut reconnaître également qu'étant donné que le contrôle du milieu intérieur fut atteint graduellement, il libère l'organisme non seulement des variations extérieures, mais aussi des troubles dus aux propres activités de l'organisme lui-même, tels que les troubles sérieux qui peuvent résulter d'un gros effort musculaire, ainsi que je l'ai déjà fait remarquer.

Les conditions qui sont maintenues constantes dans le liquide nourricier peuvent être appelées des équilibres. Cependant, ce mot a acquis une signification restreinte appliquée à des états physico-chimiques relativement simples, où des forces connues se balancent en système clos. Dans une monographie qui épuise le sujet, mon collègue, L. J. Henderson ${ }^{13}$, a récemment traité le sang à ce point de vue; c'est-à-dire qu'il a étudié le sang en fonction des substances qui peuvent le modifier, et qu'il a défini les mécanismes propres au sang lui-même qui font en sorte de maintenir stables ses fonctions respiratoires. Mais comme l'a fait remarquer J. S. Haldane, ce traité ne prend pas en considération l'influence coordinatrice et régulatrice de ces systèmes circulatoires et respiratoires ${ }^{14}$.

13 Henderson 1928

14 Haldane i929. Haldane va jusqu'à reprocher à Henderson de se réclamer
Bien qu'en cas d'hyperacidité, par exemple, la propriété tampon du système physico-chimique sanguin maintienne le $\mathrm{pH}$ du sang à un niveau très stable, ce mécanisme est de moindre importance si on le compare à la coopération effective de toute une lignée d'organes - cerveau et nerfs, cœur, poumons, reins, rate - qui entrent promptement en action quand surviennent des conditions défavorables aux fonctions respiratoires du sang. Dans les conférences de cette série je propose d'examiner plutôt les processus physiologiques que les processus physiques, qui maintiennent la stabilité.

Les réactions physiologiques coordonnées qui assurent la stabilité de la plupart des états corporels, sont si complexes et si particulières à l'organisme vivant que j'ai suggéré ${ }^{15}$ de leur

de Bernard, alors qu'il en pervertirait l'esprit : «[Henderson] traite le sang comme une simple chose qui, à la suite de diverses réactions "tampons" [buffer] se produisant en lui-même, n'est pas aussi facilement perturbée dans ses conditions physico-chimiques que d'autres liquides le seraient $[\ldots]$. Ces réactions tampons sont d'une grande importance et d'un grand intérêt, mais elles étaient inconnues de Bernard et ne modifient en rien sa conception de l'activité coordonnée des organes par laquelle les conditions dans le sang sont maintenues constantes. Cette activité coordonnée est un élément essentiel de sa conception du sang dans le corps vivant, alors que L. J. Henderson n'en tient pas compte, transformant ainsi le sang dans le corps vivant en ce qui est pour un physiologiste un simple artéfact. » (Haldane 1929, 453) Pour la controverse entre Haldane et Henderson, voir l'introduction.

15 Cannon introduit le terme "homéostasie» pour la première fois en I926, à l'occasion de la publication des actes du jubilé organisé en l'honneur de Charles Richet et tenu à l'Académie de médecine le 22 mai 1926 (Cannon I926). S'inscrivant dans la filiation de Bernard et de Richet, Cannon forge le terme afin de distinguer les processus physico-chimiques simples tendant, dans les systèmes clos, à l'équilibre, des processus physiologiques à l'œuvre dans les «systèmes ouverts" que sont les organismes vivants. Le terme «homéostasie» doit ainsi être distingué de celui d'« équilibre». Il désigne la capacité des organismes vivants à conserver leurs propriétés malgré leurs interactions continuelles avec l'environnement. Le terme «équilibre» doit être réservé aux processus physico-chimiques ne relevant pas du vivant. Cannon définit plus longuement ses choix étymologiques en juillet I929, soit quelques mois avant son séjour parisien (Cannon I929c). Anticipant les critiques, il précise bien que le terme stasis ne désigne pas «quelque chose de fixe et d'immobile, une stagnation" (Cannon I929c, 400, notre traduction), mais fait référence à la statique, soit l'étude des forces en tension. De même, le préfixe homeo, qui désigne l'identité, ne doit pas être confondu avec 
consacrer une appellation spéciale : homéostasie. Ce mot n'implique pas l'idée de permanence, d'immobilité, de stagnation. Elle signifie une condition - une condition qui peut varier, mais qui est relativement constante.

Bernard, en parlant de l'importance de la libération de l'organisme vis-à-vis des limites que tend à imposer le milieu ambiant, citait l'eau, l'oxygène, l'homéothermie, et les matières nutritives y compris les sels, les graisses et le sucre, comme étant les conditions nécessaires auxquelles le milieu interne devait pourvoir. Il est impossible de faire une liste complète des catégories homéostatiques, et tout essai de classification serait certainement gâté par les interrelations qui existeraient entre les divers points mentionnés. Mais, cependant, il y a des matériaux tels que sucres, protéines, et graisses qui doivent être fournis pour la production d'énergie ainsi que pour la croissance et la réparation. Il y a aussi l'oxygène, il y a l'eau, et les sels inorganiques (à l'exception probable du calcium qui semble être spécialement contrôlé dans l'organisme), et finalement, il y a les sécrétions internes de la thyroïde et de l'hypophyse, par exemple, qui ont des effets généraux et continus. Bien plus, il y a des facteurs qui affectent l'activité cellulaire comme la pression osmotique ${ }^{16}$, la température, et la concentration en ions hydrogène. Chacune de ces conditions est réalisée avec une fixité relative dans les liquides environnants des cellules vivantes des organismes supérieures. Il y a des oscillations, cela est bien certain, mais normalement ces oscillations n'existent qu'entre d'étroites limites. Si ces limites sont dépassées, il peut en résulter des troubles graves; il se peut aussi que les substances en excès soient

bomo, qui désigne la similarité. Tous les efforts de Cannon visant à préciser que l'homéostasie ne désigne pas un état mais les conditions de la stabilité nécessaires à la réalisation des fonctions biologiques, quelles que soient les variations environnementales, seront ignorés par ses successeurs qui, voulant dépasser le fixisme supposé du concept d'homéostasie, forgeront des concepts alternatifs - hétérostasie (Selye I975a), allostasie (Sterling/Eyer I988), homéodynamisme (Rose 1997), etc. - censés permettre de mieux conceptualiser la stabilité dans le changement.

16 La pression osmotique est la force exercée par deux solutions inégalement concentrées en molécules dissoutes et situées de part et d'autre d'une membrane perméable. éliminées. Quelques exemples montreront clairement l'importance essentielle de la limitation stricte de ces oscillations.

Si on réduit le glucose sanguin à soixante-dix (70) milligrammes environ pour cent centimètres cubes, la réaction «hypoglycémique " est déclenchée, et si le pourcentage tombe à quarante-cinq (45) milligrammes au moins, des convulsions surviennent suivies parfois de coma et de mort. D'autre part, une augmentation du taux de glucose au-dessus de cent soixante-dix (I70) à cent quatre-vingts (I80) milligrammes entraîne une élimination de ce sucre par les reins. Il est certain qu'il ne pourrait pas y avoir de grandes variations dans le taux du sucre sanguin. De même un excès d'eau dans les liquides organiques amène une « intoxication aqueuse » caractérisée par des maux de tête, nausée, vertige, sthénie, et incoordination; d'autre part, le manque d'eau occasionne la réduction de la quantité de sang, l'augmentation de sa viscosité, et l'apparition de la fièvre. Le sodium (toujours accompagné de l'ion chlore) joue un rôle spécial dans le maintien des propriétés osmotiques du plasma sanguin. Si la concentration augmente de trois dixièmes à six dixièmes pour cent, de l'eau est soutirée à la lymphe et aux cellules, et il peut en résulter de la fièvre. $\mathrm{Si}$, au contraire, la concentration est réduite, des symptômes toxiques apparaissent; il y a irritabilité réflexe très marquée, suivie de faiblesse, fièvre, parésie ${ }^{17}$ et mort. Le niveau normal du calcium sanguin est d'environ dix milligrammes pour cent. S'il tombe à la moitié de cette concentration (cinq milligrammes), des convulsions peuvent survenir; et si la concentration double (vingt milligrammes) des changements profonds ont lieu dans le sang, qui peuvent entraîner la mort. L'importance d'une température constante n'exige pas de longues explications. Les variations journalières normales de la température corporelle chez l'homme ne sont que d'un degré centigrade - de trente-six et trois dixièmes à trente-sept degrés trois dixièmes. Elle peut tomber à vingt-quatre degrés et ne pas être fatale, mais ce niveau est beaucoup trop bas pour être compatible avec une activité quelconque, et si la température

17 La parésie désigne la diminution partielle des capacités motrices d'un membre du corps. 
se maintient à quarante-deux, quarante-trois degrés, il y a danger de coagulation de certaines protéines des cellules nerveuses de l'écorce cérébrale. De plus la concentration en ions hydrogène du sang doit être gardée dans d'étroites limites. À un pH d'environ six et quatre-vingt-quinze (6.95) centièmes, le sang devient si acide que le coma et la mort en résultent, tandis qu'au-dessus de sept et sept dixièmes (7.7), il devient si alcalin que la tétanie apparaît.

J'ai cité ces exemples pour illustrer l'importance fondamentale de l'homéostasie dans les liquides organiques. Ordinairement, les variations en dessous ou au-dessus de la moyenne n'atteignent pas des extrémités qui compromettent les activités de l'organisme ou mettent en danger son existence. Avant que ces états extrêmes ne soient atteints, des mécanismes entrent automatiquement en action, qui tendent à ramener à la position moyenne l'état préalablement troublé. Dans des conférences ultérieures, j'examinerai avec vous le caractère de ces mécanismes. Au cours de cette conférence, puisque nous en avons encore le temps, je me propose de voir avec vous l'action de certains facteurs qui assurent les conditions élémentaires et essentielles ; c'est-à-dire, l'existence du milieu intérieur luimême. Dans cet exposé, je parlerai uniquement du sang, car l'importance de la lymphe peut être considérée comme secondaire à ce point de vue.

Pour que le sang puisse continuer à servir comme milieu circulant, remplissant les fonctions variées de charrieur de matières nutritives aussi bien que de déchets, et représentant l'habitat optimum des éléments vivants, il doit y avoir des mécanismes qui l'empêchent de s'échapper. Les sérieuses conséquences que peuvent avoir des opérations insignifiantes faites chez les hémophilies en sont une preuve. Je n'ai pas besoin de vous décrire ici les processus de coagulation du sang ou de vasoconstriction locale, qui se développent quand un vaisseau est lésé. Ces réactions locales bien connues n'impliquent pas les grandes réactions physiologiques qui nous intéressent. Le point sur lequel je voudrais attirer votre attention est l'accroissement de la vitesse de coagulation quand survient une hémorragie. Ce phénomène a été reconnu depuis longtemps.
Vers la fin du dix-huitième siècle, Hewson ${ }^{18}$ nota qu'au cours d'une hémorragie mortelle chez l'animal, le sang qui s'écoule en dernier lieu se coagule plus vite que celui qui s'échappait au début. Un siècle plus tard, Cohnheim ${ }^{19}$ rap-

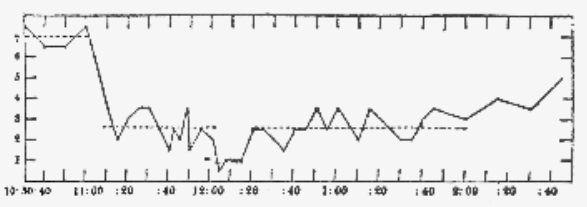

Figure 2. - Tracé montrant la diminution du temps de agie (13 0/0 du sang) à $10 \mathrm{~h} .59$ et après unc seconde hemorragie $(11$ h. 59). Les lignes en pointillé dans cette figure et dans la figure suivante indiquent les moyennes des résultats pour le temps qu'elles couvrent. porta que lorsqu'un chien est tué par soustraction du sang en portion successive, les dernières portions coagulent parfois presque instantanément. Ce témoignage fut confirmé plus tard par des observations faites au Laboratoire de Physiologie de Harvard $^{20}$. Comme le montre la projection ${ }^{21}$, le temps de coagulation mesuré au coagulomètre automatique, est en moyenne

18 Hewson I7I2, 6r. Cannon relate une des multiples expériences rapportées par Hewson. Il s'agit de l'expérience XXI, menée sur un mouton: «J'ai observé que le sang qui venait des vaisseaux immédiatement après le retrait du couteau mettait environ deux minutes à commencer à se coaguler; et que le sang prélevé plus tard, alors que l'animal s'affaiblissait, se coagulait en moins de temps.» (Hewson I712, 6I, notre traduction) William Hewson (I739-I774) est un anatomiste et physiologiste britannique, élu à la Royal Society en I77o. Il mène principalement sa carrière à la William Hunter's Anatomy School de Londres. Il étudie le système lymphatique, mais ses travaux sur les globules rouges font de lui un des pionniers de l'hématologie. Il met au jour notamment le rôle qu'une protéine, la «lymphe coagulante" (fibrogène), joue dans le processus de coagulation du sang.

19 Cohnheim I889[I877], 403. Julius Cohnheim (I839-I884) est un médecin et pathologiste originaire de Demmin, en Poméranie, ancienne province du royaume de Prusse. Il poursuit des études de médecine à Berlin et devient l'assistant du médecin et pathologiste allemand Rudolf Virchow (I82I-I902) en I865. Ses recherches sur l'inflammation lui permettent de montrer que le pus résulte de la décomposition de leucocytes. En I872, il s'installe à Breslau, où il prend part au développement de l'Institut de pathologie expérimentale. Ses expériences sur l'inoculation de la tuberculose mèneront Robert Koch (I843-I9Io) à découvrir le mycobacterium tuberculosis en I882. Cohnheim occupe enfin la chaire de pathologie de l'Université de Leipzig, de I878 à sa mort. Entre I877 et I880, il publie les Vorlesungen über allgemeine Pathologie. Ce sont ses leçons sur les pathologies, en particulier celles touchant le système cardiovasculaire, que cite Cannon.

21 Cannon projette la figure reproduite dans La Sagesse du corps (Cannon I946[1939], 32), initialement parue dans Gray and Lunt, I9I4. 


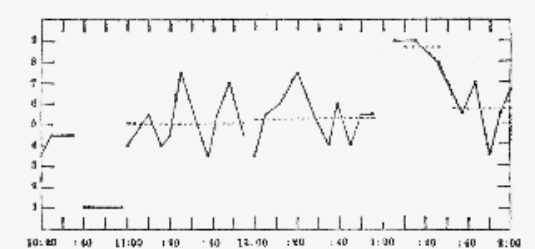

Figure 3. - Tracé montrant l'absence de coagulation rapide près hemorragie, quand la circulation est réduite a la partie du corps située en avant du diaphragme. La ligature de l'aorte et de la veine cave inferieure fut effectuée de $10 \mathrm{~h} .40$ à 10 h. 58 . A 10 h. 58 , on chaque saignée étant suivie de troubles respiratoires graves.

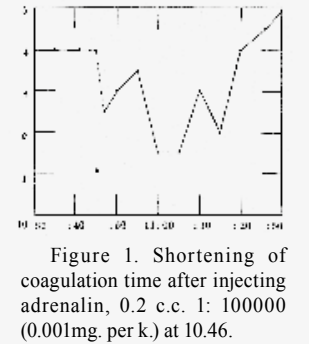

(0.001mg. per k.) at 10.46 .
Fig. $26^{22}$ d'environ sept minutes avant l'hémorragie. Quatorze pour cent Fig. $27^{24}$ du sang furent soustraits et le temps de coagulation fut réduit à deux minutes et demie environ. Une nouvelle soustraction de dix pour cent réduisit ce temps à une minute approximativement. Ensuite, le temps de coagulation fut pendant longtemps de deux minutes et demie jusqu'à ce que graduellement il revint à sa valeur primitive.

Des observations ultérieures montrèrent que l'augmentation de vitesse de coagulation n'avait pas lieu si le sang circulant était confiné dans la région susdiaphragmatique. Dans la projection suivante ${ }^{22}$, la ligne en trait plein représente le temps nécessaire pour lier l'aorte et la veine cave dans la partie inférieure du thorax. Vous noterez que des saignées ultérieures de cinq pour cent du volume sanguin estimé par le calcul ne furent pas suivies d'une coagulation plus rapide - en réalité, le temps de coagulation fut quelque peu prolongé. Ce résultat s'est trouvé d'accord avec les expériences de Nolf ${ }^{23}$, qui montra que l'hé-

Cannon projette la figure reproduite dans La Sagesse du corps (Cannon I946[I939], 33), initialement parue dans Gray/Lunt I9I4.

23 Nolf 1905-06. Pierre Nolf (I8973-I953) est un médecin et physiologiste belge, membre de l'Académie royale de médecine de Belgique. Il est diplômé de l'Université de Liège en I899. Il devient ensuite assistant à l'Institut de physiologie dirigé par Fredericq. Pendant la Grande Guerre, il est nommé médecin principal et directeur de l'Hôpital militaire de Cabour, localisé à Adinkerque. En 1925, le roi lui confie la fonction de président de la CroixRouge de Belgique. Dans l'article cité par Cannon, Nolf décrit la technique qu'il a mise au point, permettant d'éviter les problèmes liés à l'exclusion du foie chez un animal vivant : la difficulté de rétablir la circulation portale et morragie n'accélère pas le processus de coagulation après exclusion du foie.

Dans des expériences antérieures, nous avions trouvé que l'injection intraveineuse de minimes doses d'adrénaline raccourcit considérablement le temps de coagulation, ainsi que le montre la projection suivante ${ }^{24}$. Ce résultat fut confirmé par divers observateurs au Japon, en Chine, en Belgique, et aux États-Unis. En outre, quand par excitation des branches splanchniques du système sympathique, on força les glandes

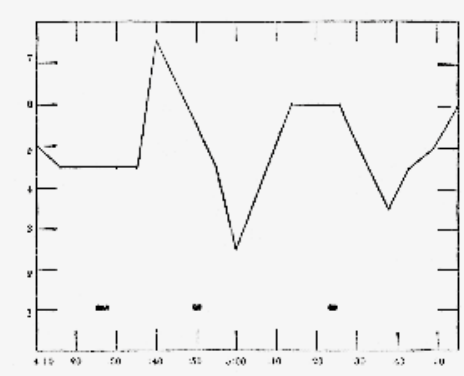

Figure 4. - Résultats de la stimulation des nerfs splanchniques gauches ( 4 h. 25 à 4 h. 28) après enlèvement de la capsule surrénale gauche de la stimulation des nerfs splanchniques droits ( 4 h. 49 à 4 h. 51 et $5 \mathrm{~h} .23$ à $5 \mathrm{~h}$. 25). La capsule surrénale droite était surrénales à décharger de l'adrénaline dans la circulation, le même phénomène se produisit. La projection suivante ${ }^{25}$ montre qu'à plusieurs reprises le sang se coagula plus rapidement après excitation des splanchniques. Il n'en est pas de même si la surrénale du côté excité a été préalablement enlevée. Et l'adrénaline le sang circulant n'entre pas dans l'abdomen. Par conséquent, il semble que l'adrénaline sécrétée, agissant sur quelque organe abdominal, probablement le foie, est l'agent normal produisant l'accélération de la coagulation.

Quand survient une hémorragie, et que la pression sanguine baisse, le système sympathique entre en action et comme Tournade et Chabrol ${ }^{26}$ l'ont montré, la médullaire surrénale est

de limiter les hémorragies des moignons hépatiques. Sa technique consiste à relier la veine porte au cœur droit et de relier la veine cave à la partie intrathoracique de la veine.

24 Cannon projette très certainement la figure parue dans Cannon/Gray, I9I4.

25 Cannon projette la figure reproduite dans La Sagesse du corps (Cannon, 1946, 34), initialement parue dans Cannon/Mendenhall, I9ı b b il ajoute à la main dans la marge gauche: «Le moment d'injection est indiqué par le point.»

26 en injection n'a aucun effet d'accélération sur la coagulation si çais. Après un doctorat de médecine obtenu à Lyon en I903, il est nommé professeur de physiologie à l'École de médecine de Marseille en Igoıg, puis à la Faculté de médecine d'Alger en 1920. En I94I, il est nommé professeur de physiologie générale du Muséum national d’histoire naturelle. Tournade a contribué à mettre au jour la fonction adrénalinogène. Maurice Chabrol était vraisemblablement un technicien travaillant aux côtés de Tournade 
excitée et secrète de l'adrénaline. En d'autres mots, les mécanismes sont tels qu'ils provoquent dans le cas qui nous occupe, une coagulation plus rapide, et cela d'une façon très efficace et physiologique ${ }^{27}$.

Cependant les expériences de Gray et Lunt ${ }^{28}$ montrent que même après ablation des glandes surrénales, l'hémorragie peut encore occasionner une coagulation plus rapide - ce résultat peut être dû à l'action directe de l'asphyxie sur les cellules hépatiques comme dans la glycogénolyse asphyxique. Le fait que l'hémorragie peut provoquer une accélération du processus de coagulation en l'absence de glandes surrénales ne doit pas cependant diminuer l'importance du système sympathico-surrénal ${ }^{29}$ dans les conditions normales, pas plus que la possibilité du cœur énervé de maintenir la circulation, ne prouve pas que les nerfs cardiaques sont d'une importance négligeable. Dans les conditions normales, la stimulation du système sympathico-surrénal par hémorragie causera automatiquement une prompte coagulation du sang extravasé. C'est-à-dire que le mécanisme conservateur naturel, la coagulation, sera rendu plus effectif, et, comme les expériences que j'ai citées l'ont indiqué, plus

à la Faculté d'Alger. Il y soutient une thèse intitulée Des mécanismes nerveux régulateurs de la pression artérielle en I92I (Chabrol $192 \mathrm{I})$. Cette référence à l'article de Tournade et de Chabrol éclaire le cheminement de Cannon depuis ses travaux sur l'adrénaline, exposés dans ses conférences sur les émotions, jusqu'à ceux sur l'homéostasie. Les auteurs notent que la pression artérielle doit être ajoutée aux facteurs d'adrénalino-sécrétion déjà connus comme l'asphyxie, l'excitation sensitive, l'émotion, l'hypoglycémie par injection d'insuline, etc. On note l'utilisation du champ sémantique de la régulation automatisée dans la conclusion de l'article: «Au total, l'hypotension stimule à la fois la sécrétion de l'adrénaline et la vasoconstriction, tandis que l'hypertension inhibe l'une et l'autre. Et la régulation de la pression nous apparaît assurée automatiquement du fait que les mécanismes nerveux et humoraux correcteurs sont opportunément mis en jeu par le trouble même à compenser» (Tournade/Chabrol 1925, 936, nous soulignons).

27 Cannon ajoute à la main, dans la marge gauche, un « «1/2» qui indique qu'il est au milieu de sa conférence.

28 Grav/Lunt, I9I4. Nous ne sommes pas parvenus à identifier Lawrence K. Lunt et Horace Gray, qui ont travaillé sous la direction de Cannon à l'École de médecine de Harvard dans les années i9i4-I9i6.

29 Tout au long de la conférence, Cannon remplace «sympathico-adrénal» par «sympathico-surrénal», afin de s’adapter aux usages français. l'hémorragie est considérable, plus le sang se coagule vite. Donc l'organisme sera protégé contre la perte d'un de ses éléments essentiels, le sang circulant, dont la conservation est une question de vie ou de mort pour les parties vivantes de l'organisme.

Cependant, il ne suffit pas d'arrêter l'écoulement du sang; il faut encore que la vie continue. Pour pouvoir accomplir ses fonctions, le sang doit couler à une vitesse suffisante pour four-

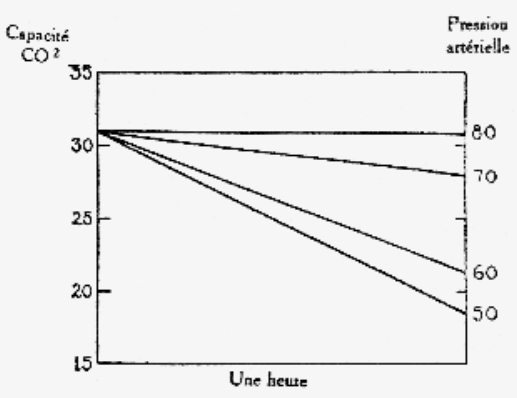

Figure 5. - Réduction progressive de la réserve alcalin (mesurée par la capacité du plasma en anhydride carbonique) quand la pression du sang tombe en dessous de 80 millimètre de mercure; par exemple, la diminution à 60 millimètres pen31 à 18,5 volumes pour cent. nir aux tissus vivants et en quantité convenable cet élément dont ils ont un si urgent besoin: l'oxygène. Des expériences faites par nous ${ }^{30}$ à Dijon ${ }^{31}$ pendant la guerre montrèrent qu'il y a un moment critique dans la chute de la tension sanguine, moment où apparaissent des signes d'insuffisance circulatoire. Comme test d'insuffisance, nous utilisions la réduction de la réserve alcaline mesurée par la quantité d'anhydride carbonique contenu dans le plasma sanguin après longue exposition à une concentration étalon de ce gaz. La projection suivante ${ }^{32}$ montre que la réduction de la pression sanguine à quatre-vingts (80) millimètres de mercure pendant une heure n'a pas d'effet sur la réserve alcaline; une chute à soixante-dix (70), soixante,

Cannon/Cattell 1922. James McKeen Cattel (199I-I983) est un médecin et physiologiste américain. Il entre en I9I7 à l'Université de Harvard, où il travaille sous la direction de Cannon. Il assiste aussi Cannon au Central Laboratories Medical Department de Dijon, durant la Grande Guerre, quand ils étudient les chocs. Il obtient son doctorat en 1920 et sa thèse de médecine en 1924. Il rejoint l'université de médecine de Cornell où il devient professeur de pharmacologie, jusqu'à sa retraite en 1959.

31 Dans le cadre du soutien que les médecins de Harvard apportent aux Alliés, durant la Grande Guerre, Cannon est affecté à Dijon du Ier avril au 25 décembre I9I8 en tant que directeur du Laboratory for Surgical Research Sur ce point, voir l'introduction.

32 Cannon projette la figure reproduite dans La Sagesse du corps (Cannon I946[I939], 36). Nous ne sommes pas parvenus à identifier la provenance de la figure originale. 


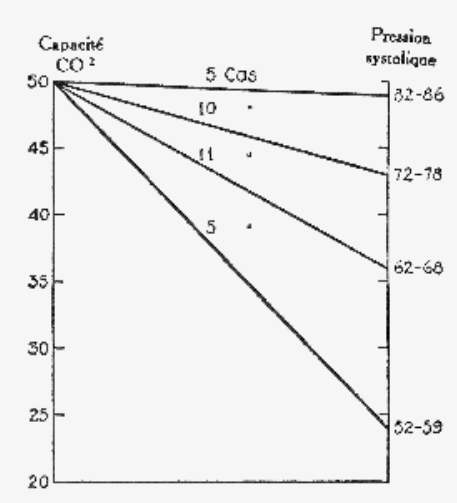

Figure 6. - Rapport entre teneur en anhydride carbonique du plasma sanguin et la pression systolique du sang dans 31 cas de choc et d'hémorragie chez des blessés de guerre. ou cinquante millimètres, maintenue pendant le même temps, a des effets de plus en plus marqués ${ }^{33}$. Nous avons attribué cette action à l'apport de plus en plus insuffisant d'oxygène aux organes actifs et par conséquent au défaut de combustion des déchets acides et non volatils. Cet acide non volatil se combinait alors avec le sodium du bicarbonate de sodium du plasma sanguin, et réduisait ainsi la réserve alcaline. Suivant

Fig. $30^{34} \quad$ cette interprétation, le niveau critique dans la chute de pression sanguine est approximativement de quatre-vingts millimètres de mercure. En dessous de ce niveau la pression n'est plus capable de maintenir une irrigation suffisante des organes actifs, permettant une oxydation normale. Des observations de blessés atteints de choc ou d'hémorragie rapportent des faits semblables. Dans la projection suivante ${ }^{34}$ sont consignés les résultats d'observations de quarante-trois de ces cas que nous avons étudiés à Béthune en mille neuf cent dix-sept ${ }^{35}$. Ils sont mis sous forme de tableau en prenant comme base la pression systolique. Comme le tableau le montre, la réserve alcaline tombe rarement en dessous de la normale (représentée par approximativement cinquante volumes pour cent d'anhydride carbonique), tant que la pression systolique n'est pas en dessous de quatre-vingts millimètres de mercure. En outre, de même que dans les expériences que je viens de décrire, plus la chute

33 Cannon ajoute à la main dans la marge gauche: «La pression de soixante millimètres, par exemple, baisse la capacité de Co à vingt-trois volumes pour cent.»

34 Cannon projette la figure reproduite dans La Sagesse du corps (Cannon I946[1939], 37). Nous ne sommes pas parvenus à identifier la provenance de la figure originale.

35 Dans le cadre du soutien que les médecins de Harvard apportent aux Alliés, durant la Grande Guerre, Cannon est affecté à Béthune, au Casualty Clearing Station $\mathrm{n}^{\circ} 33$, du 23 juin au 23 octobre I9I7. À ce sujet, voir l'introduction. de la pression sanguine en dessous du niveau critique est marquée, plus la réduction de la réserve alcaline est grande.

Que la pression en dessous du niveau critique est réellement insuffisante pour maintenir la circulation nécessaire dans les organes actifs est un fait

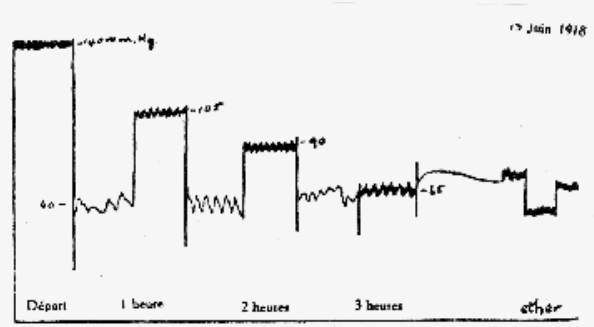

Figure 7. - Si la pression sanguine est maintenue à 60 millimètres ercure pendant des périodes successives d'une heure et qu’à la fi 作 a pression sanguine, progressivement, ne s'elève plus. que montrent des observations variées. Markwalder et Starling ${ }^{36}$ ont trouvé que les contractions cardiaques s'affaiblissent rapidement si la pression sanguine se maintient en dessous de quatre-vingts ou quatre-vingt-dix millimètres. Et un certain nombre d'observateurs en Angleterre et aux États-Unis ont montré qu'une tension sanguine basse et prolongée est si préjudiciable au système vasomoteur qu'il devient impossible d'obtenir un réflexe. Dans la projection suivante ${ }^{37}$ vous voyez les résultats d'une expérience que nous avons faite au Laboratoire de Recherche Chirurgicale à Dijon il y a douze ans. Vous remarquez que si la pression est abaissée (d'heure en heure) à soixante millimètres, à la fin de la troisième, le système vasomoteur est incapable de la moindre action réparatrice. Si nous considérons la sensibilité toute spéciale des cellules nerveuses à l'anémie même partielle, nous pouvons comprendre combien la diminution de l'intensité circulatoire peut avoir des effets nocifs extrêmement rapides.

36 Markwalder/Starling I9I3. Joseph Markwalder est un médecin et physiologiste. Nous n'avons pas trouvé d'information le concernant. Dans une biographie consacrée à Starling, John Henderson estime que « Markwalder est une figure obscure parmi les collaborateurs de Starling, car nous ne savons rien de sa vie» (Henderson 2005, 87, notre traduction).

37 Cannon projette la figure reproduite dans La Sagesse $d u$ corps (Cannon I946[I939], 38), initialement parue dans Cannon/Cattell I922. Il ajoute à la main, dans la marge gauche: «Nous avons abaissé la pression intrapéricardique cardiaque, et après chaque heure nous avons relâché la pression et permis qu'elle remonte le plus possible.» 


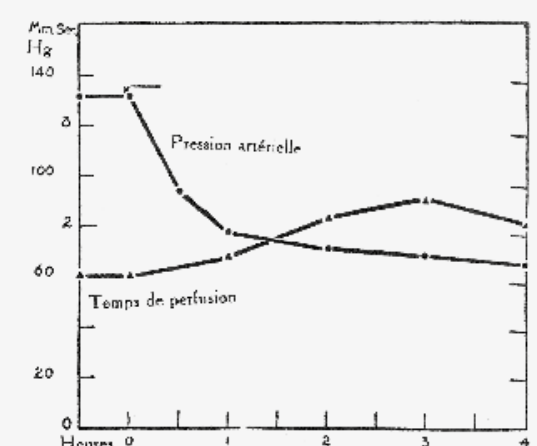

Figure 8. - Courbe donnant les résultats moyens de 6 expériences démontrant la relation entre le temps de perfusion et la pression sanguine dans l'état de choc déterminé par un broyage musculaire au moment indique par la flèche. Au fur et a mesure que la pression tombe, il y a un ralentissement de la vites
Les observations d'un de mes associés, Joseph Aub ${ }^{38}$, sont d'accord avec cette idée selon laquelle, les organes actifs souffrent d'un manque d'oxygène si la pression sanguine ne se maintient pas au-dessus d'un niveau critique. Il a étudié le métabolisme de base dans des cas de choc $^{39}$ expérimental, et il a trouvé une réduction moyenne de dix-huit et demi (I8.5) pour cent dans huit cas de choc modéré (par exemple, avec une tension sanguine

Fig. $32^{42}$ d'environ soixante-dix millimètres de mercure), et une réduction moyenne de trente-trois (33) pour cent dans huit cas de choc grave (avec pression sanguine de soixante millimètres de mercure). En maintenant la pression sanguine à un niveau de soixante millimètres environ au moyen d'une pression intrapéricardique, le métabolisme fut réduit de trente pour cent en moyenne; en d'autres mots, l'abaissement du métabolisme était dû à la diminution du flux sanguin et non aux processus responsables du choc.

Aub 1920. La méthode du « choc expérimental » consiste à écraser certain membres de chats anesthésiés à l'uréthane et dont la température corporelle est maintenue constante. Des canules placées dans la trachée, dans les deux carotides ainsi que dans la veine jugulaire permettent de mesurer la respiration ainsi que la pression sanguine de l'animal.

39 Tout au long de ses conférences sur l'homéostasie, Cannon écrit "schock" à la place de «choc", mêlant ainsi orthographes française et américaine (shock). Curieusement, cette erreur n’a pas été relevée par le traducteur Zénon Bacq, qui, à plusieurs reprises, la reconduite dans la Sagesse du corps. On peut par exemple lire: «Il a étudié le métabolisme de base (c'est-à-dire la somme des processus d'oxydation de l'organisme dans des conditions bien déterminées) dans des cas de schock expérimental avec pression san guine basse, et il a trouvé une réduction moyenne de $33 \%$ dans huit cas de schock grave (avec pression sanguine de 6o millimètres environ) » (Cannon I946[I939], 39).
J'ai quelque peu détaillé ces exemples dans lesquels nous avons vu le sang circulant empêché de remplir sa fonction primordiale - le transport d'une quantité d'oxygène suffisante pour subvenir aux besoins minimums de l'organisme - parce que je désirais insister sur les moyens employés par l'organisme pour éviter de telles circonstances et leurs conséquences déplorables.

Tout d'abord, quand survient une hémorragie, le système sympathique entre en action. Cela fut démontré par Douglas J. Pilcher et Torald Herman Sollman ${ }^{40}$ en dix-neuf cent quatorze (I9I4), quand ils observèrent qu'un organe en perfusion artificielle réagissait tout d'abord à l'hémorragie par une vasoconstriction. Ce fait fut confirmé par Bayliss et Bainbridge ${ }^{41}$. Le même phénomène apparaît quand la pression sanguine baisse pendant le développement du choc. Dans la projection suivante ${ }^{42}$

40 Pilcher/Sollmann I9I4. Nous n'avons trouvé aucune information sur J. Douglas Pilcher. Torald Herman Sollman (I874-I965) est un pharmacologue américain d'origine allemande. Il étudie la pharmacologie à l'Hôpital militaire du Val-de-Grâce à Paris, de I893 à I894, puis à la Western Reserve University Medical School à Cleveland. Il y est nommé professeur en 1904. Durant la Grande Guerre, il devient consultant de l'US Army pour ce qui touche aux brûlures dues au gaz moutarde. Il est l'auteur du premier manuel de pharmacologie expérimentale de langue anglaise (Sollmann/ Hanzlik 1928). Dans l'article cité par Cannon, les auteurs placent entre 30 et $50 \mathrm{~mm}$ de pression artérielle le shock level, c'est-à-dire le moment à partir duquel une perte de sang induit une baisse de pression fatale à l'organisme.

41 Committee 1917. Francis Arthur Bainbridge (I874-I92I) est un médecin et physiologiste britannique. Il obtient un doctorat de médecine en I904 et enseigne dès I905 la pathologie au Guy's Hospital de Londres. En I9II, il est nommé professeur de physiologie à l'Université de Durham puis, dès 1915, il revient à Londres où il occupe la chaire de physiologie du St. Bartholomew's Hospital. On lui doit la mise au jour du réflexe dit de Bainbridge, expliquant l'adaptation de la fréquence cardiaque en fonction des circonstances: les barorécepteurs dans l'oreillette droite du cœur détectent l'augmentation de la pression du sang envoyé au cœur et induit, via le nerf vague et le système nerveux central, l'activation des voies sympathiques entraînant l'augmentation de la fréquence cardiaque. John William Trevan (I887-I956) est un médecin et physiologiste britannique, diplômé de médecine en I9II. De I9I4 à 1920, il travaille comme démonstrateur en physiologie. En 1920, il devient pharmacologue pour les Wellcome Physiological Research Laboratories. En 1952, il est nommé directeur de la Wellcome Foundation.

42 Cannon projette la figure reproduite dans La Sagesse $d u$ corps (Cannon I946[I939], 40), initialement parue dans Cannon I922. 


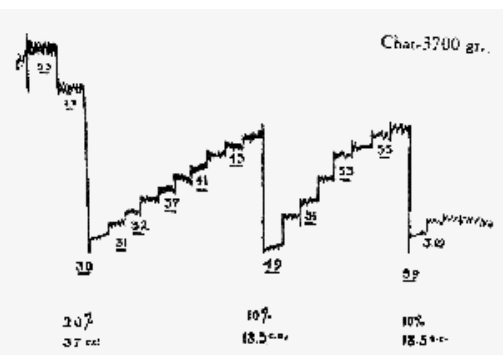

Figure 9. - Tracé montrant la rapidite du retour au niveau normal de la pression sanguine après hemorragie. Ce retour est dû cité au contenu. La limite du mécanisme regulateur fut atteinte $100 / 0$ (Cann : "Traumatic Shock $n$ D. Appleton et Cie). aune constriction des vaisseaux sanguins qui adaptent leur capa-

se voit un tracé exprimant les moyennes de six expé riences accomplies par un de mes étudiants, McKeen Cattell. Vous remarquerez qu'au fur et à mesure que la pression sanguine baissait, par suite du broyage d'un membre, le temps requis pour le passage d'une même quantité d'une solution saline à la même pression à travers les artérioles périphériques, s'allongeait graduellement. À la

Fig. $33^{43}$ fin de la troisième heure, il avait augmenté de plus de soixante pour cent. Jusqu'alors la réduction de la pression sanguine en dessous du niveau critique avait été accompagnée par une réaction du centre vasomoteur, provoquant la constriction des artérioles périphériques.

Une représentation graphique des effets de la vasoconstriction périphérique est l'objet de la projection suivante; il s'agit d'un tracé ${ }^{43}$ d'une expérience accomplie à Dijon pendant la guerre. À deux heures trente, on soustrayait vingt pour cent de la quantité de sang estimée par calcul. La pression sanguine tomba précipitamment. En quinze minutes, cependant, elle était ramenée jusqu'à son niveau primitif. Alors dix pour cent du volume sanguin furent encore soustraits. En six minutes, la pression remonta à nouveau. L'examen de concentration de l'hémoglobine dans ces cas montra que le rétablissement n'était pas dû à l'arrivée rapide de liquide provenant des tissus. Puisque l'hémorragie est accompagnée d'une constriction des vaisseaux sanguins périphériques, la montée de la pression doit être due, du moins pour une grande part, à la réduction de la capacité du système vasculaire s'adaptant à la réduction de volume de son contenu. Cette interprétation se trouve confirmée par les effets d'une troisième hémorragie; si on soustrayait encore dix

43 Cannon projette la figure reproduite dans La Sagesse $d u$ corps (Cannon, I946[I939], 4I), initialement parue dans Cannon I923. pour cent du sang à trois heures moins une minute, la limite du pouvoir que possède l'organisme d'adapter la capacité au volume était dépassée et la pression ne remontait pas ${ }^{44}$.

Il existe encore un autre effet de l'action du système sympathique qu'on peut trouver après l'hémorragie; c'est la contraction de la rate. Comme les recherches de Barcroft ${ }^{45}$ et de ses collaborateurs l'ont montré, la rate est normalement un réservoir de sang. Quand survient une hémorragie, la perte de sang au début est compensée progressivement par une contraction de la rate. Le sang mis en réserve est déchargé dans la circulation générale, comme le montre la projection suivante ${ }^{46}$, extraite d'un des ouvrages de Barcroft,

44 Cannon indique, dans la marge gauche, qu'il a atteint les «33/4» de son exposé 45 Barcroft I925. Joseph Barcroft (I872-I947) est un physiologiste britannique. Il entre au King's College de Cambridge en I894 et devient démonstrateur de physiologie sous la direction de Langley. Il devient professeur de physiologie à Cambridge en I925. Ses recherches en physiologie sont interrompues durant les deux guerres mondiales, en I9I5 puis en 1939. Il rejoint le centre de recherche militaire de Porton Down, où il étudie les effets des armes chimiques, en particulier les gaz de combat asphyxiants et toxiques. Il est surtout connu pour ses travaux sur la fonction respiratoire du sang, notamment la chimie de l'oxyhémoglobine. Ses recherches menées en grande altitude lui permettent de mettre au jour la courbe de dissociation de l'oxyhémoglobine, soit les capacités adaptatives de la fonction respiratoire du sang, qui permet de maintenir constant l'apport en oxygène des organes et l'élimination des déchets en fonction de la disponibilité de l'oxygène ambiant (Barcroft I9II). Lors de ses voyages, Barcroft a pu constater que la quantité de sang en circulation augmente en fonction de l'altitude. Dans l'article cité par Cannon, Barcroft rapporte comment il a pu tester l'hypothèse selon laquelle la rate serait un « réservoir » permettant de restituer le sang stocké en cas d'exercice ou d'hémorragie. Il ajoute des sutures métalliques sur des rates de lapins, afin de délimiter les contours de l'organe, qui seraient sans cela invisibles aux rayons X. Il constate que la taille diminue bien dans plusieurs circonstances: exercice, hémorragie, mort, etc.

46 Cannon projette la figure reproduite dans La Sagesse $d u$ corps (Cannon I946[I939], 42), initialement parue dans Barcroft I925. Cannon ajoute dans 
et c'est pour cela que le volume sanguin n'est pas réduit dans les premiers moments de l'hémorragie.

La conséquence de la vasoconstriction périphérique et de l'apport du sang splénique est que si une hémorragie même abondante survient, l'approvisionnement sanguin est assuré aux éléments extrêmement sensibles, les cellules nerveuses de l'écorce cérébrale, et aux muscles en continuelle activité, le cœur et le diaphragme, approvisionnement qui est en rapport direct avec la pression générale qui règne dans les artères. C'est seulement quand les mécanismes compensateurs sont sollicités au-delà de leurs limites, et que la pression artérielle tombe en dessous du niveau critique, que les lésions graves se produisent en dépit des agencements automatiques.

La vasoconstriction qui caractérise l'hémorragie a en outre comme effet de réduire la circulation du sang dans les vaisseaux périphériques. Cet effet, de même que l'accélération de la coagulation provoquée par la perte de sang, tendra à protéger l'organisme contre l'évasion de son plus précieux liquide.

Le mécanisme par lequel le système sympathique est mis en action par l'hémorragie semble être localisé en grande partie dans le sinus carotidien. En dix-neuf cent dix (I9I0), le professeur Hédon ${ }^{47}$ montra que l'hypotension des artères céphaliques provoque dans les vaisseaux somatiques une réaction inverse due à la vasoconstriction. Ces observations furent d'accord avec des observations antérieures de Porter et Pratt $^{48}$, qui avaient démontré cette réponse antagoniste

la marge droite: «Les figures D.E.F. montrent la diminution graduelle du volume de la rate à la suite de l'hémorragie.»

47 Hédon I9Io. Emmanuel Hédon (I863-I933) est un médecin et physiologiste français. Il obtient un doctorat de médecine en I888. Il est agrégé de phy siologie en I889, à la Faculté de médecine de Montpellier où il occupe la chaire de physiologie dès i894. Il est élu membre de l'Académie de médecine en I9I4. Il est principalement connu pour ses travaux sur le diabète expérimental, qui ont contribué à mettre au jour le rôle du pancréas dans l'apparition du diabète.

48 Porter/Pratt, I908. Pour Porter, voir l'introduction. Frederick Haven Pratt (1873-1958) est un médecin et physiologiste américain, diplômé de Harvard en 1906. Il est nommé professeur de physiologie au Wellesley College (I906-I9I2), à l'Université de Buffalo (I9I2-I9I8), puis à la Clark University (I9I9-I920). Il termine sa carrière à l'Université de Boston (I92I-I942) et des vaisseaux du pied aux modifications de la pression carotidienne. Tournade, Chabrol et Marchand ${ }^{49}$, ainsi que Anrep et Starling ${ }^{50}$, ont confirmé ces faits, et ont montré que toute chute de pression dans le cerveau est immédiatement suivie d'une vasoconstriction de tous les vaisseaux du corps, vasoconstriction qui a lieu même si les nerfs vagues ont été sectionnés. Heymans ${ }^{51}$ a approfondi ces observations, et a prouvé que la

prend la présidence de la Harvard Apparatus Company. Un dispositif photographique lui permet de montrer que la contraction des muscles fonctionne selon une loi de «tout-ou-rien» indépendante de l'intensité de la stimulation.

49 Tournade/Chabrol/Marchand I92I. Henri Marchand (I889-I974) est un médecin, physiologiste et anthropologue français. Dès I9I3, il devient préparateur à l'Institut de biologie de Tamaris-sur-mer, où il travaille sur la mytiliculture, notamment sur la fonction glycogénique de la moule. Il obtient un doctorat en I9I6. Son engagement volontaire comme médecin durant la Grand Guerre l'amène, dès I9I9, à mener des recherches en physiologie à la Faculté de médecine d'Alger, auprès d'André Tournade. Il abandonne sa carrière universitaire dans le courant des années I930. En marge de sa pratique privée de la médecine à Alger, il mène des recherches en anthropologie et en archéologie. Ses travaux lui valent de nombreux prix et distinctions. Il est notamment fait Chevalier de la Légion d'honneur en I95I.

50 Anrep/Starling I925. Gleb von Anrep (I89I-I955) est un médecin et physiologiste russe. Il est diplômé de la Faculté de science de Saint-Pétersbourg en I9I5. Élève de Pavlov, il effectue plusieurs séjours à Londres, dans le laboratoire de Starling. Durant la Révolution de I9I7, il rejoint l'armée blanche menée par Anton Ivanovitch Dénikine (I872-I947) et se bat contre les bolchéviques. La défaite de l'armée blanche le contraint à l'exil. Il devient assistant de Starling, au département de physiologie de l'University College de Londres. Après quelques années à l'Université de Cambridge, il est nommé en I930 professeur de physiologie à l'Université du Caire. En 1952, le soulèvement nationaliste entraîne la perte de son poste. Anrep est connu pour avoir contribué à populariser les travaux de Pavlov, en traduisant en anglais le livre tiré des leçons de ce dernier (Pavlov, 1927). Ses travaux avec Starling lui ont aussi permis de mettre au jour le mécanisme d'autorégulation cardiaque dit "effet Anrep » : une soudaine augmentation de la pression aortique entraîne une augmentation automatique de la contractilité ventriculaire, afin de maintenir la pression du sang constante. Ce passage est repris dans les versions anglaise et française de La Sagesse du corps. Cannon se trompe d'ailleurs dans les références, en intervertissant les chiffres de la pagination.

51 Heymans 1929. Corneille Heymans (I892-I968) est un physiologiste belge. Docteur en médecine en I92I, il effectue plusieurs séjours de recherche au Collège de France, sous la direction de Gley, à Londres auprès de 
baisse de pression agit comme un stimulus sur les terminaisons nerveuses du sinus carotidien. Les réflexes provoqués sont donc la vasoconstriction générale, la contraction de la rate, et la décharge d'adrénaline de la médullaire surrénale. Donc, tous les phénomènes que nous avons considérés comme compensateurs, correcteurs, et tendant à préserver le bienêtre de l'organisme, peuvent être expliqués comme des effets automatiques de la baisse de pression artérielle dus à la perte de sang.

Il ne faut pas oublier, cependant, que la compensation de l'hémorragie par la vasoconstriction n'est guère qu'un pis-aller. Le cerveau et le cœur reçoivent une quantité suffisante de sang, et cela est de première importance, mais les organes périphériques situés dans la région où les vaisseaux sont contractés ne sont pas normalement irrigués. La seule façon efficace de remédier à cette situation est d'augmenter le volume du sang jusqu'à remplir normalement la capacité du système vasculaire. Jusqu’à un certain point, l'augmentation de volume du sang est assurée par le passage dans le torrent circulatoire de liquide provenant des espaces intervasculaires grâce aux propriétés osmotiques des colloïdes du plasma sanguin. Ce processus devient effectif quand la pression capillaire tombe. Par conséquent, la partie très mobile du milieu intérieur, le sang, reçoit un appoint de la partie dont la circulation est plus lente, la lymphe, et petit à petit le volume sanguin revient à son niveau normal. Dans le cas de choc, un des premiers symptômes d'amélioration est la «dilution» du sang par l'addition de liquide extravasculaire.

La soustraction de liquide aux régions périphériques, ou le manque d'un apport suffisant de liquide à ces régions, provoque un certain nombre d'effets intéressants dont l'un est le phénomène de la soif. Après une bataille, la plainte unanime

Starling, ainsi que dans le laboratoire de Nicolas Maurice Arthus (I862 I945) à Lausanne. En I922, il rejoint l'Institut de pharmacologie dynamique de Gand, fondé par son père Jean-François Heymans (I859-1932). En I939, Heymans reçoit le Prix Nobel de médecine et de physiologie pour avoir mis au jour l'existence de chémorécepteurs au niveau du sinus carotidien. Ces derniers sont impliqués dans la régulation de la pression artérielle et la régulation par voie nerveuse de la teneur du sang en oxygène. des grands blessés est «à boire!». Malheureusement, ils sont souvent incapables de retenir ce qu'ils boivent. Quand l'eau est bue et absorbée, elle peut cependant être fixée en quantités étonnamment grandes. L'eau qui entre dans le corps par l'intermédiaire du tractus digestif, ainsi que l'ont montré Robertson et Bock $^{52}$, est de plus en plus efficace pour la restauration du volume sanguin réduit, que ne l'est une solution saline injectée dans les veines ou sous la peau. La soif, par conséquent, est un remarquable indicateur non seulement des besoins en eau, mais aussi du mode le plus utile d'alimentation en eau de l'organisme.

Nous nous sommes rendu compte de ce fait évident que la liberté et l'indépendance de notre existence, exposée à des conditions profondément perturbatrices provenant du monde extérieur ou de notre propre organisation, dépendent de l'existence et de la stabilité d'un milieu intérieur dans lequel baignent nos cellules vivantes. Nous avons vu que dans notre corps, des mécanismes sont prêts à intervenir qui font que, à tout danger de la part du milieu intérieur, des facteurs entrent en action pour tenter de l'écarter. Le sang coagule plus rapidement au fur et à mesure que la quantité de sang contenu dans l'organisme diminue.

Les vaisseaux sanguins périphériques se contractent; et grâce à ce mécanisme, non seulement ils réduisent la circulation du sang dans les régions où il s'échappe d'ordinaire, mais aussi assurent une irrigation continue des organes essentiels et

Robertson/Bock 19I8. Oswald Hope Robertson (I886-I966) est un médecin et physiologiste américain. Bien que diplômé de l'École polytechnique de San Francisco, il poursuit des études de médecine à l'École de médecine de Harvard et devient interniste. Durant la Grande Guerre, il rejoint les équipes de Cushing et part pour la France. En I923, il est nommé professeur de médecine et directeur du département de médecine de l'Union Medical College de Pékin. De retour aux États-Unis en I927, il occupe la fonction de professeur de médecine à l'Université de Chicago, jusqu'à sa retraite en I95I. Arlie Vernon Bock (I888-I984) est un médecin et physiologiste américain, diplômé de l'École médicale de Harvard en 1915. En 1935, il est nommé professeur d'hygiène à l'Université de Harvard. Les travaux en hématologie que les deux hommes ont menés en France visaient à étudier et à développer des techniques de préservation in vitro des cellules du sang. Ils sont considérés comme étant les premiers à avoir constitué une banque de sang à des fins de transfusion. 
sensibles, le cerveau et le cœur. Tout cela est dirigé automatiquement par le système sympathique mis en activité par la chute de pression sanguine. Ce mécanisme d'urgence est suivi par la récupération du volume sanguin grâce aux apports de liquide provenant des espaces intertissulaires, et grâce aussi à la satisfaction physiologique de la soif. Dans la prochaine conférence, nous verrons comment la soif elle-même ainsi que la faim, peuvent être considérées comme des moyens d'assurer les approvisionnements nécessaires pour le maintien des conditions homéostatiques du milieu intérieur.
Deuxième conférence

\section{Homéostasie par mise en réserve et par mise en liberté; La soif et la faim considérées comme moyens d'assurer l'approvisionnement; Inondation et séparation comme types de mise en réserve; Exemples de mise en réserve par inondation.}

L'eau est d'importance capitale pour l'organisme. Elle constitue la majorité du sang et de la lymphe, ainsi que des liquides intercellulaires. Elle sert au transport des matières alimentaires, du tractus digestif au torrent circulatoire. Elle sert de lubrifiant aux surfaces mobiles. Elle joue un rôle essentiel dans la régulation de la température corporelle. La nourriture est d'aussi grande importance. Elle doit pénétrer sans discontinuer dans l'organisme, car elle constitue la source d'énergie exigée par le travail musculaire, et le moyen de réparer et de renouveler les tissus fatigués ou blessés. Mais combien superflus sont ces détails!

Nous savons tous que l'eau et la nourriture sont des exigences fondamentales de l'organisme. Cependant, c'est continuellement que l'eau et les matières nutritives sont consommées. Les déchets non volatils passent constamment à travers le filtre rénal. Et les déchets volatils, (provenant de la combustion des matières nutritives dans les tissus), sont évacués à chaque respiration. C'est l'eau qui joue le rôle de véhicule permettant ainsi l'évacuation de ces déchets. Et il ne faut pas oublier que l'eau s'évapore constamment aux surfaces pulmonaires et cutanées.

Étant donné que l'eau et la nourriture sont continuellement consommées par l'organisme, le seul moyen d'assurer un approvisionnement constant est la mise en réserve avec libération au fur et à mesure des besoins. L'eau est accumulée dans les espaces intercellulaires et dans les cellules elles-mêmes. Les matières nutritives sont mises en réserve sous les formes bien connues de graisses, de glycogène, et probablement aussi des petites masses de matières protéiques qu'on trouve dans les cellules du foie. Quand le besoin se fait sentir, les réserves sont 
libérées pour permettre leur utilisation. Cependant, les réserves elles-mêmes doivent être renouvelées. C'est la fonction de la soif et de la faim que d'agir comme excitants automatiques pour assurer le maintien des réserves d'eau et de matières nutritives.

Tout d'abord, considérons la soif comme étant le moyen d'assurer un approvisionnement approprié en eau. La soif est une sensation rapportée à la surface interne de la bouche et de la gorge, et spécialement à la base de la langue, et à la partie postérieure du palais; elle consiste en une sensation extrêmement déplaisante de sécheresse et de quelque chose de pâteux. Elle survient habituellement dans le cas où se produit soit une évaporation rapide de la salive qui humecte la bouche, soit une diminution de la sécrétion salivaire. Par exemple, le fait de respirer un air chaud et sec, de même que de parler ou de chanter pendant un temps prolongé, ou de mâcher une nourriture sèche, amènera fatalement la sensation de soif et le désir de boire quelque chose. La peur et l'inquiétude sont également accompagnées de sécheresse de l'épithélium buccal et peuvent être la cause d'une soif insupportable.

Cependant, outre ces conditions locales, il existe certains états généraux de l'organisme qui peuvent produire cette sensation. Une sudation abondante, par exemple, ou une perte excessive de liquide au cours d'une maladie, comme la diarrhée, le choléra, ou le diabète avec abondante perte d'eau par les reins, provoqueront une soif intense. Et, ainsi que nous l'avons vu dans la conférence précédente, la soif après une hémorragie considérable devient un supplice.

D'accord avec le fait que la soif est due à une sécheresse locale de la bouche et accompagne également les états où l'organisme entier a besoin d'eau, deux ordres de théories se sont développés concernant la nature de cette sensation. Des expérimentateurs ont défendu le point de vue suivant lequel la soif est d'origine locale, périphérique et d'autres, au contraire, sont partisans de la théorie suivant laquelle la soif est une sensation générale.

Les preuves en faveur de l'idée suivant laquelle la soif est d'origine générale ou diffuse, sont tirées de certains modes de traitement général de la soif, qui en modifiant l'organisme considéré comme un tout, abolissent la sensation. Par exemple, on peut faire rapidement disparaître la soif en injectant de l'eau sous la peau ou dans l'intestin. L'introduction d'eau par ces voies n'humecte pas le pharynx et cependant la soif disparait.

Mais, le fait principal que nous devons garder à l'esprit est que l'homme assoiffé ne se plaint pas d'un malaise général, mais bien d'une gorge sèche et brûlante. Il existe encore d'autres faits qui font penser à une origine locale. Primo, des personnes souffrant d'une soif intense, par perte de grande quantité d'eau par les reins, sont délivrées de leur détresse si la sensibilité des terminaisons nerveuses de l'arrière-cavité buccale est abolie par la cocaïne. De plus, il suffit de prendre une petite gorgée d'eau et de la faire voyager dans la bouche pour arrêter la soif. De même le fait de tenir sur la langue une substance qui occasionne une sécrétion de salive - un morceau de citron, par exemple - diminuera la soif. Aucun de ces procédés ne fournit de l'eau à l'organisme, et malgré cela la détresse que peut causer la soif est adoucie. Cependant, le soulagement qu'ils procurent n'apporte aucune explication rationnelle de la corrélation entre la sécheresse locale et le manque d'eau de l'organisme entier. Nous comprendrions que la sécheresse de la bouche, condition locale, soit un moyen d'indiquer automatiquement les besoins en eau de l'organisme, et que cette sécheresse conduise automatiquement à la satisfaction de la soif. Il doit certainement y avoir un mécanisme prévu grâce auquel la bouche s'assèche quand le corps a besoin d'eau. Raisonnablement, on ne peut s'attendre à trouver ce mécanisme que chez les animaux qui perdent leur eau continuellement et rapidement et qui par suite doivent fréquemment renouveler leur provision d'eau pour se conserver une vie normale. Suivons ces directives et voyons où elles nous mènent.

Les animaux aquatiques, dont les téguments sont toujours humides, et qui font circuler l'eau à travers la bouche et les branchies, ne ressentent probablement jamais la soif. Au contraire, les animaux aériens ont une peau sèche en contact avec l'air, et comme le montre 

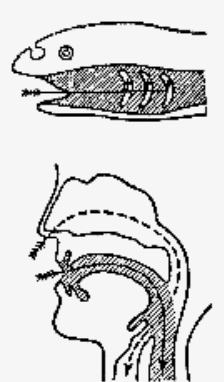

Figure 11. - Section médiane de la tête d'un poisson et de la tête d'un homme montrant les relations nouvelles du trajet vivant dans l'air. Notez que dans le pharynx, l'air va et vient à travers l'ancienne route de l'eau (qui est hachurée). (Cannon, article : « Hunger and Thirst », « Foundations of experimenta Psychology» avec l'autorisation de la «Clark University Press»)

la projection ${ }^{1}$, au lieu d'un courant d'eau dans la bouche, ils ont un courant d'air, qui leur passe par le nez et à travers les voies homologues aux branchies des poissons. Le nez et la trachée sont pourvus de glandes qu'ils humectent, mais au croisement, il y a peu de ces glandes. C'est pourquoi le fait de parler, de chanter, ou de fumer de façon prolongée - dans ces actes l'air circule par la bouche et non par le nez - aura une tendance à assécher cette région, et la sensa-

Fig. $35^{1}$ tion d'une muqueuse sèche et pâteuse est, comme nous l'avons $\mathrm{vu}$, la sensation habituelle de la soif.

Mais pourquoi cette région n'est-elle pas toujours sèche et pâteuse ? Encore une fois, si nous comparons les êtres aquatiques avec les êtres aériens, nous trouvons que seuls les animaux aériens possèdent des glandes buccales spéciales - et dans les formes les plus évoluées, des glandes salivaires.

La théorie que ces faits suggèrent, est que, quand l'eau fait défaut dans l'organisme, les glandes salivaires, de même que d'autres tissus, sont défavorablement impressionnés par l'apport d'eau devenu insuffisant; cette théorie veut que les glandes salivaires diffèrent d'autres tissus, comme les muscles, en ce qu'elles exigent une grande quantité d'eau pour l'accomplissement de leur fonction, qui est de déverser une sécrétion constituée presque uniquement d'eau ${ }^{2}$; que, de plus, elles occupent une position stratégique particulière, car si elles n'ont pas d'eau pour leur sécrétion, et pour cette raison sont incapables de secréter, la bouche et le pharynx s'assèchent et la sensation de soif apparaît. Telle est la théorie de la soif que je voudrais défendre, pour expliquer la localisation périphérique de

1 Cannon projette la figure reproduite dans La Sagesse du corps (Cannon I946[I939], 50), initialement parue dans Cannon I929a.

2 Cannon écrit à la main, dans la marge droite, « $98-99 \% »$. cette sensation dans l'arrière-bouche, quand l'organisme tout entier manque d'eau.

Essayons de prouver maintenant que la soif résulte d'une sécheresse due au défaut de salive ${ }^{3}$.

Premièrement ${ }^{4}$, le fait de mâcher pendant cinq minutes une gomme dépourvue de goût fait apparaître à chaque essai une quantité très constante de salive. Dans des observations que j'ai faites sur moi-même, cette quantité était en moyenne de quatorze centimètres cubes, comme le montre la projection ${ }^{5}$. Dans une expérience au cours de laquelle je ne bus aucun

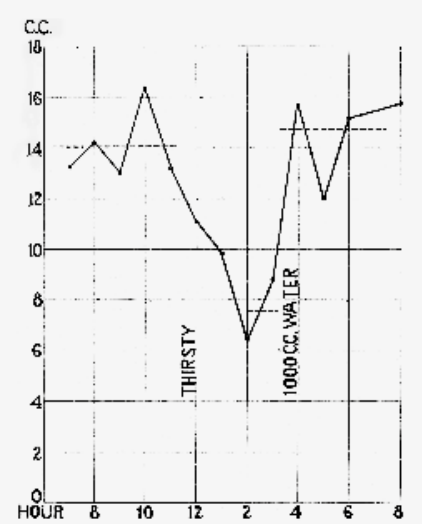

Fig. 1. - Chart showing saliva secreted each hour from 7 A.M. until 8 P.M. in consequence of chewing between $70^{\prime}$ 'clock the previous evening and 3 o'clock $^{\circ}$ P.M. For further description, see text. liquide à partir de sept heures du soir, la mastication habituelle ne montra aucune diminution de la sécrétion salivaire jusqu'à onze heures du matin suivant. À partir de ce moment, la quantité sécrétée tomba graduellement de quatorze (I4) à huit (8) centimètres cubes. Alors, à trois heures de l'après-midi je bus un litre d'eau. La récolte de salive, dont la sécrétion fut provoquée pendant les quatre heures suivantes de la même façon qu'auparavant, montra que l'émission de la salive était promptement revenue à son niveau primitif et s'y maintenait. Pendant la période durant laquelle la sécrétion salivaire était diminuée, la sensation de soif devint très marquée. Après l'ingestion d'eau et le rétablissement de la sécrétion salivaire, la sensation disparut. La coïncidence entre le besoin d'eau de l'organisme, la diminution de l'émission de salive, et la sensation de soif, appuie fortement l'idée suivant laquelle le fonctionnement défectueux des

3 L'ensemble des recherches que Cannon a menées sur la soif ont fait l'objet d'une publication (Cannon I9I8a), dérivée de la Croonian Lecture que le «major» Cannon délivre à Londres, à l'invitation de la Royal Society, durant la Grande Guerre, le 20 juin IgI8.

4 Dans la marge gauche, Cannon écrit à la main qu'il a atteint le «1/4» de son exposé.

5 Cannon projette la figure parue dans Cannon I9I8a. 
glandes salivaires signale le besoin organique en provoquant la sensation déplaisante.

Deuxièmement, en s'enveloppant dans des couvertures très chaudes et en appliquant des bouillottes, il est possible de provoquer une sudation abondante. La perte considérable en eau qui en résulte, diminue de moitié l'excrétion de salive provoquée par la mastication pendant un temps donné, si on compare cette excrétion à ce qu'elle était avant la perte d'eau. Accompagnant la diminution du flux de salive, il y a une notable sécheresse de la bouche et une sensation très déplaisante de soif. Cet état est rapidement amélioré par ingurgitation d'eau.

Troisièmement, dans une autre expérience, j’ai observé que l'injection sous-cutanée d'atropine ${ }^{6}$ provoquait la chute de la sécrétion salivaire excitée par la mastication habituelle, de treize centimètres cubes et demi à un. Cette chute survient sans une perte considérable d'eau de l'organisme. Cependant, toutes les sensations ordinaires de la soif étaient présentes. La sécheresse déplaisante de la muqueuse buccale, qui donne l'impression d'être pâteuse, la difficulté de parler et d'avaler, tous ces phénomènes particuliers à la soif se montrèrent avec la sensation générale caractéristique. Dans cette expérience, l'atropine manifesta son action périphérique habituelle qui est d'arrêter la sécrétion salivaire; et en produisant une sécheresse locale de la bouche, elle donna naissance aux sensations qui accompagnent ordinairement cette sécheresse.

Un autre fait suggestif est l'existence bien établie d'une sécrétion salivaire réflexe quand la bouche devient quelque peu sèche. Une expérience très simple est celle de mâcher une substance sans goût pendant cinq minutes et de comparer la quantité de salive ainsi obtenue à celle qui est émise si on respire tout simplement par la bouche pendant le même temps. Au début, l'entrée et la sortie d'air par la bouche assèchent petit à petit la muqueuse. Cependant, tandis que cette muqueuse devient de plus en plus sèche, de la salive est émise, et la quantité récoltée

6 Extrait naturel de plante, l'atropine a pour effet indirect d'augmenter l'ac tivité sympathique, en diminuant le tonus parasympathique. Son administration est connue pour accélérer le rythme cardiaque, diminuer les sécrétions, notamment la production de salive, et relâcher la musculature lisse. au fur et à mesure de sa production peut, comme je l'ai vu, être beaucoup plus grande que celle obtenue par mastication. La présence de ce réflexe indique qu'une des fonctions spéciales des glandes salivaires est d'humecter la bouche.

Cinquièmement, il y a longtemps que Bidder et Schmidt ${ }^{7}$, lièrent les canaux sécréteurs des glandes salivaires chez des chiens, et firent l'intéressante observation que ces animaux sont toujours prêts à boire. La ligature des canaux sécréteurs n'a évidemment aucun effet sur la quantité d'eau contenue dans l'organisme, mais elle provoque, de même que l'atropine, la sécheresse de la bouche. Dans ces circonstances, l'animal agit comme s'il était aussi assoiffé qu'un animal privé d'eau depuis longtemps. La sécheresse locale explique parfaitement ces observations.

Dernièrement, il est bien connu que la soif est une conséquence de la terreur, qui provoque un arrêt de la sécrétion salivaire. Le docteur H. J. Howard ${ }^{8}$ a rapporté d'une façon frappante les impressions qu'il ressentit quand il se crut sur le point d'être fusillé par des bandits chinois, "ainsi donc, j'allais être tué comme un chien ", écrit-il, «ma langue se mit à gonfler, et

7 Bidder/Schmidt I852. Georg Friedrich Karl Heinrich von Bidder (I8IOI894) est un physiologiste originaire du gouvernement de Livonie, dans l'actuelle Lettonie. En I834, il se voit décerner un doctorat par l'Université de Dorpat. Il y devient professeur de physiologie et de pathologie en I843. Ses recherches portent principalement sur la physiologie de la digestion et du système nerveux sympathique. Carl Ernst Heinrich Schmidt (I822I894) est un chimiste originaire de la même région. Il obtient un doctorat de l'Université de Giessen en I844. Il est nommé professeur de chimie à l'Université de Dorpat en 185 I, puis professeur de chimie en 1873 .

8 Howard 1927. Harvey James Howard (I880-1956) est un médecin ophtalmologue américain, diplômé de l'Université de Pennsylvanie en I9o8. En I9Io, il part pour la Chine, où il prend la tête du département universitaire d'ophtalmologie de l'École médicale de Canton. Durant la Grande Guerre, il développe des tests de vision destinés aux aviateurs de l'armée américaine. En I9I7, il revient en Chine, où il prend la direction du département d'ophtalmologie de l'Union Medical College de Peking. En 1926, Howard et son fils de onze ans sont kidnappés par des bandits, qui réclament une rançon de cent mille dollars. Ils sont libérés par l'armée chinoise après dix semaines de captivité. Les passages cités par Cannon sont tirés du livre à grand succès que Howard écrit afin de raconter sa mésaventure. En 1927, Howard retourne aux États-Unis, où il occupe la chaire d'ophtalmologie de l'École de médecine de l'Université de Washington. 
ma bouche devint sèche. Cette soif se fit de plus en plus violente jusqu'à ce que la langue me collât au palais; je pouvais à peine respirer. La soif m'étouffait... J'étais la proie d'une indicible terreur. ${ }^{9} \mathrm{Il}$ pria pour affronter sans crainte sa dernière heure qui approchait, et bientôt la peur le quitta quand il eut résolu de mourir comme un homme. "Aussitôt", rapporta-t-il, «ma soif commença à disparaître. En moins d'une minute elle était entièrement passée et quand nous eûmes atteint le grillage, j'étais parfaitement calme et serein. ${ }^{10}$ Vous remarquerez que cette sensation insupportable de soif intense n'était pas associée à un réel manque d'eau de l'organisme, mais résultait d'une condition locale de la muqueuse de la bouche.

Pour terminer, mentionnons les intéressantes expériences de Pack ${ }^{11}$. Il priva des lapins d'eau pendant sept jours. Alors, à certains d'entre eux il administra de la pilocarpine qui, même quand l'organisme est pauvre en eau, provoque la sécrétion des glandes salivaires. À tous ces animaux il permit alors de boire. Ceux qui salivaient grâce à l'action de la pilocarpine, ou bien refusèrent de boire, ou bien, dans deux cas, ne burent en une heure que quinze (15) à vingt-cinq (25) centimètres cubes d'eau. En une demi-heure, les autres burent de soixante-deux (62) à cent trente-sept (I37) centimètres cubes. Cette différence de comportement ne peut être expliquée que par l'humidité de la

9 «So I was going to be shot like a dog! My tongue began to swell, and my mouth to get dry. This thirst rapidly became worse until my tongue clove to the roof of my mouth, and I could scarcely get my breath. The thirst was choking me. I felt dizzy. I looked towards our destination and realized that it was only two minutes away. I was in a terrible state of fear; I was going to die like a coward» (Howard I927, 8I).

10 «Instantly my thirst began to disappear. In less than a minute it was entirely gone, and by the time we had reached the gate, I was perfectly calm and unafraid $»$ (Howard 1927,82 ).

11 Pack 1923. George T. Pack (I898-I969) est un médecin, physiologiste et oncologue américain, diplômé de l'Université de Yale en I922. Il n'a que vingt-cinq ans lorsqu'il est nommé professeur de pathologie à l'École de médecine de l'Université de l'Alabama. Son désir de pratiquer la médecine clinique l'amène à devenir interne au New York Memorial Cancer Center, où il se spécialise en oncologie. Considéré comme le plus grand oncologue de son époque, il est régulièrement consulté par des personnalités comme Franquin Delano Roosevelt (I882- I945). En I95I, il opère dans le plus grand secret Eva Perón (1919-1952). bouche, due à la sécrétion salivaire chez les animaux qui refusèrent de boire, et par la sécheresse de la bouche chez les autres. Chez tous ces animaux, le besoin général en eau de l'organisme était également grand, car on les avait tous privés d'eau pendant sept jours.

Si on rassemble toutes les observations que je viens de citer, la conclusion qui s'impose est que normalement la soif est une conséquence de la sécheresse qui survient quand les glandes salivaires ne maintiennent pas un certain degré d'humidité dans ces régions.

L'organisme perd de l'eau continuellement par les reins, par l'appareil respiratoire et la peau, et cependant ces pertes, mêmes prolongées, ne modifient pas de façon appréciable la qualité d'eau contenue dans le sang. Des observations faites par le professeur André Mayer ${ }^{12}$ ont montré qu'il peut n'y avoir à mettre en évidence aucun changement dans le sang d'un chien complètement privé d'eau depuis trois jours. Le sang, en tant que partie active du milieu interne, est maintenu constant aux dépens des réserves d'eau des tissus et des cellules des organes différenciés. Parmi les organes qui sont sollicités se trouvent les glandes salivaires. Cependant, comme nous l'avons vu, elles exigent de l'eau pour remplir leur fonction dans l'organisme. N'ayant pas d'eau disponible, elles ne peuvent accomplir leur sécrétion et par conséquent, la bouche devient désagréablement sèche. Si de l'eau est ingérée, elle est aussitôt mise à disposition des glandes salivaires, aussi bien que des autres organes, et elles récupèrent leur fonction particulière de maintenir un certain état d'humidité dans la cavité buccale. C'est ainsi que les glandes signalent les besoins en eau de l'organisme ${ }^{13}$.

Maintenant, voyons comment la faim est le mécanisme qui assure l'apport des matières nutritives. La faim a été décrite comme une sensation très désagréable, rapportée à l'épigastre ${ }^{14}$, sensation de douleur, d'angoisse, de poids, de quelque chose qui

12 Mayer 1900. Pour Mayer, voir l'introduction.

13 Dans la marge gauche, Cannon indique à la main qu'il a atteint la $« 1 / 2 »$ de son exposé.

14 L'épigastre désigne la partie moyenne et supérieure du ventre, au milieu de laquelle se trouve l'estomac. 


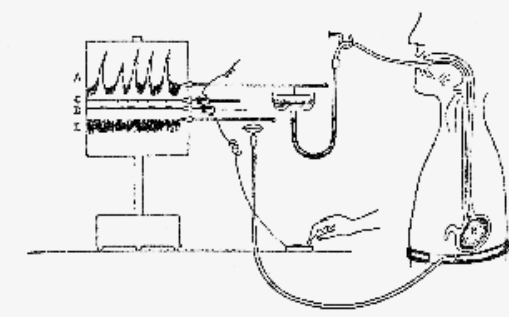

Figure 13 Diagramme montrant la technique utilisée pour phregistrer les contractions gastriques de la laim. A, tracé kymograngastrique B. C, enregistrement du temps en minutes. D, enregistrement des sensations subjectives, des crampes de la faim. E, trace du pneumographe placé à la taille. Ce tracé prouve que les contractions de la faim ne sont pas le résultat de contractions des musFoundertirs la "Clark University Press."
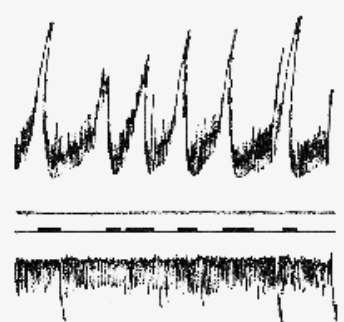

Figure 14 Copie du premier tracé de contractions de la faim, ssociées aux crampes de la faim, pris le 17 mai 1911. Réduction de moitié.

m'amenèrent, en mille neuf cent 0 nz que ces douleurs pourraient être dues à des contractions violentes et périodiques du muscle gastrique ${ }^{15}$. Peu après, l'occasion de vérifier la véracité de cette idée me fut offerte par un de mes étudiants, Washburn ${ }^{16}$, qui s'habitua à conserver un bal-

15 Cannon/Lieb igir. Nous n'avons pas trouvé d'informations concernant Clarence William Lieb (I885- I967), vraisemblablement médecin, auteur de livres grand public sur l'hygiène de vie, l'alimentation et la longévité, dont certains sont spécifiquement destinés aux fumeurs (Lieb 1953).

16 Cannon/Washburn I9I2. Arthur Lawrence Washburn (I888-I965) est un médecin américain. Il devient assistant de Cannon en I9I2. Diplômé de lon de caoutchouc dans l'estomac et un tube dans l'œsophage. Le tube raccordait le ballon à un appareil d'enregistrement ainsi que le montre la projection ${ }^{17}$.

Un pneumographe autour de

\begin{tabular}{rr}
\multicolumn{1}{c}{ Came } & Went \\
$\mathrm{I} 2-37^{-20}$ & $\mathrm{I2-38-30}$ \\
$40-45$ & $4 \mathrm{I}-\mathrm{TO}$ \\
$41-45$ & $42-25$ \\
$43^{-20}$ & $43^{-35}$ \\
$44^{-40}$ & $45-55$ \\
$4^{6-\mathrm{I}}$ & 45
\end{tabular}
l'abdomen renseignait les mouvements de l'abdomen et nous assurait de ce que les changements de pression enregistrés dans l'estomac n'étaient pas dus à la contraction de la musculature abdominale. Le sujet tenait dans la main droite une clef qu'il pressait quand il ressentait la sensation de faim. Les changements de volume du ballon gastrique, le temps en minutes, le signal annonçant la sensation et les modifications respiratoires recueillies grâce au pneumographe abdominal, furent tous enregistrés au kymographe sur une même verticale. Dans la projection ${ }^{18}$ suivante vous voyez le premier tracé obtenu en utilisant cette méthode.

Comme le montre la projection suivante ${ }^{19}$, il y a des contractions périodiques puissantes dans l'estomac vide, durant approximativement trente secondes, et revenant à des intervalles qui varient de trente (30) à quatre-vingt-dix (90) secondes, en

l'École de médecine de Harvard en I9I5, Washburn sert dans l'armée, puis renonce à la recherche pour s'installer à New York comme médecin généraliste. La préparation de l'expérience est décrite en ces termes: "L’un de nous (W.) a décidé de s'habituer à la présence d'un tube de caoutchouc dans l'œsophage. Presque tous les jours pendant plusieurs semaines, W. introduisit jusqu'à l'estomac un petit tube auquel était attaché à l'extrémité inférieure un ballon en caoutchouc souple d'environ $8 \mathrm{~cm}$ de diamètre. Le tube a donc été porté à chaque fois pendant deux ou trois heures. Après cette expérience préliminaire, l'introduction du tube et sa présence dans le tube digestif et l'œsophage n'étaient plus gênantes » (Cannon/Washburn I9I2, 449, notre traduction).

17 Cannon projette la figure reproduite dans La Sagesse $d u$ corps (Cannon I946[I939], 55), initialement parue dans Cannon I929a.

18 Cannon projette la figure reproduite dans La Sagesse du corps (Cannon I946[I939], 56), initialement parue dans Cannon/Washburn I9ı2.

19 Cannon projette le tableau paru dans Cannon/Washburn I9ז2. Ce tableau n'est pas reproduit dans La Sagesse du corps. Il ajoute à la main dans la marge gauche: « Chaque intervalle représente une minute - l'ensemble du temps est [un mot est illisible] dix min.» 


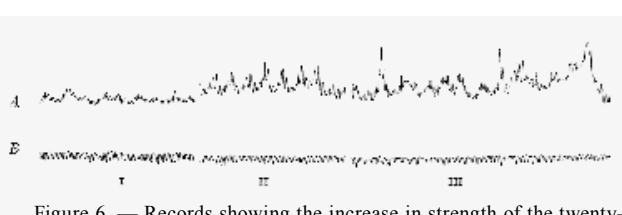

Figure 6. - Records showing the increase in strength of the twentyseconds contractions during the pause between the periodic rhythm. I, five later; III, ten minutes later. No. III shows at 4 the very beginning of the nex period. A. stomach contractions; B, respiration. Bromoform manometer.

moyenne de soixante $(60)$ secondes. Comme vous le remarquerez, le sujet ne manifesta pas de sensation pénible avant que la contraction n'eût presque atteint son maximum.

La sensation n'était donc

Fig. $40^{21}$ pas la cause de la contraction, c'était la contraction qui provoquait la sensation.

Nos observations furent bientôt confirmées par Carlson ${ }^{20}$ de l'Université de Chicago. Il étudia le phénomène de la faim chez un homme qui possédait une fistule gastrique, et aussi sur lui-même après s'être habitué au ballon stomacal comme Washburn l'avait fait. Dans une série de recherches intéressantes faites chez l'homme et sur diverses espèces d'animaux inférieurs, Carlson et ses élèves ont découvert de nombreux aspects nouveaux en ce qui concerne la relation entre les sensations de la faim et les contractions gastriques. Comme le montre le cliché ils ont trouvé ${ }^{21}$ que la faim débute ordinairement avec

20 Anton Julius Carlson (I875-1956) est un physiologiste américain d'origine suédoise. Il obtient un doctorat de physiologie à l'Université de Stanford en 1902. Il rejoint en 1904 le département de physiologie de l'Université de Chicago,où il est nommé professeur en I9I4. Il est nommé président de l'American Physiological Association de I923 à I925, ce qui l'amène à rencontrer Cannon à de nombreuses reprises. En I933, il fait partie, aux côtés de Dewey et d'autres personnalités se réclamant de l'unitarisme, des trente-quatre signataires du «manifeste humaniste» (bumanist manifesto): publié en revue (Bragg 1933) et relayé par la presse, celui-ci défend la création d'une nouvelle religion ayant vocation à remplacer les autres. Elle se veut sans dogme, mais unifiée autour de quinze principes. Selon le cinquième, la science moderne rend inacceptables les valeurs humaines fon dées sur des superstitions. Le quatorzième stipule que la société basée sur le profit, qui a fait la preuve de ses insuffisances, doit être remplacée par une société fondée sur la coopération économique, au bénéfice de tous. 1939[1932], 73), mais sans la figure. Il s'agit ties certaineme 6 , tirée de Carlson 1912. L'expérience est menée sur un jeune homme pré sentant une constriction de l'œsophage et une fistule abdominale d'origine accidentelle. D'une taille d'environ deux centimètres, la fistule permet d'introduire un ballon dans l'estomac du patient et d'étudier les contractions des contractions faibles de l'estomac vide, que ces contractions deviennent de plus en plus puissantes, et apparaissent à des intervalles de plus en plus petits, jusqu'à ce que soit atteint un maximum d'activité qui peut se terminer par un véritable spasme du muscle gastrique. Et les contractions isolées, et le spasme peuvent être accompagnés de la douleur, de l'angoisse, de la sensation de rongement caractéristiques qui depuis longtemps sont reconnus comme les signes de la faim. Après avoir atteint une acmé d'activité, l'estomac habituellement se relâche et reste inactif pendant un certain temps, après quoi il recommence à se contracter faiblement, et le cycle que nous venons de décrire se répète.

Le problème intéressant qui se pose à l'esprit, est de savoir ce qui oblige l'estomac vide à se contracter avec une vigueur plus grande que celle qu'on peut observer pendant les contractions périodiques et régulières du péristaltisme ordinaire au cours de la digestion d'un repas considérable. Il est bien connu que les substances énergétiques pour la contraction musculaire sont les hydrates de carbone - glycogène ou sucre. Il semblait possible qu'un déficit de cette matière énergétique soit signalé par des contractions violentes du muscle lisse de l'estomac. Bulatao et Carlson ${ }^{22}$ ont observé que si la glycémie sanguine est réduite de vingt-cinq (25) pour cent environ par administration d'insuline, les contractions de la faim s'intensifient - la sensation de faim a été notée chez l'homme après administration d'une dose considérable d'insuline. L'injection de sucre dans le torrent circulatoire supprime les contractions de la faim. Malgré l'importance de ce fait, on ne peut pas le considérer comme une

de l'estomac, suivant la même méthode que celle utilisée par Cannon et Washburn.

22 Bulatao/Carlson, I924. Emilio Erquiza Bulatao (I862-I96I) est un physiologiste philippin. Il occupe dès I9I5 le poste d'assistant instructeur au département de pharmacologie, biochimie et physiologie, à l'École de médecine de l'Université des Philippines à Manille. Une bourse de la Fondation Rockefeller lui permet, de 1923 à I924, de séjourner dans les laboratoires américains, période durant laquelle il cosigne des articles avec Carlson, comme ici sur la physiologie de la digestion, et avec Cannon, sur l'hyperglycémie dans les états pseudo-affectifs (Bulatao/Cannon I925b). En I930, il est nommé professeur de physiologie à l'Université des Philippines. 
preuve définitive de ce que les contractions de la faim sont dues au manque de sucre disponible, car la quantité de sucre utilisée par Bulatao et Carlson pour abolir les contractions est excessive, en comparaison avec le sucre sanguin normal, et en quantité absolue et en concentration. En outre, si on fait un estomac accessoire en isolant une partie de cet organe, les deux estomacs peuvent montrer une indépendance complète vis-à-vis des contractions de la faim. Une grande activité de l'un peut coïncider avec le repos de l'autre. Ceci suggère l'idée d'un automatisme local qui serait le facteur primaire plutôt qu'une modification du sang, car la compostion du sang qui va à l'estomac principal et à l'estomac accessoire est évidemment la même. Le problème de l'origine des contractions reste donc encore irrésolu.

On peut considérer le désir de prendre de la nourriture et de la boisson, ainsi que la faim et la soif, qui font en sorte de maintenir les réserves organiques en matières nutritives et en eau, comme des types d'autres mécanismes favorables au bienêtre de l'individu et de la race. Le comportement d'un être peut être divisé en actes qui tendent à libérer des excitations douloureuses ou pénibles, et en actes qui tendent à prolonger ou renouveler une sensation agréable. La faim et la soif appartiennent à la première catégorie. Chacun de ces états est associé à un facteur impulsif, chacun d'eux excite, pousse à l'action plus ou moins vigoureusement; tous les deux peuvent être si pénibles qu'ils forcent l'individu qui est leur proie à chercher un soulagement à cette sensation insupportable d'ennui ou de détresse. D'autre part, l'expérience peut conditionner le comportement en apprenant que le fait de prendre une nourriture ou une boisson s'accompagne d'un plaisir inattendu. C'est ainsi que s'établit le désir de répéter ces expériences: la personne en état d'appétit est tentée et non forcée à agir - elle cherche une satisfaction et non pas un soulagement. Il ne faut pas supposer que les deux mécanismes excitateurs - la douleur et le plaisirsont aussi séparés que nous les avons considérés pour faciliter notre analyse actuelle. Ils peuvent être intimement mêlés; le soulagement de la faim ou de la soif peut s'accompagner de la satisfaction de l'appétit. En ce qui concerne le maintien des réserves en matières nutritives et en eau, l'appétit ou la prise habituelle de ces aliments est le mécanisme qui intervient en premier lieu. Mais si les demandes de l'organisme ne sont pas satisfaites de cette manière douce et accidentelle, les douleurs de la faim et de la soif se font puissantes, persistantes, violentes, et exigent impérieusement la prise de nourriture et d'eau pour mettre un terme à leur tourment. Par ce mécanisme l'approvisionnement d'eau et de nourriture nécessaire pour les réserves est automatiquement assuré.

Nous pouvons maintenant reprendre l'idée de la mise en réserve comme moyen de réglage entre l'abondance fortuite, la privation ultérieure, et le besoin qui la suit. On peut distinguer deux types de mise en réserve: une submersion temporaire des interstices du tissu aréolaire par l'abondance des matières ingérées, qui peut être appelé mise en réserve par inondation; et l'inclusion des matériaux des cellules ou dans d'autres organisations relativement fixes et permanentes - mise en réserve par séparation. Considérons d'abord le premier de ces types.

L'analogie que suggère l'expression, mise en réserve par inondation est celle d'une fondrière, d'un marais, dans lequel l'eau stagne quand l'apport est considérable et dont l'eau retourne à la rivière quand le niveau de celle-ci baisse. Il semble que l'on puisse trouver un mécanisme semblable dans le tissu conjonctif lâche situé en dessous de la peau, autour des muscles, entre les faisceaux musculaires et aussi dans d'autres parties du corps. Le tissu conjonctif se distingue des autres tissus par sa richesse en colloïdes extracellulaires, par le fait qu'il est en relation intime avec les vaisseaux sanguins - en effet, il leur sert de soutien et qu'il offre une énorme surface. C'est grâce à de telles structures qu'agissent principalement les mécanismes régulateurs; ce sont elles qui rendent possible la rétention non seulement de l'eau, mais des substances qui y sont dissoutes, c'est-à-dire, les électrolytes et le sucre. Le tissu conjonctif est pauvre en cellules, il est plutôt « une toile d'araignée spongieuse aux filaments délicats ", dont chacun est composé de fibrilles minuscules agglomérées par une petite quantité de «ciment ${ }^{23}$. Dans les fines

23 Bremer I944[I927], 75. Nous ne sommes pas parvenus à identifier l'ensemble de la citation. Bien que reprise dans The Wisdom of the Body (Cannon 
mailles de ces fibres collagènes, il existe une substance mucoïde et de petites quantités d'albumine et de globuline. L'eau et les substances dissoutes paraissent être retenues dans ces mailles et leur être attachée par une certaine force. Probablement, les proportions d'eau mise en réserve, d'électrolytes et de glucose ne varient pas au-delà de limites très étroites. Cependant, nous les considérons séparément parce qu'il est certain qu'au moins l'eau et les électrolytes peuvent varier indépendamment en ce qui concerne leur rétention et leur élimination.

Lévidence de la mise en réserve d'eau est fort bien démontrée par des expériences qui soutirent l'eau de ses réservoirs, et permettent l'appréciation de la quantité qu'ils retiennent ${ }^{24}$. Après hémorragie, tous les tissus perdent de l'eau. En comparant chez le même animal un côté du corps avec l'autre, Arold Skelton ${ }^{25}$ a trouvé que la plus grande partie de l'eau qui quitte les tissus après hémorragie provient des muscles de la peau, qui sont les endroits où le tissu aréolaire est le plus abondant; cependant, si on rapporte à cent grammes de tissu, la quantité provenant des muscles est beaucoup moins grande que celle provenant de la peau. Les observations d'Engels ${ }^{26}$ sont d'accord avec celles de Skelton. Engels trouva que si quarante-huit (48) pour cent de la quantité totale d'eau de l'organisme se trouvent dans les muscles - on pouvait s'y attendre, étant donné la grande masse que représente le tissu musculaire - il y en a environ douze pour cent dans la peau,

I939[1932], 86), celle-ci n'est pas référencée. John Lewis Bremer (I874-1959) est un médecin et physiologiste américain, diplòmé de médecine en Igor à l'Université de Harvard, où il poursuit l'ensemble de sa carrière. Nommé professeur d'histologie et embryologie en $191_{5}$, il est nommé professeur d'anatomie en I93I, poste qu'il occupe jusqu'à sa retraite en I94I.

24 Dans la marge gauche, Cannon indique à la main qu'il a atteint les « $3 / 4 » d e$ son exposé.

25 Skelton I927. Nous n'avons trouvé aucune information sur ce physiologiste. Les données rapportées ici semblent issues d'une recherche effectuée dans le cadre d'un master en science achevé en 1925. Bien que cette étude soit citée dans les versions anglaise et française de La Sagesse du corps, au contraire de son habitude, Cannon ne s'est pas donné la peine de la référencer.

26 Nous n'avons pas trouvé d'informations concernant Wladimir Engels, vraisemblablement rattaché à l'Institut de pharmacologie de Heidelberg. soit presque une fois et demie la quantité d'eau trouvée dans le sang.

Les études de Mayer ${ }^{27}$ et de Wettendorf ${ }^{28}$ sur l'état du sang pendant la privation d'eau, ont démontré que l'eau emmagasinée dans les tissus était remise en liberté au fur et à mesure des besoins. Les chiens en expérience, continuaient naturellement à perdre de l'eau par les poumons et par les reins. Et cependant, un des animaux de Wettendorf fut assoiffé pendant trois jours sans modification du point de congélation du sang, un autre pendant quatre jours avec un abaissement d'un centième de degré seulement. Il est certain que cette stabilité est due au passage dans le sang de l'eau des réservoirs au fur et à mesure de son élimination.

Mais la question de savoir comment l'eau est apportée dans les réservoirs, comment elle y est retenue, et comment elle est mise en liberté quand la préservation de l'homéostasie osmotique du sang l'exige, n'a pas reçu jusqu'à présent d'explication suffisante. Il est indubitable qu'un changement dans l'équilibre, définit par Starling ${ }^{29}$, entre la pression de filtration à travers les parois capillaires et la pression osmotique des protéines, joue un rôle important. Et naturellement, les conditions modifiant la paroi capillaire augmentant sa perméabilité, élevant ou abaissant la pression du sang capillaire, ou altérant la concentration des protéines du plasma, modifieront la teneur en eau des tissus. La pression de diffusion ferait également partie de ce complexe de facteurs actifs. En outre, ainsi que l'ont

27 Mayer I9oo.

28 Wettendorff I9oI. Hugo Wettendorff (I8 57 -1935) est un physiologiste belge. Il se voit décerner le titre de docteur spécial en sciences physiologiques par l'Université libre de Bruxelles en I9I2. Il défend une thèse holiste de l'origine de la soif: «Les cellules du corps, quoique autonomes, ne sont points isolées et indépendantes; des connexions multiples et d'ordres divers, physiques, chimiques et nerveuses, les rendent tributaires l'une de l'autre; et c'est ainsi que nous concevons que la soif est le résultat de l'altération générale des tissus et qu'elle surgit, avec son allure de phénomène nerveux, indépendamment de toute localisation particulière du processus de déshydratation dans le système nerveux lui-même» (Wettendorff I9oI, 473).

29 Starling I909, IOI. 
montré Adolph ${ }^{30}$ d'une part, et Baird et Haldane ${ }^{31}$ d'autre part, l'introduction de chlorure de sodium peut influencer considérablement la rétention d'eau dans l'organisme. Probablement d'autres électrolytes jouent aussi un rôle. Il est également certain que la concentration en ions hydrogène et hydroxyle a son importance, étant donné qu'un changement du $\mathrm{pH}$ vers l'alcalinité provoque l'absorption d'eau d'imbibition par le tissu conjonctif, et une modification vers l'acidité occasionnant sa mise en liberté. Que la glande thyroïde est un agent de cette régulation, cela est indiqué par l'enrichissement en protéine du plasma et en albumine des tissus au cours du myxœdème ${ }^{32}$ et par la disparition de ces anomalies coïncidant avec la mise en liberté d'une grande quantité d'eau et de chlorure de sodium quand on administre de la thyroxine. Nous ne voyons pas clairement comment ces divers facteurs collaborent quand l'eau et le chlorure de sodium font défaut dans la circulation - après une hémorragie, par exemple. Ce domaine exige impérieusement de nouvelles recherches. Krogh ${ }^{33}$ a

30 Adolph 1923. Edward Frederick Adolph (I895-I986) est un médecin et phy siologiste américain. Il obtient un doctorat de l'Université de Harvard en I920, sous la direction de Henderson. De I920 à I92I, il mène des recherches à l'Université d'Oxford, dans le laboratoire de Haldane. Il devient professeur assistant de physiologie à l'Université de Rochester en 1925, puis professeur en 1948. De I953 à 1954, il préside l'American Physiological Association. Il s'intéresse tout particulièrement à la physiologie environnementale, notamment aux mécanismes de régulation permettant à l'organisme de s'adapter aux environnements hostiles. Il est notamment l'auteur de Physiology of Man in the Desert (Adolph 1947).

31 Baird and Haldane 1922. John Burdon Sanderson Haldane (I892-I964) est un généticien britannique. Fils de John Scott Haldane, il est nommé professeur de génétique à l'Université de Londres, puis en I937, professeur de biométrie. En 1957 il s'installe en Inde où il enseigne et poursuit ses recherches en génétique. Nous n'avons trouvé aucune information concernant M. M. Baird. Les études de Baird et de Haldane, ainsi que celles d'Adolph, ont principalement consisté à analyser le volume et la composition de l'urine de sujets soumis à diverses diètes ou ayant absorbé diverses substances comme du sel ou du bicarbonate.

32 Le myxœdème est un œdème induit par une infiltration cutanée. Caractéristique de l'hypothyroïdie, il entraine principalement une coloration de la peau et des gonflements des membres et de la face.

33 Schack August Steenberg Krogh (I874-I949) est un physiologiste danois. En ı897, il rejoint le laboratoire de physiologie médicale, sous la direction de écrit, à propos du mécanisme de la mobilisation de l'eau, «la nature d'un tel mécanisme est entièrement inconnue, et en ce qui la concerne je ne voudrais pas même avancer une conjecture ${ }^{34}$, et cependant, il est de toute première importance pour l'organisme.

Il paraît bien établi que les ions sodium et chlore peuvent varier indépendamment dans le plasma, et que des deux, l'élément basique est de loin le plus constant. C'est pourquoi dans une étude de la stabilité de la fluid matrix nous pourrions insister à bon droit sur l'homéostasie de cette base. Cependant, étant donné que la plupart des faits actuellement à notre disposition résultent d'expériences dans lesquelles le comportement du chlorure de sodium a été examiné, nous considérerons ce sel. La mise en évidence de l'existence dans l'organisme de réserves de chlorure de sodium est basée sur la rétention de ce sel dans différentes conditions. La quantité de chlorure de sodium ingérée restant constante, une perte de sel par la peau, consécutive à une sudation abondante, s'accompagne d'une forte réduction de l'élimination de chlorure par l'urine - ce fait continue à s'observer même après un régime riche en sel; en suivant cette méthode, on a pu mettre en évidence une rétention compensatrice de dix à quatorze grammes de chlorure de sodium. En outre, l'ingestion d'une solution concentrée de chlorure de sodium ne fait apparaître dans l'urine qu'une partie de la quantité du sel ingéré - la plus grande partie est retenue en réserve par l'organisme; et même si après on boit une quantité d'eau suffisante pour produire une diurèse, l'urine n'a qu'une faible concentration en sel; c'est-à-dire, que le sel n'est pas rapidement éliminé des endroits où il est mis en réserve.

Christian Bohr (I855-I9II). En I903, il soutient une thèse sur les mécanismes de respiration de la peau chez les grenouilles. Il prend sa retraite en I945 et poursuit des recherches sur l'insuline dans un laboratoire privé. Krogh a démontré que l'utilisation de l'oxygène provenant du sang augmente durant l'effort musculaire. Un processus de régulation permet d'ouvrir des capillaires, afin de maximiser la surface de diffusion de l'oxygène. Le Nobel de médecine lui est décerné en 1920 pour cette découverte.

34 Krogh 1922, 227. 
Quand on recherche les réserves en sodium de l'organisme, on trouve le plus haut pourcentage de chlorure dans la peau, et le plus bas dans les muscles - en effet, au cours d'un régime riche en sel, un tiers des chlorures de l'organisme peut se trouver dans la peau, et après injection intraveineuse d'une solution de chlorure de sodium, la peau peut retenir des quantités de sel variant dans diverses expériences entre vingt-huit (28) et soixante-dix-sept (77) pour cent de la qualité injectée. Ces faits sont corroborés par l'observation des animaux soumis à un régime pauvre en chlorure. Dans cette condition, de dix à vingt pour cent des chlorures de l'organisme sont éliminés, et de cette quantité soixante (60) à quatre-vingt-dix (90) pour cent viennent de la peau, bien que la peau ne représente que seize (I6) pour cent du poids du corps. Il est remarquable que le sang ne cède qu'une partie relativement petite de ses chlorures; notez encore une fois que le liquide circulant est maintenu constant grâce à l'apport des réserves tissulaires.

Il faut reconnaître que nous ignorons comment le chlorure de sodium est retenu dans la peau: est-ce par absorption sur les surfaces du tissu aréolaire ou à l'état de solution dans le liquide interstitiel des espaces aréolaires? Probablement est-il osmotiquement inactif. Mais il paraît bien établi qu'il existe une relation étroite entre la mise en réserve de l'eau et celle du chlorure de sodium.

Le premier dépôt temporaire du sucre en excès est, comme pour le chlorure de sodium, la peau. Quand le sucre ou d'autres hydrates de carbone rapidement assimilables constituent une grande partie du régime alimentaire, la glycémie s'élève aisément de cent à cent soixante-dix (I70) milligrammes pour cent. Pendant cette période de haute concentration en sucre sanguin, il y a également une forte concentration dans la peau. Ce fait apparaît comme un nouvel exemple de mise en réserve par inondation. Le sucre ne subit aucune modification chimique. Aucun dispositif particulier n'est requis ni pour le dépôt du sucre dans ce réservoir temporaire, ni pour sa mise en liberté. Au fur et à mesure que le sucre circulant est utilisé ou placé dans les dépôts permanents que sont les cellules hépatiques et musculaires, le niveau glycémique s'abaisse. Alors, le glucose plus concentré, qui avait envahi les espaces de la peau et peut-être d'autres régions où le tissu aréolaire est abondant, rentre dans la circulation générale et suit l'évolution habituelle du glucose sanguin, soit vers l'utilisation, soit vers la fixation.

Tels sont les moyens employés par l'organisme pour assurer la mise en réserve d'aliments, et tel est le mode de dépôt le plus simple. Dans la prochaine conférence, j'examinerai avec vous un mode beaucoup plus complexe de mise en réserve et de libération au fur et à mesure des besoins - la mise en réserve par séparation. 
Troisième Conférence

Homéostasie par la mise en réserve et libération consécutive (suite), et par inondation; Exemple de séparation avec comme type les hydrates de carbone; Problèmes relatifs aux réserves de protéines, graisse et calcium.

Aujourd'hui, nous continuerons l'examen des mécanismes de mise en réserve et de libération de ces réserves, considérées comme des moyens de maintenir la stabilité du milieu interne de l'organisme, et nous verrons tout spécialement la mise en réserve par séparation. Comme nous l'avons établi dans la dernière conférence, ce mode de mise en réserve habituel aux cellules, est stable et durable. Voyez, par exemple, les réserves d'hydrates de carbone à l'état de glycogène et de protéines à l'état de masses irrégulières dans les cellules hépatiques, les réserves de graisse que sont les tissus adipeux, les réserves de calcium que constituent les trabécules des os longs. La mise en réserve par séparation diffère du procédé par inondation en ce qu'elle est l'objet d'un contrôle beaucoup plus compliqué. La mise en réserve par inondation peut être considérée comme un processus relativement simple: les matériaux sortent du torrent circulatoire ou y restent suivant leur degré d'abondance. La mise en réserve par séparation comporte habituellement des changements dans l'état physique ou dans la structure moléculaire, et est sous la dépendance du système nerveux ou neuro-endocrinien. Nous sommes bien obligés de faire usage de cette classification plutôt hasardeuse à cause de l'état lacunaire de nos connaissances, qu'une étude ultérieure va nous révéler. Nous examinerons aujourd'hui la mise en réserve par séparation des hydrates de carbone, et incidemment nous dirons quelques mots des protéines, graisses et calcium.
Le meilleur exemple d'homéostasie par séparation, nous est offert par les mécanismes qui président à la mise en réserve et à la libération des hydrates de carbone. Il est bien connu que quand la nourriture est riche en hydrates de carbone, les réserves du foie en glycogène sont considérables. Cependant, après travail musculaire prolongé, ces réserves peuvent être presque complètement libérées. Il est intéressant de savoir que la marche de cette libération est contrôlée. Nous avons montré récemment que quand des chiens travaillent vigoureusement sur un tapis roulant pendant deux heures, le niveau moyen tombe graduellement de quatre-vingt-dix (90) environ à soixante-six (66) milligrammes pour cent centimètres cubes de sang ${ }^{1}$. En d'autres mots, pendant la période de forte utilisation du glucose, son niveau dans le sang est maintenu à des concentrations qui ne peuvent occasionner ni la perte par les reins, ni les troubles hypoglycémiques. Il doit donc exister un mécanisme qui libère le glucose du foie au fur et à mesure des besoins.

On peut avoir, par l'étude des effets de l'insuline, des renseignements sur l'action des facteurs qui évitent la chute du sucre sanguin à un niveau assez bas pour provoquer des troubles. Comme je l'ai mentionné dans ma première conférence, la réduction de la glycémie à soixante-dix milligrammes (70) environ au moyen d'insuline entraîne ce qu'on appelle la "réaction hypoglycémique ». Elle est caractérisée par la pâleur, pouls rapide, pupille dilatée, et sueur profuse. Manifestement, ce sont là les signes d'une excitation sympathique. La question qui vient naturellement à l'esprit est celle de savoir si ces phénomènes font partie d'un déploiement général d'activité de la partie sympathique du système autonome, et s'il en est ainsi, de savoir si la médullaire surrénale sécrète de l'adrénaline.

Pour répondre à cette question, nous avons utilisé le cœur énervé d'animaux anesthésiés au chloralose et aussi d'animaux non anesthésiés. Comme le montre la 


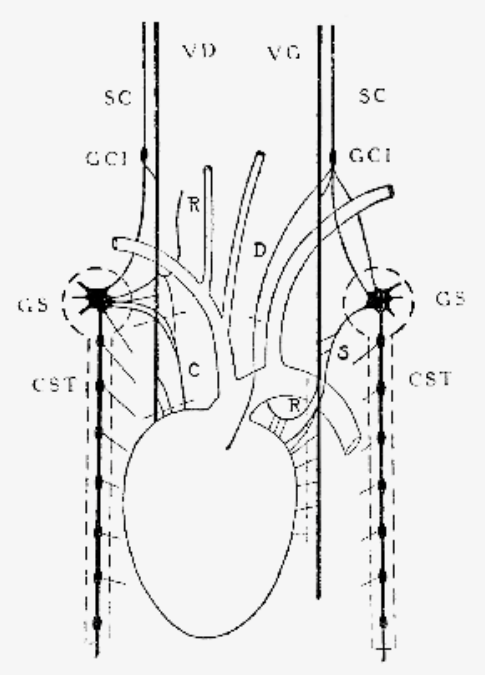

Figure 17. - Schéma de l'anatomie habituelle des nerfs cardiaques chez le chat. V D, vague droit; $\mathrm{V} \mathrm{G}$, vague gauche; $\mathrm{S} \mathrm{C}$, récurrent laryngé; D, nerf dépresseur; G S, ganglion stellaire; C, nerf commun cardiaque; S, fibre sympathique; C S T, chaîne symaits interrompus indiquent les partie sectionnées ou excisées. projection ${ }^{2}$ le coeur peut être isolé chirurgicalement du système nerveux central en enlevant (de chaque côté) la chaîne sympathique de la partie supérieure du thorax, en coupant le vague droit en dessous du récurrent laryngé, et en réséquant les branches cardiaques du vague gauche. Le récurrent laryngé droit est laissé en place pour l'innervation des muscles laryngés, et pour prévenir de cette façon les troubles de la respiration; le tronc du vague gauche continue à desservir le tractus digestif. Dans ces conditions le cœur n'est plus relié avec le reste de l'organisme que par le sang circulant. Sa vitesse n'est Fig. $41^{2} \quad$ pas affectée par des changements de pression artérielle, en effet, les seuls facteurs, à part les variations de température, qui modifient la fréquence du cœur sont des substances chimiques contenues dans le sang circulant. Le cœur énervé est exquisément sensible à une très légère augmentation de la sécrétion d'adrénaline, et il bat d'autant plus rapidement que la quantité d'adrénaline que le sang lui apporte est plus grande. La réponse du cœur est rapide, dans les dix secondes qui suivent la décharge d'adrénaline de la médullaire surrénale; et cette réponse ne survient pas après inactivation des surrénales.

En utilisant le cœur énervé comme indicateur de la sécrétion d'adrénaline, nous avons trouvé que lors de la chute de la glycémie, la concentration de soixante-dix milligrammes chez l'animal non anesthésié était un point critique. Ce point est un peu plus élevé chez l'animal anesthésié. Comme

2 Cannon projette la figure reproduite dans La Sagesse du corps (Cannon I946[1939], 87), initialement parue dans Cannon/Lewis/Britton I926, 33I

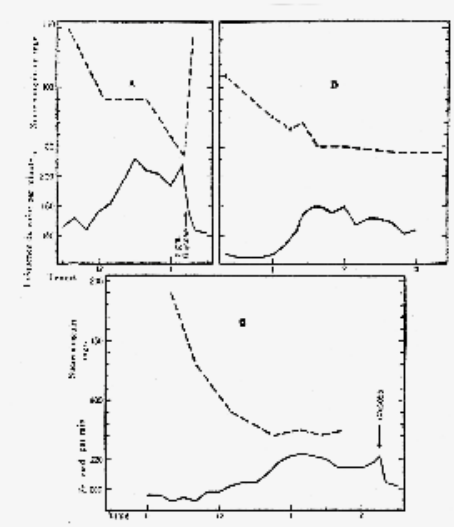

Figure 18. - Augmentation de la fréquence du ceur énervé (trat pleins) chez les animaxx aneshésís énerve (traits pleins) chez les animaux anesthésiés au (traits interrompus) passe à un point critique. Dans le cas
cas a A, l'insuline fut injectée dans la veine jugulaire à $11 \mathrm{~h}$. 33; dans le cas B, à 11 h. 8; dans le cas C, à 9 h. 30. Dans chaque cas on injecte 4 unités d'insuline par kgr.

le montre la projection ${ }^{3}$, il n'y a pas de changement dans la vitesse du cour lors de la chute de la glycémie, avant que le point critique ne soit atteint. Mais, quand on arrive à ce moment, la vitesse du cœur énervé commence à s'accélérer et si la glycémie continue à baisser, la vitesse ${ }^{4}$ augmente jusqu'à un certain maximum.

Si les surrénales ont été préalablement enlevées, ou si une des surrénales a été enlevée et l'autre énervée, une chute de la glycémie, comme le montre la projection suivante ${ }^{5}$, n'est pas accompagnée d'une augmentation de fréquence du cœur. Il est donc évident que l'accélération cardiaque montrée dans la projection précédente n'était pas due à l'action directe de l'insuline

3 Cannon projette la figure reproduite dans La Sagesse du corps (Cannon I946[I939], 88), initialement parue dans Cannon/McIver/Bliss I924. Nous n'avons pas trouvé d'informations concernant Sidney W. Bliss, vraisemblablement un candidat au doctorat de physiologie, qui assiste Cannon au début des années 1920.

4 Dans ce paragraphe ainsi que dans les suivants, Cannon biffe systématiquement le terme «chronotropisme» en faveur de «vitesse», «fréquence du cœur", "rythme cardiaque », etc.

5 Cannon projette la figure reproduite dans La Sagesse $d u$ corps (Cannon I946[I939], 90), initialement parue dans Cannon/McIver/Bliss 1924. 
sur le cour ni sur les capsules surrénales, mais bien à une exagération de la sécrétion d'adrénaline en réponse aux excitations des nerfs sympathiques.

Voici maintenant un autre fait intéressant: comme le montre la projection suivante ${ }^{6}$, si la vitesse du cœur énervé a été accélérée par l'hypoglycémie, une injection intraveineuse de glucose ramène presque instantanément le rythme cardiaque à sa valeur primitive. En d'autres mots, l'addition au sang d'une quantité suffisante de glucose abolit la réaction de cette partie de l'organisme dont l'effet est d'augmenter la concentration en glucose du sang.

Cette projection nous montre aussi que, sans aucun doute, le système sympathico-surrénal fait en sorte d'augmenter la glycémie. Vous observez que la vitesse de chute de la glycémie se ralentit; c'est-à-dire, que la courbe du sucre sanguin tend à s'aplanir dès que le système sympathico-surrénal est mis en action. Ces deux phénomènes, augmentation du rythme cardiaque et freinage de la chute de la glycémie, sont symptomatiques de l'activité du sympathique. En vérité, comme le montre le cliché suivant ${ }^{7}$, si on n'a pas administré trop d'insuline, l'accélération du cœur énervé, manifestation de l'activité du système sympathico-surrénal, s'accompagne d'une augmentation de la glycémie, et cette augmentation est à son tour suivie d'une restauration du rythme primitif.

La valeur du système sympathico-surrénal comme protection contre une chute néfaste de la glycémie est démontrée par le tableau que je vous présente ${ }^{8}$. Vous notez que des doses d'insuline variant de deux à trois unités par kilogramme, furent injectées à une série d'animaux normaux dont l'innervation des

6 Cannon projette à nouveau la figure reproduite dans La Sagesse $d u$ corps (Cannon 1946[1939], 88), figure I8, initialement parue dans Cannon/McIver/ Bliss I924.

7 Cannon projette vraisemblablement la figure parue dans Cannon/McIver/ Bliss I924. Celle-ci n'est pas reproduite dans The Wisdom of the Body.

8 Cannon projette vraisemblablement la figure reproduite dans La Sagesse $d u$ corps (Cannon I946[1939], 9I). Nous ne sommes pas parvenus à en identifier l'origine.

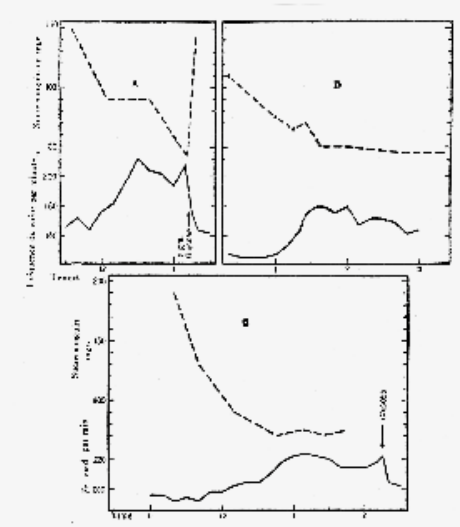

Figure 18. - Augmentation de la fréquence du ceer énervé (traits pleins) chez les animaux anesthésiés enervé (traits pleins) chez les animaux anesthésiés au chloralose quand la baisse de la teneur en sucre du sang
(traits interrompus) passe à un point critique. Dans le cas $\mathrm{A}$, l'insuline fut injectée dans la veine jugulaire à $11 \mathrm{~h}$. 33; dans le cas B, à 11 h. 8; dans le cas C, à 9 h. 30. Dan chaque cas on injecte 4 unités d'insuline par $\mathrm{kgr}$

deux surrénales était intacte. Dans un seul cas des convulsions apparurent, et seulement trois heures et demie après l'injection. Comparez cette série avec cette autre série d'animaux dont une surrénale fut enlevée et l'autre énervée ; c'est à dire, dont les surrénales étaient inactivées. Notez aussi que, sauf dans un cas, on n'utilisa que deux unités d'insuline par kilogramme; et, cependant, sauf dans trois cas, des convulsions survinrent chez tous les animaux et habituellement une heure à une heure et demie environ après l'injection.

La sécrétion d'adrénaline est la plus active pendant les accès convulsifs. Si le foie est bien approvisionné en glycogène, une telle activité du système sympathico-surrénal est capable de ramener le sucre sanguin à sa concentration normale et d'abolir, par conséquent, les conditions qui provoquent
Fig. $44^{6}$ Fig. $45^{7}$ Fig. $46^{8}$ 
les accès convulsifs. La projection suivante ${ }^{9}$, extraite d'une publication de Macleod et de ses collaborateurs ${ }^{10}$, fait ressortir cette action. Nos observations montrant que l'hypoglycémie excite le système sympathico-surrénal ont été confirmées par Abe ${ }^{11}$, qui utilisa l'iris énervé comme indicateur d'un excès de sécrétion d'adrénaline, et par Houssay, Lewis et Molinelli ${ }^{12}$, qui

9 Cannon projette la figure reproduite dans La Sagesse du corps (Cannon I946[1939], 92), initialement parue dans (McCormick/Macleod/Noble/ O'Brien I923) et (Macleod I928).

10 Edward Clark Noble (1900-1978) est un proche camarade de Charley Best (I9I5-I978), avec qui il étudie la physiologie et la chimie biologique à l'Université de Toronto. Diplômés en I92I, ils rejoignent tous deux le laboratoire de Macleod. Best et Noble auraient tiré au sort lequel des deux s'occuperait d'aider Banting lorsque ce dernier arriverait dans le laboratoire (Wright 2002). En I922, Banting et Best réalisent des pancréatectomies sur des chiens et injectent des extraits pancréatiques chez des chiens diabétiques. Ils mettent au jour le rôle de l'insuline, ce qui vaudra à Banting et Macleod de se voir décerner le Prix Nobel en I923. Après un séjour de recherche au Royaume-Uni, Noble s'installe comme généraliste à Toronto. Malgré leur implication dans les recherches de Banting, la contribution de Noble dans la découverte de l'insuline a été, encore plus que celle de Best, méconnue. Norman Arnold McCormick (I90I-I967) rejoint l'équipe de Macleod et travaille auprès de Noble sur l'extraction d'insuline de poissons, afin de réaliser des tests cliniques à l'Hôpital général de Toronto McCormick est diplômé de l'École médicale en 1926 et poursuit une carrière de chirurgien et de radiothérapeute. En I935, il est nommé directeur d'une clinique spécialisée dans le traitement des cancers à Windsor, dans l'Ontario. Nous n'avons pas trouvé d'informations concernant Yukujiro Abe médecin et physiologiste japonais, ni K. O’Brien, vraisemblablement affilié au département de physiologie de l'Université de Toronto au moment de la publication de l'article.

11 Abe I924. Nous n'avons pas trouvé d'informations concernant ce médecin physiologiste japonais, diplômé de médecine de l'Université de Keiō en 1926. L'étude citée a été vraisemblablement menée lors d'un séjour de recherche de Abe en Allemagne, à l'Institut de pharmacologie de Fribourg en Brisgau. un médecin et physiologiste argentin. Il est diplômé en IgIo de l'Université de Buenos Aires, où il est nommé en IgIg à la chaire de physiologie. Houssay montre que l'hypophyse antérieure joue un rôle central dans la régulation glycémique, en produisant une hormone antagoniste de l'insuline. Les prises de position démocratiques de Houssay, contre la dictature militaire instaurée à la suite de la révolution de I943, lui coûtent son poste. Il refuse cependant les postes qu'on lui propose à l'étranger et poursuit ses recherches dans un laboratoire privé, financé notamment par un comité américain dont Cannon était membre. Il récupère son poste en I955, lorsque

se servirent dans ce but de l'anastomose ${ }^{13}$ surrénalo-jugulaire entre deux chiens ${ }^{14}$.

En outre, le fait que les animaux privés des fonctions de la médullaire surrénale sont particulièrement sensibles à l'insuline est bien établi. Nous l'avons prouvé chez le chat, ainsi que je viens de vous le montrer. Lewis ${ }^{15}$ a également prouvé la véracité de ce fait chez le rat, Sunberg ${ }^{16}$ chez le lapin, et

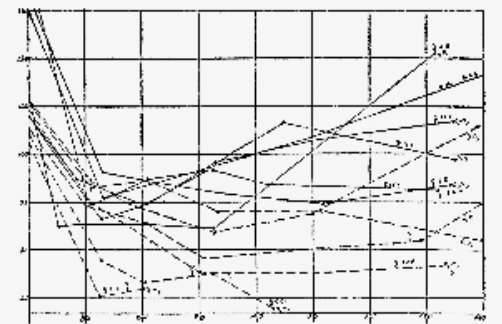

Figure 21. - Graphiques montrant l'effet de dose égales d'insuline chez des lapins bien nourris (traits pleins) ou à jeun (trai interrompus). Remarquez que le taux du sucre sanguin chez les lapins bien nourris tombe à peu pres au niveau critique de la réaction hypoglycemique et se releve ensuite dans la plupart des cas (Mac Leod: «The Fuel of life »; avec autorisation de la «Princeto University Prcss

son pays est libéré de la dictature. En I947, il se voit décerner (avec le couple Cori) le Prix Nobel de médecine, pour ses travaux sur les mécanismes de rétroaction négative impliqués notamment dans le développement du diabète. Cannon et Houssay ont eu plusieurs occasions de se lier d'amitié, notamment durant l'année I935, lorsque le second est invité en tant que Dunham Lecturer à l'Université de Harvard. Ils se sont aussi mutuellement nominés pour le Prix Nobel de médecine. Enfin, leurs intérêts communs pour l'endocrinologie a favorisé des échanges d'étudiants, comme pour Juan Treharne Lewis (I898-1975). Ce médecin et physiologiste argentin, diplômé de l'École de médecine de l'Université nationale de Buenos Aires en I920, poursuit des recherches postdoctorales dans le laboratoire de Cannon de I925 à I926, grâce à une bourse de la Rockefeller Foundation. Il est nommé professeur de physiologie à l'Université de Buenos Aires en I930, puis professeur en 1946. Nous n’avons pas réussi à identifier Ernesto A. Molinelli. Il est vraisemblablement un doctorant de Houssay. Il soutient une thèse sur l'adrénaline (Molinelli I926) à l'École de médecine de l'Université nationale de Buenos Aires.

13 L'anastomose désigne l'établissement, par voie chirurgicale, d'une communication entre deux organes. L'expérience atteste du rôle des surrénales dans la régulation de la glycémie. Elle consiste à transfuser l'ensemble du sang issu de la surrénale gauche de chiens transfuseurs, dans les veines de chiens transfusés. Puis trois unités cliniques d'insuline sont injectées par voie veineuse aux chiens transfuseurs. L'hyperglycémie constatée chez le chien transfusé démontre que, lors d'une hypoglycémie insulinique, les surrénales sécrètent de l'adrénaline et que celle-ci a un effet hyperglycémiant.

14 Dans la marge gauche, Cannon indique à la main qu'il a atteint le «1/4» de son exposé.

15 Lewis 1923

16 Sundberg I923. Nous n'avons pas trouvé d'informations concernant Carl Gustaf Sundberg. Il s'agit vraisemblablement d'un physiologiste suédois, 
Hallion et Gayet ${ }^{17}$ ont observé le même phénomène chez le chien. Les résultats obtenus par Burns ${ }^{18}$ sont d'accord avec ceux-ci. Il utilisa l'ergotamine ${ }^{19}$ comme paralysant du système sympathico-adrénal ${ }^{20}$, et trouva qu'une dose d'insuline légèrement efficace chez l'animal normal, produisait une forte hypoglycémie avec convulsions et collapsus chez l'animal injecté à l'ergotamine. Il est donc certain que le système sympathico-surrénal protège l'organisme contre les effets profondément néfastes d'une chute du niveau glycémique du milieu intérieur.

Une question assez intéressante est celle de l'importance relative des facteurs nerveux et humoraux dans la libération du glucose hépatique. Le mécanisme le plus puissant est-il la décharge des influx nerveux dans les cellules hépatiques ou l'influence d'une augmentation de l'adrénaline sanguine? Nous avons trouvé ${ }^{21}$ il y a quelques années que l'enlèvement rapide des hémisphères cérébraux, et la cessation immédiate de l'anesthésie

auteur d'une thèse sur le rôle de l'épinéphrine dans la régulation glycé mique (Sundberg 1925)

17 Louis Hallion (I862-1940) est un médecin et neurologue français. Il entre comme interne à l'Hôpital de la Salpêtrière en I888 et soutient une thèse sous la direction de Charcot en I892. En I893, il occupe le poste de chef de travaux du laboratoire de physiologie pathologique des Hautes Études à Paris. À partir de I897, il dirige le laboratoire chirurgical de l'Hôtel-Dieu. Il montre comment l'acide de l'estomac stimule, par l'intermédiaire de la sécrétine, la fonction exocrine du pancréas. Ses recherches jouent par ailleurs un rôle dans le développement de la réanimation et de l'immunologie. Pour René Gayet, voir l'introduction

18 Burn I923. Cannon ajoute un « $\mathrm{s}$ » au nom de Burn, qui est supprimé dans The Wisdom of the Body. Joshua Harold Burn (I892-I98I) est un pharmacologue britannique. Ses travaux portent principalement sur l'endocrinologie, en particulier sur l'adrénaline. Il est nommé professeur de pharmacologie à l'Université d'Oxford en I937, poste qu'il occupe jusqu'à sa retraite en I959. Il est élu à la Royal Society en I942. Dans l'article cité par Cannon, Burn fait état des effets antagonistes à l'adrénaline de plusieurs substances chimiques. Les hormones hypophysaires inhibent les effets hyperglycémiants de l'adrénaline, l'ergotoxine l'abolit et l'insuline la réduit.

19 L'ergotamine est un alcaloïde issu de l'ergot de seigle. Ce champignon, qui parasite de nombreuses céréales, peut provoquer des intoxications. Il contient l'acide lysergique dont le LSD est un dérivé synthétique.

20 Cannon oublie ici de biffer «sympathico-adrénal» en faveur de «sympathico-surrénal».

21 Cannon/Britton I925.

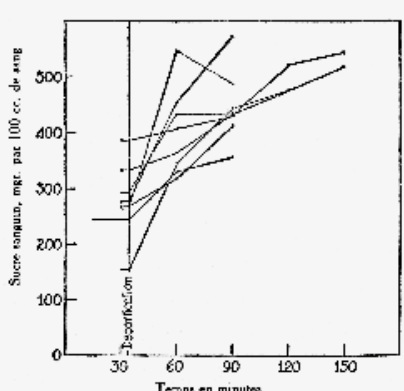

Figure 22. - Sucre sanguin chez des aniFigure 22. - Sucre sanguin chez des ani-
maux décortiqués présentant des symptômes maux décortiqu

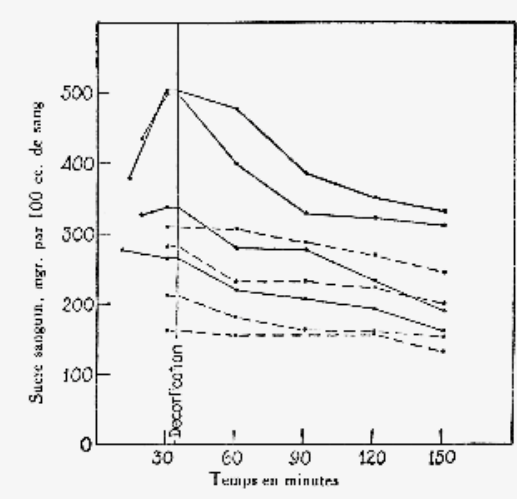

Figure 23. - Sucre sanguin chez des animaux décortiqués présentant des symptômes pseudo-affectifs; sans capsules surrénales (traits pleins), ou après enlèvement de la surenale droite et section des nerfs splanchniques gauches (trait étaient suivies par une extraordinaire exhibition de fureur - de pseudo-fureur ou de colère fausse, comme on peut l'appeler, étant donné que sans hémisphères l'animal ne peut se rendre compte de ce qui se passe ${ }^{22}$. Accompagnant l'érection des poils caractéristique de la colère, la dilatation des pupilles, l'accélération du cœur, l'augmentation de la pression sanguine, et d'autres signes d'excitation sympathique, on trouvait une augmentation de la glycémie qui pouvait atteindre le quintuple des valeurs normales, ainsi que le montre la projection suivante ${ }^{23}$. Si les surrénales étaient inactivées, les nerfs du foie étant intacts, la pseudo-fureur n'était pas accompagnée d'une augmentation du glucose sanguin, comme le montre la projection suivante ${ }^{24}$. D'autre part, si les nerfs du foie étaient coupés et si l'innervation des surrénales était intacte, les signes d'excitation étaient associés à une hyperglycémie à peu près aussi considérable chez l'animal avec innervation normale.

22 Cannon projette un cliché que nous ne sommes pas parvenus à identifier Il s'agit vraisemblablement de la photo d'un chat en état de fausse fureur, prise dans le laboratoire de Cannon.

23 Cannon projette la figure reproduite dans La Sagesse $d u$ corps (Cannon 1946[1939], 93), initialement parue dans Bulatao/Cannon 1925b.

24 Cannon projette la figure reproduite dans La Sagesse du corps (Cannon I946[I939], 94), initialement parue dans Bulatao/Cannon I925.
Fig. $48^{23}$ Fig. $49^{24}$ 


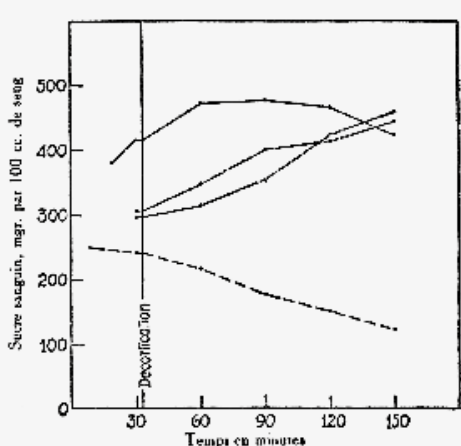
qués en etat pseudo-affectif, avec foie enerve et surn-
Figure 24. - Sucre sanguin chez des animaux décor-

C'est ce que montre la projection suivante ${ }^{25}$. Il apparaît, donc, que l'augmentation de la sécrétion d'adrénaline est un facteur de glycogénolyse plus important que l'action directe des influx nerveux. Cette conclusion a reçu l'appui de Britton ${ }^{26}$, qui a répété nos observations sur des chats opérés aseptiquement, puis effrayés par un chien aboyant. L'hyperglycémie se produisit rapidement chez les animaux avec surrénales et foies innervés, mais non si

Fig. $50^{25}$ la portion médullaire des surrénales est abolie ${ }^{27}$.

Le fait que dans les conditions normales l'adrénaline sécrétée est plus importante que l'excitation nerveuse, ne doit pas être interprété comme excluant totalement l'action des influx nerveux dans la glycogénolyse. Après enlèvement des surrénales, l'excitation des nerfs sensibles peut encore occasionner une hyperglycémie. Ces effets peuvent être expliqués comme étant dus à l'action directe des influx nerveux sur les cellules hépatiques ou peut-être l'asphyxie.

Maintenant, voyons ce qui arrive quand le sucre sanguin tend à augmenter dans le milieu intérieur. On s'aperçoit de l'efficacité des mécanismes qui s'opposent à cette tendance quand on ingère un excès de glucose. La glycémie s'élève à un niveau proche du seuil d'élimination rénale, mais normalement il ne dépasse pas souvent ce niveau. Le sucre en excès, sauf celui qui est mis de côté par inondation, est ou mis en réserve dans

Cannon projette la figure reproduite dans La Sagesse du corps (Cannon I946[1939], 95), initialement parue dans Bulatao/Cannon 1925.

26 Cannon projette la figure I et/ou 2 parue dans Britton I928. La figure n'est pas reproduite dans The Wisdom of the Body.

27 Cannon a ici grandement raturé le texte. Nous avons conservé la version supposément définitive. La version raturée est pourtant celle qui sera retenue dans The Wisdom of the Body (Cannon I939[1932], II8), ainsi que dans la traduction française (Cannon I946[I939], 95): «L'hyperglycémie se produisit rapidement chez les animaux avec surrénales innervées et foie dénervé, mais non dans les conditions inverses.» le foie et les muscles, ou converti en graisse, ou rapidement utilisé. Il est prouvé que le mécanisme de mise en réserve par séparation dans les cellules hépatiques et les muscles, dépend de l'insuline. Je ne puis que mentionner brièvement ces preuves.

Premièrement, l'extirpation du pancréas produit rapidement une hyperglycémie avec forte réduction des réserves glycogéniques.

Deuxièmement, l'administration d'insuline à un chien dépancréanté, nourri d'hydrates de carbone, ramène la glycémie à un niveau normal et provoque (à nouveau) l'accumulation de quantités considérables de glycogène dans le foie.

Troisièmement, l'insuline, (à petite dose,) provoque le dépôt d'hydrates de carbone dans le foie de chats ou de lapins phlorizinés ${ }^{28}$, même sans leur donner de sucre.

Quatrièmement, l'insuline injectée chez les chats décapités et éviscérés occasionne une augmentation considérable des dépôts de glycogène musculaire, surtout si on fournit un supplément de glucose.

Et finalement, comme preuve de ce que le pancréas intervient normalement dans le métabolisme des hydrates de carbone, il existe des changements caractéristiques dans les cellules des îlots de Langerhans ${ }^{29}$ indiquant un surcroît de travail quand de la nourriture hydrocarbonée est donnée en excès à un animal auquel on a enlevé la majeure partie du

28 La phlorizine ou phloridzine est un inhibiteur des cotransporteurs sodiumglucose. Son administration entraîne a un effet glycosurique.

29 Les «îlots de Langerhans", ou îlots pancréatiques, désignent les cellules endocrines du pancréas regroupées en amas. Ils tiennent leur nom du physiologiste allemand Paul Langerhans (I847-I888), qui découvre leur rôle dans la production d'insuline. 


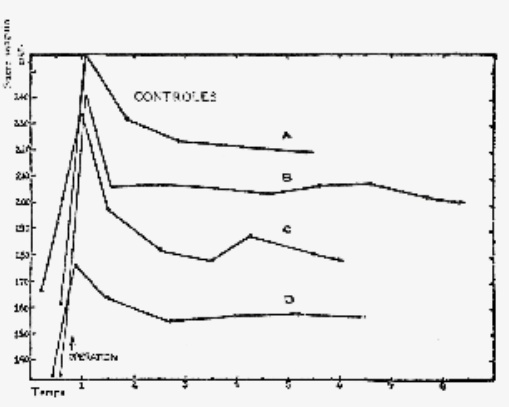

Figure 15. - A, B, C et D. Quatre expériences contrôles, montrant l'évolution du sucre sanguin pendant l'anesthéligaturée et le nerf vague droit préparé pour la stimulation. Les manœuvres opératoires déterminent invariablemen une augmentation considerable du taux du sucre sanguin piveau atteint et maintenu pendant plusieurs heures es

pancréas ; et associée à ces modifications, il y a une dégénérescence fonctionnelle des cellules.

Cependant, nous ne savons pas encore très bien comment le pancréas est excité à sécréter de l'insuline. Il n'est pas douteux que le glucose circulant a une action directe sur les cellules des îlots. C'est ce qu'indique toute une catégorie d'expériences. Une partie du pancréas peut être transplantée sous la peau, ainsi que l'a fait Minkowski ${ }^{30}$, et quand le reste de la glande est enlevé, le diabète n'apFig. $52^{34}$ paraît pas, mais si ensuite on extirpe la greffe cutanée, la maladie apparaît aussitôt. Les expériences de Gayet et Guillaumie ${ }^{31}$

30 Minkowski 1908. Oskar Minkowski (I858-193I) est un médecin et physiologiste originaire de l'actuelle Lituanie. Il soutient une thèse de doctorat en I88ז à l'Université de Königsberg. Il est nommé professeur à l'Université de Strasbourg en 1904. De I909 à 1926, il dirige le département de médecine interne de l'Université de Breslau. En I899, il réalise la première pancréatectomie et découvre ainsi le rôle que joue le pancréas dans la régulation de la glycémie. Sa découverte a été déterminante dans la découverte de l'insuline.

31 Gayet/Guillaumie I928. Maylis Anne-Cécile Guillaumie (I897-?) est une physiologiste française. En I93I, cette étudiante de la Sorbonne reçoit une bourse de la Fondation Rockefeller et rejoint le laboratoire de Cannon à l'École médicale de médecine de Harvard. Elle obtient un doctorat en sciences naturelles en I933. En I934, elle entre à l'Institut Pasteur de Paris, en tant qu'assistante de Michel Weinberg (I868-I940) qui dirige le service des anaérobies. Elle est nommée cheffe de laboratoire en 1936. À la mort de Weinberg, en I940, Guillaumie est nommée responsable du service. Elle occupe ce poste jusqu'à sa retraite, en I962. Elle est lauréate de l'Académie des sciences en 1925 et faite Chevalier de la Légion d'honneur en 1947 Létude citée fait état d'une hausse de la glycémie, lorsque des chiens ayant subi une dépancrétation totale se voient greffer un morceau de pancréas sur le trajet carotido-jugulaire. Selon les auteurs, l'expérience montre «que le tissu transplanté est le siège d'un processus de sécrétion interne d'insuline remarquablement réglé, remarquablement précis, tout à fait comparable à celui par lequel, dans les conditions physiologiques, le pancréas assure la constance de la glycémie. Par là il se confirme [...] de façon incontestable, sont d'accord avec ces résultats : ils ont montré que l'hyperglycémie diabétique est promptement réduite quand un pancréas est mis en connexion avec les vaisseaux du cou.

Mais il y a quelque évidence aussi que la sécrétion d'insuline est sous le contrôle du système nerveux. Après les expériences

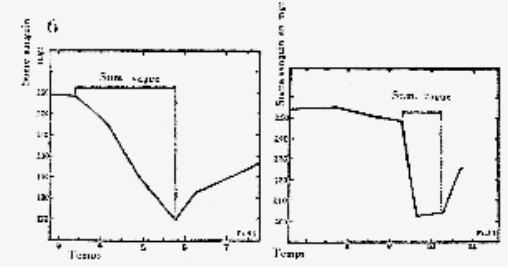

Figure 16. - Deux tracés montrant l'effet de la stimulation du vague sur le taux du sucre sanguin. La surrénale gauche était liée dans chaque cas. indécises de Corral ${ }^{32}$ et Macleod et ses collaborateurs, un de mes élèves, $\mathrm{S}$. W. Britton ${ }^{33}$, trouva que, après exclusion du système sympathico-surrénal, il pouvait obtenir un abaissement notable de la glycémie en simulant le vague droit. La projection suivante ${ }^{34}$ montre le cours de la glycémie après opération sous anesthésie à l'amytal ${ }^{35}$, sans excitation du vague. Dans la projection suivante sont figurés les effets de la stimulation du vague droit ${ }^{36}$. Ce phénomène ne se produit plus si les vaisseaux

que la glande est capable d'exercer, sans le concours du système nerveux, c'est-à-dire en obéissant à des excitations purement humorales, le rôle régulateur qui lui est dévolu» (Gayet/Guillaumie I928, 678).

32 De Corral 19i8. José María de Corral (I889-I97I) est un médecin et physiologiste espagnol. Il obtient un doctorat de la Faculté de médecine de Madrid en IgI4. De IgI6 à IgI7, il travaille pour l'Université de Berne à l'Institut de physiologie dirigé par Leon Asher (I865-I943). Il est nommé à la chaire de physiologie de l'Université de Saint-Jacques-de-Compostelle en 1923. En I940, ce proche de Juan Negrin est nommé professeur de physiologie à la Faculté de médecine de Madrid. Dans l'article cité par Cannon, Corral fait état d'expériences menées sur des chiens, qui montrent que la stimulation du nerf vague induit dans quelques cas une légère réduction du taux de sucre dans le sang. Cannon juge cependant ces résultats indécis, puisqu'il a été montré que les substances utilisées afin d'anesthésier les animaux peuvent affecter la glycémie.

33 Britton 1925

34 Cannon projette la figure reproduite dans La Sagesse $d u$ corps (Cannon I946[1939], 84), initialement parue dans Britton I925. Il ajoute à la main dans la marge gauche: «L'opération cause une augmentation du sucre sanguin mais, après, nous constatons qu'il y a peu de [illisible]. "

35 L'amytal est un barbiturique utilisé comme sédatif avant une intervention chirurgicale.

36 Cannon projette la figure reproduite dans La Sagesse du corps (Cannon 
pancréatiques ont été préalablement ligaturés. Selon Zunts et La Barre ${ }^{37}$, l'injection de glucose peut révéler le contrôle nerveux de la sécrétion d'insuline. Comme vous le savez probablement, ils ont utilisé la circulation croisée entre la veine pancréatique d'un chien A et la veine jugulaire d'un chien B, et ont trouvé que l'injection de glucose au chien A provoquait une chute de la glycémie du chien B, qui recevait le sang du pancréas de $\mathrm{A}$. Ils déclarent que ce phénomène ne se montre pas si les vagues du chien A ont été coupés ou si ce chien a été atropinisé.

Il est donc vraisemblable qu'il existe un contrôle nerveux de la sécrétion d'insuline, mais que ce contrôle n'est pas nécessaire. Dire qu'il n'est pas indispensable ne signifie pas qu'il est sans utilité. Beaucoup d'organes peuvent, bien qu'énervés, accomplir leur fonction. Il est possible d'obtenir, par asphyxie, une sécrétion d'adrénaline, même si les splanchniques ont été coupés, mais les glandes ne répondent pas aussi bien lorsqu'elles sont normalement innervées. Il se peut que le vague contrôle délicatement la sécrétion d'insuline.

Le schéma général que je viens de vous présenter implique l'action de deux mécanismes antagonistes toujours prêts à maintenir l'homéostasie du sucre sanguin. Si le niveau de la glycémie tombe en dessous d'un niveau critique, le système sympathico-surrénal entre en action et, par mobilisation des réserves hépatiques, la glycémie se relève; c'est un mécanisme de relèvement de la glycémie; et si le niveau s'élève, le système

I946[I939], 85), initialement parue dans Britton I925. Il ajoute à la main dans la marge gauche: «Vous voyez l'abaissement du sucre sanguin après chaque stimulation.»

37 Zunz/La Barre i927. Cannon écrit «Zunts » au lieu de «Zunz ». L'erreur est corrigée dans The Wisdom of the Body (Cannon I939[1932], I05). Edgar Zunz (I874-1939) est un pharmacologue belge. En I897, il soutient une thèse de doctorat à l'Université libre de Bruxelles. En I9I9, il est nommé professeur et directeur du laboratoire de pharmacologie et de thérapeutique de la Faculté de médecine de l'Université libre de Bruxelles. Il est élu à l'Académie royale de médecine de Belgique en 1920 et en devient le président en 1934. Nous n'avons pas trouvé d'informations concernant Jean La Barre, qui était vraisemblablement rattaché à l'Institut de thérapeutique de l'Université libre de Bruxelles. vago-insulaire ou le pancréas seul devient actif, et la glycémie s'abaisse; c'est un mécanisme dépressif. Par ces réglages admirables, les dangers de convulsions, de coma et de mort sont évités d'une part, et d'autre part, l'organisme prévient la perte d'un matériel de valeur ${ }^{38}$.

Les seules expériences en contradiction avec des idées que j'ai défendues sont celles que Cori et Cori ${ }^{39}$ ont rapportées récemment. Ils considèrent la mobilisation du glycogène musculaire, et une diminution de l'utilisation du sucre sanguin comme l'action la plus importante de l'adrénaline; et la déplétion des réserves de glycogène hépatique, due à une exagération de la consommation en glucose des tissus périphériques serait la conséquence inévitable des injections d'insuline. Il faut noter qu'ils ont administré des doses énormes de ces deux substances, doses équivalentes à quatorze centimètres cubes d'adrénaline et à cinq cent vingt-cinq unités d'insuline pour un homme de soixante-dix kilogrammes. Leur suggestion suivant laquelle le glycogène musculaire est "mobilisé » implique son inutile transformation en acide lactique et son retour au foie

38 Cannon ajoute à la main dans la marge gauche un «1/2», qui indique qu'il a atteint la moitié de son exposé.

39 Cori/Cori 1928. Carl Ferdinand Cori (I896-I984) est un pharmacologue américain né à Prague. Il obtient un doctorat de l'Université de médecine de Prague en 1920. En I93I, il est nommé professeur de pharmacologie à l'École de médecine de l'Université de St. Louis. Gerty Theresa Cori (I896-I957) est elle aussi née à Prague et elle obtient également un doctorat de médecine à l'Université de Prague en I920. Le couple Cori se marie en I920. Gerty travaille d'abord comme chercheuse associée auprès de Carl, puis elle est nommée professeure de biochimie en I947. La même année, le couple partage avec Bernardo Houssay le Prix Nobel de physiologie, qui récompense leurs travaux sur la métabolisation du glycogène. L'article cité par Cannon expose les termes du débat qui l'oppose au couple Cori. Il était connu que e glycogène hépatique disparaît après injection d'insuline. Il était aussi admis qu'une hypoglycémie insulinique mène à une décharge d'adrénaline et que l'adrénaline mobilise le glycogène du foie. Dans ces conditions, on pouvait supposer, comme Cannon, que l'adrénaline est responsable de la baisse du glycogène du foie. Cependant, une injection d'insuline semble faire autant diminuer le glycogène hépatique chez des animaux normaux que chez ceux ayant subi une adrénalectomie. Aussi, selon le couple Cori, l'action antagoniste de l'adrénaline ne semble pas avoir d'action directe sur le foie, comme le soutient Cannon. 
- c'est à l'opposé de tout ce que nous connaissons sur le cycle du glucose dans l'organisme. De plus, ils négligent complètement ce fait bien établi que le glycogène hépatique est rapidement décomposé dans le foie quand de l'adrénaline est injecté à petites doses. Pour s'en assurer, Colwell ${ }^{40}$, dans des recherches récentes au laboratoire de Harvard, a montré que l'injection continue de minimes quantités d'adrénaline, un millième de milligramme par kilogramme et par minute, peut enrayer l'utilisation du sucre dans l'organisme; mais d'autres expériences de Dworkin ${ }^{41}$ ont montré que lorsque l'adrénaline, injectée ou sécrétée, produit son effet de mobilisation du sucre hépatique, l'hyperglycémie persiste longtemps après la disparition de l'excès d'adrénaline du sang circulant. En d'autres mots, l'adrénaline ne persiste pas, et ne trouble pas l'utilisation du sucre. C'est pour ces diverses raisons que nous nous croyons en droit de conclure que les idées exprimées par les Coris ${ }^{42}$ ne peuvent pas nous faire abandonner les conceptions bien établies sur l'action de l'insuline et de l'adrénaline dans le maintien de l'homéostasie du glucose sanguin.

J'ai spécialement insisté sur la régulation de la glycémie, parce que cette régulation a été élucidée beaucoup plus profondément que de celle de toute autre des substances mises en réserve par séparation, et parce que, à mon avis, ce réglage peut donner des suggestions en ce qui concerne le contrôle des autres matériaux. Nous aurons le temps d'examiner brièvement quelques-uns des autres constituants permanents du sang.

Il n'est pas nécessaire d'attirer spécialement l'attention sur l'importance de la constance des protéines plasmatiques. Parce qu'elles exercent une pression osmotique et ne traversent pas dans les conditions ordinaires les parois capillaires, elles

Colwell/Bright I930.

Cette étude, absente de la version finale de The Wisdom of the Body, est mentionnée dans un autre article de Cannon (I929d) dont ce passage est tiré. L'étude en question ne semble pas avoir fait l'objet d'une publication. Simon Dworkin (I899-I978) est un médecin et physiologiste d'origine russe, diplômé de l'Université McGill en 1928. Il travaille au laboratoire de phy siologie de l'École de médecine de Harvard, sous la direction de Cannon jusqu'en 1930, avant de retourner à McGill.

42 Cannon met au pluriel le nom propre « Cori», comme cela se fait en anglais. empêchent les sels dissous dans le sang de passer librement dans les espaces intravasculaires et de s'échapper de l'organisme par les glomérules rénaux. L'effet de la réduction de ces protéines fut montré par Barcroft et Straub ${ }^{43}$. Ils enlevèrent à un lapin une grande partie de son sang, séparèrent les globules, et les réinjectèrent en suspension dans un volume égal de solution de Ringer remplaçant le plasma. Le changement apporté fut donc surtout une réduction des colloïdes du sang. La sécrétion d'urine fut quarante fois plus considérable! Mais l'homéostasie des protéines ne sert pas seulement à l'homéostasie du volume sanguin, au moins l'une d'entre elles, le fibrinogène ${ }^{44}$, est, en cas d'hémorragie, essentielle pour la conservation du sang luimême. L'existence même du milieu intérieur, de la fluid matrix, dépend par conséquent de la constance des protéines du plasma; mais habituellement elles sont remarquablement stables dans les états variés de santé ou de maladie.

Le sang est le seul tissu de l'organisme dont la matière protéique peut être quantitativement enlevée, et sa récupération consécutive étudiée. Quand par plasma-pharésis ${ }^{45}$, c'està-dire, l'enlèvement d'une partie du plasma et réinjection du reste avec les globules, les protéines du plasma sont réduites de six à deux pour cent environ, il y a une augmentation rapide de leur concentration dans les quinze minutes qui suivent, ensuite une réparation graduelle pendant les premières vingt-quatre heures jusqu'à soixante-six pour cent de la quantité normale de protéines, et une restauration complète après deux à sept jours. Il se peut que l'augmentation rapide ne soit que relative, étant due au passage de solution saline en dehors des vaisseaux,

43 Barcroft/Straub, I9IO. Hermann Straub (I882-I938) est un médecin et physiologiste allemand. Il étudie la médecine à l'Université de Tübingen et à l'Université Humboldt de Berlin. Il soutient une thèse de doctorat en I9o9. Il séjourne à l'Institut de physiologie de Londres, avant d'être nommé assistant à l'Hôpital universitaire de Tübingen. En I9I9, il prend la direction de la polyclinique médicale de Greifswald. Il démissionne peu après l'accession au pouvoir du Parti national-socialiste.

44 Le fibrinogène est une protéine du sang, impliquée dans les processus de coagulation et de formation de caillots sanguins.

45 Cannon commet ici une double erreur, car le terme employé en anglais, plasmapheresis, devrait être traduit en français par «plasmaphérèse ». 
mais cependant, des arguments ont été avancés en faveur d'une décharge brusque des protéines de réserve. La restauration plus lente dépend sans aucun doute du foie et ce pour les raisons suivantes : premièrement, si le foie a été intoxiqué par le phosphore ou le chloroforme, la restauration des protéines du plasma est retardée; deuxièmement, cette restauration peut ne pas exister dans les trois premiers jours qui suivent la plasmapharésis chez les chiens porteurs d'une fistule d'Eck ${ }^{46}$; et, troisièmement, le fibrinogène, qui habituellement est complètement rétabli dans les vingt-quatre heures, n'est pas ramené à sa concentration normale si le foie est dans l'impossibilité d'agir.

Puisqu'il est évident que le foie est un organe important pour l'homéostasie des protéines du plasma, on peut se demander si la matière protéique y est emmagasinée. Les résultats obtenus en histologie et en biochimie sont d'accord pour soutenir la conclusion selon laquelle les cellules hépatiques peuvent se charger de réserves protéiques aussi bien que de réserves d'hydrates de carbone. Les premières observations d'Afanassiew ${ }^{47}$ suivant lesquelles les cellules hépatiques du chien nourri abondamment d'albuminates s'agrandissent et contiennent des granulations protéiques entre les filaments protoplasmiques, ont été confirmées par un certain nombre d'autres chercheurs. Ces récentes études histologiques montrent que si on donne des protéines à un animal, il apparaît dans les cellules hépatiques de fines gouttelettes ou masses, qui réagissent au réactif de Million ${ }^{48}$, qui produisent la réaction à la ninhydrin ${ }^{49}$ comme

46 La réalisation d'une fistule d'Eck consiste à exclure chirurgicalement le foie de la circulation sanguine. Nikolai Vladimirovitch Eck (I847-I908) réalise pour la première fois l'opération en 1877 , afin d'explorer des voies de traitement des ascites mécaniques.

47 Michail Ivanovič Afanassiew (I850-I9Io) est un physiologiste et pathologiste russe. Il est diplômé de l'Académie médico-chirurgicale de SaintPétersbourg en 1977. Après plusieurs séjours de recherche à l'étranger, à Breslau, Leipzig ou encore Berlin, où il travaille auprès de Virchow et de Robert Koch (I843-I9IO), il est nommé en I895 professeur et directeur de l'Institut clinique de Saint-Pétersbourg.

48 Le réactif de Millon se colore en rouge si une substance qui lui est mélangée contient de la tyrosine.

49 La ninhydrine est un réactif qui a la particularité de se colorer en présence d'acides aminés. de simples protéines qui disparaissent par le jeûne, et qui réapparaissent quand l'animal est à nouveau nourri de protéines ou d'acides aminés. Les analyses biochimiques de Seitz ${ }^{50}$, qui trouva que chez des animaux non à jeun le rapport entre l'azote total du foie et du reste du corps est deux à trois fois plus grand que chez les animaux à jeun, ont été confirmées par les résultats de Tichmeneff ${ }^{51}$. Celui-ci fit jeûner des souris pendant deux jours, en tua la moitié, nourrit abondamment les autres de viande cuite, les tua à leur tour, et compara les foies des deux groupes. Exprimés en pour cent du poids du corps, les foies des animaux nourris de viande augmentèrent de vingt pour cent; la teneur en azote du foie était augmentée de cinquante-trois à soixante-dix-huit pour cent.

Bien que les expériences sur l'homéostasie des protéines du plasma indiquent que le foie est une source importante de ce matériel en cas de besoin, et bien que les témoignages que je viens de citer nous permettent de considérer le foie comme un dépôt de protéine, les mécanismes de mise en réserve et de mobilisation sont presque entièrement inconnus. En réalité, Stubel $^{52}$ a observé que les petites gouttes ou masses de protéines contenues dans les cellules hépatiques, pouvaient être considérablement réduites par l'injection sous-cutanée d'adrénaline. Si ces masses protéiques fournissent les éléments protéiques essentiels à la coagulation, comme semble l'indiquer le fait que le fibrinogène dépend du foie, leur libération par l'adrénaline et par les circonstances qui provoquent l'excitation du système

50 Seitz 1906. Nous n'avons trouvé aucune information concernant Wilhelm Seitz, qui était rattaché au laboratoire de physiologie de Bonn lorsqu'il a mené cette étude.

51 Tichmeneff rgi6. Nous n'avons pas trouvé d'information concernant ce physiologiste d'origine russe, vraisemblablement rattaché à l'Institut de chimie physiologique de Strasbourg lors de la parution de l'article.

52 Stübel 1920. Hans Stübel (1885-I96I) est un médecin et physiologiste allemand. Il se voit décerner un doctorat de médecine en I908 par l'Université de Iéna. En I923, il est nommé professeur ordinaire et directeur de l'Institut de physiologie de l'Université Tongii à Shanghai. En I95I, soit deux ans après la fondation de la République populaire de Chine, il est contraint de démissionner. De retour en Allemagne, il enseigne l'ethnologie et la sinologie à l'Université d'Erlangen, mettant à profit ses nombreux voyages à la rencontre des diverses ethnies chinoises. 
sympathico-surrénal pourrait rendre compte du phénomène d'accélération de la coagulation. Comme je l'ai montré dans ma première conférence, la coagulation est plus rapide après injection d'adrénaline, après excitation des splanchniques, mais seulement si le sang est autorisé à circuler à travers le foie et les intestins. À cette catégorie appartient également la coagulation très rapide du sang soutiré pendant la réaction hypoglycémique quand l'activité du système sympathico-surrénal est maximale.

Il est très possible que les protéines soient mises en réserve en d'autres endroits que le foie, et il est possible aussi que la glande thyroïde soit un facteur important pour le contrôle et la mise en réserve et la mobilisation. Boothby, Sandiford et Sloose ${ }^{53}$ ont rapporté que pour une ingestion d'une quantité uniforme de protéines il existe un bilan azote négatif pendant que la thyroxine élève le métabolisme à un niveau plus élevé que le niveau normal. Quand ce niveau est établi, il y a une légère rétention d'azote dans l'organisme. Et si on arrête l'administration de thyroxine, tandis que la même quantité de protéine continue à être ingérée, on a un bilan positif jusqu'à ce que soit atteint un nouveau niveau métabolique bas; c'est-àdire, que plus d'azote est retenu par l'organisme. Ces phénomènes sont beaucoup plus marqués chez une personne normale. En réalité, ainsi que l'a suggéré Boothby ${ }^{54}$, l'œdème du myxœdème peut être dû à un dépôt d'une quantité anormale de protéines à l'intérieur et en dessous de la peau. L'efficacité dans les cas de myxœdème de l'administration d'extrait thyroïdien qui réduit les quantités anormalement élevées de protéines du plasma et d'albumine des tissus, vient à l'appui de l'idée suivant laquelle la glande thyroïde intervient d'une façon ou d'une autre dans la régulation et le métabolisme de protéines.

53 Boothby/Sandiford/Sandiford/Slosse 1925. Pour Boothby et Sandiford. Cannon omet de mentionner le troisième auteur de cette publication, Kathleen Sandiford (I896-?). Cette biochimiste américaine, vraisembla blement parente d'Irene Sandiford, travaille à la Fondation Mayo de 1920 à I930. Nous n'avons pas trouvé d'informations concernant Jean Josse. Il s'agit vraisemblablement d'un chercheur belge bénéficiant d'une bourse de la Commission for Relief in Belgium (pour cette commission, voir l'introduction).

54 Boothby/Sandiford/Sandiford/Slosse I925, 746.
Bien que ces considérations aient attiré l'attention sur l'importance primordiale de l'homéostasie des protéines plasmatiques pour le maintien du volume et des caractères du milieu intérieur et intravasculaire et extravasculaire de l'organisme, et pour la protection de l'organisme contre la perte de cette partie essentielle de la fluid matrix qu'est le sang; elles révèlent surtout combien nous devons encore apprendre à ce sujet. Ici encore, comme pour toute autre substance utile, la stabilité est obtenue par la mise en réserve contrôlant l'abondance et le besoin, et dans ce cas encore, le foie joue un rôle important. Le système sympathico-surrénal semble influencer la mobilisation des réserves, et il est possible également que l'activité variable de la thyroïde soit l'agent primordial. Des mécanismes spéciaux sont-ils prévus pour le dépôt de ces réserves? Nous l'ignorons ${ }^{55}$.

Maintenant, considérons un moment les graisses. Suivant Bloor ${ }^{56}$, la concentration en graisses, cholestérol et lécithine du sang est remarquablement constante dans une même espèce, mais peut varier grandement d'une espèce à l'autre.

Bien plus, le niveau lipidique reste constant pendant de longs jours en dépit d'un jeûne relatif ou total. Ce fait implique l'existence d'un mécanisme de contrôle qui fait entrer dans la

Cannon ajoute à la main dans la marge gauche qu'il a atteint les «33/4»de son exposé.

56 Bloor 1922. « La concentration de graisse, de cholestérol et de lécithine dans le sang est assez constante pour la même espèce, mais varie considérablement d'une espèce à l'autre, la variation étant principalement perceptible dans le plasma. La concentration dans le plasma et les globules chez le même animal est différente. En général, le taux de lipoïde dans le plasma est plus élevé chez le carnivore que chez l'herbivore, étant sans doute influencé par la quantité de graisse habituellement présente dans l'alimentation. " (Bloor I922, 204, notre traduction) Walter Ray Bloor (I877-I966) est un biochimiste américain. Il obtient un doctorat à l'Université de Harvard en 1906. De I9II à I9I5, il poursuit ses recherches à l'Université de Washington, à St. Louis, avant de retourner à Harvard. En I9I8, il est nommé professeur de biochimie à l'Université de Californie. En I922, ce spécialiste du métabolisme lipidique est nommé professeur de biochimie et directeur associé de l'École de médecine à l'Université de Rochester. 
circulation des graisses de réserve. Ainsi que Lusk ${ }^{57}$ l'a remarqué, «la durée de l'existence, pendant le jeûne, dépend généralement de la quantité de graisse présente dans l'organisme au départ. ${ }^{58}$ La graisse est déposée dans le foie si la nourriture ne contient pas d'hydrate de carbone; elle est déposée également sous la peau, sous les revêtements séreux, par exemple, autour des reins, dans le grand épiploon ${ }^{59}$, et aussi entre les fibres et à l'intérieur des fibres musculaires. Nous ignorons ce qui produit chez certains individus un dépôt de graisse plus important que chez d'autres. Dans l'hypothyroïdisme, il y a généralement une obésité diffuse, obésité qui disparaît rapidement par l'administration de thyroïde. Une légère égratignure à la surface de la base du cerveau, entre les processus infundibulaires et un corps mamillaire, produit l'adiposité, tout comme une tumeur ou autre lésion de cette région. Grafe ${ }^{60}$ cite des exemples d'hypertrophie ou d'atrophie unilatérale des tissus graisseux et émet l'hypothèse suivant laquelle la répartition des graisses est sous le contrôle du sympathique gouverné par la région

57 Graham Lusk (I866-I932) est un médecin et physiologiste américain, spécialiste de la nutrition et du diabète. Diplômé de l'École des mines de l'Université de Columbia en $\mathrm{I} 887$, il reçoit un doctorat de chimie de l'Université de Munich en I89I. À son retour aux États-Unis, il est nommé instructeur de physiologie à l'Université de Yale. Il y est nommé professeur en I895. En I898, il est nommé à la chaire de physiologie de l'Université de New York et de l'Université de médecine de l'Hôpital Bellevue de New York. Il est élu à l'Académie nationale des Sciences en 1915. Lusk et Cannon se connaissent. Dans une recension parue dans Science, Lusk écrit au sujet de The Wisdom of The Body: «Ce livre se lit comme si un professeur d'université, avec un esprit riche en connaissances basées sur une vie de recherches fructueuses en laboratoire, s'était installé sous le porche, lors d'une soirée fraîche de l'été, et y avait enregistré dans un langage simple mais lumineux des pensées sur les faits et les problèmes découverts tout au long d'une vie de travail» (Lusk 1932, 640, notre traduction).

58 Lusk I928[1917], I0o. La traduction est de Cannon.

59 L'épiploon désigne un repli du péritoine, tissu tapissant la cavité abdominale et enveloppant les viscères.

60 Grafe 1927. Erich Grafe (I88I-I958) est un médecin et physiologiste allemand, spécialiste du métabolisme et du diabète. En I906, il est nommé assistant au département de médecine de l'Université de Heidelberg. Il rejoint l'Université de Rostock en I922, puis est nommé en I926 directeur du département de médecine et de neurologie de l'Université de Würzburg. hypothalamique. Cependant, nous avons enlevé d'un seul côté le système sympathique chez des jeunes chats, et nous les avons laissés vivre jusqu'à ce qu'ils aient doublé de poids, et nous avons observé aucune différence ni dans la quantité, ni dans la distribution de la graisse entre les deux côtés du corps.

Si le mécanisme de la mise en réserve des graisses est obscur, le mécanisme de la mobilisation de ces réserves l'est plus encore. Quand le maintien des énergies corporelles l'exige, la graisse est libérée des tissus adipeux jusqu'à ce que les cellules adipeuses soient pratiquement vides. Et cependant, même quand la mort survient par la suite du jeûne, la teneur en graisse des autres tissus, ainsi que l'a montré Terroine ${ }^{61}$, peut ne pas être très différente de la teneur normale. Nous ignorons ce qui produit la sortie de la graisse des tissus adipeux. La remarque de Lusk que «les organes du jeûneur attirent les graisses hors des réserves adipeuses de l'organisme, et cette graisse leur est apportées par le sang circulant ${ }^{62}$, cette remarque ne visait probablement pas à expliquer le phénomène et en effet, elle n'explique rien. Il est possible que la réaction réversible en présence de lipase, qu'ont décrite Kastle et Loevenhart ${ }^{63}$,

61 Terroine I9I4. Émile-Florent Terroine (I882-I974) est un physiologiste français. Il obtient une licence de sciences naturelles à la Sorbonne en I903. En I907, il obtient un poste de préparateur au laboratoire de chirurgie expérimentale de l'École pratique des hautes études, alors hébergé au Collège de France. Il y est nommé maître de conférences. À la fin de la Première Guerre mondiale, il soutient sa thèse de doctorat et occupe la chaire de physiologie générale de la Faculté des sciences de Strasbourg, jusqu'en 1939. Durant la Seconde Guerre mondiale, ce spécialiste de la nutrition dirige la commission sur l'alimentation. Engagé dans la résistance, il est arrêté en I944. Après la Seconde Guerre mondiale, il administre le Commissariat régional aux questions juives de Lyon. En I946, il est nommé à la direction du Centre national de coordination des études et recherches sur la nutrition et l'alimentation, puis, en 1949, à la direction des Sciences de la vie du CNRS.

62 Lusk I928[I9I7], I06. La traduction est de Cannon.

63 Kastle/Loevenhart I9oo. Joseph Hoeing Kastle (I864-I9I6) est un chimiste américain, diplômé en I886 de l'Université du Kentucky, où il est nommé professeur en I888, puis, en I9I2, directeur de la Faculté d'agriculture. Arthur Salomon Loevenhart (I878-1929) est un physiologiste et pharmacologue américain, diplômé de l'Université du Kentucky en i899. Il y mène des recherches sous la direction de Kastle. Il est nommé professeur associé 
soit un facteur important pour le maintien de l'homéostasie de la lipémie - l'enzyme favorisant le dépôt quand la lipémie s'élève et favorisant la sortie quand celle-ci s'abaisse. Mais, il serait désirable d'être mieux informé sur ces points.

Quelques mots maintenant à propos du calcium. Les utilités spéciales et variées du calcium - croissance du squelette et des dents, réparation des fractures, maintien des conditions convenables d'irritabilité nerveuse et musculaire, coagulation du sang et production d'un lait utile - en font un élément de haute importance pour l'économie de l'organisme. De même que pour le sucre, les protéines, et les graisses, le besoin en calcium peut dans des occasions exceptionnelles être considérable. Dans de telles circonstances cependant, la quantité de calcium sanguin ne doit pas être réduite de beaucoup, car de sérieuses conséquences peuvent s'en suivre - ce sont les contractions et les convulsions bien connues dont le pronostic se base sur l'importance du déficit en calcium disponible. D'autre part, si par injection d'extrait parathyroïdien la calcémie est élevée à vingt milligrammes pour cent approximativement, des changements profonds se produisent dans le sang - la viscosité est fortement augmentée, la pression osmotique s'élève, la quantité des phosphates du sang est doublée, la teneur en azote non protéique et uréique est quadruplée - condition s'accompagnant de vomissement, coma, et de déficience circulatoire. Il saute aux yeux que l'homéostasie du calcium sanguin est d'importance capitale.

de chimie physiologique et de pharmacologie à l'Université Johns Hopkins en 1906. En I908, il est nommé professeur de pharmacologie et de toxicologie à l'École de médecine de l'Université du Wisconsin. Durant la Grande Guerre, il rejoint l'unité de pharmacologie et de toxicologie du Chemical Warfare Service, à Washington. Ses recherches sur l'arsenic contribuent au développement des composants du Tryparsamide, utilisés notamment dans le traitement de la syphilis. Dans l'article cité par Cannon, Kastle et Loevenhart exposent les premières preuves de la réversibilité de l'action de la lipase, soit d'un mécanisme de régulation de l'action d'un enzyme. La lipase, sécrétée par le pancréas, a pour fonction d'hydrolyser les acides gras. L'inhibition ou l'activation de la lipase permet ainsi de réguler le taux de lipides dans le sang.
De même que l'homéostasie des autres substances, celle du calcium est assurée par des réserves accumulées aux moments d'abondance et utilisées en cas de besoin. Les études récentes d'Aub et de ses collaborateurs ${ }^{64}$ ont démontré qu'il est facile de faire disparaître les trabécules des os longs par un régime pauvre en calcium et par la croissance, et que ces trabécules réapparaissent rapidement avec un régime riche en calcium. Les animaux furent soumis au régime riche ou pauvre pendant quelques mois, puis le membre antérieur gauche fut amputé à l'épaule; on les soumit ensuite au régime inverse. Après une période semblable les animaux furent tués, et dans chaque cas l'humérus restant fut comparé à l'humérus amputé. Que les animaux soient d'abord soumis au régime riche ou à un régime pauvre, les résultats furent toujours identiques; l'os enlevé après régime riche en calcium possédait de nombreux trabécules, tandis que son homologue, après régime pauvre, n'avait que peu de trabécules. J'ai ici deux projections que le docteur Aub m'a permis d'emporter pour la démonstration de ces faits.

64 Bauer/Aub/Albright I929. Walter Bauer (I898-I963) est un médecin rhumatologue américain. Il obtient un doctorat de médecine de l'Université du Michigan en 1922. De I922 à 1923, il est interne de médecine au Long Island College Hospital de Brooklyn, à New York. Il poursuit sa carrière au Massachusetts General Hospital de Boston, où il obtient la chaire Jackson de médecine clinique en 195I. On lui doit d'avoir montré que la polyarthrite rhumatoïde est une maladie inflammatoire affectant le corps entier, et non pas seulement les os et les articulations. Bauer s'est particulièrement attaché à favoriser, au sein de l'École de médecine de Harvard, les liens entre les cliniciens et les physiologistes. Fuller Albright (I900-I969) est un médecin et endocrinologue américain. Il entre à l'École de médecine de Harvard en I920 et poursuit des recherches sur le métabolisme du calcium, sous la direction d'Aub. Après un séjour de recherche à l'Université Johns Hopkins, il retourne en 1929 à l'Hôpital général de Harvard. En I936, il y développe la division de recherche en endocrinologie. Il est surtout connu pour avoir développé les premiers traitements de l'ostéoporose par administration d'œstrogène. Ces travaux le mèneront à anticiper l'utilisation d'hormones à des fins de contraception. Il ne poursuivra pas cette ligne de recherche dès lors que, dans les années 1940, les lois du Massachussetts interdisaient le recours ainsi que la dissémination d'informations relatives à la contraception. En 1956, Allbright décide de subir une pallidectomie, afin de réduire les symptômes de la maladie de Parkinson qui lui a été diagnostiquée en I937. Victime d'une hémorragie lors de l'opération, il finit sa vie dans un mutisme akinétique à l'Hôpital général du Massachusetts. 


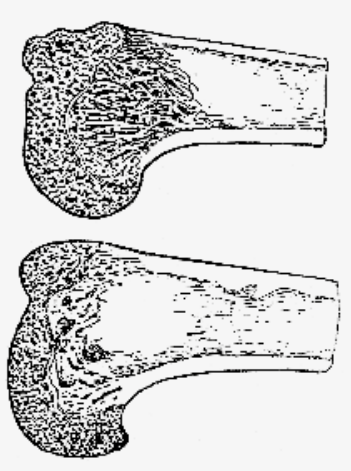

Figure 25. - Dessins d'après une photographie montrant les contient beaucoup plus de trabécules que son homologue prélevé après un régime pauvre en calcium. effets du régime alimentaire sur les trabécules de l'humérus d'un

La première projection ${ }^{65}$ montre les différences caractéristiques que je viens de décrire. Remarquez spécialement les humérus du chat trois cent quarante-quatre (344). Il fut soumis à un régime riche en calcium pendant quatre-vingts jours, et ensuite à un régime pauvre pendant trois cent soixante-dix-neuf jours. La différence entre les trabécules est frappante. Vous noterez des différences semblables dans les autres cas. Comme le montre la

Fig. $54^{65}$ projection suivante ${ }^{66}$, quand les animaux reçoivent du calcium et du rouge d'alizarine ${ }^{67}$ après une longue privation en calcium, les nouveaux trabécules sont teints en rouge. Voyez tout spécialement les os avant et après régime riche dans les cas trois cent soixantesept et soixante-huit. D'après ces remarquables recherches, il est clair que les trabécules constituent un dépôt de calcium facilement disponible.

Les mécanismes de régulation de l'homéostasie du calcium ne sont pas déterminés. Toute une série de faits bien établis permettent de considérer les glandes parathyroïdiques comme partie active de cette régulation. Mais la question de savoir comment les parathyroïdes contrôlent l'homéostasie du calcium - si elles agissent directement ou sont stimulées par des nerfs; si elles agissent seules et si par augmentation ou diminution de leur activité elles provoquent le dépôt ou

65 Cannon projette la figure reproduite dans La Sagesse du corps (Cannon I946[I939], II3), initialement parue dans Bauer/Aub/Albright I929. Seul l'humérus du chat 344 est reproduit, sous forme de dessin, dans La Sagesse $d u$ corps.

66 Cannon projette la figure parue dans Bauer/Aub/Albright 1929. La figure n'est pas reprise dans The Wisdom of the Body.

67 Colorant rouge d'origine végétale, l'alizarine rouge permet de mesurer, par colorimétrie, la quantité de dépôt calcique.

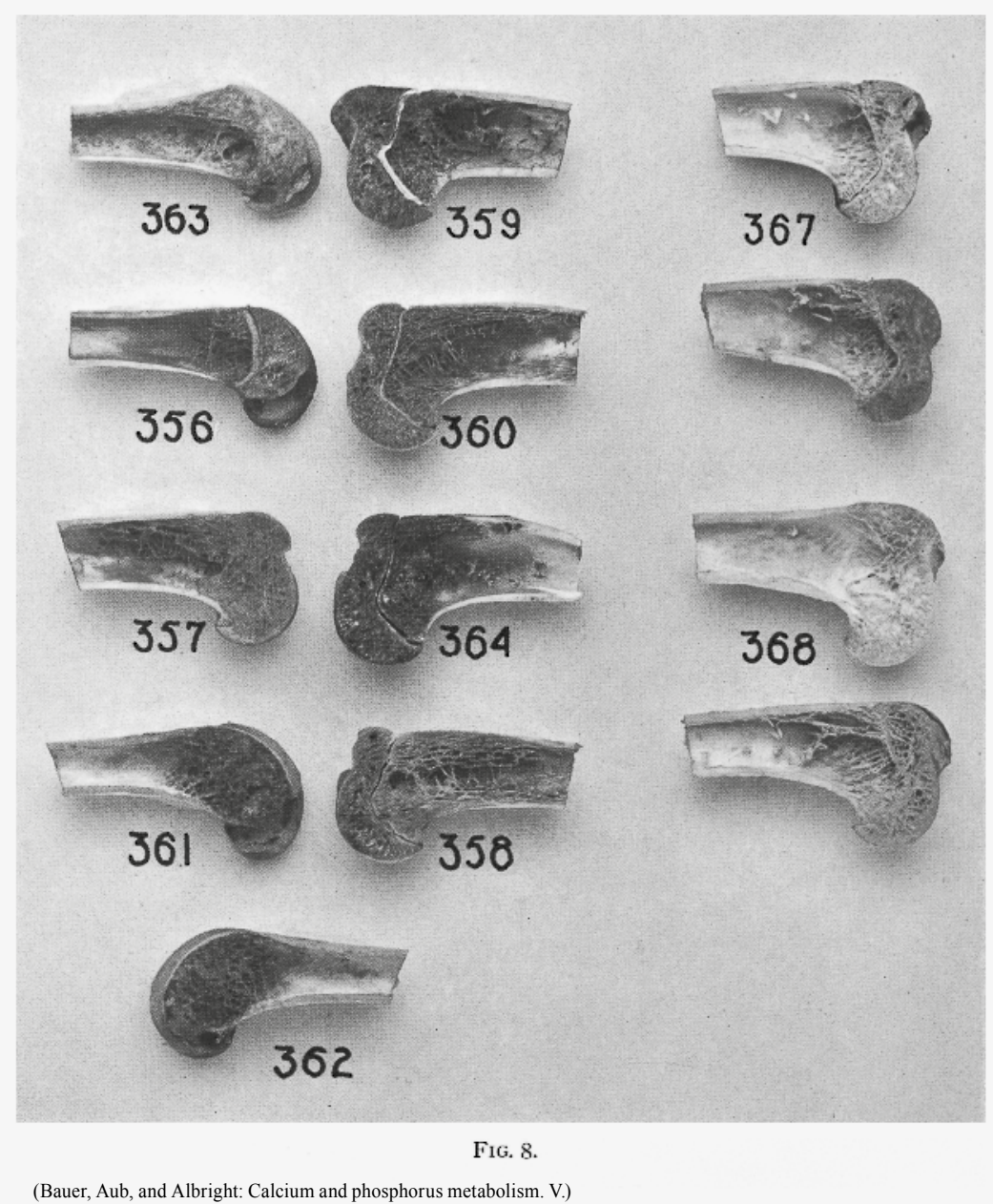

la sortie du calcium; ou bien si elles coopèrent avec d'autres Fig. $55^{66}$ mécanismes, peut-être antagonistes - tout cela nécessite de nouvelles recherches.

Dans l'examen que nous avons fait aujourd'hui du contrôle des approvisionnements mis en réserve par séparation, vous avez peut-être remarqué que j’ai fait de fréquentes allusions au fonctionnement du système sympathico-surrénal. Je vous confesse que je pose les fondements pour une interprétation ultérieure 
de l'utilité de ce système dans l'organisme. Dans la prochaine leçon j'examinerai l'homéostasie comme une conséquence de la modification de la vitesse des processus continus. Là encore, nous verrons le système sympathico-surrénal à l'œuvre. Mais ce sera l'objet de notre prochaine réunion.

\section{Quatrième conférence}

Le débordement, processus limitatif dans l'homéostasie des matériaux; Homéostasie par augmentation ou réduction de la vitesse des processus continus ; Utilité du système sympathico-surrénal dans le contrôle du pH sanguin et de la constance de la température

Avant d'entamer le sujet principal de cette conférence qui est le contrôle de l'homéostasie par la régulation de la vitesse des processus continus, je désirerais mentionner brièvement le débordement, mécanisme limitant les excès de matériaux dans le milieu intérieur de l'organisme. Quand je vous ai parlé de l'homéostasie du glucose, j'ai montré l'utilité du mécanisme de débordement comme moyen de freiner une augmentation exagérée de cette substance dans le sang. Et ce n'est pas seulement l'excès de sucre, mais aussi l'excès d'eau, d'ions sodium, potassium et chlore, qui sont déchargés par les reins. D'après la théorie moderne de la sécrétion urinaire, ce sont là des «substances à seuil ». Elles ne sont résorbées par les tubuli rénaux que jusqu'à une certaine concentration favorable au maintien de la concentration normale dans le sang. Tout ce qui est au-delà du seuil peut s'échapper du corps.

Il est intéressant de noter que ces substances sont tout d'abord mises en réserve par inondation. Cependant, quand les réserves suffisantes de ces matériaux ont été faites, la puissance du mécanisme de débordement pour maintenir l'homéostasie est merveilleuse. Haldane et Priestley ${ }^{1}$ ont rapporté un fait qui révèle non seulement l'efficacité du rein en tant que déversoir,

Haldane/Priestley igi6. Cannon cite cette référence dans plusieurs écrits et se trompe vraisemblablement de date. John Gillies Priestley (I880-I94I) est un physiologiste britannique. Il étudie la physiologie au laboratoire de physiologie d'Oxford au tournant du XX⿳亠丷厂 siècle, sous la direction de Haldane. Durant la Grande Guerre, il sert en France ainsi qu'en Belgique et reçoit la Croix de Guerre en I9I5. Après la guerre, il poursuit ses recherches à Oxford. Haldane et Priestley sont notamment connus pour avoir mis au point un pléthysmographe avec lequel ils ont mis en évidence la relation 
mais aussi les dispositions organiques pour le maintien de la constance du milieu intérieur : ils pouvaient boire cinq litres et demi d'eau en six heures, une quantité supérieure d'un tiers au volume sanguin estimé par calcul, et observer un passage tellement facile à travers les reins qu'à aucun moment il n'y a une réduction appréciable du pourcentage d'hémoglobine.

Les poumons aussi bien que les reins sont extrêmement utiles en cas de débordement. On sait très bien qu'un léger excès d'acide carbonique dans le sang artériel est suivi d'une forte augmentation de la ventilation pulmonaire. Par conséquent, l'excédent d'anhydride carbonique est si rapidement et si efficacement éliminé que l'air alvéolaire est maintenu presque constant. De cette façon, les précautions sont prises pour permettre à l'acide carbonique en excès de s'écouler du sang au-delà d'un barrage maintenu à un niveau fixe. La conséquence de ce fait est que, dans les circonstances ordinaires, la concentration en ions hydrogènes du sang reste très constante, et que les effets désastreux d'une tendance excessive à l'alcalinité ou à l'acidité sont évités. En relation directe avec cette régulation de la réaction du sang, se trouvent des mécanismes qui changent la vitesse de la circulation quand les conditions sont telles qu'il y a une menace d'augmentation de la concentration en ions hydrogène.

On ne peut exagérer l'importance de la nécessité pour un organisme de confiner les modifications du $\mathrm{pH}$ du sang dans une très petite étendue. Comme je l'ai mentionné dans notre première réunion, à un $\mathrm{pH}$ de six et neuf dixièmes environ, le sang devient si acide que le coma et la mort en résultent, et au-delà de sept et sept dixièmes il devient si alcalin que la tétanie apparaît. Une variation de sept et sept dixièmes à six et neuf dixièmes n'est pas considérable, et cependant le maintien du $\mathrm{pH}$ entre ces limites est essentiel à notre existence ${ }^{2}$. Il existe dans le sang lui-même des mécanismes extraordinairement délicats - mécanismes qui ont été décrits dans tous leurs détails par mon

entre la quantité de dioxyde de carbone dans l'air inspiré et l'intensité de la ventilation pulmonaire (Haldane/Priestley 1905).

2 Cannon ajoute à la main dans la marge gauche: «mettre les nombres en chiffres et non en lettres.» collègue, L. J. Henderson, dans son livre « Blood $»^{3}$. Cependant en arrière de ces réglages purement sanguins, il existe des mesures de protection qui anticipativement protègent le sang de tout danger. Des métabolites acides sont constamment produits dans les cellules vivantes, et s'ils pouvaient s'y accumuler, ces métabolites ${ }^{4}$ troubleraient l'activité actuelle et empêcheraient toute action ultérieure. Des arrangements détaillés sont prévus dans l'organisme pour devancer cette contingence. Il est certain que les facilités pour le contrôle de l'acide non volatile sont limitées. Mais cet acide peut être combattu de différentes façons. L'acide lactique, par exemple, (qui est produit par la contraction musculaire) est en partie promptement neutralisé - la phosphocréatine, récemment découverte par Fiske et Subbarow ${ }^{5}$, apparaît capable de fonctionner de façon très effective en neutralisant l'acide lactique à l'intérieur des cellules musculaires. Une autre partie de l'acide est bientôt oxydée, et le reste est resynthétisé à l'état de glycogène neutre. Pour permettre à ces trois dispositifs de continuer à fonctionner avec efficacité, il faut leur fournir une quantité convenable d'oxygène. Bien que les muscles, et peut-être d'autres tissus également, contractent une « dette d'oxygène » en continuant à travailler en dépit de l'acide lactique qui s'accumule, cet état est caractérisé par une diminution de la capacité de travail, diminution considérable par rapport à la dette, par une limitation de l'importance de la dette, et finalement par l'exigence de payement.

Quand l'acide non volatil est comburé jusqu'à l'état d'acide carbonique volatil, il est alors sous une forme rapidement transportable en quantités presque illimitées, avec une modification

Henderson 1928.

Cannon supprime « déchets» en faveur de «métabolites».

Fiske/Subbarow, I929. Cyrus Hartwell Fiske (I890-I978) est un médecin et biochimiste américain, diplômé en I9I4 de l'École de médecine de Harvard, où il poursuit des recherches en biochimie. Il y est nommé professeur en I937. Yellagaprada Subbarow (I896-1948) est un médecin et biochimiste d'origine indienne. Il entre en I923 à l'Université de Harvard, où il obtient un diplôme de l'École de médecine tropicale. Ses recherches s'orientent alors vers la biochimie. Il réalise son doctorat sous la direction de Fiske. En I940, il s'oriente du côté de l'industrie pharmaceutique et travaille pour les Lederle Laboratories. 


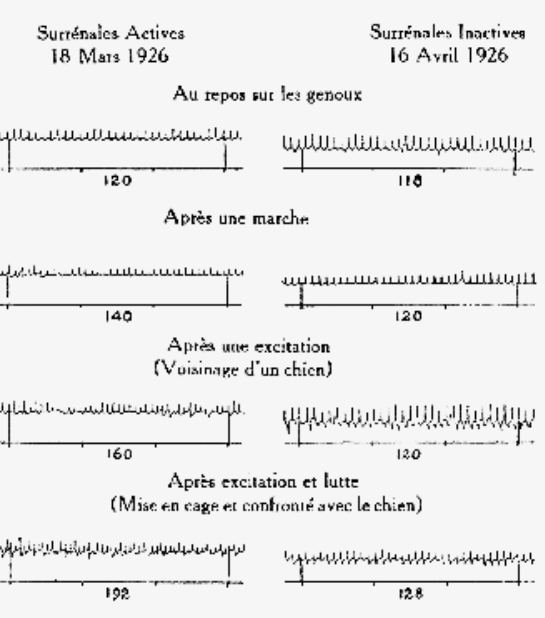

Figure 26. - Tracés originaux de la fréquence du cceur énervé du chat 27 pris avant ( 18 mars) et après ( 16 avril) inactivation des surnormale de l'animal au repos sur les genoux est comparée à la fréquence après une marche, après une excitation déterminée par la présence d'un chien et enfin après avoir été mis en cage et après avoir réagi aux aboiements du chien.

légère de la réaction du sang. Durant l'effort musculaire vigoureux, autant d'oxygène que possible doit être fourni. Il n'y a pratiquement aucune réserve d'oxygène dans l'organisme. Les animaux qui respirent de l'air sont entourés par un océan d'oxygène. Le seul problème est celui du transport de ces réserves extérieures inépuisables aux tissus exigeants. C'est dans ce but que les phénomènes circulatoires et respiratoires doivent être fortement accélérés. Heureusement, ces mécanismes nécessaires pour l'élimination de l'acide volatil sont

Fig. $56^{6} \quad$ précisément ceux qui sont requis pour apporter aux tissus l'oxygène qui permet la production d'acide volatil et son élimination rapide.

Pendant un effort musculaire vigoureux, la ventilation pulmonaire peut passer de six (6) litres par minute à soixante (6o) ou quatre-vingts litres (80) ou plus, et ce phénomène est dû à l'action de l'acidité sur le centre respiratoire. Même ${ }^{6}$ pendant de légers mouvements musculaires, le système sympathico-surrénal entre en action. Nous avons étudié l'augmentation de la rapidité du cœur énervé quand un animal se promène simplement sur le sol. Sur vingt-huit observations de cette sorte l'accélération moyenne du pouls fut de dix-sept pulsations par minute. Ceci était dû, dans ces conditions, à la seule sécrétion d'adrénaline, car, après inactivation des surrénales, la même activité musculaire ne provoqua plus aucune activation cardiaque. Quand l'animal se débattait vigoureusement, cette

6 Cannon projette la figure reproduite dans La Sagesse $d u$ corps (Cannon I946[1939], I22), initialement parue dans Cannon/Britton I927. réaction s'accompagnant d'une forte excitation, il y avait sans aucun doute une stimulation beaucoup plus grande du système sympathico-surrénal, car la vitesse du cœur énervé s'accrut en moyenne de quarante-neuf battements par minute. Nous savons à présent que les bronchioles sont sensibles à l'action du système sympathico-surrénal; celui-ci provoque la dilatation bien connue qui se démontre si dramatiquement par l'injection d'adrénaline dans le cas de l'asthme bronchique. C'est pourquoi il semble également probable que pendant un gros effort musculaire, les bronchioles sont dilatées. Ce fait aurait pour conséquence d'élargir le passage, et de faciliter de cette façon l'aller et venue des grandes quantités d'air exigées par l'effort vigoureux.

À côté de ces réglages respiratoires, et en association avec eux, il existe des mécanismes coordonnés du système circulatoire. Ils sont tellement bien connus qu'il me suffira de les énumérer pour vous rafraîchir la mémoire. (I) Pendant l'activité musculaire énergique la quantité de sang retournant au cœur par minute est plus considérable par suite de la contraction des vaisseaux splanchniques - notez encore une fois l'activité sympathico-surrénal; (2) par suite également des pressions intermittentes qu'exercent les muscles actifs sur leurs capillaires et leurs veines; (3) et aussi grâce à l'action du diaphragme qui peut être comparée à celle d'une pompe. Le cœur, donc, reçoit une charge de sang plus grande, et à chaque battement lance une quantité supérieure à la normale. Et grâce (I) à la diminution du tonus vagal, (2) à l'augmentation de la pression veineuse, et (3) à la participation des accélérateurs sympathiques, le cœur, bien chargé, peut battre deux fois plus rapidement qu'au repos. Par l'augmentation du rendement du cœur et la vasoconstriction de l'aire splanchnique, la pression artérielle est notablement élevée. Dans les muscles actifs, les artérioles sont dilatées et les capillaires qui étaient clos s'ouvrent ; dans ces canaux plus nombreux, la pression artérielle puissante dirige un flux de sang abondant. Les faits montrent que la vitesse de toute la circulation peut être quadruplée. Mais non seulement les globules rouges sont utilisés de façon plus efficace grâce à leur mouvement plus rapide, mais encore leur nombre est accru par la décharge des réserves spléniques. 
De même que Barcroft et ses collaborateurs ${ }^{7}$, Hargis et Mann ${ }^{8}$, Binet $^{9}$, Viale ${ }^{10}$, et d'autres ont montré que la rate est beaucoup plus volumineuse dans l'organisme vivant que dans le cadavre.

7 Barcroft/Poole 1927; Barcroft/Stephens, I927. John Gower Stephens (I898-?) est un radiologue australien, diplômé de médecine en I94I, à l'Université de Sydney. En 1926, il effectue un séjour de recherche dans le laboratoire de physiologie de l'Université de Cambridge. En 1929, il est nommé vice-principal de l'Andrew's College de l'Université de Sydney. Leopold Thomas Pool (I888-1965) est un médecin et pathologiste britannique. Mobilisé durant la Grande Guerre, spécialiste des pathologies induites par les armes chimiques, il est nommé directeur du War Office en I94I.

8 Hargis/Mann I925. Estes Henry Hargis (I895-I966) est un médecin et pathologiste américain. Il étudie à l'Université de Auburn de I9I5 à I9I7, ainsi qu'à la clinique Mayo de Rochester, sous la direction de Mann. Frank Charles Mann (I887-I962) est un médecin et physiologiste américain. Il étudie au Marion Normal College, dans l'Indiana, et devient en I908 assistant de physiologie à l'Université de l'Indiana. Il est diplômé de médecine en I9I3. En 1915, il est nommé directeur de l'Institut de médecine expérimentale et d'anatomie pathologique de la clinique Mayo. En 1928, il est nommé professeur à l'Université du Minnesota.

9 Binet 1927. Léon Binet (I89I-I97I) est un médecin et physiologiste français, spécialiste de la physiologie de la respiration et de réanimation. Il entre à la Faculté de médecine de Paris en Iqı, puis fait son internat des hôpitaux en I9I3. Mobilisé durant la Grande Guerre, il participe aux batailles de Verdun puis de la Somme. En I9I7, il est appelé à étudier les effets physiopathologiques induits par les gaz de combat. En I929, il soutient une thèse sur la fonction de réservoir de la rate. Il est nommé professeur titulaire de la chaire de physiologie à la Faculté de médecine de Paris, en I93. Il est élu à l'Académie de médecine en 1939 et à l'Académie des Sciences en 1942.

10 Gaetano Viale (I889-1934) est un médecin et physiologiste italien dont les études portent principalement sur la physiologie de la fatigue et sur l'endocrinologie. Assistant de Mosso au laboratoire de physiologie de Turin, Viale est nommé à la chaire de physiologie de Sassari en I925. En I926, il est nommé professeur de physiologie à l'Institut de physiologie de la Faculté de médecine de l'Université de Rosario de Santa Fe, en Argentine. Il revient en Italie en I929, pour prendre la chaire de physiologie de Gênes. Nous n'avons pas trouvé d'informations concernant $J$. De Leo Lira Rosario, très certainement un étudiant de Viale durant les trois années passées à l'Université de Rosario. Les travaux de Viale montrent que la rate régule le nombre de globules rouges dans le sang, non seulement dans certaines circonstances, lors d'un exercice par exemple, mais aussi en fonction des changements environnementaux, notamment lorsque l'oxygène se raréfie: «La rate est un organe qui sert à l'adaptation de l'organisme aux variations de la pression atmosphérique. Ce fait, ajouté à ceux qu'ont démontrés Barcroft et Binet, démontre son importance dans la régulation des globules rouges
Sa teneur en globules rouges chez le cheval peut être, selon Scheynert et Krzyswanek ${ }^{11}$, à une concentration deux fois plus forte que celle des globules rouges du sang circulant. Naturellement, ces corpuscules en excès sont mis en service quand la rate se contracte. Le professeur Barcroft ${ }^{12}$ a montré la réduction du volume de la rate sous l'effet de la course. Le nombre des érythrocytes peut augmenter de vingt pour cent ou plus à la suite de cinq minutes de violent travail. Cette réaction est aussi le résultat

circulants; elle verse des hématies dans le torrent circulatoire, selon les nécessités de l'organisme» (Viale/Di Leo Lira I927, I240).

11 Scheunert/Krzywanek 1927. L'orthographe du nom du premier auteur est fautive. Carl Arthur Scheunert (1879-1957) est un physiologiste et vétérinaire, originaire de Dresde. Il étudie la médecine vétérinaire et la chimie à l'Université de médecine vétérinaire de Dresde et aux Universités de Leipzig et de Göttingen. Il se voit décerner un doctorat de chimie physiologique en I9ıo par l'Université de Dresde, où il enseigne jusqu'en I920. De I920 à I923, il enseigne en tant que professeur de physiologie animale à l'Université agricole de Berlin, puis comme professeur de physiologie vétérinaire à la Faculté de médecine vétérinaire de l'Université de Leipzig, jusqu'en 1945. Spécialiste de la physiologie de la nutrition et proche du Parti national-socialiste allemand, Scheunert prend en I94I la direction de l'Institut du Reich pour le contrôle et l'évaluation de l'alimentation. Il y mène, ainsi qu'au camp de concentration de Dachau, des expériences visant à étudier les effets des régimes alimentaires carencés en vitamines. En I946, il est nommé professeur de physiologie vétérinaire en RDA, à l'Université de Giessen. Friedrich Karl Wilhelm Krzywanek (I896-1946) est un physiologiste et vétérinaire allemand. Il obtient un doctorat de médecine vétérinaire en I92I et devient l'assistant de Scheunert à l'Université agricole de Berlin. En I929, il est nommé professeur à la Faculté de médecine vétérinaire de Leipzig. En I933, il est nommé professeur titulaire de physiologie vétérinaire et directeur de l'Institut de physiologie vétérinaire de l'Université de médecine vétérinaire de Berlin, dont il devient le doyen en I94I. En I944, il est arrêté par l'armée soviétique et meurt en détention en 1946.

12 Cannon supprime le passage suivant: «Le cliché suivant que m’a prêté le professeur Barcroft. » Cannon projette très probablement les clichés 6 et/ou 7 parus dans Barcroft/Stephens, I927; ces passages, pas plus que les figures, ne sont reproduits dans La Sagesse du corps. 
d'une excitation; nous avons trouvé, en effet, que l'augmentation moyenne des globules rouges des chats étudiés était de vingt pour cent aussitôt après une minute d'excitation provoquée par un chien aboyant ${ }^{13}$. Ce phénomène, de même que la vasoconstriction de l'aire splanchnique et l'accélération du cœur sont le résultat de la mise en jeu du système sympathico-surrénal. Il ne survient pas après enlèvement de la partie supérieure des chaînes sympathiques abdominales et section bilatérale des nerfs splanchniques et aussi son propre transport aux poumons. C'est ainsi que la circulation locale peut arriver à être neuf fois plus importante qu'à l'état de repos, et la livraison d'oxygène à être dix-huit fois plus considérable. Donc, en dépit du fait que, en un court espace de temps, l'effort musculaire peut produire plus d'acide que n'en pourraient neutraliser les tampons du sang - condition qui cause inévitablement la mort - la réaction du sang n'est altérée qu'à un minime degré.

On ne peut citer d'exemple plus admirable d'homéostasie que celui de la concentration en ions hydrogène du milieu intérieur. Elle est assurée par l'accélération ou la diminution de la vitesse des processus continus de ventilation pulmonaire et de circulation sanguine. Les modifications physico-chimiques qui se passent dans le sang lui-même, et que je n'ai pas examinées dans cette conférence, diminuent considérablement les petites variations inévitables au cours de ces processus physiologiques. La marche de la respiration est fortement influencée par la concentration en ions hydrogène des cellules du centre respiratoire, mais celles-ci à leur tour sont influencées par un accroissement de la concentration en acide carbonique du sang. Encore une fois, le mal apporte son propre remède, et si la concentration est abaissée par une respiration intense, la respiration intense cesse. Le réglage de la circulation peut être agencé de la même façon. Laccélération cardiaque, la vasoconstriction (sauf aux surfaces actives) sont des manifestations de l'activité du système sympathico-surrénal. Comme je vous l'ai montré, une activité volontaire même légère provoque la mise en action

13 Cannon supprime en début de phrase «Comme le montre la prochaine projection...»
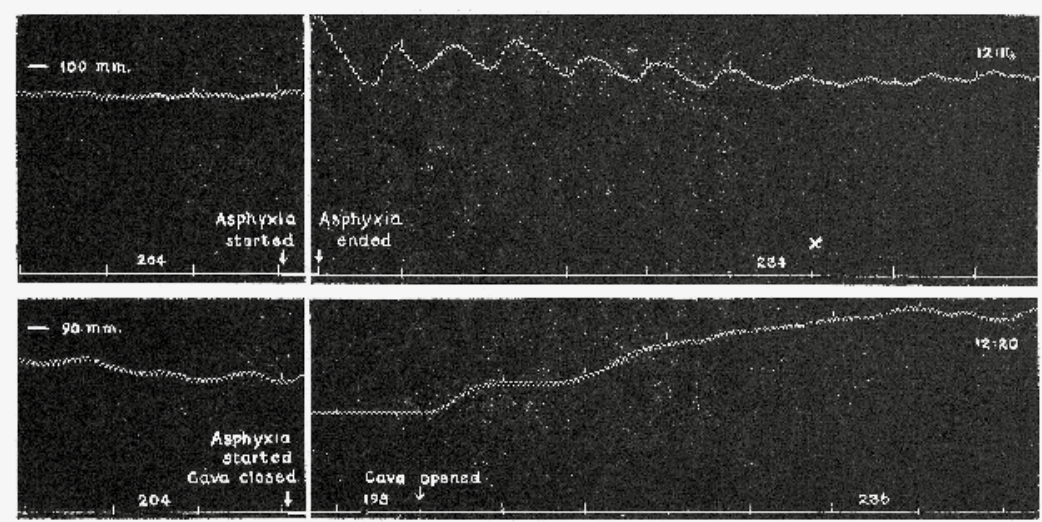

ig. 4. Beginning and end of records of the beat of the denervated heart. At 12:10, asphyxia for 45 seconds increased the heart rate 30 beats per minute. The point $X$ ( 75 seconds from the start) corresponds in time to the opening of the cava in the record for 12:20. At 12:20 asphyxia for 45 seconds, with the cava closed 75 seconds; the heart rate fell 6 beats
while venous flow was blocked, but rapidly rose 38 beats when the block was removed.

de ce système. De plus, l'asphyxie elle-même est un excitant Fig. $58^{14}$ hautement efficace du sympathique. Comme vous le voyez à l'écran, nous avons trouvé ${ }^{14}$ qu'une asphyxie de quarante-cinq secondes accroissait la vitesse du cœur énervé de trente pulsations à la minute - de deux cent quatre à deux cent trentequatre. Alors on provoqua à nouveau l'asphyxie, mais la veine cave inférieure fut fermée pour empêcher le passage vers le cœur du sang venant des surrénales. L'ouverture de la veine cave dans le tracé du bas correspond au point $\mathrm{X}$ du tracé supérieur, après le début de l'asphyxie. Notez qu'au lieu d'une augmentation de

14 Cannon projette la figure parue dans Cannon/Carrasco-Formiguera I922. Celle-ci n'est pas reprise dans The Wisdom of the Body. Rosendo CarrascoFormiguera (I892-I990) est un médecin et physiologiste espagnol, diplômé de la Faculté de médecine de Barcelone en I9I4. De I92I à I922, il mène des recherches à l'École de médecine de l'Université de Harvard, sous la direction de Cannon. Marqué par la découverte de l'insuline, il tente dès son retour à Barcelone de produire une extraction d'insuline destinée au traitement du diabète. Premier Européen à traiter un patient diabétique par injection d'insuline, il ouvre en I927 le premier centre de traitement du diabète. En I933, il est nommé professeur à l'Université de Barcelone. Il est amené à quitter son poste durant la guerre civile espagnole. Il part en exil en 1939 et poursuit ses recherches au Mexique, puis au Venezuela. CarrascoFormiguera fait partie des chercheurs de Barcelone qui accueillent Cannon lors de son voyage en Espagne, en avril 1930. 
trente battements, on obtient à ce moment une réduction de six (6) pulsations. Cependant, peu après avoir levé la pression sur la veine cave et que le sang abdominal eut atteint le cour, le pouls s'accéléra de trente-huit (38) pulsations. Nos premières expériences avaient montré que l'excitation asphyxique ne provoquait aucune accélération du cœur énervé si les surrénales avaient été préalablement enlevées. Il paraît donc bien évident que même si l'activité volontaire ne s'accompagnait pas d'activité du système sympathico-surrénal, l'asphyxie provoquée par l'effort musculaire pourrait à elle seule faire entrer ce système en action. Les centres du contrôle sympathique peuvent être influencés de la même façon que le centre respiratoire - l'acidité peut augmenter dans ces centres par suite du besoin d'oxygène ou de l'excès d'acide carbonique, et le premier résultat peut être l'excitation. Cette suggestion est appuyée par les expériences de Mathison ${ }^{15}$, montrant que l'asphyxie ainsi qu'un excès d'acide carbonique dans l'air inspiré élevait la pression artérielle, et par nos observations suivant lesquelles les métabolites musculaires excitent le système sympathico-adrénal ${ }^{16}$. Le physiologiste suédois Johansson ${ }^{17}$, vous vous en souvenez, a trouvé que le cœur énervé s'accélérait quand des métabolites musculaires étaient libérés dans la circulation. Comme le montrent les tracés supérieurs de la prochaine projection, nous avons pu confirmer

15 Gordon Clunes Mckay Mathison (I883-I9I5). Mathison est un médecin et physiologiste australien, diplômé de médecine en I905 à l'Université de Melbourne. En I908, il s'installe à Londres et poursuit ses recherches sur la physiologie de la respiration à l'Institut de physiologie de l'University College. En I9I3, il est nommé sous-directeur de l'Institut de pathologie de l'Université de Melbourne. Durant la Grande Guerre, il est engagé volontaire dans l'Australian Imperial Force. Il est tué en I9I5 durant la campagne de Gallipoli.

16 À quelques occasions, Cannon écrit «sympathico-adrénal» à la place de "sympathico-surrénal».

17 Johansson 1894 . Cette référence est présente dans The Wisdom of the Body mais Cannon indique vraisemblablement une date erronée (I895 pour I894). John Edvard Johansson (I862-1938) est un médecin et physiologiste suédois, diplômé de médecine en I89o. En I90I, il est nommé professeur de physiologie à l'Institut Caroline à Stockholm. Ses recherches portent essentiellement sur le métabolisme et l'exercice musculaire. Il préside le comité du Prix Nobel de physiologie ou de médecine jusqu'en 1926.
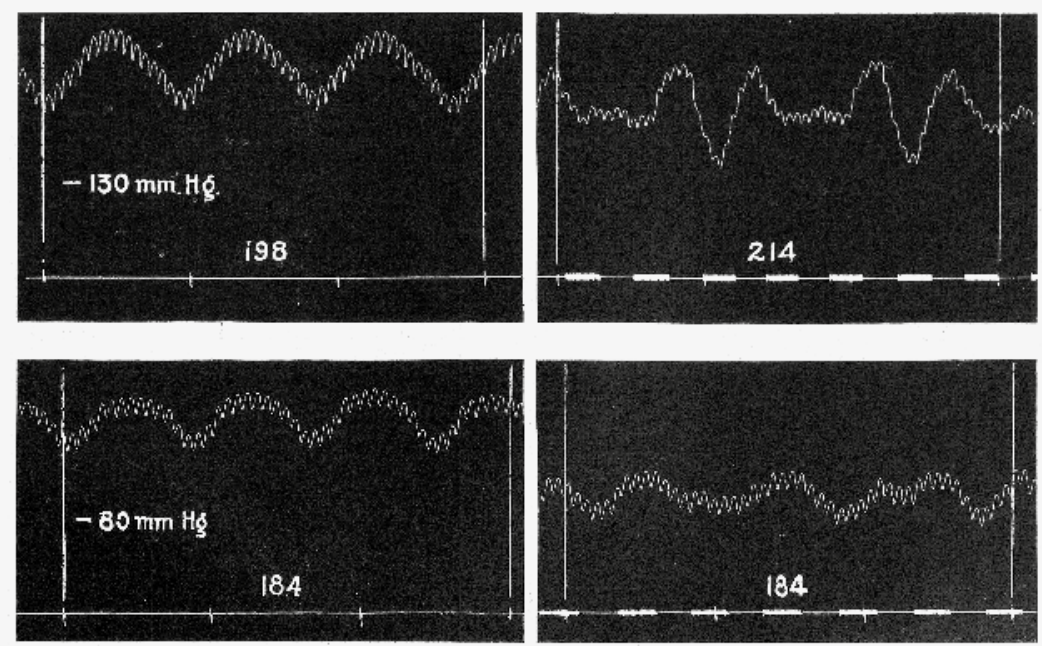

Fig. 1. Original records of rate of denervated heart. Upper record (10:40); initial rate 198 beats per minute; after 2 minutes of stimulation of peripheral ends of cut sciatics, 214 beats per minute. Base line marks 15 second intervals and minute; after 2 minutes of stimulation of peripheral ends of cut sciatics, no change. Base line marks 5 second intervals an minute; after $2 \mathrm{~m}$.
$50 \mathrm{~mm}$. mercury.

cette observation ${ }^{18}$. L'excitation des muscles des pattes postérieures par les nerfs sciatiques coupés, accroissait la fréquence du cœur de seize pulsations par minute. Après inactivation des surrénales, cependant, la même excitation fut sans effet ainsi que le démontrent les tracés inférieurs. Donc, pendant l'effort musculaire violent, la corrélation remarquablement intime entre l'adaptation des appareils respiratoire et circulatoire aux besoins de l'organisme pourrait s'expliquer ainsi : bien que les deux systèmes soient sollicités à fournir un service plus rapide par les excitations dues au travail volontaire, ils continueront à accomplir un travail supplémentaire à cause de l'augmentation de la concentration en ions hydrogène du sang, et plus tard, ils

18 Cannon projette la figure parue dans Cannon, Linton, Linton, I924. La figure n'est pas reproduite dans The Wisdom of the Body. Robert (I900-I979) et James (I899-?) Linton sont deux médecins d'origine britannique, diplômés de l'Université de Washington. Les deux frères rejoignent le laboratoire de Cannon en I92I. En 1927, Robert devient chirurgien interne de l'Hôpital général du Massachusetts. Il mène toute sa carrière dans cette institution en tant que spécialiste de chirurgie vasculaire. Nous n'avons pas trouvé d'informations concernant James Linton. 
reprendront graduellement leurs fonctions calmes habituelles, parce que leur activité excessive a eu pour résultat de réduire la concentration en ions hydrogène au niveau du repos.

Maintenant, abandonnons l'examen des facteurs qui contrôlent la réaction du sang, et voyons quels sont ceux qui maintiennent uniforme la température du milieu intérieur. Ce sujet intéressa profondément Claude Bernard ${ }^{19}$. Nous connaissons à l'heure actuelle certains des éléments du contrôle de la température dont il n'était pas averti. Ce sont ceux qui nous occupent spécialement.

Il n'est pas nécessaire d'insister sur l'importance d'une température uniforme qui fournisse des conditions favorables à la constance de l'intensité des modifications chimiques de l'organisme. Et le danger d'une élévation de température de quelques degrés au-dessus du niveau normal est aussi bien connu. Cependant, pour les considérations générales que nous discuterons bientôt, il est bon de mentionner brièvement les changements qui surviennent quand la température du corps tend à s'abaisser. Il y a constriction des vaisseaux périphériques, érection des poils et des plumes qui entourent la peau d'une couche d'air peu conducteur; il y a aussi augmentation de la glycémie. Érection des poils, vasoconstriction et hyperglycémie, ce sont là les signes d'activité de la partie sympathique du système autonome. Ce fait soulève naturellement la question de savoir si la sécrétion de la médullaire surrénale, qui est contrôlée par le sympathique, augmente quand le froid provoque une décharge d'influx sympathique. La réponse à cette question avait des conséquences physiologiques importantes, car des études antérieures faites au Laboratoire de Physiologie de Harvard par $\mathrm{Aub}^{20}$, McIver et leurs collaborateurs ${ }^{21}$ avaient montré que l'absence de sécrétion d'adrénaline abaisse le métabolisme basal et qu'une augmentation de cette sécrétion accélère les processus d'oxydation. Par conséquent, si la médullaire surrénale est

19 Voir le premier tome des Leçons sur les phénomènes de la vie, communs aux animanx et aux végétaux, en particulier la deuxième leçon portant sur «Les trois formes de la vie » (Bernard I878, 65)

20 Aub/Bright/Forman 1922.

$21 \quad$ McIver/Bright 1924. excitée par le froid, l'adrénaline sécrétée en supplément provoquerait une augmentation des combustions dans l'organisme juste au moment où celui-ci en a besoin ${ }^{22}$.

Des faits histologiques avancés par Cramer ${ }^{23}$, Vincent ${ }^{24}$ et Fujii ${ }^{25}$ avaient montré une réduction de la substance chromophile chez des animaux exposés au froid et ces faits avaient été interprétés comme preuve de la stimulation de la médullaire surrénale par le froid. Cependant, comme Trendelenburg ${ }^{26}$ le faisait remarquer, de telles observations doivent être interprétées avec prudence, car chez l'animal refroidi, la production

22 Cannon ajoute à la main, dans la marge gauche, un «1/2» qui indique qu'il a atteint la moitié de son exposé.

23 Cramer 1919. William Cramer (I878-1945) est un physiologiste et médecin, originaire de la province de Brandebourg et spécialisé dans le métabolisme et le cancer. Il soutient un doctorat à l'Université de Berlin en Igoo. Il intègre l'Imperial Cancer Research Fund de Londres. De 1905 à 1915, il enseigne la physiologie chimique à l'Université d'Édimbourg. Il revient à l'Imperial Cancer Research Fund en I9I5 et y poursuit ses recherches jusqu'à sa retraite en 1939 .

24 Vincent 1925. Thomas Swale Vincent (1868-1933) est un médecin et physiologiste britannique. Il est diplômé de médecine de l'Université de Birmingham en I892. Après un séjour de recherche à Heidelberg, il retourne à Birmingham où il est nommé démonstrateur de physiologie. En I897, il rejoint l'University College de Londres, où il travaille sous la direction d'Edward Albert Sharpey-Schäfer (I850-I935), à qui l'on doit la découverte de la fonction des surrénales et de l'adrénaline, ainsi que le néologisme «endocrine» pour désigner toute sécrétion d'hormones se déversant directement dans le sang. En I889, Vincent devient professeur assistant, sous la direction de Starling. En I90o, il part enseigner la physiologie à Cardiff. En I902, il revient dans le département de physiologie de Schäfer à Édimbourg. En I904, il obtient la chaire de physiologie de l'Université de Manitoba au Canada. En I920, il est nommé professeur de physiologie au Middlesex Hospital à Londres. Il est l'auteur d'un ouvrage de référence sur les sécrétions des glandes endocrines (Vincent I9I2).

25 Fujii I92I. Ijuro Fujii est un physiologiste japonais. En 1915, il rejoint le laboratoire de physiologie de Tohoku, dirigé par Satake. De I924 à I952, il occupe le poste de professeur de pharmacologie à l'Université préfectorale de médecine de Kyoto.

26 Trendelenburg I923. Paul Trendelenburg (I884-I93I) est un pharmacologue allemand, spécialiste du système adrénergique. Il étudie la médecine aux Universités de Grenoble et de Leipzig. En I9I9, il est nommé professeur de pharmacologie à l'Université de Rostock. De 1923 à 1927, il revient à l'Université de Fribourg, avant de terminer sa carrière à l'Université de Berlin. 
d'adrénaline peut être retardée ou diminuée ${ }^{27}$. Une preuve plus directe de l'accroissement de la sécrétion des surrénales par suite de l'exposition au froid fut fournie par mes étudiants, Hartmann, et ses collaborateurs ${ }^{28}$. Ils utilisèrent l'iris complètement énervé du chat ${ }^{29}$. Quand ces animaux étaient mouillés avec de l'eau froide ou refroidis après aspersion d'eau chaude, les pupilles se dilatèrent si les surrénales étaient en place, mais non si elles avaient été inactivées. Cependant, l'aversion naturelle du chat pour l'eau provoquait une certaine agitation, et par suite de ce fait, les effets ne furent pas absolument différenciés des conséquences de l'agitation elle-même.

Dans nos expériences, nous avons utilisé à nouveau le cœur énervé chez des animaux non anesthésiés. Nous avons utilisé diverses méthodes pour exposer les animaux au froid.

27 Ce passage nous paraît plus intelligible tel qu'il apparaît dans La Sagesse du corps: «Des faits histologiques avancés par Cramer et d'autres auteurs avaient indiqué une réduction, chez des animaux exposés au froid, de la substance dont provient l'adrénaline, et ces faits avaient été interprétés comme preuve de la stimulation de la médullaire surrénale par le froid. Mais, peut-être, chez l'animal refroidi, la production d'adrénaline est-elle retardée ou diminuée» (Cannon 1946[I939], I49). On comprend alors que les expériences de Hartman, ainsi que celles de Cannon, visent à produire des preuves plus directes de l'accroissement de la sécrétion des surrénales suivant l'exposition au froid.

28 Hartman/McCordock/Loder I923. Frank Alexander Hartman (I883-I973) est un chimiste et physiologiste américain, spécialisé en endocrinologie. En 1914, il obtient un doctorat de chimie de l'Université de Washington. De I9I5 à I9ı6, il enseigne et travaille à l'École de médecine de Harvard, sous la direction de Cannon. Il est nommé professeur à l'Université de Buffalo en I9I9 et devient en I934 professeur et directeur du département de physiologie de l'Université de l'État de l'Ohio. Howard Anderson McCordock (I895 1938) est un médecin américain. Il est nommé professeur de pathologie à l'École de médecine de l'Université de Washington. Nous n'avons pas trouvé d'informations concernant $M$. M. Loder, rattaché au département de physiologie de l'Université de Buffalo au moment de la publication de l'article

29 L'adrénaline provoque des contractions de l'iris. Les auteurs ont donc utilisé les variations de diamètre de la pupille pour mesurer le taux de sécrétion d'adrénaline. Afin de s'assurer que la variation du diamètre de la pupille était uniquement due à l'adrénaline présente dans la circulation, les auteurs ont dénervé l'iris, c'est-à-dire sectionné les nerfs ciliaires responsables de la motricité oculaire.
Notre première méthode fut de tenir l'animal au chaud dans un endroit calme sur un coussin placé près d'une fenêtre, et après avoir compté ou enregistré les pulsations cardiaques, nous ouvrions la fenêtre pour laisser entrer de l'air froid. Le grand avantage de cette méthode fut que l'élément d'agitation ou d'émotion était entièrement éliminé par ce que l'entourage était familier à l'animal et que le seul changement était l'ouverture de la fenêtre ${ }^{30}$. On a ainsi constaté

l'effet de l'exposition à l'air froid sur la vitesse du cœur dénervé. Les deux surrénales étaient intactes. La température de la chambre était de seize (I6) degrés centigrades, la température extérieure était de moins quatre (4) degrés. Vous observerez que la vitesse au départ était de cent dix-huit (II8) battements à la minute. Quatre (4) minutes après l'ouverture de la fenêtre, la vitesse cardiaque était accrue de dix (Io) battements à la minute. Treize (13) minutes après l'accroissement fut de vingtquatre (24) battements. À ce moment, la porte fut ouverte de façon à provoquer un fort courant d'air froid. À la fin des six (6) minutes qui suivirent - c'est à dire, dix-neuf (i9) minutes après le début de l'expérience - la vitesse avait atteint cent quarante-six (I46) pulsations à la minute - c'est-à-dire vingt-huit (28) pulsations au-dessus du niveau normal, soit une augmentation de vingt-quatre (24) pour cent. La fenêtre fut alors fermée. Neuf (9) minutes après, la vitesse était tombée de cent quarante-six (46) à cent trente-quatre (I34) - seize (I6) pulsations

Cannon projette la figure reproduite dans La Sagesse du corps (Cannon I946[1939], I50), initialement parue dans Cannon/Querido/Britton/ Bright 1927. 


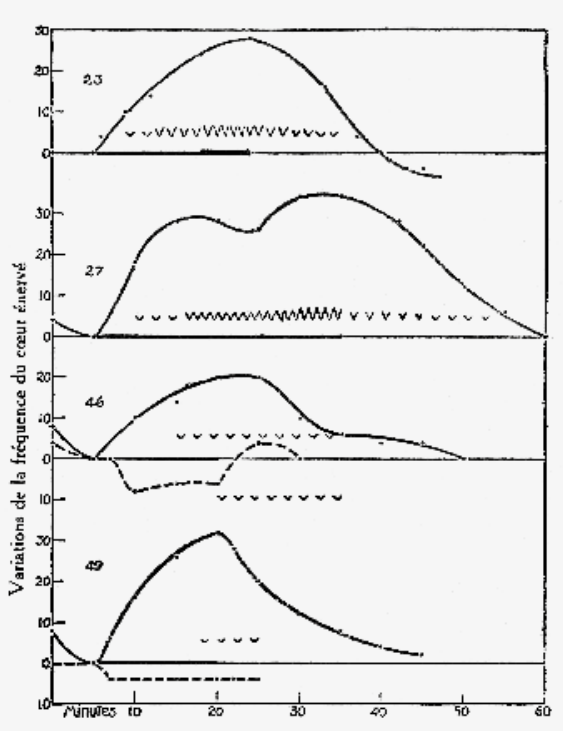

Figure 28. - Augmentations de fréquence du cceur énervé quand exposés à l'air froid. Le temps d'exposition est indiqué par un épaissisement de la ligne de base; le double épaississement du cas 23 marque le temps pendant lequel un courant d'air froid fut établi. Les frissons intermittents sont indiqués par des $\mathrm{V}$ reliés entre eux. La dimension des $\mathrm{V}$ indique grossierement l'intensite du frisson. Les lignes inter rompues des cas 46 et 48 montrent les variations de la frequence caa

Fig. $61^{31}$

La projection suivante ${ }^{31}$ montre graphiquement l'accroissement de la vitesse du cœur au-dessus d'un niveau normal chez les animaux avec surrénales intactes exposés à l'air froid de la manière que je viens de décrire. La période d'exposition est indiquée par un épaississement de la ligne du bas. Les zigzags ou les marques en forme de $\mathrm{V}$ représentent le frisson. Je vous prie d'observer particulièrement que la vitesse du cœur ne fut pas seulement accélérée pendant le frisson. Dans les cas quarante-six et quarante-neuf, les lignes brisées représentent les changements de la vitesse dans les mêmes conditions d'exposition au froid après exclusion de la sécrétion des médullaires

3

Cannon ajoute à la main, dans la marge gauche: «Supprimer tout cet alinéa.» L'image que Cannon a projetée, ou qu'il envisageait de projeter a été reproduite dans La Sagesse du corps (Cannon I946[1939], I52), initialement parue dans Cannon/Querido/Britton/Bright I927. surrénales. Remarquez que le tout premier effet fut une réduction de la vitesse au lieu d'une augmentation ${ }^{32}$.

Le défaut de la méthode que je viens de décrire gît dans sa limitation aux périodes froides de l'année. Pour posséder une méthode applicable en tout temps, nous avons imaginé introduire dans l'estomac une quantité connue d'eau froide. Naturellement, l'eau froide peut être administrée à n'importe quel moment et dans les locaux habituels. Cette méthode permet d'observer des modifications du rythme cardiaque et est quantitativement satisfaisante ${ }^{33}$.

Ce dernier avantage est très important. Le poids de l'animal, sa température rectale et sa chaleur spécifique, le volume d'eau introduite ainsi que la température et la chaleur spécifique de celle-ci, tout cela peut être connu. La chaleur de l'animal réchauffe l'eau froide dans l'estomac et l'intestin. En vérité, le sang circulant rend cette addition d'eau froide au corps approximativement équivalente au mélange de cette eau à la masse liquide qui constitue le milieu intérieur de l'organisme. Il est donc possible de calculer de combien une certaine quantité d'eau froide abaisserait la température du corps si aucune chaleur supplémentaire n'était ou produite ou conservée.

L'obligation dans laquelle se trouve l'organisme de produire de la chaleur, s'il veut maintenir sa température normale, peut donc être connue avec une belle approximation. Nous avons appelé cette obligation la «dette de chaleur». Le principal défaut de cette méthode est que l'animal au début de l'expérience est quelque peu troublé par suite de l'emploi nécessaire d'une sonde œsophagienne. Cependant, ce n'est qu'un trouble temporaire.

La dette de chaleur que nous avons établie chez des chats variait entre quinze cents (1500) et deux mille petites (2000) calories environ par kilogramme. Après que l'agitation initiale due à l'administration d'eau se fut dissipée, un plateau élevé persista dans lequel la vitesse du cœur énervé était de vingt-trois 


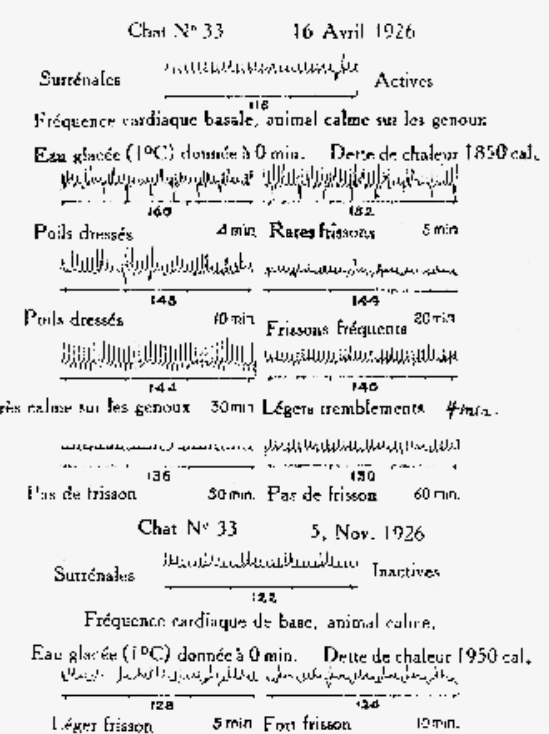

Figure 29. - Tracés originaux montrant les augmentations de fréquence du cour énervé quand le chat 33 , à surrénales actives, reçut, le 16 avril, $120 \mathrm{cc}$. d'eau à $1^{\circ}$ (dette de chaleur $=1.850$ petites calories) el quand, après inactivation des surrénales, il reçut, le 5 mai, $166 \mathrm{cc}$. d'eau $11^{\circ}$ (dette de chaleur $=1.950$ calories). En dessous de chaque trace on indique la recaction da chat en lintervalle séparant la prise du tracé
(23) à vingt-huit (28) pour cent au-dessus du niveau initial ${ }^{34}$. Un chat ${ }^{35}$ reçut une quantité d'eau à un degré centigrade, quantité qui établissait une dette de chaleur de mille huit cent cinquante (I850) petites calories. Il y eut une accélération initiale de quarante-deux pulsations. Cependant, cette accélération diminua bientôt de huit (8) battements et par la suite pendant plus d'une demi-heure, la vitesse du cour resta à un niveau élevé. En vérité, une heure après l'établissement de la dette de chaleur, la vitesse du cœur était encore supérieure de douze (I 2) pulsations à la vitesse initiale. Plus tard, après inactivation des

Fig. $62^{35}$ glandes surrénales, une dette de chaleur de mille neuf cent quarante (I940) calories fut établie, et en dix minutes, la vitesse du cœur dénervé était pratiquement revenue à sa valeur initiale.

Dans la projection suivante ${ }^{36}$ les mêmes résultats sont figurés graphiquement. Dans la ligne A, une dette de chaleur de deux mille petites calories fut établie par administration

34 Le début de la phrase a été biffé: « Le cliché suivant montre les tracés origi naux de l'augmentation du chronotropisme rythme cardiaque de la vitesse du cœur dénervé. »

35 Il s'agit du chat $\mathrm{n}^{\circ} 33$. Cannon projette la figure reproduite dans La Sagesse du corps (Cannon I946[I939], I54), initialement parue dans Cannon/Querido/ Britton/Bright I927.

36 Dans la marge gauche, Cannon ajoute: «supprimer cet alinéa » et annonce la projection d'un cliché. Cannon projette la figure reproduite dans La Sagesse du corps (Cannon I946[1939], I55), initialement parue dans Cannon/ Querido/Britton/Bright I927. d'eau à dix degrés centigrades, et dans la ligne $\mathrm{B}$, une dette de chaleur de dix-huit cent vingt calories par ingestion d'eau à un degré centigrade. Notez que l'eau la plus froide eut un effet considérablement prolongé. Dans la ligne $\mathrm{C}$, une dette de chaleur de deux cent cinquante petites calories fut établie par administration d'eau à trente-trois degrés. L'effet principal fut celui de l'agitation et vous remarquez qu'en dix minutes tout était rentré dans l'ordre.

La conclusion qu'on peut tirer de ces observations est que les conditions qui auraient une tendance naturelle à abaisser la température corporelle, provoquent une augmentation de la décharge d'adrénaline dans le sang.

Divers observateurs ont remarqué l'effet calorigénique des injections d'adrénaline. Boothby et Sandiford ${ }^{37}$ ont mesuré à ce point de vue la valeur d'un milligramme d'adrénaline et ont trouvé chez l'homme un accroissement de la production de chaleur égal à cinquante grandes calories. Diverses recherches du Laboratoire de Physiologie de Harvard ont montré que l'adrénaline sécrétée aide au maintien du métabolisme normal et qu'une sécrétion exagérée d'adrénaline augmente le métabolisme. Étant donné que la sécrétion des médullaires surrénales est augmentée quand l'organisme est exposé au danger d'une perte de chaleur trop rapide, étant donné également que l'adrénaline sécrétée en quantités physiologiques est capable d'augmenter la production de chaleur de l'organisme, il était possible de démontrer les services que cette réaction physiologique rend à l'organisme. Nous avons essayé de prouver l'utilité de ces réactions de deux manières: nous avons étudié les effets d'une certaine dette de chaleur sur le frisson quand les 
surrénales étaient actives ou inactives, et nous avons observé chez l'homme l'influence d'une dette de chaleur sur le métabolisme en absence ainsi qu'en présence du frisson.

Considérons d'abord l'effet d'une dette de chaleur sur le frisson quand les surrénales sont présentes ou absentes. Si la dette de chaleur est grande - c'est-à-dire, si elle s'élève à mille (I000) petites calories ou plus par kilogrammes, et si l'eau est à un degré centigrade et la température de la chambre d'environ vingt (20) degrés - elle est habituellement compensée par deux mécanismes calorigéniques: par une augmentation de la sécrétion d'adrénaline et par le frisson. Le frisson coïncide avec la période de la plus grosse décharge d'adrénaline ${ }^{38}$. Mais comme vous vous le rappelez, le frisson n'est pas une condition nécessaire à la décharge d'adrénaline - en vérité, le frisson peut être totalement absent tandis que la vitesse du cour s'accélère notablement ${ }^{39}$. Maintenant ${ }^{40}$, si la température environnante est de vingt (20) degrés environ, et qu'une dette de neuf cents (900) calories seulement est à acquitter, nous avons rarement vu survenir le frisson, et s'il survient, il est de courte durée. Sur quinze essais faits dans ces circonstances, le frisson n'apparut que dans deux cas, et ne dura que jusqu'à quinze, seize, et dix-sept minutes. Donc, quand la puissance calorigénique de la médullaire surrénale manque, l'organisme à recours au mécanisme du frisson.

Les autres observations récoltées dans le but d'essayer l'effet d'une dette de chaleur sur le métabolisme en l'absence de frisson, furent faites chez l'homme. Nous établîmes une dette de chaleur dont la moyenne était de quatre cent quarante-neuf petites calories par kilogramme. Dans vingt-deux

Cannon biffe le début de cette phrase: «Dans l’un des premiers clichés que je vous ai montrés aujourd'hui se trouvait démontré le fait que...» Cannon projette la figure reproduite dans La Sagesse du corps (Cannon I946[I939], I52), initialement parue dans Cannon/Querido/Britton/Bright 1927.

39 Cannon indique à la main, dans la marge gauche, qu'il a atteint les «3/4» de son exposé.

$40 \quad$ Cannon biffe le début de la phrase: «Comme le montre la projection sui vante. » Cannon projette la figure reproduite dans La Sagesse du corps (Cannon I946[I939], I57); nous ne sommes pas parvenus à en identifier la source originale.

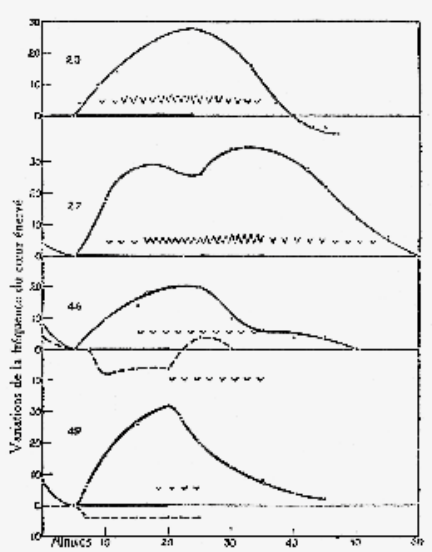

Figure28. - Augmentations def fréquenceducœurénervé quand les animaux a capsules surrénales intactes ( Nos 23, estindiqué épaississement du cas 23 marque le temps pendant lequel sont indiqués par des $\mathrm{V}$ reliés entre eux. La dimension des $\mathrm{V}$ indique grossièrement l'intensité du frisson. Les lignes expositionaufroi après exclusion de la sécrétion adrénalinique. un courant d'air froid fut établi. Les frissons intermittent interrompues des cas 46 et 48 montrent les variations de la

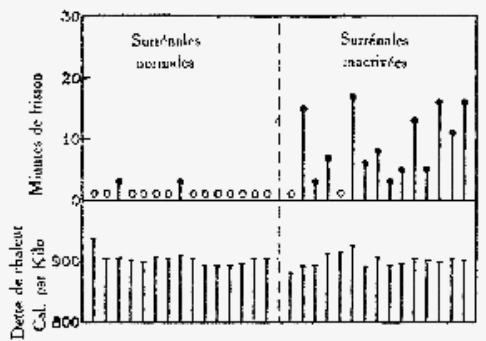

Figure31--Présenceouabsencedefrissonlorsd'unedette de chaleur de 900 calories, chez une série de chats à glandes . observations de onze sujets humains, il y eut une augmentation maximale en moyenne de métabolisme légèrement supérieure à seize (I6) pour cent, avec au-delà de cette moyenne des variations pouvant atteindre trente-huit (38) pour cent. Ces augmentations de métabolisme ne s'accompagnèrent pas du frisson. Vous pouvez supposer que l'effet fut dû au trouble que provoque la prise de l'eau froide et de la glace qui établissaient la dette de chaleur. Cependant, la plus forte augmentation survint en moyenne vingt-trois (23) minutes après la prise de l'eau et de glace, et par conséquent se produisit trop longtemps après l'ingestion pour être due à ce fait.

Vous voyez à l'écran ${ }^{41}$ les tracés de la consommation d'oxygène dans une de ces expériences ${ }^{42}$. La période préliminaire $(\mathrm{P})$ et la période finale (VI) ont approximativement la même pente.

41 Cannon projette la figure parue dans Cannon/Querido/Britton/Bright 1927. Elle n'est pas reproduite dans The Wisdom of the Body.

42 Cannon biffe cette phrase.

\section{Fig. $64^{38}$} Fig. $65^{40}$ 
Mais la pente est fortement modifiée dans la seconde période pendant laquelle l'effet de la dette de chaleur sur l'augmentation du métabolisme fut le plus considérable. En effet, l'augmentation de métabolisme dans ce cas fut approximativement de vingt-sept pour cent $(27 \%)$.

Quand une quantité d'eau chaude équivalente à la quantité d'eau froide était avalée, l'augmentation moyenne de métabolisme ne fut que de trois pour cent et un dixième $(3, \mathrm{I} \%)$, et puisque le moment d'augmentation maximale survint régulièrement dans les sept premières minutes de l'expérience, cette augmentation devait être attribuée principalement au trouble occasionné par l'ingurgitation de l'eau ${ }^{43}$. Une dette de chaleur de quarante-six (46) petites calories par kilogramme due à la prise de cinq cent vingt (520) centimètres cubes d'eau à trente degrés, provoqua chez J. L. H. une augmentation immédiate de quatre pour cent ( $4 \%$ ) du métabolisme. Plus tard, une dette de chaleur de quatre cent neuf (409) calories par kilogramme due à l'ingurgitation de trois cent cinquante-quatre (354) centimètres cubes d'eau à un degré, et de cent trente (I30) grammes de glace, déterminera une augmentation de métabolisme de treize pour cent et sept dixièmes (I3,7\%). Notez qu'une brève période de frisson fut suivie par une augmentation soudaine et passagère de cinquante-huit pour cent (58\%). Les observations sur A.J. E. sont exemptes de toute critique, car le frisson n'a pu intervenir dans le résultat final. Dans ce cas, une dette de quarante-deux (42) calories correspondent à quatre cent vingt centimètres cubes d'eau à trente degrés et deux dixièmes $(30,2)$ provoqua une augmentation immédiate de cinq pour cent (5\%) du métabolisme. Plus tard, la prise de deux cent soixante (260) centimètres d'eau à un degré et de cent trente-neuf (I39) grammes de glace, établit une dette de chaleur de quatre cent vingt-quatre (84) calories. Observez que le métabolisme augmenta graduellement jusqu'à quatorze pour cent (I4/I00) sans aucun frisson cependant.

43 La phrase suivante est biffée: «Ce fait ressort clairement de la projection suivante.» Cannon projette la figure parue dans La Sagesse du corps (Cannon I946[1939], I58), initialement parue dans Cannon/Querido/ Britton/ Bright 1927 .

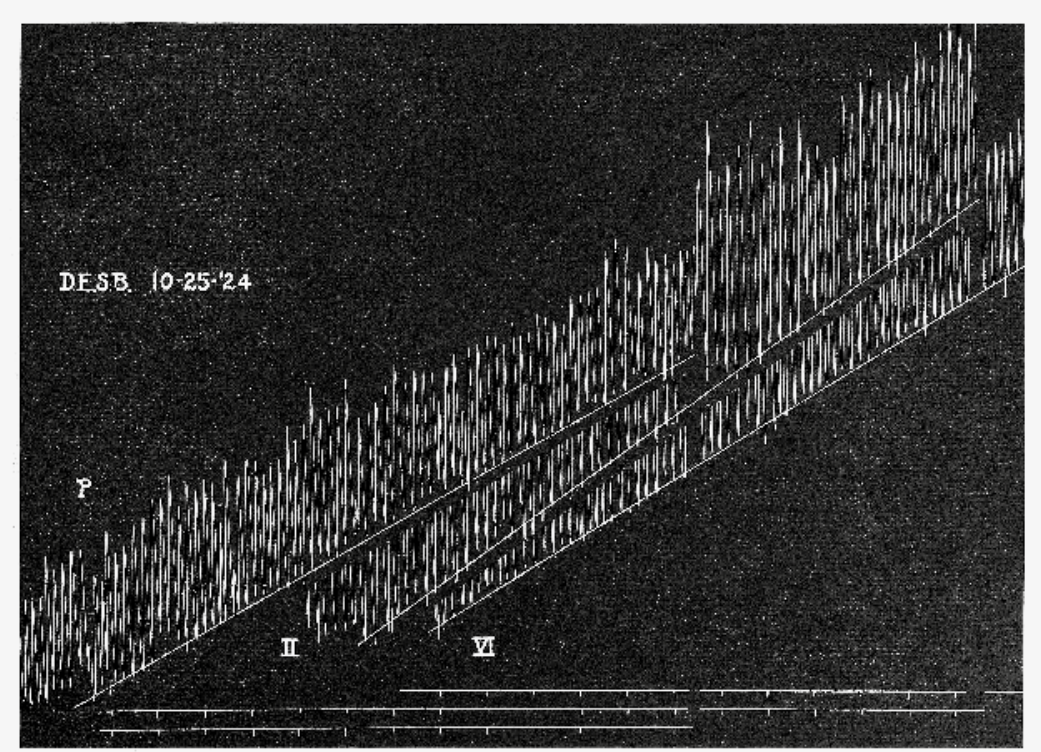

Fig. 5. Records of the preliminary period P, and of periods II and VI of the observations on D, E. S. B., October 25, 1924 (see table 4). Windows have been cut in P and II to permit the base line of the underlying records to be seen. Time in half-minutes. (n)

Les expériences précédentes ont montré que les mêmes conditions qui augmentent la sécrétion d'adrénaline chez les animaux, augmentent le métabolisme chez l'homme, et cela sans provoquer nécessairement le frisson. Il semble donc que nous ayons le droit de conclure qu'une perte de chaleur suffisante pour provoquer des troubles, stimule l'activité de la médullaire surrénale chez l'homme comme elle le fait chez l'animal, et que dans les deux organismes, l'excédent d'adrénaline sécrétée a le même effet d'accélération des combustions.

Pour résumer ce que nous venons de voir, nous pouvons dire que si la température tend à baisser, il y a érection des poils chez les mammifères - nous, avec notre peau dégénérée, nous n'avons plus que la chair de poule comme vestige de cette fonction - et un hérissement des plumes chez les oiseaux. Accompagnant ce phénomène, il y a constriction des vaisseaux superficiels. Par conséquent, la perte de chaleur par la surface du corps est fortement réduite. Si la conservation de la chaleur ne 


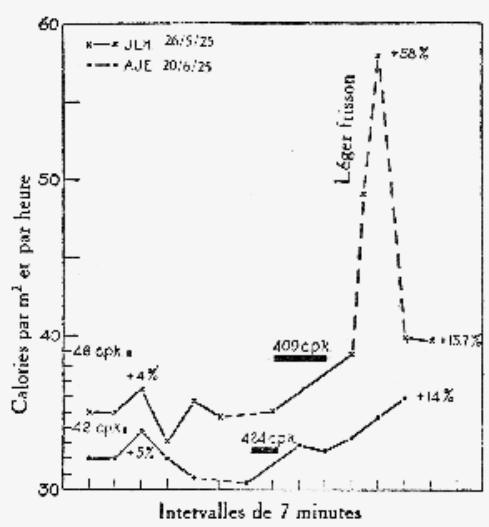

Figure 32. - Modifications du métabolisme produites par l'absorption d'eau chaude, puis d'eau froide et de glace. Une dette de cha520 cc. d'eau à $30^{\circ}$ provoqua chez J. L. H. une augmentation immé diate de $40 / 0$ du métabolisme; une dette de chaleur de 409 calories (absorption de 354 cc. d'eau à $1^{\circ}$ et de 130 gr. de glace) provoqual 'augmentation de 13.70/0. Léger frisson accompagné d'une augmentation rapide et temporaire de $580 / 0$. Une dette de chaleur de 42 calories correspondant à l'ingestion de $420 \mathrm{cc}$. d'eau à $30.2^{\circ}$ détermina chez A. J. E. une augmentation immédiate de $50 / 0$ du métabolisme; une dette de 424 calories, absorption de $260 \mathrm{cc}$. d'eau à $1^{\circ}$ et $139 \mathrm{gr}$. de Chacun des points où la ligne se brise représente une détermination du métabolisme.

peut empêcher une chute de température, il y a stimulation chimique à une production de chaleur supplémentaire par l'intermédiaire de la sécrétion des médullaires surrénales; et si ce mécanisme à son tour ne suffit pas à préserver le milieu intérieur du refroidissement, le frisson entre en jeu. Puis-je attirer votre attention sur le fait que dans toutes ces fonctions, le frisson mis à part, c'est le système sympathico-surrénal qui est en action. Comme l'ont montré des études récentes faites au Laboratoire de Physiologie de Harvard, le frisson lui-même a sa plus complète expression quand est encore intacte cette Fig. $67^{43}$ partie du cerveau, le diencéphale, qui est le centre coordinateur du système sympathique.

Le système sympathico-surrénal ne sert pas seulement à prévenir la chute de la température, il sert également à prévenir une élévation de température. Nous savons tous quelle grande quantité de chaleur est produite par un violent effort musculaire ${ }^{44}$ Si de grandes masses musculaires comme celles qu'on trouve dans les jambes et les bras fournissent un travail maximum rapidement répété, comme cela se passe dans les longues courses d'aviron, la quantité de chaleur produite peut être énorme. La quantité d'oxygène absorbé durant un exercice vigoureux et continu peut augmenter de vingt-cinq centièmes de litre $(0,25 \mathrm{l})$ à quatre litres et cinq dixième $(4,51)$ - soit une augmentation de dix-huit (I8) fois. Cela suffirait, si la chaleur n'était perdue, à élever la température du corps de six (6) degrés centigrades en seize (I6) minutes environ. Cette élévation serait dangereuse, car il existe des cellules nerveuses dans le cerveau qui ne pourraient supporter pendant une période prolongée des températures supérieures à quarante-deux degrés sans subir des modifications chimiques irréversibles. L'élévation de la température dans le milieu intérieur, dans la fluid matrix, est donc un fait de grande importance. Cependant, quand ce danger apparaît, le système sympathique entre en action. Il y a relâchement des vaisseaux sanguins périphériques, dont le tonus est contrôlé par le sympathique, et il y a excrétion de sueur. Le sang chaud de l'intérieur afflue dans les vaisseaux périphériques relâchés et se refroidit par convection et conduction si les objets extérieurs sont plus froids que le sang. La sueur s'évapore, si l'air n'est pas trop humide, et de cette façon refroidit la peau à travers laquelle le sang chaud circule. Donc, la tendance à l'élévation de la température est évitée par l'activité du système sympathique.

Si nous reprenons les deux phénomènes que nous avons considérés aujourd'hui - la production continue d'acide dans l'organisme et la production continue de chaleur - nous notons que dans ces deux exemples, l'homéostasie du milieu intérieur est maintenue par des processus continus qui ordinairement, à l'état de repos, fournissent un travail routinier. Cependant, aussitôt que la balance penche d'un côté ou de l'autre, ces processus continus se modifient de façon à ramener l'organisme à son état antérieur. Les mécanismes stabilisants dans les deux directions, comprennent l'activité de la partie sympathique du système autonome. Dans la prochaine conférence, je me propose d'examiner l'utilité générale de ce système comme régulateur du milieu interne, nous verrons également ce que devient la physiologie des animaux dont le sympathique a été enlevé ${ }^{45}$.

45 Cannon biffe la dernière phrase : «Je montrerai aussi un film de ces animaux, " 
Cinquième conférence

Revue des conditions homéostatiques et rôle du système sympathico-adrénal dans leur maintien; Physiologie des animaux dont le système sympathique a été enlevé; Division extérofective et intérofective du système nerveux; Interprétation des fonctions intérofectives du système autonome

Dans des publications récentes ${ }^{1}$, j’ai suivi Claude Bernard en insistant sur le fait que nous ne vivons pas dans l'atmosphère qui nous entoure. Nous sommes séparés de cette atmosphère par une couche de cellules mortes ou bien par une pellicule de mucus ou de solution saline. Tout ce qui vit à l'intérieur de ces surfaces sans vie est immergé dans les liquides du corps, le sang et la lymphe, qui forment le milieu intérieur. Des exemples frappants des dangers qui surviennent quand le milieu intérieur, ou fluid matrix, est fortement altéré, démontrent clairement l'importance primordiale du maintien aussi parfait que possible de sa constance.

Comme Claude Bernard l'a défini explicitement, par lesquels cette stabilité est assurée ${ }^{2}$. Comme je l'ai déjà dit, Claude Bernard fut le premier à suggérer un trait hautement important dans ce contrôle de la stabilité. Dans ses conférences sur la patbologie expérimentale, données en mille huit cent cinquante-neuf et soixante ${ }^{3}$, il montra qu'il y a deux milieux pour les corps animés - un milieu général qu'ils partagent

Cannon I929c; r929e.

La phrase est incomplète. On trouve cependant une phrase dont la construction est proche dans la version publiée des conférences: « Comme Claude Bernard l'a défini explicitement, la préservation de la stabilité est la condition d'une vie libre et indépendante " (Cannon I930b, 4I)

3 Comme Bernard l'indique dans l'avant-propos des Leçons de patbologie expérimentale (Bernard 1872), ces leçons furent délivrées au Collège de France en I859-1860. Initialement publiées en anglais dans la Medical Times and Gazette (Bernard I860), elles paraissent en I872 retraduites en français, réorganisées et complétées. avec les objets inanimés et qui environne l'organisme considéré comme un tout; et un milieu intérieur dans lequel les éléments vivants du corps trouvent leur habitat optimum. Tout d'abord, il considéra seulement le plasma sanguin comme représentant le milieu intérieur. Plus tard il parla du plasma sanguin et de la lymphe comme constituant le milieu intérieur, et finalement dans son traité sur les phénomènes de la vie, il classa sous ce nom la «totalité des liquides circulants de l'organisme».

Ce fut une remarquable contribution que Bernard apporta à la physiologie quand il reconnut que le plasma sanguin et les liquides interstitiels ou intra-organiques fournissaient aux éléments vivants de l'organisme un milieu approprié et favorable. Il montra que ce mécanisme permettait la nutrition des cellules cachées dans la profondeur des tissus, loin des surfaces qui séparent la structure vivante du monde extérieur inanimé, et qu'il fournissait un véhicule pour le transport des déchets, de ces cellules aux endroits d'excrétion. Il fit remarquer de bonne heure que ce fluide nourricier cette matrice liquide - fluid matrix - comme nous pouvons l'appeler est un produit de l'organisme lui-même et qu'il est sous le contrôle de mécanismes qui le maintiennent constant. Il comprit clairement que pour autant que cette stabilité est conservée, l'organisme est libéré des vicissitudes extérieures. "C'est la fixité du milieu intérieur qui est la condition d'une vie libre et indépendante ${ }^{4}$, dit-il. Cette fixité suppose dans l'organisme des mécanismes si parfaitement ajustés que toute tendance à la variation est rapidement compensée.

Ces vues fréquemment défendues par Bernard dans les diverses séries de conférences qu'il fit dans les vingt dernières années de sa vie, trouvèrent un bon accueil dans l'esprit d'autres physiologistes. En mille huit cent quatre-vingt-cinq, Léon Frédéric déclarait: "L'être vivant est agencé de telle manière que chaque influence perturbatrice provoque d'elle-même la mise en activité de l'appareil compensateur qui doit neutraliser et réparer le dommage. À mesure qu'on s'élève dans l'échelle

4 Bernard I878, II3 
des êtres, ces appareils régulateurs deviennent plus nombreux, plus parfaits, plus compliqués; ils tendent à affranchir complètement l'organisme des influences nuisibles et des changements survenus dans le milieu extérieur. $»^{5}$

Plus tard en dix-neuf cents, Richet attira l'attention sur ce phénomène général. «L'être vivant est stable », écrit-il « et il faut qu'il le soit pour n'être pas détruit, dissous, désagrégé par les forces colossales, souvent adverses, qui l'entourent. Mais par une sorte de contradiction qui n'est qu'apparente, il ne maintient sa stabilité que s'il est excitable et capable de se modifier suivant les irritations du dehors, et de conformer sa réponse à l'irritation; de sorte qu'il n'est stable que, parce qu'il est modifiable ${ }^{6}$ - cette instabilité modérée est la condition nécessaire de la véritable [stabilité], la préservation de cette constance est la condition d'une vie libre et indépendante. Quand nous nous déplaçons, nous emportons avec nous notre climat interne, et en conséquence, des modifications de la température, de l'humidité, du pourcentage d'oxygène du monde qui nous entoure, ont peu d'effets sur le royaume dans lequel nous vivons, si ces modifications ne sont pas excessives.

Nous avons remarqué, cependant, que cette stabilité confortable ou boméostasie comme j'ai proposé de l'appeler, n'est pas assurée sans la coopération de mécanismes admirables qui normalement sont toujours prêts à prévenir le désastre.

Cependant, des conditions peuvent survenir à l'intérieur ou à l'extérieur de l'organisme qui, s'il leur était permis de l'emporter, produiraient rapidement des troubles profonds.

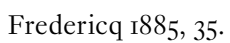

Le guillemet fermant est absent et la suite de la phrase semble incomplète. Cannon cite très librement les propos de Richet, que nous restituons afin de rendre tout ce passage intelligible: «En somme, l'être vivant est stable; et il faut qu'il le soit pour n'être pas détruit, dissous, désagrégé, par les forces colossales, souvent adverses, qui l'entourent. Mais, par une sorte de contradiction qui n'est qu'apparente, il ne maintient sa stabilité que s'il est excitable, capable de se modifier suivant les irritations du dehors et de conformer sa réponse à l'irritation; de sorte qu'il n'est stable que parce qu'il est modifiable. La défense n'est compatible qu'avec une certaine instabilité. Celle-ci doit s'exercer sans cesse, mais dans d'étroites limites; et cette modérée instabilité est la condition nécessaire de la véritable stabilité de l'être» (Richet I899, 72I).
Dans cette conférence, je présenterai des évidences que c'est la portion sympathique du système nerveux autonome qui agit pour maintenir la stabilité de l'organisme. Il fournit aux muscles et aux glandes des viscères - à l'estomac et aux intestins, au cœur et aux vaisseaux sanguins, au foie, aux muscles érecteurs des poils, aux glandes sudoripares, aux bronchioles et à la portion médullaire des capsules surrénales. Si on excite le sympathique, on détermine aussitôt la production par ces capsules d'une décharge de leur produit de sécrétion, l'adrénaline, dans le courant sanguin. Il existe une relation remarquable entre cette substance et les organes innervés par le sympathique.

Elle est capable de produire au niveau de ces organes le même effet que l'excitation des nerfs sympathiques. De plus, cette substance est extraordinairement active. Par exemple, diluée dans le sang à un pour un milliard quatre cents millions, elle a le pouvoir d'accélérer le cœur isolé du chat. Puisque l'adrénaline est déchargée dans le sang au moment où les nerfs sympathiques envoient des influx aux viscères, elle agit avec le mécanisme nerveux. Le mécanisme nerveux et le mécanisme humoral coopèrent donc à étendre leurs effets dans l'organisme, nous pouvons parler d'un système sympathico-surrénal. Maintenant considérons les effets de ce système.

Une blessure peut ouvrir les vaisseaux sanguins et permettre au milieu intérieur lui-même de s'échapper. Cependant, la coagulation du sang apparaît comme un mécanisme protecteur. Et par l'action du système sympathico-surrénal, l'efficacité de la coagulation en tant que moyen d'arrêter l'hémorragie, est augmentée grâce à l'accélération du processus de coagulation. Et si par hasard l'hémorragie est si forte qu'il y a perte considérable de sang, le système sympathico-surrénal à nouveau s'active et contracte les vaisseaux périphériques diminuant ainsi la circulation dans les endroits où l'hémorragie a le plus de chance de survenir et assurant aussi un apport de sang continu aux organes essentiels et constamment actifs, le cour et le cerveau.

Autre exemple le froid extérieur peut soutirer de la chaleur à l'organisme et par suite provoquer un abaissement de la 
température du milieu interne. Le système sympathique surrénal agit rapidement de façon à éloigner ce danger. Il contracte les vaisseaux périphériques et diminue ainsi le passage du sang chaud à la surface. Il cause l'érection des poils et des plumes chez des animaux pourvus de ces moyens de protection, de telle sorte qu'une couche d'air peu conducteur entoure le corps. Il libère l'adrénaline dans la circulation, adrénaline qui augmente l'intensité des processus d'oxydation de l'organisme au moment où un supplément de chaleur est nécessaire pour empêcher la température de tomber.

Il peut aussi se faire que l'apport d'oxygène soit réduit, soit à cause d'un séjour en haute altitude, soit à cause de l'action de certains poisons comme par exemple l'oxyde de carbone. Encore une fois, le système sympathico-surrénal vient soutenir l'homéostasie. Le cœur, sous son action, bat plus fréquemment, et il y a vasoconstriction de la région splanchnique de telle sorte que le flux sanguin est plus rapide dans les organes essentiels; la rate se contracte également et décharge au service de l'organisme des myriades de globules rouges supplémentaires.

Grâce à ces fonctions du système sympathique, ce qui pourrait être un trouble sérieux est diminué dans une proportion considérable.

Mais ce ne sont pas seulement les conditions extérieures qui affectent le milieu intérieur. L'activité du corps lui-même peut troubler l'homéostasie, et sans mécanismes protecteurs des désordres marquants peuvent en résulter.

Parmi les conditions intérieures pouvant provoquer des troubles, l'effort musculaire violent est au premier plan. Revoyons les modifications causées par un gros travail, et rendons-nous compte de la façon dont elles sont mises dans l'impossibilité d'altérer le milieu intérieur.

Lactivité musculaire s'accompagne d'utilisation de sucre. Une course prolongée réduit considérablement la glycémie et peut épuiser les réserves de glycogène hépatique. La réduction de la glycémie à quarante-cinq milligrammes pour cent, soit par l'extirpation du foie, soit par injection d'insuline, provoque des convulsions, et si la chute n'est pas arrêtée, le coma et la mort suivent. Cependant, avant que ne soit atteinte la période convulsive, le système sympathico-surrénal est mis en action, et du glucose se libère du foie. Et il s'en libère une quantité suffisante pour que le danger soit écarté, si toutefois l'agent hypo-glycémiant n'est pas d'une puissante écrasante, et le système sympathico-surrénal retombe en inactivité. Donc, en dépit de la grosse consommation de sucre des muscles au travail, la glycémie est maintenue à un niveau supérieur à la région dangereuse.

Une vigoureuse activité musculaire s'accompagne d'une production de chaleur. La quantité de chaleur produite quand les grandes masses musculaires du corps donnent un effort violent et prolongé, peut être incroyablement grande. À moins que cette chaleur supplémentaire ne soit dissipée, la température du milieu intérieur peut atteindre un niveau dangereux. Des températures de quarante degrés et demi chez l'homme ont été rapportées à la suite de l'effort. Deux degrés de plus avec prolongation, peuvent entraîner des désastres à cause de la sensibilité de certaines cellules du cerveau. En guise de protection contre ce danger, le système sympathique est appelé à la rescousse. Les vaisseaux périphériques se relâchent, la sueur abonde, et l'élimination de la chaleur est fortement accélérée. Par conséquent, une élévation de température est rapidement arrêtée et compensée, et les effets néfastes d'une température élevée sont évités.

En outre, quand on est engagé dans un violent effort musculaire, la réaction du sang tend à passer du côté acide. Cela est dû à une augmentation des acides carboniques et lactiques, produits de l'activité neuromusculaire. La production d'acide n'est pas sans danger. Il est bien connu que dans certains cas pathologiques l'acidose peut être telle que les fonctions du système nerveux sont altérées, et que, si cette acidose ne disparaît pas, le coma et la mort s'ensuivent. Bien avant que cet état ne soit atteint à la suite de l'effort musculaire, des processus correcteurs et protecteurs sont déclenchés et vigoureusement continués. Les points importants de la défense sont d'abord un apport rapide d'oxygène supplémentaire pour brûler l'acide lactique non volatil, qui autrement persisterait et altérerait le sang à l'état d'acide volatil carbonique 
qui peut être facilement éliminé; en second lieu, une accélération de la respiration de telle sorte que l'anhydride carbonique est emporté et que l'oxygène supplémentaire est attiré dans les poumons.

En résumé, les systèmes respiratoire et circulatoire doivent donner tout ce qu'ils peuvent. Encore une fois, le système sympathico-surrénal s'avance pour épargner tout trouble grave au milieu intérieur. Les agencements circulatoires - constriction des vaisseaux splanchniques, accélération du cœur, décharge des globules de réserve de la rate - sont tous réglés par l'intermédiaire du système sympathico-surrénal. De plus, ce système facilite probablement les processus respiratoires, car il a été démontré qu'il peut provoquer une dilatation rapide et efficace des bronchioles et par conséquent réduire la résistance qu'elles offrent aux mouvements de va-et-vient de l'air respiré. Grâce à ces bons offices variés, le système sympathico-surrénal jour un rôle des plus importants dans la préservation de l'homéostasie de l'équilibre acide-base du sang au moment où celui-ci est dangereusement menacé.

Le regard que nous venons de jeter sur cette question a montré que non seulement l'état du milieu extérieur mais aussi les réponses de l'organisme lui-même aux conditions de ce milieu ambiant, s'accompagnent de troubles du milieu interne. Ce milieu personnel, individuel, que nous emportons partout avec nous-mêmes, ne doit pas changer si nous voulons continuer une existence effective. Par conséquent, pour la stabilité du milieu intérieur, chaque mouvement considérable en relation avec le monde extérieur, doit être suivi d'un processus rectificatif dans le monde intérieur de l'organisme. Le principal agent de ces processus rectificatifs est comme nous venons de l'illustrer maintes fois, la partie sympathique du système autonome.

Maintenant que nous avons insisté sur la grande importance du sympathique ou des mécanismes sympathico-surrénaux, je me propose de vous parler de la physiologie des animaux dont cette partie du système nerveux autonome a été enlevée. Comme vous le savez fort bien, et la projection suivante $^{7}$ en est une illustration, une des caractéristiques des neurones sympathiques, est la disposition de leurs corps cellulaires en une chaîne de ganglions situés le long de chaque côté de la colonne vertébrale, du ganglion cervical supérieur haut dans le cou, au ganglion terminal dans le bassin. Les ganglions sont en connexion avec la moelle épinière depuis le premier nerf thoracique jusqu'un deuxième ou troisième lombaire. L'agglomération des centres nourriciers (les corps cellulaires) des neurones éloignés, dans la chaîne ganglionnaire, spécialement dans le thorax et l'abdomen, où se font les communications directes avec nerveux autonome. Le cerveau et la moelle épinière sont représence schéma Les fibres préganglionnaires sont en traits interronpus, les fibres postganglionnaires en traits pleins. Pour détails, voir texte. (Bard, d'après Cannon, « Foundations of experimental Psychology », avec l'autorisation de la « Clark University

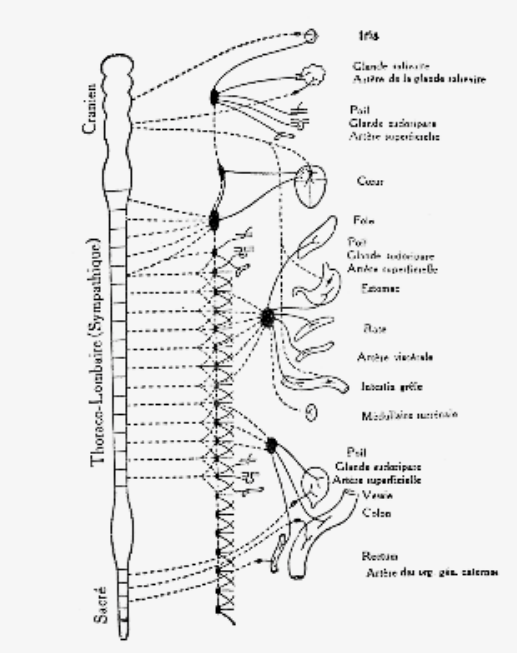

Figure 34. - Diagramme de l'anatomie générale du système

la moelle, permet l'extirpation totale de ce système. Si les ganglions sont enlevés, les fibres post-ganglionnaires, venant du système nerveux central, sont incapables d'entrer en connexion effective avec les organes normalement innervés par des fibres postganglionnaires. C'est pourquoi l'enlèvement des chaînes sympathiques doit avoir pour résultat inévitable de supprimer de façon permanente, tout contrôle du système nerveux central sur ces organes.

Nous avons utilisé ${ }^{8}$ deux méthodes pour l'extirpation des chaînes sympathiques. Tout d'abord nous enlevions séparément les parties cervicale, thoracique et abdominale.

7 Cannon projette la figure reproduite dans La Sagesse $d u$ corps (Cannon I946[1939], 203). Cette figure est aussi reproduite dans Bodily Changes in Pain, Hunger, Fear and Rage (Cannon 1915, 454).

8 Cannon/Newton/Bright/Menkin/Moore 1929. Harlan F. Newton et Robert M. Moore sont deux étudiants en chirurgie de Harvard, qui assistent Cannon à partir de 1929 . 


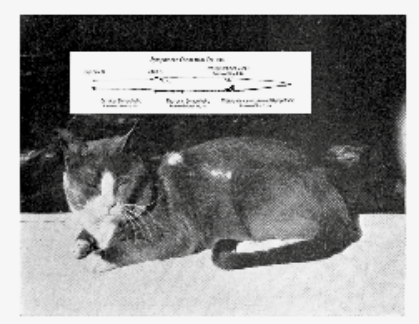

Fig. 5. Photograph of cat 104 taken February 12, 1927, nine ponths after removal of the semilunar ganglia and the las mounted on the card above the cat.

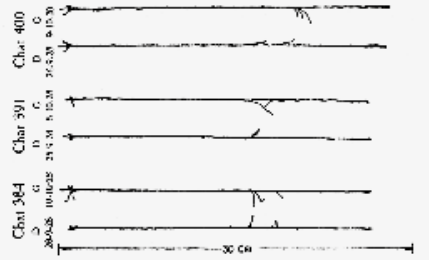

Figure 36. - Exemples de chaines sympad'une seule pièce. Elles furent séchées, mevertées sur collodion et disposées sur un carton avant d'être photographiées.
Fig. $69^{9}$ Fig. $70^{10}$
Le cliché ${ }^{9}$ suivant vous montrera le résultat de ces opérations. C'est la photographie d'un chat, neuf mois après enlèvement du ganglion semi-lunaire et de la dernière partie de chaîne sympathique. Les parties extirpées furent montées sur une carte, et la carte est photographiée en même temps que l'animal qui les possédait avant l'intervention. La date de l'enlèvement de chaque partie est indiquée, comme vous pouvez le noter. Le défaut de cette méthode est qu'on peut parfois négliger d'exciser un ou deux ganglions de la partie inférieure du thorax près du diaphragme. Pour éviter cette possibilité, nous avons enlevé les chaînes abdominales et thoraciques d'une seule pièce depuis les ganglions stellaires jusqu'aux ganglions pelviens. Le cliché suivant ${ }^{10}$ montre des exemples de cette extirpation totale. On ne peut mettre en doute le fait que le système sympathique en tant qu'appareil fonctionnel est enlevé en totalité Je dis en tant «qu'appareil fonctionnel » car le sympathique cervical est laissé en place. Cependant, les fibres préganglionnaires des ganglions sympathiques cervicaux passent par le ganglion stellaire, et par conséquent l'enlèvement des stellaires isole nécessairement les ganglions cervicaux du système nerveux central. Comme preuve de ce que les ganglions cervicaux

9 Cannon projette la photographie parue dans Cannon/Newton/Bright/ Menkin/Moore 1929. La photographie n'est pas reprise dans La Sagesse du corps.

10 Cannon projette la figure reproduite dans La Sagesse du corps (Cannon I946[1939], 216), initialement parue dans Cannon/Newton/Bright/Menkin/ Moore I929. ne fonctionnent pas dans de telles circonstances, veuillez remarquer la projection suivante ${ }^{11}$. La chaîne thoracique et abdominale droite a été extirpée in toto. L'animal placé dans le froid, les poils encore innervés du côté gauche sont hérissés. Notez que les poils de la tête du côté droit, bien qu'encore innervés par le ganglion cervical isolé, ne participent pas à la réponse au froid. C'est pourquoi il est certain que l'enlèvement des parties thoraciques et abdominales de la chaîne élimine complètement tout contrôle du sympathique sur les viscères.

Ce qui au premier abord frappe l'observateur, c'est que ces animaux sympathectomisés continuent à vivre sans difficulté apparente. Je vous ai montré à l'écran des illustrations de ce fait. Nous possédons présentement au Laboratoire de Harvard un animal qui n'a plus aucune partie du système sympathique depuis le dix octobre mille neuf cent vingt-sept. Ces faits que je viens de relater anéantissent certaines idées émises par des chercheurs antérieurs. Meltzer, par exemple, enlevait les ganglions cervicaux supérieurs de lapins et de chats et à cause de la très grande mortalité, il concluait que ces ganglions contiennent un principe essentiel au maintien de la vie. Plus tard, Spadolini ${ }^{12}$ déclara que l'extirpation complète et étendue

II Cannon projette la photographie reproduite dans La Sagesse du corps (Cannon I946[1939], 2I8 bis), initialement parue dans Cannon/Newton/Bright/ Menkin/Moore I929.

12 Spadolini 1926. "L'extirpation complète et étendue de ces nerfs est incompatible avec la vie. La survie des animaux oscille, en général, entre des limites plutôt vastes: de 3 jours à un mois environ. Les phénomènes généraux les plus caractéristiques de cette lésion consistent dans une dépression accentuée de l'état général de l'animal, dans son dépérissement progressif et grave, dans la diminution de la température du corps et de la fréquence des actes respiratoires.» (Spadolini I926, I8) Igino Spadolini (I887-I966) est un physiologiste italien, dont les principaux travaux portent sur les mécanismes antagonistes des systèmes sympathique et parasympathique. Il enseigne la chimie biologique à l'Université de Florence dès I92I. En I926, il est nommé professeur à l'Université de Sienne, puis à l'Université de Pise en I927. En I942, il rejoint l'Université de Florence. 
des nerfs mésentériques du chat est incompatible avec la continuation de l'existence.

Il observa une dépression générale, un amaigrissement grave, l'abaissement de la température corporelle, et des lésions sérieuses du tractus gastro-intestinal. Bien que nous ayons enlevé les chaînes sympathiques abdominales, y compris les nerfs splanchniques de plus de cent chats, et bien qu'à certains d'entre eux nous ayons enlevé également le ganglion semi-lunaire, dépouillé le tronc coliaque et l'artère mésentérique de leurs filets nerveux ${ }^{13}$, nous n'avons jamais vu aucun symptôme sérieux consécutif à cette intervention.

Finalement, Abel ${ }^{14}$ a prétendu que le tissu chromophile, trouvé dans les ganglions sympathiques, était nécessaire à la vie. Cependant, nous avons enlevé tous les ganglions sympathiques, depuis le ganglion cervical supérieur jusqu'au ganglion pelvien, nous avons disséqué et enlevé le ganglion semi-lunaire, nous avons extirpé le surrénal droit et aspiré la médullaire de la surrénale gauche. L'animal (un chat) a vécu normalement dans le laboratoire plusieurs mois avant que nous le sacrifiions. L'autopsie montra que les extirpations avaient bien été totales. Aucun tissu chromophile n'avait pu être oublié. Toutes ces observations nous forcent à conclure que le ganglion cervical supérieur, ainsi que les ganglions sympathiques et les nerfs mésentériques, ne sont pas indispensables, et que de même l'existence de tissu chromophile dans l'organisme n'est pas d'importance vitale. Vous pourriez penser que le système végétatif est important pour la croissance. Cependant, nous avons pratiqué la sympathectomie unilatérale chez de jeunes chats et nous les avons laissés croître jusqu'à l'état adulte. Nous les avons alors sacrifiés, et nous avons comparé les poids de différents organes et formations à symétrie bilatérale, y compris les os. Il est impossible de mettre en évidence aucune différence entre les deux côtés. Il semble donc que le système sympathique n'intervient pas dans la croissance du squelette ou des organes internes.

13 Le ganglion semi-lunaire, ou ganglion trigéminal, est le ganglion du nerf trijumeau. Le tronc cœliaque désigne l'artère qui approvisionne le foie en sang oxygéné. L'artère mésentérique vascularise quant à elle l'intestin.

14 Abel I926.

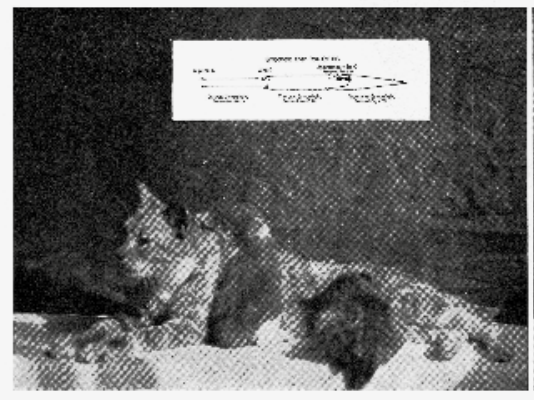

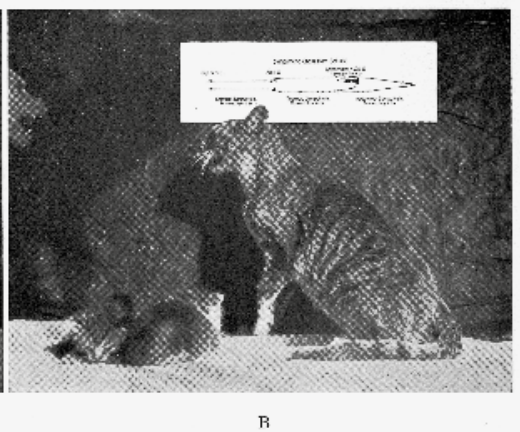

Fig. 3. Photographs of cat 106; her sympathetic ganglionated strands - cervical, thoracic and abdominal (including the semilunar ganglia) - mounted on the card above; and also her kittens born after she was completely sympathectomized.

A. One of the kittens nursing, the other playing on the mother's back.
B. A barking dog at the left has roused her to protect her kittens. Note no erection of the hairs on her neck, back, or tail.

Nous avons mesuré soigneusement le métabolisme basal avant l'opération et après l'enlèvement de chacune des trois portions des chaînes sympathiques - cervicale, thoracique et abdominale. Habituellement, il existe une légère chute de métabolisme après sympathectomie. Nos observations montrent que ce fait apparut le plus fréquemment après extirpation de la portion cervicale. Cependant, il est certain que l'opération ne réduit pas le métabolisme basal de plus de dix pour cent. Et en vérité, cette réduction peut être considérée comme négligeable.

Mais en voilà assez sur le manque d'altération frappante après sympathectomie. Voyons un peu quelques-uns des défauts de l'organisme sympathectomisé.

Dans un mémoire publié l'année dernière, je disais que la sympathectomie n'empêche pas la femelle d'accomplir ses fonctions de reproduction et de lactation ${ }^{15}$. L'observation sur laquelle cette déclaration était basée fut faite chez un animal sympathectomisé peu de temps avant la naissance de sa progéniture. Le cliché ${ }^{16}$ suivant montre cet animal avec ses deux petits; elle en nourrit un. Puis-je vous demander de noter également

15 Cannon/Newton/Bright/Menkin/Moore 1929, Io6.

16 Cannon projette la photographie 3.A, parue dans Cannon/Newton/Bright/ Menkin/Moore I929, 9I bis). La photographie est reproduite dans La Sagesse $d u$ corps (Cannon I946[I939], 2I8).

$$
\text { Fig. } 72^{16}
$$


l'autre côté de cette image sur laquelle l'animal réagit à un chien aboyant en rétractant les oreilles, montrant les dents, et levant la patte prête à frapper ${ }^{17}$. Observez que les poils du dos et de la queue ne sont pas hérissés. Au-dessus de l'animal se trouvent ses deux chaînes sympathiques. Maintenant revenons à la lactation. Cet animal, en vérité nourrit un de ses petits jusqu'à ce qu'il fût capable de vivre par lui-même. Plus tard, cependant, une autre femelle dont le sympathique avait été enlevé depuis vingt mois donna naissance à trois chatons. Et chose extraordinaire, les mamelles n'étaient pas préparées à la lactation, et un fait plus extraordinaire encore fut l'absence totale de soins maternels de la part de la mère. Elle ne faisait aucunement attention à ses petits. Quand on la faisait se coucher et qu'on lui apportait des chatons à nourrir, elle se levait immédiatement et les quittait sans avoir le moindre soin de leur bien-être ${ }^{18}$. Des nouvelles études sur la relation possible entre les troubles de la lactation et l'extirpation du sympathique sont à l'heure actuelle en cours au Laboratoire de Physiologie de Harvard ${ }^{19}$.

Quand les animaux sont complètement sympathectomisés, il y a une forte réduction de leur capacité de travail. Nous avons complètement sympathectomisé un chien qui avait été entraîné à courir sur un tapis roulant jusqu'à l'épuisement ${ }^{20}$. Le tapis roulant étant animé d'une vitesse uniforme, la course durait approximativement deux heures, avec de courtes interruptions vers la fin pour ménager quelques périodes de repos. Après sympathectomie, la capacité de travail de l'animal fut réduite de trente-cinq

Dans La Sagesse du corps, seule la partie de gauche (A) est reprise avec cette légende: "Photographie du chat ro6; ses chaînes ganglionnaires sympathiques - cervicale, thoracique et abdominale, ganglions semi-lunaires inclus - sont exposés sur le carton au-dessus de l'animal: on voit aussi les petits qu'elle a mis monde après avoir été complètement sympathectomisée. Un des petits chats est en train de téter, l'autre joue sur le dos de sa mère.»

18 Cannon/Bright I93I.

19 Ces travaux sont détaillés dans The Wisdom of the Body (Cannon 1939[I932], 273), ainsi que dans sa traduction française (Cannon I946[I939], 2I9). Cannon se réfère très certainement aux études en cours de Bacq, qui montreront que la sympathectomie impacte les capacités reproductives des rongeurs (Bacq 193I), ainsi que le développement de la lactation chez le rat (Bacq 1932). pour cent à peu près. Bien entendu, les nerfs vagues étaient intacts et par diminution du tonus vagal, le cœur pouvait s'accélérer quelque peu. Cependant, la redistribution du sang dans l'organisme, l'élévation de la pression sanguine, la dilatation des bronchioles et la libération d'adrénaline, faisaient défaut, nous avons vu que tous ces facteurs favorisaient l'effort musculaire prolongé. Dans ces conditions, le fait que l'animal ne peut plus fournir la même course qu'avant l'opération est très compréhensible.

En plus de l'absence d'adaptation du système circulatoire, la libération du sucre hépatique fait défaut.

Ce fait [a été] prouvé en déterminant les effets de l'excitation émotive sur la glycémie avant et après sympathectomie. Comme la prochaine projection ${ }^{21}$ vous le montre, une agitation de trois à dix minutes provoque chez l'animal normal une augmentation moyenne du sucre sanguin de trente-quatre pour cent. D'autre part, chez les animaux sympathectomisés une même durée d'excitation ne provoque aucune élévation appréciable de la glycémie.

Accompagnant cette déficience évidente dans la fonction du système sympathique l'augmentation normale des globules rouges qui suit l'agitation fait défaut. Cette absence de poly-globule est due à la non-coopération de la rate. La section des nerfs de la rate laisse sans fonction cet organe si important pour la décharge de globules supplémentaires en cas de besoin. Toutes ces différences (déficiences circulatoires, défauts des

21 Cannon projette la figure reproduite dans La Sagesse du corps (Cannon I946[I939], 222). Nous ne sommes pas parvenus à en identifier l'origine. 
mécanismes qui libèrent du sucre sanguin et qui déchargent des globules rouges de réserve) jouent un rôle indubitable dans la réduction de l'efficacité de l'effort musculaire de l'animal. Il faut admettre que beaucoup des détails de ce tableau doivent être approfondis avant de pouvoir établir définitivement leur valeur quantitative, mais je suis persuadé que les faits principaux sont bien tels que je les ai indiqués.

Les animaux sympathectomisés ne sont pas seulement déficients quant au travail musculaire, ils le sont également si on les expose au froid. Nous avons déjà vu aujourd'hui un cliché qui montrait qu'après sympathectomie, l'exposition au froid n'est plus suivie d'érection des poils ; c'est-à-dire que le froid n'a plus pour effet de contracter les muscles pilomoteurs. De même les muscles lisses des vaisseaux sanguins ne sont plus sous le contrôle du système nerveux, et encore une fois, le froid n'a plus pour effet la contraction locale des vaisseaux. Pour ces deux raisons, la perte de la chaleur n'est plus contrôlée. Bien plus, l'organisme a été privé des bénéfices d'une sécrétion d'adrénaline qui augmente la production de chaleur quand la température corporelle tend à tomber. Pour ces divers motifs, l'animal sympathectomisé, mis dans la nécessité de maintenir sa température normale, est physiologiquement déficient. Le comportement d'un tel animal par temps froid est en conformité avec son état défectueux. Il montre une antipathie marquée pour le froid et les courants d'air. Pendant la période froide de l'hiver, il se blottit dans le voisinage des sources de chaleur et ne les quitte qu'au moment des repas.

Pour apprécier la réaction au froid des animaux sympathectomisés, nous ${ }^{22}$ les avons placés dans une chambre froide, dont la température était de huit dixièmes à six degrés centigrades, et nous avons observé la température rectale et le frisson. Des résultats typiques sont l'objet de la projection suivante ${ }^{23}$. Les lignes en trait plein marquent les changements de température des animaux normaux pendant un séjour de deux heures environ

22 Cannon/Newton/Bright/Menkin/Moore I929.

23 Cannon projette la figure reproduite dans La Sagesse du corps (Cannon I946[1939], 223), initialement parue dans Cannon/Newton/Bright/Menkin/ Morre 1929 dans le milieu froid. Les lignes brisées représentent les modifications chez les animaux sympathectomisés mis dans les mêmes conditions.

Notez que les observations furent faites sur les chats trois cent quatre-vingt-treize et trois cent quatre-vingt-seize et avant et après sympathectomie. Comme le montrent clairement les tracés de l'animal trois cent quatre-vingt-treize, il y a une différence frappante entre les réponses de l'animal avant et après l'intervention. Après sympathectomie il se produisit une chute rapide de température jusqu'à un point bas auquel elle put se maintenir assez constante. Le frisson fut également beaucoup plus

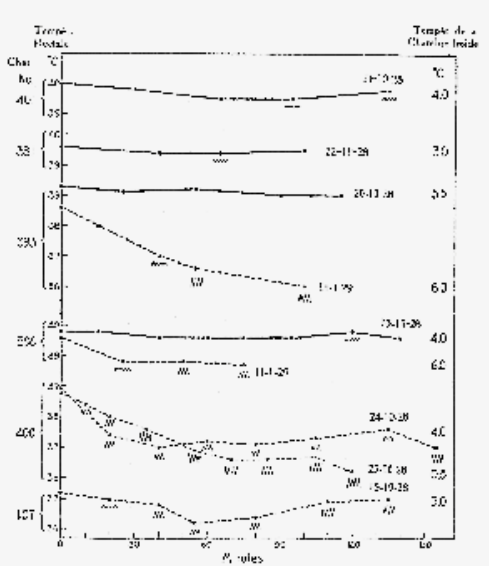

Figure 40. - Tracés de la température interne de chats gardés dans une chambre froide. Les traits pleins montrentle comportement des chats normaux; les trait sontmarques pardeszigzags, au-dessous dutracé; 'linportancedeces
du frisson. pointillés, celuideschatssympathectomisés. Lesfrisson

fréquent et plus violent. Cette réaction, indiquée par les lignes en zigzag, n'apparut pas du tout chez le chat trois cent quatrevingt-treize, avant sympathectomie, mais fut très marquée après l'opération. Ce phénomène fut très visible, comme vous pouvez le remarquer, chez les autres animaux privés de leur système sympathique. Puisqu'à part l'exercice le frisson était la seule ressource laissée à ces animaux pour les protéger contre un abaissement considérable de la température, le niveau réellement bas qui fut maintenu, comme par exemple chez le chat quatre cent, est probablement l'indication d'un équilibre entre la perte de chaleur dans le milieu froid et la production de chaleur par le processus du frisson. J'attire spécialement votre attention sur le chat cent sept. C'est l'animal dont j'ai parlé auparavant, qui n'avait plus de système sympathique depuis octobre dix-neuf cent vingt-sept. Cet animal paraît s'être habitué à frissonner, cette réaction étant sa protection principale contre une chute de température. Placé dans la chambre froide, il se mit très rapidement à frissonner avec vigueur, et de cette façon prévint une chute marquée de sa température et en réalité s'arrangeait pour la rétablir à son niveau normal de température. 
Il n'y a pas seulement difficultés à arrêter la tendance à la chute de température. Nous avons des preuves de la difficulté à freiner la tendance à l'élévation. Pour prouver qu'un animal qui prend habituellement la position verticale peut survivre à la sympathectomie, nous avons enlevé les chaînes sympathiques chez les singes. Et pour permettre à ces singes de jouir du soleil et du grand air, nous en avons mis un certain nombre dans la cour proche du laboratoire en juillet dernier. Les animaux ne furent nullement inquiétés par la chaleur. Mais l'animal sans sympathique fut trouvé peu de temps après avec une température extrêmement élevée en réalité, il avait un coup de chaleur dont il mourut.

En repassant les résultats que je viens de vous rapporter vous avez pu être impressionnés plus fortement pas les conséquences bénignes de l'enlèvement du système sympathique que par le phénomène de réelle déficience. Il faut admettre, cependant, que ces conséquences bénignes peuvent être trompeuses. Les animaux continuent à vivre, cela est certain, mais ils vivent dans les limites protectrices du laboratoire, où il n'y a pas de modifications importantes de température quelle que soit la saison, où il ne faut ni se battre pour la nourriture, ni échapper aux ennemis, ou le danger d'hémorragie est inexistant en réalité, c'est là une existence très spéciale et très limitée. Si l'on en juge par de telles observations, on peut aisément être amené à conclure que le système sympathique est d'importance minime pour le propre fonctionnement de l'organisme. Nous croyons qu'une telle conclusion est erronée. Si ces animaux étaient mis en liberté dans le monde extérieur et devaient rencontrer les exigences de la lutte, il n'y aurait aucune élévation de la glycémie pour le cas où le glucose deviendrait nécessaire, aucune polyglobulie, aucune vasoconstriction splanchnique avec l'augmentation de la pression sanguine et de la vitesse de la circulation qui en résulte, aucune accélération considérable du cœur, aucune modification circulatoire considérable du cœur, aucune modification circulatoire au bénéfice des muscles en contraction, aucune sécrétion d'adrénaline pour hâter la coagulation et pour venir en aide aux muscles fatigués.
Les déficiences de l'animal sympathectomisé sont évidentes quand il est exposé au froid ou au chaud. Quand des nécessités surviennent, il est incapable de préserver la constance de son milieu intérieur.

En dix-neuf cent quatorze ${ }^{24}$, je faisais remarquer que les trois divisions du système autonome ont des fonctions générales distinctives. La partie crâniale détermine un groupe de réflexes dont la fonction est protectrice, conservatrice et constructive. Il donne au cœur l'occasion de se reposer et de récupérer des forces en réduisant sa vitesse pendant les moments calmes, il favorise les mouvements gastro-intestinaux; c'est lui qui excite la sécrétion des sucs digestifs et par là assure les réserves en matériel capable de fournir de l'énergie; c'est lui encore qui paraît collaborer au mécanisme vago-insulaire qui aide à la mise en réserve de quelques-uns de ces matériaux. Cependant, les parties sacrées et crâniennes ne jouent qu'un rôle indirect et quelque peu lointain dans la protection de l'homéostasie.

C'est la division médiane ou thoraco-lumbaire ${ }^{25}$ du système autonome qui agit directement et rapidement pour prévenir les modifications du milieu intérieur. En mobilisant les réserves de l'organisme et en modifiant la vitesse des processus continus, cette partie fait en sorte de conserver la stabilité du milieu interne et peut donc être considérée à juste titre comme l'organisation spéciale et immédiate de l'homéostasie.

Dans nos connaissances des conditions homéostatiques et des mécanismes qui les conditionnent, il y a de grosses lacunes. D'innombrables questions demeurent sans réponse. Quels sont les stimuli effectifs de telles réactions hémostatiques bien connues? Comment les réactions homéostatiques sont-elles déterminées comme, par exemple, les globules rouges supplémentaires qui prennent naissance dans les organismes vivant à de grandes altitudes, et la croissance d'un pelage épais qui se produit par temps froid prolongé - réactions dont nous n'avons pas parlé antérieurement? Qu'est-ce qui maintient la constance

24 Cannon I9I4b

25 Cannon traduit librement le terme thoraco-lumbar. Dans La Sagesse du corps, nous lisons «thoraco-lombaire» (Cannon I946[I939], 2I0), soit la structure fibreuse située dans la région lombaire postérieure. 
des produits endocriniens dans le sang circulant, la constance de la thyroxine par exemple, comme l'indique la stabilité du métabolisme basal? Les questions que soulèvent ces conditions et réponses homéostatiques et bien d'autres encore utiles à la préservation de l'uniformité du milieu intérieur, offrent un champ fascinant à la recherche.

Dans la conférence que je vous ai présentée, je sais que la stabilité des organismes a été décrite d'une façon tout à fait inadéquate et provisoire. J'espère, cependant, que ces défauts me seront pardonnés à cause de l'importance du sujet qui nous a occupés. Ma plus grande satisfaction serait de vous avoir intéressés à ces faits et à leur importance. Ils ont, à mon avis, une grande signification comme aspects primordiaux de l'organisation biologique et de l'activité. Ils sont également significatifs en nous donnant la connaissance et la compréhension des désordres complexes de l'organisme.

Ils ont encore une signification en physiologie comparée, car l'homéostasie chez les animaux supérieurs est probablement le résultat d'essais innombrables au cours de l'évolution organique; et la connaissance de la stabilité qui a été finalement atteinte est éminemment suggestive en comparaison avec les mécanismes moins efficaces opérant chez les animaux inférieurs.

Finalement ${ }^{26}$, les analyses ultérieures de processus biologiques exprimées en termes physiques et chimiques doivent attendre une compréhension complète des voies que suivent les processus homéostatiques pour remplir leurs fonctions et retourner ensuite à l'inactivité.

En réalité, la régulation de l'organisme est le problème central de la physiologie.

Ces dernières considérations, opposant toute approche physico-chimique à la physiologie homéostatique de Cannon, attestent que ce dernier souscrit aux principes de la «nouvelle physiologie» développée par Haldane (à ce sujet, voir l'introduction): la physiologie n'est pas une science des composants biologiques, mais une science de la coordination des parties entre elles. Cannon ajoute ici l'idée d'un ordre des découvertes: une recherche sur les composants physico-chimiques de l'organisme ne peut contribuer à la compréhension des processus vitaux qu'à la condition que les processus homéostatiques aient été préalablement mis au jour.
Pour toutes ces raisons, il est désirable de voir s'engager de nouvelles recherches sur les agissements des mécanismes qui maintiennent l'homéostasie biologique. 


\section{Bibliographie}

Abel,John J., "On Epinephrin, the Active Constituent of the Suprarenal Capsule and Its Compounds ", Proceedings of the Physical Society 3 (I898) 3-5

"Some Recent Advances in Our Knowledge of the Ductless Glands", Bulletin of the Johns Hopkins Hospital 38 (1926) I-32

Adolph, Edward F., «The Excretion of Water by the Kidneys ", American Journal of Physiology 65 (I923)

Adolph, Edward F./Associates, Physiology of Man in the Desert (New York 1947)

Alvarez, Walter C., «Ways in Which Emotion Can Affect the Digestive Tract», Journal of the American Medical Association 92 (I929) I23I-I 237

Angell, James R., "A Reconsideration of James' Theory of Emotion in the Light of Recent Criticisms », Psychological Review 23 (I9I6) 25I-26I

Anrep, Gled von/Ernest H. Starling, "Central and Reflex Regulation of the Circulation ", Proceedings of the Royal Society 97 (1925) 463-487

Arminjon, Mathieu, «Birth of the Allostatic Model: From Cannon's Biocracy to Critical Physiology ", Journal of the History of Biology 49 (2016) 397-423

Arminjon, Mathieu, «The American Roots of Social Epidemiology and its Transnational Circulation. From the African-American Hypertension Enigma to the WHO's Recommendations ", Gesnerus. Swiss Journal for the History of Medicine and Science 77 (2020) 35-63

Aronson, Jeffrey K., «"Where Name and Image M eet"- the Argument for "Adrenaline" ", British Medical Journal 320 (2000) 506-509

Aub, Joseph C., «Studies in Experimental Traumatic Shock. I. The Basal Metabolism ", American Journal of Physiology 54 (1920) 388-407

Aub, Joseph C./Elizabeth M. Bright/Jonathan Forman, «The Metabolic Effect of Adrenalectomy Upon the Urethanized Cat», American Journal of Physiology 6I (I922) $349-368$

Aub, Joseph C./Jonathan Forman/Elizabeth M. Bright, «The Effect of Adrenalectomy Upon the Total Metabolism of the Cat ", American Journal of Physiology 6I (I922) 326-348 
Bacq, Zenon M., "Impotence of the Male Rodent After Sympathetic Denervation of the Genital Organs ", American Journal of Physiology 96 (I93I) 32I-330

«The Effect of Sympathectomy on Sexual Functions, Lactation, and the Maternal Behavior of the Albino Rat ", American Journal of Pbysiology 99 (I932) $444-453$

Lettre à Walter B. Cannon du 25 novembre I938, Walter Bradford Cannon Papers, Box: 57, Folder: 75I, Countway Library of Medicine

Lettre à Walter B. Cannon du 23 février I939a, Walter Bradford Cannon Papers, Box: 57, Folder: 752, Countway Library of Medicine

Lettre à Walter B. Cannon du 23 janvier I939b, Walter Bradford Cannon Papers, Box: 57, Folder : 752, Countway Library of Medicine

Lettre à Walter B. Cannon du 23 mars I939c, Walter Bradford Cannon Papers, Box: 57, Folder : 752, Countway Library of Medicine

Lettre à Walter B. Cannon du 28 janvier I94I, Walter Bradford Cannon Papers, Box: 57, Folder : 752, Countway Library of Medicine

Baird, M. M./John B. S. Haldane, "Salt and Water Elimination in Man", The Journal of Physiology 56 (I922) 259-262

Baker Eddy, Mary, Science and Health (Boston I875)

Science et santé avec la clef des écritures (Boston I9I7[1875])

Barbara, Jean-Gaël, «Les heures sombres de la neurophysiologie à Paris (I909I939)", Lettre des neurosciences 29 (2005) 3-6

Barcroft, Joseph, «The Effect of Altitude on the Dissociation Curve of Blood», The Journal of Physiology 42 (I9I I) 44-63

«Recent Knowledge of the Spleen ", The Lancet 205 (1925) 319-322

Barcroft, Joseph/Léonard T. Poole, «The Blood in the Spleen Pulp ", The Journal of Physiology 64 (I927) 23-29

Barcroft, Joseph/John G. Stephens, «Observations Upon the Size of the Spleen »,

The Journal of Physiology 64 (1927) I-22

Barcroft, Joseph/Hermann Straub, "The Secretion of Urine ", The Journal of Physiology 4I (I9I0) I45-I67

Bard, Philip, «A Diencephalic Mechanism for the Expression of Rage with Specia Reference to the Sympathetic Nervous System ", American Journal of Physiology 84 (I928) $490-5$ I5
Bauer, Walter/Joseph C. Aub/Fuller Albright, "Studies of Calcium and Phosphorus Metabolism: V. A Study of the Bone Trabeculæ as a Readily Available Reserve Supply of Calcium ", Journal of Experimental Medicine 49 (I929) I45-I62

Bazett, Henry C./Wilder G. Penfield, «A Study of the Sherrington Decerebrate Animal in the Chronic as Well as the Acute Condition ", Brain 45 (I922) I85-265

Becker, Howard S., La bonne focale. De l'utilité des cas particuliers en sciences sociales (Paris 2016[1988])

Beer, François-Joachim, « L'histoire du concept biologique de stress », Histoire des sciences médicales II (I977) I35-I40

Bekhthrev, Vladimir, «Die Bedeutung Der Sehhügel Auf Grund von Experimentellen Und Pathologischen Daten ", Virchow's Archiv Für Patbologische Anatomie Und Physiologie 2 (I887) 322-365

Benhamou, Edmond/Raoul Marchioni/Nouchy, « La splénocontraction à l'émotion chez l'homme normal ", Comptes rendus hebdomadaires des séances et mémoires de la société de biologie et de ses filiales 102 (Paris I929) 46I-462

Benison, Saul/A. Clifford Barger/Elin L. Wolfe, Walter B. Cannon. The Life and Times of a Young Scientist (Cambridge, Mass 1987)

Bergson, Henri, Lettre à James H. Hyde du 24 janvier I930 ", in: André Robinet (éd.), Bergson. Correspondances (Paris I930) I320-I32I

Berkman, Lisa F./Ichiro Kawachi, "A Historical Framework for Social Epidemiology", in: Lisa F. Berkman/Ichiro Kawachi (éds.), Social Epidemiology (New York/Oxford 2000) 3-12

Bernard, Claude, Leçons de pathologie expérimentale (Paris 1872)

Lectures on Experimental Pathology and Operative Physiology, Delivered at the Collège de France, During the Winter Session I859-60 2 (Londres I860).

Leçons sur les phénomènes de la vie, communs aux animaux et aux végétaux I (Paris I878)

Leçons sur les phénomènes de la vie, communs aux animaux et aux végétaux 2 (Paris I879)

Berton, Olivier/Pierre Mormède, "Connaître et comprendre les inégalités sociales de santé", in: Annette Leclerc/Didier Fassin/Hélène Grandjean/Monique Kaminski/Thierry Lang (éds.) Les inégalités sociales de santé (Paris 2000) 377-90

Bidder, Friedrich/Carl Schmidt, Die Verdauungssäfte Und Der Stoffwechsel (Mitau/ Leipzig I852)

Binet, Alfred, «W. James La Théorie de l'émotion », L'année psychologique 9 (1902) $388-40$ I 
Binet, Alfred/Nicolas Vaschide, «Données anatomiques. Capacité vitale et vitesse du cœur chez 40 jeunes gens", L'année psychologique 4 (I897) 225-232

Binet, Léon, La Physiologie de la rate (d'après les travaux récents) (Paris I927)

Bloor, Walter R., "Normal Fat Metabolism», in: Lewellys P. Barker (éd.) Endocrinology and Metabolism 3 (New York/Londres I922) I83-2II

Boas, Ernst P./Nikolai Michelsohn, The Challenge of Chronic Diseases (New York I929) Boothby, Walter M./Irene Sandiford, «The Calorigenic Action of Adrenalin Chlorid ", American Journal of Physiology 66 (1923) 93-123

Boothby, Walter M./Irene Sandiford/Kathleen Sandiford/Jean Slosse, «The Effect of Thyroxin on the Respiratory and Nitrogenous Metabolism of Normal and Myxedematous Subjects », Ergebnisse Der Physiologie 24 (I925) 728-756

Bragg, Raymond, «The Humanist Manifesto ", The Nerw Humanist 6 (I933) I-5

Bremer, John L., Textbook of Histology: Arranged Upon an Embryological Basis (Philadelphie/Londres 1944[1927])

Brissaud, Édouard, Lecons sur les maladies nerveuses. Salpêtrière, I893-I894. Recueillies et publiées par Henry Meige (Paris 1895 )

Britton, Sidney W., «Studies on the Conditions of Activity in Endocrine Glands. XVII. The Nervous Control of Insulin Secretion ", American Journal of Physiolog $y$ 74 (I925) 29I-308

"Neural and Hormonal Factors in Bodily Activity the Prepotency of

Medulliadrenal Influence in Emotional Hyperglycemia ", American Tournal of Physiology 86 (I928) 340-352

Brooks, Chandler McCuskey/Kiyomi Koizumi/James O. Pinkston (éds.) The Life and Contributions of Walter Bradford Cannon, I87 I-I945 (Albany, N. Y. I975)

Brousseau, Albert, Essai sur la peur aux armées, I9I4-I9I8 (Paris I920)

Brown-Séquard, Charles-Édouard, « Nouvelles recherches sur l'action du chloroforme appliqué sur la peau ", Gazette bebdomadaire de médecine et de chirurgie I8 (I88I) $27-28$

«Expérience démontrant la puissance dynamogénique chez l'homme d'un liquide extrait de testicules d'animaux ", Archives de Physiologie Normale et Pathologique I (Paris I889) 65I-658

Bulatao, Emilio/Walter B. Cannon, "Studies on the Conditions of Activity in Endocrine Glands. The Role of the Adrenal Medulla in Pseudaffective Hyperglycemia ", American Journal of Physiology 72 (1925a) 295-313

«Studies on the Conditions of Activity in Endocrine Glands. XVI. The Role of the Adrenal Medulla in Pseudoaffective Hyperglycemia ", American Journal of Physiology 72 (1925b) 295-3I3
Bulatao, Emilio/Anton J. Carlson, «Contributions to the Physiology of the Stomach. Influence of Experimental Changes in Blood Sugar Level on Gastric Hunger Contractions ", American Journal of Physiology 69 (I924) I07-I I 5

Burn, Joshuha H., «The Modification of the Action of Insulin by Pituitary Extract and Other Substances", The Journal of Physiology 57 (I923) 318-329

Cabot, Richard, «Religion and Health ", Harvard Alumni Bulletin 28 (1925) 384-392

Campos, F. A. de M./Walter B. Cannon/H. Lundin/T. T. Walker, «Some Conditions Affecting the Capacity for Prolonged Muscular Work», American Journal of Physiology 87 (I929) 680-70 I

Canguilhem, Georges, Le Normal et Le Pathologique (Paris I966[1943])

«Sur les normes organiques chez l'homme ", in: Le Normal et le Pathologique (Paris I966[I943]) 247-265

"La régulation comme réalité et comme fiction", in: Fin des normes ou crise des régulations? Trois conférences, Louvain, 20-22 Mars 1973, CAPHES (ENS) Archives de Georges Canguilhem, GC.25.17, (1973)

«Régulation (Épistémologie)», Encyclopæedia Universalis (1974[I968]) 40I-403 «La question de la normalité dans l'histoire de la pensée biologique», in: Idéologie et rationalité dans l'histoire des sciences de la vie. Nouvelles études d'bistoire et de philosophie des sciences (Paris 1977[I973]) I22-I39

La formation du concept de réflexe aux XVII'et XVIII' siècles (Paris 1977)

«Le problème des régulations dans l'organisme et la société», in: Écrits sur la médecine. (Paris 2002[I955]) I20-I23

Cannon, Walter B., "The Case Method of Teaching Systematic Medicine", The Boston Medical and Surgical Journal I42 (I900) 3I-36

Lettre à William James du 4 juin I909, Walter Bradford Cannon Papers, Box: I, Folder: 5, Countway Library of Medicine

Laboratory Course in Physiology (Cambridge, MA I9I0)

«The Emergency Function of the Adrenal Medulla in Pain and the Major Emotions ", American Journal of Physiology 33 (I9I4a) 356-372

"The Interrelations of Emotions as Suggested by Recent Physiological Researches ", The American Tournal of Psychology 25 (1914b) 256-282 Bodily Changes in Pain, Hunger, Fear and Rage (New York 1915)

« Some Disorders Supposed to Have an Emotional Origin », New York Medical Journal I04 (I916) 870-873 
«Croonian Lecture: The Physiological Basis of Thirst", Proceedings of the Royal Society of London. Series B, Containing Papers of a Biological Character 20 (I9I8a) 283-30 I

Lettre à Charles Richet du 2I mai I9ı8b, Walter Bradford Cannon Papers, Box: 55, Folder : 713, Countway Library of Medicine

«Rapport destiné à servir de base pour une discussion sur le choc traumatique ", Comptes rendus hebdomadaires des séances et mémoires de la société de biologie et de ses filiales 8I (Paris 19I8c) 854-863

Bodily Changes in Pain, Hunger, Fear and Rage. An Account of Recent Researches into the Function of Emotional Excitement, (New York/Londres 1922[1915])

«Studies in Experimental Traumatic Shock: IV. Evidence of a Toxic Factor in Wound Shock ", Archives of Surgery 4 (1922) I-22

\section{Traumatic Shock (New York/Londres I923)}

«Physiological Regulation of Normal States: Some Tentative Postulates Concerning Biological Homeostatics", in: Auguste Pettit (éd.), À Charles Richet: ses amis, ses collègues, ses élèves (Paris 1926) 9I-93

«The James-Lange Theory of Emotions: A Critical Examination and an Alternative Theory ", The American Journal of Psychology 39 (I927) I06-I 24

«Neural Organization for Emotional Expression », in: Martin L. Reymert (éd.), Feelings and Emotions: The Wittenberg Symposium (Worcester I928a) 257-268

"The Mechanism of Emotional Disturbance of Bodily Functions", New England Journal of Medicine 198 (1928b) 877-984

«Hunger and Thirst», in: Carl Murchison (éd.), The Foundations of Experimental Psychology (Worcester I929a) 434-448

Lettre à William W. Norton du 28 mai I929b, Walter Bradford Cannon Papers, Box: Io6, Folder: I457, Countway Library of Medicine

«Organization for Physiological Homeostasis", Physiological Revierws 9 (I929c) 399-43I

«Pharmacological Injections and Physiological Inferences», Science 70 (I929d) 500-501

«The Sympathetic Division of the Autonomic System in Relation to Homeostasis ", Archives of Neurology $\approx$ Psycbiatry 22 (I929e) 282-294

Lettre à Bradford Cannon du 20 décembre I929f, Walter Bradford Cannon Papers, Box: 162, Folder : 2296, Countway Library of Medicine

L'Homéostasie. Cours professé à la Faculté des sciences de Paris, cours des facultés. Certificat d'études supérieures de physiologie générale. I929-I930 I (Paris 1930a)
L'Homéostasie. Cours professé à la Faculté des Sciences de Paris, cours des facultés. Certificat d'etudes supérieuers de physiologie générale. I920-I930 2 (Paris I93ob)

«Quelques observations sur le comportement maternel d'animaux privés du système sympathique ", in: Pierre Janet/Georges Dumas (éds.), Journal de Psychologie Normale et Pathologique 27 (Paris I930c) 486-488

«Rôle du sympathique dans le maintien de la stabilité organique", Revue médicale de l'Est 58 (I930d) 689-704

«The Linacre Lecture. On the Autonomic Nervous System: An Interpretation", The Lancet 2I5 (I930e) II09-III5

Lettre à Georges Dumas du 3 juin I93ıa, Walter Bradford Cannon Papers, Box: 56 , Folder : 738 , Countway Library of Medicine

Lettre à William W. Norton du 3 décembre I93Ib, Walter Bradford Cannon Papers, Box: I06, Folder: I 458, Countway Library of Medicine

Lettre à William W. Norton 7 août I93Ic, Walter Bradford Cannon Papers, Box: I06, Folder: I458, Countway Library of Medicine

Lettre à William W. Norton du I2 mai I93Id, Walter Bradford Cannon Papers, Box: I06, Folder: I457, Countway Library of Medicine

Lettre à William W. Norton du 29 avril I93ıe, Walter Bradford Cannon Papers, Box: 106, Folder: I457, Countway Library of Medicine

Lettre à William W. Norton du 4 janvier 1932a, Walter Bradford Cannon Papers, Box: I06, Folder: I459, Countway Library of Medicine

Lettre à William W. Norton du 9 janvier 1932b, Walter Bradford Cannon Papers, Box: I06, Folder: I459, Countway Library of Medicine

Lettre à William W. Norton du Io février I932c, Walter Bradford Cannon Papers, Box: 106, Folder: I459, Countway Library of Medicine The Wisdom of the Body (New York I932d)

«Biocracy. Does the Human Body Contain the Secret of Economic Stabilization?», The Technology Revierw, 35.6 (I933a), 203-227

Lettre à William W. Norton Du 8 février I933b, Walter Bradford Cannon Papers, Box: Io6, Folder: I46I, Countway Library of Medicine

«Stresses and Strains of Homeostasis», Journal of the Medical Sciences I89 (I935) I3-I4

Lettre à William W. Norton du 6 juillet I938, Walter Bradford Cannon Papers, Box: 106, Folder: I467, Countway Library of Medicine

The Wisdom of the Body (New York I939[1932])

Lettre à Zénon M. Bacq du 2 janvier 1940, Walter Bradford Cannon Papers, Box: 57, Folder: 752, Countway Library of Medicine 
Lettre à William Norton du 29 mars I94Ia, Walter Bradford Cannon Papers, Box: I06, Folder: I469, Countway Library of Medicine

«The Body Physiologic and the Body Politic», Science 93 (I94Ib) I-Io

«"Voodoo" Death", American Antbropologist 44 (I942) I69-I8I

«Biographical Memoir of Lawrence Joseph Henderson, I878-I942. Presented to the Academy at the Autumn Meeting, I943", National Academy of Sciences of the United States of America $\mathrm{I}_{3}$ (I943) $3 \mathrm{3}^{\mathrm{I}-58}$

The Way of an Investigator. A Scientist's Experience in Medical Researcb (New York I945)

La Sagesse du corps (Paris I946[1939])

Cannon, Walter B./Elizabeth M. Bright, «A Belated Effect of Sympathectomy on Lactation ", American Journal of Physiology 97 (I93I) 3I9-32I

Cannon, Walter B./Sidney W. Britton, "Studies on the Conditions of Activity in Endocrine Glands. XV. Peseudoaffective Medulliadrenal Secretion", American Journal of Pbysiology 72 (1925) 283-294

«Studies on the Conditions of Activity in Endocrine Glands XX. The Influence of Motion and Emotion on Medulliadrenal Secretion", American Journal of Physiology 79 (1927) 433-465

Cannon, Walter B./Rosendo Carrasco-Formiguera, «Studies on the Condition of Activity in Endocrine Glands: XI. Further Evidence for Reflex and Asphyxial Secretion of Adrenin ", The Journal of Physiology 6I (I922) 215-227

Cannon, Walter B./Horace Gray, «Factors Affecting the C oagulation Time of Blood. II. The Hastening or Retarding of Coagulation by Adrenalin Injections », American Journal of Physiology 34 (1914) 232-242

Cannon, Walter B./Roy G. Hoskins, «The Effects of A sphyxia, H yperpnoea, and Sensory Stimulation on Adrenal Secretion ", American Journal of Physiology 29 (I9II) 274-279

Cannon, Walter B./Juan T. Lewis/Sidney W. Britton, «Studies on the Conditions of Activity in Endocrine Glands. XVII. A Lasting Preparation of the Denervated Heart for Detecting Internal Secretion, with Evidence for Accessory Accelator Fibers from the Thoracic Sympathethic Chains ", American Journal of Physiology 77 (I926) $326-352$

Cannon, Walter B./Clarence W. Lieb, «The Receptive Relaxation of the Stomach », American Journal of Physiology 29 (I9I I) 267-273

Cannon, Walter B./James R. Linton/Robert R. Linton, « Conditions of Activity in Endocrine Glands. XIV. The Effects of Muscle Metabolites on Adrenal Secretion», American Journal of Physiology 7I (I924) I53-I62
Cannon, Walter B./Monroe A. McIver/Sidney W. Bliss, «Studies on the Conditions of Activity in Endocrine Glands. XIII. A Sympathetic and Adrenal Mechanism for Mobilizing Sugar in Hypoglycemia ", American Journal of Physiolog 69 (1924) 46-66

Cannon, Walter B./James McKeen Cattell, «Studies in Experimental Traumatic Shock: V. The Critical Level in a Falling Blood Pressure", Arcbives of Surgery 4 (I922) $300-323$

Cannon, Walter B./Walter L. Mendenhall, « Factors Affecting t he Coagulation Time of Blood. I. The Graphic Method of Recording Coagulation Used in These Experiments ", American Journal of Physiology 34 (I9I4a) 225-231

«Factors Affecting the Coagulation Time of Blood. III. The Hastening of Coagulation by Stimulating the Splanchnic Nerves", American Journal of Physiology 34 (I9I 4 b) 243-250

«Factors Affecting the Coagulation Time of Blood. IV. The Hastening of Coagulation in Pain and Emotional Excitement ", American Journal of Physiology 34 (I9I4C) 25I-26I

Cannon, Walter B./Albert Moser, "The Movements of the Food in the Oesophagus ", American Journal of Physiolog $\gamma_{\text {I }}$ (I898) 435-444

Cannon, Walter B./Harlan F. Newton/Elizabeth M. Bright/Valy Menkin/Moore M., "Some Aspects of the Physiology of Animals Surviving Complete Exclusion of Sympathetic Nerve Impulses ", American Journal of Physiology 89 (I929) 84-I07

Cannon, Walter B./Daniel de la Paz, «Emotional Stimulation of Adrenal Secretion ", American Tournal of Physiologv 28 (I9I I) 64-70

Cannon, Walter B./J. R. Pereira, "Increase of Adrenal Secretion in Fever», Proceedings of the National Academy of Sciences of the United States of America Io (1924) $247-248$

Cannon, Walter B./Arie Querido/Sidney W. Britton/Elizabeth M. Bright, "Studies on the Conditions of Activity in Endocrine Glands. XXI. The Role of Adrenal Secretion in the Control of Body Temperatures " American Journal of Physiology 79 (1927) 466-507

Cannon, Walter B./Alfred T. Shohl/W. S. Wright, "Emotional Glycosuria», American Journal of Physiology 29 (I9I I) 280-287

Cannon, Walter B./Arthur L. Washburn, « An Explanation of Hunger », American Tournal of Physiology 29 (I9I2) 44I-454

Carlson, Anton J., «Contributions to the Physiology of the Stomach. I. The Character of the Movements of the Empty Stomach in Man ", American Journal of Physiology 3I (I9I2) I5I-I68

Cassel, John, «The Contribution of the Social Environment to Host Resistance », American Journal of Epidemiology I04 (I976) I07-I23 
Chabrol, Maurice, «Des mécanismes nerveux régulateurs de la pression artérielle: étude expérimentale par la méthode des circulations céphaliques croisées» (Alger I92I)

Chauveau, Auguste/Maurice Kaufmann, « La glycose, le glycogène, la glycogénie, en rapport avec la production de la chaleur et du travail mécanique dans l'économie animale. Deuxième étude : calorification dans es organes en travail », Comptes rendus hebdomadaires des séances de l'Académie des sciences I03 (Paris I886) I39-I064

Cohnheim, Julius, Lectures on General Pathology. A Handbook for Practitioners and Students (Londres I889[1877])

Colwell, Arthur R./Elizabeth M. Bright, «The Use of Constant Glucose Injections for the Study of Induced Variations in Carbohydrate Metabolism IV. Suppression of Glucose Combustion by Continuous Prolonged Epinephrin Administration », American Journal of Physiology 92 (1930) 555-567

Committee, The Medical Research, "Memorandum Upon Surgical Shock and Some Allied Conditions ", British Medical Journal I (I9I7) 38I-383

Comte, Auguste, Système de politique positive (Osnabrück 1967[185I])

Cori, Carl F./Gerty T. Cori, "The Mechanism of Epinephrine Action. I The Influence of Epinephrine on the Carbohydrate Metabolism of Fasting Rats, with a Note on New Formation of Carbohydrate ", Journal of Biological Chemistry 79 (I928) 309-319

Corral, José de, «Die Abhängigkeit der inneren Sekretion des Pankreas vom Nervensystem ", Zeitscbrift für Biologie 68 ( I918) 395-4I8

Cramer, William, «Observations on the Functional Activity of the Suprarenal Gland in Health, and in Disease», Sixth Scientific Report g be Imperial Cancer Research Fund 5I (I9I9) I-2 I

\section{D - E}

Damasio, Antonio R., William James and the Modern Neurobiology of Emotion (Oxford 2004)

Self Comes to Mind. Constructing the Conscious Brain (New York 2010)

Darwin, Charles, L'expression des émotions chez l'homme et les animaux (Paris I890[I872]) The Expression of the Emotions in Man and Animals (Londres 1872)

Desmarez, Pierre, «La sociologie de Lawrence J. Henderson, ou Pareto perverti », Rerue européenne des sciences sociales 24 (I986) I73-199

Diedrich, Maria, Cornelia James Cannon and the Future American Race (Amherst 20I I) Dubos, René, Mirage of Health : Utopias, Progress, and Biological Change (Londres 1959)
Dumas, Georges, "L'expression des émotions", Rerue philosophique de la France et de l'étranger 93 (I922) 32-72

La vie affective. Physiologie - psychologie - socialisation (Paris 1948)

Dusser de Barenne, Joannes Gregorius, Recherches expérimentales sur les fonctions du système nerveux central, faites en particulier sur deux chats, dont le néopallium a été enlevé (Amsterdam 1919)

Éditions Masson, Lettre à Walter B. Cannon du i6 juin I930a, Walter Bradford Cannon Papers, Box: 56, Folder : 736, Countway Library of Medicine

Lettre à Walter B. Cannon du I8 octobre I930b, Walter Bradford Cannon Papers, Box: 56, Folder: 736, Countway Library of Medicine

Eliot, Charles W., "The New Education », The Atlantic Montbly 23 (I869)

Emerson, Charles P., «The Emotional Element in the Production of Organic Disease ", Transactions of the Association of American Physicians 42 (Atlantic City, 1927) $246-355$

\section{$\mathrm{F}-\mathrm{G}$}

Fassin, Didier, «Another Politics of Life Is Possible», Theorv, Culture $\sigma$ Societv 26 (2009) $44-60$

Fassin, Didier/Hélène Grandjean/Monique Kaminski/ Thierry Lang/Annette Leclerc, "Connaître et comprendre les inégalités sociales de santé », in: Annette Leclerc/Didier Fassin/Hélène Grandjean/Monique Kaminski/Thierry Lang (éds.), Les inégalités sociales de santé (Paris 2000) $\mathrm{I} 3-24$

Ferrari, Giulio C., «Ricerche globulimetriche negli stati emozionali », Rivista di patologia nervosa e mentale 2 (I897) 306-3 I I

Fisher, Irving/United States National Conservation Commission, A Report on National Vitality, Its Wastes and Conservation (Washington I909)

Fiske, Cyrus H./Yellapragada Subbarow, « Phosphocreatine », Journal of Biological Chemistry 8I (I929) 629-679

Folin, Otto/Willey Denis/Wilson G. Smillie, « Some Observations on "Emotional Glycosuria" in Man ", Journal of Biological Chemistry I7 (1914) 519-520

Foucault, Michel, Il faut défendre la société. Cours au collège de France (Paris 1997[1976]) Fraser, John, «The Clinical Pathology of Surgical Shock», Edinburgh Medical Journal 25 (I920) $260-278$

Fredericq, Léon, «Influence du m ilieu a mbiant s ur la c omposition d u s ang d es animaux aquatiques ", in: Henri de Lacaze-Duthiers (éd.), Archives de zoologie expérimentale et générale 3 (Paris I885) 34-37 
Freud, Sigmund, «The Origin and Development of Psychoanalysis », The American Journal of Psychology 2I (I9I0) I8I-2I8

Cinq leçons sur la psychanalyse (Payot I923)

«Au-delà du principe de plaisir », in: Essai de psychanalyse (Paris I98I[I923]) $4 \mathrm{I}-\mathrm{II} 5$

Fujii, Ijuro, « Über Fesselungshyperglykämie und -Glykosurie beim Kaninchen », The Toboku Journal of Experimental Medicine 2 (1921) 9-64

Fulton, John Farquhar/Franc D. Ingraham, «Emotional Disturbances Following Experimental Lesions of the Base of the Brain (Pre-Chiasmal)", Journal of Physiology (London) 67 (I929) XXVII-XXVIII

Galdston, Iago, Beyond the Germ Theory: The Roles of Deprivation and Stress in Health and Disease (New York 1954)

Gallavardin, Louis/Jean Haour, "Baisse systolique de la tension et notation sphygmomanométrique ", Archives des maladies du cœur et des vaisseaux 5 (Paris I9I 2) 8

Gayet, René, Lettre à Walter B. Cannon du 22 novembre I932, Walter Bradford Cannon Papers, Box: 55, Folder: 717, Countway Library of Medicine

Gayet, René/Mayliss Guillaumie, "La régulation de la glycémie des chiens diabétiques par des quantités variées de tissus pancréatiques transplantés ", Comptes rendus hebdomadaires des séances et mémoires de la société de biologie et de ses filiales 98 (Paris I928) 584-586

Geroulanos, Stefanos/Todd Meyers, The Human Body in the Age of Catastrophe. Brittleness, Integration, Science, and the Great War (Chicago 2018)

Gibbs, J. Willard, « On the Equilibrium of Heterogeneous Substances», Transaction of the Connecticut Academy of Arts and Sciences 3 (I878) 343-520

Gley, Eugène/Alfred Quinquaud, «La fonction des surrénales. I. Du rôle phy siologique supposé de l'adrénaline", Journal de physiologie et de pathologie générale, I7 (Paris I9I8) 807-835

Goltz, Friedrich, "Der Hund ohne Grosshirn ", Archiv für die gesamte Physiologie des Menschen und der Tiere 5I (I892) 570-6I

Grafe, Erich, «Die nervose Regulation des Stoffwechsels», in: Carl Oppenheimer (éd.) Handbuch der Biochemie 9 (Jena 1927) 67-70

Gray, Horace/Lawrence K. Lunt, «Factors Affecting the Coagulation Time of Blood. V. The Effect of Hemorrhage Before and After Exclusion of Abdomina Circulation, Adrenals, or Intestine ", American Journal of Physiology 34 (I9I4) 332-5I

Gruber, Charles M., «Studies in Fatigue. II. The Threshold Stimulus as Affected by Fatigue and Subsequent Rest », American Journal of Physiology 32 (I9I3) 438-449
«Studies in Fatigue. III. The Fatigue Threshold as Affected by Adrenalin and by Increased Arterial Pressure ", American Journal of Physiology 33 (I9I4) $335-355$

«Studies in Fatigue XI. The Effect of Intravenous Injection of Adrenalin Upon Skeletal Muscle Undergoing Fatigue ", American Journal of Physiology 6I (I922) 475-492

Haldane, John S., «The New Physiology », Science 44 (I9I6) 6I9-631 Respiration (New Haven 1922)

"Claude Bernard's Conception of the Internal Environment ", Science 69 (I929) $453-454$

The Pbilosophical Basis of Biology. Donnellan Lectures, University of Dublin, I93o (Garden City, N.Y. I93I)

Haldane, John S./John G. Priestley, «The Regulation of the Lung-Ventilation », The Journal of Physiology 32 (1905) 225-266

"The Regulation of Excretion of Water by the Kidneys", The Journal of Physiology 50 (I916) 296-303

Hall, G. Stanley, «A Synthetic Genetic Study of Fear: Chapter I ", The American Journal of Psychology 25 (19I4) I49-200

Hargis, Estes H./Frank C. Mann, "A Plethysmographic Study of the Changes in the Volume of the Spleen in the Intact Animal ", American Journal of Physiology 75 (I925), I $80-200$

Hartman, Frank A./Howard A. McCordock/Loder M. M., «Conditions Determining Adrenal Secretion ", American Journal of Physiology 64 (I923) I-34

HCSP, Les inégalités sociales de santé: sortir de la fatalité. Groupe de travail : Thierry Lang (président), Gérard Badeyan, Chantal Cases, Pierre Chauvin, Marc Duriez, Isabelle Gremy, Virginie Halley Des Fontaines, Éric Jougla, Lucile Olier, Rapport du Haut Conseil de la santé publique (HCSP) (Paris 2009)

Head, Henry, «Croonian Lecture: Release of Function in the Nervous System », Proceedings of the Royal Society of London. Series B, Containing Papers of a Biological Character 92 (I92I) I84-200

Studies in Neurology 2 (Londres 1920)

Head, Henry/Gordon Holmes, «Researches into Sensory Disturbances from Cerebral Lesions", The Lancet I79 (I9I2) 79-83

Hédon, Emmanuel, «Transfusion sanguine réciproque entre deux animaux par anastomose carotidienne», Archives internationale de physiologie Io (I9I0) 192-207 
Henderson, John, A Life of Ernest Starling (New York 2005)

Henderson, Lawrence J., Blood. A Study in General Physiology (New Haven 1928)

Pareto's General Sociology : A Physiologist's Interpretation (Cambridge, Mass. I935)

Henry, James P./John C. Cassel, «Psychosocial Factors in Essential Hypertension Recent Epidemiologic and Animal Experimental Evidence », American Journal of Epidemiology 90 (1969) I7 I-200

Herzog, Johann J./Philip Schaff/Bert Hauck, The New Schaff-Herzog Encyclopedia of Religious Knowledge (New York/Londres I909)

Hewson, William, Experimental Inquiries into the Properties of the Blood (Londres I772)

Heymans, Cornelle, Le sinus carotidien et les autres zones vasosensibles réflexogènes (Paris 1929)

Horton, John, «Order and Conflict Theories of Social Problems as Competing Ideologies », American Journal of Sociology I7 (I966) 70I-7I3

Houssay, Bernardo A./Juan T. Lewis/Ernesto A. Molinelli, «Rôle de la sécrétion d'adrénaline pendant l'hypoglycémie produite par l'insuline ", Comptes rendus des séances et mémoires de la société de biologie et de ses filiales 9I (Paris I924) I0I I-IOI3

Howard, Harvey J., Ten Weeks with Chinese Bandists (New York 1927)

Hurst, Arthur F., "Observations on the Etiology and Treatment of War Neuroses », British Medical Journal 2 (I9I7) 409-4I 4

Huteau, Michel, Psychologie, psychiatrie et société sous la Troisième République. La biocratie d'Édouard Toulouse (I865-I947) (Paris 2002)

Hyde, James H., Lettre à Walter B. Cannon du 28 décembre I928, Walter Bradford Cannon Papers, Box: 56, Folder : 73I, Countway Library of Medicine

Lettre à Walter B. Cannon du i6 janvier i930, Walter Bradford Cannon Papers, Box: 56, Folder: 73I, Countway Library of Medicine

\section{$\mathbf{I}-\mathbf{K}$}

Isenschmid, G. Robert, «Physiologie der Wärmeregulation», in : A. Bethe/G von Bergmann/G Embden/A. Ellinger (éds), Handbuch der normalen und patbologischen Physiologie Correlationen III (J/XVI-XXI. Wärme-und Wasserbaushalt Umweltfaktoren - Scblaf. Altern und Sterben . Konstitution und Vererbung) (Berlin/Heidelberg I926) 3-

Izquierdo, José J./Walter B. Cannon, «Studies on the Conditions of Activity in Endocrine Glands. XXIII. Emotional Polycythemia in Relation to Sympathetic and Medulliadrenal Action on the Spleen ", American Journal of Physiology 84 (I 928 ) $545-562$

Jackson, Huglings, «Discussion », Brain I I (I888) 386-393

Jackson, Mark, The Age of Stress: Science and the Search for Stability (Oxford 2013)
James, William, «What Is an Emotion? », Mind 9 (I884) I88-205

Principi di psichologia (Milano I9ог[I890])

The Principles of Psychology 2 (Londres I89I)

«The Energies of Men", in: Memories and Studies (New York I9 I ) 229-64 La théorie de l'émotion par William James, précédé d'une introduction par le $D$ Georges Dumas (I9I7[1884])

James-Cannon, Cornelia, Red Rust (Boston 1928)

A Servant of Science, Walter Bradford Cannon [tapuscrit inédit] Walter Bradford Cannon Papers, Box: 209, Countway Library of Medicine

Jasanoff, S heila, « Ordering K nowledge, O rdering S ociety ", in S heila J asanoff (éd.), States of Knowledge: The Co-Production of Science and Social Order (Londres 2004)

Johansson, John E., «Ueber die Einwirkung der Muskelthätigkeit auf die Athmung und die Herzthätigkeit ", Skandinavisches Archiv für Physiologie 5 (I894) 20-66

Karplus, Johann P./Alois Kreidl, «Gehirn und Sympathicus», Pflügers Archiv European Journal of Physiology $\mathrm{I} 35$ (I9I0) 40I-4I6

Kastle, Joseph H./Salomon Loevenhart, « Concerning Lipase, the Fat-Splitting Enzyme, and the Reversibieity of Its Action. ", in: Ira Remsen (éd.), American Chemical Journal, 24 (Esaton I900) 49I-527

Kirilzev, S., "Cases of Affections of the Optic Thalamus ", Reviewed in Neurologisches Centralblatt Io (I89I) 3 IO

Kovarsky, Véra, «Quelques applications de la méthode du profil psychologique ", in: Pierre Janet/Georges Dumas (éds.), Journal de psychologie normale et patbologique 27 (Paris I930) 805-8I5

Krieger, Nancy, Epidemiology and the People's Health : Theory and Context (New York/ Oxford 20II)

Krogh, August, The Anatomy and Physiology of Capillaries (New Haven 1922)

Kruse, Harry D., «The Interplay of Noxious Agents, Stress, and Deprivation in the Engenderment of Disease», The Milbank Memorial Fund Quarterly 3I (I953) 93-I24

\section{$\mathbf{L}-\mathbf{M}$}

Landis, Carney, «Studies of Emotional Reactions. II. General Behavior and Facial Expression ", Journal of Comparative Psychology 4 (1924) 447-509

"Studies of Emotional Reactions IV. Metabolic Rate», American Journal of Physiology 74 (1925) I88-203

Lange, Carl G., Om Sindsbervagelser, et Psyko-Fysiologisk Studie (Copenhague 1885)

«The Emotions", in: Knight Dunlap (éd.), The Emotions I (Baltimore I922[I885]) $33-90$ 
Les émotions. Etude psychopbysiologique (Paris I895[1885])

Lange, Carl G./William James, The Emotions I, in: Knight Dunlap (éds.), (Baltimore 1922)

Lapicque, Louis, «Essai d'une nouvelle théorie de l'émotion », in: Pierre Janet/ Georges Dumas (éds.), Journal de psychologie normale et pathologique 7 (Paris I9I I) I-8

La macbine nerveuse (Paris 1942)

Le Roux, Ronan, «L'homéostasie sociale selon Norbert Wiener », Revue d'histoire des sciences bumaines $\mathrm{I} 6$ (2007) II $3^{-1} 35$

Lelle, Fernand, Lettre à William W. Norton I8 juillet I938a, Walter Bradford Cannon Papers, Box: 106, Folder : I467, Countway Library of Medicine

Lettre à William W. Norton du I5 juin I938b, Walter Bradford Cannon Papers, Box: I06, Folder: I467, Countway Library of Medicine

Lévy-Bruhl, Lucien, Lettre à Walter B. Cannon du 26 octobre I934, Walter Bradford Cannon Papers, Box: 56, Folder: 738, Countway Library of Medicine

Lewis, Juan T., "Action de l'insuline sur les rats privés de surrénales », in Comptes rendus des séances et mémoires de la société de biologie et de ses filiales 89 (Paris I 923) I I 8 -III9

Lieb, Clarence W, Safer Smoking: What Every Smoker Should Know and Do (New York I953)

Lupien, Sonia, «L'histoire de la science du stress : de Hans Selye à la découverte des anti-inflammatoires », Santé mentale au Québec 40 (2015) 275-286

Lusk, Graham, The Elements of the Science of Nutrition (Philadelphie/Londres I928[I9I7])

«The Wisdom of the Body », Science 75 (I932) 640-64I

Macleod, John J. R., The Fuel of Life (Princeton I928)

Mann, Frank C./Thomas B. Magath, "Studies on the Physiology of the Liver II. The Effect of the Removal of the Liver on the Blood Sugar Level », Archives of Internal Medicine 30 (1922) 73-84

Marañon, Gregorio, «Le facteur émotionnel dans la pathogénie des états hyperthyroïdiens ", Annales de médecine 9 (I92I) 8I-93

"Contribution à l'étude de l'action émotive de l'adrénaline », Rerue francaise d'endocrinologie 2 (1924) 30I-325

Markwalder, Josef/Ernest H. Starling, "A Note on Some Factors Which Determine the Blood-Flow Through the Coronary Circulation ", The Journal of Physiology 47 (I9I3) 275-285

Marmot, Michael/Richard Wilkinson (éds), Social Determinants of Health (Oxford Press, 2005)
Martín-Moruño, Dolores, «Ressentiment, médecine et société dans la biographie biologique de Gregorio Marañón", Histoire, médecine et santé 4 (2013) I05-II8

Martínez-Vidal, Alvar/Jon Arrizabalaga (à paraître), «Medicine, Religion, and the Humanitarian Ethos: Walter B. Cannon, the Unitarian Church, and the Care of Spanish Republican Refugees in France»

Mayer, André, «Variations de la tension osmotique du sang chez les animaux privés de liquides", Comptes rendus hebdomadaires des séances et mémoires de la société de biologie et de ses filiales 52 (Paris I900) ${ }^{1} 53-155$

McCormick, Norman A./John J. R. Macleod/Edouard C. Noble/K. O'brien, «The Influence of the Nutritional Condition of the Animal on the Hypoglycæmia Produced by Insulin ", The Journal of Physiology 57 (1923) 234-252

McDougall, William, An Introduction to Social Psychology (Londres 1908)

McEwen, Bruce S./John C. Wingfield, "What Is in a Name? Integrating Homeostasis, Allostasis and Stress », Hormones and Behavior 57 (2010) I05-III

McIver, Monroe A./Elizabeth M. Bright, "Studies on Conditions of Activity in Endocrine Glands », American Journal of Physiology 68 (I924) 622-644

Mellone, Sidney H., Unitarian Christianity and Christian Science (Londres I927)

Meltzer, Samuel J., «Studies on the "Paradoxical" Pupil-Dilatation Caused by Adrenalin. II.- On the Influence of Subcutaneous Injections of Adrenalin Upon the Eyes of Cats After R emoval of $\mathrm{t}$ he $\mathrm{S}$ uperior $\mathrm{C}$ ervical $\mathrm{G}$ anglion ", American Journal of Physiology II (I 904) 37-39

"Comment on "Some Disorders Supposed to Have an Emotional Origin"

by Walter B. Cannon ", New York Medical Journal I04 (I916) 873

Menkin, Valy, «Emotional Relative Mononucleosis », American Journal of Physiology $\underline{8}$ (I928) $489-497$

\section{Dynamics of Inflammation (New York I940)}

Meyerson, Ignace, "Compte rendu de la séance du 22 février 1930 ", in: Pierre Janet/Georges Dumas (éds.), Journal de psychologie normale et patbologique 27 (Paris 1930) 485

Meynert, Theodor, Psychiatrie : clinique des maladies du cerveau antérieur basée sur sa structure, ses fonctions et sa nutrition I (Bruxelles I888[I884])

Minkowski, Oscar, «Zur Kenntnis der Funktion des Pankreas beim Zuckerverbrauch ", Archiv für experimentelle Pathologie und Pharmakologie 59 (Leipzig 1908), 395

Molinelli, Ernesto A., La secreción de adrenalina [Thèse] (Buenos Aires I926)

Morat,Jean Pierre/E. Dufourt, « Consommation du sucre par les muscles, origine probable du glycogène musculaire ", Archives de physiologie 24 (Paris I892) 327-339

Mosso, Angelo, La paura (Milan I884). 
La Peur: étude psycho-physiologique (Paris I886[I884])

Fear (Londres I896[1884])

$\mathbf{N}-\mathbf{P}$

Nolf, Pierre, «Des modifications de la coagulation du sang chez le chien après extirpation du sang ", Archives internationales de physiologie 3 (1905) I-43

Norton, Jeffrey/Philip S. Barie/Ralph R. Bollinger/Alfred E. Chang/Stephen Lowry/Sean J. Mulvihill/Harvey L. Pass/Robert W. Thompson, Surgery: Basic Science and Clinical Evidence (New York 2009)

Norton, William W., Lettre à Walter B. Cannon du 24 mai I929, Walter Bradford Cannon Papers, Box: I06, Folder: I457, Countway Library of Medicine

Lettre à Walter B. Cannon du 24 novembre I930, Walter Bradford Cannon Papers, Box: 106, Folder: I457, Countway Library of Medicine

Lettre à Walter B. Cannon du I I mai I93ra, Walter Bradford Cannon Papers, Box: I06, Folder: I457, Countway Library of Medicine

Lettre à Walter B. Cannon du I2 août I93Ib, Box: Io6, Walter Bradford Cannon Papers, Folder: I458, Countway Library of Medicine

Lettre à Walter B. Cannon du 27 avril I93Ic, Walter Bradford Cannon Papers, Box: I06, Folder: I457, Countway Library of Medicine

Lettre à Walter B. Cannon du 4 août I933a, Walter Bradford Cannon Papers, box: I06, Folder: I46I, Countway Library of Medicine

Lettre à Walter B. Cannon du 7 février I933b, Walter Bradford Cannon Papers, Box: I06, Folder: I46I, Countway Library of Medicine

Lettre à Walter B. Cannon du I5 février I933c, Walter Bradford Cannon Papers, Box: 106, Folder: I46I, Countway Library of Medicine

Lettre à Walter B. Cannon du 2 juin I938a, Walter Bradford Cannon Papers, Box: 106, Folder: I467, Countway Library of Medicine

Lettre à Walter B. Cannon du 24 mai I938b, Walter Bradford Cannon Papers, Box: I06, Folder: I467, Countway Library of Medicine

Lettre à Walter B. Cannon du 28 mars I94I, Walter Bradford Cannon Papers, Box: I06, Folder: I469, Countway Library of Medicine

Ohayon, Annick, "Les médecins hygiénistes français face à la politique raciale allemande. I933-I939", L'Évolution psychiatrique 66 (2001) 348-356

Oliver, George/Edward A. Schäfer, « On the Physiological Action of Extract of the Supra Renal Capsules ", Journal of Physioly (London) I6 (I894) i-iv

Orbeli, Leon A., «Sympathetic Innervation of Skeletal Musculature (Dedicated to I.P. Pavlov », Izv. Nauchn. Inst. Im. P.F. Lesgafta 6 (1923) I 87-197
Pack, George T., "New Experiments on the Nature of the Sensation of Thirst ", American Journal of Physiology 65 (I923) 346-349

Panella, A., « Action du principe actif surrénal sur la fatigue musculaire », Archives italiennes de biologie 48 (1907) 430-463

Papez, James. W., «A Proposed Mechanism of Emotion », Journal of Neuropsychatry Z (I937) I03-I2

Pavlov, Ivan Petrovitch, Conditioned Reflexes an Trvestigation of the Physiological Activity of the Cerebral Cortex (Oxford 1927)

Payot, Lettre à William W. Norton du 4 novembre 1932, Walter Bradford Cannon Papers, Box: I06, Folder: I460, Countway Library of Medicine

Lettre à William W. Norton du 8 mai I933, Walter Bradford Cannon Papers, Box: I06, Folder: I46I, Countway Library of Medicine

Peabody, Francis W./Cyrus C. Sturgis/Edna M. Tompkins/Joseph T. Wearn, «Epinephrin Hypersensitiveness and Its Relation to Hyperthyroidism ", The American Journal of the Medical Sciences I6I (I92I) 508-5I7

Penfield, Wilder/Edwin Boldrey, «Somatic Motor and Sensory Representation in the Cerebral Cortex of Man as Studied by Electrical Stimulation», Brain 60 (1937) $389-443$

Perrin C. Galpin, lettre à Walter B. Cannon du I 5 octobre I929, Walter Bradford Cannon Papers, Box: 57, Folder : 759, Countway Library of Medicine

Perry, Ralph B., The Thought and Character of William James as Revealed in Unpublished Correspondence and Notes, Together with his Published Writings I-2 (Londres I935-1936).

General Theory of Value. Its Meaning and Basic Principles Construed in Terms of Interest (Cambridge I950[1926])

Petit-Dutaillis, Charles, Lettre à Cannon du I 5 mars I930, Walter Bradford Cannon Papers, Box: 56, Folder: 73I, Countway Library of Medicine

Piéron, Henri, "Emotion in Animals and Man », in: Martin L. Reymert (éd.), Feelings and Emotions: The Wittenberg Symposium (Worcester 1928) 284-294

Pilcher, James D./Torald Sollmann, «Studies on the Vasomotor Centre: The Effects of Hemorrhage and Reinjection of Blood and Saline Solution ", American Journal of Physiology 35 (I I I4) 59-69

Pollock, Kristian, «On the Nature of Social Stress: Production of a Modern Mythology", Social Science \& Medicine 26 (I988) 38I-392

Porter, William T./Frederick H. Pratt, «Proceedings of the American Physiological Society. The Reactions of Peripheral Vasomotor Areas ", American Journal of Physiology 2 I ( 1908$) \mathrm{xvi}$ 
$\mathrm{Q}-\mathrm{S}$

Quinquaud, Alfred, "Expériences sur la contraction musculaire et la chaleur animale ", Comptes rendus hebdomadaires des séances et mémoires de la société de biologie et de ses filiales 38 (Paris I886) $4 \mathrm{IO}-4 \mathrm{II}$

Redfield, Alfred C./Elizabeth M. Bright, « The Relative Physiological Effects of $\beta$-Rays of Different Velocities », The Journal of General Physiology 2 (I9I9) 3I-47

Renault, Emmanuel, «Biopolitique, médecine sociale et critique du libéralisme », Multitudes, 34 (2008) 195-205

Richet, Charles, «Défense (Fonction De)», in: Charles Richet (éd.), Dictionnaire de physiologie 4 (Paris 1899) 699-721

La sélection bumaine (Paris i919a)

L'Homme stupide (Paris I9I9b)

Traité de métapsychique (Paris 1922)

Lettre à Walter B. Cannon du io janvier I930, Walter Bradford Cannon Papers, Box: 55, Folder : 7I4, Countway Library of Medicine

Robertson, Oswald H./Arlie V. Bock, Memorandum on Blood Volume after Hhemorraghe. Medical Reserach Comittee on Surgical Schock (Londres I918)

Rose, Steven, Lifelines: Biology Freedom, Determinism (Londres 1997)

Rothmann, Hans, «Zusammenfassender Bericht über den rothmannschen gro〉 hirnlosen Hund nach klinischer und anatomischer Untersuchung ", Zeitschrift fuir die gesamte Neurologie und Psychiatrie 87 (I923) 247-313

Ryan, Kathy L., «Walter B. Cannon's World War I Experience: Treatment of Traumatic Shock Then and Now ", Advances in Physiology Education 42 (2018) 267-276

Sataké, Yasutaro/Masanosuké Watanabé/Tadashi Sugawara, «Effect of Fastening and of Sensory Stimulation Upon the Rate of Epinephrine Output from the Suprarenal Gland in Dogs ", The Tohoku Journal of Experimental Medicine 9 (I927) I-40

Schäfer, Edward A., «Oliver-Sharpey Lectures On the Present Condition of Our Knowledge Regarding the Functions of the Suprarenal Capsules: Delivered before the Royal College of Physicians of London on April 7th and 9th, I908* British Medical Journal I (I908) I277-I28I

Scheunert, Arthur/Wilhelm Krzywanek, «Über die Beziehungen der Milz zu den Schwankungen der Menge der roten Blutkörperchen ", Pflügers Archiv für die gesamte Physiologie des Menschen und der Tiere 215 (1927) I87-190

Segal, Harry L./Herbert F. Binswanger/Solomon Strouse, "The Effect of Emotion on Basal Metabolism ", Archives of Internal Medicine 4I (I 928) 834-842

Seitz, Wilhelm, «Die Leber als Vorrathskammer für Eiweissstoffe », Arcbiv fir die gesamte Physiologie des Menschen und der Tiere I II (1906) 309-334
Selye, Hans, The Physiology and Patbology of Exposure to Stress (Montréal I950)

The Stress of Life (New York 1956)

Stress Without Distress (Philadelphie I974)

«Homeostasis and Heterostasis», in: Stacey B. Day (éd.), Trauma (New York/Londres 1975a) 25-29

«Homeostasis and the Reaction to Stress : A Discussion of Walter B. Cannon's Contribution», in: Chandler MacCuskey Brooks/Kiyomi Koizumi/James O. Pinkston (éds.), The Life and Contributions of Walter Bradford Cannon, I87I-I945 (Albany, N. Y., I975b) 89-I07

Selye, Hans/Thomas McKeown/James B. Collip, «Studies on the Physiology of the Maternal Placenta in the Rat ", Proceedings of the Roval Society of London. Series B - Biological Sciences I I9 (I935) I-3I

Sherrington, Charles S., The Integrative Action of the Nervous System (New York I906)

«Experiments on the Value of Vascular and Visceral Factors for the Genesis of Emotion ", Proceedings of the Royal Society of London 66 (I899) 390-403

Simondon, Gilbert, Du mode d'existence des objets techniques (Paris 1989[1958])

Sinding, Christiane, Une utopie médicale: la sagesse du corps (Arles I989)

Skelton, Harold, « The Storage of Water by Various Tissues of the Body », Archives of Internal Medicine 40 (I927) I40-52

Sollmann, Torald H./Paul J. Hanzlik, An Introduction to Experimental Pharmacology (Philadelphie 1928)

Spadolini, Igino, «Sur les lésions expérimentales des nerfs mésentériques et de l'appareil parathyroïdien par rapport aux intoxications d'origine intestinales ", Arcbives italiennes de biologie 77 (Pise I926) I7-27

Starling, Ernest H., Croonian Lectures on the Chemical Correlation of the Functions of the Body. Delivered Before the Royal College of Physicians of London on June 2oth, 22nd, 27th G 29th, I905 (Londres 1905)

\section{The Fluids of the Body (Londresigo9)}

«The Wisdom of the Body. The Harveian Oration, Delivered before The Royal College of Physicians of London, St. Luke Day, I923 », British Medical Journal (1923) 685-690

Starobinski, Jean, «"La sagesse du corps", et la maladie comme égarement : le "stress" ", Critique 8 (1952) 347-360

Sterling, Peter, «Principles of Allostasis: Optimal Design, Predictive Regulation, Pathophysiology, and Rational Therapeutics", in: Jay Schulkin (éd.) Allostasis, Homeostasis, and the Cost of Physiological Adaptation (Cambridge, MA 2004) I7-64 
Sterling, Peter/Joseph Eyer, «Allostasis: A New Paradigm to Explain Arousal Pathology", in: Shirley Fisher/James Reason (éds.), Handbook of Life Stress, Cognition, and Health (New York 1988) 629-649

Stewart, George N., A Manual of Physiology: With Practical Exercises (Baillière I895) Stewart, George N./Julius M. Rogoff, «The Spontaneous Liberation of Epinephrin from the Adrenals", Journal of Pharmacology and Experimental Therapeutics 8 (I916) $479-524$

Stratton, George M., "The Function of Emotion as Shown Particularly in Excitement ", Psychological Review 35 (I928) 35I-366

Straus, Robert, "The Nature and Status of Medical Sociology", American Sociological Review 22 (I957) 200-204

Stübel, Hans, «Die Wirkung des Adrenalins auf das in der Leber gespeicherte Eiweiß", Pflügers Archio für die gesamte Physiologie des Menschen und der Tiere I 85 (I920) $74-85$

Sundberg, Carl G., "Sur l'action de l'insuline après l'extirpation des capsules surrénales ", Comptes rendsu hebdomadaires des séances et mémoires de la société de biologie et de ses filiales 89 (Paris 1923) 807-810

Studien über die Blutzuckerregulation bei epinephrektomierten Tieren (Stockholm I925

Takamine, Jokichi, «The Isolation of the Active Principle of the Suprarena Gland », Journal of Physiology (London) 27 (I90I) XXIX-XXX

Tansey, Elizabeth M., "What's in a Name? Henry Dale and Adrenaline, I906 ", Medical History 39 (I995) 459-476

Terroine, émile F., «Le transport des graisses. I. Variations lipo-cholestériné miques au cours de l'inanition et de l'alimentation ", Journal de physiologie et de patbologie générale I6 (Paris 1914) 386-397

«The French Honor Mrs. Eddy », The New York Times I December (New York 1907) I Thomas, James, Three Years Among the Indians and Mexicans (Saint-Louis I9I6[I846]) Tichmeneff, N., «Über Eiweißspeicherung in der Leber», Biochemische Zeitschrift. Beiträge zur chemischen Physiologie und Pathologie (Berlin 1916) 326-332

Tilney, Frederick/Francis Morrison, «Pseudo-Bulbar Palsy, Clinically and Pathologically Considered, with the Clinical Report of Five Cases », The Journal of Nervous and Mental Disease 39 (1912) 505-535

Toulouse, Édouard, La question sociale (Paris I92I)

Lettre à Cannon du 27 février 1930, Walter Bradford Cannon Papers, Box : 56, Folder : 733, Countway Library of Medicine
Toulouse, Edouard/Georges Genil-Perrin, « L'organisation technique de la prophylaxie mentale", Annales médico-psychologiques. Journal de l'aliénation mentale et de la médecine légale des aliénés $\mathrm{I} 2$ (Paris 1920$) 5^{\mathrm{IO}}-520$

Tournade, André/Maurice Chabrol, «Effets des variations de la pression artérielle sur la sécrétion de l'adrénaline", Comptes rendus hebdomadaires des séances et mémoires de la société de biologie et de ses filiales 93 (Paris 1925) 934-936

Tournade, André/Maurice Chabrol/Henri Marchand, «Des mécanismes nerveux régulateurs de la pression artérielle. I. La régulation centrale", Comptes rendus hebdomadaires des séances et mémoires de la société de biologie et de ses filiales 84 (Paris I92I) 6I0-6I2

Trendelenburg, Paul, «Die Adrenalinsekretion unter normalen und gestörten Bedingungen ", Ergebnisse der Physiologie 2I (I923) 500-557

Troy, Michele K., Strange Bird: The Albatross Press and the Third Reich (New Haven, Connecticut 2017)

\section{$\mathrm{V}-\mathrm{W}$}

Valenstein, Elliot S., The War of the Soups and the Sparks: The Discovery of Neurotransmitters and the Dispute Over How Nerves Communicate (New York 2005)

Vaschide, Nicolas, Le sommeil et les rềves (Paris I9I8)

Viale, Gaetano/J. Di Leo Lira, « Rôle de la rate dans l'hyperglobulie par l'air raréfié », Comptes rendus hebdomadaires des séances et mémoires de la société de biologie et de ses filiales 97 (Paris I927) I 239-I 240

Vincent, Swale, Internal Secretion and the Ductless Glands (Londres I9I2)

"The Effects of Fatigue and Temperature on the Adrenal Bodies of the Rat ", Quarterly Journal of Experimental Physiology I5 (I925) 319-326

Vosburgh, Charles H./Alfred N. Richards, "An Experimental Study of the Sugar Content and Extravascular Coagulation of the Blood after Administration of Adrenalin ", American Journal of Physiology 9 (1903) 35-5I

Wang, Ging Hsi, «Johann Paul Karplus (I866-I936) and Alois Kreidl (I864-I928): Two Pioneers in the Study of Central Mechanisms of Vegetative Function », Bulletin of the History of Medicine 39 (1965) 529-539

Watson, John B., Bebaviorism (Londres 1925)

Watson, John B./Rayner R., "Conditioned Emotional Reactions", Journal of Experimental Psychology 3 (1920) I-I4

Weisz, George, Chronic Disease in the Twentieth Century. A History (Baltimore 2014)

Wells, Frederic L., «Reactions to Visual Stimuli in Affective Settings », Journal of Experimental Psychology 8 (1925) 64-76 
Wettendorff, Hugo, «Modifications du sang sous l'influence de la privation d'eau: contribution à l'étude de la soif", in Travaux du laboratoire de physiologie, institut solvay 4 (Bruxelles I90I) 353-484

Wiener, Norbert, Cybernetics or Control and Communication in the Animal and the Machine (Cambridge, Mass. I96r[1948])

Wilson, Alexander K., «Some Problems in Neurology. No. II. Pathological Laughing and Crying ", Journal of Neurology and Psychopatbology 4 (I924) 299-333

Wolfe, Elin L./Abraham Clifford Barger/Saul Benison, Walter B. Cannon, Science and Society (Cambridge, Mass. 2000)

Woodworth, Robert S./Charles S. Sherrington, «A Pseudaffective Reflex and Its Spinal Path", The Journal of Physiology 3I (1904) 234-243

Woodyatt, Rollin T., «Psychic and Emotional Factors in General Diagnosis and

Treatment", Journal of the American Medical Association 89 (I927) IOI3-IOI4

Wright, James R., "Almost Famous: E. Clark Noble, the Common Thread in the Discovery of Insulin and Vinblastine ", Canadian Medical Association Journal I67 (2002) I39I-I396

$$
\mathbf{Y}-\mathbf{Z}
$$

Young, Allan, «The Discourse on Stress and the Reproduction of Conventional Knowledge ", Social Science שr Medicine I4 (1980) I33-I46

\section{The Harmony of Illusions: Inventing Post-Traumatic Stress Disorder (Princeton} 1995)

Zunz, Edgard/Jean La Barre, «Sur l'augmentation de la teneur en insuline du sang veineux pancréatique après l'hyperglycémie provoquée par injection de glucose ", Comptes rendus hebdomadaires des séances et mémoires de la société de biologie et de ses filiales 96 (Paris 1927 ) $42 \mathrm{I}-423$

\section{Table des figures et crédits}

Francis A. Countway Library of Medicine, Havard University, Cambridge, Massachusetts

Illustration I, p.2I, illustration 2, p.63, illustration 3 p.64

The Wisdom of the Body (New York 1932d)

Fig.I p.I26, fig.2 p.I 27, fig.3 p.I27, fig.I 2 p.I4I, fig.I 4 p.I72, fig.I5 p.I74, fig.I7 p.I76, fig.23 p.213, fig. 25 p.243, fig.26 p.244, fig.27 p.244, fig.28 p.245, fig.30 p.248, fig.3I p.249, fig.32 p.250, fig.33 p.252, fig.34 p.253, fig.35 p.262, fig.37 p.268, fig.38 p.268, fig.40 p.27o, fig.4I p.282, fig.42 p.283, fig.43 p.283, fig.44 p.285, fig. 45 p.285, fig.46 p.285, fig. 47 p. 28 , fig. 48 p. 289 , fig.49 p.289, fig.50 p.29o, fig.52 p.292, fig.53 p.293, fig.54 p.306, fig.56 p.3I 2, fig.60 p.323, fig.6I p.324, fig.62 p.326, fig.63 p.327, fig.64 p.329, fig.65 p.329, fig.67 p.332, fig.68 p.34I, fig.70 p.342, fig.7I p.343, fig.73 p.347, fig.74 p.349

American Journal of Physiology

Fig.4 p.I28, fig.5 p.I29, fig.6 p.I30, fig.7 pI32, fig.8 pi33, fig.9 pi36, fig.Io pı37, fig.II p.I40, fig.I3 pI42, fig.I6 p.I75, fig.I8 pI79, fig.I9 p.I79, fig.20 p.I8o, fig.29 p. 247 , fig.39 p.269, fig.57 p.315, fig.58 p.3I7, fig.59 p.319, fig.66 p.33I, fig.69 p.342, fig. 72 p. 345

Journal of Neurology and Psychopatbology

Fig. 22 p.183

Journal of Experimental Medicine

Fig.55 p.307

The Royal Society of London

Fig. 36 p. 263

Bodily Changes in Pain, Hunger, Fear and Rage

Fig.2I p.I82, fig.24 p.2I4 


\section{Index nominum}

L’astérisque signifie que le prénom est inconnu

Abe, Yukujiro, 286, 286nIo-I2

Abel, John, 34, 34n49, 344, 344nI 4

Abetz, Otto, 88

Adolph, Edward Frederick, 276, 276n30

Adrian, Edgar, $396_{3}$

Afanassiew, Michail Ivanovič, $298 \mathrm{n} 47$

Albright, Fuller, 305n64, 306n65-66, 307

Alvarez, Walter Clement, 2I6, 2I6n9, 2I7, 2I7ng

Anrep, Gleb von, $255,255 n_{50}$

Arthus, Nicolas Maurice, 69, 69nI32-I33, 256n9I

Asher, Leon, 69, 69ni 34, 293n32

Aub, Joseph Charles, I43, I43n56, 249, 249n38, 305, 305n64, 306n65-66, 307, $320,320 n 20$

Bacq, Zénon Marcel, I3, I3n2, 65, 82, 86-89, 86n I8I, 87nI82-I86, 88ni 88, 88niqo, 236n9, 250n39, 346nig

Bainbridge, Francis Arthur, 25I, 25In4I

Baker Eddy, Mary, 226n 24

Barcroft, Joseph, 253, 253n45-46, 297, 297n43, 3I4-3I5, 3I 4n7, 3I 4nIo, 3I5nI2

Bard, Philip, I3, I3n3, 47n8o, 49, I22, I74-176, I74nI2, I75nI3, I76, 34I

Bauer, Walter, 305n64, 306n65-66, 307

Bayliss, William, 30, 30n38, 3on 40, 3I, 39, 4I, 25

Bazett, Henry Cuthbert, I73, I73nIo

Bekhterev, Vladimir, 56n98, I8I, I8In23

Bell, Charles, I $205_{5}$
Bergson, Henri, 67, 67nI 25

Bernard, Claude, 22, 22n25, 27, 32-33, 32n44, 40n64, 43, 43n7i, 65nir7, 7inI37, 72, 74-75, 75n $45,86,89$, I62, 233, 235-236, 235n3-6, 236n7-8, 238, 239ni 4-I5, 240, $320,320 \mathrm{n} 19,334-335,334 \mathrm{n} 2-3,335 \mathrm{n} 4$

Best, Charley, 286nio

Bidder, Georg Friedrich Karl Heinrich von, 265, 265n7

Binet, Alfred, 55n97, I32, I32n28, I34n34

Binet, Léon, 3I4, 3I4n9-I0

Binswanger, Herbert F., I 44 n60

Bliss, Sidney W., I95nio, 283n3, 285n5, 284n6-7

Bloor, Walter Ray, 30I, $30 \mathrm{In}_{5} 6$

Boothby, Walter M.*, I I2, I 42n55, 300, 300n53-54, 326, 326n37

Bremer, John Lewis, 273-274n 23

Breuer, Joseph, I76ni6

Bright, Elizabeth, I37n44, I43, I43n56-57, I5InIo, I78nI9, I79n20-2I, I8on22, I95n9, 296n4o, 320n20-2I, 323n3o, 324n 3 I, 326n 35-36, 328n 38, 329n4I, 33on 43 , 34In8, 342n9-Io, 343nII, 345nI5-I6, 346nis, 348n22-23

Brissaud, Édouard, I85n29

Britton, Sidney William, I26n20, I28n20-2I, I30, I30n24-25, I7on7, I95n9, 282n2, 288n2I, 290, 290n26, 293, 293n33-34, 294n36, 3I 2n6, 323n3o, 324n3I, 326n35-36, $328 n_{3} 8,329 n_{4}$ I, 330n 43

Brousseau, Albert, 86, 86ni79, I62-I63n22

Brown-Séquard, Charles-Édouard, I62-I63n22

Bulatao, Emilio Erquiza, I72n8, 27I-272, 27In22, 289n23-24, $290 \mathrm{n} 25$

Burdon, John, 276n3I

Burn, Harold, 288, 288ni 8

\section{C}

Cabot, Richard Clarke, 26, 27n33, 60, 217, 217nio

Campos, F. A. de M.*, I38n45, I38n48, 28InI, 346n20

Canguilhem, Georges, I5, I5n7-8, 90-96, 90n195-I99, 9In200, 92n203, 93n205206, 94n207-210, 95n2II-2I 2, 96n213, I08n248

Carlson, Anton Julius, 270-272, 270n20-2I, 27In2I

Carrasco-Formiguera, Rosendo, $3 \mathrm{I} 7 \mathrm{nI} 4$ 
Carrel, Alexis, 38, I32n 29

Carver, Thomas Nixon, 80-8I

Cassel, John, I09, I09n254

Chabrol, Maurice, 245, 245-246n 26, 255, 255n 49

Charcot, Jean Martin, 50, I85n29, 288nI7

Chauveau, Jean-Baptiste Auguste, I49, I49n5

Cohnheim, Julius, 243, 243nis

Collip, James Bertram*, I8nı6

Colter, John, I60-I6I, I60-I6InI8

Colwell, Arthur Ralph Sr., I5Inı, 296, 296n 40

Comte, Auguste, 92, I03n235

Cori, Carl Ferdinand, 287, 295, 295n39, 296n 42

Cori, Gerty Theresa, 287, 295, 295n39, 296n 42

Corral, José María de, 293, 293n32

Cotton, Frederic Jay, I $42 n_{52}$

Cramer, William, 32I, 32In23, 322n27

Crile, George Washington, 38, 38n6o, 4I, 49

\section{D}

Dale, Henry, 34, 39, 41

Damasio, Antonio, 47-48n8I

Darwin, Charles, 44, 49, 50n87, 56, 99, I20-I2I, I44n58, I58, I58nI3-I4, I 77, I77ni7 de La Paz, Daniel, $36 n_{5} 6$

Dénikine, Anton Ivanovitch, 255

Denis Willey Glover, I30-I3In26

Dewey, John, I92n5, 270 n20 $^{2}$

Dhéré, Charles, 69, 69ni33

Dubos, René, I09, I09n253

Duchenne De Boulogne, Guillaume-Benjamin, I2on 5

Dufourt, E.*, I 49, I49n7

Dumas, Georges, 49, 50n86, 64, 83, 99, 99n223, 99n224-225, I00, Ioon227-228, I $47, I_{47} n_{3}, I_{5} 8 n_{12}$
Dunlap, Knight, I9In3

Dusser de Barenne, Johannes Gregorus, I70, I7on 5

Dworkin, Simon, 296, 296n4I

$$
\mathrm{E}-\mathrm{F}
$$

Eck, Nikolai Vladimirovitch, 298, 298n46

Edsall, David Linn, 62

Eliot, Charles William, 35, 35n53-54, 36, I30n 26

Elliott, Thomas Renton, I39-I40, I39n5o

Emerson, Charles Phillips, 6I, 6Iniog-I Io, 6InII 2, 220, 220 I7

Engels, Wladimir, 274, 274n26

Enoch, Kurt, 84-85, 85nI75

Eyer, Joseph, I09, I09n256, 240nI5

Ferrari, Giulio Cesare, I34, I34n 34

Ferrier, David, I69n3

Fisher, Irving, 98, $98 \mathrm{n} 220$

Fiske, Cyrus Hartwell, 3I I, 3IIn 5

Florkin, Marcel, 63

Folin, Otto, I30-I 3 In 26

Foucault, Michel, I04n 242

Frédéricq, Léon, 236, 236n9, 237nı, 244n23, 336n 5

Fujii, Ijuro, 32I, 32 In 25

Fulton, John Farquhar, I75, I75nI4

\section{$\mathbf{G}-\mathbf{H}$}

Galdston, Iago, I09, I09n25I

Gallavardin, Louis Bénédict, I32, I32n29

Galpin, Perrin Comstock, 68

Gay, Edwin Francis, 80

Gayet, René, 83, 83ni68-169, 86, 86ni8o, 288, 288ni7, 292, 292-293n3I

Genil-Perrin, Georges, I04n 238

Gibbs, Willard, 7InI37 
Gley, Emile Marcel Eugène, 65, 65nI 20, 99n226, I24, I24-I25nI7, 255

Goldstein, Kurt, 94, I69n4

Goltz, Friedrich, 39n63, I69, I69n3, I70, I73

Grafe, Erich, 302, $302 n 60$

Grant, Banting Frederick, $33 n_{4} 6$

Gray, Horace, 243-244n20-22, 245n24,246, 246n28

Gregg, Alan, 66

Gruber, Charles Michael, I36, I36-I37n4I-43, I38, I38n47

Guillaumie, Maylis, Anne-Cécile, 292, 292-293n3I

Haldane, John Burdon Sanderson, $276 \mathrm{n}_{3} \mathrm{I}$

Haldane, John Scott, 2I, 2I-22n23-24, 7I, 72-74, 72nI38-39, 73nI40-I 42, 76, 238 , 238-239ni4, 309, 309-3IOnI

Hall, Granville Stanley, 59n I07, I65n24

Hallion, Louis, 83ni68, 288ni7

Haour, Jean, I32, I32n 29

Hargis, Estes Henry, 3I 4, 3I 4n8

Hartman, Frank Alexander, 322, 322n27-28

Harvey, Williams Cushing, 38, 38n6r, 79, 265n8

Head, Henry, I85, I85n30, I86, 20I-203, 205

Hédon, Emmanuel, 254, 254n 47

Henderson, Lawrence Joseph, 65, 7I-74, 7InI37, 73nI43, 238, 238-239ni3-I4, 249n36, 276n30, 3I I, 3IIn3

Henry Pickering Bowditch, 27

Heredia, Marcelle de, 63 ni 6

Hess, Walter Rudolf, 69 n 135

Hewson, William, 243, 243n I8

Heymans, Corneille Jean-François, 255, 255-256n5I

Hofmeister, Franz, 7 I

Holmes, Gordon Morgan, $\mathrm{I}_{5} \mathrm{n}_{3} \mathrm{O}$

Holroyd-Reece, John, 84-85, 85nI75

Hoover, Herbert, 68nI28, I07

Hoskins, Roy Graham, 36n57, I24n I6, I56nI I

Houssay, Bernardo Alberto, 286, 286-287nI2, 295n39
Howard, Harvey James, 265, 265n8, 266n9-I0, 322n28

Hunt, Reid, I37, I37n44

Hurst, Arthur Frederick, 53n94, I35, I35n37

Hyde, James Hazen, 67, 67nI24, 67n 26

$$
\mathbf{I}-\mathbf{K}
$$

Ingraham, Franc Douglas, I75, I75n 4

Isenschmid, Georg Robert, I76, I76nI5

Izquierdo, Joaquín, I33n30, I33n32

Jackson, John Hughlings, I70n6, I85, I85n30, 305n64

James Rowland Angell, I92, I92-19375, I96, I96nI I

James, William, 26-27, 26n3o, 27n4, 45-49, 45n77-78, 46n78-8o, 47n8o-8I, 48n8I$83,49,50 n 85,62,62$ nII3-II 4, 99, I02n232, I2I, I In 7 , I 32 n28, I34n34, I 43 n58, I46, I48n4, I59n15, I6oni8, I62, I62n2I, I65n24, I72n9, I85n30, I87, I89-I9I, I9oni, I9In3, I92n5, I93-194, I93n5-7, I94, I96, I98, 200-208, 200ni7, 200nI9, 205n23, 223n2I, 247n3o, 265n8, 3I I9ni 8

James-Cannon, Cornelia, 62, 62nII3-II5, 70, IOI-IO2n232

Janet, Pierre, 50 n 86,56 n 98

Johansson, John Edvard, 3I 8 , 3I8nI7

Jokichi, Takamine, 34

Josse, Jean, $3^{\text {oon }} 53$

Karplus, Johann Paul, i76, I76ni6

Kastle, Joseph Hoeing, 303, 303n63, 304

Kaufmann, Maurice, I49, I49n5

Kirilzev, B.,, I85, I85n26

Koch, Robert, 243ni9, 298n 47

Kovarsky, Véra, I05 243

Kreidl, Alois, I76, I76ni6

Krogh, Schack August Steenberg, 276, 276-277n33, 277n34

Kruse, Harry, I09, I09n252

Krzywanek, Friedrich Karl Wilhelm, 315n I 


$$
\mathbf{L}-\mathbf{M}
$$

Landis, Carney, I43, I $43^{-1} 44 n_{5} 8$

Lange, Carl Georg, 45n77, 46, 46n79-80, 47n80-8I, 99, 99n223, I43n58, I59nI5, I87, I89-I9I, I9on2, I9In3, I93-I94, I96, I98, 200, 204-208

Langley, John Newport, I39-I 40n5o, 253n 45

Lapicque, Louis, 63-66, 63-64nII6, I39, I39n49, 233

Laugier, Henri, 66

Lelle, Fernand, 84, 84nI73, 86, 86ni7 8

Leo Lira, Rosario, J. De, 314-315nIo

Lévy-Bruhl, Lucien, 57nio3, 65, 65ni 8

Lewis, Juan Treharne, I6onI8, 274n23, 282n2, 286-287, 286-ni2, 287ni2-I3

Lieb, Clarence William, 268nI5

Linton, James, 3I9n I8

Linton, Robert R., 319nI8

Loder, M. M.*, $322 \mathrm{n} 28$

Loevenhart, Arthur Salomon, 303, 303-304n63

Lundin, H.*, I38n45, I38n48, 282nI, 346n20

Lunt, Lawrence K., 243n20-2I, 244n22, 246, 246n28

Lusk, Graham, 302-303, 302n57-58, 303n62

Macleod, John James Richard, 33, 33-34n46, 286, 286n9-Io, 293

Magath, Thomas Byrd, I50, I50n9

Magendie, François, 22n 25

Malthus, Thomas, 92

Mann, Frank Charles, I50, I50n9, 3I 4, 3I4n8

Marañon, Gregorio, 54, 54n95, 68 I99, I99ni6, 220, 220nI5-I6

Marchand, Henri, 255, 255n 49

Marchioni, Raoul, I34, I34n35

Markwalder, Joseph, 249, 249n36

Marmot, Michael, I09, I Ion258

\section{Marx, Karl, 92}

Mathison, Gordon Clunes Mckay, 3I8, 3I8n I5

Max Christian, Wegner, 84
Mayer, André, 65, 65nil7-I I8, 267. 267ni2, 275, 275n27

McCordock, Howard Anderson, $322 \mathrm{n} 28$

McCormick, Norman Arnold, 286

McDougall, William, 49, 49-50n85, 56n98, I48, I48n4, I59nI5, 202n20, 208

McEwen, Bruce, I09, I09n257

McKeen Cattel, James, 247n30, 252

McKeown, Thomas, I6

Meltzer, Samuel James, 27, 27-28n34, 343

Mendenhall, Walter Leslie, I 40n52, I 4 In 53 , I 42n $54,245_{25}$

Menkin, Valy, I34, I34n36, I78ni9, I79n20-2I, I8on23, 34In8, 342n9-Io, 343ni I, 345 nI5-I6, 348n22-23

Meyerson, Ignace, 66

Meynert, Theodor Hermann, 48, 48n82, $201 n$ I9

Minkowski, Oskar, 292, $292 n 30$

Molinelli, Ernesto A.", 286, 286-287nI2

Morat, Jean-Pierre, I 49, I 49 n

Morrison, Francis, I84, I $84 \mathrm{n} 28$

Moser, Albert, 28, 28n35-36

Mosso, Angelo, 50, 50n87-88, I58ni3, 210, 210n3, 3I 4 nIo

\section{$\mathbf{N}-\mathbf{Q}$}

Negrin, Juan, 68, 68ni30, 293n32

Newton, Harlan Fay, I 40n5I, I78nig, I79n20-2I, I8on22, 34In8, 342n9-Io, 343ni I, 345 nI5-I6, 348n22-23

Noble, Edward Clark, 286n9-I0, 32In26

Nolf, Pierre, 244, 244n 23

Norton, William Warder, 62nII5, 70nI36, 77-81, 77nI48, 77nI5I, 77nI53, 78ni56, 83-88, 83ni7I-I72, 86ni76, 88ni9i, I03n236, 2IIn5

Nouchy", I34n 35

O'Brien, K.", 286n9-IO

Oliver, George, $34,34 \mathrm{n} 48$

Orbeli, Leon Abgarovich, I38, I38n 46

Pack, George T.*, 266, 266n I I 
Panella, A.*, I36, I36n39

Papez, James, 49, 49n84

Pareto, Wilfredo, 7 InI 37

Paul Trendelenburg, 32 In 26

Pavlov, Ivan Petrovitch, 30, 30-3In4I, I38n46, I82n23, 222nI9, 223, 255n5o

Peabody, Francis Weld, I98nI5

Penfield, Wilder Graves, I73, I73n Io

Pereira, J. R.", I95n8

Perry, Ralph Barton, 45, 45-46n78, I93, I93n6, I96, I96ni2, 202n20

Peter, Sterling, I09, I09n256, 240nI5

Petit-Dutaillis, Charles, 67, 67nI 27

Piéron, Henri, 55-56, 55n97, 56n98-100, 209, 209n I

Pilcher, J. Douglas*, 25I, $25 \mathrm{In} 40$

Poole, Leopold Thomas., 3 I 4 n7

Popielski, Léon, 3 I

Porter, William, $35^{-} 36,35^{n} 53,254,254^{n} 4^{8}$

Pratt, Frederick Haven, 254, 254n 48

Priestley, John Gillies, 309, 309-310n I

Querido, Arie, I95n9, 323n3o, 324n3I, 326n35-36, 328n38, 329n4I, 330n 43

Quinquaud, Alfred, 65nI 20, 99n226, I24, I24nI7, I49, I49n6

$$
\mathrm{R}-\mathrm{S}
$$

Redfield, Alfred Clarence, I37n44, I43n56

Richards, Alfred Newton, I40, I40n5 I

Richet, Charles, 40, 40n64, 65, 65nI 19, 75, 75nI44, IOI-I03, IOIn229-23I, IO2n233,

I05, II0, I63n22, 237, 237nit, 239ni5, 336, 336n6

Robert, M. Moore*, 3I9ni8, 34In8

Robertson, Oswald Hope, $257 \mathrm{n}_{5} 2$

Rogoff, Julius Moses, 99n236, I24-I25, I24nI6, I25nI8, I40n50

Roosevelt, Theodore, 98, 107, 107n247, 266nII

Rosenblueth, Arturo, 108n248

Rothmann, Hans, I69-I70, I69n4
Sandiford, Irene, I42-I $43 \mathrm{n} 55,300,327,327 \mathrm{n} 27$

Sandiford, Kathleen, 300n $53^{-} 54$

Sanger, Margaret, IOI-I02n 232

Satake, Yasutaro, I 25nI8, 32 In 25

Schäfer, Edward, 34, 34n48, 34n 5 I, 32 In 24

Scheunert, Carl Arthur, $315 \mathrm{nI}$ I

Schlesinger, Arthur Meier Jr., I07

Schmidt, Carl Ernst Heinrich, 265, 265n7

Segal, Harry Louis, I44, I44n6o

Seitz, Wilhelm, 299, 299n50

Selye, Hans, I3-I9, I3-I4n4, I4n5, I8nI6, 23, 8I, 85-90, 94, 96-97, 240

Sharpey-Schäfer, Edward Albert, 32 In 24

Sherrington, Charles Scott, 39, 39n63, I72-I73, I72n9-Io, I75nI4, I9I-I93, I92n4, I93n $5-7$

Shohl, Alfred Theodore, 37n58, 52n92, I29n23

Sidney Ringer, I30n24, I49n8, I95nIo, 283n3

Sigmund, Freud, $5^{8}$

Simondon, Gilbert, 93n206

Skelton, Harold, 274, 274n 25

Sollman, Torald Herman, 25I, 25In40

Sorokin, Pitirim, 80-8I

Spadolini, Igino, 343, 343n I 2

Spencer, Herbert, 49, 99, I58ni3, I7on6

Starling, Ernest Henry, 30-34, 30n38-40, 32n 42-44, 33n 45, 34n 47, 37, 39, 4I, 72, $74,78,79,80,249,249 \mathrm{n} 36,255,255^{n} 50,256 \mathrm{n} 5 \mathrm{I}, 275^{\mathrm{n}} 29,275,32 \mathrm{In} 24$

Starobinski, Jean, 96-97, 96n216, 97n217-218

Stephens, John Gower, 314n7, 315nI2

Stewart, George Neil, 99n226, I24-I 25, I 24ni6, I25ni 8, I40n5o

Stratton, George Malcolm, 56 n98, I59, I59ni5-16

Straub, Hermann, 297, 297n 43

Straus, Robert, 6I, 6InIII

Strouse, Solomon, I 44 n6o

Stübel, Hans, 299n52 
Sturgis, Cyrus Cressey, I98-I99nI5

Subbarow, Yellagaprada, 3II, 3 IIn 5

Sugawara, Tadashi, I25nI8

Sundberg, Carl Gustaf, 287-288ni6

$$
\mathbf{T}-\mathbf{Z}
$$

Taussig, Frank William, 80

Terroine, Émile-Florent, 303, 303n6I

Thorndike, Edward Lee, I72n9

Tichmeneff, N.*, 299, 299n5I

Tilney, Frederick, I84, I84n28

Tompkins, Edna M.*, I98-I99nI5

Toulouse, Edouard, 66, 66nI22-I23, I02-I06, I02n234, I03n235, I03n237, I04n23824I, I05n242-243, I06, IIO

Tournade, André, 245, 245-246n26, 255, 255n 49

Trevan, John William, 25In4I

Vaschide, Nicolas, I32, I32n28

Vernon, Bock Arlie, $257 \mathrm{n}_{52}$

Viale, Gaetano, 3I4, 3I4-3I5nIo

Vincent, Thomas Swale, 32I, 32 In 24

Virchow, Rudolf, 243nI9, 298

Vosburgh, Charles H.*, I40, I40n5I

Walker, T. T.*, I38n45, I38n48, 28Ini, 346n20

Walpole, Horace, 28

Washburn, Arthur Lawrence, 268, 268ni6, 269ni6-I9, 270, 27In2I

Watanabe, Masanosuke, I25nI 8

Watson, John Broadus, 96, 222, 222nI9, 223n2I

Wearn, Joseph Treloar, I98-I99n I5

Weinberg, Michel, 292n3I

Wells, Frederic Lyman, I7, I97nI3, I98

Wettendorff, Hugo, $275^{\mathrm{n} 2} 2$

Wiener, Norbert, Io 8 n 248
Wilkinson, Richard, I09, I Ion 258

Wilson George Smillie, I3In26

Wilson, Alexander Kinnier, I84, I84n27

Woodworth, Robert Sessions, 56n98, I72-I73, I72-I73n9

Woodyatt, Rollin Turner, 5I, 5In89, 52-53, 52n90-93, 6o, I5InIo, 2I9, 2I9nI3, $220 n_{4} 4$

Wright, W. S.", 37n58, 52n92, I29n23, 296nio

Wundt, Wilhelm, 99, I59nI2, I59ni5, I65n24

Zunz, Edgar, 294n37, 380 


\section{Parutions}

\section{Bibliothèque d'histoire de la médecine et de la santé}

Le mouvement peut-il guérir? Les usages médicaux de la gymnastique au Ig siècle G. Quin, 352 p., 2019

Visages. Histoires, représentations, créations Édité par L. Guido, M. Hennard Dutheil de la Rochère, B. Maire, F. Panese et N. Roelens, avec un prélude de J.-J. Courtine, XXII et 4 Io p., 2017

Les mots du corps. Expérience de la maladie dans des lettres de patients à un médecin du I Ie $^{\text {siècle: }}$ Samuel Auguste Tisso

S. Pilloud, avec une préface par O. Faure, XVIII et 374 p., 2013

Le compas G le bistouri. Architectures de la médecine et du tourisme curatif. L'exemple vaudois (I760-1940)

D. Lüthi, avec une préface par A.-M. Châtelet, XXII et 548 p., 2012

Body, Disease and Treatment in a Changing World. Latin Texts and Contexts in Ancient and Medieval Medicine

Édité par D. R. Langslow et B. Maire, XVIII et 404 p., 2010

Anatomie d'une institution médicale. La Faculté de médecine de Genève (I876-I920) Ph. Rieder, XII et 392 p., 2009

Le style des gestes. Corporéité et kinésie dans le récit littéraire

G. Bolens, avec une préface par A. Berthoz, XIV et I56 p., 2008

La médecine dans l'Antiquité grecque et romaine

H. King et V. Dasen, XII et Izo p., 2008

L'Ombre de César. Les chirurgiens et la construction du système hospitalier vaudois (I840-I960)

P.-Y. Donzé, avec une préface par J. V. Pickstone, XX et 369 p., 2007

Medicina, soror philosophiae. Regards sur la littérature et les textes médicaux antiques (1975-2005)

Ph. Mudry. Édité par B. Maire, avec une préface par J. Pigeaud, XXIV et 545 p., 2006

La formation des infirmiers en psychiatrie. Histoire de lécole cantonale vaudoise d'infirmières et d'infirmiers en psychiatrie I96I-Ig96 (ECVIP)

J. Pedroletti, VIII et 23I p., 2004
Bâtir, gérer, soigner. Histoire des établissements hospitaliers de Suisse romande P.-Y. Donzé, 388 p., 2003

Visions du rềve

Édité par V. Barras, J. Gasser, Ph. Junod, Ph. Kaenel et O. Mottaz, 288 p., 2002

Rejetées, rebelles, mal adaptées. Débat sur l'eugénisme. Pratique de la stérilisation non volontaire en Suisse romande au $20^{e}$ siècle

G. Heller, G. Jeanmonod et J. Gasser, 482 p., 2002

Médecins voyageurs. Théorie et pratique du voyage médical au début du Ig siècle D. Vaj, 348 p., 2002

La médecine à Genèrve jusqu'à la fin du I $8^{e}$ siècle

L. Gautier, réédition, avec une préface par J. Starobinski

et une introduction par V. Barras et M. Louis-Courvoisier, 746 p., 200

L'Avènement de la médecine clinique moderne en Europe I750-I8I5.

Politique, institutions et savoirs

O. Keel, 544 p., 2001

Soigner et consoler. La vie quotidienne dans un bôpital à la fin de l'Ancien Régime (Genève I750-I820)

M. Louis-Courvoisier, 336 p., 2000

\section{Sources en perspectives}

C. G. Jung. Comptes rendus critiques de la psychologie francophone. Introduction, traduction et notes par F. Serina, 204 p., 2020

Maqari, Le Recueil des vertus de la médecine ancienne. La médecine gréco-arabe en Mauritanie

Édition, introduction et notes par B. Graz, V. Barras, A.-M. Moulin et C. Fortier, 36r p., 20I7

L'Usage du sexe. Lettres au Dr Tissot, auteur de «L'Onanisme» (I760) Édition, introduction et notes par P. Singy, X et 278 p., 2014

Samuel Auguste Tissot, De la Médecine civile ou de la Police de la Médecine Édition par M. Nicoli, introduction par D. Tosato-Rigo et M. Nicoli,

LXX et I6o p., 2009

Gabriel Tarde, «Sur le sommeil. Ou plutôt sur les rềves». Et autres textes inédits Édition, introduction et notes par J. Carroy et L. Salmon, VIII et 228 p., 2009

Se soigner par les plantes. Les «Remèdes» de Gargile Martial

Edition, traduction et notes par B. Maire avec un avant-propos par 
K. Hostettmann et un dossier iconographique par M. Fuchs, XXXVI et I36 p., 2007

\section{Hors-série}

La Société Suisse d'Orthopédie et de Traumatologie au tournant du nouveau millénaire M. Kaba, 200 p., 2020

L'Hôpital Riviera-Chablais. Enjeux et défis d'une collaboration intercantonale M. Kaba et A. Cochand, 308 p., 2019

Une histoire de l'orthopédie. L'Hôpital orthopédique de la Suisse romande dans le contexte international ( $I 8^{e}-2 I^{e}$ siècle)

M. Kaba, 284 p., 2018

La Maternité de Lausanne. Un patrimoine pour la vie

Édité par R. Fuschetto, II 2 p., 2017

75 ans de pédopsychiatrie à Lausanne. Du Bercail au Centre psychothérapeutique

T. Garibian, avec un avant-propos par J.-M. Henny, une préface par

F. Ansermet et une postface par O. Halfon et Ph. Nendaz,

XVIII et I30 p., 2015

Anatomies. De Vésale au virtuel

Édité par V. Barras, I04 p., 20I4, coédition avec T. Schaap éditeur

Migration et système de santé vaudois, du I $9^{e}$ siècle à nos jours

M. Garibian et V. Barras, XVI et 72 p., 20I2

L'Hôpital de l'enfance de Lausanne. Histoire d'une institution pionnière de la pédiatrie suisse

M. Tavera et V. Barras, XII et I88 p., 20I I

\section{Ebooks et bases de données en libre accès}

Walter B. Cannon Conférences sur les émotions et l'boméostasie, Paris, I93o Édition, introduction et notes par M. Arminjon, 400 p., 2020 (également disponible en version papier) Collection Sources en perspectives

Le mouvement peut-il guérir? Les usages médicaux de la gymnastique au Ige siècle G. Quin, 352 p., 2019 (également disponible en version papier) Collection Bibliothèque d'histoire de la médecine et de la santé

L'Imprimé scientifique. Enjeux matériels et intellectuels

Édité par M. Nicoli, I86 p., 2014

Collection Bibliothèque d'histoire de la médecine et de la santé

Archives du corps et de la santé au I ${ }^{e}$ siècle: les lettres de patients au $D^{r}$ Samuel Auguste Tissot (I728-I797)

S. Pilloud, M. Louis-Courvoisier et V. Barras, 2013

Base de données en ligne: www.chuv.ch/iuhmsp/ihm_bhms

Documenter l'bistoire de la santé et de la maladie au siècle des Lumières: les consultations épistolaires adressées au $D^{r}$ Samuel Auguste Tissot (I728-I797)

S. Pilloud, 50 p., 2013

Collection Sources en perspective

Maladies en lettres, $I 7^{e}-2 I^{e}$ siècles

Édité par V. Barras et M. Dinges, 266 p., 2013

Collection Bibliothèque d'histoire de la médecine et de la santé

\section{Cartes}

La Maternité de Lausanne. Vues historiques

Sept cartes A6 (I05 × I48 mm), cartes_BHMS 2, 2017

Fleurs animées $\sigma$ Flore médicale

Douze cartes A5 $\left(\mathrm{I}_{5} \times 2 \mathrm{I} \mathrm{cm}\right)$, cartes_BHMS I, 2012 


\section{À paraître}

Histoire de la médecine

J. Starobinski, avec une préface de V. Barras

Collection Hors-série en coédition avec les Éditions Héros-Limite

I 28 p., 25 ill., 2020

Pierre Decker, médecin et collectionneur

G. Monney, C. Noverraz et V. Barras

Collection Hors-série

240 p., 200 ill., 2020

Le laboratoire des troubles mentaux: expérimentation animale et recherche thérapeutique

à l'ère de la psychopharmacologie

L. Gerber

Collection Bibliothèque d'histoire de la médecine et de la santé

300 p., 202I

Galien, Tempéraments

Introduction, traduction et notes par V. Barras et T. Birchler

Collection Sources en perspective

200 p., 2021 
Walter B. Cannon (I87I-I945) est l'une des figures majeures de la physiologie moderne. Ces deux séries de conférences inédites qu'il délivre en français lors d'un séjour à Paris au début de l'année 1930, représentent un moment charnière dans son parcours intellectuel. Elles lui serviront de base à la rédaction de son ouvrage phare La sagesse du corps. Capitales pour l'histoire de la physiologie et de la médecine du $20^{\mathrm{e}}$ siècle, ces sources éclairent l'émergence du concept de stress, qui, avec les problématiques médicales et sociales qu'il soulève, trouve un écho particulier dans les sciences biomédicales contemporaines.

Mathieu Arminjon est adjoint scientifique à la Haute école de santé Vaud (HESAV), Haute école spécialisée de Suisse occidentale (HES-SO), et médecine (CHUV-UNIL).

Philosophe et historien des sciences, ses recherches

portent sur les sciences biomédicales, le soin et les inégalités de santé. 